\title{
Interventions for treating proximal humeral fractures in adults (Review)
}

\author{
Handoll HHG, Brorson S
}

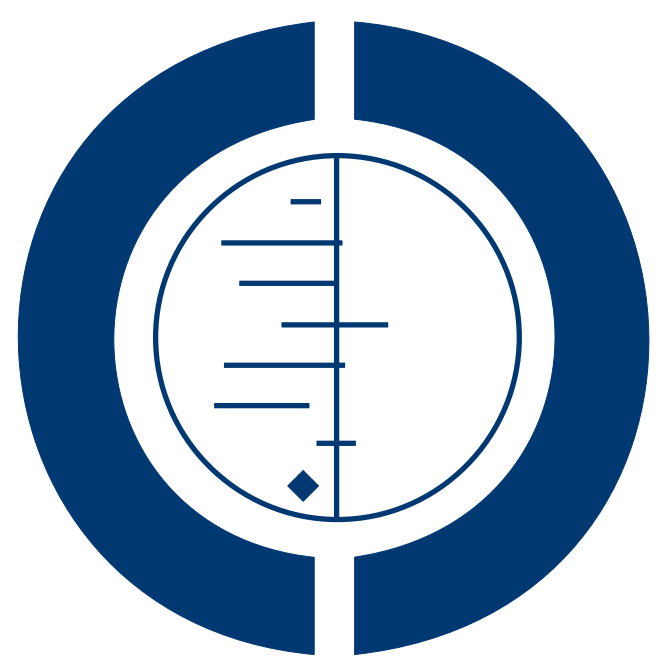

THE COCHRANE COLLABORATION $^{\circledR}$

This is a reprint of a Cochrane review, prepared and maintained by The Cochrane Collaboration and published in The Cochrane Library 2015, Issue 11

http://www.thecochranelibrary.com

\section{WILEY}

Interventions for treating proximal humeral fractures in adults (Review)

Copyright $\odot 2015$ The Cochrane Collaboration. Published by John Wiley \& Sons, Ltd. 
TABLE OF CONTENTS

HEADER . . . . . . . . . . . . . . . . . . . . . . . . . . . . . . . . . . . . . . .

ABSTRACT . . . . . . . . . . . . . . . . . . . . . . . . . . . . . . . . . . . . . . . . . . 1

PLAIN LANGUAGE SUMMARY . . . . . . . . . . . . . . . . . . . . . . . . . . . . . . . . . . . . . . . 2

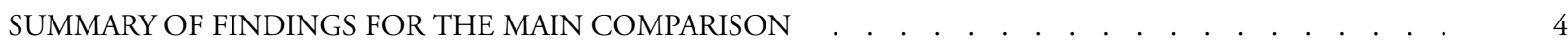

BACKGROUND . . . . . . . . . . . . . . . . . . . . . . . . . . . . . . . . . . . . . . . . . . . 9

OBJECTIVES . . . . . . . . . . . . . . . . . . . . . . . . . . . . . . . . . . . . . . . . . . . . . . .

METHODS . . . . . . . . . . . . . . . . . . . . . . . . . . . . . . . . . . . . . . . . .

RESULTS . . . . . . . . . . . . . . . . . . . . . . . . . . . . . . . . . . . . . . . . . . . .

Figure 1. . . . . . . . . . . . . . . . . . . . . . . . . . . . . . . . . . . . . . 15

Figure 2. . . . . . . . . . . . . . . . . . . . . . . . . . . . . . . . . . 20

Figure 3. . . . . . . . . . . . . . . . . . . . . . . . . . . . . . . . . . . . . . . . .

Figure 4. . . . . . . . . . . . . . . . . . . . . . . . . . . . . . . . . . . . . . 425

ADDITIONAL SUMMARY OF FINDINGS . . . . . . . . . . . . . . . . . . . . . . . . . . . . .

DISCUSSION . . . . . . . . . . . . . . . . . . . . . . . . . . . . . . . . . . . . . . . . . . . . . .

AUTHORS' CONCLUSIONS . . . . . . . . . . . . . . . . . . . . . . . . . . . . . . . . . . . . . .

ACKNOWLEDGEMENTS . . . . . . . . . . . . . . . . . . . . . . . . . . . . . . . . . 44

REFERENCES . . . . . . . . . . . . . . . . . . . . . . . . . . . . . . . . . . . . . . 45

CHARACTERISTICS OF STUDIES . . . . . . . . . . . . . . . . . . . . . . . . . . . . . . . . 56

DATA AND ANALYSES . . . . . . . . . . . . . . . . . . . . . . . . . . . . . . . . . . . . . . . . . . . . . . . . 140

Analysis 1.1. Comparison 1 Early mobilisation (within or up to 1 week) versus immobilisation for 3 or 4 weeks, Outcome

1 Shoulder disability: Croft Shoulder Disability Score. . . . . . . . . . . . . . . . . . . . . 155

Analysis 1.2. Comparison 1 Early mobilisation (within or up to 1 week) versus immobilisation for 3 or 4 weeks, Outcome

2 Croft shoulder disability score: individual problems at 2 years. . . . . . . . . . . . . . . . . . . . . . . 156

Analysis 1.3. Comparison 1 Early mobilisation (within or up to 1 week) versus immobilisation for 3 or 4 weeks, Outcome

3 Number of treatment sessions (until independent function achieved). . . . . . . . . . . . . . . . . . . .

Analysis 1.4. Comparison 1 Early mobilisation (within or up to 1 week) versus immobilisation for 3 or 4 weeks, Outcome

4 SF-36 scores: pain \& physical dimensions. . . . . . . . . . . . . . . . . . . . . . . . . . .

Analysis 1.5. Comparison 1 Early mobilisation (within or up to 1 week) versus immobilisation for 3 or 4 weeks, Outcome

5 Quality of life assessment: EuroQol 5D (0: dead to 1: best health). . . . . . . . . . . . . . . . . . 158

Analysis 1.6. Comparison 1 Early mobilisation (within or up to 1 week) versus immobilisation for 3 or 4 weeks, Outcome

6 Adverse events. . . . . . . . . . . . . . . . . . . . . . . . . . . . . . . . . . . . . . . . . . . . . 15

Analysis 1.7. Comparison 1 Early mobilisation (within or up to 1 week) versus immobilisation for 3 or 4 weeks, Outcome 7 Mortality. . . . . . . . . . . . . . . . . . . . . . . . . . . . . . . . . . . . . . . 161

Analysis 1.8. Comparison 1 Early mobilisation (within or up to 1 week) versus immobilisation for 3 or 4 weeks, Outcome 8 Constant shoulder score (ratio of affected/unaffected arm).

Analysis 1.9. Comparison 1 Early mobilisation (within or up to 1 week) versus immobilisation for 3 or 4 weeks, Outcome

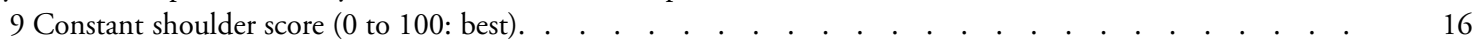

Analysis 1.10. Comparison 1 Early mobilisation (within or up to 1 week) versus immobilisation for 3 or 4 weeks, Outcome 10 Pain VAS (0 to 100: worst pain). . . . . . . . . . . . . . . . . . . . . . . . . . . 163

Analysis 1.11. Comparison 1 Early mobilisation (within or up to 1 week) versus immobilisation for 3 or 4 weeks, Outcome 11 Changes in pain intensity $(\mathrm{mm})$ from baseline: $100 \mathrm{~mm}$ visual analogue scale (positive change = less pain). . 164

Analysis 1.12. Comparison 1 Early mobilisation (within or up to 1 week) versus immobilisation for 3 or 4 weeks, Outcome 12 Range of motion at 6 months (degrees): difference between two shoulders. . . . . . . . . . . . . . . . . 165

Analysis 1.13. Comparison 1 Early mobilisation (within or up to 1 week) versus immobilisation for 3 or 4 weeks, Outcome 13 Patient dissatisfied with treatment. . . . . . . . . . . . . . . . . . . . . . . . . . . . . . . 16

Analysis 1.14. Comparison 1 Early mobilisation (within or up to 1 week) versus immobilisation for 3 or 4 weeks, Outcome 14 Patient satisfaction (0 to 10: higher scores - greater satisfaction). . . . . . . . . . . . . . . . . . . . . . 166

Analysis 2.1. Comparison 2 Gilchrist bandage versus 'Classic' Desault bandage, Outcome 1 Problems with bandages. 166 Analysis 2.2. Comparison 2 Gilchrist bandage versus 'Classic' Desault bandage, Outcome 2 Fracture displacement by 3 weeks.

Interventions for treating proximal humeral fractures in adults (Review)

Copyright $\odot 2015$ The Cochrane Collaboration. Published by John Wiley \& Sons, Ltd. 
Analysis 2.3. Comparison 2 Gilchrist bandage versus 'Classic' Desault bandage, Outcome 3 Poor or bad rating by patient at fracture consolidation. . . . . . . . . . . . . . . . . . . . . . . . . . . . . . . . . $\quad . \quad 167$

Analysis 3.1. Comparison 3 Instructed self-exercise versus conventional physiotherapy, Outcome 1 Pain at one year (scale 0 to 8: maximum pain). . . . . . . . . . . . . . . . . . . . . . . . . . . . . . . . . . . . . . . . .

Analysis 3.2. Comparison 3 Instructed self-exercise versus conventional physiotherapy, Outcome 2 Severe or moderate pain at 3 months. . . . . . . . . . . . . . . . . . . . . . . . . . . . . . . 168

Analysis 3.3. Comparison 3 Instructed self-exercise versus conventional physiotherapy, Outcome 3 Requested change of therapy. . . . . . . . . . . . . . . . . . . . . . . . . . . . . . . . . . . . .

Analysis 3.4. Comparison 3 Instructed self-exercise versus conventional physiotherapy, Outcome 4 Adverse events (frozen shoulder: 1 v 2; unexplained prolonged pain: $0 \vee 1)$. . . . . . . . . . . . . . . . . . . . . . . . . .

Analysis 3.5. Comparison 3 Instructed self-exercise versus conventional physiotherapy, Outcome 5 Neer's rating (0 to 100 :

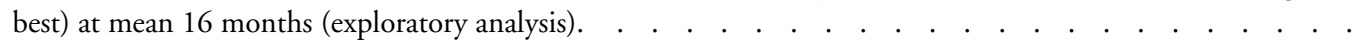

Analysis 3.6. Comparison 3 Instructed self-exercise versus conventional physiotherapy, Outcome 6 Active gleno-humeral elevation (degrees). . . . . . . . . . . . . . . . . . . . . . . . . . . . . . . . . . . . . . . . . . . . . . . .

Analysis 4.1. Comparison 4 Surgical versus non-surgical treatment, Outcome 1 Functional scores at 12 months (higher = better outcome). . . . . . . . . . . . . . . . . . . . . . . . . . . . . . . . . . . . . . . . . .

Analysis 4.2. Comparison 4 Surgical versus non-surgical treatment, Outcome 2 Functional scores at 24 months (higher $=$ better outcome).

Analysis 4.3. Comparison 4 Surgical versus non-surgical treatment, Outcome 3 Oxford Shoulder Score (0 to 48: best outcome).

Analysis 4.4. Comparison 4 Surgical versus non-surgical treatment, Outcome 4 DASH (0 to 100: worst disability). .

Analysis 4.5. Comparison 4 Surgical versus non-surgical treatment, Outcome 5 American Shoulder and Elbow Surgeons score (0 to 24 : best).

Analysis 4.6. Comparison 4 Surgical versus non-surgical treatment, Outcome 6 Simple Shoulder Test (0 to 12: best function).

Analysis 4.7. Comparison 4 Surgical versus non-surgical treatment, Outcome 7 Activities of daily living.

Analysis 4.8. Comparison 4 Surgical versus non-surgical treatment, Outcome 8 Quality of life assessment: EuroQol (0: dead to 1 : best health). .

Analysis 4.9. Comparison 4 Surgical versus non-surgical treatment, Outcome 9 Quality of life assessment (Fjalestad 2010 and 2014 data).

Analysis 4.10. Comparison 4 Surgical versus non-surgical treatment, Outcome 10 Quality of life: SF-12 Physical Component Score (0 to 100: best).

Analysis 4.11. Comparison 4 Surgical versus non-surgical treatment, Outcome 11 Quality of life: SF-12 Mental Component Score $(0$ to 100 : best). . . . . . . . . . . . . . . . . . . . . . . . . . . . . . . . . . . . . . . . $\quad 180$

Analysis 4.12. Comparison 4 Surgical versus non-surgical treatment, Outcome 12 Mortality. . . . . . . . . . . 181

Analysis 4.13. Comparison 4 Surgical versus non-surgical treatment, Outcome 13 Additional surgery (re-operation or secondary surgery). . . . . . . . . . . . . . . . . . . . . . . . . . . . . . . . . . .

Analysis 4.14. Comparison 4 Surgical versus non-surgical treatment, Outcome 14 Adverse events / complications.

Analysis 4.15. Comparison 4 Surgical versus non-surgical treatment, Outcome 15 Dependent in activities of daily living (or dead) at 6 months.

Analysis 4.16. Comparison 4 Surgical versus non-surgical treatment, Outcome 16 Constant scores (overall: 0 to 100: best score).

Analysis 4.17. Comparison 4 Surgical versus non-surgical treatment, Outcome 17 Constant scores (difference between injured and uninjured shoulder): Normal $=0$..

Analysis 4.18. Comparison 4 Surgical versus non-surgical treatment, Outcome 18 Poor or unsatisfactory function at 1 year

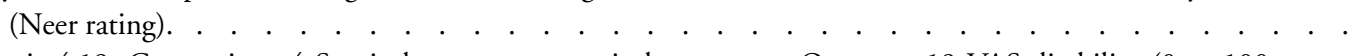

Analysis 4.19. Comparison 4 Surgical versus non-surgical treatment, Outcome 19 VAS disability (0 to 100: no restrictions).

Analysis 4.20. Comparison 4 Surgical versus non-surgical treatment, Outcome 20 Pain: VAS (0 to 100: worst pain). .

Analysis 4.21. Comparison 4 Surgical versus non-surgical treatment, Outcome 21 Constant score at 50 months: overall

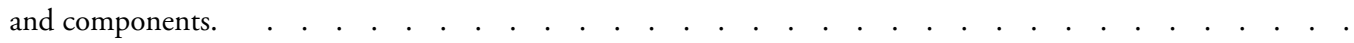

Analysis 4.22. Comparison 4 Surgical versus non-surgical treatment, Outcome 22 Constant (often severe) pain at 6 months.

Interventions for treating proximal humeral fractures in adults (Review)

Copyright $\odot 2015$ The Cochrane Collaboration. Published by John Wiley \& Sons, Ltd. 
Analysis 4.23. Comparison 4 Surgical versus non-surgical treatment, Outcome 23 Failure to recover $75 \%$ muscle power relative to other arm (survivors) at 6 months.

Analysis 4.24. Comparison 4 Surgical versus non-surgical treatment, Outcome 24 Range of movement impairments in survivors at 6 months.

Analysis 4.26. Comparison 4 Surgical versus non-surgical treatment, Outcome 26 Total costs including indirect costs (Euros) at 1 year. . . . . . . . . . . . . . . . . . . . . . .

Analysis 5.1. Comparison 5 Locking plate versus locking intramedullary nail, Outcome 1 American Shoulder and Elbow Surgeons (ASES) score $(0$ to 100 : best). . . . . . . . . . . . . . . . . . . . . . . . . . . . . . . . . . .

Analysis 5.2. Comparison 5 Locking plate versus locking intramedullary nail, Outcome 2 Death, re-operation and adverse events.

Analysis 5.4. Comparison 5 Locking plate versus locking intramedullary nail, Outcome 4 Constant score (0 to 100: best). . . . . . . . . . . . . . . . . . . . . . . . . . . . . . . . . . . .

Analysis 5.5. Comparison 5 Locking plate versus locking intramedullary nail, Outcome 5 Active range of motion (at 3 years).

Analysis 5.7. Comparison 5 Locking plate versus locking intramedullary nail, Outcome 7 Strength of suprapinatus (relative to opposite side) $\%$ - at 3 years. . . . . . . . . . . . . . . . . . . . . . . . . . . . . . . . . . . . . . . . .

Analysis 5.8. Comparison 5 Locking plate versus locking intramedullary nail, Outcome 8 Operation times and blood loss.

Analysis 5.9. Comparison 5 Locking plate versus locking intramedullary nail, Outcome 9 Intra-operative complication.

Analysis 6.1. Comparison 6 Locking plate versus intramedullary nails (Zifko method), Outcome 1 Complications and [slight] malunion. . . . . . . . . . . . . . . . . . . . . . . . . . . . . . . . . . . . . . .

Analysis 6.2. Comparison 6 Locking plate versus intramedullary nails (Zifko method), Outcome 2 Constant score (\% of healthy limb) at mean 2 years. . . . . . . . . . . . . . . . . . . . . . . . . . . . . . . . . .

Analysis 6.3. Comparison 6 Locking plate versus intramedullary nails (Zifko method), Outcome 3 Time to union and time to recover upper limb function (weeks). . . . . . . . . . . . . . . . . . . . . . . . . . . . . . . . . .

Analysis 6.4. Comparison 6 Locking plate versus intramedullary nails (Zifko method), Outcome 4 Operation and fluoroscopic times. . . . . . . . . . . . . . . . . . . . . . . . . . . . . . . . . . . .

Analysis 6.5. Comparison 6 Locking plate versus intramedullary nails (Zifko method), Outcome 5 Length of hospital stay (days). . . . . . . . . . . . . . . . . . . . . . . . . . . . . . . . . . . . . . .

Analysis 7.1. Comparison 7 Replacement (hemiarthroplasty) versus fixation (tension band wiring; plate fixation) (4 part fractures), Outcome 1 DASH score (0 to 100: worst disability).

Analysis 7.2. Comparison 7 Replacement (hemiarthroplasty) versus fixation (tension band wiring; plate fixation) (4 part fractures), Outcome 2 EQ-5D score (0 to 1: best quality of life). $\quad . \quad$. . . . . . . . . . . . . . . . . . . . . . .

Analysis 7.3. Comparison 7 Replacement (hemiarthroplasty) versus fixation (tension band wiring; plate fixation) (4 part fractures), Outcome 3 Re-operation. . . . . . . . . . . . . . . . . . . . . . . . . . .

Analysis 7.4. Comparison 7 Replacement (hemiarthroplasty) versus fixation (tension band wiring; plate fixation) (4 part fractures), Outcome 4 Dead at 2 years. . . . . . . . . . . . . . . . . . . . . . . . . . . . . . .

Analysis 7.5. Comparison 7 Replacement (hemiarthroplasty) versus fixation (tension band wiring; plate fixation) (4 part fractures), Outcome 5 Implant removal at 1 year.

Analysis 7.6. Comparison 7 Replacement (hemiarthroplasty) versus fixation (tension band wiring; plate fixation) (4 part fractures), Outcome 6 Constant score (0 to 100: best score). . . . . . . . . . . . . . . . . . . . . . . . . . .

Analysis 7.7. Comparison 7 Replacement (hemiarthroplasty) versus fixation (tension band wiring; plate fixation) (4 part fractures), Outcome 7 Pain VAS (0 to 100: worst pain) at 24 months..$\quad$. . . . . . . . . . . . . . . . . . .

Analysis 7.8. Comparison 7 Replacement (hemiarthroplasty) versus fixation (tension band wiring; plate fixation) (4 part fractures), Outcome 8 Pain at 1 year. . . . . . . . . . . . . . . . . . . . . . . . . . . . . .

Analysis 7.9. Comparison 7 Replacement (hemiarthroplasty) versus fixation (tension band wiring; plate fixation) (4 part fractures), Outcome 9 Range of motion at 24 months. . . . . . . . . . . . . . . . . . . . . . . . . . . . . . .

Analysis 8.1. Comparison 8 Reverse shoulder arthroplasty (RSA) versus hemiarthroplasty (HA), Outcome 1 Shoulder function scores at 24 to 49 months. $.7 . \quad$. . . . . . . . . . . . . . . . . . . . . . . . . . . . . . . . . . .

Analysis 8.2. Comparison 8 Reverse shoulder arthroplasty (RSA) versus hemiarthroplasty (HA), Outcome 2 Reoperation. . . . . . . . . . . . . . . . . . . . . . . . . . . . . . . . . . . . . . . . . . .

Analysis 8.3. Comparison 8 Reverse shoulder arthroplasty (RSA) versus hemiarthroplasty (HA), Outcome 3 Death. . 
Analysis 8.4. Comparison 8 Reverse shoulder arthroplasty (RSA) versus hemiarthroplasty (HA), Outcome 4 Composite (objective and subjective) shoulder function scores at 24 to 49 months. . . . . . . . . . . . . . . . . . . . . .

Analysis 8.5. Comparison 8 Reverse shoulder arthroplasty (RSA) versus hemiarthroplasty (HA), Outcome 5 Constant score at 24 to 49 months: overall and components.

Analysis 8.6. Comparison 8 Reverse shoulder arthroplasty (RSA) versus hemiarthroplasty (HA), Outcome 6 Complications.

Analysis 8.7. Comparison 8 Reverse shoulder arthroplasty (RSA) versus hemiarthroplasty (HA), Outcome 7 Radiological assessment findings. . . . . . . . . . . . . . . . . . . . . . . . . . . . . . . . . .

Analysis 8.8. Comparison 8 Reverse shoulder arthroplasty (RSA) versus hemiarthroplasty (HA), Outcome 8 Range of motion (degrees) at 24 to 49 months.

Analysis 9.1. Comparison 9 Deltoid-split versus deltopectoral approaches for plate fixation, Outcome 1 Re-operation.

Analysis 9.2. Comparison 9 Deltoid-split versus deltopectoral approaches for plate fixation, Outcome 2 Dead at 1 year.

Analysis 9.3. Comparison 9 Deltoid-split versus deltopectoral approaches for plate fixation, Outcome 3 Complications.

Analysis 9.4. Comparison 9 Deltoid-split versus deltopectoral approaches for plate fixation, Outcome 4 Constant score $(0$ to 100: best score)

Analysis 9.5. Comparison 9 Deltoid-split versus deltopectoral approaches for plate fixation, Outcome 5 Pain (VAS 0 to 10 : intolerable pain). . . . . . . . . . . . . . . . . . . . . . . . . . . . . . . . . .

Analysis 9.6. Comparison 9 Deltoid-split versus deltopectoral approaches for plate fixation, Outcome 6 Operation and fluoroscopic times. . . . . . . . . . . . . . . . . . . . . . . . . . . . . . . . . . . . . .

Analysis 9.7. Comparison 9 Deltoid-split versus deltopectoral approaches for plate fixation, Outcome 7 Length of hospital stay (days).

Analysis 10.1. Comparison 10 Polyaxial versus monoaxial screw insertion in plate fixation, Outcome 1 DASH score at 12 months (0 to 100 : greatest disability).

Analysis 10.2. Comparison 10 Polyaxial versus monoaxial screw insertion in plate fixation, Outcome 2 Simple shoulder test (0 to 12 : best outcome).

Analysis 10.3. Comparison 10 Polyaxial versus monoaxial screw insertion in plate fixation, Outcome 3 Re-operation.

Analysis 10.4. Comparison 10 Polyaxial versus monoaxial screw insertion in plate fixation, Outcome 4 Dead at 1 year.

Analysis 10.5. Comparison 10 Polyaxial versus monoaxial screw insertion in plate fixation, Outcome 5 Constant score at 12 months (\% of contralateral limb).

Analysis 10.6. Comparison 10 Polyaxial versus monoaxial screw insertion in plate fixation, Outcome 6 Complications (radiological assessment).

Analysis 10.7. Comparison 10 Polyaxial versus monoaxial screw insertion in plate fixation, Outcome 7 Range of motion (degrees) at 12 months.

Analysis 10.8. Comparison 10 Polyaxial versus monoaxial screw insertion in plate fixation, Outcome 8 Operation and fluoroscopic times.

Analysis 11.1. Comparison 11 Medial support screws versus control for locking plate fixation, Outcome 1 Adverse events. . . . . . . . . . . . . . . . . . . . . . . . . . . . . . . . . . . . . . . .

Analysis 11.2. Comparison 11 Medial support screws versus control for locking plate fixation, Outcome 2 Constant score ( 0 to 100 : best) at 2.5 years. . . . . . . . . . . . . . . . . . . . . . . . . . . . . . . . . . . . . .

Analysis 12.1. Comparison 12 MultiLoc Proximal Humeral Nail (MPHN) versus Polarus nail, Outcome 1 Adverse events. . . . . . . . . . . . . . . . . . . . . . . . . . . . . . . . . . . . .

Analysis 12.2. Comparison 12 MultiLoc Proximal Humeral Nail (MPHN) versus Polarus nail, Outcome 2 Constant score (0 to 100: best outcome) at 14 months $(6$ to 22 months). . . . . . . . . . . . . . . . . . . . . . . . . .

Analysis 12.3. Comparison 12 MultiLoc Proximal Humeral Nail (MPHN) versus Polarus nail, Outcome 3 Range of shoulder motion (degrees).

Analysis 12.4. Comparison 12 MultiLoc Proximal Humeral Nail (MPHN) versus Polarus nail, Outcome 4 Lengths of surgery and hospital stay. . . . . . . . . . . . . . . . . . . . . . . . . . . . . . . . . . . . . . .

Analysis 13.1. Comparison 13 Hemiarthoplasty: EPOCA prosthesis versus HAS prosthesis, Outcome 1 Adverse events.

Analysis 13.2. Comparison 13 Hemiarthoplasty: EPOCA prosthesis versus HAS prosthesis, Outcome 2 Radiological assessment findings. . . . . . . . . . . . . . . . . . . . . . . . . . . . . . . . . . . . . . . . . .

Analysis 14.1. Comparison 14 Hemiarthroplasty: tenodesis of long head of biceps (LHB) versus LHB tendon left intact, Outcome 1 Complications and further surgery. . . . . . . . . . . . . . . . . . . . . . . 
Analysis 14.2. Comparison 14 Hemiarthroplasty: tenodesis of long head of biceps (LHB) versus LHB tendon left intact, Outcome 2 Constant score ( 0 to 100 : best function) at 2 years. . . . . . . . . . . . . . . . . . . . . . .

Analysis 14.3. Comparison 14 Hemiarthroplasty: tenodesis of long head of biceps (LHB) versus LHB tendon left intact, Outcome 3 Shoulder pain at 2 year follow-up. $\quad . \quad$. . . . . . . . . . . . . . . . . . . . . . . . . . . .

Analysis 14.4. Comparison 14 Hemiarthroplasty: tenodesis of long head of biceps (LHB) versus LHB tendon left intact,

Outcome 4 Active shoulder elevation (degrees) at 2 years. . . . . . . . . . . . . . . . . . . . . . . .

Analysis 15.1. Comparison 15 Post-operative (percutaneous fixation) immobilisation for 1 week versus 3 weeks, Outcome

1 Neer score $\leq 80$ points (unsatisfactory or failure) at 6 months. . . . . . . . . . . . . . . . . . . . . . . .

Analysis 15.2. Comparison 15 Post-operative (percutaneous fixation) immobilisation for 1 week versus 3 weeks, Outcome

2 Premature removal of Kirschner wires. . . . . . . . . . . . . . . . . . . . . . . . . . . . . . . . . . . . . $\quad . \quad 232$

Analysis 16.1. Comparison 16 Post-operative (hemiarthroplasty) mobilisation: early ( 2 weeks immobilisation) versus late (6 weeks), Outcome 1 Oxford Shoulder Score at 1 year (adjusted: 0 to 100 best). . . . . . . . . . . . . . . . 232

Analysis 16.2. Comparison 16 Post-operative (hemiarthroplasty) mobilisation: early ( 2 weeks immobilisation) versus late (6 weeks), Outcome 2 Constant shoulder score (at 1 year). . . . . . . . . . . . . . . . . . . . . . . .

Analysis 16.3. Comparison 16 Post-operative (hemiarthroplasty) mobilisation: early ( 2 weeks immobilisation) versus late (6 weeks), Outcome 3 Radiological assessment findings. . . . . . . . . . . . . . . . . . . . . . . . 234

Analysis 16.4. Comparison 16 Post-operative (hemiarthroplasty) mobilisation: early ( 2 weeks immobilisation) versus late (6 weeks), Outcome 4 Range of motion at 1 year. . . . . . . . . . . . . . . . . . . . . . . 234

ADDITIONAL TABLES . . . . . . . . . . . . . . . . . . . . . . . . . . . . . . . . . . . . . 235

APPENDICES . . . . . . . . . . . . . . . . . . . . . . . . . . . . . . . . . . . . . . 238

WHAT'S NEW . . . . . . . . . . . . . . . . . . . . . . . . . . . . . . . . . . . . . . 245

HISTORY . . . . . . . . . . . . . . . . . . . . . . . . . . . . . . . . . . . . . . . . 246

CONTRIBUTIONS OF AUTHORS . . . . . . . . . . . . . . . . . . . . . . . . . . . . . . . . . . . . . . . . . . . . . .

DECLARATIONS OF INTEREST . . . . . . . . . . . . . . . . . . . . . . . . . . . . . . . . . . . . .

SOURCES OF SUPPORT . . . . . . . . . . . . . . . . . . . . . . . . . . . . . . . . . . . . . . . . . . . . . . .

DIFFERENCES BETWEEN PROTOCOL AND REVIEW . . . . . . . . . . . . . . . . . . . . . . . . . . . . 249

INDEX TERMS . . . . . . . . . . . . . . . . . . . . . . . . . . . . . . . . . . . . . 249

Interventions for treating proximal humeral fractures in adults (Review)

Copyright @ 2015 The Cochrane Collaboration. Published by John Wiley \& Sons, Ltd. 


\title{
[Intervention Review] \\ Interventions for treating proximal humeral fractures in adults
}

\author{
Helen HG Handoll ${ }^{1}$, Stig Brorson ${ }^{2}$ \\ ${ }^{1}$ Health and Social Care Institute, Teesside University, Middlesbrough, UK. ${ }^{2}$ Department of Orthopaedic Surgery, Herlev University \\ Hospital, Herlev, Denmark \\ Contact address: Helen HG Handoll, Health and Social Care Institute, Teesside University, Middlesbrough, Tees Valley, TS1 3BA, \\ UK. h.handoll@tees.ac.uk.H.Handoll@ed.ac.uk. \\ Editorial group: Cochrane Bone, Joint and Muscle Trauma Group. \\ Publication status and date: New search for studies and content updated (conclusions changed), published in Issue 11, 2015. \\ Review content assessed as up-to-date: 10 November 2014.
}

Citation: Handoll HHG, Brorson S. Interventions for treating proximal humeral fractures in adults. Cochrane Database of Systematic Reviews 2015, Issue 11. Art. No.: CD000434. DOI: 10.1002/14651858.CD000434.pub4.

Copyright (C) 2015 The Cochrane Collaboration. Published by John Wiley \& Sons, Ltd.

\begin{abstract}
A B S T R A C T
Background

Fracture of the proximal humerus, often termed shoulder fracture, is a common injury in older people. The management of these fractures varies widely. This is an update of a Cochrane Review first published in 2001 and last updated in 2012.
\end{abstract}

Objectives

To assess the effects (benefits and harms) of treatment and rehabilitation interventions for proximal humeral fractures in adults.

\section{Search methods}

We searched the Cochrane Bone, Joint and Muscle Trauma Group Specialised Register, the Cochrane Central Register of Controlled Trials (CENTRAL), MEDLINE, EMBASE, and other databases, conference proceedings and bibliographies of trial reports. The full search ended in November 2014.

\section{Selection criteria}

We considered all randomised controlled trials (RCTs) and quasi-randomised controlled trials pertinent to the management of proximal humeral fractures in adults.

\section{Data collection and analysis}

Both review authors performed independent study selection, risk of bias assessment and data extraction. Only limited meta-analysis was performed.

\section{Main results}

We included 31 heterogeneous RCTs (1941 participants). Most of the 18 separate treatment comparisons were tested by small singlecentre trials. The main exception was the surgical versus non-surgical treatment comparison tested by eight trials. Except for a large multicentre trial, bias in these trials could not be ruled out. The quality of the evidence was either low or very low for all comparisons except the largest comparison.

Nine trials evaluated non-surgical treatment in mainly minimally displaced fractures. Four trials compared early (usually one week) versus delayed (three or four weeks) mobilisation after fracture but only limited pooling was possible and most of the data were from 
one trial (86 participants). This found some evidence that early mobilisation resulted in better recovery and less pain in people with mainly minimally displaced fractures. There was evidence of little difference between the two groups in shoulder complications (2/127 early mobilisation versus 3/132 delayed mobilisation; 4 trials) and fracture displacement and non-union (2/52 versus 1/54; 2 trials).

One quasi-randomised trial (28 participants) found the Gilchrist-type sling was generally more comfortable than the Desault-type sling (body bandage). One trial (48 participants) testing pulsed electromagnetic high-frequency energy provided no evidence. Two trials (62 participants) provided evidence indicating little difference in outcome between instruction for home exercises versus supervised physiotherapy. One trial (48 participants) reported, without presentable data, that home exercise alone gave better early and comparable long-term results than supervised exercise in a swimming pool plus home exercise.

Eight trials, involving 567 older participants, evaluated surgical intervention for displaced fractures. There was high quality evidence of no clinically important difference in patient-reported shoulder and upper-limb function at one- or two-year follow-up between surgical (primarily locking plate fixation or hemiarthroplasty) and non-surgical treatment (sling immobilisation) for the majority of displaced proximal humeral fractures; and moderate quality evidence of no clinically important difference between the two groups in quality of life at two years (and at interim follow-ups at six and 12 months). There was moderate quality evidence of little difference between groups in mortality in the surgery group (17/248 versus 12/248; risk ratio (RR) 1.40 favouring non-surgical treatment, $95 \%$ confidence interval (CI) 0.69 to 2.83 ; $\mathrm{P}=0.35 ; 6$ trials); only one death was explicitly linked with the treatment. There was moderate quality evidence of a higher risk of additional surgery in the surgery group (34/262 versus 16/261; RR 2.06, $95 \%$ CI 1.18 to 3.60 ; P = $0.01 ; 7$ trials). Although there was moderate evidence of a higher risk of adverse events after surgery, the $95 \%$ confidence intervals for adverse events also included the potential for a greater risk of adverse events after non-surgical treatment.

Different methods of surgical management were tested in 12 trials. One trial (57 participants) comparing two types of locking plate versus a locking nail for treating two-part surgical neck fractures found some evidence of slightly better function after plate fixation but also of a higher rate of surgically-related complications. One trial (61 participants) comparing a locking plate versus minimally invasive fixation with distally inserted intramedullary K-wires found little difference between the two implants at two years. Compared with hemiarthroplasty, one trial (32 participants) found similar results with locking plate fixation in function and re-operation rates, whereas another trial (30 participants) reported all five re-operations occurred in the tension-band fixation group. One trial (62 participants) found better patient-rated (Quick DASH) and composite shoulder function scores at a minimum of two years follow-up and a lower incidence of re-operation and complications after reverse shoulder arthroplasty (RSA) compared with hemiarthroplasty.

No important between-group differences were found in one trial (120 participants) comparing the deltoid-split approach versus deltopectoral approach for non-contact bridging plate fixation, and two trials (180 participants) comparing 'polyaxial' and 'monaxial' screws in locking plate fixation. One trial (68 participants) produced some preliminary evidence that tended to support the use of medial support locking screws in locking plate fixation. One trial (54 participants) found fewer adverse events, including re-operations, for the newer of two types of intramedullary nail. One trial (35 participants) found better functional results for one of two types of hemiarthroplasty. One trial ( 45 participants) found no important effects of tenodesis of the long head of the biceps for people undergoing hemiarthroplasty.

Very limited evidence suggested similar outcomes from early versus later mobilisation after either surgical fixation (one trial: 64 participants) or hemiarthroplasty (one trial: 49 participants).

\section{Authors' conclusions}

There is high or moderate quality evidence that, compared with non-surgical treatment, surgery does not result in a better outcome at one and two years after injury for people with displaced proximal humeral fractures involving the humeral neck and is likely to result in a greater need for subsequent surgery. The evidence does not cover the treatment of two-part tuberosity fractures, fractures in young people, high energy trauma, nor the less common fractures such as fracture dislocations and head splitting fractures.

There is insufficient evidence from RCTs to inform the choices between different non-surgical, surgical, or rehabilitation interventions for these fractures.

\section{PLAIN LANGUAGE SUMMARY}

\section{Interventions for treating shoulder fractures in adults}

\section{Background}

Interventions for treating proximal humeral fractures in adults (Review)

Copyright () 2015 The Cochrane Collaboration. Published by John Wiley \& Sons, Ltd. 
Fracture of the top end of the upper arm bone (proximal humerus) is a common injury in older people. It is often called a shoulder fracture. The bone typically fractures (breaks) just below the shoulder, usually after a fall. Most of these fractures occur without breaking the skin lying over the fracture. The injured arm is often supported in a sling until the fracture heals sufficiently to allow shoulder movement. More severe (displaced) fractures may be treated surgically. This may involve fixing the fracture fragments together by various means. Alternatively, the top of the fractured bone may be replaced (half'shoulder' replacement: hemiarthroplasty). More rarely, the whole joint, thus including the joint socket, is replaced (total 'shoulder' replacement). Physiotherapy is often used to help restore function.

\section{Results of the search}

We searched medical databases up to November 2014 and included 31 randomised studies with a total of 1941 participants. Most of the 18 treatment comparisons were tested by one study only. The best evidence was from eight studies, one of which was a relatively large multicentre study; these investigated whether surgery gave a better result than non-surgical treatment for displaced fractures.

\section{Key results}

Nine trials evaluated non-surgical treatment in usually less severe fractures. One trial found a type of arm sling was generally more comfortable than a type of body bandage. There was some evidence that early mobilisation (within one week), compared with delayed mobilisation (after three weeks), resulted in less pain and faster recovery in people with 'stable' fractures. Two studies provided weak evidence that many patients could generally achieve a satisfactory outcome when given sufficient instruction to pursue exercises on their own.

Eight studies, involving 567 participants with displaced fractures, compared surgical versus non-surgical treatment. Pooled results from the five most recent trials showed that there were no important differences between the two approaches for patient-reported measures of function and quality of life at 6,12 and 24 months. There was little difference between the two groups in mortality. Twice as many surgical group patients had additional or secondary surgery. More surgical group patients had adverse events.

Twelve trials (744 participants) tested different methods of surgical treatment. There was weak evidence of some differences (e.g. in complications) between some interventions (e.g. different devices or different ways of using devices).

There was very limited evidence suggesting similar outcomes for early versus delayed mobilisation after either surgical fixation or hemiarthroplasty.

\section{Quality of the evidence}

Most of the 31 studies had weaknesses that could affect the reliability of their results. We considered that the evidence was either of high or moderate quality for the results of the surgical versus non-surgical treatment comparison, which means that we are pretty certain these results are reliable. We considered that the evidence for other comparisons was of low or very low quality, which means we are unsure of these results.

\section{Conclusions}

Surgery does not result in a better outcome for the majority of people with displaced proximal humeral fractures and is likely to result in a greater need for subsequent surgery. Otherwise, there is not enough evidence to determine the best non-surgical or, when selected, surgical treatment for these fractures. 


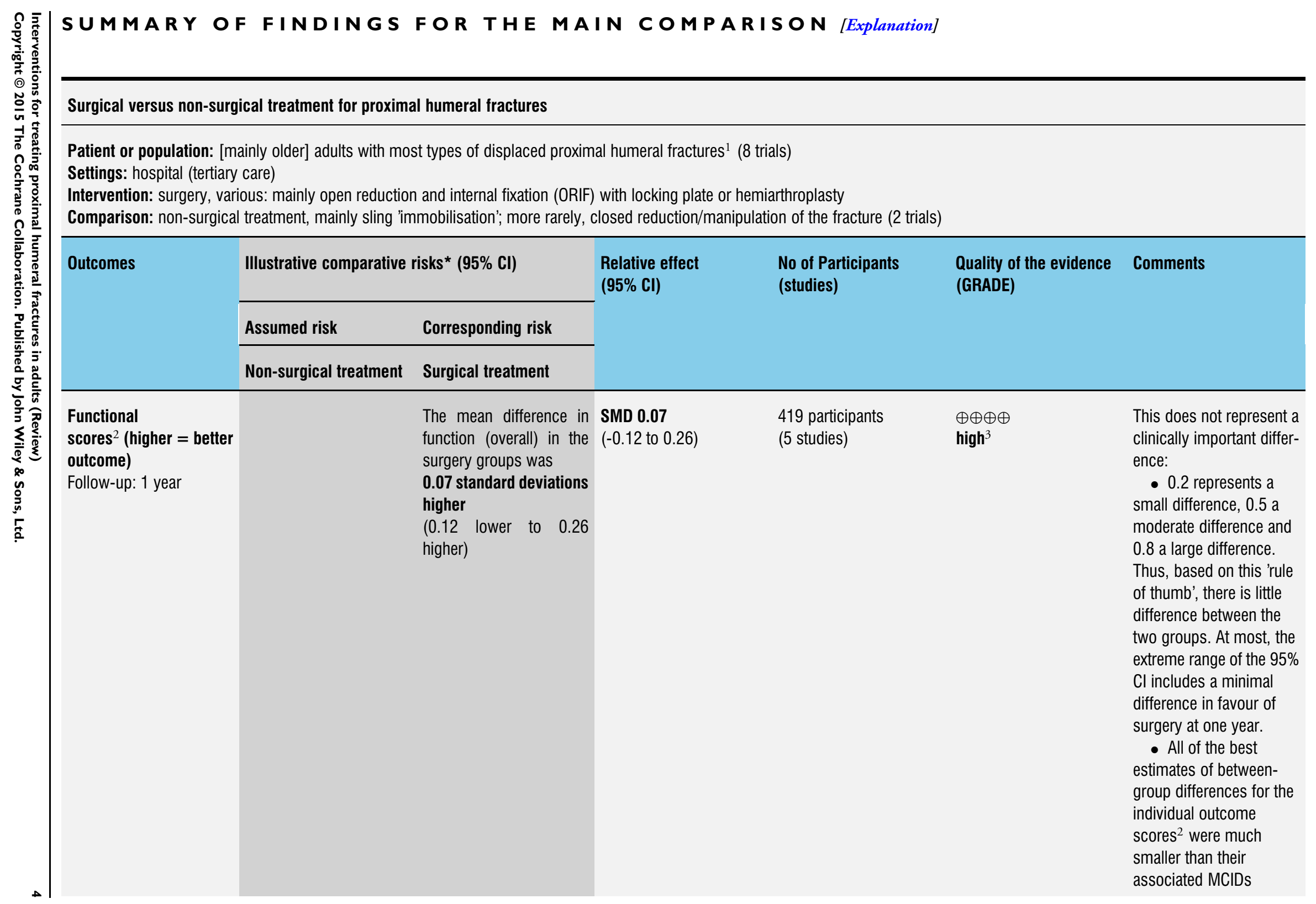




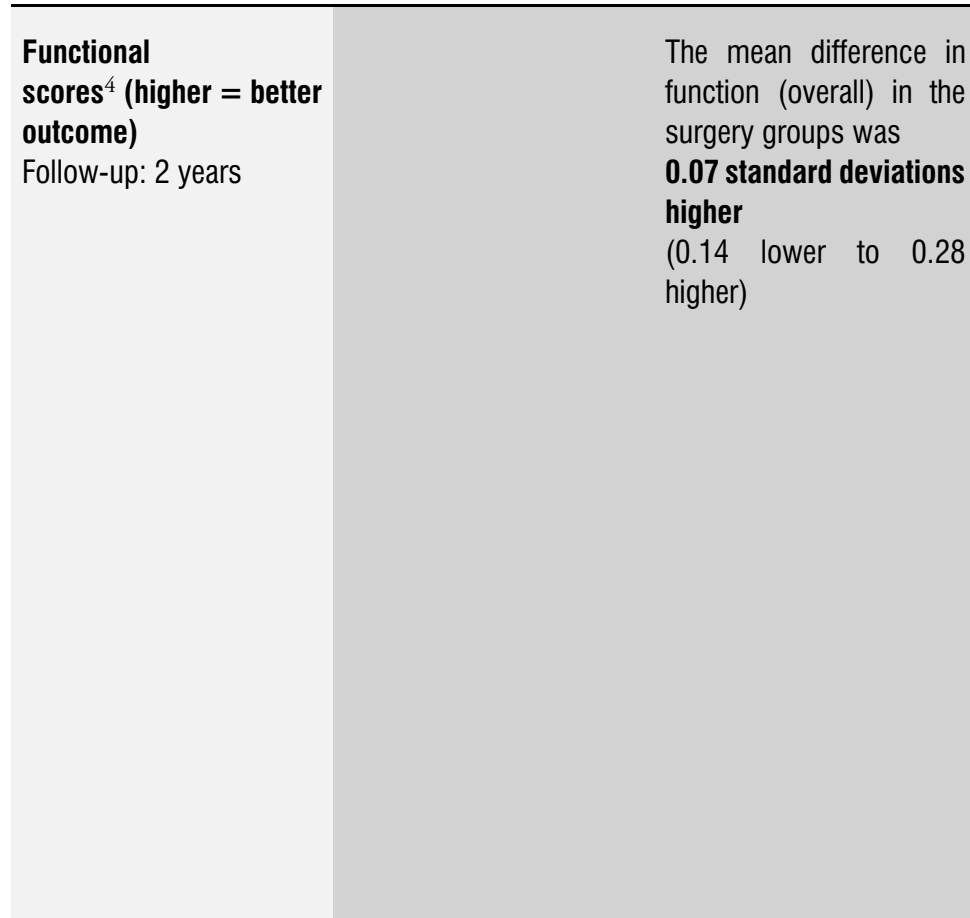

Quality of life assess- The mean EuroQol score The mean EuroQol score ment: EuroQol (0: dead ranged across control in the surgery groups was to 1 : best health)

Follow-up: 2 years groups from 0.7 to 0.85 0.03 higher,

(0.01 lower to 0.08 higher)
(SMD 0.07, 95\% $\mathrm{Cl}-0.14351$ participants (4 studies)

$\oplus \oplus \oplus \oplus$ high $^{5}$

surgery groups was

\section{higher}

higher)

\section{to 0.28$)$}

higher)

Quality of life: SF- The mean SF-12PCS was The mean SF-12 PCS in 12 Physical Component $44.1 \quad$ the surgery group was Score (0 to 100: best) 1.10 higher (1.99 lower Follow-up: 2 years to 4.19 higher)

This does not represent a clinically-important difference.

- 0.2 represents a small difference, $0.5 \mathrm{a}$ moderate difference and 0.8 a large difference.

Thus, based on this 'rule of thumb', there is little difference between the two groups. At most, the extreme range of the $95 \%$

$\mathrm{Cl}$ includes a minimal difference in favour of surgery at two years.

- All of the best estimates of betweengroup differences for the individual outcome scores ${ }^{4}$ were much smaller than their associated MCIDs

The MCID of 0.12 was outside the $95 \% \mathrm{Cl}$ at this time period and at 6 months (MD 0.04, 95\% $\mathrm{Cl} 0.01$ to 0.08 ) and 12 months (MD 0.02, 95\% $\mathrm{Cl}-0.02$ to 0.06 )

A similar lack of clinically important difference ${ }^{8}$ was noted at 6 and 12 months. This measure may not be sensitive to recovery from this injury 


\begin{tabular}{|c|c|c|c|c|c|c|}
\hline $\begin{array}{l}\text { Mortality } \\
\text { Follow-up: up to } 2 \text { years }\end{array}$ & 52 per $1000^{8}$ & $\begin{array}{l}\mathbf{7 3} \text { per } 1000 \\
\text { (4 to } 147)\end{array}$ & RR 1.40 (0.69 to 2.83) & $\begin{array}{l}596 \text { participants } \\
\text { (6 studies) }\end{array}$ & $\begin{array}{l}\oplus \oplus \oplus \bigcirc \\
\text { moderate }{ }^{9}\end{array}$ & $\begin{array}{l}\text { Surgery resulted in } 21 / \\
1000 \text { more deaths up to } \\
2 \text { years ( } 95 \% \mathrm{Cl} 48 \text { fewer } \\
\text { to } 95 \text { more) } \\
\text { Where reported, none of } \\
\text { the deaths was related to } \\
\text { their fracture or treatment } \\
\text { with the exception of one } \\
\text { early death due to venous } \\
\text { thromboembolism in the } \\
\text { surgical group of one trial }\end{array}$ \\
\hline $\begin{array}{l}\text { Additional surgery (re- } \\
\text { operation or secondary } \\
\text { surgery) } \\
\text { Follow-up: up to } 2 \text { years }\end{array}$ & 40 per $1000^{9}$ & $\begin{array}{l}83 \text { per } 1000 \\
(47 \text { to } 144)\end{array}$ & $\begin{array}{l}\text { RR 2.06 } \\
\text { (1.18 to } 3.60)\end{array}$ & $\begin{array}{l}523 \text { participants } \\
\text { (7 studies) }\end{array}$ & $\begin{array}{l}\oplus \oplus \oplus \bigcirc \\
\text { moderate }^{10}\end{array}$ & $\begin{array}{l}\text { Surgery resulted in } 43 \text { / } \\
1000 \text { more patients hav- } \\
\text { ing additional surgery up } \\
\text { to } 2 \text { years ( } 95 \% \mathrm{Cl} 7 \text { to } \\
104 \text { more) } \\
\text { One trial ( } 250 \text { par- } \\
\text { ticipants) also reported } \\
\text { on additional shoulder- } \\
\text { related therapy ( } 7 / 1254 \\
\text { versus } 4 / 125 ; \quad \mathrm{RR} 1 . \\
75 \text { favouring non-surgical } \\
\text { therapy, } 95 \% \mathrm{Cl} 0.53 \text { to } \\
5.83)\end{array}$ \\
\hline $\begin{array}{l}\text { Adverse events / com- } \\
\text { plications - Number of } \\
\text { patients with complica- } \\
\text { tions } \\
\text { Follow-up: } 2 \text { years }\end{array}$ & 184 per $1000^{9}$ & $\begin{array}{l}239 \text { per } 1000 \\
\text { (147 to } 389)\end{array}$ & $\begin{array}{l}\text { RR } 1.30 \\
\text { (0.80 to 2.11) }\end{array}$ & $\begin{array}{l}250 \text { participants } \\
\text { (1 study) }\end{array}$ & $\begin{array}{l}\oplus \oplus \oplus \bigcirc \\
\text { moderate }^{11}\end{array}$ & $\begin{array}{l}\text { Surgery resulted in } 55 \text {, } \\
1000 \text { more patients hav- } \\
\text { ing adverse events up to } \\
2 \text { years ( } 95 \% \mathrm{Cl} 37 \text { fewer } \\
\text { to } 205 \text { more) } \\
\text { All } 8 \text { trials reported on } \\
\text { individual complications, } \\
\text { the pattern of distribution } \\
\text { generally reflecting the } \\
\text { expected: e.g. infection 8/ }\end{array}$ \\
\hline
\end{tabular}


*The basis for the assumed risk (e.g. the median control group risk across studies) is provided in footnotes. The corresponding risk (and its $95 \%$ confidence interval) is based on the assumed risk in the comparison group and the relative effect of the intervention (and its $95 \% \mathrm{Cl}$ ).

Cl: confidence interval; MCID: minimal clinically important differences; RR: risk ratio; SMD: standardised mean difference

GRADE Working Group grades of evidence

High quality: Further research is very unlikely to change our confidence in the estimate of effect.

Moderate quality: Further research is likely to have an important impact on our confidence in the estimate of effect and may change the estimate.

Low quality: Further research is very likely to have an important impact on our confidence in the estimate of effect and is likely to change the estimate.

Very low quality: We are very uncertain about the estimate.

1. The inclusion/exclusion criteria varied among the trials: one (30 participants) included 2-, 3- or 4-part fractures; one (60 participants) included only 3-part fractures that included surgical neck; two (90 participants) included 3- or 4-part fractures, three (137 participants) included only 4-part fractures. The final trial (250 participants) included " displaced fracture of the proximal humerus that involved the surgical neck", resulting in a few 1-part (but confirmed as still ' "displaced") as well as 2-, 3- and 4-part fractures. The majority of the fractures $(146 / 250=58.4 \%)$ in the largest trial were either 2-part (128) or 1-part (18) fractures. Several trials included further criteria; for example, the largest trial explicitly excluded fracture dislocations (i.e. fractures with an associated dislocation of the injured shoulder joint). Consideration is also needed of other inclusion and exclusion criteria, including multiple trauma, clear indications for surgery (severe soft-tissue compromise), and co-morbidities precluding surgery or anaesthesia

2. Patient-reported functional scores were the Disability of the Arm, Shoulder, and Hand questionnaire (DASH; 2 trials), the Oxford Shoulder Score (OSS; 1 trial); the American Shoulder and Elbow Surgeons (ASES; 1 trial) and Simple Shoulder Test (SST; 1 trial)

3. Although the evidence was first downgraded by one level for study limitations, reflecting a high risk of performance bias relating to lack of blinding in four single-centre trials, the consistency in the results of these and the fifth and largest trial, where the analysis indicated that the study design limited the risk of bias relating to the inevitable lack of blinding, resulted in an upgrade

4. Patient-reported functional scores were the Disability of the Arm, Shoulder, and Hand questionnaire (DASH; 2 trials), the Oxford Shoulder Score (OSS; 1 trial); and the American Shoulder and Elbow Surgeons (ASES; 1 trial)

5. The evidence was downgraded by one level for study limitations, reflecting a high risk of performance bias relating to lack of blinding in 3 single-centre trials. There was, however, consistency in the results of these and the fourth and largest trial, where the analysis indicated that the study design limited the risk of bias relating to the inevitable lack of blinding, resulting in an upgrade

6 . The evidence was downgraded by one level for inconsistency, reflecting the statistical heterogeneity (Chi ${ }^{2}=6.76, \mathrm{df}=3(P=0.08)$; $\mathrm{I}^{2}=56 \%$ ), but also data from two trials (102 participants) from the same centre that found minimal clinically important differences favouring surgery

7. The evidence was downgraded one level for imprecision, reflecting that these data were from one trial alone

8. A minimal clinically important difference for the SF-12 PCS was assumed to be 6.5. Notably, a similar finding applied for the betweengroup differences in SF-12 Mental Component Scores, but the direction of effect favoured non-surgical treatment 


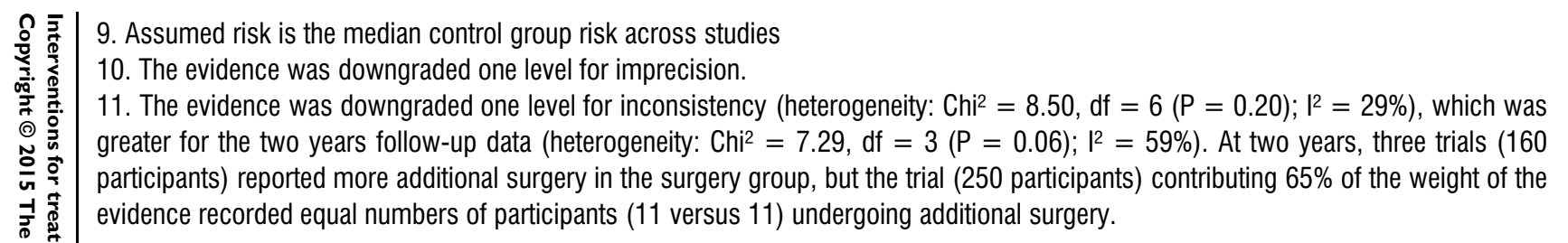




\section{B A C K G R O U N D}

\section{Description of the condition}

Proximal humeral fractures account for approximately six per cent of all adult fractures (Court-Brown 2006). Their incidence rapidly increases with age, and women are affected between two and three times as often as men (Court-Brown 2006; Lind 1989). Many patients who sustain a proximal humeral fracture are old and their bones are osteoporotic. Court-Brown 2001 found that $87 \%$ of these fractures in adults resulted from falls from standing height. Palvanen 2006 found that the incidence of osteoporotic-related fractures of the proximal humerus in Finland had tripled between 1970 and 2002 to 105 per 100,000 people aged 60 or above. An epidemiological study of upper-limb fractures occurring in 2009 in the USA reported an incidence of 60 proximal humeral fractures per 100,000 people overall, with four-fold increased incidence of 253 per 100,000 in those aged 65 or older (Karl 2015).

Most proximal humeral fractures are closed fractures in that the overlying skin remains intact. The most commonly used classification of shoulder fractures is that of Neer (Neer 1970). Neer considered four anatomical segments of the proximal humerus the articular part, the greater tuberosity, the lesser tuberosity and the humeral shaft. These may be affected by fracture lines but are only considered as a 'part' if displaced by more than one centimetre or 45 degrees angulation from each other. Fractures, regardless of the number of fracture lines present, which did not meet the criteria for displacement of any one segment with respect to the others were considered 'minimally displaced'; these are sometimes referred to as one-part fractures. Neer's other categories, two-part, three-part and four-part fractures all involved the displacement or angulation of some or all of the above four segments. Each of these fracture types may be potentially associated with an anterior or posterior humeral head dislocation.

At initial presentation, it may be difficult to delineate the exact pathoanatomical pattern of the fracture even with sophisticated imaging. In any event, this may not correlate with the extent to which the vascularity (blood supply) of the humeral head is compromised. The vascularity of the proximal humerus is a primary focus of another widely used classification system for these fractures, the AO classification system (Muller 1991), which was updated in conjunction with the OTA classification in 2007 (Marsh 2007). There are three main types (A, B, C), which in turn are further divided into three groups, each with a further three subgroups. Type A fractures are "extra-articular, unifocal, with intact vascular supply"; type B fractures are "extra-articular, bifocal, with possible vascular compromise"; and type $\mathrm{C}$ fractures are "articular, with a high likelihood of vascular compromise" (Robinson 2008). Many proximal humeral fractures are only minimally displaced. Neer's estimate (Neer 1970) that approximately $85 \%$ of all proximal humeral fractures are minimally displaced, in that no bone fragment is displaced by more than one centimetre, or angulated by more than 45 degrees is often cited (Koval 1997). However, a lower figure of $49 \%$ was reported in a prospective consecutive series of over 1000 proximal humeral fractures (Court-Brown 2001).

\section{Description of the intervention}

Non-surgical (conservative) treatment is generally the accepted treatment option for minimally displaced fractures, and often used also for people with displaced fractures. Non-surgical treatment usually involves a period of immobilisation, such as in an arm sling, followed by physiotherapy and exercises. Non-surgical treatment can include closed reduction, where the displaced bone fragments are reduced using various manoeuvres while the arm is under traction. Various aspects of non-surgical treatment, such as the arm sling and collar and cuff, are illustrated online (AO 2015). Older types of bandages, such as the Desault and Velpeau, are illustrated in Brorson 2011a.

Surgery is usually reserved for displaced and unstable fractures and those with more complicated fracture patterns. Surgical interventions include:

- closed reduction and percutaneous stabilisation using pins or wires;

- external fixation;

- open reduction and plating, for example buttress plates, angle blade plates and proximal humeral locking plates;

- open reduction and fixation using a tension-band principle;

- intramedullary nailing, either antegrade or retrograde insertion (intramedullary nails usually offer the option of locking screws, which are inserted into fracture fragments then transverse the nail, providing additional fracture stability);

- hemiarthroplasty (replacement of the humeral head);

- total shoulder replacement (replacement of the entire joint; both the 'ball' (humeral head) and 'socket' (glenoid)). There are two distinct types: anatomical and reverse shoulder arthroplasty. In reverse arthroplasty the joint polarity is reversed such that the ball is on glenoid side and the socket (fixed on a stem) on the humeral side.

Post-operative treatment generally involves a period of immobilisation followed by physiotherapy and exercises.

\section{How the intervention might work}

Immobilisation of the injured limb provides support and pain relief during healing. However, there is a risk of the shoulder becoming stiff and painful with substantial reduction of function. Subsequent physiotherapy and exercises aim to restore function and mobility of the injured (or operated) arm. Malunion of proximal humeral fractures may result in impingement or compromised function of the 'rotator cuff' of muscles and tendons that surrounds the shoulder joint. Persistent pain and painful pseudoparalysis are common indications for late surgery. 
After reduction or repositioning of the fractured parts, surgical fixation using various techniques aims to stabilise the reduced fracture and restore joint integrity. Surgical stabilisation of the fracture may also allow earlier movement of the shoulder and elbow, preventing stiffness. Surgeons have often followed Neer's premise (Neer 1975) that head avascular necrosis is virtually guaranteed in a four-part fracture and have usually offered these patients a hemiarthroplasty, where the humeral head is replaced by an artificial part. An exception is often made for a specific type of four-part fracture, the valgus impacted four-part fracture, which was not mentioned initially in Neer's classification. This fracture, where the fractured parts are compressed towards each other, is less likely to lead to avascular necrosis of the humeral head, provided the lateral displacement of the head fragment is not excessive (Jakob 1991; Resch 1997). Bone quality also influences the appropriateness of any intervention and hence the long term clinical outcome. Furthermore, the patient's frailty may lead to a low rehabilitation drive and delay any recovery from both the initial trauma and any subsequent management.

\section{Why it is important to do this review}

Proximal humeral fractures are increasing in incidence, particularly in older people, and the short and long term consequences for individuals with these injuries and society are substantial (Palvanen 2006). There is considerable variation in practice, both in terms of definitive treatment such as surgical treatment for displaced fractures (Guy 2010) and rehabilitation (Hodgson 2006). Variation in practice includes that of the uptake of new implants, typically before their effectiveness has been evaluated, as illustrated for reverse shoulder arthoplasty in the USA (Schairer 2015). The costs of treating these fractures are also substantial and growing. The direct health-care costs, adjusted to 2007 prices, in the Netherlands of upper arm fractures, the majority of which were proximal humeral fractures, were EUR 4,440 per case with an overall annual cost of approximately EUR 40M (Polinder 2013). Polinder 2013 suggested that the increase in the cost of fracture care in 'elderly women' from a previous report of costs in the Netherlands was partly because of a higher incidence of surgery. This trend to increased surgery also applies in other countries such as the USA (Bell 2011). A very recently published report by the same team in the Netherlands estimated the medical costs per case in 2012, including hospitalisation, rehabilitation and nursing care, and, primarily in patients aged over 80 years, home care costs, were EUR 11,224 (Mahabier 2015). The estimated costs for lost productivity including time off work and other costs for those in work was EUR 20,374 per case in 2012. Schairer 2015 found the estimated mean hospital costs in 2011 in the USA were significantly higher for reverse shoulder arthroplasty than for hemiarthroplasty (USD 21,723 versus USD 18,122), a difference which was almost three times greater when it came to mean hospital charges (USD $75,849$ versus USD 65,477$)$. The often poor treatment outcome and the increasing incidence of these fractures, the increasing use of surgery and of reverse shoulder arthoplasty (Han 2015), high treatment costs and variations in practice all endorse the need for this review update.

The last two versions of this review noted the insufficiency of the evidence to inform practice, but also located ongoing trials that could potentially help to address this deficiency (Handoll 2007; Handoll 2010). This update continues the systematic review of the evidence for managing these fractures.

\section{O B J E C T I V E S}

To assess the effects (benefits and harms) of treatment and rehabilitation interventions for proximal humeral fractures in adults.

We defined a priori the following broad objectives:

- to compare different methods of non-surgical treatment (including rehabilitation);

- to compare surgical versus non-surgical treatment;

- to compare different methods of surgical treatment;

- to compare different methods of rehabilitation after surgical treatment.

We planned to study the outcomes in different age groups (initially, under versus over 65 years) and for different types of proximal humeral fractures.

\section{METHODS}

\section{Criteria for considering studies for this review}

\section{Types of studies}

We included randomised or quasi-randomised (method of allocating participants to a treatment that is not strictly random; e.g. by hospital record number) trials which compared two or more interventions in the management of fractures of the proximal humerus in adults.

\section{Types of participants}

We included adults with a fracture of the proximal humerus. Stratification was planned by fracture type (e.g. based on the Neer classification (Neer 1970) or the AO classification (Muller 1991)) and by age (under versus over 65 years) if possible. Trials including children were included provided either separate data for skeletally 
mature participants were available or the proportion of children was small and, preferably, balanced in intervention groups.

\section{Types of interventions}

Non-surgical and surgical interventions, as exemplified in Description of the intervention, used in the treatment and rehabilitation of fractures of the proximal humerus. Pharmacological trials were excluded.

\section{Types of outcome measures}

The primary focus is on long term functional outcome, preferably measured at one year or more.

\section{Primary outcomes}

- Functional outcomes: patient-reported measures of upperlimb function (e.g. the Disability of the Arm, Shoulder, and Hand questionnaire (DASH), the Oxford Shoulder Score (OSS; Dawson 1996; Dawson 2009), and other validated shoulder rating scales).

- Activities of daily living and health-related quality-of-life scores (e.g. EuroQol (EQ-5D); Short-Form 36 (SF-36) and Short-Form 12 (SF-12; Ware 1996).

- Serious adverse events (e.g. death, deep infection, avascular necrosis, complex regional pain syndrome type 1) and need for substantive treatment, such as an operation.

\section{Secondary outcomes}

- Composite scores of subjectively and objectively rated function and overall outcome (e.g. Constant and Murley's score (Constant 1987); Neer's rating (Neer 1970)).

- Pain.

- Upper limb strength and range of movement.

- Less serious complications/adverse events of limited duration and impact (e.g. superficial infection, transient paraesthesia, skin irritation).

- Patient satisfaction with treatment, including cosmetic outcomes.

- Anatomical outcomes (e.g. radiological deformity).

Economic outcomes: each trial report was reviewed for cost and resource data, such as length of hospital stay and number of outpatient attendances, that would enable economic evaluation.

We based our judgement of clinically important between-group mean differences in measures of pain and function using the following minimal clinically important differences (MCID); alternative sources are listed after the main selected item in bold.

- ASES (0 to 100: best outcome) (rotator cuff disease): $\mathbf{1 2 . 0 1}$ (function-based) (Tashjian 2010).

- Constant score (0 to 100: best outcome) (proximal humerus fracture): 11.6 (anchor-based), 5.1 (distribution-based)
(Van de Water 2014); (upper limb proximal diagnosis): MCID 10.2 (Schmitt 2004)

- DASH (0 to 100: worst outcome) (proximal humerus fracture): 13.0 (anchor-based), 8.1 (distribution-based) (Van de Water 2014); 15 recommended in DASH/QuickDASH

- EQ-5D (0 to 1: best outcome) (proximal humerus fracture): $\mathbf{0 . 1 2}$ (assessed in relation to a DASH MCID of 10) (Olerud 2011c)

- OSS (0 to 48: best outcome) (proximal humerus fracture): 11.4 (anchor-based), 5.1 (distribution-based) (Van de Water 2014)

- QuickDASH (0 to 100: worst outcome): 16 in DASH/ QuickDASH; 8 (shoulder pain) (Mintken 2009)

- SF-12-PCS (0 to 100: best outcome) (physical component score) (upper limb proximal diagnosis): MCID 6.5 (Schmitt 2004)

- SST (0 to 12: best outcome) (rotator cuff disease): 2.05

(Tashjian 2010)

- UCLA (2 to 35: best outcome) (proximal humerus fracture): 2.4 (anchor-based), 2.0 (distribution-based) (Van de Water 2014)

\section{Search methods for identification of studies}

\section{Electronic searches}

We searched the Cochrane Bone, Joint and Muscle Trauma Group Specialised Register (10 November 2014), the Cochrane Central Register of Controlled Trials (CENTRAL) (The Cochrane Library 2014, Issue 10), MEDLINE (1966 to October Week 5 2014), MEDLINE In-Process \& Other Non-Indexed Citations (7 November 2014), EMBASE (1988 to 2014 Week 45), CINAHL (Cumulative Index to Nursing and Allied Health Literature) (10 November 2014), AMED (Allied and Complementary Medicine) (1985 to 10 November 2014), and PEDro Physiotherapy Evidence Database (10 November 2014).

In MEDLINE, we combined subject-specific terms with the sensitivity-maximizing version of the Cochrane Highly Sensitive Search Strategy for identifying randomised trials (Lefebvre 2011) (Appendix 1). Search strategies for CENTRAL, EMBASE, CINAHL, AMED and PEDro can also be found in Appendix 1. For this update, the search results were limited from January 2012 onwards. Details of the search strategies used for previous versions of the review are given in Handoll 2007, Handoll 2010 and Handoll 2012. We applied no language or publication restrictions.

We searched the WHO International Clinical Trials Registry Platform Search Portal, the ISRCTN registry, and ClinicalTrials.gov to identify ongoing and recently completed trials (10 November 2014) (see Appendix 1). 


\section{Searching other resources}

We searched the reference lists of articles. We also included the findings from handsearches of the British Volume of the Journal of Bone and Joint Surgery supplements (1996 to 2006) and electronic searches of the The Bone and Joint Journal Orthopaedic Proceedings (10 November 2014) (see Appendix 1). We searched abstracts of the British Elbow and Shoulder Society annual meetings (2001 to 2013), the American Orthopaedic Trauma Association annual meetings (1996 to 2014), American Academy of Orthopaedic Surgeons annual meetings (2005, 2006, 2014), and the British Trauma Society annual scientific meetings (2012 and 2014). Prior to this update, we handsearched various orthopaedic proceedings and screened weekly downloads from AMEDEO (to 2007), the details of which can be found in Handoll 2012.

\section{Data collection and analysis}

\section{Selection of studies}

For this update, both review authors independently screened search results and assessed potentially eligible studies for inclusion. The initial decisions of trial eligibility were based on citations and, where available, abstracts and indexing terms. We obtained full articles and, where necessary to ascertain trial methods and status, one author $(\mathrm{HH})$ sent requests for information to trial investigators. Study inclusion was by consensus. Titles of journals, names of authors or supporting institutions were not masked at any stage. Both authors performed independent study selection on the trials for which the other author was an investigator.

\section{Data extraction and management}

Both review authors independently completed a data extraction tool, which had been used in the previous version of the review, for each newly included trial. We recorded details of the study methods, participants, interventions and outcome assessment and results. Any differences that were clearly not transcription errors were discussed between review authors. Data management and entry into Review Manager (RevMan 2014) was mainly by one author $(\mathrm{HH})$ with checks made by both review authors. When necessary, additional details of trial methodology or data, or both were requested from trialists.

\section{Assessment of risk of bias in included studies}

Both review authors independently assessed risk of bias for newly included trials, without masking of the source and authorship of the trial reports. HH checked between-rater and between-versions consistency in assessment at data entry. All inter-rater differences were resolved by discussion. We used the tool outlined in the Cochrane Handbook for Systematic Reviews of Interventions (Higgins 2008a). This tool incorporates assessment of randomisation (sequence generation and allocation concealment), blinding (of participants, treatment providers and outcome assessment), completeness of outcome data, selection of outcomes reported and other sources of bias. We considered subjective and functional outcomes (e.g. functional outcomes, pain, clinical outcomes, complications) and 'hard' outcomes (death, reoperation) separately in our assessment of blinding and completeness of outcome data. We assessed two additional sources of bias: bias resulting from major imbalances in key baseline characteristics (e.g. age, gender, type of fracture); and performance bias such as resulting from lack of comparability in the experience of care providers.

Additionally, we assessed four other aspects of trial quality and reporting that would help us judge the applicability of the trial findings. The four aspects were: definition of the study population; description of the interventions; definition of primary outcome measures; and length of follow-up.

\section{Measures of treatment effect}

For each trial, risk ratios (RR) and $95 \%$ confidence intervals (CIs) were calculated for dichotomous outcomes, and mean differences (MD) and 95\% CIs were calculated for continuous outcomes. Standardised mean differences (SMD) rather than mean differences were used when pooling data from continuous outcome measures based on different scoring schemes.

\section{Unit of analysis issues}

We remained aware of potential unit of analysis issues arising from inclusion of participants with bilateral fractures, and presentation of outcomes, such as total complications, by the number of outcomes rather than participants with these outcomes. There was just one patient with bilateral fractures (Kristiansen 1988) but there was insufficient information to quantify the small difference this would have made to study findings. We avoided the second described unit of analysis problem, mainly by reporting on incidences of individual complications.

\section{Dealing with missing data}

We contacted trialists for missing information, including for denominators and standard deviations. We performed intention-totreat analyses where possible. Where there were missing standard deviations, we calculated these from other data (standard errors, 95\% CIs, exact P values) where available. We did not impute missing standard deviations.

\section{Assessment of heterogeneity}

We assessed heterogeneity for pooled data from comparable trials by visual inspection of the analyses along with consideration of the chi $^{2}$ test for heterogeneity and the $\mathrm{I}^{2}$ statistic (Higgins 2003). The 
main quantitative assessment of heterogeneity was based on the $\mathrm{I}^{2}$ statistic where the following interpretation from the Cochrane Handbook for Systematic Reviews of Interventions was used: $0 \%$ to $40 \%$ might not be important; $30 \%$ to $60 \%$ may represent moderate heterogeneity; $50 \%$ to $90 \%$ may represent substantial heterogeneity; and $75 \%$ to $100 \%$ considerable heterogeneity ( Deeks 2011).

\section{Assessment of reporting biases}

There are insufficient data thus far (a minimum of 10 trials is required) to merit the production of funnel plots to explore publication bias. The search for trials via conference proceedings and trial registration, together with the contacting of authors for information of trial status and progress has provided some insights on unpublished trials, which generally were abandoned because of poor recruitment.

\section{Data synthesis}

Where the data allowed, the results of comparable groups of trials were pooled using both fixed-effect and random-effects models; the selection of the model for presentation was determined by the consideration of the extent of the heterogeneity.

\section{Subgroup analysis and investigation of heterogeneity}

We set out a priori two subgroup analyses: by age (primarily, under versus over 65 years) and by types of fracture (primarily, minimally displaced versus displaced, based on the Neer classification). To test whether the subgroups are statistically significantly different from one another, we planned to inspect the overlap of confidence intervals and perform the test for subgroup differences available in Review Manager.

\section{Sensitivity analysis}

We planned sensitivity analyses based on aspects of trial and review methodology, including the effects of missing data, the inclusion of studies at high or unclear risk of bias (primarily, selection bias with reference to allocation concealment), the inclusion of studies only reported in abstracts and using fixed-effect versus randomeffects models for pooling.

\section{'Summary of findings' tables and quality assessment of the evidence}

We produced 'Summary of findings' tables only for the two comparisons where a more substantive body of evidence had accrued. We used the GRADE approach to assess the quality of evidence related to each of the key outcomes listed in the Types of outcome measures for each comparison (see the Cochrane Handbook for Systematic Reviews of Interventions Section 12.2, Schunemann 2011).

\section{RE S U L T S}

\section{Description of studies}

\section{Results of the search}

The search was updated from January 2012 to November 2014. We screened a total of 796 records from the following databases: the Cochrane Bone, Joint and Muscle Trauma Group Specialised Register (26), CENTRAL (91), MEDLINE (114), EMBASE (199), CINAHL (129), AMED (5), PEDro (55), WHO Trials Registry (61), ISRCTN registry (66) and ClinicalTrials.gov (50). We also identified four potentially eligible studies from other sources (abstracts of American Academy of Orthopaedic Surgeons annual meeting 2014 (331), the American Orthopaedic Trauma Association annual meetings (2012 to 2014) (96), The Bone and Joint Journal Orthopaedic Proceedings (13); British Elbow and Shoulder Society annual meetings (2011 to 2013) (23), British Trauma Society Annual Scientific Meeting 2014 (37); and reports for three other trials from the review authors (4)). Subsequent notification of an ongoing study was received from a trialist (Torrens). One other ongoing study was identified from a subsequent trials registry search. Overall, 32 new studies were identified. Of these, eight were included (Boons 2012; Buecking 2014; Cai 2012; Lopiz 2014; ProFHER 2015 (5 references, including 1 trial registration and trial protocol); Sebastiá-Forcada 2014; Soliman 2013 (2 references, including 1 trial registration); Torrens 2012 (1 reference and unpublished data)), 12 were excluded (Cigni 2012; Elidrissi 2013; Erdoğ an 2014; Fan 2012; IRCT2013052313435N1; Maniscalco 2014a (2 references); Martetschlager 2012; NCT00384852; NCT01532076; NCT02122315; NTR2186; Zuckerman 2012), eight were placed in ongoing trials (NCT01524965; NCT01847508; NCT01984112; NCT02075476; NTR4019; ROTATE (2 references, including 1 trial registration); SHeRPA; Torrens) and four await classification (Liu 2011 (2 papers); NCT02052206; Wang 2013; Zhu 2014).

Further information was obtained for several studies in the previous version (Handoll 2012); this included the two-year follow-up report (Fjalestad 2014a) of functional outcome for Fjalestad 2010, and an additional article (Ockert 2014), which reported on 48 additional participants for Ockert 2010. A trial registration document and published protocol (Fjalestad 2014b) were found for a newly designated ongoing trial (DELPHI), previously Fjalestad (RCT proposal) in studies awaiting classification. Published protocols were also found for two ongoing trials (HOMERUS ( Verbeek 2012); TPHF (Launonen 2012)). Additional information from updated trial registration documentation was added in for seven ongoing trials (HURA; NCT00438633; NCT00818987; NCT00999193; NCT01113411; NCT01557413; TPHF). Ad- 
ditional information was also available for Brorson 2009 which was moved from ongoing to studies awaiting classification.

Summaries of the trial populations of past and the present versions of this review as well as the changes between updates are presented in Appendix 2.

In all, 31 trials are now included, 38 trials are excluded, 21 trials are listed as ongoing and seven are in Studies awaiting classification. A flow diagram summarising the study selection process is shown in Figure 1. 
Figure I. 8 ( 9 articles) new ongoing studies; additional materials (9 articles) for 7 already ongoing studies 4 new studies (5 articles) awaiting classificationChange in status: I previously ongoing study to awaiting classification (I extra article) I study previously awaiting classification to ongoing ( 2 extra articles)Study flow diagram

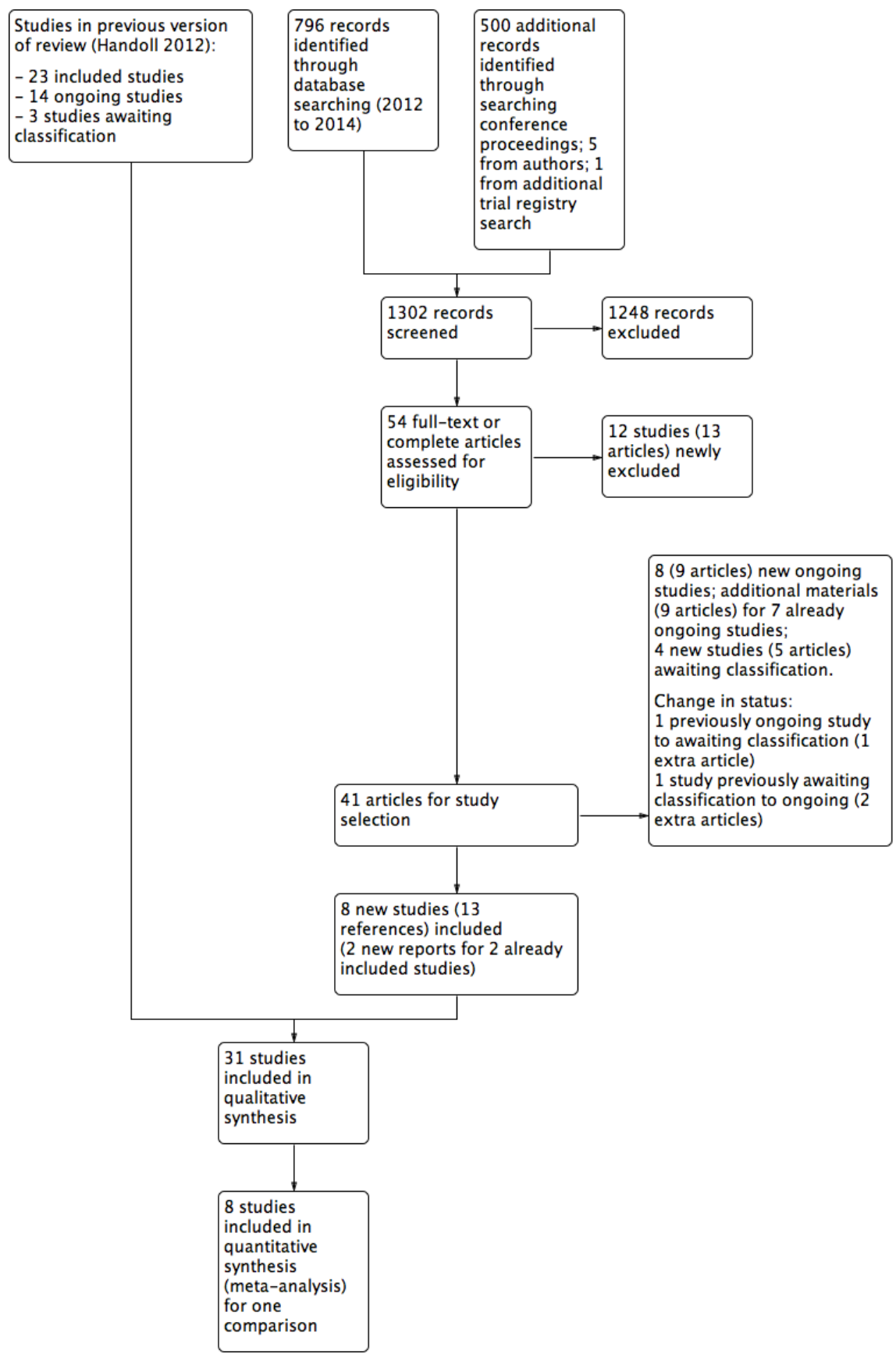




\section{Included studies}

Thirty included trials were published as full reports in journals, their availability ranging from 1979 (Lundberg 1979) to 2015 (ProFHER 2015). The remaining trial was published as a conference abstract only (Torrens 2012). Additional information via other publications, conference abstracts, trial registration details and communications from trial investigators were available for 15 trials (Agorastides 2007; Boons 2012; Fjalestad 2010; Hodgson 2003; Hoellen 1997; Lefevre-Colau 2007; Ockert 2010; Olerud 2011a; Olerud 2011b; ProFHER 2015; Soliman 2013; Torrens 2012; Voigt 2011; Zhang 2011; Zyto 1997); these sometimes preceded the availability of the main report. Details of study methods, participants, interventions and outcome measurement for the individual studies are provided in the Characteristics of included studies and summarised below.

\section{Design}

Thirty trials were randomised clinical trials, although seven of these provided no details of their method of randomisation and thus the use of quasi-randomised methods for sequence generation cannot be ruled out (Cai 2012; Hoellen 1997; Kristiansen 1988; Kristiansen 1989; Lundberg 1979; Stableforth 1984; Wirbel 1999). Rommens 1993 was a quasi-randomised trial using alternation for treatment allocation. Livesley 1992 was double-blinded. Of note is that the design of ProFHER 2015, a multicentre trial that compared surgical versus non-surgical treatment, was purposefully pragmatic such as in the requirement for individual surgeons to use surgical methods and implants with which they were familiar.

\section{Sample sizes}

The 31 included trials involved a total of 1941 participants. Study size ranged from 20 participants (Bertoft 1984) to 250 participants (ProFHER 2015). One trial (Kristiansen 1989) included one person with bilateral fractures; the treatment allocation for this participant is unclear.

\section{Setting}

Thirty of the 31 included trials were single centre studies conducted in 13 different countries: Austria (1 trial); Belgium (1); China (3); Czech Republic (1); Denmark (2); Egypt (1); France (1); Germany (5); The Netherlands (1); Norway (1); Spain (3); Sweden (6) and UK (4). (Though essentially a single centre trial, the interventions in Hodgson 2003 were undertaken at two centres within an NHS Trust in the UK.) The remaining trial was a multicentre trial conducted in the UK (ProFHER 2015). Details of the timing or duration or both of trial recruitment provided for 26 included trials (see the Characteristics of included studies) show Stableforth 1984 to have the earliest start date (1970) and longest period of recruitment (11 years).

\section{Participants}

With the exception of one trial (Soliman 2013), the majority of participants in each trial were women $(67 \%$ to $94 \%$ of their trial population). Most participants were aged 60 and above; two trials included a small number of children (Livesley 1992; Wirbel 1999). Seventeen trials set lower age limits. In 10 of these (Boons 2012; Cai 2012; Fialka 2008; Fjalestad 2010; Hodgson 2003; Hoellen 1997; Olerud 2011a; Olerud 2011b; Sebastiá-Forcada 2014; Voigt 2011), the age limit restricted the population to older adults; the most extreme was Sebastiá-Forcada 2014, where only people who were 70 years or over were included. Zyto 1997 specified that participants should be "elderly". Exceptionally, the participants of Soliman 2013 were aged between 45 to 60 years, with the majority (78\%) being male.

Five trials included only minimally displaced fractures (Bertoft 1984; Hodgson 2003; Livesley 1992; Lundberg 1979; Revay 1992), whereas 22 selected only people with displaced fractures (Agorastides 2007; Boons 2012; Buecking 2014; Cai 2012; Fialka 2008; Fjalestad 2010; Hoellen 1997; Kristiansen 1988; Lopiz 2014; Ockert 2010; Olerud 2011a; Olerud 2011b; ProFHER 2015; Sebastiá-Forcada 2014; Smejkal 2011; Soliman 2013; Stableforth 1984; Voigt 2011; Wirbel 1999; Zhang 2011; Zhu 2011; Zyto 1997). The majority of fractures were minimally displaced in Kristiansen 1989 and Rommens 1993. Lefevre-Colau 2007 included either minimally displaced or "stable" impacted fractures, the latter included two-part and three-part fractures. Torrens 2012 included either minimally displaced or displaced fractures (two-part or three-part fractures were reported). Fractures were graded using the Neer classification system (Neer 1970) in 28 trials, together with the AO classification system (Muller 1991) in Fialka 2008, Lefevre-Colau 2007 and Smejkal 2011. A modification of the AO classification system was described in Wirbel 1999 , and no specific classification system was referred to in the remaining two trials (Bertoft 1984; Rommens 1993).

\section{Interventions}

Eleven trials evaluated non-surgical treatment; however, this was post-surgical treatment in two of these. Eight trials compared surgical with non-surgical treatment and 12 compared two methods of surgery. A list of the comparisons, associated trials and numbers of trial participants, grouped according to the main objectives presented in the Objectives is given below. 
Methods of non-surgical management (including rehabilitation)

\section{Initial treatment, including immobilisation}

- "Immediate" physiotherapy within one week of fracture versus delayed physiotherapy after three weeks of immobilisation in a collar and cuff sling: Hodgson 2003 (86 participants).

- Immobilisation in sling and body bandage for one week versus three weeks: Kristiansen 1989 (85 participants).

- Physiotherapy started within three days of fracture versus delayed physiotherapy after three weeks of immobilisation in a sling: Lefevre-Colau 2007 (74 participants).

- Immobilisation in sling for one week versus four weeks; all followed same "progressive rehabilitation" regimen: Torrens 2012 (42 participants).

- Gilchrist arm sling versus "classic" Desault bandage: Rommens 1993 (28 participants).

\section{Continuing management (rehabilitation) after initial sling immobilisation}

- Instructed self-exercise versus conventional physiotherapy: Bertoft 1984 (20 participants); Lundberg 1979 (42 participants).

- Swimming pool treatment plus self-training versus selftraining alone: Revay 1992 (48 participants).

- Apparatus supplying pulsed electromagnetic field versus dummy apparatus: Livesley 1992 (48 participants).

\section{Surgical treatment versus non-surgical treatment}

The currently available trials fall into three subcategories but are all treated together in Effects of interventions.

\section{Fracture fixation versus non-surgical treatment}

- Percutaneous reduction and external fixation versus closed manipulation and sling: Kristiansen 1988 (30 participants).

- Internal fixation using surgical tension band or cerclage wiring versus sling: Zyto 1997 (40 participants; three more were recorded in Tornkvist 1995, another report of Zyto 1997).

- Surgery involving open reduction and fixation with a locking plate and metal cerclages versus non-surgical treatment starting with immobilisation of the injured arm in a modified Velpeau bandage: Fjalestad 2010 (50 participants).

- Surgery involving open reduction and fixation with a PHILOS plate and nonabsorbable sutures versus non-surgical treatment starting with arm immobilisation in a sling: Olerud 2011a (60 participants).

\section{Arthroplasty versus non-surgical treatment}

- Hemiarthroplasty using the Neer prosthesis versus closed manipulation and sling: Stableforth 1984 (32 participants).

- Humeral head replacement with the Global Fx prosthesis versus non-surgical treatment starting with arm immobilisation in a sling: Olerud $2011 \mathrm{~b}$ (55 participants).

- Humeral head replacement with the Global Fx prosthesis versus arm immobiliser alone: Boons 2012 (50 participants)

Surgery (surgeon's choice of method according to their experience) versus non-surgical treatment

- Surgery involving internal fixation (primarily locking plate fixation, most commonly PHILOS plate) or hemiarthroplasty versus sling: ProFHER 2015 (250 participants)

\section{Different methods of surgical management}

\section{Comparisons of different categories of surgical intervention}

- Open reduction with internal fixation using a locking plate (LPHP or PHILOS) versus a locking nail (PHN): Zhu 2011 (57 participants).

- Open reduction and internal fixation using a PHILOS plate versus the Zifko method of minimally invasive fixation with intramedullary K-wire (Kirschner wire) insertion (distally inserted): Smejkal 2011 (61 participants).

- Hemiarthroplasty using a DuPuy prosthesis versus open reduction and PHILOS plate fixation: Cai 2012 (32 participants)

- Hemiarthroplasty using a Global prothesis versus tension band wiring: Hoellen 1997 (30 participants); an additional nine participants were reported in another report of this trial (Holbein 1999).

- Reverse shoulder arthroplasty using the SMR Reverse prosthesis versus hemiarthroplasty using the SMR Trauma prosthesis: Sebastiá-Forcada 2014 (62 participants).

\section{Comparisons of different methods of performing an intervention in the same category}

- Deltoid-split approach versus deltopectoral approach for non-contact bridging plate fixation: Buecking 2014 (120 participants)

- Polyaxial versus monoaxial locking plate fixation. NCB-PH plate versus PHILOS plate: Ockert 2010 (76 participants; 124 in a later report of this trial (Ockert 2014)); and HSP plate versus PHILOS plate: Voigt 2011 (56 participants).

- Open reduction with internal fixation with PHILOS locking plate with or without the use of medial support locking screws: Zhang 2011 (72 participants). 
- MultiLoc proximal humeral nail (MPHN) - a straight nail versus Polarus humeral nail - a curved nail: Lopiz 2014 (54 participants)

- Hemiarthroplasty using an EPOCA prosthesis versus hemiarthroplasty using a HAS prosthesis: Fialka 2008 (40 participants).

- Hemiarthroplasty with tenodesis of the long head of the biceps (LHB) versus hemiarthroplasty with LHB tendon left intact: Soliman 2013 (45 participants)

\section{Continuing management (including rehabilitation) after surgical intervention}

- Immobilisation in sling for one week versus three weeks after percutaneous fixation: Wirbel 1999 (77 participants).

- Early active-assisted mobilisation (after two weeks) versus late mobilisation (after six weeks) after cemented hemiarthroplasty: Agorastides 2007 (59 participants).

\section{Outcomes}

Many trials in previous versions of this review preceded the availability of validated patient-reported outcome measures (e.g. DASH, Oxford Shoulder Score (Dawson 1996)) for assessing function. From the 2012 update of this review (Handoll 2012), data for these types of outcome have become available from a growing number of trials (Boons 2012; Cai 2012; Fjalestad 2010; Olerud 2011a; Olerud 2011b; ProFHER 2015; Sebastiá-Forcada 2014; Voigt 2011). All trials except Ockert 2010 assessed functioning and pain, but often reported these as part of a combined overall assessment, such as that of Neer (Neer 1970) and Constant (Constant 1987), that included other measures. Most trials reported on adverse events or complications. Exceptionally, Fjalestad 2010 and ProFHER 2015 reported on costs. Livesley $1992 \mathrm{did}$ not provide outcomes split by treatment group.

\section{Excluded studies}

Brief details and reasons for exclusion for 26 studies are given in the Characteristics of excluded studies. It is noteworthy that 11 excluded studies were trials that were registered (usually in the now archived National Research Register, UK) but either did not take place (Mechlenburg 2009) or were abandoned due to lack of or poor recruitment (Brownson 2001; Dias 2001; Flannery 2006; Hems 2000; Sinopidis 2010; Wallace 2000; Welsh 2000) or perhaps both of these (Pullen 2007); or not put forward for publication due to compromised methods or data (Bing 2002; Martin 2000). Edelson 2008 also reported an abandoned randomised trial because of lack of patient consent.

\section{Ongoing studies}

Details of the 21 ongoing trials are given in the Characteristics of ongoing studies. Two trials, both with three interventions under test, appear in two comparisons (NCT00999193; TPHF). Just two trials (aim 140 participants in total) compare different interventions, early versus late mobilisation or physiotherapy, for non-surgically treated patients (NCT00438633; Torrens). There are four trials (aim 580 participants in total) comparing surgical versus non-surgical treatment; three are multicentre trials (NCT00818987; ProCon; TPHF) and one is a singlecentre trial (NCT00999193). Three trials (aim 248 participants in total) are comparing nailing versus plating (NCT01557413; NCT01984112; NTR4019); three trials (aim approximately 385 participants in total) are comparing hemiarthroplasty versus plating (NCT00999193; HOMERUS; TPHF); one trial (aim 120 participants) is comparing reverse shoulder arthroplasty versus plating (DELPHI); and three trials (aim 142 participants in total) are comparing reverse shoulder arthroplasty versus hemiarthroplasty (NCT02075476; NTR3208; SHeRPA). Four trials are comparing different methods of performing an intervention in the same category of which two trials (aim 180 participants in total) are comparing minimally invasive versus usual methods of locking plate fixation (ACTRN12610000730000; HURA), one trial (aim 128 participants) is evaluating screw augmentation of locking plate fixation (NCT01847508) and one trial (aim 40 participants) is comparing two designs of reverse shoulder arthroplasty (NCT01086202). Lastly, two trials (aim 180 participants in total) are evaluating early versus standard rehabilitation after locking plate fixation (NCT01113411; NCT01524965) and one trial is comparing an external rotation brace versus a polysling with the arm in internal rotation (ROTATE).

\section{Studies awaiting classification}

Seven studies await classification (see the Characteristics of studies awaiting classification). There are insufficient data for Battistella 2011, reported in a conference abstract only. Brorson 2009, which was listed as ongoing in the 2012 version of the review was stopped after recruiting 25 participants; the use of these data is under discussion. Requests for clarification on study design have been sent to the contact authors of three trials testing bone grafts or substitutes (Liu 2011; Wang 2013; Zhu 2014). The full report of Luo 2008, which tests acupuncture and reports limited findings at one month follow-up, is in Chinese and we will seek translation of this article for a future update. NCT02052206, which is an ongoing trial, also includes patients with osteoarthritis.

\section{New studies found at this update}

Eight trials, including a total of 601 participants, were newly included in this update. One trial compared different methods of non-surgical treatment (Torrens 2012); two trials compared surgical with non-surgical treatment (Boons 2012; ProFHER 2015), 
and the other five trials compared different methods of surgery (Buecking 2014; Cai 2012; Lopiz 2014; Sebastiá-Forcada 2014; Soliman 2013).

\section{Risk of bias in included studies}

The risk of bias judgements on nine items for the individual trials are summarised in Figure 2 and described in the risk of bias tables in the Characteristics of included studies. A 'Yes' (+) judgement means that the authors considered there was a low risk of bias associated with the item, whereas a 'No' (-) means that there was a high risk of bias. Frequently assessments resulted in an 'Unclear' (?) verdict; this often reflected a lack of information upon which to judge the item (see Figure 3). However, lack of information on blinding for functional outcomes was always taken to imply that there was no blinding and rated as a 'No'. 
Figure 2. Risk of bias summary: review authors' judgements about each risk of bias item for each included study.

\begin{tabular}{|c|c|c|c|c|c|c|c|c|c|}
\hline & 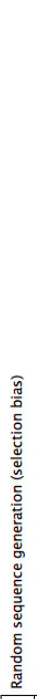 & 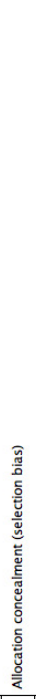 & 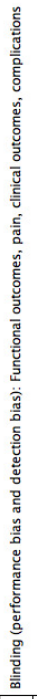 & 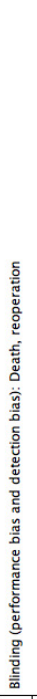 & 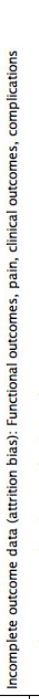 & 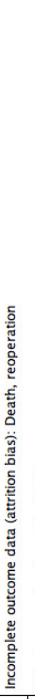 & 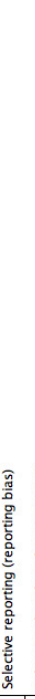 & 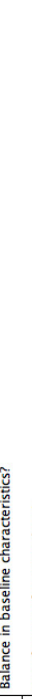 & 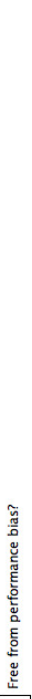 \\
\hline Agorastides 2007 & ? & $?$ & $?$ & $?$ & ? & - & - & $?$ & $?$ \\
\hline Bertoft 1984 & \begin{tabular}{|l|}
+ \\
\end{tabular} & 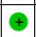 & $?$ & $\odot$ & $?$ & ๑ & $?$ & $?$ & $\odot$ \\
\hline Boons 2012 & (๑) & $\odot$ & O & $?$ & ๑ & $\odot$ & $?$ & $\oplus$ & $\odot$ \\
\hline Buecking 2014 & ๑) & 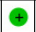 & - & - & ○ & $\odot$ & $?$ & + & (†) \\
\hline Cai 2012 & $?$ & $?$ & - & $?$ & $?$ & ๑ & + & $?$ & ๑ \\
\hline Fialka 2008 & \begin{tabular}{|l|l|}
$?$ \\
\end{tabular} & $?$ & ? & $?$ & - & $?$ & $?$ & $?$ & $\odot$ \\
\hline Fjalestad 2010 & + & + & - & $?$ & $?$ & ๑ & $?$ & $?$ & $\oplus$ \\
\hline Hodgson 2003 & \begin{tabular}{|l|}
$?$ \\
\end{tabular} & 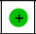 & $?$ & $?$ & (๑) & $\odot$ & $?$ & $?$ & + \\
\hline Hoellen 1997 & $?$ & $?$ & - & $?$ & - & ? & ○ & $?$ & $?$ \\
\hline Kristiansen 1988 & $?$ & $?$ & - & $?$ & - & ๑ & $?$ & + & $?$ \\
\hline Kristiansen 1989 & \begin{tabular}{|l|}
$?$ \\
\end{tabular} & $?$ & $?$ & ? & - & ○ & $?$ & $?$ & $?$ \\
\hline Lefevre-Colau 2007 & ๑) & $\oplus$ & $?$ & $?$ & \begin{tabular}{|l|l} 
\\
\end{tabular} & $?$ & $?$ & $\oplus$ & (4) \\
\hline Livesley 1992 & \begin{tabular}{|l|}
$?$ \\
\end{tabular} & $\oplus$ & + & $?$ & - & $?$ & $\odot$ & $?$ & $?$ \\
\hline Lopiz 2014 & + & 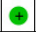 & - & $?$ & $?$ & $\oplus$ & $?$ & $\oplus$ & $\oplus$ \\
\hline Lundberg 1979 & \begin{tabular}{|l|}
$?$ \\
\end{tabular} & $?$ & - & $?$ & $?$ & $?$ & $?$ & $\oplus$ & + \\
\hline Ockert 2010 & \begin{tabular}{|l|}
$?$ \\
\end{tabular} & $?$ & ? & $?$ & - & $?$ & $\theta$ & $?$ & $\oplus$ \\
\hline Olerud 2011a & ๑) & $\odot$ & - & ? & ๑ & $\odot$ & $?$ & + & ( \\
\hline Olerud $2011 \mathrm{~b}$ & ๑ & ๑ & - & $?$ & ๑ & 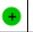 & $?$ & ๑ & $?$ \\
\hline ProFHER 2015 & ๑ & $\oplus$ & $?$ & (4) & ๑ & $\odot$ & ๑ & $\oplus$ & $\odot$ \\
\hline Revay 1992 & $?$ & $?$ & $?$ & $?$ & - & $?$ & $?$ & $?$ & $?$ \\
\hline Rommens 1993 & (? & ○ & అ & $?$ & \begin{tabular}{|l|l|}
$?$ \\
\end{tabular} & $?$ & - & $?$ & $?$ \\
\hline Sebastiá-Forcada 2014 & + & $\oplus$ & $?$ & (๑) & + & $\odot$ & $?$ & $?$ & ๑ \\
\hline Smejkal 2011 & + & 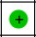 & ○ & $?$ & - & ? & $?$ & $?$ & $?$ \\
\hline Soliman 2013 & + & $?$ & $\oplus$ & $?$ & - & † & ? & $?$ & ๑ \\
\hline Stableforth 1984 & $?$ & $?$ & - & $?$ & - & $?$ & $?$ & $?$ & $?$ \\
\hline Torrens 2012 & \begin{tabular}{|c|}
+ \\
\end{tabular} & $?$ & ? & $?$ & (†) & 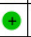 & $?$ & $?$ & $?$ \\
\hline Voigt 2011 & + & + & - & $?$ & $?$ & + & $?$ & $?$ & $?$ \\
\hline Wirbel 1999 & $?$ & $?$ & ? & $?$ & (2) & $?$ & $?$ & $\oplus$ & $\oplus$ \\
\hline Zhang 2011 & + & $?$ & $?$ & $\oplus$ & $?$ & $?$ & $?$ & $?$ & $\oplus$ \\
\hline Zhu 2011 & + & $?$ & - & $?$ & (†) & ๑ & $?$ & $?$ & 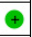 \\
\hline Zyto 1997 & $?$ & $?$ & (1) & $?$ & $?$ & ○ & ○ & $\oplus$ & ๑ \\
\hline
\end{tabular}


Figure 3. Risk of bias graph: review authors' judgements about each risk of bias item presented as percentages across all included studies.

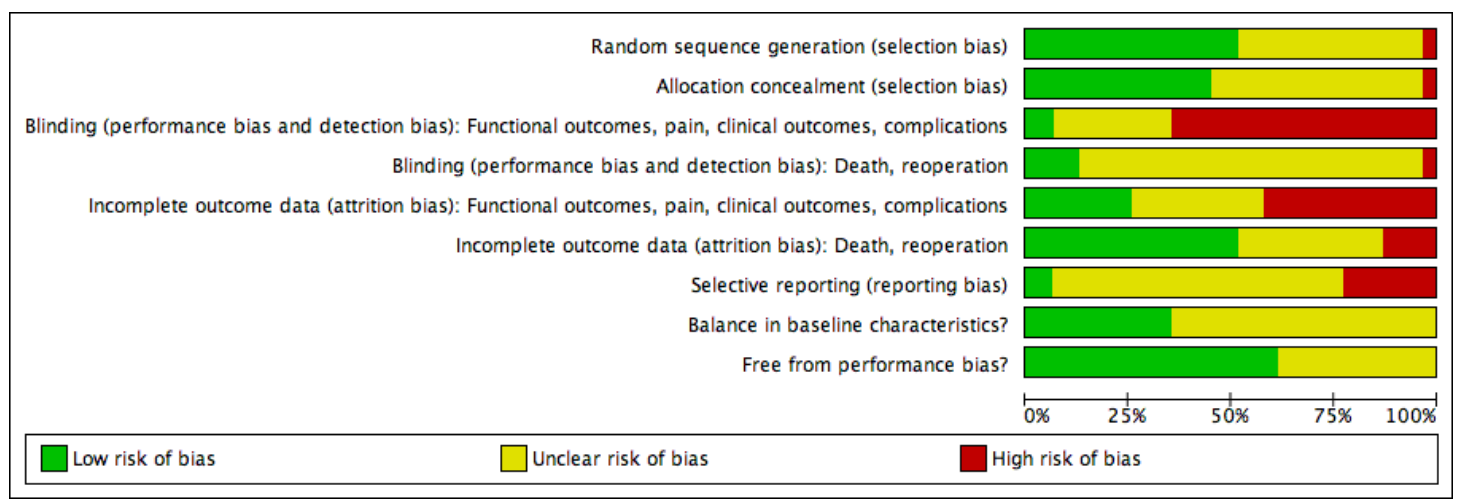

\section{Allocation}

Twelve trials were judged at low risk of selection bias resulting from adequate sequence generation and allocation concealment (Bertoft 1984; Boons 2012; Buecking 2014; Fjalestad 2010; Lefevre-Colau 2007; Lopiz 2014; Olerud 2011a; Olerud 2011b; ProFHER 2015; Sebastiá-Forcada 2014; Smejkal 2011; Voigt 2011); and a further two trials also took adequate measures to safeguard allocation concealment (Hodgson 2003; Livesley 1992). Based on its postrandomisation application of exclusion criteria, Ockert 2010 was judged at high risk of selection bias; as was Rommens 1993, which was a quasi-randomised trial using alternation.

\section{Blinding}

A low risk of detection bias for functional outcomes resulting from assessor and participant blinding was judged for Livesley 1992, which used sham controls, and Soliman 2013, where the intervention was very likely to have remained unknown to the blinded assessor of Constant scores. While several other trials reported blinded assessors, the lack of reporting of adequate safeguards and the lack of blinding of participants or care providers meant that the risk of bias was considered unclear. A high risk of bias reflecting no reporting or indication of blinding was likely in 21 trials. Exceptionally, ProFHER 2015, which did not blind trial participants, personnel or outcome assessment, was rated at 'unclear' risk of bias. This is because statistical tests showed a lack of a significant effect of baseline patient preferences on the primary outcome results (Oxford Shoulder Score).

\section{Incomplete outcome data}

Eight trials were considered to be at low risk of bias from the incompleteness of data on functional outcomes (Boons 2012; Hodgson 2003; Olerud 2011a; Olerud 2011b; ProFHER 2015; Sebastiá-Forcada 2014; Torrens 2012; Zhu 2011). Thirteen trials were deemed at high risk of bias, usually reflecting large losses to follow-up and post-randomisation exclusions.

\section{Selective reporting}

The lack of trial registration details and protocols hindered the appraisal of the risk of bias from selective reporting. Seven trials were considered at high risk of selective reporting bias (Agorastides 2007; Hoellen 1997; Livesley 1992; Ockert 2010; Rommens 1993; Soliman 2013; Zyto 1997).

\section{Other potential sources of bias}

\section{Baseline characteristics}

No trial was considered at high risk of bias because of confounding resulting from major imbalances in baseline characteristics. However, low risk of bias judgements were given for only 11 trials (Boons 2012; Buecking 2014; Kristiansen 1988; Lefevre-Colau 2007; Lopiz 2014; Lundberg 1979; Olerud 2011a; Olerud 2011b; ProFHER 2015; Wirbel 1999; Zyto 1997). 


\section{Care programmes}

Risk of performance bias from important differences in care programmes other than the trial interventions, or differences in the experience of care providers, was judged either low (19 trials) or unclear (in the other 12 trials), usually based on inadequate information.

\section{Effects of interventions}

See: Summary of findings for the main comparison Summary of findings: surgical versus non-surgical treatment for proximal humeral fractures; Summary of findings 2 Summary of findings: early versus delayed mobilisation for non-surgically treated proximal humeral fractures

Where available, outcome data reported at final follow-up for individual trials are presented in the analyses.

We based our judgement of clinically important between-group mean differences in the various patient-reported outcome measures (PROMS) using the following minimal clinically important differences (MCID); alternative sources and values are listed in the Primary outcomes. We decided that we would rescale MCIDs where a scoring system was rescaled but would not use these where the scoring instruments were modified, such as question removal.

- ASES (0 to 100: best outcome): 12.01 (Tashjian 2010; rotator cuff disease)

- Constant score (0 to 100: best outcome): 11.6 (Van de Water 2014; proximal humerus fracture)

- DASH (0 to 100: worst outcome): 13.0 (Van de Water 2014; proximal humerus fracture)

- EQ-5D (0 to 1: best outcome): 0.12 (Olerud 2011c; proximal humerus fracture)

- OSS (0 to 48: best outcome): 11.4 (Van de Water 2014; proximal humerus fracture)

- QuickDASH (0 to 100: worst outcome): 16 (DASH/ QuickDASH; general)

- SF-12-PCS (physical component score) (0 to 100: best outcome): 6.5 (Schmitt 2004; upper limb proximal diagnosis)

- SST (0 to 12: best outcome): 2.05 (Tashjian 2010; rotator cuff disease)

- UCLA (2 to 35: best outcome): 2.4 (Van de Water 2014; proximal humerus fracture)

\section{Methods of non-surgical management}

\section{Initial treatment, including immobilisation}

Five trials reported outcomes following initial treatment for nonsurgically managed proximal humeral fractures (Hodgson 2003; Kristiansen 1989; Lefevre-Colau 2007; Rommens 1993; Torrens 2012). All or most fractures were described as minimally displaced in three of these trials (Hodgson 2003; Kristiansen 1989; Rommens 1993). Both Lefevre-Colau 2007 and Torrens 2012 included displaced (two- or three-part) fractures; these were described as "stable" in Lefevre-Colau 2007 while Torrens 2012 put an upper limit to fracture displacement.

\section{Early mobilisation versus delayed mobilisation}

Although four trials compared early versus delayed mobilisation (Hodgson 2003; Kristiansen 1989; Lefevre-Colau 2007; Torrens 2012), the timing of the start of early mobilisation varied as, where described, did the nature and intensity of the physiotherapy provided. Notable is the long (two hour) duration of individual physiotherapy sessions of Lefevre-Colau 2007. With three exceptions, the lack of comparable outcome measurement and data precluded data pooling and so the results of the individual trials are presented separately below.

Hodgson 2003 compared commencing physiotherapy within one week of fracture versus delayed physiotherapy after three weeks of immobilisation in a collar and cuff sling in 86 people with minimally displaced fractures. The results, presented in Hodgson 2007 for self-reported shoulder disability using the Croft Shoulder Disability Questionnaire (Croft 1994), show a tendency for less disability in the early mobilisation group at one year (e.g. disability ( 1 or more problems): $18 / 42$ versus 29/40; RR 0.59 , 95\% CI 0.40 to 0.88 ; severe disability ( 5 or more problems): $13 / 42$ versus $17 /$ 40; RR 0.73 , 95\% CI 0.41 to 1.30 ), continuing improvement and recovery between one and two years, and also reveal that, overall, a substantial proportion of participants continued to report some or severe disability at two years (see Analysis 1.1). Results at two years for eight of the 22 questions of the Croft questionnaire are shown in Analysis 1.2. These are presented to give an indication of the variety of problems experienced by these patients and the variation in the responses. There was some evidence supporting a quicker recovery in the early group as trial participants given early physiotherapy attended fewer treatment sessions (see Analysis 1.3: mean difference (MD) -5.00 sessions; $95 \%$ (CI) -8.25 to 1.75) until they and their physiotherapists agreed that independent shoulder function had been achieved. As can be seen in Analysis 1.4 , participants of the early group had significantly better healthrelated quality-of-life scores at 16 weeks in two dimensions of the SF36 (role limitation physical: MD 22.20, 95\% CI 3.82 to 40.58; and pain: MD $12.10,95 \%$ CI 3.26 to 20.94 ). There were no statistically significant differences between the two treatment groups in the other six dimensions (e.g. physical functioning) of the SF36 at 16 weeks, and in all eight dimensions at one year. There were no complications arising from fracture displacement. The only recorded complication in the trial was a frozen shoulder in a participant of the delayed physiotherapy group (see Analysis 1.6). Shoulder function, relative to the unaffected shoulder, measured using the Constant score (Constant 1987) was better at 8 and 16 weeks in the early group (see Analysis 1.8: mean difference in ratio affected/unaffected arm $0.16 ; 95 \%$ CI 0.07 to 0.25$)$. The betweengroup differences were smaller at one year and the confidence 
intervals crossed the line of no effect (MD $0.07,95 \%$ CI -0.03 to $0.17)$.

Kristiansen 1989, which tested the duration of immobilisation in a sling and body bandage (one week versus three weeks) in 85 people with mainly undisplaced fractures, provided insufficient followup data to allow any test for statistical significance. The authors reported that while pain, function and mobility at six months and over were similar in both groups, the patients who started early mobilisation at one week suffered less pain in the first three months than those who kept their bandaging for three weeks. One case of complex regional pain syndrome type 1 (CRPS-1) occurred in each group (see Analysis 1.6).

Lefevre-Colau 2007 compared commencing physiotherapy within three days of fracture with delayed physiotherapy after three weeks of immobilisation in a sling in 74 people with minimally displaced or "stable" impacted fractures. Ten trial participants withdrew from the trial because of difficulties in reaching the hospital for treatment. Participants were discharged from physiotherapy at six months. Shoulder function measured using the Constant score was statistically significantly better in the early group at six weeks and three months (see Analysis 1.9), with the differences probably including a clinically relevant effect; the differences at six months and end of treatment, though favouring the early group, were smaller and not statistically significant (MD 6.10, 95\% CI -0.22 to 12.42 ). Although the early group had significantly reduced pain compared with the three weeks group by three months follow-up, there was no difference at six months (see Analysis 1.11). Active range of motion, measured relative to the opposite arm, also did not differ significantly between the two groups at six months (see Analysis 1.12). There were no cases of fracture non-union or displacement. One participant from each group received treatment for subacromial impingement (see Analysis 1.6). All participants attended at least $70 \%$ of the supervised physiotherapy sessions; and very few expressed dissatisfaction with their treatment (see Analysis 1.13).

Torrens 2012 compared sling immobilisation for one week versus four weeks in 42 people with minimally displaced or displaced two- or three-part fractures; all participants had the same "progressive rehabilitation" regimen. Results were reported at 3, 6 and 12 months. Participants in the four-weeks group had consistently higher quality-of-life scores (EuroQol 5D) at all three follow-ups (e.g. MD -0.09, 95\% CI -0.21 to 0.03 ; see Analysis 1.5). All three results include a clinically important difference in quality of life in favour of the four-weeks group but also the possibility of a much smaller and clinically unimportant effect favouring the early group. Torrens 2012 reported no complications aside from noting that the three participants (two early mobilisation versus one, four-weeks immobilisation) experiencing a "significant displacement" of their fracture did not require surgical treatment (see Analysis 1.6). One person had died in the four-weeks group by 12 months follow-up (see Analysis 1.7). The evidence from Torrens 2012 did not confirm differences between the two groups at any of the three follow-ups in Constant scores (see Analysis 1.9), pain (see Analysis 1.10) or patient satisfaction (see Analysis 1.14). Of note though is that the confidence intervals of the pain score results at 12 months included a clinically important difference in favour of the four-weeks group (MD 10.80, 95\% CI -4.59 to 26.19); these also crossed the line of no effect.

The exceptions in terms of pooling were data for the adverse events, when pooled under 'shoulder complications' and 'fracture complications', for four and two trials respectively, and the secondary outcomes of pain and Constant scores available from two trials. Data pooled for reported shoulder complications that comprised frozen shoulder, CRPS-1 and treated subacromial impingement showed little difference between the two groups (2/127 versus 3/ 132; RR 0.73 , 95\% CI 0.15 to 3.63; 4 trials, 259 participants). Two trials reporting on fracture complications found no cases of non-union and only one trial (Torrens 2012) reported actual cases of fracture displacement (2/52 versus 1/54; RR 2.20, 95\% CI 0.22 to $22.45 ; 2$ trials; 106 participants). Both analyses of Constant score and pain (see Analysis 1.9; Analysis 1.10) display evidence of statistical heterogeneity, which may in part reflect clinical heterogeneity of the contributing trials (Lefevre-Colau 2007; Torrens 2012).

\section{Gilchrist arm sling versus the Desault bandage}

Rommens 1993 compared the use of two types of immobilisation, the Gilchrist arm sling versus the Desault bandage, worn for two to three weeks in 28 patients with mainly minimally displaced fractures. Reporting up until fracture consolidation, Rommens 1993 reported, without presenting data, that they had found no differences in the end result, either in terms of functional outcome or fracture healing. More people found the initial application of a Desault bandage uncomfortable and severe skin irritation prompted premature removal of the bandage in two people in this group (see Analysis 2.1). Pain during immobilisation was also reported to be greater in the Desault group. Slight displacement of the fracture in the first week was reported in two participants of the Gilchrist group (see Analysis 2.2). At fracture consolidation, patients' rating of their assigned bandage was significantly more favourable in the Gilchrist group (see Analysis 2.3 "Poor or bad rating by patient at fracture consolidation": $2 / 14$ versus $8 / 14$; risk ratio (RR) 0.25 , $95 \%$ CI 0.06 to 0.97 ).

\section{Continuing management (rehabilitation) after initial sling immobilisation}

Two small trials (Bertoft 1984; Lundberg 1979) compared selfdirected exercise following a course of instruction versus conventional physiotherapy during the 12 weeks following trauma in a total of 62 patients with minimally displaced fractures. In both trials there were no statistically significant differences between those receiving instruction for exercises at home and those undergoing 
supervised physiotherapy in any of the outcomes recorded (see Analysis 3.1, Analysis 3.2, Analysis 3.3, Analysis 3.4, Analysis 3.5 and Analysis 3.6). It should be noted that since Lundberg 1979 did not report whether there had been any loss to long-term follow-up at an average of 16 months, the results for Neer's score presented in Analysis 3.5 are for illustrative purposes only.

Revay 1992, which included 48 participants with minimally displaced fractures, reported that the addition of supervised exercises in a swimming pool to self-treatment did not enhance long term outcome. Participants of the control group (self-treatment only) were reported as having significantly better functional movements, joint mobility and activities of daily living at two and three month follow-up. However, there were no significant differences at one year. Revay 1992 suggested that those using the pool may have neglected their home exercises, but the authors did not evaluate compliance.

Livesley 1992, which included 48 patients with minimally displaced fractures, reported that there was no difference in outcome between the two groups (pulsed electromagnetic high frequency energy (PHFE) versus placebo) at any stage of the trial, but provided no quantitative data. All trial participants were reported as achieving a "good" result as converse to a "poor" one.

\section{Surgical treatment versus non-surgical treatment}

Eight heterogeneous trials, with a total of 567 participants and 568 fractures, evaluated surgical intervention for displaced fractures, of which over $73 \%$ were three- or four-part fractures (Neer classification). Table 1 gives a brief summary of their characteristics. The methods of surgery varied between the trials, being restricted to hemiarthroplasty in three trials (Boons 2012; Olerud 2011b; Stableforth 1984), internal fixation in three trials (Fjalestad 2010; Olerud 2011a; Zyto 1997) and external fixation in Kristiansen 1988. Most surgery involved internal fixation in ProFHER 2015, where the surgeons used methods with which they were experienced. Non-surgical treatment was predominantly sling immobil- isation; this was preceded by closed manipulation in all participants in two trials (Kristiansen 1988; Stableforth 1984) and in eight participants in Fjalestad 2010.

\section{Primary outcomes}

Pooled results of four different patient-reported functional scores reported by five trials (Boons 2012; Fjalestad 2010; Olerud 2011a; Olerud 2011b; ProFHER 2015) at 12 months follow-up showed no statistically significant difference between the two groups (standardised mean difference (SMD) 0.07 favouring surgery, 95\% CI -0.12 to $0.26 ; \mathrm{P}=0.46 ; 419$ participants, see Analysis 4.1; and Figure 4). The same finding, which was based on data for three scores reported by four trials, of no significant between-group difference applied at 24 months (SMD 0.07 favouring surgery, 95\% CI -0.14 to $0.28 ; \mathrm{P}=0.50 ; 351$ participants; see Analysis 4.2). The Oxford Shoulder Score (OSS) results for ProFHER 2015 showed no clinically important (the MCID for the OSS was set at 5 points in this trial) or statistically significant differences between the two groups over the two-year follow-up (MD 0.75, 95\% CI 1.68 to $3.18 ; \mathrm{P}=0.55 ; 231$ participants) or at 6,12 or 24 months (see Analysis 4.3). Pooled DASH scores from Olerud 2011a and Olerud 2011b showed no statistically significant differences between the two groups at four months, or at one or two years (see Analysis 4.4); although the scores favoured surgery, the best estimate at 24 months was still lower than the MCID (10 points) for DASH (0 to 100: worst function): MD $-7.43,95 \%$ CI -16.26 to 1.41 ; 99 participants). Fjalestad 2010 found no significant differences between the two groups in the American Shoulder and Elbow Surgeons (ASES: 0 to 24: best function) scores at either 6, 12 or 24 months follow-up (see Analysis 4.5). Boons 2012 found no significant differences between the two groups in the Simple Shoulder Test scores at 3 or 12 months (see Analysis 4.6). There were no statistically significant differences in subjective assessment of function between the two groups of Zyto 1997 at either one or three years (see Analysis 4.7). 
Figure 4. Forest plot of comparison: 4 Surgical versus non-surgical treatment, outcome: 4.I Functional scores at 12 months (higher = better outcome).

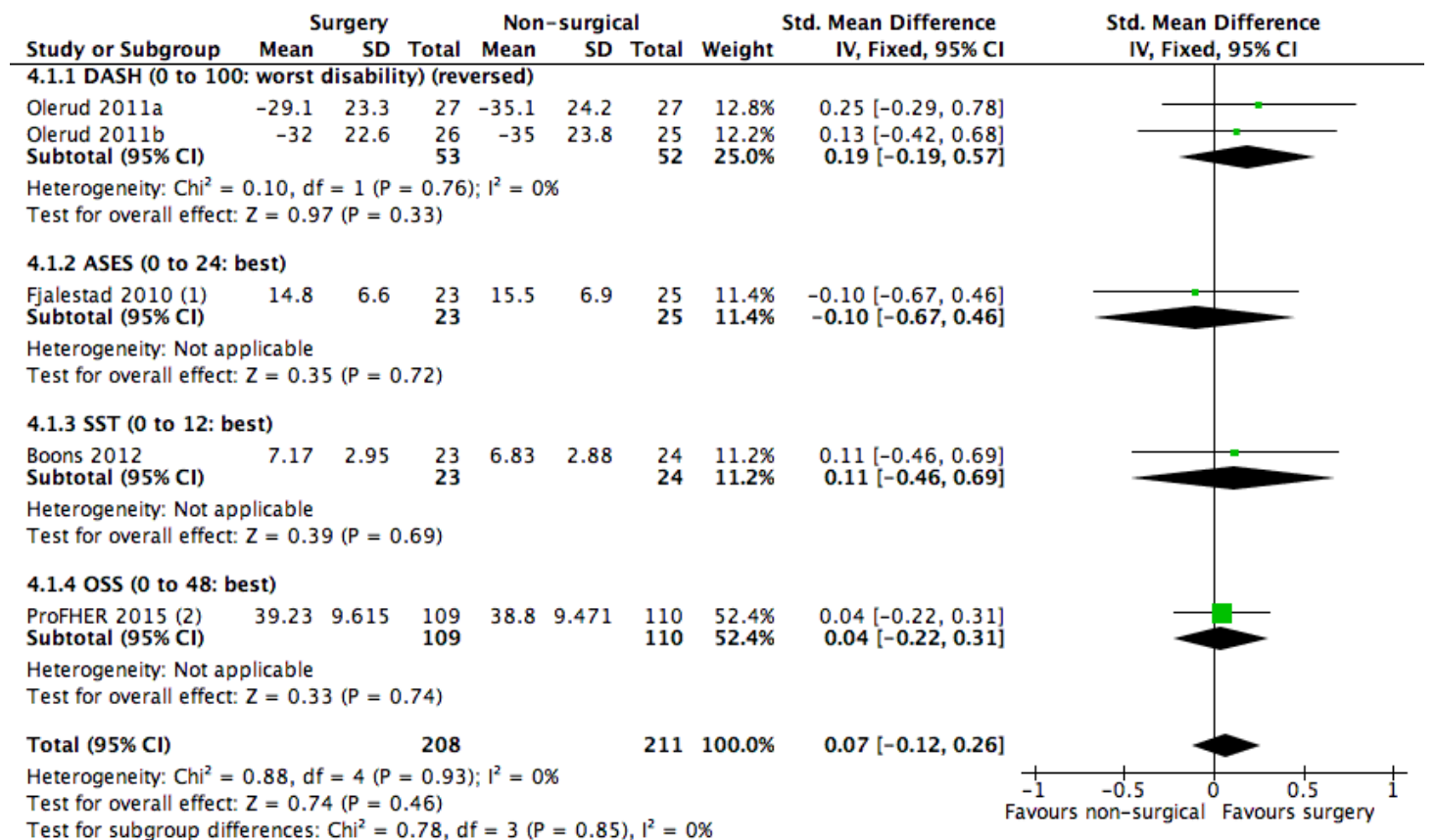

Quality of life based on the EuroQol scores from three trials (Olerud 2011a; Olerud 2011b; ProFHER 2015) and 15D (Sintonen 2001) results from Fjalestad 2010 were slightly higher in the surgery group but none of the between-group differences were clinically important including the statistically significant finding at six months (MD 0.04, 95\% CI 0.01 to $0.08 ; \mathrm{P}=0.02 ; 381$ participants; see Analysis 4.8). A separate breakdown of the results from Fjalestad 2010, which include the number of quality of life years (QALYs), showed no differences in any quality-of-life outcomes for this trial (see Analysis 4.9). Based on data adjusted for covariates, ProFHER 2015 reported there were also no significant between-group differences over two years in the mean SF12 physical component score (MD 1.77 favouring surgery, 95\% $\mathrm{CI}-0.84$ to 4.39 points; reported $\mathrm{P}=0.18$ ) and the mean SF-12 mental component score (1.28 favouring non-surgical treatment, $95 \% \mathrm{CI}-3.80$ to 1.23 ; reported $\mathrm{P}=0.32$ ). The $\mathrm{SF}-12$ physical component scores ( 0 to 100 : best outcome) were slightly higher in the surgery group at all three follow-ups (see Analysis 4.10) and, conversely, the SF-12 mental component scores (0 to 100 : best outcome) were slightly higher in the non-surgical treatment group at all three follow-ups (see Analysis 4.11). None of these differences was statistically significant and the confidence interval limits are less than the minimal clinically important difference. There was no significant difference between the two groups in mortality (17/248 versus 12/248; RR 1.40 favouring non-surgical treatment, $95 \%$ CI 0.69 to 2.83 ; $\mathrm{P}=0.35 ; 6$ trials, see Analysis 4.12). Where reported, none of the deaths was related to their fracture or treatment with the exception of one early death due to venous thromboembolism in the surgical group of ProFHER 2015. Notably, the two deaths that occurred within three months of surgery in Fjalestad 2010 were people with underlying health problems. In Zyto 1997, eight of the 11 missing participants had died at 50 months, but no information on group allocation or causes of death was provided.

Significantly more surgical group patients had additional or secondary surgery (34/262 versus 16/261; RR 2.06, 95\% CI 1.18 to 3.60; 7 trials; see Analysis 4.13). In Boons 2012, one surgical group participant underwent revision surgery after one week because of head-stem separation. A non-surgically treated participant in Boons 2012 who had surgery at 13 months, thus outside the trial's follow-up period, because of shoulder pain and impairment was not included in this analysis. In Fjalestad 2010, treatment failure resulting in an operation occurred in eight surgical group participants, one of whom had re-fixation plus bone grafting at six months and seven whose implants were removed because of screw penetration into the joint space; and one non-surgically treated patient, who had surgery because of fracture redisplacement at two 
weeks. In Kristiansen 1988, the three cases of treatment failure were the removal of pins due to infection in one surgical group participant and a change of method resulting from a poor initial fracture reduction in two non-surgical group participants. The reasons for re-operations in the surgical group of Olerud 2011a were deep infection (two cases), non-union (one case), impingement (two cases), avascular necrosis (one case), screw penetration into joint (one case) and stiffness (two cases). One non-surgically-treated patient in Olerud 2011a had surgery because of impingement. Not included in this analysis is another non-surgically-treated patient with non-union who abstained from surgery partly because of a late diagnosis of axillary nerve palsy. The reasons for additional surgery in Olerud 2011b were screw penetration of the joint (for one patient treated with a locking plate), stiffness and impingement and displaced greater tuberosity respectively in three surgical group patients, and for complete displacement of the humeral shaft without bony contact in one non-surgically treated patient. Not included in this analysis is another non-surgically-treated patient who refused surgery for a non-union. The reasons for further surgery in the surgical group of ProFHER 2015 were avascular necrosis (two cases), metalwork problems (seven cases) and post-traumatic stiffness (two cases). The reasons for subsequent surgery in the non-surgical treatment group of ProFHER 2015 were avascular necrosis (one case), malunion (two cases), nonunion (four cases), post-traumatic stiffness (one case), rotator cuff tear (one case), severe pain (one case) and not-reported (one case). In Stableforth 1984, one surgical group participant had their prosthesis removed because of a deep infection. Only ProFHER 2015 reported on additional shoulder-related therapy, which occurred in slightly more participants of the surgery group (7/125 versus $4 /$ 125; RR 1.75 favouring non-surgical treatment, $95 \%$ CI 0.53 to 5.83; $\mathrm{P}=0.36$; see Analysis 4.14).

The numbers of people in each group with one or more adverse events or complications were available only in ProFHER 2015 (30/125 versus 23/125; RR 1.30 favouring non-surgical treatment, $95 \%$ CI 0.80 to $2.11 ; \mathrm{P}=0.28$; see Analysis 4.14). Analysis 4.14 also presents the available data for individual complications. Unsurprisingly, surgery-related complications (e.g. infection and screw penetration of the joint) were predominant in the surgery treatment group. While non-union was more common in the nonsurgical treatment group $(\mathrm{P}=0.05)$, none of the differences between the two groups in the radiologically detected outcomes of avascular necrosis and signs of osteoarthritis were statistically significant. For avascular necrosis, data favouring surgery from Boons 2012 and Olerud 2011b needs to be seen in the context that these were only likely to be detected in non-surgically treated patients, given that surgery involved the replacement of the humeral head. Additionally, some of these outcomes were without symptoms or minor in extent. For instance, in Fjalestad 2010, both cases of nonunion in the non-surgical treatment group were without symptoms, and 22 of the 27 participants with radiographically-detected avascular necrosis were asymptomatic.
In Stableforth 1984, fewer participants of the prosthesis group needed some help with activities of daily living or had died by six months (see Analysis 4.15: 2/16 versus 9/16; RR 0.22, 95\% CI 0.06 to 0.87$)$.

\section{Secondary outcomes}

The differences between the two groups in the Constant scores (0 to 100: best outcome) at four different time points (3-4, 12, 24 and 50 months) were all small and clinically not important (e.g. the most data were for 12 months: MD 2.81, 95\% CI 2.20 to 7.82 ; 199 participants, 4 trials; see Analysis 4.16). The same lack of differences between the two groups applied to the Constant scores of the injured arm in Fjalestad 2010 at 6, 12 and 24 months follow-up (see Analysis 4.17). At one year followup in Kristiansen 1988, fewer participants of the surgical group had a poor or unsatisfactory rating of function assessed using the Neer score (3/11 versus 6/10; RR 0.45, 95\% CI 0.15 to 1.35 ; see Analysis 4.18).

Boons 2012 reported similar results in the two groups for patientassessed disability based on a 0 to 100 VAS scale; where the maximum score equated to "no restrictions". The clinical relevance of the results, which were in favour of the surgical group, is uncertain (see Analysis 4.19).

Boons 2012 reported lower pain scores, measured using VAS (0 to 100: higher scores mean worse pain), in the hemiarthroplasty group at three months (MD -18.00, 95\% CI -29.03 to -6.97; 49 participants; see Analysis 4.20) than in the non-surgical group; this difference is likely to be clinically important. In contrast, there were similar results in the two groups at 12 months (median 23 in the surgery group versus 25 in the non-surgical group; reported $\mathrm{P}=0.725$ ). Pooled results from two trials (Olerud 2011a; Olerud 2011b) showed slightly less pain at two year follow-up in the surgery group (MD -6.38 ; 95\% CI -14.18 to $1.41 ; 101$ participants; see Analysis 4.20); the clinical importance of this result is questionable. Nearly all trial participants in Stableforth 1984 had shoulder pain but fewer in the prosthesis group reported constant pain that impaired sleep or function (see Analysis 4.22: 2/15 versus 9/15; RR 0.22 , 95\% CI 0.06 to 0.86 ). The categorisation of pain is not clear in the trial report nor whether pain was assessed for all participants. Assuming the latter is the case, the difference between the two groups is less marked when all those with more than occasional pain are included (4/15 versus 9/15; RR 0.44, 95\% CI 0.17 to 1.13 ; analysis not shown). Zyto 1997 , which provided a breakdown of the Constant score into the separate components (activities of daily living, pain, range of motion, strength), did not confirm a significant difference between the two groups in the pain component, which was in favour of the non-surgical treatment group, at 50 months (see Analysis 4.21).

Reduced muscle strength and restricted mobility were less frequent in the prosthesis group survivors of Stableforth 1984 (see Analysis 4.23 and Analysis 4.24) than in the group receiving closed manipulation and sling. Zyto 1997 found no difference between the two 
groups in strength ('power') at 50 months follow-up. The clinical relevance of the three point difference in the range of motion component of the Constant score in favour of non-surgical treatment is questionable (see Analysis 4.21). In Boons 2012, abductor strength, reported as a percentage of the opposite shoulder, was lower in the surgery group at both three months (median values: $20 \%$ versus $30 \%$; reported $\mathrm{P}=0.015$ ) and 12 months (median values: $24 \%$ versus $42 \%$; reported $\mathrm{P}=0.008)$. Boons 2012 also found that forward flexion (median 68 versus 88 degrees; reported $\mathrm{P}=0.001$ ) and abduction (median 61 versus 78 degrees; reported $\mathrm{P}=0.02$ ) were worse in the surgery group at three months. There were no between-group differences in external rotation and internal rotation at this time, nor for all four range of motion measures at 12 months).

Fjalestad 2010 found no differences at one year between the two groups in costs (see Analysis 4.25 and Analysis 4.26). The base case economic analysis of ProFHER 2015 showed that at two years, the cost of surgical intervention was, on average, GBP 1,780.73 more per patient (95\% CI GBP 1,152.71 to GBP 2,408.75).

\section{Different methods of surgical management}

\section{Comparisons of different categories of surgical intervention}

Five trials compared different methods of surgical management (Cai 2012; Hoellen 1997; Sebastiá-Forcada 2014; Smejkal 2011; Zhu 2011).

\section{Open reduction with internal fixation using a locking plate versus a locking nail}

Zhu 2011 compared open reduction with internal fixation using a locking plate (LPHP or PHILOS) versus a locking nail (PHN) in 57 participants with two-part surgical neck fractures. The American Shoulder and Elbow Surgeon's scores were statistically significantly better in the plate group at one year (MD 7.20; 95\% CI 1.48 to 12.92 ) and three years (MD $4.00 ; 95 \%$ CI 0.01 to 7.99 ) (see Analysis 5.1). The clinical importance of these findings, however, is uncertain given the MCID for ASES is included only in the 95\% CI at one year follow-up. One participant of the nail group died of unrelated causes. While complications were not described in full, significantly more patients in the plate group had a complication (9/29 versus $1 / 28$; RR 8.69 , 95\% CI 1.18 to 64.19; see Analysis 5.2). This included five patients in the plate group who had a re-operation for screw penetration into the articular surface of the humeral head. Zhu 2011 found a statistically significant but probably not a clinically important difference in favour of the plate group in pain at one year but not at three years (see Analysis 5.3). There were no statistically significant differences between the two groups in the Constant scores at the two follow-up times (see Analysis 5.4) or in range of motion measures at either one year (not shown) or three years (see Analysis 5.5 and Analysis 5.6). Although the plate group had greater muscle strength at one year, the difference between the two groups was no longer statistically significant at three years (see Analysis 5.7). Both duration of surgery (MD 24.90 minutes, 95\% CI 5.97 to 43.83 minutes) and blood loss were statistically significantly greater in the plate group (see Analysis 5.8). Consistent with the finding of an increased blood loss in the plate group, more people in this group had a blood transfusion but the difference between the two groups was not statistically significant (see Analysis 5.9).

\section{Open reduction with internal fixation using a locking plate versus minimally invasive fixation with distally inserted intramedullary K-wires}

Smejkal 2011 compared open reduction and internal fixation using a PHILOS plate versus the Zifko method of minimally invasive fixation with distally inserted intramedullary K-wires (Kirschner wires) in 61 participants with two- or three-part fractures. Smejkal 2011 did not report patient-reported function or activities of daily living. The account of the complications seemed incomplete, with no indication of how many required a re-operation but this was perhaps partly due to difficulties in translation from Czech to English. There was no significant difference between the two groups in the overall numbers of participants incurring a complication (11/28 versus 9/27; RR 1.18, 95\% CI 0.58 to 2.38; see Analysis 6.1 The recorded nature of the complications reflected the type of implant, with four cases of screw protrusion in the plate group that resulted in impingement and migration of $\mathrm{K}$-wires, a distal humeral fracture and a nerve injury in the Zifko group. Smejkal 2011 found no difference between the two groups in Constant scores relative to the healthy limb at a mean two years follow-up (MD -0.81\%, 95\% CI -7.45\% to 5.83\%; see Analysis 6.2). Three participants of each group had a 'poor' Constant score. Analysis 6.3 shows there were no statistically significant differences between the two groups in time to union (MD 2.10 weeks, 95\% CI -2.25 to 6.45 weeks) or in a vaguely-described measure of time to recover normal upper limb function (27.2 versus 21.4 weeks; MD 5.80 weeks; $95 \%$ CI -0.16 to 11.76 weeks). Smejkal 2011 suggested that the greater time to recover in the plate group reflected a greater impact of complications in this group. The duration of operation was significantly greater in the plate group (MD 44.74 minutes, 95\% CI 32.23 to 57.25 minutes; see Analysis 6.4), but with a non-significant tendency for less X-ray exposure. The tendency for longer hospital stays for plate group patients did not achieve statistical significance (MD 1.20 days; 95\% CI -0.34 to 2.74; see Analysis 6.5).

\section{Hemiarthroplasty versus internal fixation}

Two small heterogeneous trials compared humeral head replacement versus internal fixation for four-part fractures (Cai 2012; 
Hoellen 1997). Only data for re-operation were available for pooling; these favoured hemiarthroplasty ( $3 / 34$ versus $8 / 28$; RR 0.32 , $95 \%$ CI 0.10 to 1.10 ) but were moderately heterogeneous (heterogeneity: $\mathrm{Chi}^{2}=1.82$, degrees of freedom $(\mathrm{df})=1(\mathrm{P}=0.18) ; \mathrm{I}^{2}$ $=45 \%$; see Analysis 7.3). Given this, the results of the two trials are presented separately.

\section{Hemiarthroplasty versus open reduction and locking plate fixation:}

Cai 2012, which compared hemiarthroplasty with open reduction and PHILOS plate fixation in 32 participants with four-part fractures, reported outcome at 4,12 and 24 months. Although DASH scores at one and two years favoured the hemiarthroplasty group, the mean differences were smaller than the MCID of 13 for DASH (at 12 months: MD -7.30, 95\% CI -16.70 to 2.10, 28 participants; at 24 months: MD $-6.10,95 \%$ CI -11.03 to 1.17, 27 participants; see Analysis 7.1). Although favouring the hemiarthroplasty group, the differences between the two groups in quality of life measured via the EQ-5D were not clinically or statistically significant at any of the three follow-up times (see Analysis 7.2). Re-operations were reported for three participants in the hemiarthroplasty group (one dislocation, one prosthesis loosening, one infection) and three participants in the fixation group (one non-union, two fixation failure); RR $0.68,95 \%$ CI 0.16 to 2.88; see Analysis 7.3). One person in the hemiarthroplasty group had died by two years (see Analysis 7.4). The Constant scores were higher in the hemiarthroplasty group at all three follow-ups; in particular, the $95 \%$ confidence interval at two years included a clinically important effect (MD 12.20, 95\% CI 2.85 to 21.55; 27 participants; see Analysis 7.6). While the results at two years for pain and range of motion favoured hemiarthroplasty, Cai 2012 found no statistically significant between-group differences in either of these outcomes (see Analysis 7.7 and Analysis 7.9). The mean time of surgery was slightly longer in the hemiarthroplasty group (93 minutes versus 86 minutes).

\section{Hemiarthroplasty versus tension band wiring}

Hoellen 1997 compared hemiarthroplasty versus reduction and stabilisation of the fracture using tension band wiring. All $30 \mathrm{pa}-$ tients reported in Hoellen 1997 had four-part fractures. Patients with three-part fractures were also eligible according to a later report of the trial (Holbein 1999), which reported on 39 patients. However, until we obtain further information from the trialists, we will continue to report the results from Hoellen 1997. In Hoellen 1997, results for only 18 of the 30 trial participants were available at one year. There were no serious peri-operative or post-operative complications such as pulmonary embolism. No participants of the replacement group required further surgery compared with five participants of the osteosynthesis group (the wires displaced in four participants and the fracture completely dislocated in one participant): RR 0.09, 95\% CI 0.01 to 1.51 (see Analysis 7.3). Implants were removed in four participants of the osteosynthesis group (see Analysis 7.5). The mean Constant scores (minus the power component) for the 18 people available at one year follow-up were similar in the two groups ( 48 versus 49 points out of a maximum of 75). Two participants of the hemiarthroplasty group and one in the fixation group reported pain at one year (see Analysis 7.8). Though we have not obtained clarification on the inadequately reported results presented in Holbein 1999, these did not appear to differ in a major way from those in Hoellen 1997.

\section{Reverse shoulder arthroplasty versus hemiarthroplasty}

Sebastiá-Forcada 2014 compared reverse shoulder arthroplasty with hemiarthroplasty in 62 participants with either three- or fourpart fractures, some of which included dislocation. Follow-up was between 24 and 49 months. Patient-reported upper-limb function pain assessed using the Quick DASH (0 to 55: worst outcome) was superior in the reverse arthroplasty group: MD -6.90, 95\% CI -10.81 to -2.99 (see Analysis 8.1). One participant in the reverse arthroplasty group was re-operated because of deep infection compared with six participants in the hemiarthroplasty group reoperated because of proximal migration of implant (1/31 versus 6/ 31; RR 0.17 favouring reverse arthroplasty, $95 \%$ CI 0.02 to 1.30 ; see Analysis 8.2). All seven participants received a reverse shoulder arthroplasty. No deaths occurred in this trial. University of California-Los Angeles scores and Constant and adjusted Constant scores all favoured the reverse arthroplasty group (see Analysis 8.4). A similar finding applied to pain, range of motion, power and activities of daily living components of the Constant score (see Analysis 8.5). Fewer participants had a complication in the reverse arthroplasty group compared with the hemiarthroplasty group (2/ 31 versus 10/30; RR 0.19, 95\% CI 0.05 to 0.81 ; see Analysis 8.6 footnotes for actions taken to treat the individual complications). The findings of radiological assessment (see Analysis 8.7) did not confirm a difference between the two groups in malunion or resorption of tuberosities. The one case of scapular notching in the reverse arthroplasty group was without clinical consequence, as were the 11 cases of heterotopic ossification. Anterior forward and abduction were superior in the reverse arthroplasty group (Analysis 8.8).

\section{Comparisons of different methods of performing an intervention in the same category}

Seven trials compared different types or methods in the same intervention category (e.g. plating) (Buecking 2014; Fialka 2008; Lopiz 2014; Ockert 2010; Soliman 2013; Voigt 2011; Zhang 2011). 


\section{Deltoid-split approach versus deltopectoral approach for non-contact bridging plate fixation}

Buecking 2014, which made this comparison in 120 people with Neer two-, three- or four-part fractures, reported results for activity of daily living at 6 and 12 months based on a score by Lawton (Lawton 1969). However, the trialists appear not to have used the scoring system correctly and reported scores that are greater than the maximum score of 8 . There was no statistically significant difference between the mean scores at six months or 12 months (18 for the deltoid-split versus 17 for the deltopectoral) but the clinical relevance of these scores is questionable. Similar numbers of participants in the two groups had a re-operation for a complication or a fall (9/60 versus $8 / 60$; RR $1.13,95 \%$ CI 0.47 to 2.72 ; see Analysis 9.1); the same observation applies to the numbers of participants requesting plate removal (see Analysis 9.1). By one year follow-up, one person had died in the deltoid-split group versus three in the deltopectoral group (see Analysis 9.2). Analysis 9.3 presents the data for the complications, all present resulted in a re-operation, reported for this trial. The Constant scores favoured the deltoid-split group, but the mean differences were smaller than the MCID for the Constant score and the confidence intervals crossed the line of no effect (see Analysis 9.4). A similar finding applied to the pain VAS results (see Analysis 9.5). There were no significant between-group differences in duration of operation or fluoroscopy time (see Analysis 9.6). The mean length of stay in hospital was 10 days in both groups (see Analysis 9.7).

\section{Polyaxial versus monoaxial locking plate fixation}

Two trials made this comparison (Ockert 2010; Voigt 2011). Ockert 2010, which reported on outcome for patients (66 patients in their 2010 publication; 124 patients in their later publication (Ockert 2014)) with Neer two-, three- or four-part fractures, did not report on functional outcome. Voigt 2011 found no statistically significant differences at one year (48 patients with Neer three- or four-part fractures) between the two groups in their DASH scores (see Analysis 10.1: RR 2.10, 95 CI -6.24 to 10.44), nor at 3, 6 or 12 months in the 'simple shoulder test' (see Analysis 10.2).

Since the extended trial report of Ockert 2010 (Ockert 2014) reported only on re-operation at 12 months, the data from the more detailed report of re-operations and complications occurring up to six months from the 2010 publication are also presented in the following. Neither trial found statistically significant differences between the two groups in participants having a re-operation; either at six months (data from Ockert 2010: 2/29 versus 3/37) or at one year (see Analysis 10.3: 15/83 versus 16/97; RR $1.10,95 \%$ CI 0.58 to 2.08 ). In the initial six months follow-up report for Ockert 2010, one participant of the polyaxial group had a loosened screw taken out at 10 weeks; one participant of each group had early hardware removal (at five months) because of subacromial impingement from poor plate positioning; and two monoaxial group participants had early hardware removal and a revision respectively because of intra-articular screw protrusion. In the recruitment and follow-up extension of Ockert 2010 (Ockert 2014), five polyaxial group versus nine monaxial group participants had revision because of secondary varus displacement with subsequent intra-articular screw protrusion; four versus two participants had revision because of subacromial impingement; and one monoaxial group participant had revision surgery because of an infection. In Voigt 2011, one person in each group had an early "prosthetic replacement" and three participants in the polyaxial group and one in the monoaxial group had refixation. The two other re-operated polyaxial group participants of Voigt 2011 had a corrective osteotomy and a screw removal respectively, while two other re-operated monoaxial group participants both had early implant removals.

Similarly, neither trial found statistically significant differences between the two groups in their other reported outcome measures. The available data are shown for death (see Analysis 10.4), participants with any or individual complications (see Analysis 10.6), the Constant score relative to the uninjured arm (see Analysis 10.5), range of motion (see Analysis 10.7) or duration of operation or fluoroscopy time (see Analysis 10.8).

\section{Locking plate: use of medial support locking screws}

Zhang 2011 tested the use of the medial support locking screws in 72 people with Neer two-, three- or four-part fractures treated with open reduction with internal fixation using the PHILOS locking plate. They reported results for 68 participants. In the medial support group, locking screws were introduced through the plate so as to run up the inferior portion of the humeral neck providing support to the calcar. In the control group, these screw holes were left empty. One participant in the medial screw group had early failure of fixation due to plate breakage compared with nine participants with early fixation failure (six varus collapse; three screw penetration) in the control group; however, this difference did not reach statistical significance (see Analysis 11.1: RR 0.15, 95\% CI 0.02 to 1.11 ). Seven of these patients, including the patient in the medial screw group, consented to have a re-operation (RR 0.22; $95 \%$ CI 0.03 to 1.11). One patient in the medial screw group had asymptomatic osteonecrosis. The medial screw group had statistically significantly higher Constant scores (0 to 100: best score) at 31 month follow-up (see Analysis 11.2: MD 9.00, 95\% CI 2.41 to 15.59$)$.

\section{MultiLoc proximal humeral nail (MPHN) - a straight nail - versus Polarus humeral nail - a curved nail}

Lopiz 2014 compared these two types of intramedullary nails in 54 people with Neer two- or three-part fractures, reporting results at a mean of 14 months (range 6 to 22 months). Of the two excluded participants, who were both in the MPHN group, one had died 
and one was lost to follow-up. Patient-reported outcome measures were not reported in this trial. Adverse events including re-operations are presented in Analysis 12.1. Significantly fewer participants in the MPHN group had a re-operation (3/26 versus 11/26; RR 0.27 favouring MPHN, $95 \%$ CI 0.09 to 0.87 ; $=0.03$ ). All reoperations involved hardware removal of either a loose screw (one versus seven) or the whole nail (two versus four). One participant of the Polarus group had a non-union; subsequent to nail removal, this patient had a reverse shoulder arthroplasty. Fewer participants in the MPHN group had rotator cuff symptoms (9/26 versus 19/ 26; RR $0.47,95 \%$ CI 0.27 to 0.84 ) or shoulder impingement (2/ 26 versus 5/26; RR 0.40, 95\% CI 0.09 to 1.88 ). Both the unadjusted and age- and sex-adjusted Constant scores were higher in the MPHN group; e.g. adjusted Constant score: MD 10.60, 95\% CI 1.71 to 19.49; Analysis 12.2). Although the MDs were a little smaller than the MCID (11.2) for the Constant score, the $95 \%$ confidence intervals included a clinically relevant difference in favour of the MPHN. There were no significant between-group differences in range of shoulder motion (see Analysis 12.3), length of surgery or length of hospital stay (see Analysis 12.4).

\section{Hemiarthroplasty: comparison of two types}

Fialka 2008 compared two types of hemiarthroplasty, the EPOCA prosthesis versus the HAS prosthesis, which differ in a number of ways including the method of fixation of the tuberosities. Fialka 2008 reported results at one year for 35 of the 40 trial participants. The treatment allocations of three participants who had died and the two who were lost to follow-up were not reported. Significantly better functional results, including range of motion, at one year were reported for the EPOCA prosthesis group. The relative (compared with the patient's uninjured shoulder) individual Constant score results were $70.4 \%$ (range $38 \%$ to $102 \%$ ) for the EPOCA group versus $46.2 \%$ (range $15 \%$ to $80 \%$ ) for the HAS group (reported $\mathrm{P}=0.001$ ). Reported complications were two patients with deep infection in the EPOCA group, two patients with persistent pain scheduled for a reoperation in the HAS group (see Analysis 13.1), and a periprosthetic fracture that occurred in one of the three patients who had died by one year. Radiological findings, except for heterotopic ossification where there were contradictory data, are shown in Analysis 13.2. These tended to favour the EPOCA prosthesis. Fialka 2008 noted some association between the bony resorption of the tuberosities and a decreased Constant outcome score. Results for range of motion are shown in Analysis 13.3.

\section{Hemiarthroplasty: tenodesis of the long head of the biceps (LHB) versus LHB tendon left intact}

Soliman 2013 compared tenodesis of the LHB versus leaving the LHB tendon intact in 45 people undergoing hemiarthroplasty. By deduction from the study report, four participants in each group were excluded because they had a complication within three months of follow-up. These were reported to be tuberosity malposition (three participants); inferior subluxation of the prosthesis (two participants), loss of reduction of the greater tuberosity (two participants) and deep infection that required surgical debridement (one participant). Data for complications split by treatment group are shown in Analysis 14.1. Of these complications, only deep infection resulted in further surgery. At two years, the difference between the two groups in the Constant scores in favour of the tenodesis group was below the MCID and thus unlikely to be clinically important (MD 4.60, 95\% CI 0.38 to 8.82 ; see Analysis 14.2). Three participants reported mild pain in the tenodesis group and six participants reported pain (four mild and two moderate pain) in the tendon intact group (3/19 versus 6/18; RR $0.47,95 \%$ CI 0.14 to 1.62 ; see Analysis 14.3). Both participants with moderate pain went on to have a mini-open biceps tenodesis at 18 and 28 months after diagnosis of an inflamed and scarred biceps tendon. There was no difference between the two groups in active shoulder elevation results at two years (see Analysis 14.4).

\section{Continuing management (including rehabilitation) after surgical treatment}

Wirbel 1999 tested the duration of immobilisation (one week versus three weeks) before starting physiotherapy after closed reduction and percutaneous fixation of displaced fractures in $77 \mathrm{pa}-$ tients. Wirbel 1999 reported that there were no statistically significant differences between the two trial groups in their functional results, assessed using the Neer score, at 3, 6 or at an average of 14.2 months. Data provided for unsatisfactory or worse outcome, as defined by the Neer score, at six months are consistent with this claim (see Analysis 15.1: 9/32 versus 10/32; RR $0.90,95 \%$ CI 0.42 to 1.92 ; 64 participants). Premature removal of Kirschner wires because of loosening occurred in the five people in each group (see Analysis 15.2); these results, however, were not provided for the whole study population nor was it reported the treatment groups of five people who underwent open revision or hemiarthroplasty. Though similar numbers (three versus two) of people underwent removal of screws due to subacromial impingement after six months, the numbers of people in each group whose displaced tuberosity fractures were fixed with cannulated screws were not reported. Of the 21 participants followed up for more than two years, one developed partial necrosis of the humeral head but was symptom-free and had a full range of motion of his affected shoulder.

Agorastides 2007 reported the findings of early active-assisted mobilisation (after two weeks) versus late mobilisation (after six weeks) after cemented hemiarthroplasty in 49 of the 59 participants recruited in their trial. At one year follow-up, there were no significant differences between the two groups in function as rated by the Oxford shoulder score (see Analysis 16.1; MD -6.0, $95 \%$ CI -16.53 to 4.53 ; scale was 0 to 100 ) or the overall Con- 
stant score (see Analysis 16.2). Two non-unions occurred in the early group but none of the differences in radiologically-assessed outcomes between the two groups was statistically significant (see Analysis 16.3). The differences between the two groups at one year in elevation and external rotation were neither statistically nor clinically significant (see Analysis 16.4). 
ADDITIONALSUMMARYOF F INDINGS [Explanation]

\section{Early versus delayed mobilisation for non-surgically treated proximal humeral fractures}

Patient or population: adults with minimally displaced or displaced (2-part or 3-part) proximal humeral fractures (4 trials)

Settings: various, including fracture clinics and physiotherapy

Intervention: early (within or at one week) mobilisation

Comparison: delayed (usual) mobilisation or physiotherapy after three or four weeks immobilisation

\begin{tabular}{|c|c|c|c|c|c|c|}
\hline \multirow[t]{3}{*}{ Outcomes } & \multicolumn{2}{|c|}{ Illustrative comparative risks* $(95 \% \mathrm{Cl})$} & \multirow{3}{*}{$\begin{array}{l}\text { Relative effect } \\
(95 \% \mathrm{Cl})\end{array}$} & \multirow{3}{*}{$\begin{array}{l}\text { No of Participants } \\
\text { (studies) }\end{array}$} & \multirow{3}{*}{$\begin{array}{l}\text { Quality of the evidence } \\
\text { (GRADE) }\end{array}$} & \multirow[t]{3}{*}{ Comments } \\
\hline & Assumed risk & Corresponding risk & & & & \\
\hline & $\begin{array}{l}3 \text { to } 4 \text { weeks immobili- } \\
\text { sation }\end{array}$ & $\begin{array}{l}\text { Early mobilisation ( } \leq 1 \\
\text { week) }\end{array}$ & & & & \\
\hline $\begin{array}{l}\text { Shoulder disability: } \\
\text { Croft Shoulder Disability } \\
\text { Score - Disability (1 or } \\
\text { more problems) } \\
\text { Follow-up: } 1 \text { year }\end{array}$ & 725 per $1000^{1}$ & $\begin{array}{l}\mathbf{4 2 8} \text { per } 1000 \\
(290 \text { to } 638)\end{array}$ & RR 0.59 (0.40 to 0.88$)$ & $\begin{array}{l}82 \text { participants } \\
\text { (1 study) }\end{array}$ & $\begin{array}{l}\oplus \oplus \bigcirc \bigcirc \\
\text { low }^{2}\end{array}$ & $\begin{array}{l}\text { Early mobilisation re- } \\
\text { sulted in } 297 / 1000 \text { fewer } \\
\text { people with one or more } \\
\text { problems at } 1 \text { year ( } 95 \% \\
\text { Cl } 87 \text { fewer to } 435 \text { fewer) } \\
3\end{array}$ \\
\hline $\begin{array}{l}\text { Number of treatment } \\
\text { sessions (until indepen- } \\
\text { dent function achieved) } \\
\text { Follow-up: as described }\end{array}$ & $\begin{array}{l}\text { The mean number of ses- } \\
\text { sions was } 14 \text { in the usual } \\
\text { timing group }\end{array}$ & $\begin{array}{l}\text { The mean number of ses- } \\
\text { sions in the early group } \\
\text { was } \\
\mathbf{5 . 0} \text { lower ( } 1.75 \text { to } 8.25 \\
\text { sessions lower) }\end{array}$ & & $\begin{array}{l}86 \text { participants } \\
\text { (1 study) }\end{array}$ & $\begin{array}{l}\oplus \oplus \bigcirc \bigcirc \\
\text { low }\end{array}$ & $\begin{array}{l}\text { This pertains to early re- } \\
\text { covery to a level that may } \\
\text { vary with individual pa- } \\
\text { tients }\end{array}$ \\
\hline $\begin{array}{l}\text { SF-36 scores: pain \& } \\
\text { physical dimensions - } \\
\text { all } 3 \text { dimensions } 0-100 \text { : } \\
\text { higher scores mean bet- } \\
\text { ter quality of life) } \\
\text { Follow-up: } 16 \text { weeks }\end{array}$ & $\begin{array}{l}\text { The mean values for } 3 \\
\text { dimensions in the delayed } \\
\text { group }{ }^{4} \text { were: } \\
\text { Physical functioning } \mathbf{6 9 . 2} \\
\text { Role limitation physical } \\
\mathbf{3 9 . 7} \\
\text { Pain } \mathbf{5 9 . 9}\end{array}$ & $\begin{array}{l}\text { The mean values in the } \\
\text { early group were: } \\
\text { Physical functioning } \mathbf{0 . 7 0} \\
\text { higher ( } 9.91 \text { lower to } 11 \text {. } \\
31 \text { higher) } \\
\text { Role limitation physical } \\
22.2 \text { higher ( } 3.82 \text { to } 40 \text {. } \\
58 \text { higher) }\end{array}$ & & $\begin{array}{l}81 \text { participants } \\
\text { (1 study) }\end{array}$ & $\begin{array}{l}\oplus \oplus \bigcirc \bigcirc \\
\text { low }^{6}\end{array}$ & $\begin{array}{l}\text { An overall score was not } \\
\text { available. } \\
\text { General physical func- } \\
\text { tioning was high and } \\
\text { comparable in the two } \\
\text { groups. It is likely that the } \\
\text { results for role limitation }\end{array}$ \\
\hline
\end{tabular}




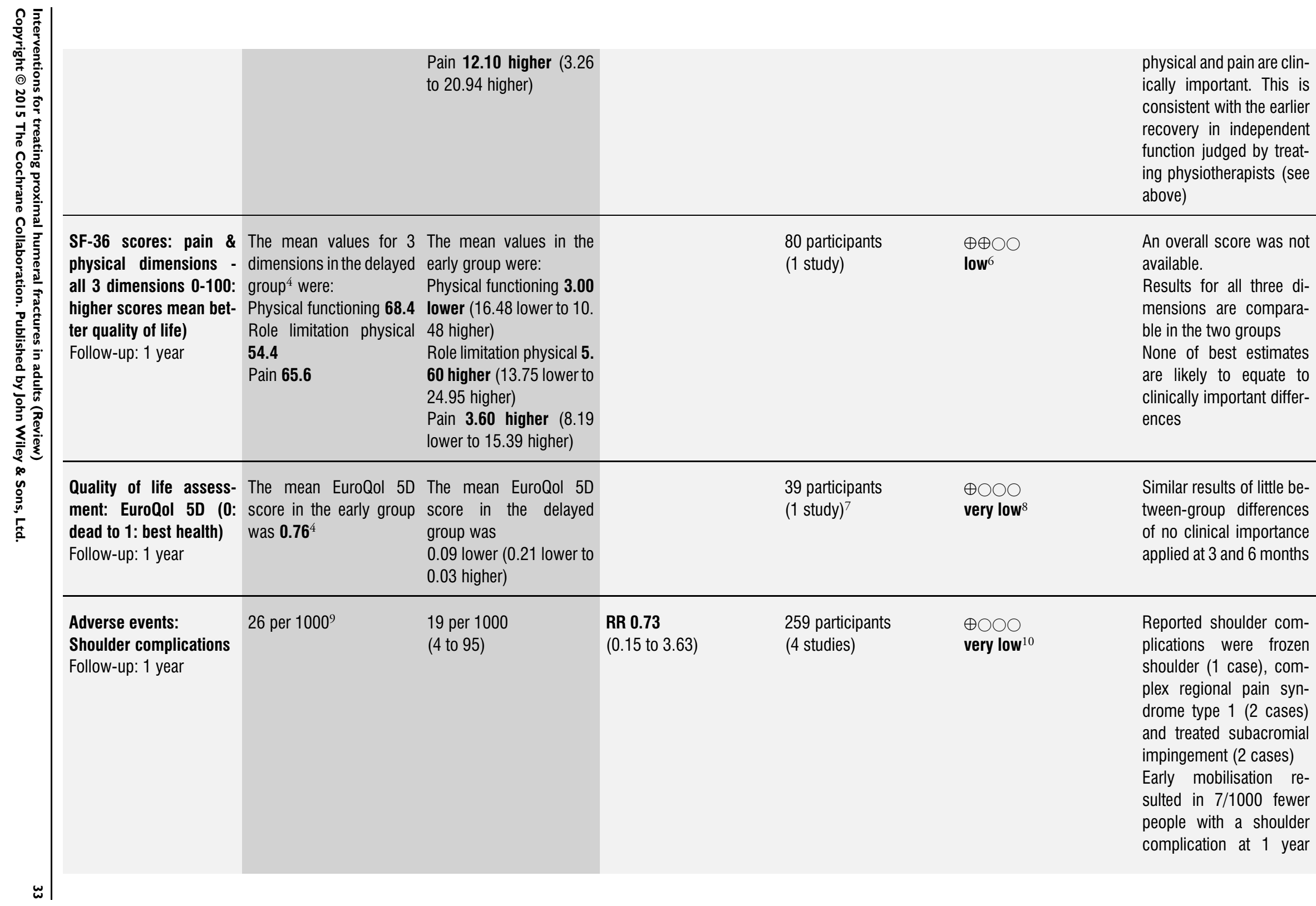




\begin{tabular}{|c|c|c|c|c|c|c|}
\hline & & & & & & $\begin{array}{l}\text { (95\% Cl } 22 \text { fewer to } 69 \\
\text { more) }\end{array}$ \\
\hline \multirow[t]{2}{*}{$\begin{array}{l}\text { Adverse events: } \\
\text { Fracture displacement } \\
\text { and non-union } \\
\text { Follow-up: } 1 \text { year }\end{array}$} & 23 per $1000^{9}$ & $\begin{array}{l}51 \text { per } 1000 \\
(5 \text { to } 517)\end{array}$ & RR 2.20 (0.22 to 22.45) & $\begin{array}{l}106 \text { participants } \\
\text { (2 studies) }\end{array}$ & $\begin{array}{l}\oplus \bigcirc \bigcirc \bigcirc \\
\text { very low } 10\end{array}$ & $\begin{array}{l}\text { There were no cases } \\
\text { of non-union. All three } \\
\text { fracture displacements } \\
\text { (none of which required } \\
\text { surgery) occurred in one } \\
\text { trial that included dis- } \\
\text { placed fractures }\end{array}$ \\
\hline & & & & & & $\begin{array}{l}\text { Early mobilisation re- } \\
\text { sulted in } 28 / 1000 \text { more } \\
\text { people with a fracture dis- } \\
\text { placement at } 1 \text { year (95\% } \\
\text { Cl } 18 \text { fewer to } 494 \text { more) }\end{array}$ \\
\hline
\end{tabular}

*The basis for the assumed risk (e.g. the median control group risk across studies) is provided in footnotes. The corresponding risk (and its $95 \%$ confidence interval) is based on the assumed risk in the comparison group and the relative effect of the intervention (and its $95 \% \mathrm{Cl}$ ).

CI: Confidence interval; RR: Risk Ratio

GRADE Working Group grades of evidence

High quality: Further research is very unlikely to change our confidence in the estimate of effect.

Moderate quality: Further research is likely to have an important impact on our confidence in the estimate of effect and may change the estimate.

Low quality: Further research is very likely to have an important impact on our confidence in the estimate of effect and is likely to change the estimate.

Very low quality: We are very uncertain about the estimate.

1. Control risk based on study data

2. Evidence downgraded one level for one level for imprecision (single small trial) and one level for indirectness (question over outcome measure's validity; the importance of individual problems will vary)

3. Two-year follow-up data from the same trial (74 participants) showed that based on a control risk of 595 per 1000 in the delayed group, early mobilisation resulted in 160/1000 fewer people with one or more problems at two years ( $95 \% \mathrm{Cl} 321$ fewer to 90 more); very low quality evidence (see above footnote)

4. Data from control group of study

5. Evidence downgraded one level for imprecision (single small trial data) and one level for indirectness ('independent function' and physiotherapy discharge depicts an intermediate outcome)

6. Evidence downgraded one level for study limitations (several domains at unclear risk of bias) and one level for imprecision (single small trial data) 


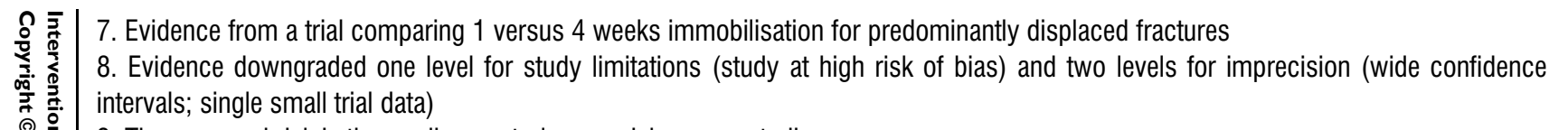

9. The assumed risk is the median control group risk across studies

10. Evidence downgraded one level for study limitations and two levels for imprecision (sparse data and wide confidence intervals) 


\section{DISCUSSION}

\section{Summary of main results}

mThis review, which covers all non-pharmacological treatment and rehabilitation interventions for proximal humeral fractures in adults, now includes 31 trials involving a total of 1941 participants. The only multicentre trial recruited 250 participants (ProFHER 2015). With the increased availability of trials, we have been able to undertake further pooling of data compared with the last version, but this is still limited to four comparisons. We have undertaken substantive pooling in only one comparison, that of surgical versus non-surgical treatment, including patient-reported outcome measures of function and quality of life. This is presented first, below. The main results of the comparisons falling within the three other main treatment categories are then presented in turn. Where data allow, we have given the main results of individual comparisons in terms of the listed Primary outcomes.

\section{Surgical treatment versus non-surgical treatment}

Eight heterogeneous trials, with a total of 567 participants and 568 predominantly displaced fractures evaluated surgical intervention for displaced fractures, of which 73\% (415) were three- or fourpart fractures (Neer classification). Of note is that the majority of the fractures $(146 / 250=58.4 \%)$ in ProFHER 2015 were either two-part (128) or one-part (18) fractures; the other seven twopart fractures were included in Kristiansen 1988. Table 1 summarises the main fracture types, the interventions and length of follow-up of the individual trials. Six trials specifically limited their trial populations to older people. Although ProFHER 2015 recruited adults of any age, the majority of trial participants were over 65 years $(142 / 250=57 \%)$. Data for patient-reported functional scores and quality-of-life scores were available from the five more recent trials that are thus more likely to represent current practice. The main results of this comparison are presented in Summary of findings for the main comparison. The results apply to the majority of displaced proximal humeral fractures involving the surgical neck, but note should be taken of clear exceptions, such as where surgery is required for severe soft-tissue compromise, as well as the exclusion of fracture-dislocations, in ProFHER 2015. There was high quality evidence of no clinically important difference in patient-reported shoulder and upper-limb function at one- or twoyear follow-up between surgical (primarily locking plate fixation or hemiarthroplasty) and non-surgical treatment (sling 'immobilisation') for the majority of displaced proximal humeral fractures. There was moderate quality evidence of no clinically important difference between the two groups in quality of life at two years. While this observation applied to interim follow-ups at six and 12 months, pooled data from four studies at six months showed a statistically significant effect. There was moderate quality evidence of little difference between the groups in mortality: although there were slightly more deaths in the surgery group, the $95 \%$ confidence interval also included the potential for a higher mortality after nonsurgical treatment. Also of note is that, where reported, only one death was explicitly linked with treatment (surgery). There was moderate quality evidence of a higher risk of additional surgery in the surgery group: based on an illustrative risk of 40 subsequent operations per 1000 non-surgically treated patients, this amounts to an extra 43 subsequent operations per 1000 surgically treated patients ( $95 \%$ CI 7 to 104 more). There was also moderate quality evidence of a higher overall risk of adverse events after surgery; however, the $95 \%$ confidence intervals for adverse events also included the potential for a greater risk of adverse events after nonsurgical treatment.

\section{Methods of non-surgical management (including rehabilitation)}

Non-surgical management, generally involving a period of arm immobilisation followed by physiotherapy, of (mainly) minimally displaced fractures is the focus of nine trials. Exceptionally, Torrens 2012 included a higher percentage of displaced fractures $(81 \%=$ $34 / 42$ fractures). There was a general recognition of the impaired function and serious complications, such as complex regional pain syndromes, that could follow a proximal humeral fracture.

\section{Initial treatment, including immobilisation}

When considering the extent and duration of initial immobilisation after a fracture, a balance is needed between the advantages of pain relief and avoidance of fracture displacement, and the consequences of immobilisation, notably joint stiffness and muscle atrophy.

\section{Early versus delayed mobilisation}

Of the four heterogeneous trials comparing early versus delayed mobilisation for minimally displaced or displaced fractures (Hodgson 2003; Kristiansen 1989; Lefevre-Colau 2007; Torrens 2012), only limited data, mainly for secondary outcomes, could be pooled from Lefevre-Colau 2007 and Torrens 2012.

Summary of findings 2 summarises the data relating to primary outcome measures for early versus delayed mobilisation in nonsurgically treated fractures. With the exception of adverse event data provided by all four trials, most of these data are from Hodgson 2003. There was low quality evidence in favour of early mobilisation in terms of fewer people with shoulder problems at one year, of the need for fewer sessions of physiotherapy to achieve independent function, and of a better quality of life at 16 weeks in terms of less pain and less limitation of physical function. There was low quality evidence of no difference between early and delayed mobilisation in physical and pain aspects of quality of life at one year. There was very low quality evidence of no clinically important between-group differences in quality-of-life scores for people with mainly displaced fractures. There was very low quality 
evidence of little difference between the two groups in shoulder complications and fracture displacement and non-union; the incidences of individual complications were low.

\section{Type of bandage}

The one quasi-randomised trial (28 participants with mainly minimally displaced fractures) provided very low quality evidence on the relative effects of two types of bandages, the Gilchrist arm sling versus the Desault body bandage (Rommens 1993). There was no report of PROMS nor data to support the claims of no betweengroup differences in functional outcome or fracture healing. More participants found the arm sling comfortable and acceptable compared with the body bandage.

\section{Continuing management (rehabilitation) after initial treatment involving sling immobilisation}

\section{Instructions for home exercises versus physiotherapy}

Two small trials including a total of 62 participants with minimally displaced fractures compared home exercises after receiving instructions versus supervised physiotherapy (Bertoft 1984; Lundberg 1979). Neither trial reported on PROMS for function or quality of life. There was very low quality evidence from single trials of little difference between the two groups in pain, change of therapy, adverse events, and range of motion.

Supervised exercises in a swimming pool plus home exercises versus home exercises alone

The trial making this comparison in 48 participants with minimally displaced fractures did not provide evidence that could be presented or tested in the analyses (Revay 1992). Revay 1992 claimed that the self-treatment group had better activities of daily living and joint mobility in the first two to three months but that the two groups had similar results at one year. Revay 1992 suggested that the supervised group had neglected their home exercises, which effectively undermines the aim of this trial.

\section{Pulsed electromagnetic high frequency energy (PHFE)}

Livesley 1992 hypothesised that pain was associated with contracture of the capsule of the glenohumeral joint and that PHFE would reduce inflammation and swelling, improving the end functional result. However, the trial (48 participants with minimally displaced fractures) failed to provide any quantitative data to support or refute this hypothesis.

\section{Different methods of surgical management}

\section{Comparisons of different categories of surgical intervention}

Five trials compared different methods of surgical management (Cai 2012; Hoellen 1997; Sebastiá-Forcada 2014; Smejkal 2011; Zhu 2011).

Open reduction with internal fixation using a locking plate versus a locking nail

There is very low quality evidence from one trial (Zhu 2011: 57 participants with two-part surgical neck fractures) of marginally better function (higher American Shoulder and Elbow Surgeon's scores) and slightly less pain after locking plate fixation compared with locking nail fixation at one year but not at three years. There was very low quality evidence of a higher rate of complications, including re-operation for screw penetration into the humeral head after plate fixation.

Open reduction with internal fixation using a locking plate versus minimally invasive fixation with distally inserted intramedullary K-wires

There is very low quality evidence from one trial (Smejkal 2011: 61 participants with two- or three-part fractures) of no difference between these two interventions for numbers of participants incurring a complication or in the Constant scores at two years follow-up.

\section{Hemiarthroplasty versus internal fixation}

With minimal opportunity for pooling, data from two small heterogeneous trials testing this comparison were presented separately.

\section{Hemiarthroplasty versus open reduction and locking plate fixation}

The very low quality evidence from one trial (Cai 2012: 32 participants with four-part fractures) of lower DASH scores (better function) and slightly higher EQ-5D scores (better quality of life) at one and two years may not equate to clinically important differences in either of these outcomes between hemiarthroplasty and locking plate fixation. Three participants in each group had a reoperation.

\section{Hemiarthroplasty versus tension band wiring}

There is very low quality evidence from one trial (Hoellen 1997: 30 participants with four-part fractures) of no differences between the two groups in the Constant scores or pain (18 participants). At one-year follow-up, all five reoperations occurred in the fixation group. 
Reverse shoulder arthroplasty versus hemiarthroplasty

There is low quality evidence from one trial (Sebastiá-Forcada 2014: 62 participants with either three- or four-part fractures) of better patient-rated (Quick DASH) and composite shoulder function scores (UCLA and Constant scores) at a minimum of two years follow-up in the reverse shoulder arthroplasty (RSA) group. Although a condition-specific minimal clinically important difference is not available for QuickDASH, it is likely that the difference would have been clinically important to some extent. The clinically important differences favouring RSA in the Constant and UCLA scores will in part reflect the greater range of motion in the RSA group. Fewer people in the reverse arthroplasty group had a re-operation (one versus six) or had a complication (two versus 10)

\section{Comparisons of different methods of performing an intervention in the same category}

Seven trials compared different types or methods in the same intervention category (e.g. plating) (Buecking 2014; Fialka 2008; Lopiz 2014; Ockert 2010; Soliman 2013; Voigt 2011; Zhang 2011).

\section{Deltoid-split approach versus deltopectoral approach for non-contact bridging plate fixation}

There is very low quality evidence from one trial (Buecking 2014: 120 participants with two-, three- or four-part fractures) of no differences between groups in activities in daily living, re-operations or complications, Constant scores or pain at one year.

\section{Polyaxial versus monoaxial locking plate fixation}

Although two trials (Ockert 2010 and Voigt 2011: 180 participants with two-, three- or four-part fractures) made this comparison, most of the data were from Voigt 2011 (48 participants for function) and only data for re-operation were pooled. There was very low quality evidence of no between-group differences in function (DASH and simple shoulder test scores), re-operations and complications.

\section{Locking plate: use of medial locking screws}

There is very low quality evidence from one trial (Zhang 2011: 68 participants with two-, three- or four-part fractures) of medial locking screws resulting in fewer early losses of fixation and reoperations. However, the $95 \%$ CI results also included a higher risk of re-operations in the medial locking screws group. Based on the control risk of 154 re-operations per 1000 participants, medial locking screws resulted in 120 fewer re-operations (95\% CI 149 fewer to 117 more). Although the medial screw group had statistically significantly higher Constant scores at 31-month follow-up, only part of the $95 \%$ CI included the minimal clinically important difference.

\section{Nails: comparison of two types}

There is low quality evidence from one trial (Lopiz 2014: 54 participants with two- or three-part fractures) of fewer adverse events, including re-operations and impingement, for the MPHN nail compared with the Polarus nail. Based on the control (Polarus nail) group risk of 423 re-operations per 1000 participants, the MPHN resulted in 308 fewer re-operations ( $95 \%$ CI 55 to 385 fewer). Of note is the very low quality evidence, as half as many participants in the MPHN group had rotator cuff symptoms. The MPHN group had higher Constant scores (very low quality evidence), which the authors linked with the lower incidence of rotator cuff symptoms in this group.

\section{Hemiarthroplasty: comparison of two types}

There was very low quality evidence from one trial (Fialka 2008: 35 out of 40 people with four-part fractures) for better function (Constant scores and range of motion) at one year for the EPOCA prosthesis when compared with the HAS prosthesis. Two participants in each group had a serious complication or pain requiring further treatment.

\section{Hemiarthroplasty: tenodesis of the long head of the biceps (LHB) versus LHB tendon left intact}

There was very low quality evidence from one trial (Soliman 2013: 45 people with four-part fractures undergoing hemiarthroplasty) of no between-group differences in complications at three months follow-up, in function (Constant score), in numbers of participants with shoulder pain or range of motion.

\section{Continuing management (including rehabilitation) after surgical intervention}

The need for and duration of immobilisation before commencing physiotherapy after surgery for displaced fractures was tested in two small heterogeneous trials for fixation and hemiarthroplasty respectively. There was very low quality and incomplete evidence from one trial (Wirbel 1999: 64 participants (of the 77 recruited)) of no difference between one week versus three weeks immobilisation after percutaneous fixation in the numbers of participants with an unsatisfactory or worse outcome based on the Neer outcome score at six months or incurring premature removal of $\mathrm{K}$ wires failure (five in each group). There was very low quality evidence from one trial (Agorastides 2007: 49 participants (of the 59 recruited)) of no difference between participants mobilised after two weeks (which was current practice) after hemiarthroplasty versus those mobilised after six weeks in function (Oxford shoulder score or Constant score), radiological outcomes and range of motion at one year. 


\section{Overall completeness and applicability of evidence}

To inform consideration of applicability of the evidence from individual trials, we give quite extensive details in the Characteristics of included studies on the study populations and interventions. Additionally, Table 2 shows our assessments for each trial of four aspects of relevance to ascertaining external validity: definition of the study population, description of the interventions, definition of primary outcome measures and length of follow-up. Clearly unhelpful is where there are incomplete descriptions of study inclusion (10 trials) and interventions (five trials). Five trials had less than one year's follow-up: Lefevre-Colau 2007 (six months), Livesley 1992 (six months), Ockert 2010 (six months) and Rommens 1993 (until fracture consolidation - time unspecified). Additionally, the minimum follow-up was six months in Lopiz 2014. Despite the claims of longer follow-up, the results seemed to apply to six months at most in Stableforth 1984. In Wirbel 1999, though follow-up of 21 participants was more than two years, the main results applied to the set follow-up at six months. Our setting of our criterion to one-year follow-up as acceptable is arbitrary and mainly reflects a reasonable timing for assessment of function. However, it should be noted that in terms of a full outcome assessment, data at oneyear follow-up must be considered preliminary results only given that complications such as avascular necrosis and device failure may not become evident until later and functional recovery can still be ongoing.

The measurement of outcome was variable, though generally comprehensive. In most of the older trials, there was frequent use of non-validated or, at best, partly validated scoring systems such as the Neer (Neer 1970) and Constant (Constant 1987) systems, but also of simple rating systems for individual outcomes. Validated schemes such as the Oxford Shoulder Score (Dawson 1996) and Shoulder Rating Questionnaire (L'Insalata 1997) for subjective assessment of symptoms and function were not available at the time for the trials in earlier versions of this review. Nonetheless, some consideration of interobserver reproducibility and other aspects of validity was evident in the establishment of the Constant score in two trials (Lundberg 1979; Zyto 1997). Non-validated outcome assessment schemes, often with arbitrary criteria for grading overall outcome (excellent, good, fair, poor), are probably best viewed as 'blunt' and flawed instruments. This needs to be noted when viewing the results of many of the older included trials; in particular Kristiansen 1989, whose outcome assessment is almost completely based on the Neer scoring system. As noted also in our 2012 update, more recent trials continue to be better in this respect. Four of the eight newly included trials reported on PROMS for function: for example, Boons 2012 reported on the Simple Shoulder test; Cai 2012 on DASH; ProFHER 2015, the Oxford Shoulder Score; and Sebastiá-Forcada 2014, the QuickDASH. The continued use of the Constant score is notable, being reported by the newly included trials with the exception of ProFHER 2015, which did not conduct additional clinical examinations for the collection of such data.

The majority of the trials used Neer's fracture classification (Neer 1970). Problems, such as poor interobserver reproducibility and intraobserver reliability, with the classification of fractures according to the Neer and AO systems have been shown for both radiographs and computerised tomographic scans (Bernstein 1996; Brorson 2008; Sidor 1993; Siebenrock 1993; Sjoden 1997). This variation in the classification of fractures and hence diagnosis needs to be considered when interpreting the results of trials, both in respect to the comparability and composition of the intervention groups and in the applicability of the trial's findings. The limitations of the Neer classification scheme were also demonstrated by the identification of the valgus impacted four-part fracture as a separate category with a lower risk of avascular necrosis (Jakob 1991). Ideally a fracture classification system should act as a guide to treatment as well to enable the comparison of results from studies of patients with similar fracture patterns. However, other factors, such as osteoporotic bone, associated soft tissue injury and the patient's overall health and motivation, will also influence treatment choices and outcome. A recent study (Brorson 2012) looking at the agreement of surgeons' treatment recommendations in conjunction with the Neer classification concluded that the low observer agreement on the Neer classification may have less clinical importance than previously assumed. However, it noted that interobserver agreement on treatment did not exceed moderate levels. The purposefully pragmatic inclusion criteria used in ProFHER 2015 is noteworthy in this regard. These stipulated that the degree of displacement had to be sufficient for the treating surgeon to consider surgical intervention but did not have to meet the displacement criteria of Neer for inclusion in the trial. Post-recruitment classification by two independent surgeons of the baseline $\mathrm{X}$-rays, resulted in the identification of 18 one-part fractures (see Table 1). Nonetheless these exceptions were judged sufficiently displaced that they would have been considered for surgical intervention in practice; where the exact observation of Neer's arbitrary criteria is rare.

While it is possible that all 31 trials are relevant to current practice somewhere in the world, it is likely that some interventions are now rarely used. These include body bandages as tested in Rommens 1993. Nowadays it is much more common practice to use either a 'collar and cuff' sling or a 'poly-sling' (these incorporate a chest strap that can be passed around the body). Additionally, the applicability of the findings from older trials, such as Stableforth 1984, is potentially less given subsequent changes in practice including the availability of new implants. These include locking plates, which are being increasingly used and promoted for these fractures (Thanasas 2009). Previously, we noted that the increasing use of locking plates for these fractures was reflected in the use of locking plates in more recently included trials (Handoll 2012). Another more recent development has been the use of reverse shoulder arthroplasty (RSA), typically for more complex four-part fractures in older people. This was tested in a 
newly included trial (Sebastiá-Forcada 2014), with evidence pending from three ongoing trials comparing RSA with hemiarthroplasty (NTR3208; NCT02075476; SHeRPA), one ongoing trial comparing RSA with plating (DELPHI) and one ongoing trial comparing two types of RSA (NCT01086202).

\section{Comments on individual comparisons}

\section{Surgical treatment versus non-surgical treatment}

In our previous commentary for this comparison we noted that "Trials comparing surgical versus non-surgical interventions, or indeed different surgical interventions, risk losing currency as different implants and methods become available and fashionable." (Handoll 2012). We also noted the impact of surgical decisionmaking in favour of locking plating systems, which allow for stronger constructs and fixation of more complex fracture patterns in osteopenic bone with the potential for less soft-tissue stripping and compromise to the blood supply (Thanasas 2009). More recently for more complex (predominantly four-part) fractures, reverse shoulder arthroplasty is being promoted, as illustrated by its increasing use, for instance in the USA (Han 2015; Schairer 2015). These illustrate how evolving technology (and marketing forces) mitigates against applying the findings of these types of trials. However, more emphasis can be given to the evidence from the five more recent trials (Boons 2012; Fjalestad 2010; Olerud 2011a; Olerud 2011b; ProFHER 2015), all of which report patient-reported outcome measures of function and quality of life. When considering the validity and applicability of surgical trials, account needs to be taken also of fundamental variations in surgical practice, including facilities and operator expertise. In particular, operator expertise and the linked issue of the surgical learning curve, play a pivotal role in the validity and applicability of surgical trial findings. Awareness of these issues was behind the pragmatic decision in ProFHER 2015 for surgeons to use methods with which they are familiar rather than stipulate the type of surgery. Indeed, the pragmatic multicentre design of ProFHER 2015 , including the constant emphasis on good standard practice and surgery by experienced surgeons (predominantly consultants), means that its results have immediate applicability at least in the setting where it was conducted (UK NHS trauma hospitals) and most likely in many other countries with similar surgical practice. Because of the dominance of the evidence from ProFHER 2015, particular note should be taken of its exclusion criteria (such as of fracture dislocations and two-part greater tuberosity fractures and other patterns not involving the surgical neck) and its study population, the composition of which shows the treatment uncertainty covered by this trial applied to the majority of displaced fractures of the proximal humerus. Additionally the lack of subgroup differences in ProFHER 2015, either for age (threshold of 65 years) or fracture type (tuberosity involvement or not; or Neer one- or two-part versus three- or four-part) strengthens the case for not differentiating treatment (use of surgery) on the basis of these characteristics. Nonetheless, in this trial and the other seven trials, six of which purposefully excluded younger adults, the evidence is predominantly from older people. This reflects the population distribution for these fractures (Karl 2015) but also the population for which the main treatment uncertainty applies.

\section{Initial treatment, including immobilisation}

Most of the evidence for the comparison of early versus delayed mobilisation came from Hodgson 2003 and thus applies primarily to the less severe fractures (minimally displaced two-part fractures). A survey sent to senior hospital physiotherapists working directly with orthopaedic patients revealed large variation in rehabilitation, in particular with regards to routine immobilisation, duration of immobilisation and timing of first contact with a physiotherapist, within and between hospitals in the UK (Hodgson 2003a; Hodgson 2006). A survey sent to the participating centres of ProFHER 2015, which included displaced fractures, found the recommended duration of arm immobilisation for non-surgically treated patients ranged from two to six weeks, with 29 (91\%) of 32 UK hospitals recommending immobilisation of $\geq 3$ weeks (Handoll 2015). This variation also needs to be viewed in the context of the type of arm immobilisation used, as methods such as collar and cuff provide support rather than rigid immobilisation. As noted by McKee 2007 in his commentary on Lefevre-Colau 2007 , the applicability of this trial is limited by the intensive physiotherapy regimen used in both groups. Both practically and financially the 32 two-hour sessions of physiotherapy may be difficult for patients and health providers; notably, 10 participants withdrew from the trial because of difficulties in attending. In contrast, the mean numbers of treatment sessions in Hodgson 2003 were nine and 14 respectively in the two groups.

As stated above the body bandages tested in Rommens 1993, which compared the Gilchrist arm sling with the Desault body bandage, is rarely used in practice. The above-mentioned survey of practice carried out as part of ProFHER 2015 confirms this in the UK, where 'collar and cuff' slings, poly-slings and more rarely broadarm slings are used (Handoll 2010).

\section{Continuing management (rehabilitation) after initial treatment involving sling immobilisation}

The three trials in this category that examined supervised versus home exercises were based in Sweden and possible differences in conventional physiotherapy regimens within and between countries, then and now, need to be taken into account when considering the application of trial findings. If they work, self-instruction and home-based exercise programmes are attractive for patients and conserve health care resources. There is some evidence from a Cochrane Review on fall prevention that older people, if well instructed and with intensive support (regular phone calls etc), can maintain a home-based exercise programme (Gillespie 2003; 
Gillespie 2009). However, there will still be some patients with insufficient understanding or motivation to perform the required exercises.

\section{Different methods of surgical management}

Most of the recent research activity, in both published and registered trials, evaluates different types of surgery. We now distinguish between trials comparing different categories of surgical interventions (tested by five trials) and trials comparing different methods of performing an intervention in the same category (tested by seven trials).

\section{Comparisons of different categories of surgical intervention}

The variety of available implants in the same category can limit the applicability and usefulness of trials comparing different categories of surgical intervention by comparisons of specific implants. Nonetheless, the comparison by Zhu 2011 of one of two locking plates versus a locking nail is very pertinent in terms of providing a useful investigation of the appropriateness of the current trend from locking nails to plates. This trial is too small to establish the superiority of one method over the other but it does provide some evidence of better function in the plate group at one year, and possibly for longer, although at a potentially greater risk of surgical complications and initially more invasive surgery. The comparison by Smejkal 2011 of a locking plate versus minimally invasive fixation with distally inserted multiple intramedullary K-wires (the Zifko method of minimally invasive fixation) is of relevance to current practice but, while data from Smejkal 2011 lend support to the use of the Zifko method in terms of it being a less extensive surgical procedure with potentially an earlier recovery than plate fixation, there were inadequate data on longer term function and outcome.

Again, the trial comparing hemiarthroplasty versus open reduction and locking plate fixation was too small to inform practice (Cai 2012). The absence of intraoperative conversions for the open reduction and internal fixation (ORIF) group to hemiarthroplasty or early failures is notable for a series of 13 displaced four-part fractures and fracture-dislocations and may indicate differences in assessing and dealing with problematic or failed fixation in this centre compared with other centres. Hoellen 1997, a flawed trial with only one-year follow-up, considered only one of several shoulder prostheses now available (the prosthesis was cemented in place) in their comparison with tension band wiring.

The comparison of reverse shoulder arthroplasty (RSA) versus hemiarthroplasty tested in Sebastiá-Forcada 2014 is very topical, as shown also by the three ongoing trials that are making the same comparison (NCT02075476; NTR3208; SHeRPA). The prostheses compared within each of these four trials come from only one manufacturer. However, the three ongoing trials examines prosthesis from three different manufacturers. Prostheses made by different manufacturers will differ to some extent; however, the variation between prostheses from different manufacturers is likely to be of lesser importance clinically than the large differences between RSA and hemiarthroplasty. In terms of applicability, Sebastiá-Forcada 2014 is mainly limited by being a single-centre trial with the participants being operated on by two surgeons.

\section{Comparisons of different methods of performing an intervention in the same category}

The trial (Buecking 2014) comparing two approaches (deltoidsplit approach versus deltopectoral) for non-contact bridging plate fixation had two notable limitations in terms of external validity. One was the absence of criteria for excluding patients for whom hemiarthroplasty was planned. The second was the inappropriate interpretation of the Lawson quality-of-life score. The two trials (Ockert 2010; Voigt 2011) comparing 'polyaxial' (where surgeons had greater control in their positioning of screws into the bone) versus 'monoaxial' locking plate fixation found no difference between the two methods. With no report of functional outcome, Ockert 2010 contributed relatively little to this question. Voigt 2011, which was a stronger trial but still insufficient to be conclusive, pointed out that the "majority of surgeons chose the same screw directions for the polyaxial screws as already exist in the fixed angle plate”. In their 2014 publication, Ockert 2010 also found that polyaxial screws were placed similarly to monoaxial screws. Of note also is the differences in the types of screws in the two implants in Voigt 2011, which could in some respects alter the question. Zhang 2011 tested the use of medial support locking screws for fixation using the PHILOS locking plate. While Zhang 2011 did not provide conclusive evidence of clinical benefit of the enhanced stabilisation of this commonly used plate, the direction of effect is consistent with the theoretical advantages for medial support screws.

In their comparison of the MultiLoc Proximal Humeral Nail (MPHN) versus the Polarus nail, Lopiz 2014 found the newer "straight" nail (the MPHN) resulted in fewer adverse events (screw loosening, impingement, rotator cuff symptoms) than the "curved" Polarus nail. This is plausible given the different design features of the MPHN that attempt to avoid the various problems, including impingement, that have been identified when using the Polarus nail. However, some consideration is also required of the rather high incidence of adverse events for the Polarus nail and the general inadequacies of tests for rotator cuff symptoms (Hanchard 2013).

Fialka 2008 compared two shoulder prostheses but although the authors ascribed the different functional outcomes to tuberosity fixation, other design differences may account for these results. These include a different stem finish and a more accurate recreation of pre-operative humeral geometry with the EPOCA prosthesis. The study population of Soliman 2013, which compared tenodesis of the long head of the biceps (LHB) versus LHB tendon left intact in people undergoing hemiarthroplasty, was exceptional in being younger (aged 45 to 60 years) than all other trial 
populations in this review and younger than the population for whom hemiarthroplasty is more typically used. Although the inclusion criteria included more severe injuries such as head-splitting fractures, Soliman 2013 provided insufficient criteria on which to judge participant suitability for hemiarthroplasty. Additionally of note, is the absence of spontaneous ruptures of the long head of biceps.

\section{Continuing management (including rehabilitation) after surgical intervention}

The need for and duration of immobilisation before commencing physiotherapy after surgical treatment is likely to depend on the method of fixation or type of prosthesis; and also other factors such as bone quality. While neither trial (Agorastides 2007; Wirbel 1999) found conclusive evidence for early mobilisation, such as offering any functional advantage, it can also be observed that the evidence was inconclusive for later mobilisation too, such as avoiding destabilisation of the fracture fixation after percutaneous fixation or tuberosity fixation after hemiarthroplasty.

\section{Quality of the evidence}

As noted in Handoll 2012 and continues to apply in this update, more recent trials generally have better study design (e.g. they have appropriate random sequence generation and allocation concealment, and thus are at low risk of selection bias) and reporting (e.g. including participant flow diagrams). Nonetheless, as shown by Figure 2, many of the included trials had serious shortcomings and are at high risk of bias that could affect the validity of their findings. The main but generally unavoidable shortcoming in trials testing physical and surgical interventions was the lack of blinding, which is unavoidable to a great degree. Twenty-one trials were considered at high risk of outcome assessment bias for function and other subjective outcomes. The risk of bias resulting from a high loss to follow-up or exclusion of participants from the analyses was considered high in 13 trials, two of which were new to this version (Buecking 2014; Soliman 2013). Most comparisons were carried out in small single trials only; there is clearly a need for caution in interpreting the results of small trials which demonstrate 'no evidence of an effect' rather than 'evidence of no effect'. Insufficiencies in quantity and quality of the evidence still preclude the drawing of robust conclusions for most of the comparisons evaluated by the included trials.

Only one of the eight newly included trials had prospective trial registration and a published protocol (ProFHER 2015). While this is discouraging at present, it is notable that the increased research activity in this previously overlooked area is also associated with far more prospective trial registration as well as publication of trial protocols. Both these items show the greater use of robust methodology that is required to minimise bias. Additionally, there is a growing interest in multicentre trials. Seven of the 21 ongoing trials are multicentre.

The results of the GRADE assessment of the quality of evidence for the individual comparisons are summarised below. With the exception of the evidence for the comparison of surgical versus non-surgical treatment, most of the GRADE assessments for the other comparisons were low or very low quality. This typically reflects the insufficiency of the evidence from small single trials which have limitations in design, conduct, analysis and reporting, putting them at high risk of bias.

\section{Surgical treatment versus non-surgical treatment}

- The quality of evidence assessments for difference outcomes for this comparison ranged from moderate to high (for details please see Summary of findings for the main comparison).

\section{Initial treatment, including immobilisation}

- Early versus delayed mobilisation: the quality of evidence assessments for difference outcomes for this comparison ranged from very low to low (for details please see Summary of findings 2).

- Type of bandage (Gilchrist arm sling versus the Desault body bandage): the quality of evidence assessments for all reported outcomes were very low. The evidence was downgraded two levels for serious study limitations reflecting a serious risk of bias (including selection bias: quasi-randomised trial) and one level for imprecision (single small trial: 28 participants).

\section{Continuing management (rehabilitation) after initial treatment involving sling immobilisation}

- Instructions for home exercises versus physiotherapy: the quality of evidence assessments for all reported outcomes were very low. The evidence was downgraded one level for study limitations reflecting a high risk of bias and two levels for imprecision (evidence from single small trials: 20 and 42 participants).

- Supervised exercises in a swimming pool plus home exercises versus home exercises alone: the quality of evidence assessments for all reported outcomes were very low. There is no quantitative evidence available for this comparison.

- Pulsed electromagnetic high frequency energy (PHFE): the quality of evidence assessments for all reported outcomes were very low. There is no quantitative evidence available for this comparison.

\section{Different methods of surgical management}

Comparisons of different categories of surgical intervention 
- Open reduction with internal fixation using a locking plate versus a locking nail: the quality of evidence assessments for all reported outcomes were very low. The evidence was downgraded one level for study limitations reflecting a high risk of bias and two levels for imprecision (evidence from 57 participants in one trial).

- Open reduction with internal fixation using a locking plate versus minimally invasive fixation with distally inserted intramedullary K-wires: the quality of evidence assessments for all reported outcomes were very low. The evidence was downgraded two levels for study limitations reflecting a serious risk of bias and one level for imprecision (evidence from 55 participants in one trial).

- Hemiarthroplasty versus open reduction and locking plate fixation: the quality of evidence assessments for all reported outcomes were very low. The evidence was downgraded one level for study limitations reflecting a high risk of bias and two levels for imprecision (evidence from 32 participants in one trial).

- Hemiarthroplasty versus tension band wiring: the quality of evidence assessments for all reported outcomes were very low. The evidence was downgraded two levels for study limitations reflecting a serious risk of bias and one level for imprecision (evidence from 30 participants in one trial).

- Reverse shoulder arthroplasty versus hemiarthroplasty: the quality of evidence assessments for all reported outcomes were low. The evidence was downgraded one level for study limitations reflecting unclear risk of bias in several domains and one level for imprecision (evidence from 62 participants in one trial).

\section{Comparisons of different methods of performing an intervention in the same category}

- Deltoid-split approach versus deltopectoral approach for non-contact bridging plate fixation: the quality of evidence assessments for all reported outcomes were very low. The evidence was downgraded two levels for study limitations reflecting a serious risk of bias and one level for imprecision (wide confidence intervals; evidence from 120 participants in one trial). The evidence would have been further downgraded one level for indirectness because of the possible misapplication of the Lawson quality-of-life score.

- Polyaxial versus monoaxial locking plate fixation: the quality of evidence assessments for all reported outcomes were very low. The evidence was downgraded one level for study limitations reflecting a serious risk of bias and two levels for imprecision (wide confidence intervals; evidence for function from 48 participants in one trial).

- Locking plate - use of medial locking screws: the quality of evidence assessments for all reported outcomes were very low. The evidence was downgraded one level for study limitations reflecting unclear risk of bias in several domains and two levels for imprecision (wide confidence intervals; evidence from 68 participants in one trial).

- Intramedullary nails: MPHN versus Polarus: the quality of evidence assessments for the reported outcomes were low or very low. The evidence was downgraded one or two levels for study limitations reflecting the high risk of outcome assessment bias and the unclear risk of bias relating to detection given the large range in follow-up (6 to 22 months) for some outcomes, and one level for imprecision (wide confidence intervals; evidence from 54 participants in one trial).

- Hemiarthroplasty - comparison of the EPOCA versus the HAS prosthesis: the quality of evidence assessments for all reported outcomes were very low. The evidence was downgraded two levels for study limitations reflecting a serious risk of bias and one level for imprecision (inadequate data presented; evidence from 35 participants in one trial).

- Hemiarthroplasty - tenodesis of the long head of the biceps (LHB) versus LHB tendon left intact: the quality of evidence assessments for all reported outcomes were very low. The evidence was downgraded two levels for study limitations reflecting a serious risk of bias and one level for imprecision (evidence from 45 participants in one trial).

\section{Continuing management (including rehabilitation) after surgical intervention}

- One week versus three weeks immobilisation after percutaneous fixation: the quality of evidence assessments for all reported outcomes were very low. The evidence was downgraded two levels for study limitations reflecting a serious risk of bias and one level for imprecision (evidence at six months from 64 participants in one trial).

- Mobilisation after two weeks versus six weeks following hemiarthroplasty: the quality of evidence assessments for all reported outcomes were very low. The evidence was downgraded two levels for study limitations reflecting a serious risk of bias and one level for imprecision (evidence from 49 participants in one trial).

\section{Potential biases in the review process}

While our search was comprehensive it is likely that we have failed to identify some randomised trials, particularly those reported only in abstracts or in non-English language publications. We may also have overlooked mixed-population trials that included proximal humeral fractures as a subgroup. However, we are almost certain that we have not overlooked trials that would provide definitive evidence that could inform practice. It is clear, from the growing awareness and imperative of trial registration, that such trials are now in progress. We prepared the review using systematic processes throughout, including contacting trial investigators for clarification and missing data. We describe the dilemma presented 
in the pooling of data from clearly heterogeneous trials for the surgical treatment versus non-surgical treatment comparison in the Effects of interventions. This is, however, compatible with the overall question and notably the pooled analyses did not result in statistically significant heterogeneity.

\section{Agreements and disagreements with other studies or reviews}

Several new systematic reviews, none of which cover all treatment options, were identified via the search update. The only rehabilitation review examined the effects of exercise in people with select upper limb fractures including proximal humeral fractures (Bruder 2011). Two reviews compared surgical versus non-surgical intervention (Li 2013; Mao 2014). Li 2013 limited surgery to internal fixation. One review compared arthroplasty versus 'joint preservation' that was either non-surgical treatment or internal fixation (Zhang 2014). Two compared arthroplasty versus internal fixation (Dai 2014; Gomberawalla 2013). Dai 2014 limited internal fixation to locking plate fixation. Two reviews compared reverse shoulder arthroplasty versus hemiarthroplasty (Mata-Fink 2013; Namdari 2013). All eight reviews, four of which included evidence from a broader spectrum of study designs (Dai 2014; Gomberawalla 2013; Mata-Fink 2013; Namdari 2013), reported the limitations in the available evidence. Unlike our review, with its later search date, none of the three reviews comparing surgical versus non-surgical treatment included ProFHER 2015 and neither of the two reviews comparing RSA with hemiarthroplasty included the first randomised trial on this topic (Sebastiá-Forcada 2014).

\section{AUTHORS' CONCLUSIONS}

\section{Implications for practice}

There is high or moderate quality evidence that, compared with non-surgical treatment, surgery does not result in a better outcome at one and two years after injury for people with displaced proximal humeral fractures involving the humeral neck and is likely to result in a greater need for subsequent surgery. The evidence does not cover the treatment of two-part tuberosity fractures, fractures in young people, high energy trauma, nor the less common fractures such as fracture dislocations and head splitting fractures.

There is insufficient evidence from randomised controlled trials to inform the choices between different non-surgical interventions, different surgical interventions, or different rehabilitation interventions for these fractures.

\section{Implications for research}

The availability of high quality evidence primarily from a sufficiently powered multicentre randomised trial (ProFHER 2015) is the key reason why this review can now inform on the use of surgery for the majority of displaced fractures. There is a need for similar trials to help address other key treatment uncertainties. Decisions on priority topics should consider the coverage of the current evidence base as well as the topics covered by the ongoing trials. Of particular note is that three ongoing trials are already comparing reverse shoulder arthoplasty versus hemiarthoplasty.

Although the identification of priority topics requires input from others, including patients, we suggest that research should be focused primarily on optimising non-surgical treatment. Where randomised trials are warranted, these should use standard and validated outcome measures, including patient-reported measures of functional outcome and quality of life, and also assess resource implications. They should also meet the CONSORT criteria for design and reporting of non-pharmacological studies (Boutron 2008) and subsequent developments including the adequate reporting of interventions (Hoffmann 2014).

This Cochrane review should be maintained and updated as further randomised controlled trials become available. The authors would be pleased to receive information about any other randomised controlled trials relating to the treatment of these fractures.

\section{ACKNOWLEDGEMENTS}

We thank Mario Lenza and David Limb for their helpful feedback at editorial review. We thank Lindsey Elstub for her editorial support. We thank Joanne Elliott for patiently supplying several search downloads for this version and for her advice on searching other databases.

We thank the following for further information on their research in this area for this update: Harm Boons, Stig Brorson, Omar Soliman and Carlos Torrens.

We thank Benjamin Ollivere and Katie Rollins for their contribution to the previous version of this review. The acknowledgements for former versions of the review are presented in Appendix 3.

This project was supported by the National Institute for Health Research via Cochrane Infrastructure funding to the Cochrane Bone, Joint and Muscle Trauma Group. The views and opinions expressed therein are those of the authors and do not necessarily reflect those of the Systematic Reviews Programme, NIHR, NHS or the Department of Health. 


\section{R E F E R E N C E S}

\section{References to studies included in this review}

Agorastides 2007 \{published and unpublished data\}

* Agorastides I, Sinopidis C, El Meligy M, Yin Q, Brownson P, Frostick SP. Early versus late mobilization after hemiarthroplasty for proximal humeral fractures. Journal of Shoulder \& Elbow Surgery 2007;16(3 Suppl):S33-8.

Frostick S. Randomised prospective study to evaluate sling immobilisation versus early active assisted mobilisation following hemiarthroplasty for fractures of the proximal humerus. In: National Research Register, Issue 1, 2003. Oxford; Update Software.

Frostick S. Randomised prospective study to evaluate sling immobilisation versus early active assisted mobilisation following hemiarthroplasty for fractures of the proximal humerus. http://www.controlled-trials.com/mrct/trial/ 483871 (accessed August 6 2010).

Bertoft 1984 \{published data only\} Bertoft ES, Lundh I, Ringqvist I. Physiotherapy after fracture of the proximal end of the humerus. Comparison between two methods. Scandinavian Journal of Rehabilitation Medicine 1984;16(1):11-6.

Boons 2012 \{published data only\}

Boons H. personal communication March 122015. * Boons HW, Goosen JH, Grinsven S, Susante JL, Loon CJ. Hemiarthroplasty for humeral four-part fractures for patients 65 years and older: a randomized controlled trial. Clinical Orthopaedics and Related Research 2012;(470): 3483-91.

Buecking 2014 \{published data only\} Buecking B, Mohr J, Bockmann B, Zettl R, Ruchholtz $S$. Deltoid-split or deltopectoral approaches for the treatment of displaced proximal humeral fractures?. Clinical Orthopaedics and Related Research. 2014;472(5):1576-85.

Cai 2012 \{published data only\}

Cai M, Tao K, Yang C, Li S. Internal fixation versus shoulder hemiarthroplasty for displaced 4-part proximal humeral fractures in elderly patients. Orthopedics 2012;35 (9):e1340-6.

Fialka 2008 \{published data only\}

Fialka C, Stampfl P, Arbes S, Reuter P, Oberleitner G, Vecsei V. Primary hemiarthroplasty in four-part fractures of the proximal humerus: Randomized trial of two different implant systems. Journal of Shoulder and Elbow Surgery 2008;17(2):210-5.

Fjalestad 2010 \{published and unpublished data\} Fjalestad T. personal communication April 122010. Fjalestad T. personal communication December 32006. Fjalestad T. Comminuted proximal humeral fractures. A randomised study of surgical versus conservative treatment. http://clinicaltrials.gov/ct2/show/record/NCT00863473 (accessed June 24 2009).

Fjalestad T, Hole M. Displaced proximal humeral fractures: operative versus non-operative treatment-a 2- year extension of a randomized controlled trial. European Journal of Orthopaedic Surgery \& Traumatology 2014;24(7): 1067-73.

Fjalestad T, Hole MO, Hovden IA, Blucher J, Stromsoe K. Surgical treatment with an angular stable plate for complex displaced proximal humeral fractures in elderly patients: a randomized controlled. Journal of Orthopaedic Trauma 2012;26(2):98-106.

* Fjalestad T, Hole MO, Jorgensen JJ, Stromsoe K, Kristiansen IS. Health and cost consequences of surgical versus conservative treatment for a comminuted proximal humeral fracture in elderly patients. Injury 2010;41(6): 599-605.

Fjalestad T, Stromsoe K, Blucher J, Tennoe B. Fractures in the proximal humerus: functional outcome and evaluation of 70 patients treated in hospital. Archives of Orthopaedic and Trauma Surgery 2005;125(5):310-6.

Fjalsted T. personal communication April 272012.

Kristiansen IS, Jorgensen JJ, Fjalestad T, Stromsoe K. Fracture of the proximal humerus. Randomised clinical study at the Aker University Hospital - economic evaluation [Fraktur i øvre humuserende. Randomisert klinisk studie ved Aker Universitetssykehus HF- Økonomisk evaluering]. University of Oslo website: http://www.hero.uio.no/ prosjekter/prosjekt6.13.html (Accessed November 13 2006).

\section{Hodgson 2003 \{published and unpublished data\}}

Campbell M. Early versus late physiotherapy in fractured proximal neck of humerus: a randomised controlled study. In: National Research Register, Issue 3, 2000. Oxford: Update Software.

Hodgson S, Stanley D. A randomised controlled trial investigating functional outcome, with early and late physiotherapy, on patients sustaining a fractured proximal humerus. British Elbow and Shoulder Society (BESS) Annual Scientific Meeting and Instructional Course; 2000 May 4-5; Nottingham (UK). 2000.

Hodgson S, Stanley S, Mawson S. Timing of physiotherapy in management of fractured proximal humerus:

Randomised controlled trial [Abstract]. Physiotherapy 2002; 88(12):763.

Hodgson S, Stanley S, Mawson S. Timing of physiotherapy in the management of the fractured proximal humerus: a randomised controlled trial. Extending the boundaries. Chartered Society of Physiotherapy Congress 2001; 2001 Oct 19-21; Birmingham (UK). 2001.

Hodgson SA. A controlled, randomised study investigating functional outcome, with early and late physiotherapy, on patients following a fractured proximal humerus. National Research Register (http://www.update-software.com/ National/nrr-frame.html) 2000, issue 3.

Hodgson SA, Mawson SJ, Saxton JM, Stanley D. Rehabilitation of two-part fractures of the neck of the humerus (two-year follow-up). Journal of Shoulder \& Elbow 
Surgery 2007;16(2):143-5.

* Hodgson SA, Mawson SJ, Stanley D. Rehabilitation after two-part fractures of the neck of the humerus. Journal of Bone and Joint Surgery. British Volume 2003;85(3):419-22. Stanley D. A prospective, controlled randomised study investigating functional outcome, with early and late physiotherapy, on patients following a fractured proximal humerus. http://www.controlled-trials.com/mrct/trial/ 485287 (accessed August 06 2010).

Stanley D. A prospective, controlled, randomised study investigating functional outcome, with early and late physiotherapy, on patients following a fractured proximal humerus. In: National Research Register, Issue 2, 2001. Oxford: Update Software.

\section{Hoellen 1997 \{published data only\}}

Bauer G, Hoellen I, Hohlbein O. Primary prosthetic humerus head replacement in dislocated multiple fragment fracture of the humerus head in over 60-year-olds [Der primare prothetische Humeruskopfersatz bei der dislozierten Humeruskopfmehrfragmentfraktur bei uber 60jahrigen]. Hefte zur der Unfallchirurg 1999;272:169-70.

* Hoellen IP, Bauer F, Holbein O. Primary endoprosthesis in comminuted fractures of the proximal humerus - an alternative treatment for elderly patients? [Der prothetische humeruskipfersatz bei der dislozierten humerusmehrfragmentfraktur des alten menschen - eine alternative zur minimalosteosynthese]. Zentralblatt fur Chirurgie 1997;122(11):994-1001.

Hoellen IP, Holbein O, Bauer G. Prosthetic humerus head replacement in dislocated humerus head multiple fracture in older people: An alternative to minimal osteosynthesis? [Der prothetische Humeruskopfersatz bei der dislozierten Humeruskopfmehrfragmentfraktur des alten Menschen: Eine Alternative zur Minimalosteosynthese?]. Hefte zur der Unfallchirurg 1997;268:49-51.

Holbein O, Bauer G, Hoellen I, Keppler P, Hehl G, Kinzl L. Is primary endoprosthetic replacement of the humeral head an alternative treatment for comminuted fractures of the proximal humerus in elderly patients? [Stellt die primare Implantation einer Humeruskopfprothese bei der dislozierten Humeruskopfmehrfragmentfraktur des alten Menschen eine Alternative zur Minimalosteosynthese dar?]. Osteosynthese International 1999;7 Suppl 2:207-10. Holbein O, Hehl G, Keppler P, Kinzl L. Treatment of proximal humerus multiple fracture in old and very old people [Die Behandlung der Mehrfragmentfraktur des proximalen Humerus bei alten und uralten Menschen]. Hefte zur der Unfallchirurg 2000;275:207-8.

Pokar S, Holbein O, Kinzl L, Hehl G. Primary head of humerus prosthetic vs. minimal osteosynthesis in the treatment of head of humerus multiple-fragment fractures in older patients [Primare Humeruskopfprothese vs. Minimalosteosynthese in der Behandlung von Humeruskopfmehrfragmentfrakturen des alteren Patientenatients]. Hefte zur der Unfallchirurg 2001;283: $375-6$.
Kristiansen 1988 \{published data only\}

Kristiansen B, Kofoed H. Transcutaneous reduction and external fixation of displaced fractures of the proximal humerus. A controlled clinical trial. Journal of Bone and Joint Surgery. British Volume 1988;70(5):821-4.

\section{Kristiansen 1989 \{published data only\}}

Kristiansen B, Angermann P, Larsen TK. Functional results following fractures of the proximal humerus. A controlled clinical study comparing two periods of immobilization. Archives of Orthopaedic and Trauma Surgery 1989;108(6): 339-41.

\section{Lefevre-Colau 2007 \{published and unpublished data\}} Lefevre-Colau M-M. Efficacy of shoulder mobilisation versus conventional immobilisation for nonsurgically proximal humerus fracture. http://clinicaltrials.gov/show/ NCT00326794 (accessed September 22 2006).

* Lefevre-Colau MM, Babinet A, Fayad F, Fermanian $\mathrm{J}$, Anract $\mathrm{P}$, Roren $\mathrm{A}$, et al. Immediate mobilization compared with conventional immobilization for the impacted nonoperatively treated proximal humeral fracture. A randomized controlled trial. Journal of Bone and Joint Surgery - American Volume 2007;89(12):2582-90.

\section{Livesley 1992 \{published data only\}}

Livesley PJ, Mugglestone A, Whitton J. Electrotherapy and the management of minimally displaced fracture of the neck of the humerus. Injury 1992;23(5):323-7.

Lopiz 2014 \{published data only\}

Lopiz Y, Garcia-Coiradas J, Garcia-Fernandez C, Marco F. Proximal humerus nailing: a randomized clinical trial between curvilinear and straight nails. Journal of Shoulder \& Elbow Surgery 2014;23(3):369-76.

\section{Lundberg 1979 \{published data only\}}

Lundberg BJ, Svenungson-Hartvig E, Wikmark R. Independent exercises versus physiotherapy in nondisplaced proximal humeral fractures. Scandinavian Journal of Rehabilitation Medicine 1979;11(3):133-6.

\section{Ockert 2010 \{published data only\}}

Biberthaler P, Braunstein V, Kirchoff C, Kroetz M, Kettler M, Mutschler W. Surgical therapy of humeral head fractures with a locking plate: analysis of 176 cases. Journal of Bone \& Joint Surgery - British Volume 2009;91(Suppl 1):40. Ockert B. personal communication June 182012.

* Ockert B, Braunstein V, Kirchhoff C, Korner M, Kirchhoff S, Kehr K, et al. Monoaxial versus polyaxial screw insertion in angular stable plate fixation of proximal humeral fractures: radiographic analysis of a prospective randomized study. Journal of Trauma 2010;69(6):1545-51. Ockert B, Pedersen V, Geyer L, Wirth S, Mutschler W, Grote $S$. Position of polyaxial versus monoaxial screws in locked plating for proximal humeral fractures: analysis of a prospective randomized study. European Journal of Orthopaedic Surgery and Traumatology 2014;24(5):747-52. 
Olerud 2011a \{published data only\}

Olerud P. personal communication April 192012.

Olerud P. personal communication May 222012.

* Olerud P, Ahrengart L, Ponzer S, Saving J, Tidermark J. Internal fixation versus nonoperative treatment of displaced 3-part proximal humeral fractures in elderly patients: a randomized controlled trial. Journal of Shoulder \& Elbow Surgery 2011;20(5):747-55.

Olerud 2011b \{published data only\}

Olerud P. personal communication April 192012.

Olerud P. personal communication May 222012.

* Olerud P, Ahrengart L, Ponzer S, Saving J, Tidermark

J. Hemiarthroplasty versus nonoperative treatment of displaced 4-part proximal humeral fractures in elderly patients: a randomized controlled trial. Journal of Shoulder \& Elbow Surgery 2011;20(7):1025-33.

ProFHER 2015 \{published data only\}

Handoll H, Brealey S, Rangan A, Keding A, Corbacho B, Jefferson L, et al. The ProFHER (PROximal Fracture of the Humerus: Evaluation by Randomisation) trial - a pragmatic multicentre randomised controlled trial evaluating the clinical effectiveness and cost-effectiveness of surgical compared with non-surgical treatment for proximal fracture of the humerus in adults. Health Technology Assessment 2015;19(24):1-280.

Handoll H, Brealey S, Rangan A, Torgerson D, Dennis L, Armstrong A, et al. Protocol for the ProFHER (PROximal Fracture of the Humerus: Evaluation by Randomisation) trial: a pragmatic multi-centre randomised controlled trial of surgical versus non-surgical treatment for proximal fracture of the humerus in adults. BMC Musculoskeletal Disorder 2009;10:140.

Handoll HH, Goodchild L, Brealey SD, Hanchard NC, Jefferson L, Keding A, et al. Developing, delivering and documenting rehabilitation in a multi-centre randomised controlled surgical trial: experiences from the ProFHER trial. Bone \& Joint Research 2014;3(12):335-40.

Rangan A. Multi-centre randomised trial evaluating surgery for displaced fractures of the proximal humerus. http:// www.controlled-trials.com/ISRCTN50850043 (accessed June 18 2010).

* Rangan A, Handoll H, Brealey S, Jefferson L, Keding A, Corbacho Martin B, et al. Surgical vs nonsurgical treatment of adults with displaced fractures of the proximal humerus: The PROFHER randomized clinical trial. JAMA 2015;313 (10):1037-47.

Revay 1992 \{published data only\}

Revay S, Dahlstrom M, Dalen N. Water exercise versus instruction for self-training following a shoulder fracture. International Journal of Rehabilitation Research 1992;15(4): 327-33.

Rommens 1993 \{published data only\}

Deldycke J, Rommens PM, Heyvaert G, Broos PL. Conservative treatment of sub-capital humerus fractures. A comparative study between the classical Desaultbandage and the new Gilchrist-bandage [Die konservative Behandlung von subkapitalen Humerusfrakturen.
Eine vergleichende Studie zwischen dem klassischen Desault-Verband und der neuen Gilchrist-Bandage]. Hefte zur der Unfallchirurg 1993;232:145-6.

* Rommens PM, Heyvaert G. Conservative treatment of subcapital humerus fractures. A comparative study of the classical Desault bandage and the new Gilchrist bandage [Die konservative behandlung subkapitaler humerusfrakturen. Eine vergleichende studie zwischen dem klassischen Desault-verband und der neuen Gilchrist-bandage]. Unfallchirurgie 1993;19(2):114-8.

Sebastiá-Forcada 2014 \{published data only\} Sebastiá-Forcada E, Cebrián-Gómez R, Lizaur-Utrilla A, Gil-Guillén V. Reverse shoulder arthroplasty versus hemiarthroplasty for acute proximal humeral fractures. A blinded, randomized, controlled, prospective study. Journal of Shoulder and Elbow Surgery 2014;23(10):1419-26.

Smejkal 2011 \{published data only\}

Smejkal K, Didek T, Zvak I, Trlica J, Folvarsky J. Operation treatment of 2-3 fragments proximal humeral fractures using Zifko versus Philos [abstract]. European Journal of Trauma \& Emergency Surgery 2008;34(Suppl 1):44-5.

* Smejkal K, Lochman P, Dedek T, Trlica J, Koci J, Zvak I. Surgical treatment for proximal humerus fracture [Czech] [Operacni lecba zlomenin proximalniho humeru]. Acta Chirurgiae Orthopaedicae et Traumatologiae Cechoslovaca 2011;78(4):321-7.

\section{Soliman 2013 \{published data only\}}

Soliman O. personal communication May 32015.

Soliman O. personal communication May 62015.

Soliman O, Koptan W. Proximal humeral fractures treated with hemiarthroplasty. Does tendodesis of the long head of the biceps improve results?. http://apps.who.int/trialsearch/ Trial2.aspx?TrialID=PACTR201205000381245 (accessed February 10 2015).

* Soliman OA, Koptan WMT. Proximal humeral fractures treated with hemiarthroplasty: does tenodesis of the long head of the biceps improve results?. Injury 2013;44(4): $461-4$.

Stableforth 1984 \{published data only\} Stableforth PG. Four-part fractures of the neck of the humerus. Journal of Bone and Joint Surgery. British Volume 1984;66(1):104-8.

Torrens 2012 \{published data only\}

Torrens C. Personal communication June 042015. Torrens C. Personal communication June 102015.

* Torrens C, Perez FS, Rigol P, Puig L. Management of conservatively treated proximal humeral fractures: Prospective randomized study [abstract]. American Academy of Orthopaedic Surgeons. 2012 Mar 7-11; San Francisco, California. http://www.aaos.org/education/ anmeet/prevmeet.asp (accessed 26/02/13). San Francisco, 2012.

Voigt 2011 \{published data only\}

Voigt C. personal communication May 302012.

* Voigt C, Geisler A, Hepp P, Schulz AP, Lill H. Are polyaxially locked screws advantageous in the plate 
osteosynthesis of proximal humeral fractures in the elderly? A prospective randomized clinical observational study. Journal of Orthopaedic Trauma 2011;25(10):596-602.

\section{Wirbel 1999 \{published data only\}}

* Wirbel R, Knorr V, Saur B, Duhr B, Mutschler W. Minimally invasive fixation of displaced proximal humeral fractures. Orthopaedics and Traumatology 1999;7(1):44-53. Wirbel RJ, Knorr V, Mutschler W. Minimal invasive therapy in dislocated proximal humerus fractures. Influence of post-operative immobilisation on the function outcome [Minimal invasive Therapie bei dislozierten proximalen Humerusfrakturen. Einfluss der postoperativen Immobilisation auf das funktionelle Ergebnis]. Hefte zur der Unfallchirurg 1997;268:678-81.

Zhang 2011 \{published data only\}

Zhang D. personal communication May 142012.

* Zhang L, Zheng J, Wang W, Lin G, Huang Y, Zheng J, et al. The clinical benefit of medial support screws in locking plating of proximal humerus fractures: a prospective randomized study. International Orthopaedics 2011;35(11): 1655-61.

\section{Zhu 2011 \{published data only\}}

Zhu Y, Lu Y, Shen J, Zhang J, Jiang C. Locking intramedullary nails and locking plates in the treatment of two-part proximal humeral surgical neck fractures: a prospective randomized trial with a minimum of three years of follow-up. Journal of Bone \& Joint Surgery - American Volume 2011;93(2):159-68.

\section{Zyto 1997 \{published data only\}}

Karladani HA. Treatment of displaced proximal humeral fractures in elderly patients [Letter and reply]. Journal of Bone and Joint Surgery. British Volume 1999;81(1):181-2. Tornkvist H, Ahrengart L, Sperber A. Tension band wiring vs. non-operative treatment of displaced proximal humerus fractures. A prospective randomized study [abstract]. Orthopaedic Transactions 1997;21(2):592.

Tornkvist H, Ahrengart L, Sperber A. Tension band wiring vs. nonoperative treatment of proximal humerus fractures - a prospective randomized study [Abstract]. Acta Orthopaedica Scandinavica. Supplementum 1995;66(265): $40-1$.

Zyto K. personal communication March 211997.

* Zyto K, Ahrengart L, Sperber A, Tornkvist H. Treatment of displaced proximal humeral fractures in elderly patients. Journal of Bone and Joint Surgery. British Volume 1997;79 (3):412-7.

\section{References to studies excluded from this review}

\section{Bing 2002 \{unpublished data only\}}

Armstrong A. personal communication July 172010.

* Bing AJF, Eastwood G, Sharma R, Cross R, Taylor GJS, Harper WM. A randomised prospective trial comparing Polarus nail and Rush pins for fixation of proximal humeral fractures - Poster A5. British Orthopaedic Association
Annual Congress; 2002 Sept 18-20; Cardiff (UK). London: British Orthopaedic Association, 2002:2.

Sharma R. A prospective, randomised clinical trial to compare Rush pins fixation with Polaris nail fixation of displaced two part fractures of the proximal humerus. In: National Research Register, Issue 2, 2000. Oxford: Update Software.

\section{Bolano 1995 \{published data only\}}

Bolano LE. Operative treatment of humeral shaft fractures: A prospective, randomized study of intramedullary nailing versus dynamic compression plating [abstract]. Orthopaedic Transactions 1995;19(1):33.

\section{Brownson 2001 \{unpublished data only\}}

Brownson P. A prospective randomised trial comparing conservative treatment and the Halder Humeral Nail for displaced fractures of the surgical neck and shaft of the humerus. In: National Research Register, Issue 2, 2000. Oxford: Update Software.

Carbone 2012 \{published data only\}

Carbone S, Tangari M, Gumina S, Postacchini R, Campi A, Postacchini F. Percutaneous pinning of three- or four-part fractures of the proximal humerus in elderly patients in poor general condition: MIROS® versus traditional pinning. International Orthopaedics 2012;36(6):1267-73.

\section{Chapman 1997 \{published data only\}}

* Chapman J, Weber TG, Henley B, Benca PJ. Randomized prospective study of humerus fixation: nails vs. plates [abstract]. Orthopaedic Transactions 1997;21(2):594. Chapman J, Weber TG, Henley MB, Benca P. Randomized prospective study of humerus fixation: Nails vs. plates [abstract]. Orthopaedic Transactions 1996;20(1):10.

Chiu 1997 \{published data only\} Chiu FY, Chen CM, Lin CF, Lo WH, Huang YL, Chen TH. Closed humeral shaft fractures: a prospective evaluation of surgical treatment. Journal of Trauma 1997;43(6):947-51.

\section{Cigni 2012 \{published data only\}}

Cigni S, Battista E, Chessa A, Pietrogrande L. Stability plate surgery in proximal humerus fractures: Deltoidpectoral approach versus direct lateral transdeltoid approach [abstract]. Journal of Orthopaedics and Traumatology 2012; 13(Suppl 1):S20.

De Boer 2003 \{unpublished data only\}

Booth C. personal communication March 202003.

De Boer P. personal communication March 122003.

* De Boer P. Plating of proximal humerus fracture: a blind comparative study. In: National Research Register, Issue 1, 2003. Oxford: Update Software.

\section{Dias 2001 \{unpublished data only\}}

Armstrong A. personal communication July 172010. Der Tavitian J. The management of comminuted proximal humeral fractured, a randomised prospective trial. https: //portal.nihr.ac.uk/Profiles/NRR.aspx?Publication $\cdot \mathrm{ID}=$ N0123137861) (accessed Aug 09 2010).

Dias J. personal communication November 162001. 
Edelson 2008 \{published data only\}

Edelson G, Safuri H, Salami J, Vigder F, Militianu D. Natural history of complex fractures of the proximal humerus using a three-dimensional classification system. Journal of Shoulder \& Elbow Surgery 2008;17(3):399-409.

Elidrissi 2013 \{published data only\}

Elidrissi M, Bensaad S, Shimi M, Elibrahimi A, Elmrini A. Surgical treatment of proximal humeral fractures anatomical plate versus palm tree pinning (26 cases) [Le traitement chirurgical des fractures de l'extrémité supérieure de l'humérus : plaque anatomique versus embrochage en palmier, à propos de 26 cas]. Chirurgie de la Main. 2013;32 (1):25-9.

Erdoğ an 2014 \{published data only\}

Erdoğ an M, Desteli EE, i mren Y, Üztürk A, K, 1 I ç M, Sezgin H. The effect of inferomedial screw on postoperative shoulder function and mechanical alignment in proximal humerus fractures. European Journal of Orthopaedic Surgery \& Traumatology 2014;24(7):1055-9.

Fan 2012 \{published data only\} Fan Y, Wang S, Luo Y. Effectiveness comparison of operative and non-operative treatment for complex proximal humeral fractures in elderly patients [Chinese]. Chung-Kuo Hsiu Fu Chung Chien Wai Ko Tsa Chih/Chinese Journal of Reparative \& Reconstructive Surgery 2012;26(9):1029-32.

Flannery 2006 \{unpublished data only\}

Flannery M. personal communication November 162006. * Flannery M. A prospective randomised trial for the treatment of four part fractures of proximal humerus: "conservative vs hemiarthroplasty". In: National Research Register, Issue 3, 2006. Oxford; Update Software. Turner R. personal communication November 192006.

Gradl 2009 \{published data only\} Gradl G, Dietze A, Kaab M, Hopfenmuller W, Mittlmeier T. Is locking nailing of humeral head fractures superior to locking plate fixation?. Clinical Orthopaedics and Related Research 2009;467(11):2986-93.

Hems 2000 \{unpublished data only\} Hems T. A prospective randomised trial comparing conservative treatment and the Halder Humeral Nail for displaced fractures of the surgical neck and shaft of the humerus. In: National Research Register, Issue 3, 2000. Oxford: Update Software.

IRCT2013052313435N1 \{unpublished data only\} Majd DA, Nouraei MH. Comparing review of the treatment results based on surgical or nonsurgical methods in the patients with fracture of proximal humerus referred to Al-Zahra and Kashani Hospitals (Isfahan) during 1388 1392 (years). http://apps.who.int/trialsearch/Trial2.aspx? TrialID=IRCT2013052313435N1 (accessed February 11 2015).

* Nouraei MH, Majd DA, Zamani F. Comparing the treatment results of proximal humerus fracture based on surgical or nonsurgical methods. Advanced Biomedical Research 2014;3(253):1-9.
Liao 2009 \{published data only\}

Liao C, Wang P, Xie Y, Fan T, Li P, Liang W. Different surgical methods for treatment of senile osteoporotic comminuted proximal humerus fracture. Zhongguo xiu fu chong jian wai ke za zhi = Zhongguo xiufu chongjian waike zazhi $=$ Chinese Journal of Reparative and Reconstructive Surgery 2009;23(12):1443-6.

Maniscalco 2014a \{published data only\}

Maniscalco P, Caforio M. The importance of early rehabilitation in proximal humeral fracture: A randomized controlled clinical trial of efficacy and safety of endomedullary Diphos nail [abstract]. Journal of Orthopaedics and Traumatology 2014;15(Suppl 1):S32-3.

Martetschlager 2012 \{published data only\} Martetschlager F, Siebenlist S, Weier M, Sandmann G, Ahrens P, Braun K, et al. Plating of proximal humeral fractures. Orthopedics 2012;35(11):e1606-12.

\section{Martin 2000 \{published data only\}}

Heath C. The effect of interfential current on pain following proximal fracture of the humerus. A single blind, randomised, controlled clinical trial. In: National Research Register, Issue 4, 2001. Oxford: Update Software.

* Martin D, Palmer S, Heath C. Interferential current as an adjunct to exercise and mobilisation in the treatment of proximal humerus fracture pain: Lack of evidence of an additional effect [Abstract]. Physiotherapy 2000;86(3):147. Palmer S. personal communication May 92003.

Mechlenburg 2009 \{unpublished data only\}

* Mechlenburg I. Comparison of the Philos plate and cemented hemiprosthesis (Bigliani) in the treatment of three- and four-part fractures of the proximal humerus: a prospective, randomised migration and bone density study. http://clinicaltrials.gov/show/NCT00408291 (assessed June 24 2009).

Mechlenburg I. Evaluation of the Winsta PH osteosynthesis device in the treatment of three- and four-part fractures of the proximal humerus: a prospective migration and bone density study. http://clinicaltrials.gov/show/NCT00408291 (assessed May 17 2010).

Mechlenburg IB. personal communication May 252010.

\section{NCT00384852 \{unpublished data only\}}

Lo K. personal communication February 122015.

* Wyeth (Pfizer). A study of rhBMP-2/CPM in closed fractures of the humerus. https:/clinicaltrials.gov/ct2/ show/NCT00384852 (accessed January 19 2015).

\section{NCT01532076 \{unpublished data only\}}

Saxer F, Jakob M. Effectiveness of adipose tissue derived mesenchymal stem cells as osteogenic component in composite grafts (ROBUST). https://www.clinicaltrials.gov/ ct2/show/NCT01532076 (accessed January 19 2015).

\section{NCT02122315 \{unpublished data only\}}

Fernández-de-las-Peñas C. Dry needling in post-operative shoulder pain. https://www.clinicaltrials.gov/ct2/show/ NCT02122315 (accessed January 19 2015). 
NTR2186 \{unpublished data only\}

Loon CJ, Grinsven S. Delta Xtend reversed shoulder prosthesis. http://apps.who.int/trialsearch/Trial2.aspx? TrialID=NTR2186 (accessed Februrary 11 2015).

Parnes 2005 \{published data only\} Parnes N, Pritsch T, Mozes G. Is surgery the best choice in the treatment of complex fractures of proximal humerus? Preliminary study on 50 cases [Abstract]. Journal of Bone \& Joint Surgery - British Volume 2005;87(Suppl 3):384.

Pullen 2007 \{unpublished data only\} Pullen H. Comparison of the T2 procimal humeral nail and PHILOS system in the treatment of $2+3$ part proximal humeral fractures. https://portal.nihr.ac.uk/Profiles/ NRR.aspx?Publication ID=M0044170200 (accessed Aug 09 2010).

Rodriguez-Merchan 95 \{published data only\} Rodriguez-Merchan EC. Compression plating versus Hackethal nailing in closed humeral shaft fractures failing nonoperative reduction. Journal of Orthopaedic Trauma 1995;9(3):194-7.

Shah 2003 \{unpublished data only\}

Shah N. personal communication April 12003.

* Shah N. Shoulder function following four part fractures of proximal humerus: A prospective randomised trial for treatment of four part fractures of proximal humerus conservative vs hemiarthroplasty. In: National Research Register, Issue 1, 2003. Oxford: Update Software.

Sinopidis 2010 \{published data only\}

* Sinopidis C. Prospective randomised study of reverse shoulder prosthesis and hemiarthroplasty for elderly patients with proximal humeral fractures. http://www.controlledtrials.com/ISRCTN32335957 (accessed June 18 2010). Smith M. personal communication April 272012.

Wallace 2000 \{unpublished data only\}

Wallace WA. A prospective randomised trial of the management of displaced surgical neck and displaced shaft fractures of the humerus with the Halder Humeral Nail. In: National Research Register, Issue 3, 2000. Oxford: Update Software.

Wan 2005 \{published data only\}

Wan L, Wang G-X. Interventional effect of improved mobilization on dysfunction of fractured shoulder joint. Zhongguo Linchuang Kangfu [Chinese Journal of Clinical Rehabilitation] 2005;9(26):10-1.

Warnecke 1999 \{published data only\} Warnecke J, Jansen T, Oestern H-J. Surgical treatment of proximal humerus fractures [Operative behandlung proximaler humerusfrakturen - $\mathrm{AO}$ multicenterstudie]. Deutsche Gesellschaft fur Chirurgie 1999;Suppl Kongressband II:1021-4.

Welsh 2000 \{unpublished data only\}

Kulkarni R. Welsh proximal humeral fracture project. In: National Research Register, Issue 2, 2000. Oxford: Update Software.
Yang 2006 \{published data only\}

Yang L. personal correspondence March 92010.

* Yang L, Li B, Pan XY, Li C, Huang JW, Wang ZW, et

al. Percutaneous reduction and fixation of osteoporotic fractures for the proximal humerus in a geriatric population. Zhonghua wai ke za zhi [Chinese journal of surgery] 2006;44 (12):830-2.

Zhang 2010 \{published data only\}

Zhang JH, Di ZL, He ZY, Feng JX, Xu RM. Comparison of humeral head replacement and internal fixation for the treatment of 3 parts and 4 parts fractures of proximal humerus in the elderly. Zhongguo gu shang = China Journal of Orthopaedics and Traumatology 2010;23(6):435-9.

Zuckerman 2012 \{published data only\} Zuckerman JD. Hemiarthroplasty improved healthrelated quality of life more than nonoperative treatment in older patients with four-part proximal humeral fractures: commentary. Journal of Bone and Joint Surgery - American Volume 2012;94(10):942.

\section{References to studies awaiting assessment}

\section{Battistella 2011 \{published data only\}}

Battistella F, Oldani M, Tajana MS, Prestamburgo D. Treatment of proximal humerus fractures with locking plate by minimally invasive technique. Journal of Orthopaedics and Traumatology 2011;12(Supplement):S15-6.

\section{Brorson 2009 \{published data only\}}

Brorson S. personal communication February 32015.

Brorson S. personal communication January 282015.

Brorson S. personal communication June 112012.

Brorson S. personal communication May 222015.

Brorson S. Effect of osteosynthesis, primary hemiarthroplasty, and non-surgical management for displaced four-part fractures of the proximal humerus in elderly: a multi-centre, randomised clinical trial. clinicaltrials.gov/ ct2/show/record/NCT00835562 (accessed January 19 2015).

Brorson S. Effect of osteosynthesis, primary hemiarthroplasty, and non-surgical management for displaced four-part fractures of the proximal humerus in elderly: a multi-centre, randomised clinical trial. clinicaltrials.gov/ ct2/show/record/NCT00835562 (accessed June 24 2009). * Brorson S, Olsen BS, Frich LH, Jensen SL, Johannsen HV, Sorensen AK, et al. Effect of osteosynthesis, primary hemiarthroplasty, and non-surgical management for displaced four-part fractures of the proximal humerus in elderly: a multi-centre, randomised clinical trial. Trials 2009;10:51.

Liu 2011 \{published data only\}

* Liu ZZ, Zhang GM, Ge T. Use of a proximal humeral internal locking system enhanced by injectable graft for minimally invasive treatment of osteoporotic proximal humeral fractures in elderly patients. Orthopaedic Surgery 2011;3(4):253-8.

Liu ZZ, Zhang GM, Ge T, Yang YF. Proximal humeral internal locking system and injectable calcium sulfate graft 
for the treatment of osteoporotic proximal humeral fractures in elderly patients [Chinese]. Chinese Journal of Tissue Engineering Research 2012;16(51):9679-83.

Luo 2008 \{published data only\}

Luo KM, Hou Z, Yang L. Observation on therapeutic effect of electroacupuncture on activity disturbance of the shoulder joint after operation of fracture. Zhongguo zhen jiu = Chinese Acupuncture \& Moxibustion 2008;28(10):727-9.

NCT02052206 \{unpublished data only\}

Vlachopoulos L. Accuracy evaluation of shoulder replacement surgery. https://www.clinicaltrials.gov/ct2/ show/NCT02052206 (accessed January 22 2015).

Wang 2013 \{published data only\} Wang ZH, Deng D, Chen LQ, Zhang WK, Yan HB, Chen $\mathrm{XY}$, et al. Case-control studies on therapeutic effects of combined methods of minimally invasive percutaneous proximal humerus locked osteosynthesis plate with injectable bone for the treatment of proximal humerus fractures in elderly patients [Chinese]. Zhongguo gu shang = China Journal of Orthopaedics and Traumatology 2013;26 (5):404-7.

Zhu 2014 \{published data only\} Zhu L, Liu Y, Yang Z, Li H, Wang J, Zhao C, et al. Locking plate fixation combined with iliac crest bone autologous graft for proximal humerus comminuted fracture. Chinese Medical Journal 2014;127(9):1672-6.

\section{References to ongoing studies}

\section{ACTRN12610000730000 \{unpublished data only\}}

Stanley J. Minimally invasive versus standard open reduction of proximal humerus fractures. http://www.anzctr.org.au/ ACTRN12610000730000.aspx (accessed April 10 2012).

\section{DELPHI \{unpublished data only\}}

* Fjalestad T, Iversen P, Hole MO, Smedsrud M, Madsen JE. Clinical investigation for displaced proximal humeral fractures in the elderly: a randomized study of two surgical treatments: reverse total prosthetic replacement versus angular stable plate Philos (The DELPHI-trial). $B M C$ Musculoskeletal Disorders 2014;15:323.

Fjalestad T, Madsen JE. Displaced proximal humeral fractures: Delta prothesis or Philos plate? (DELPHI). https: //www.clinicaltrials.gov/show/NCT01737060 (accessed 19 January 2015).

Fjalsted T. personal communication April 272012.

HOMERUS \{unpublished data only\}

Diercks RL, Verbeek PA. Hemiarthroplasty versus osteosynthesis in humeral fractures (HOMERUS): A multicenter randomized trial. http://www.trialregister.nl/ trialreg/admin/rctview.asp?TC=2461 (accessed April 10 2012).

* Verbeek PA, Van den Akker-Scheek I, Wendt KW, Diercks RL. Hemiarthroplasty versus angle-stable locking compression plate osteosynthesis in the treatment of threeand four-part fractures of the proximal humerus in the elderly: design of a randomized controlled trial. $B M C$ Musculoskeletal Disorders 2012;13:(16).

\section{HURA \{unpublished data only\}}

Rouleau DM, Poirier M-F. Lateral mini approach vs anterior approach for plating of proximal humerus fracture (HURA). http://clinicaltrials.gov/show/NCT00612391 (accessed January 19 2015).

* Rouleau DM, Poirier M-F. Lateral mini approach vs anterior approach for plating of proximal humerus fracture (HURA). http://clinicaltrials.gov/show/NCT00612391 (accessed June 18 2010).

\section{NCT00438633 \{unpublished data only\}}

Ring D. personal communication April 162012.

Ring D. Comparison of early and late therapy for adults with non-operatively treated proximal humerus fractures. http://clinicaltrials.gov/show/NCT00438633 (accessed January 19 2015).

* Ring D. Comparison of early and late therapy for adults with non-operatively treated proximal humerus fractures. http://clinicaltrials.gov/show/NCT00438633 (accessed June 18 2010).

\section{NCT00818987 \{unpublished data only\}}

Guy P. Operative versus non operative treatment of proximal humerus (shoulder joint) fractures. http://clinicaltrials.gov/ show/NCT00818987 (accessed January 19 2015).

* Guy P. Operative versus non operative treatment of proximal humerus (shoulder joint) fractures. http:// clinicaltrials.gov/show/NCT00818987 (accessed June 24 2009).

\section{NCT00999193 \{unpublished data only\}}

* Lähdeoja T, Paavola M. Treatment of comminuted fractures of the proximal humerus. A randomised, controlled study. http://clinicaltrials.gov/ct2/show/record/ NCT00999193 (accessed August 6 2010).

Lähdeoja T, Paavola M, Pajarinen J. Treatment of comminuted fractures of the proximal humerus. A randomised, controlled study. http://clinicaltrials.gov/ct2/ show/record/NCT00999193 (accessed January 19 2014).

\section{NCT01086202 \{unpublished data only\}}

Phipatanakul W. Clinical outcome comparison between medial and lateral offset reverse shoulder arthroplasty. http: //clinicaltrials.gov/show/NCT01086202 (accessed August 6 2010).

\section{NCT01113411 \{unpublished data only\}}

Pelet $S$, Arteau A. Effectiveness of intensive rehabilitation on shoulder function after proximal humerus fracture. http: //clinicaltrials.gov/show/NCT01113411 (accessed August 6 2010).

* Pelet S, Arteau A. Effectiveness of intensive rehabilitation on shoulder function after proximal humerus fracture. http: //clinicaltrials.gov/show/NCT01113411 (accessed January 19 2015).

\section{NCT01524965 \{unpublished data only\}}

Lahdeoja $T$. The effect of the timing of postoperative mobilisation after locking plate osteosynthesis of fractures of the surgical neck of the humerus. https://clinicaltrials.gov/ ct2/show/NCT01524965 (accessed January 19 2015). 


\section{NCT01557413 \{unpublished data only\}}

* Boyer P. Randomised study between intramedullary locking nails and locking plates for treatment of proximal humerus fractures (HUMERUS). http://clinicaltrials.gov/ show/NCT01557413 (accessed April 10 2012).

Boyer P. Randomised study between intramedullary locking nails and locking plates for treatment of proximal humerus fractures (HUMERUS). http://clinicaltrials.gov/show/ NCT01557413 (accessed January 19 2015).

NCT01847508 \{unpublished data only\}

Kralinger F, Hanson BP. PHILOS augmented - a multicenter randomized controlled trial. https://www.clinicaltrials.gov/ show/NCT01847508 (accessed January 19 2015).

NCT01984112 \{unpublished data only\}

Gracitelli M. Proximal humerus fractures: randomized study between locking nails and locking plates for Neer 2 and 3 parts. https://www.clinicaltrials.gov/ct2/show/ NCT01984112 (accessed January 19 2015).

NCT02075476 \{unpublished data only\}

Alvarez C, Mombiela F. The effectiveness between two surgical techniques for reconstruction of humeral proximal extremity fractures or fractures luxation in three or four fragments. https://clinicaltrials.gov/ct2/show/ NCT02075476 (accessed January 19 2015).

NTR3208 \{unpublished data only\}

Engelsma Y. Arthroplasty in three- or four-part proximal humerus fracture: hemi or reverse?. http:// www.trialregister.nl/trialreg/admin/rctview.asp?TC=3208 (accessed April 10 2012).

\section{NTR4019 \{unpublished data only\}}

Bransen J. PROMOTION-trial: A PROspective randomized Multicenter trial for the treatment of dislocated 3-part proximal humerus fractures: Open reduction and internal fixaTION versus intramedullary nailing. http:/ /apps.who.int/trialsearch/Trial2.aspx?TrialID=NTR4019 (accessed February 11 2015).

ProCon \{published and unpublished data\}

Den Hartog D. Primary hemiarthroplasty versus conservative treatment for comminuted fractures of the proximal humerus in the elderly (ProCon) - A multicenter randomized trial. http://www.trialregister.nl/trialreg/ admin/rctview.asp?TC=2040] (accessed May 25 2010). * Den Hartog D, van Lieshout EM, Tuinebreijer WE, Polinder S, Van Beeck EF, Breederveld RS, et al. Primary hemiarthroplasty versus conservative treatment for comminuted fractures of the proximal humerus in the elderly (ProCon): A multicenter randomized controlled trial. BMC Musculoskeletal Disorders 2010;11:97.

ROTATE \{unpublished data only\}

* Conboy V. Return of function and external rotation post proximal humerus fracture fixation with neutral rotation brace (ROTATE). clinical.trials.gov http://clinicaltrials.gov/ ct2/show/NCT02073695 (accessed 1 December 2014). Kassam AM, Ainsworth B, Hawken R, Ramesh R, Conboy $\mathrm{V}$. Return of function and external rotation post-proximal humerus fracture fixation with neutral rotation brace (abstract). Bone and Joint Journal 2013;95(Suppl 9):31.

SHeRPA \{published data only\}

Watts A. Comparison of two shoulder replacement methods after trauma. http://www.isrctn.com/ISRCTN21981284 (accessed 11 August 2015).

Torrens \{published data only\}

Torrens C. Personal communication June 42015.

Torrens C. Personal communication May 252015.

TPHF \{unpublished data only\}

Laitinen M, Launonen A, Rissanen P, Flinkkila T, Ojanpera J, Lepola V, et al. Treatment of proximal humeral fractures (TPHF). http://clinicaltrials.gov/show/NCT01246167 (accessed April 10 2012).

* Launonen AP, Lepola V, Flinkkila T, Strandberg N, Ojanpera J, Rissanen P, et al. Conservative treatment, plate fixation, or prosthesis for proximal humeral fracture. A prospective randomized study. BMC Musculoskeletal Disorders 2012;13:(167).

\section{Additional references}

\section{AO 2015}

Jaeger M, Leung F, Li W. Nonoperative treatment. AO Surgery Reference: https://www2.aofoundation.org/ $\mathrm{wps} /$ portal/surgery?showPage=redfix \&bone=Humerus $\&$ segment $=$ Proximal $\&$ classification $=11-A 3 \&$ treatment $=\&$ method=Nonoperative $\% 20$ treatment\&implantstype $=\&$ approach $=\&$ redfix $\cdot$ url $=1301989095781 \&$ Language $=$ en $\#$ stepUnit-2 (accessed 11 September 2015).

Bell 2011

Bell JE, Leung BC, Spratt KF, Koval KJ, Weinstein JD, Goodman DC, et al. Trends and variation in incidence, surgical treatment, and repeat surgery of proximal humeral fractures in the elderly. Journal of Bone and Joint Surgery American Volume 2011;93(2):121-31.

Bernstein 1996

Bernstein J, Alder LM, Blank JE, Dalsey RM, Williams GR, Iannotti JP. Evaluation of the Neer system of classification of proximal humeral fractures with computerized tomographic scans and plain radiographs. Journal of Bone and Joint Surgery. American Volume 1996;78(9):1371-5.

\section{Biberthaler 2009}

Biberthaler P, Braunstein V, Kirchoff C, Kroetz M, Kettler M, Mutschler W. Surgical therapy of humeral head fractures with a locking plate: analysis of 176 cases. Journal of Bone \& Joint Surgery - British Volume 2009;91(Suppl 1):40.

\section{Boutron 2008}

Boutron I, Moher D, Altman DG, Schulz KF, Ravaud P, CONSORT Group. Extending the CONSORT statement to randomized trials of nonpharmacologic treatment: explanation and elaboration. Annals of Internal Medicine 2008;148(4):295-309.

\section{Brorson 2008}

Brorson S, Hrobjartsson A. Training improves agreement among doctors using the Neer system for proximal 
humeral fractures in a systematic review. Journal of Clinical Epidemiology 2008;61(1):7-16.

\section{Brorson 2011a}

Brorson S. Management of proximal humeral fractures in the nineteenth century: an historical review of preradiographic sources. Clinical Orthopaedics and Related Research 2011;469(4):1197-206.

\section{Brorson 2012}

Brorson S, Olsen BS, Frich LH, Jensen SL, Sorensen AK, Krogsgaard MR, et al. Surgeons agree more on treatment recommendations than on classification of proximal humeral fractures. BMC Musculoskeletal Disorders 2012;13: 114. [DOI: $10.1186 / 1471-2474-13-114]$

\section{Bruder 2011}

Bruder A, Taylor NF, Dodd KJ, Shields N. Exercise reduces impairment and improves activity in people after some upper limb fractures: a systematic review. Journal of Physiotherapy 2011;57(2):71-82.

\section{Constant 1987}

Constant CR, Murley AHG. A clinical method of functional assessment of the shoulder. Clinical Orthopaedics and Related Research 1987;(214):160-4.

\section{Court-Brown 2001}

Court-Brown CM, Garg A, McQueen MM. The epidemiology of proximal humeral fractures. Acta Orthopaedica Scandinavica 2001;72(4):365-71.

\section{Court-Brown 2006}

Court-Brown CM, Caesar B. Epidemiology of adult fractures: A review. Injury 2006;37(8):691-7.

\section{Croft 1994}

Croft P, Pope D, Zonca M, O’Neill T, Silman A. Measurement of shoulder related disability: results of a validation study. Annals of the Rheumatic Diseases 1994;53 (8):525-8.

\section{Dai 2014}

Dai J, Chai Y, Wang C, Wen G. Meta-analysis comparing locking plate fixation with hemiarthroplasty for complex proximal humeral fractures. European Journal of Orthopaedic Surgery \& Traumatology 2014;24(3):305-13.

\section{Dawson 1996}

Dawson J, Fitzpatrick R, Carr A. Questionnaire on the perceptions of patients about shoulder surgery. Journal of Bone and Joint Surgery - British Volume 1996;78(4): 593-600.

\section{Dawson 2009}

Dawson J, Rogers K, Fitzpatrick R, Carr A. The Oxford shoulder score revisited. Archives of Orthopaedic and Trauma Surgery 2009;129(1):119-23.

\section{Deeks 2011}

Deeks JJ, Higgins JPT, Altman DG (editors). Chapter 9: Analysing data and undertaking meta-analyses. In: Higgins JPT, Green S (editors). Cochrane Handbook for Systematic Reviews of Interventions Version 5.1.0 (updated March 2011). The Cochrane Collaboration, 2011. Available from www.cochrane-handbook.org.

\section{Der Tavitian 2006}

Der Tavitian J. The management of comminuted proximal humeral fractured, a randomised prospective trial. https: //portal.nihr.ac.uk/Profiles/NRR.aspx?Publication $\cdot \mathrm{ID}=$ N0123137861). National Research Register Archive (accessed August 09 2010).

\section{Fjalestad 2012}

Fjalestad T, Hole MO, Hovden IA, Blucher J, Stromsoe K. Surgical treatment with an angular stable plate for complex displaced proximal humeral fractures in elderly patients: a randomized controlled trial. Journal of Orthopaedic Trauma 2012;26(2):98-106.

\section{Fjalestad 2014a}

Fjalestad T, Hole M. Displaced proximal humeral fractures: operative versus non-operative treatment - a 2-year extension of a randomized controlled trial. European Journal of Orthopaedic Surgery \& Traumatology 2014;24(7): 1067-73.

\section{Fjalestad 2014b} Fjalestad T, Iversen P, Hole MO, Smedsrud M, Madsen JE. Clinical investigation for displaced proximal humeral fractures in the elderly: a randomized study of two surgical treatments: reverse total prosthetic replacement versus angular stable plate Philos (The DELPHI-trial). BMC Musculoskeletal Disorders 2014;15:(323).

\section{Gillespie 2003} Gillespie LD. personal communication August 082003.

\section{Gillespie 2009} Gillespie LD, Robertson MC, Gillespie WJ, Lamb SE, Gates S, Cumming RG, Rowe BH. Interventions for preventing falls in older people living in the community. Cochrane Database of Systematic Reviews 2009, Issue 2. [DOI: 10.1002/14651858.CD007146.pub2]

\section{Gomberawalla 2013}

Gomberawalla M, Miller B, Coale R, Bedi A, Gagnier J. Meta-analysis of joint preservation versus arthroplasty for the treatment of displaced 3- and 4-part fractures of the proximal humerus. Injury 2013;44(11):1532-9.

\section{Guy 2010}

Guy P, Slobogean GP, McCormack RG. Treatment preferences for displaced three- and four-part proximal humerus fractures. Journal of Orthopaedic Trauma 2010;24 (4):250-4.

Han 2015

Han RJ, Sing DC, Feeley BT, Ma CB, Zhang AL. Proximal humerus fragility fractures: recent trends in nonoperative and operative treatment in the Medicare population. Journal of Shoulder and Elbow Surgery 2015 Oct 1;pii: S1058-2746(15)00389-4:[Epub ahead of print]. [DOI: $10.1016 /$ j.jse.2015.07.015]

\section{Hanchard 2013}

Hanchard NCA, Lenza M, Handoll HHG, Takwoingi Y. Physical tests for shoulder impingements and local lesions of bursa, tendon or labrum that may accompany impingement. Cochrane Database of Systematic Reviews 2013, Issue 4. [DOI: 10.1002/14651858.CD007427.pub2] 


\section{Handoll 2015}

Handoll H, Brealey S, Rangan A, Keding A, Corbacho B, Jefferson L, et al. The ProFHER (PROximal Fracture of the Humerus: Evaluation by Randomisation) trial - a pragmatic multicentre randomised controlled trial evaluating the clinical effectiveness and cost-effectiveness of surgical compared with non-surgical treatment for proximal fracture of the humerus in adults. Health Technology Assessment 2015;19(24):1-280.

Higgins 2003

Higgins JP, Thompson SG, Deeks JJ, Altman DG. Measuring inconsistency in meta-analyses. BMJ 2003;327 (7414):557-60.

Higgins 2008a

Higgins JPT, Altman DG (editors). Chapter 8: Assessing risk of bias in included studies Table 8.5a. Chapter 8: Assessing risk of bias in included studies. In: Higgins JPT, Green S (editors). Cochrane Handbook for Systematic Reviews of Interventions Version 5.0.1 (updated September 2008). The Cochrane Collaboration, 2008. Available from www.cochrane-handbook.org.

Higgins 2008b

Higgins JPT, Green S (editors). Cochrane Handbook for Systematic Reviews of Interventions Version 5.0.1 [updated September 2008]. The Cochrane Collaboration, 2008. Available from www.cochrane-handbook.org.

Hodgson 2003a

Hodgson SS. personal communication May 142003.

Hodgson 2006

Hodgson S. Proximal humerus fracture rehabilitation. Clinical Orthopaedics \& Related Research 2006;(442):131-8.

Hodgson 2007

Hodgson SA, Mawson SJ, Saxton JM, Stanley D. Rehabilitation of two-part fractures of the neck of the humerus (two-year follow-up). Journal of Shoulder \& Elbow Surgery 2007;16(2):143-5.

\section{Hoffmann 2014}

Hoffmann TC, Glasziou PP, Boutron I, Milne R, Perera R, Moher D, et al. 348 :g1687. Better reporting of interventions: template for intervention description and replication (TIDieR) checklist and guide. BMJ 2014;348: g1687. [: http://dx.doi.org/10.1136/bmj.g1687]

\section{Holbein 1999}

Holbein O, Bauer G, Hoellen I, Keppler P, Hehl G, Kinzl L. Is primary endoprosthetic replacement of the humeral head an alternative treatment for comminuted fractures of the proximal humerus in elderly patients? [Stellt die primare Implantation einer Humeruskopfprothese bei der dislozierten Humeruskopfmehrfragmentfraktur des alten Menschen eine Alternative zur Minimalosteosynthese dar?]. Osteosynthese International 1999;7 Suppl 2:207-10.

\section{Jakob 1991}

Jakob RP, Miniaci A, Anson PS, Jaberg H, Osterwalder A, Ganz R. Four-part valgus impacted fractures of the proximal humerus. Journal of Bone and Joint Surgery. British Volume 1991;73(2):295-8.

Karl 2015

Karl JW, Olson PR, Rosenwasser MP. The epidemiology of upper extremity fractures in the United States, 2009. Journal of Orthopaedic Trauma 2015;29(8):e242-4.

Koval 1997

Koval KJ, Gallagher MA, Marsicano JG, Cuomo F, McShinawy A, Zuckerman JD. Functional outcome after minimally displaced fractures of the proximal part of the humerus. Journal of Bone and Joint Surgery. American Volume 1997;79(2):203-7.

\section{L'Insalata 1997}

L'Insalata JC, Warren RF, Cohen SB, Altchek DW, Peterson MG. A self-administered questionnaire for assessment of symptoms and function of the shoulder. Journal of Bone and Joint Surgery. American Volume 1997;79(5):738-748.

Launonen 2012

Launonen AP, Lepola V, Flinkkila T, Strandberg N, Ojanpera J, Rissanen P, et al. Conservative treatment, plate fixation, or prosthesis for proximal humeral fracture. A prospective randomized study. BMC Musculoskeletal Disorders 2012;13:(167).

\section{Lawton 1969}

Lawton MP, Brody EM. Assessment of older people: selfmaintaining and instrumental activities of daily living. Gerontologist 1969;9(3):176-86.

\section{Lefebvre 2011}

Lefebvre C, Manheimer E, Glanville J. Chapter 6: Searching for studies. In: Higgins JPT, Green S (editors). Cochrane Handbook for Systematic Reviews of Interventions Version 5.1.0 (updated March 2011). The Cochrane Collaboration, 2011. Available from www.cochrane-handbook.org.

Li 2013

Li Y, Zhao L, Zhu L, Li J, Chen A. Internal fixation versus nonoperative treatment for displaced 3-part or 4-part proximal humeral fractures in elderly patients: a metaanalysis of randomized controlled trials. PLoS ONE 2013;8 (9):e75464.

Lind 1989

Lind T, Kroner K, Jensen J. The epidemiology of fractures of the proximal humerus. Archives of Orthopaedic and Trauma Surgery 1989;108(5):285-7.

Lo 2012

Lo KW, Ulery BD, Ashe KM, Laurencin CT. Studies of bone morphogenetic protein-based surgical repair. Advanced Drug Delivery Reviews 2012;64(12):1277-91.

\section{Mahabier 2015}

Mahabier KC, Hartog DD, Van Veldhuizen J, Panneman MJ, Polinder S, Verhofstad MH, et al. Trends in incidence rate, health care consumption, and costs for patients admitted with a humeral fracture in The Netherlands between 1986 and 2012. Injury 2015;46(10):1930-7. 


\section{Maniscalco 2014b}

Maniscalco P, Caforio M, Del Vecchio EO, D'Ascola J, Crainz E, Ferrata P. Preliminary experience with Diphos Nail in the treatment of proximal humerus fractures [Esperienza preliminare con il chiodo Diphos nail nel trattamento delle fratture prossimali di omero]. GIOT 2014;39(April):48-55.

Mao 2014

Mao Z, Zhang L, Zhang L, Zeng X, Chen S, Liu D, et al. Operative versus non operative treatment in complex proximal humeral fractures. Orthopedics 2014;37(5): e410-9.

Marsh 2007

Marsh JL, Slongo TF, Agel J, Broderick JS, Creevey W, DeCoster TA, et al. Fracture and dislocation classification compendium - 2007: Orthopaedic Trauma Association classification, database and outcomes committee. Journal of Orthopaedic Trauma 2007;21(Suppl 10):S1-133.

Mata-Fink 2013

Mata-Fink A, Meinke M, Jones C, Kim B, Bell JE. Reverse shoulder arthroplasty for treatment of proximal humeral fractures in older adults: a systematic review. Journal of Shoulder \& Elbow Surgery 2013;22(12):1737-48.

\section{McKee 2007}

McKee MD. Commentary and perspective on "Immediate mobilization compared with conventional immobilization for the impacted nonoperatively treated proximal humeral fracture. A randomized controlled trial". JBJS-Online: www.ejbjs.org/Comments/2007/cp' dec07' mckee.dtl (accessed Jan 08 2008).

\section{Mintken 2009}

Mintken PE, Glynn P, Cleland JA. Psychometric properties of the shortened disabilities of the Arm, Shoulder, and Hand Questionnaire (QuickDASH) and Numeric Pain Rating Scale in patients with shoulder pain. Journal of Shoulder and Elbow Surgery 2009;18(6):920-6.

\section{Muller 1991}

Muller M, Allgower M, Schneider R, Willenegger $\mathrm{H}$. Manual of internal fixation: techniques recommended by the AO-ASIF Group. 3rd Edition. Berlin: Springer-Verlag, 1991.

\section{Namdari 2013}

Namdari S, Horneff JG, Baldwin K. Comparison of hemiarthroplasty and reverse arthroplasty for treatment of proximal humeral fractures: a systematic review. Journal of Bone \& Joint Surgery - American Volume 2013;95(18): $1701-8$.

Neer 1970

Neer CS. Displaced proximal humeral fractures.

Classification and evaluation. Journal of Bone and Joint Surgery. American Volume 1970;52(6):1077-89.

Neer 1975

Neer CS. Four segment classification of displaced proximal humeral fractures. Instructional Course Lectures. The
American Academy of Orthopaedic Surgeons. Vol. 24, Saint Louis: The C. V. Mosby Company, 1975:160-8.

\section{Ockert 2014}

Ockert B, Pedersen V, Geyer L, Wirth S, Mutschler W, Grote $S$. Position of polyaxial versus monoaxial screws in locked plating for proximal humeral fractures: Analysis of a prospective randomized study. European Journal of Orthopaedic Surgery and Traumatology. 2014;24(5):747-52.

\section{Olerud 2011c}

Olerud P, Tidermark J, Ponzer S, Ahrengart L, Bergstrom G. Responsiveness of the EQ-5D in patients with proximal humeral fractures. Journal of Shoulder \& Elbow Surgery 2011;20(8):1200-6.

\section{Palvanen 2006}

Palvanen M, Kannus P, Niemi S, Parkkari J. Update in the epidemiology of proximal humeral fractures. Clinical Orthopaedics and Related Research 2006;(442):87-92.

\section{Polinder 2013}

Polinder S, Lordens GI, Panneman MJ, Eygendaal D, Patka P, Den Hartog D, et al. Trends in incidence and costs of injuries to the shoulder, arm and wrist in the Netherlands between 1986 and 2008. BMC Public Health 2013;13: (531). [DOI: 10.1186/1471-2458-13-531]

\section{Resch 1997}

Resch H, Povacz P, Frohlich R, Wambacher M. Percutaneous fixation of three- and four-part fractures of the proximal humerus. Journal of Bone and Joint Surgery - British Volume 1997;79(2):295-300.

\section{RevMan 2014}

The Nordic Cochrane Centre, The Cochrane Collaboration. Review Manager (RevMan). 5.2. Copenhagen: The Nordic Cochrane Centre, The Cochrane Collaboration, 2014.

\section{Robinson 2008}

Robinson BC, Athwal GS, Sanchez-Sotelo J, Rispoli DM. Classification and imaging of proximal humerus fractures. Orthopedic Clinics of North America 2008;39(4):393-403.

\section{Schairer 2015}

Schairer WW, Nwachukwu BU, Lyman S, Craig EV, Gulotta LV. Reverse shoulder arthroplasty versus hemiarthroplasty for treatment of proximal humerus fractures. Journal of Shoulder and Elbow Surgery 2015;24(10):1560-6. [DOI: http://dx.doi.org/10.1016/j.jse.2015.03.018]

\section{Schmitt 2004}

Schmitt JS, Di Fabio RP. Reliable change and minimum important difference (MID) proportions facilitated group responsiveness comparisons using individual threshold criteria. Journal of Clinical Epidemiology 2004;57(10): $1008-18$.

\section{Schunemann 2011}

Schunemann HJ, Oxman AD, Vist GE, Higgins JPT, Deeks JJ, Glaziou P, et al. Chapter 12: Interpreting results and drawing conclusions. In: Higgins JPT, Green S (editors), Cochrane Handbook for Systematic Reviews of Interventions Version 5.1.0 (updated March 2011). 
The Cochrane Collaboration, 2011. www.cochranehandbook.org.

\section{Sidor 1993}

Sidor ML, Zuckerman JD, Lyon T, Koval K, Cuomo F, Schoenberg N. The Neer classification system for proximal humeral fractures. An assessment of interobserver reliability and intraobserver reproducibility. Journal of Bone and Joint Surgery - American Volume 1993;75(12):1745-50.

Siebenrock 1993

Siebenrock KA, Gerber C. The reproducibility of classification of fractures of the proximal end of the humerus. Journal of Bone and Joint Surgery - American Volume 1993;75(12):1751-5.

Sintonen 2001

Sintonen $\mathrm{H}$. The 15D instrument of health-related quality of life: properties and applications. Annals of Medicine 2001;33(5):328-36

\section{Sjoden 1997}

Sjoden GO, Movin T, Guntner P, Aspelin P, Ahrengart L, Ersmark $\mathrm{H}$, et al. Poor reproducibility of classification of proximal humeral fractures. Additional CT of minor value. Acta Orthopaedica Scandinavica 1997;68(3):239-42.

\section{Tashjian 2010}

Tashjian RZ, Deloach J, Green A, Porucznik CA, Powell AP. Minimal clinically important differences in ASES and simple shoulder test scores after non operative treatment of rotator cuff disease. Journal of Bone and Joint Surgery American Volume 2010;92(2):296-303.

\section{Thanasas 2009}

Thanasas C, Kontakis G, Angoules A, Limb D, Giannoudis P. Treatment of proximal humerus fractures with locking plates: a systematic review. Journal of Shoulder and Elbow Surgery 2009;18(6):837-44.

\section{Tornkvist 1995}

Tornkvist H, Ahrengart L, Sperber A. Tension band wiring vs. nonoperative treatment of proximal humerus fractures - a prospective randomized study [Abstract]. Acta Orthopaedica Scandinavica. Supplementum 1995;66(265): $40-1$.

\section{Van de Water 2014}

Van de Water AT, Shields N, Davidson M, Evans M, Taylor NF. Reliability and validity of shoulder function outcome measures in people with a proximal humeral fracture. Disability and Rehabilitation 2014;36(13):1072-9.

\section{Verbeek 2012}

Verbeek PA, Van den Akker-Scheek I, Wendt KW, Diercks RL. Hemiarthroplasty versus angle-stable locking compression plate osteosynthesis in the treatment of threeand four-part fractures of the proximal humerus in the elderly: design of a randomized controlled trial. $B M C$

Musculoskeletal Disorders 2012;13:(16).

\section{Ware 1996}

Ware JE, Kosinski N, Keller SD. The 12-Item ShortForm Health Survey (SF-12): construction of scales and preliminary tests of reliability and validity. Medical Care 1996;34(3):220-33.

Zhang 2014

Zhang HY, Zhao Y, Xie C, Cheng YT, Wang CC. Arthroplasty versus joint preservation for displaced 3and 4-part proximal humeral fractures: A meta-analysis [Chinese]. Chinese Journal of Tissue Engineering Research 2014;18(26):4241-7.

\section{References to other published versions of this review}

\section{Gibson 2001}

Gibson JNA, Handoll HHG, Madhok R. Interventions for treating proximal humeral fractures in adults. Cochrane Database of Systematic Reviews 2001, Issue 1.

\section{Gibson 2002a}

Gibson JNA, Handoll HHG, Madhok R. Interventions for treating proximal humeral fractures in adults. Cochrane Database of Systematic Reviews 2002, Issue 2.

\section{Gibson 2002b}

Gibson JNA, Handoll HHG, Madhok R. Interventions for treating proximal humeral fractures in adults. Cochrane Database of Systematic Reviews 2002, Issue 3.

\section{Handoll 2003a}

Handoll HHG, Gibson JNA, Madhok R. Interventions for treating proximal humeral fractures in adults. Cochrane Database of Systematic Reviews 2003, Issue 4. [DOI: 10.1002/14651858.CD000434]

Handoll 2007

Handoll HHG, Madhok R. Interventions for treating proximal humeral fractures in adults. Cochrane Database of Systematic Reviews 2003, Issue 4. [DOI: 10.1002/ 14651858.CD000434]

Handoll 2010 Handoll HHG, Ollivere BJ. Interventions for treating proximal humeral fractures in adults. Cochrane Database of Systematic Reviews 2010, Issue 12. [DOI: 10.1002/ 14651858.CD000434.pub2]

\section{Handoll 2012}

Handoll HHG, Ollivere BJ, Rollins KE. Interventions for treating proximal humeral fractures in adults. Cochrane Database of Systematic Reviews 2012, Issue 12. [DOI: 10.1002/14651858.CD000434.pub3]

* Indicates the major publication for the study 


\title{
CHARACTERISTICS OF STUDIES
}

\section{Characteristics of included studies [ordered by study ID]}

\author{
Agorastides 2007
}

Methods

Randomised using sequentially numbered sealed envelopes

Assessor blinding: stated for Constant Shoulder Assessment and Oxford scores at 6 and 12 months

Loss to follow-up at 1 year: 10 (all exclusions: 4 wrong prosthesis; 1 pathological fracture; 1 deep infection requiring further procedure; 2 initial greater tuberosity malpositioning; 2 did not attend follow-up visits)

Participants
Reyal Liverpool Hospital, Liverpool, UK
Period study recruitment: October 2002 to October 2003
59 patients with displaced proximal humeral fractures, 3-part or 4-part or articular
fractures who were treated with cemented hemiarthroplasty. Isolated non-pathologic
fractures < 6 weeks old. Physiologically old patients with poor bone quality. Informed
consent.
Exclusion criteria: no extra information
Of $49: 39$ female, 10 male; mean age 70 years, range 34 to 85 years

Interventions $\quad$ Intervention started post surgery (mean 10 days; range 1 to 30 days after injury)

1. Early active-assisted mobilisation (after 2 weeks). Arm kept in sling in neutral rotation for 2 weeks; only pendulum and elbow exercises allowed. Between weeks 3 and 6 , progressed to active-assisted exercises; from week 7 , to active exercises.

2. Late mobilisation (after 6 weeks). Arm kept in sling in neutral rotation for 6 weeks; only elbow exercises allowed. From week 7 to week 12, progressed from pendulum to active-assisted exercises; from week 13, to active exercises

Both mobilisation protocols were supervised by a team of specialist shoulder physiotherapists

Outcomes

Length of follow-up: 1 year; also assessed at 2 and 6 weeks, and 3 and 6 months (coinciding with outpatient visits)

Oxford shoulder score

Constant shoulder score (mobility, strength, pain, activities of daily living)

Range of motion: elevation, external and internal rotation

Complications

Radiological assessment: greater tuberosity migration; superior luxation of prosthesis

Notes The early mobilisation regimen represented normal practice at the hospital

Risk of bias

Bias Authors' judgement

Support for judgement

Random sequence generation (selection Unclear risk bias)

No description of method: "Patients were randomly allocated"

Interventions for treating proximal humeral fractures in adults (Review)

Copyright () 2015 The Cochrane Collaboration. Published by John Wiley \& Sons, Ltd. 
Agorastides 2007 (Continued)

\begin{tabular}{|c|c|c|}
\hline Allocation concealment (selection bias) & Unclear risk & $\begin{array}{l}\text { "Randomization took place in the operat- } \\
\text { ing theater after the procedure, by use of } \\
\text { sequentially numbered, sealed envelopes." }\end{array}$ \\
\hline $\begin{array}{l}\text { Blinding (performance bias and detection } \\
\text { bias) } \\
\text { Functional outcomes, pain, clinical out- } \\
\text { comes, complications }\end{array}$ & Unclear risk & $\begin{array}{l}\text { "At the 6-and 12-month visits, an inde- } \\
\text { pendent blinded observer completed the } \\
\text { Constant Shoulder Assessment and Ox- } \\
\text { ford scores." However, care providers and } \\
\text { participants were not blind to allocation } \\
\text { and assessment of complications was not } \\
\text { blinded either }\end{array}$ \\
\hline
\end{tabular}

Blinding (performance bias and detection Unclear risk bias)

Death, reoperation

No accounting of these, but lack of blinding unlikely to affect reporting of these

Incomplete outcome data (attrition bias) High risk

Functional outcomes, pain, clinical out-

comes, complications

\begin{tabular}{|c|c|c|}
\hline comes, complications & & analyses \\
\hline $\begin{array}{l}\text { Incomplete outcome data (attrition bias) } \\
\text { Death, reoperation }\end{array}$ & High risk & $\begin{array}{l}\text { No accounting of these outcomes, but in- } \\
\text { complete account of participant flow, with } \\
\text { exclusion of } 10 \text { participants from the anal- } \\
\text { yses }\end{array}$ \\
\hline Selective reporting (reporting bias) & High risk & $\begin{array}{l}\text { No protocol available. May have been } \\
\text { stopped early, greater tuberosity migration } \\
\text { not specifically listed in brief trial entry in } \\
\text { the National Research Register (UK) }\end{array}$ \\
\hline Balance in baseline characteristics? & Unclear risk & $\begin{array}{l}\text { Incomplete data to back up claims of lack } \\
\text { of baseline differences as these given only } \\
\text { for } 49 \text { ( } 10 \text { excluded) but a } 5 \text {-year difference } \\
\text { in mean age ( } 72 \text { versus } 67 \text { years) }\end{array}$ \\
\hline Free from performance bias? & Unclear risk & $\begin{array}{l}\text { Although } 3 \text { upper limb surgeons perform- } \\
\text { ing the operations agreed to the same pro- } \\
\text { cedures a different uncemented prosthesis } \\
\text { was used in } 4 \text { subsequently excluded par- } \\
\text { ticipants. } \\
\text { "Both mobilization protocols were super- } \\
\text { vised by a team of specialist shoulder phys- } \\
\text { iotherapists." }\end{array}$ \\
\hline
\end{tabular}

Interventions for treating proximal humeral fractures in adults (Review)

Copyright () 2015 The Cochrane Collaboration. Published by John Wiley \& Sons, Ltd. 
Methods

Participants

Use of permutation table, single-blind, independently administered Assessor blinded

Loss to follow-up at 1 year: 7/20 (2 excluded)

Central hospital, Vasteras, Sweden

Period of study recruitment: not stated

20 patients with non or minimally displaced proximal humeral fractures ( 7 had fracture of the greater tubercle); sling for 10 days.

Exclusion criteria: no information

17 female, 3 male; mean age 64 years, range 50 to 75 years

Interventions

Interventions started 10 to 12 days post injury, after removal of sling.

1. Instructed self-exercise: patients instructed to train 5 to 10 minutes, 4 to 5 times daily. They had three training sessions (day 1 , weeks $3 \& 8$ post injury)

2. Conventional physiotherapy: 9 sessions (average 20 to 30 minutes), 1 to 2 times each week, over 10 to 12 weeks. No thermoelectrotherapy.

Assigned: 10/10

Completed ( $>1$ year): $7 / 6$

Outcomes

Length of follow-up: 1 year; also assessed at 3, 8, $16 \& 24$ weeks

Range of motion: forward flexion (graph), abduction, internal \& external rotation

Functional movements: placing hand on neck, placing hand on back

Pain: when placing hand on neck: combing hair (graph)

Isometric muscle strength: vertical \& horizontal pushing

Change of treatment requested

Notes

The 2 excluded participants were in the control group: 1 died and 1 underwent an operation

\section{Risk of bias}

\begin{tabular}{|c|c|c|}
\hline Bias & Authors' judgement & Support for judgement \\
\hline $\begin{array}{l}\text { Random sequence generation (selection } \\
\text { bias) }\end{array}$ & Low risk & $\begin{array}{l}\text { Mention of "permutation table" and "ran- } \\
\text { domized controlled" trial }\end{array}$ \\
\hline Allocation concealment (selection bias) & Low risk & $\begin{array}{l}\text { "A third person was responsible for the ran- } \\
\text { domization procedure and kept the key to } \\
\text { the permutation table" }\end{array}$ \\
\hline
\end{tabular}

Blinding (performance bias and detection Unclear risk bias)

Functional outcomes, pain, clinical outcomes, complications
"A second physiotherapist examined the patients. She did not know to which group the patient belonged, and the patients were instructed not to tell her." However, there is no guarantee of blinding and, for practical reasons, neither participants nor care provider were blinded 


\section{Bertoft 1984 (Continued)}

\begin{tabular}{|c|c|c|}
\hline $\begin{array}{l}\text { Blinding (performance bias and detection } \\
\text { bias) } \\
\text { Death, reoperation }\end{array}$ & Low risk & $\begin{array}{l}\text { Lack of blinding unlikely to affect assess- } \\
\text { ment of these outcomes }\end{array}$ \\
\hline $\begin{array}{l}\text { Incomplete outcome data (attrition bias) } \\
\text { Functional outcomes, pain, clinical out- } \\
\text { comes, complications }\end{array}$ & Unclear risk & $\begin{array}{l}\text { Participant flow provided but large loss to } \\
\text { follow-up }(7 / 20=35 \%)\end{array}$ \\
\hline $\begin{array}{l}\text { Incomplete outcome data (attrition bias) } \\
\text { Death, reoperation }\end{array}$ & Low risk & Participant flow provided \\
\hline Selective reporting (reporting bias) & Unclear risk & Insufficient information to judge this. \\
\hline Balance in baseline characteristics? & Unclear risk & $\begin{array}{l}\text { Incomplete data to back up claims of lack of } \\
\text { baseline differences but a } 4 \text {-year difference } \\
\text { in mean age between groups ( } 66 \text { versus } 62 \\
\text { years) }\end{array}$ \\
\hline Free from performance bias? & Low risk & No indication of performance bias. \\
\hline
\end{tabular}

Boons 2012

Methods

Participants
Method of randomisation: computer-generated randomisation sequence; sealed opaque envelopes stored in statistician's room

Assessor blinding: not blinded

Loss to follow-up at 12 months: 3/50 (2 withdrawn; 1 died)

\begin{tabular}{|c|c|}
\hline Participants & $\begin{array}{l}\text { Rijnstate Hospital, Arnhem, The Netherlands } \\
\text { Period of study recruitment: June } 2004 \text { to July } 2009 \\
50 \text { patients with an acute displaced (based on Neer's criteria) 4-part proximal humeral } \\
\text { fractures ( } 8,4 \text { in each group, had valgus impacted fractures; no mention of fracture- } \\
\text { dislocations). Age } 65 \text { or older. Informed consent. } \\
\text { Exclusion criteria: pre-existing mental disorders (dementia) or unable to provide in- } \\
\text { formed consent or answer the questionnaires; disabling disorder or additional trauma to } \\
\text { the affected arm; pathological or open fracture; associated neurovascular injury; pre-ex- } \\
\text { isting impairment of the contralateral shoulder; unable to understand Dutch; unable to } \\
\text { participate in the rehabilitation protocol; contraindicated for surgery (American Society } \\
\text { of Anesthesiologists [ASA] physical status 4) } \\
47 \text { female, } 3 \text { male; mean age } 78 \text { years, range not stated }\end{array}$ \\
\hline
\end{tabular}

Interventions
Randomisation was performed in the first week after fracture.

1. Surgery: operation within 7 days of injury. Under general anaesthesia. Humeral head replacement using a deltopectoral approach with the Global Fx shoulder fracture endoprosthesis (DePuy, Leeds, UK). All prostheses were cemented. Cancellous bone graft from the head fragment was applied on the proximal stem before restoration of the tuberosities. Nonabsorbable sutures used to encircle the tuberosities to "enhance anatomic restoration". (All patients had prophylactic antibiotics.) Post surgery: shoulder immobiliser for 6 weeks. 
Boons 2012 (Continued)

2. Non-surgical treatment: shoulder immobiliser for 6 weeks.

Rehabilitation was same in both groups: Experienced shoulder physical therapists instructed the patients for 40 -minute sessions three times a week up to 12 weeks. Up to 2 weeks light passive ROM movements; between 2 and 6 weeks, passive ROM up to 45 degrees forward flexion and abduction and active ROM up to 30 degrees forward flexion and abduction were allowed if pain control adequate; no external rotation. After 6 weeks, unlimited passive glenohumeral exercise, with active ROM up to 90 degrees in forward flexion and abduction. External rotation was allowed up to 30 degrees. After the 3-month visit, patients were seen by the physical therapist every month until the 12month follow-up, with an emphasis on maximizing ROM, strength and return to daily activities.

Assigned: 25/25

Completed (at 1 year): 23/24 (based on text account)

Outcomes

Length of follow up: 1 year; also assessed at $1 \& 6$ weeks and 3 months

Constant shoulder score (contralateral shoulder measured at 1 week follow-up as reference)

SST (Simple Shoulder Test)

Pain (VAS)

Disability (VAS)

Abduction strength (contralateral shoulder measured at 1 week follow-up as reference)

Range of motion: abduction, flexion, external rotation, internal rotation (lumbar level)

Complications: Non-union, osteonecrosis, pain and impingement, heterotopic ossification, infection, implant dislocation (head stem separation), secondary migration of greater tuberosity, secondary rotator cuff tear (from migration of hemiarthroplasty), nonunion of greater tuberosity

Subsequent surgery (reasons: head stem separation and pain and impingement)

Notes

Additional information on group of a withdrawn participant (had deteriorating condition) and SST scores (incorrect in Table 2 in article) requested from Dr van Loon (14 Feb 2015). Response from Dr Boons (12 March 2015) identified group of the withdrawn participant (hemiarthroplasty) and provided the raw data for STT

Risk of bias

\begin{tabular}{|c|}
\hline Bias \\
\hline
\end{tabular}

Random sequence generation (selection Low risk bias)

"patients were randomly allocated to nonoperative treatment or hemiarthroplasty. The randomization list was generated by an independent statistician". "A computergenerated variable block schedule was used.

Allocation concealment (selection bias) Low risk

"the resulting treatment allocations were stored in sealed opaque envelopes in the statistician's room." 
Boons 2012 (Continued)

Blinding (performance bias and detection High risk bias)

Functional outcomes, pain, clinical outcomes, complications
No mention of independent or blinded assessment. Initial care providers could not be blinded for these contrasting interventions

Lack of blinding unlikely to affect assessment of these outcomes
Blinding (performance bias and detection Unclear risk

bias)

Death, reoperation

Incomplete outcome data (attrition bias) Low risk

Functional outcomes, pain, clinical out-

comes, complications
Incomplete outcome data (attrition bias) Low risk

Death, reoperation
Active and systematic surveillance. Low loss to follow-up (6\%). Author's response resolved problem over contradictory statements in trial report on group allocation for one participant

Active and systematic surveillance. Low loss to follow-up (6\%). Author's response resolved problem over contradictory statements in trial report on group allocation for one participant

No protocol. Outcome measures well described but the problems with the STT scores (incorrect data and direction of effect) gave slight cause for concern

No difference in baseline characteristics, including in numbers of valgus impacted (4 versus 4) fractures

Standard procedure performed by two experienced shoulder surgeons. Same rehabilitation protocols including during shoulder immobilisation

Buecking 2014

Methods

(a)

Method of randomisation: block randomisation stratified by type of fracture; pre-sealed randomisation envelope given by study staff to surgeon before surgery

Assessor blinding: not blinded (independent observer)

Loss to follow-up at 12 months: 13/120 (9 lost to follow-up; 4 died)
Participants
University hospital of Giessen and Marburg, Marburg, Germany

Period of study recruitment: December 2009 to November 2011

120 patients with displaced proximal humeral fractures (Neer 2-part; and 3- or 4-part). Age 18 or older. Written consent.

Exclusion criteria: glenohumeral dislocation, concomitant ipsilateral fractures of the arm or forearm, malignancy-related fractures, multiple trauma, other surgery planned 
Buecking 2014 (Continued)

(prosthesis or a longer plate)

92 female, 28 male; mean age 68 years, range 63 to 72

Interventions

Randomisation was performed prior to surgery; timing not stated

All participants received a plate osteosynthesis with the non-contact bridging plate for the proximal humerus. In addition to the plate, a cable wire was used to fix the greater tuberosity in 3 and 4 part fractures. All patients received a single-shot antibiotic.

1. Deltoid-split approach: anterolateral $3 \mathrm{~cm}$ deltoid split with two small incisions for the three locking screws in the humeral shaft.

2. Deltopectoral approach: fracture was exposed through a classical anterior approach; a 10 to $12 \mathrm{~cm}$ incision was begun at the tip of the coracoid process and run medially in the direction of the deltoid muscle

Rehabilitation after surgery was same in both groups. Operated shoulder immobilised for first 2 days; then passive and limited active motion started. For 3- and 4-part fractures, only limited assisted abduction up to $90^{\circ}$ was allowed for first 6 weeks

Assigned: 60/60

Completed (at 1 year): 48/42

Outcomes

Length of follow-up: 1 year; also assessed at 6 weeks and 6 months

Constant shoulder score (normalised)

Activities of daily living (Lawton 1969)

Pain (VAS)

Complications: humeral head necrosis (0), axillary nerve damage (0), deep infection, screw perforations, implant loosening at head or shaft, inadequate reduction, implant failure from subsequent fall

Change during primary operation: primary prosthesis inserted

Re-operations (for complications and at request of patient)

Fluoroscopy use

Length of surgery

Hospital stay

Notes

The range of scores for Lawton's instrumental activities of daily living score is from 0 to 8 (best score); those reported in this trail report include mean scores of 11 up to 21 . Since there is no explanation for this in the trial report, the meaning of the data is unclear and they are not presented in the review

\section{Risk of bias}

Bias

Random sequence generation (selection Low risk bias)
Authors' judgement

\section{Support for judgement}

"With the use of a block randomization stratified by type of fracture (two-part fractures versus three- and four part fractures), patients were randomized to either the deltoid-split or the deltopectoral approach." Not quite enough detail to be certain; although likely 


\section{Buecking 2014 (Continued)}

\begin{tabular}{|c|c|c|}
\hline Allocation concealment (selection bias) & Low risk & $\begin{array}{l}\text { "Presealed randomization envelopes were } \\
\text { given by the study staff to the attending } \\
\text { surgeon before surgery." }\end{array}$ \\
\hline $\begin{array}{l}\text { Blinding (performance bias and detection } \\
\text { bias) } \\
\text { Functional outcomes, pain, clinical out- } \\
\text { comes, complications }\end{array}$ & High risk & $\begin{array}{l}\text { "Standardised } \\
\text { follow-up examinations were performed at } \\
6 \text { weeks, } 6 \text { months, and } 12 \text { months after } \\
\text { surgery by the same independent observer. } \\
. . \text { " However, the incision would still have } \\
\text { been obvious }\end{array}$ \\
\hline $\begin{array}{l}\text { Blinding (performance bias and detection } \\
\text { bias) } \\
\text { Death, reoperation }\end{array}$ & High risk & $\begin{array}{l}\text { Unclear if choices for primary or secondary } \\
\text { surgery were influenced by prior knowl- } \\
\text { edge. No revisions to hemiarthroplasty in } \\
\text { the deltoid-split group could indicate that } \\
\text { reoperation was only considered if a del- } \\
\text { topectoral approach could be enlarged }\end{array}$ \\
\hline $\begin{array}{l}\text { Incomplete outcome data (attrition bias) } \\
\text { Functional outcomes, pain, clinical out- } \\
\text { comes, complications }\end{array}$ & High risk & $\begin{array}{l}\text { Inappropriate post-randomisation exclu- } \\
\text { sions: primary prosthesis ( } 2 \text { versus } 4) \text {, sec- } \\
\text { ondary prosthesis ( } 5 \text { versus } 2 \text { ) and en- } \\
\text { larged deltopectoral approach during revi- } \\
\text { sion surgery ( } 0 \text { versus } 4)\end{array}$ \\
\hline $\begin{array}{l}\text { Incomplete outcome data (attrition bias) } \\
\text { Death, reoperation }\end{array}$ & Low risk & $\begin{array}{l}\text { Inappropriate post-randomisation exclu- } \\
\text { sions: primary prosthesis }(2 \text { versus } 4 \text { ), sec- } \\
\text { ondary prosthesis ( } 5 \text { versus } 2 \text { ) and en- } \\
\text { larged deltopectoral approach during revi- } \\
\text { sion surgery }(0 \text { versus } 4) \text {. However, these } \\
\text { were known for these outcomes. Similar } \\
\text { numbers were not reachable ( } 4 \text { versus } 5)\end{array}$ \\
\hline Selective reporting (reporting bias) & Unclear risk & $\begin{array}{l}\text { No protocol. Although systematic data col- } \\
\text { lection and all outcomes reported, the dis- } \\
\text { parity between the reported data for the } \\
\text { Lawton instrumental activities of daily liv- } \\
\text { ing score (maximum } 8 \text { ) and the actual } \\
\text { scores presented in the text is of some con- } \\
\text { cern }\end{array}$ \\
\hline Balance in baseline characteristics? & Low risk & Good balance in baseline characteristics \\
\hline Free from performance bias? & Low risk & $\begin{array}{l}\text { Operations were performed by } 3 \text { senior } \\
\text { surgeons who were trained in both tech- } \\
\text { niques. Equivalent care programmes in } \\
\text { both groups }\end{array}$ \\
\hline
\end{tabular}

Interventions for treating proximal humeral fractures in adults (Review)

Copyright @ 2015 The Cochrane Collaboration. Published by John Wiley \& Sons, Ltd. 
Method of randomisation: no details

Assessor blinding: not blinded (independent observer)

Loss to follow-up at 24 months: 5/32 (4 lost to follow-up; 1 died)

Participants

Shanghai Tenth People's Hospital of Tongji University, Shanghai, China

Period of study recruitment: April 2005 to March 2010

32 patients with acute displaced 4-part proximal humeral fracture of the surgical neck (Neer classification). At least one tubercle needed to be displaced more than $10 \mathrm{~mm}$ in relation to the head fragment but the other did not need to meet this criterion (thus 3-part fractures were also acceptable); see Notes. Age 67 or older with low energy trauma. Independent living conditions (not institutionalised), and no severe cognitive dysfunction (3 or more correct answers on a 10-item Short Portable Mental Status Questionnaire [SPMSQ])

Exclusion criteria: completely displaced shaft in relation to the head fragment, such as a fracture without bony contact; valgus impacted fracture, previous shoulder problems 27 female, 5 male; mean age 72 years, range 67 to 86

Interventions

Randomisation was performed after clearance by an anaesthetist prior to surgery; timing not stated

All patients received a single dose of antibiotic preoperatively.

1. Hemiarthroplasty using the DuPuy prosthesis with suturing of tuberosities. Cemented stem. Bone graft from removed humeral head used to restore the humeral offset

2. Open reduction and internal fixation with Philos plate. Suturing of tuberosities Postoperative arm sling for 4 weeks (optional thereafter). All patients referred to physiotherapy. Pendulum exercises and passive elevation/abduction up to $90^{\circ}$ were started on postoperative day 1 . After 4 weeks, the patients were allowed free active range of motion Assigned: $19 / 13$

Completed (at 2 years): $15 / 12$

Outcomes

Length of follow-up: 2 years; also assessed at 4 and 12 months

DASH

Constant shoulder score

Pain (VAS)

Complications (relating to re-operations): non-union, fixation failure, dislocation, infection, prosthesis loosening

Re-operations (for complications)

Length of surgery

Notes

One participant initially had a 3-part greater tuberosity fracture but at surgery, the lesser tuberosity was also found to be displaced $>1 \mathrm{~cm}$. Hence all had 4-part fractures. Three of 32 participants had fracture dislocations

Sent email to $\mathrm{Dr} \mathrm{Li}$ requesting details of the randomisation method and clarification on deaths on 24 May 2015

Risk of bias

Bias

Authors' judgement

Support for judgement

Interventions for treating proximal humeral fractures in adults (Review) 
Cai 2012 (Continued)

\begin{tabular}{|c|c|c|}
\hline $\begin{array}{l}\text { Random sequence generation (selection } \\
\text { bias) }\end{array}$ & Unclear risk & $\begin{array}{l}\text { "the patients were randomized". No other } \\
\text { details. }\end{array}$ \\
\hline Allocation concealment (selection bias) & Unclear risk & $\begin{array}{l}\text { "the patients were randomized". No other } \\
\text { details. }\end{array}$ \\
\hline
\end{tabular}

Blinding (performance bias and detection High risk

bias)

Functional outcomes, pain, clinical out-

Not blinded even though there was some independent assessment at final follow-up: "Final 24-month follow-up was performed comes, complications by an independent orthopedic surgeon $(\mathrm{K}$. T.) not involved in treatment."

Blinding (performance bias and detection Unclear risk bias)

Death, reoperation

Lack of blinding less likely to affect assessment of these outcomes. Standardisation of assessment

Incomplete outcome data (attrition bias) Unclear risk Functional outcomes, pain, clinical outcomes, complications

Active and systematic surveillance and clear participants flow diagram. However, more participants lost to follow-up in the hemiarthroplasty group (4 (21\%) versus $1(8 \%)$ ). There are also some incorrect percentages that give rise to concern

Incomplete outcome data (attrition bias) Low risk

Death, reoperation

Active and systematic surveillance and clear participants flow diagram. It is likely that patients with complications would have returned

Selective reporting (reporting bias) Low risk

No protocol. However, systematic data collection and reporting of all outcomes

Balance in baseline characteristics? Unclear risk

Where reported, the baseline characteristics were balanced in the two groups. However, the baseline distribution of the fracture types, which included three 4-part fracture dislocations, was not reported

Free from performance bias? Low risk

"All patients underwent surgery performed by 1 of 2 orthopedic surgeons (M.C., S.L. ), both experienced in shoulder surgery." Same rehabilitation. 
Methods

Participants
Method of randomisation: referral to random list and randomisation timed at surgery Assessor blinding: no

Loss to follow-up at 1 year: 5/40 (3 deaths, 2 lost to follow-up)

Vienna General Hospital, Austria

Period of study recruitment: not stated - lasted 22 months

40 patients with acute 4-part (Neer) proximal humeral fractures (type C: AO/ASIF classification), aged $>50$ years, no history of previous problems in either shoulder, informed consent

Exclusion criteria: concomitant vascular or neurological injuries of involved limb; prior operative procedures; neurologic or mental disorders; or drug abuse

30 female, 10 male; mean age 75 years; of 35 : range 56 to 88 years
Surgery started 7.3 days of injury ( 0 to 26 days). General anaesthesia used in all cases. Stems were cemented in place and bone grafting was performed using cancellous bone from patient's humeral head.

1. Hemiarthroplasty using EPOCA prosthesis (Argomedical). Fixation of tuberosities using wire cables threaded through a medial and lateral hole in the stem.

2. Hemiarthroplasty using HAS prosthesis (Stryker). Fixation of tuberosities using transosseous braided sutures tied to lateral fin of the stem.

Same general rehabilitation protocol used for both groups: shoulder kept for 2 weeks in immobiliser to prevent active external rotation, passive movement for 15 minutes per day by physiotherapist to avoid contractures and shoulder stiffness. Then, active range of motion increased to horizontal level. Active external rotation initiated after another 2 weeks.

Assigned: number in each group not known

Completed (at 1 year): 18/17

Outcomes

Length of follow-up: 1 year; also assessed at 12 days, $3 \& 6$ weeks, and 6 months Functional assessment (individual Constant score, where results were relative to patient's unaffected shoulder)

Range of motion (active forward flexion, abduction, external rotation)

Radiological assessment: resorption of tuberosities, superior migration of prosthesis, anterior subluxations, glenoid erosion, aseptic stem loosening, secondary dislocation of the tuberosities, heterotopic ossification

Deep infection

Periprosthetic fracture

Reoperation \& scheduled for reoperation (persistent pain)

Mortality

Notes

Differences between the two prostheses include the type and position of fixation of the tuberosities and the volume of the stem in the metaphyseal area, thus allowing different amounts of additional (autologous) cancellous bone grafting.

The data for heterotopic ossification were contradictory and not used here.

Request for information sent to contact trialist on 19 February 2010 
Fialka 2008 (Continued)

\begin{tabular}{|c|c|c|}
\hline $\begin{array}{l}\text { Random sequence generation (selection } \\
\text { bias) }\end{array}$ & Unclear risk & $\begin{array}{l}\text { "The random list was designed to finally } \\
\text { produce } 2 \text { groups of equal size." }\end{array}$ \\
\hline Allocation concealment (selection bias) & Unclear risk & $\begin{array}{l}\text { "Each surgeon was informed at the begin- } \\
\text { ning of the operation as to which implant } \\
\text { had randomly been selected." }\end{array}$ \\
\hline $\begin{array}{l}\text { Blinding (performance bias and detection } \\
\text { bias) } \\
\text { Functional outcomes, pain, clinical out- } \\
\text { comes, complications }\end{array}$ & High risk & No blinding. \\
\hline $\begin{array}{l}\text { Blinding (performance bias and detection } \\
\text { bias) } \\
\text { Death, reoperation }\end{array}$ & Unclear risk & $\begin{array}{l}\text { Lack of blinding unlikely to affect assess- } \\
\text { ment of these outcomes. Standardisation of } \\
\text { assessment }\end{array}$ \\
\hline $\begin{array}{l}\text { Incomplete outcome data (attrition bias) } \\
\text { Functional outcomes, pain, clinical out- } \\
\text { comes, complications }\end{array}$ & High risk & $\begin{array}{l}\text { The group allocation and baseline data } \\
\text { were not provided for } 5 \text { participants: } 2 \text { lost } \\
\text { to follow-up and } 3 \text { who had died. Standard } \\
\text { deviations not provided }\end{array}$ \\
\hline $\begin{array}{l}\text { Incomplete outcome data (attrition bias) } \\
\text { Death, reoperation }\end{array}$ & Unclear risk & $\begin{array}{l}\text { Group allocation not provided for those } \\
\text { who had died. }\end{array}$ \\
\hline Selective reporting (reporting bias) & Unclear risk & Insufficient information to judge this. \\
\hline Balance in baseline characteristics? & Unclear risk & $\begin{array}{l}\text { Incomplete baseline data ( } 5 \text { excluded) to } \\
\text { confirm baseline comparability of those in } \\
\text { analysis }\end{array}$ \\
\hline Free from performance bias? & Low risk & $\begin{array}{l}\text { No indication of performance bias: a "gen- } \\
\text { eral rehabilitation protocol was used for all } \\
\text { patients regardless of the type of implant. } \\
\text { "; each of the } 4 \text { participating surgeons was } \\
\text { experienced in joint replacement surgery }\end{array}$ \\
\hline
\end{tabular}

Fjalestad 2010

\begin{tabular}{ll} 
Methods & $\begin{array}{l}\text { Method of randomisation: use of computer software by independent hospital statistician; } \\
\text { block size 12; use of numbered opaque sealed envelopes } \\
\text { Assessor blinding: no, but assessment by two independent physiotherapists } \\
\text { Loss to follow-up at } 1 \text { year: } 2 / 50 \text { (2 deaths); at } 2 \text { years: } 8 / 50 \text { (3 deaths) }\end{array}$ \\
\hline Participants & $\begin{array}{l}\text { Olso University Hospital, Oslo, Norway } \\
\text { Period of study recruitment: May } 2003 \text { to May } 2008 \\
\text { 50 patients with displaced proximal humeral fractures, AO group B2 or C2 (displaced } \\
\text { 3-part and 4-part fractures) who were admitted to hospital. Malposition was at least }\end{array}$ \\
\hline
\end{tabular}


Fjalestad 2010 (Continued)

$45^{\circ}$ angular deviation in the true frontal (inclination) or transthoracic radiographic projections, regardless of whether the fracture was impacted or not. The greater or lesser tuberosity had to be displaced at least $10 \mathrm{~mm}$. Furthermore, the displacement between the head and metaphyseal/diaphyseal main fragments should not exceed $50 \%$ of the diaphyseal diameter. Age 60 years or over. Written informed consent. Resident in Oslo. Exclusion criteria: non-Scandinavian ethnicity, previous history of injury or illness of the injured or contralateral shoulder, injury of the other part of the humerus or the contralateral upper extremity, alcohol or drug abuse, dementia or neurological disease or severe cardiovascular disease that would contraindicate surgery.

44 female, 6 male; mean age 73 years, range 60 to 88 years

Interventions

Interventions (and randomisation) started after hospital admission. (On admission to the hospital, all patients were immobilized in a modified Velpeau bandage.)

1. Surgery: operation occurred within the first week after admission to hospital. Open reduction and fixation using a minimally open deltopectoral approach with an interlocking plate device (Locking Compression Plate (LCP) of the AO basic type, Synthes, Switzerland) and metal cerclages to secure the tuberosities. Surgery was performed under fluoroscopic control. Then immobilisation in a modified Velpeau bandage until selfexercises and instructed physiotherapy was started on the third postoperative day.

2. Non-surgical treatment: all patients stayed in the hospital for at least 1 day and received the same instructions from the physiotherapist as those allocated to surgery. If the displacement between the head and metaphyseal fragment (main fragments) exceeded $50 \%$ of the diaphyseal diameter (subsequent to randomisation), closed reduction was performed in the operating room under general anaesthesia within 48 hours of admission. Immobilisation in a modified Velpeau bandage for 2 weeks before self exercises and instructed physiotherapy started on day 15 .

The same self-training programme and instructed physiotherapy programme used for both groups, although the non-surgical treatment group started 12 days later. Both groups progressed to strengthening exercises at the 6-week time point. Physical therapy and self-exercise were recommended for at least 6 months.

Assigned: 25/25

Completed (at 1 year): 23/25

Outcomes

Length of follow-up: 2 years; also assessed at 2, 8, 12, 26 and 52 weeks

Constant shoulder score (both shoulders) (3, 6, $12 \& 24$ months)

ASES (American Shoulder and Elbow Surgeons) questionnaire (sports domains not included - maximum 24 points) $(6,12 \& 24$ months)

Quality of life score: Harri Sintonen 15D instrument (sexual function domain not included)

Mortality

Fixation failure or redisplacement - subsequent operation

Radiographic outcomes including avascular necrosis (score $2=$ no changes; 1 = changes to normal trabecular organisation $<50 \%$ of humeral head; $0=>50 \%$ or partial collapse) ; and post-traumatic glenohumeral osteoarthritis

Check for axillary nerve injury

Health economic outcomes, including direct (cost of surgery; cost of hospital stays) and indirect costs (sick leave, family use of time to assist patient)

Length of stay in acute hospital and hospital rehabilitation centre 
Fjalestad 2010 (Continued)

Information on the trial received December 2006 from Dr Tore Fjalestad.
Currently only some results for one year follow-up are published. Communication from
Dr Tore Fjalestad in April 2010 indicated that the two-year follow-up was likely to
be finished during 2010. Further information from Dr Tore Fjalestad in April 2012
indicated that the two-year follow-up had been submitted to another journal (estimated
publication during 2012)
More details on non-surgical treatment were provided in Fjalestad 2012. Tore Fjalestad
also provided in an email (April 2012) the following clarification on the use of closed
reduction for 8 non-surgically treated participants (this had not been described in the
protocol): "The primary X-rays were assessed for classification and decision-making
for closed reduction. Those eight patients had a new radiographic examination after
allocation to non-surgical treatment and after the procedure in the operating room, to
confirm an acceptable position of the fragments. If not acceptable, the patients had
to be treated with ORIF. Surprisingly, only one patient demonstrated unacceptable re-
displacement after two weeks, and was analyzed according to intention-to-treat principle
in the non-surgical group at one year."
Two-year follow-up results published in 2014 (Fjalestad 2014a).

\section{Risk of bias}

\begin{tabular}{|c|c|c|}
\hline Bias & Authors' judgement & Support for judgement \\
\hline $\begin{array}{l}\text { Random sequence generation (selection } \\
\text { bias) }\end{array}$ & Low risk & $\begin{array}{l}\text { "The [randomisation] procedure was de- } \\
\text { signed by the statistician at the hospital re- } \\
\text { search centre using the computer software } \\
\text { S-PLUS } 6.0 \text { for Windows } 2002 \text {... Ran- } \\
\text { domisation was based on equal blocks of } \\
\text { length } 12 \text {, with the exception of the last } \\
\text { one, which was interrupted due to } 50 \text { pa- } \\
\text { tients." }\end{array}$ \\
\hline Allocation concealment (selection bias) & Low risk & $\begin{array}{l}\text { "Randomisation was performed by means } \\
\text { of consecutively numbered and sealed non- } \\
\text { translucent envelopes containing each par- } \\
\text { ticipant's allocation to surgery or to non- } \\
\text { surgical treatment." Independent statisti- } \\
\text { cian }\end{array}$ \\
\hline
\end{tabular}

Blinding (performance bias and detection High risk

bias)

Functional outcomes, pain, clinical out-

comes, complications

Blinding (performance bias and detection Unclear risk

bias)

Death, reoperation
Two trained physiotherapists performed the $15 \mathrm{D}$ interviews. The physiotherapists were not blinded to group assignment. No provider or participant blinding

Lack of blinding unlikely to affect assessment of these outcomes, but may affect decisions for subsequent surgery 


\section{Fjalestad 2010 (Continued)}

\begin{tabular}{|c|c|c|}
\hline $\begin{array}{l}\text { Incomplete outcome data (attrition bias) } \\
\text { Functional outcomes, pain, clinical out- } \\
\text { comes, complications }\end{array}$ & Unclear risk & $\begin{array}{l}\text { Participant flow diagram provided and in- } \\
\text { tention-to-treat analysis conducted } \\
\text { However, reported in } 2014 \text { : "Missing data } \\
\text { were handled according to single imputa- } \\
\text { tion by the last observation for each indi- } \\
\text { vidual." Also, some imbalance in loss to fol- } \\
\text { low-up } 2(8 \%) \text { (surgery) versus } 6 \text { ( } 24 \%) \\
\text { (non-surgical) }\end{array}$ \\
\hline
\end{tabular}

Incomplete outcome data (attrition bias) Low risk

Death, reoperation

Selective reporting (reporting bias) Unclear risk
Participant flow diagram provided and intention-to-treat analysis conducted

Trial registered after completion. Small discrepancies in trial inclusion criteria
Balance in baseline characteristics? Unclear risk

Free from performance bias?
Low risk
Statistically non significant imbalance in gender ( 5 females versus 1 male) and baseline quality-of-life scores (higher in surgical group)

All the operations were performed by three surgeons experienced in the procedure performed

\section{Hodgson 2003}

Methods

Participants
Randomised using sequentially numbered sealed envelopes

Assessor blinding: yes, on review of patients at home or clinic appointment

Loss to follow-up at 1 year: 4 (1 death); at 2 years: 12 (3 deaths)

Royal Hallamshire Hospital, Sheffield, UK

Period of study recruitment: November 1998 to April 2000

86 patients, over 40 years old, with minimally displaced 2-part fractures (Neer), including isolated fractures of the greater tuberosity

Exclusion criteria: inability to understand written or verbal information

70 female, 16 male; mean age 70 years

Interventions
Intervention started: at arrival at A\&E.

1. Early physiotherapy (within 1 week of the fracture). Most patients were seen by a physiotherapist at clinic the day after their fracture. Patients received a sling for comfort but were instructed to take their arm out of the sling and to perform gradual, assisted movements of the upper limb.

2. Late physiotherapy after 3 weeks of immobilisation in a collar and cuff sling. Both groups received same rehabilitation programme. First 2 weeks: education and instruction for home exercises; weeks 2 to 4: progression to full passive flexion and light functional exercises; week 4: start of progressive functional exercises. Discharge when both patient and physiotherapist thought independent shoulder function was achieved 
Hodgson 2003 (Continued)

\begin{tabular}{ll}
\hline Outcomes & $\begin{array}{l}\text { Length of follow-up: } 2 \text { years, also } 8 \text { and } 16 \text { weeks and } 1 \text { year } \\
\text { Functional assessment (Constant score) } \\
\text { Patients' perceived health status: SF36 (physical function, physical role limitation, pain) } \\
\text {; Croft shoulder disability questionnaire } \\
\text { Complications } \\
\text { Number of physiotherapy treatment sessions }\end{array}$ \\
\hline Notes & $\begin{array}{l}\text { Information on this trial received from Mr Hodgson on several occasions. This included } \\
\text { draft report of the 2-year follow-up and notice of their plan to extend follow-up to } 5 \\
\text { years }\end{array}$ \\
\hline
\end{tabular}

Risk of bias

$\begin{array}{lll}\text { Bias } & \text { Authors' judgement } & \text { Support for judgement }\end{array}$

Random sequence generation (selection Unclear risk bias)

No details: "using sequentially numbered sealed envelopes we randomly allocated patients"

Allocation concealment (selection bias) Low risk

"using sequentially numbered sealed envelopes we randomly allocated patients". Also from phone conversation (08/08/ 2001): "physio opened envelopes when details entered on envelope"

Blinding (performance bias and detection Unclear risk bias)

Functional outcomes, pain, clinical out-

comes, complications

Blinding (performance bias and detection Unclear risk bias)

Blinded assessor of function but patients and care providers were not blinded

Death, reoperation

Incomplete outcome data (attrition bias) Low risk Functional outcomes, pain, clinical outcomes, complications

\begin{tabular}{l|l|l}
\hline $\begin{array}{l}\text { Incomplete outcome data (attrition bias) } \\
\text { Death, reoperation }\end{array}$ & Low risk & Participant flow provided. \\
\hline Selective reporting (reporting bias) & Unclear risk & $\begin{array}{l}\text { Trial registration was incomplete and dif- } \\
\text { fered slightly from final reports }\end{array}$ \\
\hline Balance in baseline characteristics? & Unclear risk & $\begin{array}{l}\text { More males in the early mobilisation group } \\
\text { (11 versus 5). }\end{array}$
\end{tabular}

Lack of blinding unlikely to affect assessment of these outcomes

A full account of loss to follow-up provided. While $14 \%$ at 2 years $(12 / 86)$, it was under $5 \%(4 / 86)$ at 1 year

(11 versus 5). 
Hodgson 2003 (Continued)

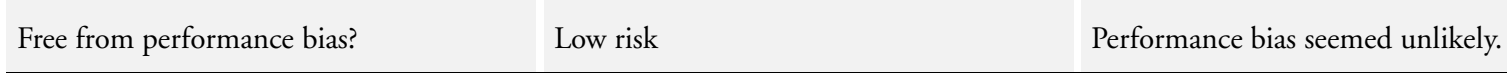

Hoellen 1997

\begin{tabular}{ll} 
Methods & $\begin{array}{l}\text { Randomisation method unknown } \\
\text { Assessor blinding: not stated } \\
\text { Loss to follow-up at } 1 \text { year: } 12 / 30 \text { (3 deaths) }\end{array}$ \\
\hline Participants & University Clinic Ulm, Germany \\
& Period of study recruitment: $1 / 12 / 1994$ to $30 / 06 / 1996$ in Hoellen 1997 report (to $31 /$ \\
& $08 / 1998$ in Holbein 1999 report) \\
& $30^{*}$ patients with 4 -part fractures (Neer). * ${ }^{*}$ ee Notes. \\
& Exclusion criteria: age $<65$ years, $>14$ days since fracture, rheumatoid arthritis, previous \\
shoulder injury, terminally ill & 24 female, 6 male; mean age 74 years \\
\hline
\end{tabular}

Interventions

Interventions started within 14 days of fracture.

1. Hemiarthroplasty (Global prosthesis, DePuy, US) - cemented

2. "Minimal osteosynthesis": tension band wiring - 2 pins + figure of 8 wire

All were given low dose heparin for DVT prophylaxis. The same post-operative treatment was used in both groups. A Glichrist bandage was used for temporary rests. Passive moving exercises started from first postoperative day, with active exercises postponed until after 6 weeks. Referral to rehabilitation clinic for 3 to 4 weeks post discharge.

Assigned: $15 / 15$

Completed (1 year): 9/9

$\begin{array}{ll}\text { Outcomes } & \text { Length of follow-up: 1 year } \\ \text { Functional assessment (Constant score) } \\ \text { Mobility (component of Constant score) } \\ \text { Pain (ditto) } \\ \text { Power } \\ \text { Haematoma } \\ \text { Infection } \\ \text { Implant failure } \\ \text { Medical complications } \\ \text { Re-operation } \\ \text { Time on ward } \\ \text { Discharge location } \\ \text { Mortality }\end{array}$

Notes

The plan for longer term follow-up was announced in the Hoellen 1997 trial report. Further abstracts and a trial report (Holbein 1999) were identified for the review update (Issue 4, 2003). Holbein 1999 reported on 39 patients (19 versus 20), with 3- and 4part fractures, 31 (number in each group not known) of whom had been followed up for 1 year and 24 (number in each group not known) for 2 years. Requests (June 2003) for further information, including for denominators, resulted in the discovery that both Dr Holbein and Dr Hoellen were no longer at Ulm 
Hoellen 1997 (Continued)

\section{Risk of bias}

\begin{tabular}{l|l|l}
\hline Bias & Authors' judgement & Support for judgement \\
\hline $\begin{array}{l}\text { Random sequence generation (selection } \\
\text { bias) }\end{array}$ & Unclear risk & No details: prospective randomised trial \\
\hline $\begin{array}{l}\text { Allocation concealment (selection bias) } \\
\text { Blinding (performance bias and detection } \\
\text { bias) }\end{array}$ & Unclear risk & Nigh risk \\
$\begin{array}{l}\text { Functional outcomes, pain, clinical out- } \\
\text { comes, complications }\end{array}$ & & No blinding. \\
\hline
\end{tabular}

Blinding (performance bias and detection Unclear risk bias)

Lack of blinding unlikely to affect assess-

Death, reoperation

Incomplete outcome data (attrition bias) High risk

Functional outcomes, pain, clinical outment of these outcomes

comes, complications

Incomplete outcome data (attrition bias) Unclear risk

Death, reoperation

Une risk

\begin{tabular}{|c|c|c|}
\hline Selective reporting (reporting bias) & High risk & $\begin{array}{l}\text { Insufficient information to judge this but } \\
\text { the pragmatic removal of the power com- } \\
\text { ponent of the Constant score was post hoc. } \\
\text { Also non addressed difference in trial in- } \\
\text { clusion criteria between the two reports of } \\
\text { this trial }\end{array}$ \\
\hline Balance in baseline characteristics? & Unclear risk & $\begin{array}{l}\text { No information on baseline characteristics } \\
\text { of the two treatment groups but inclusion } \\
\text { criteria rule out some confounders }\end{array}$ \\
\hline Free from performance bias? & Unclear risk & $\begin{array}{l}\text { Same post-operative treatment but in all } \\
\text { there is insufficient information to assess } \\
\text { performance bias }\end{array}$ \\
\hline
\end{tabular}

Participant flow provided but large loss to follow-up $(12 / 30=40 \%)$; and potential exclusions

Participant flow provided but large loss to follow-up $(12 / 30=40 \%)$. Serious outcomes though are less likely to be missed

Insufficient information to judge this but the pragmatic removal of the power component of the Constant score was post hoc. clusion criteria between the two reports of this trial

No information on baseline characteristics of the two treatment groups but inclusion there is insufficient information to assess performance bias 
Methods

Participants
Method of randomisation: unknown, "randomly selected"

Assessor blinding: unlikely

Loss to follow-up at 1 year: 10/31 (4 failed to attend, 2 died, 4 excluded)

Rigshospitalet, Copenhagen, Denmark

Period of study recruitment: not stated

30 patients with 31 displaced 2-part (7 fractures), 3-part (19) and 4-part (5 fractures)

proximal humeral fractures (Neer).

Exclusion criteria: no information

(of 31 fractures) 22 female, 9 male; age range 30 to 91 years

Interventions

Interventions started: not stated.

1. Surgery: Percutaneous reduction (using Steinmann pin under image intensifier control) and external fixation ( 2 half pins with continuous threads into humeral head and 2 or 3 pins into the humeral shaft, and neutralising bar applied; Steinmann pin removed) 2. Non-surgical treatment: closed manipulation under general anaesthesia $\&$ sling Assigned: $15 / 16$

Completed (at 1 year): 11/10

Outcomes

Length of follow-up: 12 months; also assessed at $3 \& 6$ months

'Treatment failure': poor reduction, pin removal due to loosening

Non-union

Quality of fracture reduction: good, fair, poor

Functional overall score: excellent, satisfactory, unsatisfactory, poor. Neer (without anatomical section)

Complications: avascular humeral head necrosis, deep infection, radiographic pseudarthrosis, refracture

Reoperations

Mortality

Notes

In both groups, functional exercises were started under instruction during the first week. Excluded participants were: 1 treatment failure (deep infection) in the surgical group; and 2 treatment failures (poor reduction) and 1 refracture in the non-surgical treatment group

Risk of bias

Bias

Authors' judgement

Support for judgement

Random sequence generation (selection Unclear risk bias)

Allocation concealment (selection bias) Unclear risk

No details: "randomly selected for treatment"

No details: "randomly selected for treatment"

Blinding (performance bias and detection High risk

No blinding reported. bias)

Functional outcomes, pain, clinical out-

comes, complications 


\section{Kristiansen 1988 (Continued)}

\begin{tabular}{|c|c|c|}
\hline $\begin{array}{l}\text { Blinding (performance bias and detection } \\
\text { bias) } \\
\text { Death, reoperation }\end{array}$ & Unclear risk & Unlikely to be affected by lack of blinding \\
\hline $\begin{array}{l}\text { Incomplete outcome data (attrition bias) } \\
\text { Functional outcomes, pain, clinical out- } \\
\text { comes, complications }\end{array}$ & High risk & $\begin{array}{l}\text { Exclusion of data for participants with } \\
\text { treatment failure and early refracture from } \\
12 \text { month review. Large loss to follow-up } \\
(10 / 31=32 \%)\end{array}$ \\
\hline $\begin{array}{l}\text { Incomplete outcome data (attrition bias) } \\
\text { Death, reoperation }\end{array}$ & Low risk & Participant flow provided. \\
\hline Selective reporting (reporting bias) & Unclear risk & Insufficient information to judge this. \\
\hline Balance in baseline characteristics? & Low risk & $\begin{array}{l}\text { No information on the patient with bilat- } \\
\text { eral fractures but a relatively minor unit of } \\
\text { analysis issue }\end{array}$ \\
\hline Free from performance bias? & Unclear risk & $\begin{array}{l}\text { No information on operator competencel } \\
\text { expertise. }\end{array}$ \\
\hline
\end{tabular}

\section{Kristiansen 1989}

\begin{tabular}{|c|c|}
\hline Methods & $\begin{array}{l}\text { Method of randomisation: unknown } \\
\text { Assessor blinding: yes at } 2 \text {-year follow-up } \\
\text { Loss to follow-up at } 2 \text { years: } 46 / 85 \text { ( } 18 \text { deaths, } 28 \text { non-attenders) }\end{array}$ \\
\hline Participants & $\begin{array}{l}\text { Hvidovre University Hospital, Denmark } \\
\text { Period of study recruitment: } 1983 \\
85 \text { patients with proximal humeral fractures; } 74 \% \text { minimally displaced (Neer). } \\
\text { Exclusion criteria: no information } \\
60 \text { female, } 25 \text { male; median age } 72 \text { years ( } 1 \text { week group), } 70 \text { years ( } 3 \text { weeks group) }\end{array}$ \\
\hline Interventions & $\begin{array}{l}\text { Interventions started immediately or after closed or open manipulation. } \\
\text { 1. One week immobilisation in sling and body bandage. } \\
\text { 2. Three weeks immobilisation in sling and body bandage. } \\
\text { At the end of immobilisation, instructions were given to perform Codman's pendulum } \\
\text { exercises as well as active movements of the elbow and hand. } \\
\text { Assigned: } 42 / 43 \\
\text { Completed (at } 2 \text { years): } 18 / 21\end{array}$ \\
\hline Outcomes & $\begin{array}{l}\text { Length of follow-up: } 2 \text { years; also assessed at } 1,3,6 \& 12 \text { months } \\
\text { Overall score (Neer without anatomic section) } \\
\text { Mobility: overall from Neer score (range of motion: flexion, extension, abduction, in- } \\
\text { ternal \& external rotation) } \\
\text { Function: overall from Neer score (strength, reaching, stability) } \\
\text { Pain: overall from Neer score (none to disabling) }\end{array}$ \\
\hline
\end{tabular}


Kristiansen 1989 (Continued)

Complex regional pain syndrome type 1 (this was referred to as reflex sympathetic dystrophy in the trial report)

$\begin{array}{ll}\text { Notes } & \text { Post immobilisation for both groups: instructions given for Codman's pendulum exercises } \\ \text { as well as active movements of elbow and hand }\end{array}$

\section{Risk of bias}

\begin{tabular}{|c|c|c|}
\hline Bias & Authors' judgement & Support for judgement \\
\hline $\begin{array}{l}\text { Random sequence generation (selection } \\
\text { bias) }\end{array}$ & Unclear risk & $\begin{array}{l}\text { No details: "Random allocation to immo- } \\
\text { bilization for } 1 \text { to } 3 \text { weeks was performed" }\end{array}$ \\
\hline Allocation concealment (selection bias) & Unclear risk & No details. \\
\hline $\begin{array}{l}\text { Blinding (performance bias and detection } \\
\text { bias) } \\
\text { Functional outcomes, pain, clinical out- } \\
\text { comes, complications }\end{array}$ & Unclear risk & $\begin{array}{l}\text { Only claimed for outcome assessors at fi- } \\
\text { nal follow-up: "The } 2 \text {-year follow-up ex- } \\
\text { amination was blind, as the examiners had } \\
\text { no knowledge of the period of immobiliza- } \\
\text { tion." }\end{array}$ \\
\hline
\end{tabular}

Blinding (performance bias and detection Unclear risk

bias)

Death, reoperation

Incomplete outcome data (attrition bias) High risk

Functional outcomes, pain, clinical out-

comes, complications
No blinding but may not have affected appraisal of mortality (which was not split by treatment group)

Large loss to follow-up ( $46 / 85=54 \%)$. Numbers given for those available at follow-up but incompletely reported data: only medians

Although numbers given for those available at follow-up, only overall mortality data provided (extracted from graph)

Death, reoperation

High risk

Insufficient information to judge this.

\begin{tabular}{ll} 
Selective reporting (reporting bias) Unclear risk \\
\hline
\end{tabular}

Balance in baseline characteristics?

Unclear risk

Unclear risk

Although there appeared to be comparability between treatment groups in age and gender, the percentage of minimally displaced fractures (79\% versus $70 \%$ : 33/42 versus 30/43) differed between the two groups and no information was available on the numbers who had open manipulation (thus entailing surgery)
Lack of information to judge on performance bias. 
Methods
Randomised using block randomisation (under supervision of a statistician) and telephone to an independent researcher with patient details.

Assessor blinding: yes

Loss to follow-up at 6 months: 10 (all had difficulties in travelling to the hospital for scheduled sessions)

Cochlin Hospital, Paris, France

Period of study recruitment: October 2002 to March 2005

74 patients, over 20 years old, with non-operatively treated impacted ("stable") fractures, including 34 minimally displaced (1-part fracture); 16 2-part (surgical neck or greater tuberosity (1)); and 24 3-part (surgical neck and greater tuberosity) (Neer). (AO classification also given). Written consent.

Exclusion criteria: pre-existing shoulder pathology, neurological upper limb disorder, indication for shoulder surgery, multiple injuries, high-energy trauma, or difficulties with language or unable to understand rehabilitation programme or other treatment information.

54 female, 20 male; mean age 63 years

Interventions

Intervention started within 72 hours after fracture.

1. Early mobilisation: active rehabilitation begun within 72 hours of fracture: 2 -hour sessions supervised by a physiotherapist, 5 times a week. Progressing from physical techniques to manage pain, then passive motion, performed by physiotherapist, in a) abduction, with arm suspension and patient supine (session 1); passive range of motion in forward elevation with the patient in a lateral supine position (session 2), with addition of external rotation with the patient in a seated position at session 8. After 3 weeks, sessions occurred twice a week without arm suspension. Patients wore a sling between sessions for 4 to 6 weeks, depending on the level of pain. After 6 weeks, active range of motion was begun during weekly sessions. Strengthening began at 3 months in twicemonthly sessions. Patients underwent a total of 32 sessions.

2. Usual care, starting with 3 weeks of sling immobilisation. Then 2-hour sessions supervised by a physiotherapist 4 times a week for 4 weeks. Passive mobilisation in all planes without arm suspension was performed by physiotherapist. Patients kept their arm in a sling between sessions for 1 to 3 additional weeks, depending on pain level. Then sessions were scheduled 2 times weekly for 5 weeks. Active range-of-motion exercises began after 6 weeks. After 9 weeks of rehabilitation, sessions occurred twice monthly until 6 months. Each patient underwent a total of 33 sessions

Patients used oral analgesics to manage pain. After 4 to 6 weeks, patients were advised to perform daily exercises at home. Patients were discharged from the study at 6 months

Outcomes

Length of follow-up: 6 months, also 6 weeks and 3 months

Functional assessment (Constant score: split into subjective and objective components)

Pain

Patient satisfaction

Range of motion: abduction, anterior elevation, lateral rotation

Complications: non-union (0); fracture displacement (0); treatment (injection) for subacromial impingement syndrome

Compliance

Notes

Interventions for treating proximal humeral fractures in adults (Review) 


\section{Risk of bias}

\begin{tabular}{|c|c|c|}
\hline Bias & Authors' judgement & Support for judgement \\
\hline $\begin{array}{l}\text { Random sequence generation (selection } \\
\text { bias) }\end{array}$ & Low risk & $\begin{array}{l}\text { "Block randomization involved choosing } \\
\text { randomly from among blocks of lengths } 4 \\
\text { and } 2 \text { to prevent the risk of predictability." }\end{array}$ \\
\hline Allocation concealment (selection bias) & Low risk & $\begin{array}{l}\text { "After completion of the trial entry details, } \\
\text { an independent researcher responsible for } \\
\text { treatment allocation was contacted by tele- } \\
\text { phone." }\end{array}$ \\
\hline $\begin{array}{l}\text { Blinding (performance bias and detection } \\
\text { bias) } \\
\text { Functional outcomes, pain, clinical out- } \\
\text { comes, complications }\end{array}$ & Unclear risk & $\begin{array}{l}\text { "Outcome measures were recorded by two } \\
\text { physicians, including one of the authors (F. } \\
\text { F.), who were blinded to the treatment as- } \\
\text { signments." However, care providers and } \\
\text { participants were not blinded to allocation }\end{array}$ \\
\hline $\begin{array}{l}\text { Blinding (performance bias and detection } \\
\text { bias) } \\
\text { Death, reoperation }\end{array}$ & Unclear risk & Not reported. \\
\hline $\begin{array}{l}\text { Incomplete outcome data (attrition bias) } \\
\text { Functional outcomes, pain, clinical out- } \\
\text { comes, complications }\end{array}$ & Unclear risk & $\begin{array}{l}\text { Data were unavailable for } 10 \text { participants } \\
\text { ( } 5 \text { in each group) who were lost to fol- } \\
\text { low-up because of difficulties in travelling } \\
\text { to the hospital. Their characteristics were } \\
\text { reported not to differ from those who at- } \\
\text { tended }\end{array}$ \\
\hline $\begin{array}{l}\text { Incomplete outcome data (attrition bias) } \\
\text { Death, reoperation }\end{array}$ & Unclear risk & Not reported. \\
\hline Selective reporting (reporting bias) & Unclear risk & $\begin{array}{l}\text { Insufficient information to judge this; ret- } \\
\text { rospective trial registration }\end{array}$ \\
\hline Balance in baseline characteristics? & Low risk & Good balance in baseline characteristics \\
\hline Free from performance bias? & Low risk & $\begin{array}{l}\text { Rehabilitation was standardised and "deliv- } \\
\text { ered by physiotherapists who were experi- } \\
\text { enced in the field" }\end{array}$ \\
\hline
\end{tabular}


Livesley 1992

\begin{tabular}{ll} 
Methods & $\begin{array}{l}\text { Method of randomisation: unknown, double-blind } \\
\text { Assessor blinding: likely as code only broken at end of trial } \\
\text { Loss to follow-up at } 6 \text { months: } 3 / 48\end{array}$ \\
\hline Participants & $\begin{array}{l}\text { Mansfield District General Hospital, Mansfield, UK } \\
\text { Period of study recruitment: November } 1988 \text { to May } 1990 \\
48 \text { patients with minimally displaced humeral neck fractures (all Neer Group 1); } 4 \text { had } \\
\text { epiphyseal fractures } \\
\text { Exclusion criteria: able to co-operate with treatment and attend daily therapy for the } \\
\text { first } 10 \text { working days. } \\
37 \text { female, } 11 \text { male; age range } 11 \text { to } 85 \text { years }\end{array}$ \\
\hline
\end{tabular}

Interventions

Interventions started on average 8.6 days since injury, upon referral to physiotherapy department.

1. Pulsed high frequency electromagnetic field ('Curapulse'), 30 minutes/day for first 10 working days. (Intensity setting 3, pulse repetition frequency 35, maximum pulse power 300 watts.)

2. Dummy apparatus (deactivated machine).

Assigned: 22/26

Completed (at 6 months): $21 / 24$

Outcomes

Length of follow-up: 6 months; also assessed at $1 \& 2$ months

No data provided in report

Range of movement of glenohumeral \& scapulothoracic joints

Pain scores, at rest, on movement, analgesia requirement

Muscle wasting and strength

Overall functional assessment score

Subjective opinion of treatment

Overall estimation of treatment (a 'good result')

Time to discharge

Notes

All patients received the same standardised physiotherapy regimen.

No data provided in report for comparison between the two interventions

\section{Risk of bias}

\begin{tabular}{l|l|l} 
Bias & Authors' judgement & Support for judgement \\
\hline $\begin{array}{l}\text { Random sequence generation (selection } \\
\text { bias) }\end{array}$ & Unclear risk & $\begin{array}{l}\text { No details provided: "patients were ran- } \\
\text { domized into two groups" }\end{array}$ \\
\hline $\begin{array}{l}\text { Allocation concealment (selection bias) } \\
\text { Low risk }\end{array}$ & Low & $\begin{array}{l}\text { "double-blind", and randomisation code } \\
\text { was only broken at end of the trial period } \\
\text { to permit analyses }\end{array}$ \\
\hline $\begin{array}{l}\text { Blinding (performance bias and detection } \\
\text { bias) } \\
\text { Functional outcomes, pain, clinical out- } \\
\text { comes, complications }\end{array}$ & Low risk & "double-blind", use of sham control \\
\hline
\end{tabular}

Interventions for treating proximal humeral fractures in adults (Review)

Copyright @ 2015 The Cochrane Collaboration. Published by John Wiley \& Sons, Ltd. 


\section{Livesley 1992 (Continued)}

\begin{tabular}{l|l|l|l}
$\begin{array}{l}\text { Blinding (performance bias and detection } \\
\text { bias) } \\
\text { Death, reoperation }\end{array}$ & No report of these outcomes \\
\hline
\end{tabular}

Incomplete outcome data (attrition bias) High risk

Functional outcomes, pain, clinical outcomes, complications
Although loss to follow-up reported, no results were presented for the trial groups

Incomplete outcome data (attrition bias) Unclear risk

No report of these outcomes

Death, reoperation

\begin{tabular}{ll} 
Selective reporting (reporting bias) High risk \\
\hline
\end{tabular}

Balance in baseline characteristics? Unclear risk

Results not presented.

\begin{tabular}{|l|l|l} 
& $\begin{array}{l}\text { the article claims "patients ... were referred } \\
\text { to the physiotherapy department without } \\
\text { delay", the ranges for average time from in- } \\
\text { jury to start treatment were } 0 \text { to } 17 \text { days } \\
\text { (active) and } 0 \text { to } 27 \text { days (sham) }\end{array}$ \\
\hline Free from performance bias? & Unclear risk & $\begin{array}{l}\text { "Standardized physiotherapy } \\
\text { However, although the article claims "pa- } \\
\text { tients ... were referred to the physiotherapy } \\
\text { department without delay", the ranges for } \\
\text { average time from injury to start treatment } \\
\text { were } 0 \text { to } 17 \text { days (active) and } 0 \text { to } 27 \text { days } \\
\text { (sham) }\end{array}$ \\
\hline
\end{tabular}

Lopiz 2014

Methods

Method of randomisation: used sequentially numbered, opaque, sealed envelopes

Assessor blinding: not done

Loss to follow-up: 2 participants of the MultiLoc nail group were excluded (one died and one was lost to follow-up)

Participants

$\mathrm{Cl}_{1}$ nico San Carlos Hospital, Madrid, Spain

Period of study recruitment: March 2011 and September 2012

54 patients with displaced Neer 2- or 3-part proximal humerus fractures

Exclusion criteria: pathological or open fractures, 4-part fractures, concomitant fractures in the same upper limb, or the opposite and previous surgery on that shoulder. Lack of consent

Of 52: 41 female, 11 male; mean age 70 years, range 38 to 89 years

Interventions

All had general anaesthesia and intrascalene block. Mainly minimally invasive (percutaneous) - small deltoid-splitting incision ( 5 were open reduction with extended superior incision)

1. MultiLoc proximal humeral nail (MPHN) (Synthes-DePuy, Solothurn, Switzerland)

Interventions for treating proximal humeral fractures in adults (Review) 
- a straight nail
2. Polarus humeral nail (Acumed LLC, Hillsboro, OR, USA) - a curved nail

Postoperatively, patients were immobilised with a sling. Passive range-of-motion exercises were allowed 24 to 48 hours after surgery, followed as soon as possible by active-assisted motion

Assigned: $28 / 26$

Completed: 26/26 (mean 14 months)

Outcomes

Length of follow-up: mean 14 months (6 to 22 months); formally 1, 3, 6 and 12 months Constant score (categories excellent; good; satisfied; fair; poor)

Constant score - adjusted for age and sex

Physical tests to assess evidence of rotator cuff disease for entry point morbidity

Non-union, protrusion of the osteosynthesis material (subacromial impingement or articular surface intrusion of the screws), final alignment of the healed fracture (malunion) Re-operation (hardware removal for complications; reverse arthroplasty)

Length of operation

Intraoperative complications (none)

Length of hospital stay

Mortality

Notes

Request for information sent to Dr Lopiz on 20 October 2014 requesting clarification on method of sequence generation, details on the 2 excluded participants, query on tuberosity involvement of 3-part fractures in the MultiLoc nail group, clarification on whether 1 of 2 participants had a reverse shoulder replacement and length of follow-up times for each group. No response received

\section{Risk of bias}

\begin{tabular}{|c|c|c|}
\hline Bias & Authors' judgement & Support for judgement \\
\hline $\begin{array}{l}\text { Random sequence generation (selection } \\
\text { bias) }\end{array}$ & Low risk & $\begin{array}{l}\text { "assistant generated the random allocation } \\
\text { sequence, which was concealed from the } \\
\text { authors." "Patients were randomly assigned } \\
\text { to } 2 \text { parallel groups, initially at a } 1: 1 \text { ratio, } \\
\text { " } \\
\text { description raises the concern that the se- } \\
\text { quence may have been predictable (not ran- } \\
\text { dom) in the early stages - but was probably } \\
\text { OK }\end{array}$ \\
\hline Allocation concealment (selection bias) & Low risk & $\begin{array}{l}\text { "Randomization was carried out with use } \\
\text { of sequentially numbered, opaque, sealed } \\
\text { envelopes." } \\
\text { "All patients were randomized by a research } \\
\text { co-ordinator who was not involved subse- } \\
\text { quently in the study." }\end{array}$ \\
\hline
\end{tabular}




\section{Lopiz 2014 (Continued)}

\begin{tabular}{|c|c|c|}
\hline $\begin{array}{l}\text { Blinding (performance bias and detection } \\
\text { bias) } \\
\text { Functional outcomes, pain, clinical out- } \\
\text { comes, complications }\end{array}$ & High risk & $\begin{array}{l}\text { "The health care providers involved with } \\
\text { subsequent patient care were not blinded } \\
\text { to the treatment." } \\
\text { No mention of independent or blinded } \\
\text { outcome assessment }\end{array}$ \\
\hline
\end{tabular}

Blinding (performance bias and detection Unclear risk

bias)

Death, reoperation
"The health care providers involved with subsequent patient care were not blinded to the treatment."

However, it is unlikely that lack of blinding will affect the reporting of these outcomes

Incomplete outcome data (attrition bias) Unclear risk Functional outcomes, pain, clinical outcomes, complications

Variable follow-up with no confirmation of similar follow-ups in the two groups. Additionally, data lost from 2 participants in the Polarus nail group ( 1 died +1 lost to follow-up)

Incomplete outcome data (attrition bias) Low risk

Death, reoperation

Data lost from 2 participants in the Polarus nail group (1 died +1 lost to follow-up). Unlikely to bias the results

\begin{tabular}{|c|c|c|}
\hline Selective reporting (reporting bias) & Unclear risk & $\begin{array}{l}\text { Insufficient information to judge this. No } \\
\text { protocol found. }\end{array}$ \\
\hline
\end{tabular}

Balance in baseline characteristics? Low risk

Although baseline data were not presented for 2 participants in the MultiLoc nailing group, there were no major imbalances in baseline characteristics between the two groups: "No statistically significant differences were found between the 2 groups."

Free from performance bias?

Low risk
"All surgeries were performed by 1 of the 3 senior trauma surgeons in the unit." Post-operative care was the same in both groups.

Lundberg 1979

Methods

Method of randomisation: unknown

Assessor blinding: no, but mention of independent assessors

Loss to follow-up at 3 months: 0/42; not known for final assessment

Participants

Gavle, Sweden

Period of study recruitment: not stated

42 patients with undisplaced proximal humeral fractures (all Neer Group 1) fixed with a sling; 13 had avulsion of the greater tuberosity.

Exclusion criteria: no information 
Lundberg 1979 (Continued)

37 female, 5 male; mean age 65 years

\begin{tabular}{ll} 
Interventions & Interventions started 7 days post injury, after removal of sling. \\
& 1. Instructed self-exercise: patients instructed to train 5 to 10 minutes, 4 to 5 times \\
They had 3 visits (day 1 , and $1 \& 3$ months) to physiotherapist for instructions \\
checks. At 1 month, patients were told how to extend their exercises to same level \\
physiotherapy group. \\
2. Conventional physiotherapy: 9 visits (average 20 to 30 minutes) between 2 \\
months; patients encouraged to continue exercise at home. At about 4 weeks, treatm \\
was intensified. \\
Assigned: $20 / 22$ \\
Completed (at 3 months): 20/22; (at mean 16 months): number in each group \\
known \\
\hline Length of follow-up: $>1$ year (mean 16 months); also assessed at $1 \& 3$ months \\
Range of movement: abduction, shoulder elevation - active $\&$ passive \\
Pain (insignificant, moderate, severe), longstanding \\
Lifting power of shoulder \\
Frozen shoulder (secondary) \\
Neer score (at final evaluation) including failure category \\
Hand grip strength
\end{tabular}

Notes

No indication in the report of any loss to follow-up at last follow-up (> 1 year), but cannot be assumed

\section{Risk of bias}

Bias

Random sequence generation (selection Unclear risk bias)

Allocation concealment (selection bias)

Unclear risk

Blinding (performance bias and detection High risk

bias)

Functional outcomes, pain, clinical out-

comes, complications

Blinding (performance bias and detection Unclear risk bias)

Death, reoperation

Incomplete outcome data (attrition bias) Unclear risk Functional outcomes, pain, clinical outcomes, complications

\section{Support for judgement}

No details of method: "In all, 42 patients were randomly assigned into two groups."

No details of method: "In all, 42 patients were randomly assigned into two groups."

No blinding, although independent assessment claimed: "Examination was made by physicians and physiotherapists independently at 1 month and 3 months.."

Not reported.

Full data provided for 1 and 3 months follow-up; but denominators not stated for long-term (mean 16 months) follow-up

Interventions for treating proximal humeral fractures in adults (Review)

Copyright @ 2015 The Cochrane Collaboration. Published by John Wiley \& Sons, Ltd. 
Lundberg 1979 (Continued)

\begin{tabular}{l|l|l}
\hline $\begin{array}{l}\text { Incomplete outcome data (attrition bias) } \\
\text { Death, reoperation }\end{array}$ & Unclear risk & Data not reported for these outcomes \\
\hline Selective reporting (reporting bias) & Unclear risk & Insufficient information to judge this. \\
\hline Balance in baseline characteristics? & Low risk & $\begin{array}{l}\text { No major imbalances in baseline character- } \\
\text { istics }\end{array}$ \\
\hline Free from performance bias? & Low risk & No indications of performance bias. \\
\hline
\end{tabular}

\section{Ockert 2010}

Methods
Method of randomisation: used closed envelopes

Assessor blinding: unknown

Loss to follow-up (2010 publication): 10 patients excluded from analysis following randomisation; 6 with polytrauma, 2 with neurologic deficiency and 2 (1 versus 1 ) who were converted to shoulder arthroplasty intraoperatively. There was no mention of group allocation at randomisation or evaluation in the paper - these (8 versus 2 ) were notified after contact with the lead trial investigator

Loss to follow-up (2014 publication): not stated
Ludwig-Maximilians University, Munich, Germany

Period of study recruitment: August 2006 to July 2008 (extended to February 2010 for 2014 publication)

2010 publication: 76 patients, aged over 18 years, with displaced proximal humeral fractures with displacement $>1 \mathrm{~cm}$ and angulation of fragments $>45$ degrees (Neer criteria)

Exclusion criteria: poly-traumatised patients, neurologic deficit or intra-operative conversion to shoulder arthroplasty. (Paper noted there were no open or pathological fractures.)

Of 66: 48 female, 18 male; mean age 68 years, range 29 to 92 years

2014 publication: 124 patients with displaced proximal humeral fractures with displacement $>1 \mathrm{~cm}$ and angulation of fragments $>45$ degrees (Neer criteria)

Exclusion criteria: open or pathological fractures, poly-traumatised patients, primary nerve palsy (given as examples)

89 female, 35 male; mean age 71 years, range not given
1. Polyaxial angular stable plate fixation (Non-contact bridging - Proximal Humerus (NCB-PH)). Polyaxial plating allows a range of 0 to 15-degree angle off-centre. After insertion, a threaded screw cap locks the axis of the screw.

2. Monoaxial angular stable plate fixation with Proximal Humeral Internal Locking System (PHILOS) Synthes GmbH. Monoaxial locking plate technique is characterized by fixed divergent and convergent screw orientation due to threaded screw holes A deltopectoral approach was used for open reduction and internal fixation of all fractures. All patients received prophylactic intravenous antibiotic immediately before surgery. "The postoperative rehabilitation protocol included immediate passive- and active-assisted range of motion (ROM) up to 60-degree angle of abduction and elevation without forced external rotation for 6 weeks. Full ROM with active exercises was started 6 weeks 
Ockert 2010 (Continued)

after operation." (2010 publication)

Assigned: $39 / 37$ (2010 publication); 58/66 (2014 publication but post-randomisation exclusions may have occurred)

Completed: 29/37; 58/66 (2014 publication)

Outcomes

2010 publication: Length of follow-up: 6 months (X-rays 1 day, 6 weeks, 3 months and 6 months)

Secondary varus displacement ( $>10$ degrees)

Delayed union (due to osteonecrosis)

Intra-articular screw cut out

Re-operation: revision surgery and early hardware removal

Infection (none)

Neurovascular injuries (none)

2014 publication: Length of follow-up: 12 months (X-rays 1 day, 6 weeks, and 3, 6 and 12 months)

Revision surgery (reasons given: secondary varus displacement, subacromial impingement, intra-articular screw cut out, infection)

Screw position in different region of the humeral head

Request for information sent to Dr Ockert on 2 June 2012. Repeated on 8 June 2012, in email Peter Biberthaler regarding identification and further information on ongoing trial referred to in conference abstract (Biberthaler 2009) - it seems highly likely that the ongoing trial was this trial. However, this was not clear from email from Ben Ockert on 18 June 2012; this also provided details on the method of randomisation, the numbers allocated and analysed in each group

The 2014 publication of this trial (Ockert 2014) reported on an additional 48 participants, reflecting an extended period of trial recruitment, and a longer follow-up. Only the revision surgery data from Ockert 2014 were used in this review.

\section{Risk of bias}

\begin{tabular}{|c|c|c|}
\hline Bias & Authors' judgement & Support for judgement \\
\hline $\begin{array}{l}\text { Random sequence generation (selection } \\
\text { bias) }\end{array}$ & Unclear risk & $\begin{array}{l}\text { "consecutive patients ... were prospectively } \\
\text { randomized“. No description of sequence } \\
\text { generation }\end{array}$ \\
\hline Allocation concealment (selection bias) & Unclear risk & $\begin{array}{l}\text { "consecutive patients ... were prospectively } \\
\text { randomized". Contact from trialist re- } \\
\text { vealed they "used closed envelope tech- } \\
\text { nique for randomization". (Exclusion cri- } \\
\text { teria appeared to be applied post-randomi- } \\
\text { sation.) } \\
\mathbf{2 0 1 4} \text { publication: "Randomization was } \\
\text { performed by closed envelope technique." }\end{array}$ \\
\hline $\begin{array}{l}\text { Blinding (performance bias and detection } \\
\text { bias) } \\
\text { Functional outcomes, pain, clinical out- }\end{array}$ & High risk & $\begin{array}{l}\text { No mention of blinding. Radiographic as- } \\
\text { sessment performed by two trained radi- } \\
\text { ologists twice in separate sessions } 8 \text { weeks }\end{array}$ \\
\hline
\end{tabular}




\section{Ockert 2010 (Continued)}

comes, complications

bias)

Death, reoperation

Incomplete outcome data (attrition bias) High risk

Functional outcomes, pain, clinical out-

comes, complications apart. Consensus decision for osteonecrosis and implant-related failure. Criteria for healing stated

No mention of blinding, but unlikely to affect this.

"Follow-up rate was $71 \%$ of all radiographs taken 1 day, 6 weeks, 3 months, and 6 months after surgery."

Numbers of patients allocated or assessed by intervention group provided after personal communication. Post-randomisation exclusions $(10 / 76=13 \%)$, was imbalanced (8 versus 2 ) and other loss to follow-up not accounted for

2014 publication: Concerns on post-randomisation exclusions continue for this publication

Incomplete outcome data (attrition bias) Unclear risk

Death, reoperation

As above. Paper described cases of revision surgery and early removal of metalwork; however, group allocation not given. Information provided subsequently

Selective reporting (reporting bias) High risk

No protocol available. The extension of the recruitment, incomplete results and lack of full listing of exclusion criteria are of concern in the Ockert 2014 publication.
Balance in baseline characteristics? Unclear risk

Low risk

"The fracture types were equally distributed in both study groups." However, this applied to 66 participants. Does not state how many patients in each group or compare demographics

Age and gender were comparable in the two groups in the Ockert 2014 publication. There was no mention of fracture type.

Free from performance bias?
Six experienced surgeons performed the surgery: "In advance of this study, all surgeons were trained in the respective monoaxial and polyaxial locking plate system"

Same antibiotic regimen and post-op management. 
Methods

Participants
Method of randomisation: opaque, sealed envelopes

Assessor blinding: no, but mention of independent surgeon

Loss to follow-up at 24 months: 7/60 (1 excluded themselves; 2 lost; 4 died)

Stockholm Söder Hospital, Stockholm, Sweden

Period of study recruitment: April 2003 to March 2008

60 patients with acute displaced (based on Neer's criteria) 3-part proximal humeral fractures (all had displaced surgical neck fracture, all bar one had a displaced greater tuberosity; the exception had a displaced lesser tuberosity). Age 55 or older with a fracture sustained after a low-energy trauma (e.g. a simple fall). Independent living conditions

Exclusion criteria: patients with a completely displaced shaft in relation to the head fragment or with a valgus impact fracture. Institutionalised, severe cognitive dysfunction ( $<3$ correct answers on a 10-item Short Portable Mental Status Questionnaire).

Of 59 ( 1 patient excluded themselves): 48 female, 11 male; mean age 74 years, range 56 to 92 years (operations were performed within a mean of 6 (SD 4.1) days after the injury)

Interventions

Interventions (and randomisation) started after hospital admission.

1. Surgery: operation occurred at mean of 6.1 days of injury. Open reduction and fixation using a deltopectoral approach with a PHILOS plate (Synthes, Stockholm, Sweden) and with nonabsorbable sutures used to fix displaced/unstable lesser and/or greater tuberosity fractures. The reduction and position of the implant was checked with the aid of an Xray image intensifier. (All patients had pre-operative antibiotics.) Post surgery, the arm was placed in a sling and patients were referred to a physiotherapist. The sling was used for 4 weeks; afterwards, the patients were allowed to use it at their own convenience. Pendulum exercises and passive elevation/abduction up to 90 degrees were started from the first postoperative day. After 4 weeks, the patients were allowed a free active range of movement.

2. Non-surgical treatment: arm immobilisation in a sling for 2 weeks, after which they were allowed to use it at their own convenience. After 2 weeks, the patients were referred to a physiotherapist, and pendulum exercises and passive elevation/abduction up to 90 degrees were started. After 4 weeks, they were allowed a free active range of movement. Assigned: $30 / 30$

Completed (at 2 years): $27 / 26$

Outcomes

Length of follow up: 2 years

Constant shoulder score (both shoulders)

DASH (Disabilities of the Arm, Shoulder and Hand) questionnaire

Quality of life score: EQ-5D

Mortality

Pain

Range of motion: abduction, flexion

Fixation failure, redisplacement, non-union, malunion

Subsequent surgery (reasons including deep infection, etc)

Radiographic outcomes including avascular necrosis and osteoarthritis

Notes

Trial run concurrently with Olerud 2011b.

Additional information on randomisation and trial location obtained from Dr Olerud

(April 2012). Pain data received May 2012 
Olerud 2011a (Continued)

Risk of bias

\begin{tabular}{|c|c|c|}
\hline Bias & Authors' judgement & Support for judgement \\
\hline $\begin{array}{l}\text { Random sequence generation (selection } \\
\text { bias) }\end{array}$ & Low risk & $\begin{array}{l}\text { "After clearance by an anesthetist, the } \\
\text { patients were randomized (independently } \\
\text { prepared opaque, sealed envelopes) to open } \\
\text { reduction and internal fixation with a lock- } \\
\text { ing plate or nonoperative treatment." trial } \\
\text { report } \\
\text { "the patients were randomised by num- } \\
\text { bered sealed opaque envelopes drawn con- } \\
\text { secutively } \\
\text { The envelopes were independently pre- } \\
\text { pared and thoroughly mixed. After that the } \\
\text { envelopes were numbered by another per- } \\
\text { son. At the time of randomisation the en- } \\
\text { velopes were drawn in numerical order." } \\
\text { personal communication }\end{array}$ \\
\hline Allocation concealment (selection bias) & Low risk & See above \\
\hline $\begin{array}{l}\text { Blinding (performance bias and detection } \\
\text { bias) } \\
\text { Functional outcomes, pain, clinical out- } \\
\text { comes, complications }\end{array}$ & High risk & $\begin{array}{l}\text { No assessor blinding, although "The final } \\
24 \text {-month follow-up was performed by an } \\
\text { independent orthopaedic surgeon not pre- } \\
\text { viously involved in the treatment." } \\
\text { No provider or participant blinding. }\end{array}$ \\
\hline
\end{tabular}

Blinding (performance bias and detection Unclear risk bias)

Death, reoperation

Lack of blinding unlikely to affect assessment of these outcomes, but may affect decisions for subsequent surgery

Incomplete outcome data (attrition bias) Low risk Functional outcomes, pain, clinical outcomes, complications

"In the outcome analyses, all patients remained in their randomization group regardless of secondary procedures according to the intention-to-treat principle." Participant flow provided; no cause for concern

$\begin{array}{lll}\text { Incomplete outcome data (attrition bias) } & \text { Low risk } & \text { As above. }\end{array}$

Death, reoperation

Selective reporting (reporting bias) Unclear risk

Insufficient information to judge this. No protocol found.

Balance in baseline characteristics? Low risk

No imbalances: baseline comparability.

Interventions for treating proximal humeral fractures in adults (Review)

Copyright @ 2015 The Cochrane Collaboration. Published by John Wiley \& Sons, Ltd. 
Olerud 2011a (Continued)

\begin{tabular}{|c|c|c|}
\hline Free from performance bias? & Low risk & $\begin{array}{l}\text { "All operations in patients randomized to } \\
\text { surgery were performed by } 1 \text { of } 2 \text { or- } \\
\text { thopaedic surgeons, both well experienced } \\
\text { in shoulder surgery." } \\
\text { While all surgical patients were referred to a } \\
\text { physiotherapist after their surgery and non- } \\
\text { surgically treated patients were referred af- } \\
\text { ter } 2 \text { weeks, this was unlikely to influence } \\
\text { results. Otherwise, similar exercise / reha- } \\
\text { bilitation schedules }\end{array}$ \\
\hline
\end{tabular}

\section{Olerud 2011b}

\section{Methods}

Participants
Method of randomisation: opaque, sealed envelopes

Assessor blinding: no, but mention of independent surgeon

Loss to follow-up at 24 months: 6/55 (1 lost; 5 died)

\begin{tabular}{|c|c|}
\hline & $\begin{array}{l}\text { Period of study recruitment: April } 2003 \text { to March } 2008 \\
55 \text { patients with an acute displaced (based on Neer's criteria) 4-part proximal humeral } \\
\text { fractures (all had displaced surgical neck, greater and lesser tuberosity fractures). Age } \\
55 \text { or older with a fracture sustained after a low-energy trauma (e.g. a simple fall), no } \\
\text { previous shoulder problems. Independent living conditions. } \\
\text { Exclusion criteria: patients with a completely displaced shaft in relation to the head } \\
\text { fragment or with a valgus impact fracture. Institutionalised, severe cognitive dysfunction } \\
\text { (<3 correct answers on a } 10 \text {-item Short Portable Mental Status Questionnaire). } \\
47 \text { female, } 8 \text { male; mean age } 77 \text { years, range } 58 \text { to } 92 \text { years }\end{array}$ \\
\hline Interventions & $\begin{array}{l}\text { Interventions (and randomisation) started after hospital admission. } \\
\text { 1. Surgery: operation occurred at mean of } 6.0 \text { days of injury. Humeral head replacement } \\
\text { using a deltopectoral approach with the Global Fx prosthesis (DePuy, Sollentuna, Swe- } \\
\text { den); this is a modular prosthesis with a fixed angle and a conventional head - it has } \\
3 \text { fins. Heavy nonabsorbable sutures were tagged on the bone tendon interface of both } \\
\text { tuberosities } \\
\text { Cancellous bone graft from the head fragment was placed between the shaft and the } \\
\text { tuberosities. (All patients had pre-operative and } 2 \text { doses post-operative antibiotics.) Post } \\
\text { surgery, the arm was placed in a sling and patients were referred to a physiotherapist. } \\
\text { The sling was used for } 6 \text { weeks; afterwards, the patients were allowed to use it at their } \\
\text { own convenience. Pendulum exercises and passive elevation/abduction up to } 90 \text { degrees } \\
\text { were started from the first postoperative day. After } 6 \text { weeks, the patients were allowed a } \\
\text { free active range of movement. Strengthening exercises were begun after } 3 \text { months. } \\
2 \text {. Non-surgical treatment: arm immobilisation in a sling for } 2 \text { weeks, after which they } \\
\text { were allowed to use it at their own convenience. After } 2 \text { weeks, the patients were referred } \\
\text { to a physiotherapist, and pendulum exercises and passive elevation/abduction up to } 90 \\
\text { degrees were started. After } 4 \text { weeks, they were allowed a free active range of movement. } \\
\text { Assigned: } 27 / 28 \\
\text { Completed (at } 2 \text { years): } 24 / 25\end{array}$ \\
\hline
\end{tabular}

Stockholm Söder Hospital, Stockholm, Sweden

Period of study recruitment: April 2003 to March 2008

55 patients with an acute displaced (based on Neer's criteria) 4-part proximal humeral previous shoulder problems. Independent living conditions.

Exclusion criteria: patients with a completely displaced shaft in relation to the head fragment or with a valgus impact fracture. Institutionalised, severe cognitive dysfunction ( $<3$ correct answers on a 10-item Short Portable Mental Status Questionnaire).

47 female, 8 male; mean age 77 years, range 58 to 92 years

Interventions (and randomisation) started after hospital admission. den); this is a modular prosthesis with a fixed angle and a conventional head - it has 3 fins. Heavy nonabsorbable sutures were tagged on the bone tendon interface of both tuberosities

Cancellous bone graft from the head fragment was placed between the shaft and the uberosities. (All patients had pre-operative and 2 doses post-operative antibiotics.) Post surgery, the arm was placed in a sling and patients were referred to a physiotherapist. 90 degrees were started from the first postoperative day. After 6 weeks, the patients were allowed a free active range of movement. Strengthening exercises were begun after 3 months.

2. Non-surgical treatment: arm immobilisation in a sling for 2 weeks, after which they were allowed to use it at their own convenience. After 2 weeks, the patients were referred to a physiotherapist, and pendulum exercises and passive elevation/abduction up to 90 Assigned: $27 / 28$

Completed (at 2 years): 24/25 
Olerud 2011b (Continued)

\begin{tabular}{ll}
\hline Outcomes & Length of follow up: 2 years \\
& Constant shoulder score (both shoulders) \\
& DASH (Diasabilities of the Arm, Shoulder and Hand) questionnaire \\
& Quality of life score: EQ-5D \\
& Mortality \\
& Pain \\
& Range of motion: abduction, flexion \\
& Fixation failure, redisplacement, non-union, malunion \\
& Subsequent surgery (reasons including non-union, etc) \\
& Radiographic outcomes including avascular necrosis and osteoarthritis \\
\hline \multirow{2}{*}{ Notes } & Trial run concurrently with Olerud 2011a. \\
& $\begin{array}{l}\text { Additional information on randomisation and trial location obtained from Dr Olerud } \\
\text { (April 2012). Pain data received May 2012 }\end{array}$ \\
\hline
\end{tabular}

Risk of bias

Bias

Random sequence generation (selection Low risk bias)
Authors' judgement

\section{Support for judgement}

"After clearance by an anesthesiologist, the patients were randomized (opaque sealed envelopes prepared independently) to a primary HA or nonoperative treatment." trial report

"the patients were randomised by numbered sealed opaque envelopes drawn consecutively

The envelopes were independently prepared and thoroughly mixed. After that the envelopes were numbered by another person. At the time of randomisation the envelopes were drawn in numerical order." personal communication

See above

No assessor blinding, although "The final 24-month follow-up was performed by an independent orthopaedic surgeon not previously involved in the treatment."

No provider or participant blinding.

Lack of blinding unlikely to affect assessment of these outcomes, but may affect decisions for subsequent surgery 
Olerud 2011b (Continued)

\begin{tabular}{|c|c|c|}
\hline $\begin{array}{l}\text { Incomplete outcome data (attrition bias) } \\
\text { Functional outcomes, pain, clinical out- } \\
\text { comes, complications }\end{array}$ & Low risk & $\begin{array}{l}\text { "In the outcome analyses, all patients re- } \\
\text { mained in their randomization group re- } \\
\text { gardless of secondary procedures according } \\
\text { to the intention-to-treat principle." Partic- } \\
\text { ipant flow provided; no cause for concern }\end{array}$ \\
\hline $\begin{array}{l}\text { Incomplete outcome data (attrition bias) } \\
\text { Death, reoperation }\end{array}$ & Low risk & As above. \\
\hline Selective reporting (reporting bias) & Unclear risk & $\begin{array}{l}\text { Insufficient information to judge this. No } \\
\text { protocol found. }\end{array}$ \\
\hline Balance in baseline characteristics? & Low risk & No imbalances: baseline comparability. \\
\hline Free from performance bias? & Unclear risk & $\begin{array}{l}\text { "In patients randomized to surgery, all op- } \\
\text { erations were performed by } 1 \text { of } 2 \text { ortho- } \\
\text { pedic surgeons, both well experienced in } \\
\text { shoulder surgery ..." } \\
\text { While all surgical patients were referred to a } \\
\text { physiotherapist after their surgery and non- } \\
\text { surgically treated patients were referred af- } \\
\text { ter } 2 \text { weeks, this was unlikely to influence } \\
\text { results. As was the differences in timing for } \\
\text { free ROM ( } 6 \text { versus } 4 \text { weeks). However, it } \\
\text { was only reported for the surgical group } \\
\text { that strengthening exercises were begun af- } \\
\text { ter } 3 \text { months }\end{array}$ \\
\hline
\end{tabular}

ProFHER 2015

Methods

Method of randomisation: remote randomisation computer programme with 1:1 allocation, stratifying

by tuberosity involvement (yes or no) and using random block sizes of 4, 8, and 12

Assessor blinding: no, except for blinded independent coding

Loss to follow-up at 24 months: $32 / 250$ (12 no response, 6 withdrew ( 1 who died), 14 died)

Participants

33 acute UK National Health Service hospitals, UK

Period of study recruitment: September 2008 and April 2011

250 patients aged 16 years or older presenting within 3 weeks after sustaining a displaced fracture of the proximal humerus that involved the surgical neck. The degree of displacement had to be sufficient for the treating surgeon to consider surgical intervention but did not have to meet the displacement criteria of Neer $\left(1 \mathrm{~cm}\right.$ or $45^{\circ}$ angulation of displaced parts, or both) for inclusion in the trial. Written consent

Exclusion criteria: patients who had associated dislocation of the injured shoulder joint, open fracture, insufficient mental capacity to understand the trial or instructions for rehabilitation, co-morbidities precluding surgery or anaesthesia, clear indication for surgery such as severe soft-tissue compromise, multiple injuries (upper limb fractures), patho-

Interventions for treating proximal humeral fractures in adults (Review)

Copyright @ 2015 The Cochrane Collaboration. Published by John Wiley \& Sons, Ltd. 
ProFHER 2015 (Continued)

logical fracture (other than osteoporotic), terminal illness, or not resident in the hospital catchment area

192 female, 58 male; mean age 66 years, range 24 to 92 years

Interventions

Outcomes
Interventions (and randomisation) started after presentation at the hospital

1. Surgery: either internal fixation, such as with plate and screws (majority were Philos plates),or joint replacement (hemiarthroplasty).

2. Non-surgical treatment: patients were given a sling for the injured arm for as long as deemed necessary (3 weeks was suggested), followed by active early rehabilitation

Delivery of care and rehabilitation, which was freely available for all patients, incorporated three set measures to ensure good standards of care within the NHS: provision of an information leaflet on personal care during sling immobilisation; a basic treatment protocol to guide physiotherapy; and promotion of home exercises. Rehabilitation care was provided by physiotherapists in inpatient, outpatient and/or community settings

Length of follow-up: 2 years, also 3 (for EQ-5D), 6 and 12 months

Oxford Shoulder Score

SF-12 (12-item short form health survey)

Euroqol (EQ-5D)

Complications, including surgical complications (wound infection, implant failure, shoulder dislocation, septicaemia); early medical complications, i.e. chest infection, confirmed myocardial infarction or stroke, treated deep vein thrombosis and pulmonary embolism

Mortality

Subsequent referral for operation or substantive treatment

Data for economic evaluation: NHS and societal costs

Notes Published protocol.

\section{Risk of bias}

\section{Bias}

Random sequence generation (selection Low risk bias)
Allocation concealment (selection bias)
Low risk

Blinding (performance bias and detection Unclear risk bias)

Functional outcomes, pain, clinical outcomes, complications

\section{Support for judgement}

"Randomization was done with a computer programme using 1:1 allocation, stratifying by tuberosity involvement (yes or no) and using random block sizes of 4,8 and 12."

"research associates randomly allocated individual patients to surgical or non-surgical treatment using an independent remote randomization service"

"There was no blinding of trial participants, clinicians, or assessment of outcomes." "Coding was performed by at least 2 independent coders blinded to treatment allocation."

Discussion: "Although lack of blinding of 
ProFHER 2015 (Continued)

patient-reported outcome assessment is unavoidable, similarities in the 2 groups in patient return of questionnaires and baseline characteristics at 24 months, and the lack of a significant effect of baseline patient preferences on the OSS results suggest this did not introduce a bias."

Blinding (performance bias and detection Low risk bias)

Death, reoperation

Incomplete outcome data (attrition bias) Low risk Functional outcomes, pain, clinical outcomes, complications
As above. Additionally, lack of blinding unlikely to affect assessment of these outcomes

Loss to follow-up balanced in the two groups. Trial reports: "Overall, 41 patients (16\%) had missing follow-up data on at least 1 time point. Using complete data derived by multiple imputation resulted in comparable treatment effect estimates to the primary analysis with no overall statistically significant group difference $(\mathrm{P}=.48)$

..... Nonresponse (none or intermittent) was not associated with any demographic or fracture characteristics."
Incomplete outcome data (attrition bias) Low risk Death, reoperation

Low risk

Low risk

Very high return of hospital forms: 249 of $250(99.6 \%)$ at 1-year follow-up forms and 234 of $250(93.6 \%) 2$-year (but 2-year forms not sent for those who had already died before 1 year)

Selective reporting (reporting bias) Low risk

Balance in baseline characteristics?

Free from performance bias?
Prospective trial registration, publication of trial protocol and trial analysis plan

The baseline characteristics were well balanced except for smoking status (there were more smokers in the non-surgical group). However, this was shown not to impact on the results

"It was emphasized that good standards care, both surgical and nonsurgical, should be provided throughout the treatment pathway for the injury, including surgical care or management of the sling, postoperative care, and rehabilitation in both groups. Participating hospitals did not introduce new or experimental interventions for these fractures during the study."

"To avoid learning curve problems, sur- 
ProFHER 2015 (Continued)

geons and physiotherapists used surgical interventions and procedures with which they were familiar."

"Physiotherapy treatment log data demonstrated equal access and implementation between groups, with similarly high numbers of participants recorded as performing home exercises in both groups."

Revay 1992

Methods

Randomisation from closed envelopes

Assessor blinded

Loss to follow-up at 1 year: 1/48

Participants

Danderyd Hospital, Danderyd, Sweden

Period of study recruitment: not stated

48 patients with 2-, 3- or 4-part minimally displaced proximal humeral fractures $(<1$ $\mathrm{cm}$ or $<45$ degrees; Neer Group 1) treated non-surgically with sling immobilisation for 1 week.

Exclusion criteria: patients with skin diseases and/or chlorine allergy, non-ambulatory

39 female, 9 male; mean age 66 years

Interventions

Interventions started 5 to 10 days post-injury after removal of sling.

1. Swimming pool training (30 minutes each session, up to 20 sessions maximum) in groups (6 to 8 patients) plus instructions for self-training (see below).

2. Instructions for self-training: exercises to be performed at least 4 times a day for 10 to 15 minutes each time, use of hand on injured side for activities of daily living, advice on relaxation and resting positions.

Assigned: 25/23

Completed: number in each group not known

\begin{tabular}{ll} 
Outcomes & Length of follow-up: 1 year; also assessed at 3 weeks, $2 \& 3$ months \\
& Pain (analogue scale) \\
& $\begin{array}{l}\text { Activities of daily living: subjective assessment of } 9 \text { activities each rated on a } 5 \text { point scale } \\
\text { Functional scale: } 6 \text { point scale } \\
\text { Joint movement (abduction, flexion, internal rotation) }\end{array}$ \\
\hline Notes & Means (probably) presented without standard deviations.
\end{tabular}

Risk of bias

\begin{tabular}{lll}
\hline Bias & Authors' judgement & Support for judgement
\end{tabular}

Random sequence generation (selection Unclear risk bias)
No details: "patients were randomized into two groups" 
Revay 1992 (Continued)

\begin{tabular}{|c|c|c|}
\hline Allocation concealment (selection bias) & Unclear risk & $\begin{array}{l}\text { Insufficient details of safeguards: "random- } \\
\text { ized and given instructions in a sealed en- } \\
\text { velope" }\end{array}$ \\
\hline $\begin{array}{l}\text { Blinding (performance bias and detection } \\
\text { bias) } \\
\text { Functional outcomes, pain, clinical out- } \\
\text { comes, complications }\end{array}$ & Unclear risk & $\begin{array}{l}\text { "All patients were examined by a physio- } \\
\text { therapist who did not know which group } \\
\text { each patient belonged to". However, no } \\
\text { participant or care provider blinding nor } \\
\text { mention of ways to prevent disclosure to } \\
\text { assessor }\end{array}$ \\
\hline $\begin{array}{l}\text { Blinding (performance bias and detection } \\
\text { bias) } \\
\text { Death, reoperation }\end{array}$ & Unclear risk & Not reported. \\
\hline $\begin{array}{l}\text { Incomplete outcome data (attrition bias) } \\
\text { Functional outcomes, pain, clinical out- } \\
\text { comes, complications }\end{array}$ & High risk & $\begin{array}{l}\text { The treatment group of the participant lost } \\
\text { to follow-up was not stated. Standard devi- } \\
\text { ations not provided. Graphs only provided } \\
\text { for female participants - denominators not } \\
\text { provided for these }\end{array}$ \\
\hline $\begin{array}{l}\text { Incomplete outcome data (attrition bias) } \\
\text { Death, reoperation }\end{array}$ & Unclear risk & $\begin{array}{l}\text { Not reported. The treatment group of the } \\
\text { participant lost to follow-up was not stated }\end{array}$ \\
\hline Selective reporting (reporting bias) & Unclear risk & Insufficient information to judge this. \\
\hline Balance in baseline characteristics? & Unclear risk & Baseline data not provided for gender. \\
\hline Free from performance bias? & Unclear risk & $\begin{array}{l}\text { Uncertainty if any compensatory advice } \\
\text { given for the control group }\end{array}$ \\
\hline
\end{tabular}

Rommens 1993

\begin{tabular}{ll}
\hline Methods & $\begin{array}{l}\text { Method of randomisation: alternation } \\
\text { Assessor blinding: unlikely } \\
\text { Loss to follow up at } 3 \text { weeks: } 0 / 28\end{array}$ \\
\hline Participants & $\begin{array}{l}\text { Leuven University Hospital, Belgium } \\
\text { Period of study recruitment: } 1991 \\
28 \text { patients with acute } 2 \text { - and 3-part proximal humeral fractures (but most were non or } \\
\text { minimally displaced). } \\
\text { Exclusion criteria: those indicated for surgical intervention, age }<15 \text { years, with multiple } \\
\text { injuries or other fractures at same site } \\
22 \text { female, } 6 \text { male; mean age } 69 \text { years, range } 25 \text { to } 100 \text { years }\end{array}$ \\
\hline Interventions & $\begin{array}{l}\text { Interventions started immediately. } \\
1 . \text { Gilchrist bandage, } 2 \text { to } 3 \text { weeks. The arm was bandaged with mesh type tubing and } \\
\text { held by two slings: one round the shoulder and neck and the other which immobilised }\end{array}$ \\
\hline
\end{tabular}


the distal part of the upper arm. (Bandage allowed wrist and hand exercises.) 2. Desault bandage, 2 to 3 weeks. Arm was immobilised to the chest using a circular elastic body bandage. (Some had one or more strips of plaster to stop the bandage slipping.) Assigned: $14 / 14$

Completed (at fracture consolidation): 14/14

Outcomes

Length of follow-up: until fracture consolidation; also assessed at $1 \& 3$ weeks

Functional results: overall result, no data

Pain: patient questionnaire, 0 (none) to 100 (significant) scale

Displacement of fracture

Complication: skin irritation

Removal of bandage

Surgeon assessment of ease of application of bandage

Patient assessment of bandage

Notes

Two fractures in the Gilchrist group required reduction. Seven participants had other fractures: 3 in group 1 ( 2 rib, 1 vertebra); 4 in group 2 ( 1 ankle, 1 hip, 1 rib, 1 vertebra) Trial reports in German; translation obtained.

\section{Risk of bias}

\begin{tabular}{|c|c|c|}
\hline Bias & Authors' judgement & Support for judgement \\
\hline $\begin{array}{l}\text { Random sequence generation (selection } \\
\text { bias) }\end{array}$ & High risk & Quasi-randomised: alternation \\
\hline Allocation concealment (selection bias) & High risk & Alternation \\
\hline $\begin{array}{l}\text { Blinding (performance bias and detection } \\
\text { bias) } \\
\text { Functional outcomes, pain, clinical out- } \\
\text { comes, complications }\end{array}$ & High risk & No mention of blinding \\
\hline
\end{tabular}

Blinding (performance bias and detection Unclear risk

Not reported

bias)

Death, reoperation

Incomplete outcome data (attrition bias) Unclear risk Functional outcomes, pain, clinical outcomes, complications intention-to-treat analyses seemed to have
been done, no data on function were presented nor were the criteria for judging fracture consolidation

Incomplete outcome data (attrition bias) Unclear risk Not reported

Death, reoperation 
Rommens 1993 (Continued)

\begin{tabular}{|l|l|l}
\hline Balance in baseline characteristics? & Unclear risk & $\begin{array}{l}\text { Small discrepancies (e.g. in other injuries or } \\
\text { having fracture reduction) can have bigger } \\
\text { consequences for small group sizes }\end{array}$
\end{tabular}

Free from performance bias? Unclear risk

Differences in care programmes cannot be ruled out.

Sebastiá-Forcada 2014

Methods

Method of randomisation: sequentially numbered opaque sealed envelopes

Assessor blinding: yes, independent surgeons who did not know which type of prothesis was used

Loss to follow-up at minimum 24 months: 1/62 (1 died)

Participants

Hospital Universitario de Elda, Elda, Alicante, Spain

Period of study recruitment: 2009 to 2011

62 older patients with acute complex proximal humeral fractures (Neer's: 3-part, 4-part and 4-part + dislocation). Age $>70$ years. Candidate for shoulder arthroplasty: indications for shoulder arthroplasty were complex fractures not amenable to reconstruction, including displaced 4-part fractures, fracture-dislocations with 3-part fractures, and head-splitting fractures with more than $40 \%$ articular surface involvement. (All had computed tomography.) Informed consent.

Exclusion criteria: contraindications to surgery, prior surgery in the shoulder, associated ipsilateral upper limb fracture and neurologic disorder

53 female, 9 male; mean age 74 years, range 70 to 85 years (operations were performed within a mean of 5.1 (range 1 to 12 ) days after the injury)

Interventions

A modular shoulder replacement system (SMR; Lima, Udine, Italy) was used in both groups. The system allows the choice of cementless shoulder prostheses: hemiarthroplasty, reverse and anatomic arthroplasty). A common cementless humeral stem with porous coating titanium was assembled with one of two prostheses. The same deltopectoral approach and basic surgical technique was used at each shoulder; the tuberosities were repositioned as anatomically as possible and reattached with nonabsorbable sutures. Regional anaesthesia. In both groups, a suction drain was placed postoperatively for 24 hours. Standard antibiotic and antithrombotic prophylaxis was given

1. Reverse shoulder arthroplasty (RSA): an SMR Reverse prosthesis was used in all shoulders. Of note is that the reverse liner of polyethylene (cross-link) had a chamfer in its inferior portion designed to decrease the risk of impingement and the consequent scapular notching. The proximal humeral body was in titanium alloy with a hole to allow suture of the tuberosities. Shoulders were postoperatively immobilised in sling for 2 weeks in a regimen similar to that of the HA group. Patients then continued with physiotherapy in a rehabilitation centre for at least 4 weeks to perform deltoid activation exercises and activities as tolerated

2. Hemiarthroplasty: an SMR Trauma prosthesis was implanted. The proximal humeral body had holes to allow suture of the tuberosities to the stem, and the modular head was in titanium alloy. Rotator cuff tears repaired if possible. Sling immobilisation after surgery, gradually discontinued around 3 weeks. Passive mobilisation and pendulum exercises were allowed immediately. At week 2, passive- and active-assisted exercises were 
Sebastiá-Forcada 2014 (Continued)

allowed in a rehabilitation centre with forward elevation and abduction limited to $100^{\circ}$ and external rotation limited to $30^{\circ}$. When consolidation of tuberosities was observed on the radiographs (around 6 weeks), active and resisted exercises were started Assigned: $31 / 31$

Completed (at 2 years): $31 / 30$

Outcomes

Length of follow-up: mean 28.5 months (range: 24 to 49 months); also followed-up but no data for 6 weeks, 3, 6 and 12 months (and then yearly)

QuickDASH

University of California-Los Angeles (UCLA) score

Constant score (absolute and adjusted for age and gender)

Mortality

Complications (intra-operative fracture, infection, haematoma, neurological, severe stiffness, proximal migration of implant)

Re-operations

Range of motion (anterior forward; abduction)

Tuberosity healing, malunion, non-union resorption

Strength (not reported)

Radiographs: acromiohumeral distance; scapular notching, loosening, heterotopic ossi-

fication, proximal migration, radiolucent lines

Notes

Risk of bias

\begin{tabular}{ll|l}
\hline Bias & Authors' judgement & Support for judgement \\
\hline $\begin{array}{l}\text { Random sequence generation (selection } \\
\text { bias) }\end{array}$ & Low risk & $\begin{array}{l}\text { Method not stated, although seems likely } \\
\text { that an appropriate method was used }\end{array}$ \\
\hline Allocation concealment (selection bias) & Low risk & $\begin{array}{l}\text { "Randomization to the HA or RSA } \\
\text { group was based on sequentially num- } \\
\text { bered opaque sealed envelopes. The sur- } \\
\text { geons were not involved in the randomiza- } \\
\text { tion process." }\end{array}$ \\
\hline
\end{tabular}

Blinding (performance bias and detection Unclear risk bias)

Functional outcomes, pain, clinical outcomes, complications
"All postoperative functional evaluation forms were completed at each visit by an independent experienced surgeon (A.L.U. ) who had not participated in the surgeries and did not know which type of prosthesis had been used"

However, there was no blinding of care providers or participants

Surgeons were experienced.

"clinical and radiologic evaluations were performed by independent observers who had not participated in the surgeries" 


\begin{tabular}{|c|c|c|}
\hline $\begin{array}{l}\text { Incomplete outcome data (attrition bias) } \\
\text { Functional outcomes, pain, clinical out- } \\
\text { comes, complications }\end{array}$ & Low risk & $\begin{array}{l}\text { One loss to follow-up (death) only. Interim } \\
\text { follow-up data not reported }\end{array}$ \\
\hline $\begin{array}{l}\text { Incomplete outcome data (attrition bias) } \\
\text { Death, reoperation }\end{array}$ & Low risk & One loss to follow-up (death) only. \\
\hline Selective reporting (reporting bias) & Unclear risk & $\begin{array}{l}\text { No trial registration or published protocol. } \\
\text { Marginal but some arbitrary definitions of } \\
\text { outcomes. No data on interim follow-ups }\end{array}$ \\
\hline Balance in baseline characteristics? & Unclear risk & $\begin{array}{l}\text { Baseline characteristics were balanced in } \\
\text { the two groups. (Characteristics of } 1 \text { partic- } \\
\text { ipant not provided.) Difference in cuff tears } \\
\text { between groups accounted for and tested }\end{array}$ \\
\hline Free from performance bias? & Low risk & $\begin{array}{l}\text { All operations were performed by } 2 \text { sur- } \\
\text { geons experienced in shoulder surgery; the } \\
\text { modular shoulder replacement system was } \\
\text { already in use at centre before the study. } \\
\text { Same approach and operating methods and } \\
\text { conditions; regional anaesthesia etc } \\
\text { Comparable rehabilitation - differences ap- } \\
\text { propriate for different procedures }\end{array}$ \\
\hline
\end{tabular}

\section{Smejkal 2011}

Methods

Participants
Method of randomisation: computer-generated block randomisation with sealed envelopes

Assessor blinding: no mention in the paper

Loss to follow-up: 4 lost to follow-up and 2 died of breast cancer during the study period

University Hospital in Hradec Králové, Czech Republic

Period of study recruitment: January 2006 to January 2010

61 patients with $\mathrm{AO}$ type A2, A3, B1 and C1 (2-part and 3-part) proximal humerus fractures aged between 18 and 80 years able to give informed consent

Exclusion criteria: open fracture, associated injury (AIS $>2$ ), open growth plates, or patient's health would limit the extent of surgery

Of 55: 45 females, 10 males; mean age 61 years, range 21 to 81 years

Interventions

Interventions started 0 to 24 days after injury.

1. Open reduction and internal fixation group: consisted of patients undergoing open reduction with angle-stable osteosynthesis using a PHILOS plate (Synthes, Switzerland) 2. Minimally invasive group: Zifko method of minimally invasive osteosynthesis with intramedullary K-wire ((Kirschner wire) insertion (distally inserted) - figure in article shows 8 wires inserted into humeral head along medullary canal

Assigned: number in each group not known (total 61) 
Smejkal 2011 (Continued)

Completed: $28 / 27$

\begin{tabular}{ll}
\hline Outcomes & Length of follow-up: mean 2 years \\
& Days to operation \\
& Constant-Murley score (relative to healthy limb) \\
& Time to recover normal upper limb function \\
& Complications \\
& Time to radiographically assessed recovery \\
& Anatomical position \\
& X-ray exposure \\
& Length of operation \\
& Length of hospital stay \\
\hline Notes & \\
\hline
\end{tabular}

Risk of bias

\begin{tabular}{|c|c|c|}
\hline Bias & Authors' judgement & Support for judgement \\
\hline $\begin{array}{l}\text { Random sequence generation (selection } \\
\text { bias) }\end{array}$ & Low risk & $\begin{array}{l}\text { "The patients were randomised to the } \\
\text { groups by a computer programme which } \\
\text { facilitates the maintenance of homogeneity } \\
\text { of the groups compared." } \\
\text { Web-based translation implied use of ran- } \\
\text { dom numbers and permuted blocks so as } \\
\text { to get similar numbers on each group. Pro- } \\
\text { duced independently by a statistical com- } \\
\text { pany }\end{array}$ \\
\hline Allocation concealment (selection bias) & Low risk & $\begin{array}{l}\text { The sealed envelopes were created by a pro- } \\
\text { fessional statistical company (Pharm test s. } \\
\text { r. o., Hradec Králové): in accordance with } \\
\text { randomization sheet each envelope their } \\
\text { number and sealed inside information," } \\
\text { zifko" or "LCP." The sealed envelopes were } \\
\text { opened sequentially }\end{array}$ \\
\hline
\end{tabular}

Blinding (performance bias and detection High risk

Not possible to blind patient/providers. No bias)

Functional outcomes, pain, clinical out-

mention of outcome assessment

comes, complications

Blinding (performance bias and detection Unclear risk bias)

Death, reoperation

May not affect assessment

Incomplete outcome data (attrition bias) High risk Functional outcomes, pain, clinical outcomes, complications

Incomplete data (and group of 6 excluded participants not noted) 


\section{Smejkal 2011 (Continued)}

\begin{tabular}{l|ll}
\hline $\begin{array}{l}\text { Incomplete outcome data (attrition bias) } \\
\text { Death, reoperation }\end{array}$ & High risk & $\begin{array}{l}\text { Incomplete data (and group of 2 deaths not } \\
\text { stated) }\end{array}$ \\
\hline Selective reporting (reporting bias) & Unclear risk & No protocol \\
\hline Balance in baseline characteristics? & Unclear risk & $\begin{array}{l}\text { Aside from age - no details or confirmation } \\
\text { of this }\end{array}$ \\
\hline Free from performance bias? & Unclear risk & $\begin{array}{l}\text { No details - including of surgeon's experi- } \\
\text { ence }\end{array}$ \\
\hline
\end{tabular}

Soliman 2013

Methods

Participants

Interventions
Method of randomisation: use of computer-generated random numbers table

Assessor blinding: yes, blinded observer for Constant score, pain and range of motion Loss to follow-up: 8 post-randomisation exclusions

\section{Cairo University Hospital, Cairo, Egypt}

Period of study recruitment: 2005 to 2009

45 patients treated with hemiarthroplasty for 4-part fractures of the proximal humerus, fracture dislocations or head splitting fractures presenting within the first five days after injury. Informed consent

Exclusion criteria: not available. Exclusion criteria applied to post-randomisation exclusions of participants with complications

13 females, 32 males; mean age 52 years (of 37 participants), range 45 to 60 years

(Note: This was a young population with very severe injuries. Predominantly males and presumably high-energy trauma. The biceps tendon is stronger in younger patients.)

Interventions started within 5 days after injury. Same prosthesis (Johnson and Johnson) and surgical technique used in both groups. "A standard deltopectoral approach was used and the coracoacromial ligament was preserved in all patients." The operative technique is described at length in the article.

1. Hemiarthroplasty and tenodesis of long head of the biceps (LHB): LHB tendon was divided at its insertion and tenodesed by Ethibond sutures into the insertion of the pectoralis major

2. Hemiarthroplasty: LHB tendon left intact.

Post-surgery, the arm was immobilised in a position of neutral rotation for 4 weeks. This was followed by the same physiotherapy protocol for all participants

Assigned: 23/22

Completed (2 years): 19/18

Outcomes

Length of follow-up: mean 2 years (range 21 to 27 months)

Constant score ("modified"; not clear how)

Pain (VAS, then categorised to none, mild, moderate, severe)

Re-operation

Complications (these were excluded - see Notes)

Anterior shoulder elevation 
Soliman 2013 (Continued)

Post-randomisation exclusions: "Eight patients were excluded from the study within
the first 3 months of follow-up due to tuberosity malposition (three patients), inferior
subluxation of the prosthesis (two patients), loss of reduction of the greater tuberosity
(two patients) and deep infection, which slowed down the physiotherapy protocol and
required surgical debridement (one patient)." (page 262 in report)
Contact with the lead author resulted in no clarification of the method of randomisation
("we enrolled the patients in a random number"), but information on the manufacturer
of the implant, a breakdown of the numbers of participants with specific complications
in each group and clarification that there were no re-operations aside from debridement
for a deep infection

\section{Risk of bias}

\begin{tabular}{l|l|l}
\hline Bias & Authors' judgement & Support for judgement \\
\hline $\begin{array}{l}\text { Random sequence generation (selection } \\
\text { bias) }\end{array}$ & Low risk & $\begin{array}{l}\text { "Patients were randomly assigned to either } \\
\text { hemiarthroplasty or hemiarthroplasty and } \\
\text { tenodesis of the LHB, according to a com- } \\
\text { puter-generated random number sequence. }\end{array}$ \\
\hline
\end{tabular}

Allocation concealment (selection bias) Unclear risk

"After enrolment, cases were sequentially arranged and plotted on the random number table to determine to which group they will be assigned"

No mention of safeguards

Blinding (performance bias and detection Low risk bias)

Functional outcomes, pain, clinical outcomes, complications
All patients were evaluated by a blinded observer using the Constant score

Blinding (performance bias and detection Unclear risk bias)

Death, reoperation

Incomplete outcome data (attrition bias) High risk Functional outcomes, pain, clinical outcomes, complications
Lack of blinding unlikely to affect reporting

Inappropriate exclusion of eight participants. Although the number of post-randomisation exclusions was four in each group, the loss to follow-up was $17 \%$ ( 4 / 23 in the tenodesis group) and $18 \%(4 / 22$ in the intact LHB tendon group) and all eight participants were more likely to have had poor results

Data from author clarified that only the participant with deep infection had subsequent treatment for a complication 
Soliman 2013 (Continued)

\begin{tabular}{|c|c|c|}
\hline Selective reporting (reporting bias) & High risk & $\begin{array}{l}\text { Retrospective trial registration. Inadequate } \\
\text { description of outcomes, including the cat- } \\
\text { egorisation of pain. Strength was measured } \\
\text { but not reported. Pain categories and mea- } \\
\text { surement not defined sufficiently }\end{array}$ \\
\hline Balance in baseline characteristics? & Unclear risk & $\begin{array}{l}\text { No separate data aside from age. While bal- } \\
\text { anced for age, these data apply to } 37 \text { of } 45 \\
\text { participants. No details on fracture severity } \\
\text { and cuff integrity, both of which could af- } \\
\text { fect result }\end{array}$ \\
\hline Free from performance bias? & Low risk & $\begin{array}{l}\text { Same surgeon operated with same prosthe- } \\
\text { sis. Same post-surgical care and rehabilita- } \\
\text { tion }\end{array}$ \\
\hline
\end{tabular}

Stableforth 1984

Methods

Method of randomisation: unknown, "randomly selected"

Assessor blinding: unlikely

Loss to follow-up at 18 months to 12 years: $2 / 32$ (2 deaths)

Participants

Bristol Royal Infirmary, Bristol, UK

Period of study recruitment: 1970 to 1981

32 patients with displaced 4-part proximal humeral fractures (Neer).

Exclusion criteria: impacted or minimally displaced fractures

25 female, 7 male; mean age 68 years, range 52 to 88 years

Interventions Interventions started: within 5 days for surgery.

1. Neer prosthesis, uncemented

2. Non-surgical treatment: closed manipulation

All were placed in sling, mobilisation of hand encouraged, shoulder flexion rotation exercises after 2 to 3 days. Supervised physiotherapy for 3 to 6 months.

Assigned: $16 / 16$

Completed (at 1 year): $15 / 15$ (but totals given as $16 / 16$ in tables in the trial report)

Outcomes

Length of follow-up: stated as 18 months to 12 years; but also assessed regularly up to 6 months

Dependent in activities of daily living

Range of motion (flexion, medial rotation, lateral rotation)

Pain

Muscle strength (flexion, abduction, lateral rotation)

Complications: haematoma, cellulitis, deep sepsis, early shoulder stiffness

Mortality

Notes

Risk of bias

Interventions for treating proximal humeral fractures in adults (Review)

Copyright $\Subset 2015$ The Cochrane Collaboration. Published by John Wiley \& Sons, Ltd. 
Stableforth 1984 (Continued)

\begin{tabular}{|c|c|c|}
\hline Bias & Authors' judgement & Support for judgement \\
\hline $\begin{array}{l}\text { Random sequence generation (selection } \\
\text { bias) }\end{array}$ & Unclear risk & $\begin{array}{l}\text { No details: "assigned by pre-arranged ran- } \\
\text { dom selection" }\end{array}$ \\
\hline Allocation concealment (selection bias) & Unclear risk & $\begin{array}{l}\text { No details: "assigned by pre-arranged ran- } \\
\text { dom selection" }\end{array}$ \\
\hline $\begin{array}{l}\text { Blinding (performance bias and detection } \\
\text { bias) } \\
\text { Functional outcomes, pain, clinical out- } \\
\text { comes, complications }\end{array}$ & High risk & Not blinded \\
\hline $\begin{array}{l}\text { Blinding (performance bias and detection } \\
\text { bias) } \\
\text { Death, reoperation }\end{array}$ & Unclear risk & $\begin{array}{l}\text { No blinding but may not have affected ap- } \\
\text { praisal of mortality }\end{array}$ \\
\hline $\begin{array}{l}\text { Incomplete outcome data (attrition bias) } \\
\text { Functional outcomes, pain, clinical out- } \\
\text { comes, complications }\end{array}$ & High risk & $\begin{array}{l}\text { Large loss to follow-up }(46 / 85=54 \%) \text {. } \\
\text { Numbers given for those available at fol- } \\
\text { low-up but incompletely reported data: } \\
\text { only medians }\end{array}$ \\
\hline $\begin{array}{l}\text { Incomplete outcome data (attrition bias) } \\
\text { Death, reoperation }\end{array}$ & Unclear risk & $\begin{array}{l}\text { Slight discrepancy in trial report that } 2 \\
\text { deaths are reported, one in each group, but } \\
\text { long term denominators are as at baseline }\end{array}$ \\
\hline Selective reporting (reporting bias) & Unclear risk & $\begin{array}{l}\text { Insufficient information to judge this, but } \\
\text { the protracted nature of this trial makes se- } \\
\text { lective reporting more likely }\end{array}$ \\
\hline Balance in baseline characteristics? & Unclear risk & $\begin{array}{l}\text { Surgical group on average } 4.5 \text { years } \\
\text { younger, but uncertainties mainly reflect } \\
\text { Inadequate information in terms of other } \\
\text { co-morbidities and injuries for this broad } \\
\text { category of patients }\end{array}$ \\
\hline Free from performance bias? & Unclear risk & $\begin{array}{l}\text { Inadequate information on care pro- } \\
\text { gramme comparability especially given the } \\
\text { protracted nature of the trial recruitment. } \\
\text { However, one surgeon operated through- } \\
\text { out }\end{array}$ \\
\hline
\end{tabular}

Interventions for treating proximal humeral fractures in adults (Review)

Copyright () 2015 The Cochrane Collaboration. Published by John Wiley \& Sons, Ltd. 
Methods

Participants

Castelldefels, Barcelona, Spain

Period of study recruitment: not known

42 patients with displaced or non-displaced proximal humeral fractures that were not considered for surgery or patient refused surgery. (Included: 8 non-displaced fractures, 11 2-part and 23 3-part fractures.) Written informed consent

Exclusion criteria: incapacity to understand or complete the tests or sign informed consent form; no contact of humeral head and humeral shaft, fracture dislocation, posterior displacement of the greater tuberosity $\geq 1.5 \mathrm{~cm}$

32 female, 10 male; mean age 70 years, range 60 to 80 years

Interventions

Interventions started: probably very soon after patients attended with their fracture

1. Functional one week immobilisation regimen using arm sling in internal rotation

2. Conventional four weeks immobilisation regimen, using arm sling in internal rotation

Both groups followed the same progressive rehabilitation programme

Assigned: 20/22

Completed (at 1 year): 19/20

Outcomes

Length of follow-up: 1 year (also 1 week, and 3 and 6 months)

Pain (VAS: 0 to 10: higher scores = worse pain)

Constant shoulder functional score

Satisfaction score (VAS: 0 to 10: higher scores = greater satisfaction)

Euroqol-5D

Mortality

Secondary surgery and complications

Further 'significant' displacement

Notes

Conference abstract (2012) presented data for 42 patients (mean age 70 years), 32 of whom had displaced fractures. A query on publication status was sent 23 May 2015, with response from Carlos Torrens received 25 May 2015: "Unfortunately this study was stopped because of lack of money so we just could recruit 40 patients." This included notification of an ongoing trial (Torrens) testing the same comparison.

A data collection form sent to Carlos Torrens for this trial on June 52015 was returned completed by him on June 102015

\section{Risk of bias}

Bias Authors' judgement

Random sequence generation (selection Low risk bias)

\section{Support for judgement}

"Randomisation was done by the Statistics that gave us a computer generated random numbers list.” (email June 10 2015)

No mention of safeguards. 
Torrens 2012 (Continued)

\author{
Blinding (performance bias and detection High risk \\ No mention of blinding. \\ bias) \\ Functional outcomes, pain, clinical out- \\ comes, complications
}

Blinding (performance bias and detection Unclear risk

bias)

Death, reoperation

Not blinded but less likely that these outcomes would be affected

Incomplete outcome data (attrition bias) Low risk

One lost to follow-up in each group.

Functional outcomes, pain, clinical out-

comes, complications

Incomplete outcome data (attrition bias) Low risk

Data provided by trialist. One lost to fol-

Death, reoperation

low-up in each group

\begin{tabular}{ll} 
Selective reporting (reporting bias) & Unclear risk \\
\hline Balance in baseline characteristics? & Unclear risk
\end{tabular}

No protocol or trial registration

were fewer 'non-displaced' fractures in the 1 week immobilisation group ( 1 versus 7). However, this was not statistically significant and the changes in the reported fracture distribution between abstract (4 2-part; 26 3-part; 10 non-displaced) and unpublished data (11 2-part; 23 3-part; 8 non-displaced) may indicate some intra- or inter-rater discrepancies in applying (if applied) the Neer classification system. Abstract reported "no differences as far as age, gender and displacement between conventional and functional groups". Insufficient information to confirm this

Free from performance bias?

Unclear risk

There is insufficient information to confirm this. However, it seems likely because both groups followed the same rehabilitation regimen

Voigt 2011

\begin{tabular}{ll} 
Methods & $\begin{array}{l}\text { Method of randomisation: drawing balls from a bag by an independent person } \\
\text { Assessor blinding: assessor blinding } \\
\text { Loss to follow-up at } 12 \text { months: } 8 / 56 \text { (did not complete follow-up: } 2 \text { deaths, } 4 \text { drop- } \\
\text { outs, } 2 \text { excluded because of early secondary arthroplasty) }\end{array}$ \\
\hline Participants & $\begin{array}{l}\text { Friederikenstift Hospital Hannover, Hannover, Germany } \\
\text { Period of study recruitment: conducted over } 18 \text { month period (no dates) } \\
56 \text { patients with isolated Neer type 3- and 4-part proximal humeral fractures, aged }>60\end{array}$
\end{tabular}

Interventions for treating proximal humeral fractures in adults (Review)

Copyright () 2015 The Cochrane Collaboration. Published by John Wiley \& Sons, Ltd. 
Voigt 2011 (Continued)

years

Exclusion criteria: fractures older than 2 weeks, open fractures, pathological fractures, refractures, neurologic disease and patients who would be clearly non-compliant (e.g. alcoholics, patients of no fixed address)

Of 48: 38 female, 10 male; Of 56: mean age 74 years, range 60 to 87 years

Interventions

Interventions started: at surgical fixation (time to surgery from injury not given)

1) Polyaxial locked screws: Humeral Suture Plate (HSP) (Arthrex, Naples, FL) with polyaxially locked screws. Screws were blunt-ended (considered better in the prevention of glenoid erosions in case of screw perforations)

2) Non-polyaxial (monoaxial) implant: Proximal Humerus Internal Locking System (PHILOS) (Synthes, Bettlach, Switzerland) with nonpolyaxially locked screws. (Screws were pointed in the PHILOS plate.)

All surgery performed under general anaesthesia using deltopectoral approach. Tuberosity fragments reduced with fibre wire, different approaches for head fragment depending on whether valgus or varus. Allocated plate positioned anatomically and fixed with a shaft screw

Patients' shoulders were immobilised in a sling for 2 days. Then, active-assisted motion beyond 90 degrees flexion and abduction were initiated avoiding the provocation of pain. At 7 weeks, free range of motion was allowed

Assigned: 25/31

Completed: 20/28 (at 12 months)

Outcomes

Length of follow-up: 12 months

Simple shoulder test

Disability of the Arm, Shoulder and Hand (DASH) score

Constant score (relative to contralateral limb)

Death

Complications

Re-operation

Range of active shoulder motion (flexion, abduction, internal rotation, external rotation)

Fracture healing - AP and axillary radiographs

Duration of operation

Fluoroscopy time

Notes

Additional information and clarification of 8 participants who did not complete followup and gender data for those who completed follow-up obtained from Dr Voigt (May 2012)

Risk of bias

Bias

Authors' judgement

Support for judgement

Random sequence generation (selection Low risk bias)

"The randomization technique was blinded by drawing balls from a bag: one ball for HSP and the other ball for PHILOS by an independent person." 
Voigt 2011 (Continued)

\begin{tabular}{|c|c|c|}
\hline Allocation concealment (selection bias) & Low risk & $\begin{array}{l}\text { "The random- } \\
\text { ization technique was blinded by drawing } \\
\text { balls from a bag: one ball for HSP and the } \\
\text { other ball for PHILOS by an independent } \\
\text { person." }\end{array}$ \\
\hline
\end{tabular}

Blinding (performance bias and detection High risk bias)

Functional outcomes, pain, clinical outcomes, complications

No mention of blinding of patients or personnel other than assessor blinding: "Follow-up evaluations postoperatively were performed in a standardized fashion by an independent trauma surgeon"

Blinding (performance bias and detection Unclear risk

Unlikely to influence this.

bias)

Death, reoperation

Incomplete outcome data (attrition bias) Unclear risk Functional outcomes, pain, clinical outcomes, complications

Although clarification on loss to follow-up (8 patients: 5 versus 3 ) received from author, the impact on the results for functional outcomes is unclear

Incomplete outcome data (attrition bias) Low risk

Death, reoperation

Clarification received from author on the loss to follow-up: 2 were deaths and 2 were replacement arthroplasty

\begin{tabular}{|c|c|c|}
\hline Selective reporting (reporting bias) & Unclear risk & No protocol provided \\
\hline Balance in baseline characteristics? & Unclear risk & $\begin{array}{l}\text { Balance in 3- versus 4-part fractures, prob- } \\
\text { ably age and pre-operative DASH. Incom- } \\
\text { plete data on gender, } 2 \text { versus } 6 \text { with dia- } \\
\text { betes (but no frozen shoulder) }\end{array}$ \\
\hline Free from performance bias? & Unclear risk & No details of surgeon experience. \\
\hline
\end{tabular}

\section{Wirbel 1999}

Methods

Participants
Method of randomisation: unknown, "random allocation"

Assessor blinding: unlikely

Loss to follow-up at 6 months: 13/77; also 14 months (9 to 36 months): 18/77

University Hospital, Homburg/Saar, Germany

Period of study recruitment: January 1995 to March 1998

77 patients with displaced (separation exceeds $1 \mathrm{~cm}$; fragment angulation $>30$ degrees, or when tuberosity fragment is separated by $>3 \mathrm{~mm}$ ) subcapital humeral fractures of type A1, A3, B and $\mathrm{C} 1$ (modified AO classification) treated by closed reduction and percutaneous fixation.

Exclusion criteria: Extensive local skin infection. Impacted fractures of type A2 (treated non-surgically). Not fit enough to undergo anaesthesia and X-ray of affected shoulder 
Wirbel 1999 (Continued)

in anterior-posterior plane. Closed reduction not feasible.

54 female, 23 male; mean age 63 years, range 6 to 89 years

Interventions

Interventions started post-operatively after percutaneous fixation (Kirschner wires plus in 38 cases, cannulated screws).

1. 1 week immobilisation in Gilchrist sling

2. 3 weeks immobilisation in Gilchrist sling

Active mobilisation of elbow from first post-operative day. Active and passive physiotherapy of the shoulder (optional continuous passive motion) after removal of sling. Removal of Kirschner wires after 4 to 6 weeks, with post-procedure continuation of active exercises.

Assigned: 38/39

Completed (at 6 months): $32 / 32$

Outcomes

Length of follow-up: 9 to 36 (mean 14 months) months (in 59 participants), but also assessed at 1, 3 and 6 months

Neer score

Complications: avascular necrosis, local infection/haematoma, premature removal of Kirschner wires, screw removal due to subacromial impingement

Notes

Short report (1997) from conference proceedings gave interim results for 51 patients. Full report and some results provided by Dr Wirbel (February 2003).

Most of the results given in the trial report were either for the whole study population or split by basic $\mathrm{AO}$ fracture type

Risk of bias

\begin{tabular}{l|l|l}
\hline Bias & Authors' judgement & Support for judgement \\
\hline $\begin{array}{l}\text { Random sequence generation (selection } \\
\text { bias) }\end{array}$ & Unclear risk & $\begin{array}{l}\text { No details: "a random allocation of patients } \\
\text { in 2 groups was done" }\end{array}$ \\
\hline $\begin{array}{l}\text { Allocation concealment (selection bias) } \\
\text { Blinding (performance bias and detection } \\
\text { bus) } \\
\text { Functional outcomes, pain, clinical out- } \\
\text { comes, complications }\end{array}$ & High risk & $\begin{array}{l}\text { No details: "a random allocation of patients } \\
\text { in 2 groups was done" }\end{array}$ \\
\hline
\end{tabular}

Blinding (performance bias and detection Unclear risk bias)

Not blinded but less likely that these out-

Death, reoperation comes would be affected

Incomplete outcome data (attrition bias) High risk

Functional outcomes, pain, clinical out-

comes, complications
Limited data on function using a non-validated assessment instrument with a moderate loss to follow-up at 6 months $(13 / 77$ $=17 \%)$ 
Wirbel 1999 (Continued)

\begin{tabular}{l|l|l}
\hline $\begin{array}{l}\text { Incomplete outcome data (attrition bias) } \\
\text { Death, reoperation }\end{array}$ & Unclear risk & $\begin{array}{l}\text { Incomplete data. Although loss to follow- } \\
\text { up reported, reoperations were not suffi- } \\
\text { ciently reported by treatment group }\end{array}$ \\
\hline Selective reporting (reporting bias) & Unclear risk & Insufficient information to judge this. \\
\hline Balance in baseline characteristics? & Low risk & No indication of any major baseline imbal- \\
\hline Free from performance bias? & Low risk & $\begin{array}{l}\text { No indication of performance bias from } \\
\text { differences in care programmes }\end{array}$ \\
\hline
\end{tabular}

\section{Zhang 2011}

Methods

Method of randomisation: computer generated random numbers

Assessor blinding: likely, "independent" assessor at follow-up

Loss to follow-up: 4 patients within the first year after surgery due to moving out of the area and change of telephone number

Participants

The Third Affiliated Hospital of Whenzou, Whenzou, China

Period of study recruitment: October 2007 to September 2008

72 patients aged over 18 years with an acute closed 2-, 3- or 4-part fracture (Neer classification) of the proximal humerus treated with open reduction and internal fixation using a locking plate

Exclusion criteria: pathological fractures, primary or metastatic tumour and fracture with non-union

Of 68 followed-up: 46 female, 22 male; mean age 63 years, range 32 to 78 years

Interventions

Interventions started: both at surgery, time from injury not stated

1. ORIF with PHILOS locking plates (Synthes, Switzerland). Standard deltopectoral approach; reduction enabled with a K-wire under fluoroscopy. Locking plate was placed $10 \mathrm{~mm}$ posterior to the intertubercular groove and $10 \mathrm{~mm}$ distal to the tip of greater tubercle. A cortical screw was inserted initially to fix the distal fragment. Four or five locking screws were used for the fixation of the proximal fragment. All proximal screws were inserted $5 \mathrm{~mm}$ below subchondral bone. One or two additional locking screws were inserted obliquely into the medio-inferior region of the humeral head in this group The tubercular fragments and rotator cuff tendon were fixed using Ethibond sutures. Autograft bone was used in comminuted fractures where there was a mass defect and for reconstruction of the medial support structures. Fracture reduction and screw length were finally assessed with fluoroscopy

2. As above without medial support locking screws.

All patients received prophylactic intravenous antibiotics before the procedure. Passive abduction and clock-wise rotation exercises were allowed on the day after surgery. Active rehabilitation was started six weeks postoperatively

Assigned: 32/40 (total: 72)

Completed (2+ years): 29/39 


\section{Zhang 2011 (Continued)}

\begin{tabular}{ll} 
Outcomes & $\begin{array}{l}\text { Length of follow-up: average } 30.8 \text { months (also } 4,8,12 \text { weeks, } 6,9 \text { and } 12 \text { months and } \\
\text { yearly) } \\
\text { Shoulder function (Constant shoulder score) } \\
\text { Union } \\
\text { Complications: osteonecrosis of the humeral head, early failure and loss of fixation } \\
\text { Re-operation }\end{array}$ \\
\hline Notes & $\begin{array}{l}\text { Personal contact (email } 14 / 05 / 2012) \text { clarified method of randomisation, group of pa- } \\
\text { tients who were lost to follow-up; and complications }\end{array}$ \\
\hline
\end{tabular}

Risk of bias

\begin{tabular}{|c|c|c|}
\hline Bias & Authors' judgement & Support for judgement \\
\hline $\begin{array}{l}\text { Random sequence generation (selection } \\
\text { bias) }\end{array}$ & Low risk & $\begin{array}{l}\text { "The patients were randomized into two } \\
\text { groups for study according to computer- } \\
\text { generated random numbers" (Group sizes, } \\
\text { however, were unequal.) }\end{array}$ \\
\hline Allocation concealment (selection bias) & Unclear risk & $\begin{array}{l}\text { There were insufficient safeguards (selec- } \\
\text { tion according to odd and even random } \\
\text { numbers) to confirm allocation conceal- } \\
\text { ment }\end{array}$ \\
\hline $\begin{array}{l}\text { Blinding (performance bias and detection } \\
\text { bias) } \\
\text { Functional outcomes, pain, clinical out- } \\
\text { comes, complications }\end{array}$ & Unclear risk & $\begin{array}{l}\text { It is possible that the participants did not } \\
\text { know which group they were in. The dif- } \\
\text { ference between the two interventions was } \\
\text { not large. } \\
\text { "Complications, shoulder function and ra- } \\
\text { diological measurement were recorded by } \\
\text { an independent junior doctor (YJH) who } \\
\text { did not participate in the surgery." }\end{array}$ \\
\hline
\end{tabular}

Blinding (performance bias and detection Low risk bias)

Death, reoperation

Incomplete outcome data (attrition bias) Unclear risk Functional outcomes, pain, clinical outcomes, complications

Incomplete outcome data (attrition bias) Unclear risk Death, reoperation

Selective reporting (reporting bias)
Unclear risk
These outcomes are fairly robust regarding blinding.

Active surveillance but missing data for 4 participants lost to follow-up. Personal correspondence gave details on complications

Missing data for 4 participants. Personal correspondence provided information on re-operations

Insufficient data to judge this. No protocol available. 
Zhang 2011 (Continued)

\begin{tabular}{|c|c|c|}
\hline Balance in baseline characteristics? & Unclear risk & $\begin{array}{l}\text { Although the baseline characteristics of } 68 \\
\text { participants were comparable, data were } \\
\text { missing for } 4 \text { participants loss to follow-up }\end{array}$ \\
\hline Free from performance bias? & Low risk & $\begin{array}{l}\text { "Operations were performed by two senior } \\
\text { surgeons." All participants received same } \\
\text { rehabilitation }\end{array}$ \\
\hline
\end{tabular}

\section{Zhu 2011}

Methods

Participants
Method of randomisation: computer-generated random numbers list reviewed by nurse before surgery

Assessor blinding: no, but mention of independent observer

Loss to follow-up at 3 years: $6 / 57$ (5 lost; 1 died)

Beijing Ji Shui Tan Hospital, Beijing, China

Period of study recruitment: November 2004 to December 2006

57 skeletally mature patients with an acute 2-part surgical neck fracture of the proximal humerus (Neer's classification) treated surgically within 21 days of the injury. Patient consent.

Exclusion criteria: open physes, fracture and displacement involving the greater or lesser tuberosity or extension of the fracture line distally beyond the deltoid tubercle, associated musculoskeletal injuries to the same upper extremity, open fracture, and prior surgery on the affected shoulder.

Of 51 followed up: 34 female, 17 male; mean age 53 years

Interventions

Interventions started: surgery on average 9 days after injury (randomisation before surgery)

1. Open reduction with internal fixation using a locking plate: Locking Proximal Humeral Plate (LPHP; Synthes) or the Proximal Humeral Internal Locking System (PHILOS; Synthes). General anaesthesia combined with an interscalene block. Indirect reduction under image intensifier, with reduced fracture temporarily fixed by a Kirschner wire. After placement, position of the locking plate checked with the image intensifier intraoperatively, and the plate was fixed with locking screws. Finally, a thorough fluoroscopic screening was done to ensure that no screw was penetrating the articular surface of the humeral head

2. Open reduction with internal fixation using a locking nail: the Proximal Humeral Nail (PHN; Synthes). An interscalene brachial plexus block was used. Nail was inserted under image control without reaming after the fracture was fully reduced. After insertion of the spiral blade and the distal locking screws, an end cap was screwed in to lock the spiral blade. The rotator cuff tendon and the deltoid were carefully repaired during wound closure

The affected extremity was protected by a sling for six weeks postoperatively. Passive range-of-motion exercises, supervised by a physical therapist, were initiated on the first postoperative day. Active and active-assisted exercises began after six weeks, when early callus formation could be seen on radiographs. Strengthening exercises were started three months after the surgery.

Assigned: 29/28 
Zhu 2011 (Continued)

Completed (at 3 years): 26/25

\begin{tabular}{ll} 
Outcomes & Length of follow up: 3 years (also 1 year) \\
& ASES (American Shoulder and Elbow Surgeons) score \\
& Constant shoulder score (both shoulders) \\
& Pain (VAS) \\
& Mortality \\
& Complications (overall, infection (none), heterotopic ossification, screw penetration, \\
& pseudothorax) \\
& Re-operation \\
& Range of motion (active flexion, external rotation, internal rotation) \\
& Strength \\
& Duration of surgery \\
& Blood loss and transfusion \\
Radiographic outcomes including avascular necrosis, union, and degenerative change \\
(osteoarthritis) \\
\hline \multirow{2}{*}{ Notes } & \\
\hline & \\
\hline
\end{tabular}

Risk of bias

\begin{tabular}{|c|c|c|}
\hline Bias & Authors' judgement & Support for judgement \\
\hline $\begin{array}{l}\text { Random sequence generation (selection } \\
\text { bias) }\end{array}$ & Low risk & $\begin{array}{l}\text { "Randomization was accomplished with } \\
\text { use of a random numbers list generated by } \\
\text { software and kept by the operating room } \\
\text { nurse." }\end{array}$ \\
\hline Allocation concealment (selection bias) & Unclear risk & $\begin{array}{l}\text { "Before the surgery, the circulating nurse } \\
\text { reviewed the random-numbers list. Patients } \\
\text { who had been assigned an odd number } \\
\text { were subsequently treated with a locking } \\
\text { nail, and those who had been assigned an } \\
\text { even number were managed with a locking } \\
\text { plate." }\end{array}$ \\
\hline
\end{tabular}

Blinding (performance bias and detection High risk bias)

Functional outcomes, pain, clinical outcomes, complications
No mention of blinding. However:

"All of the follow-up physical examinations and radiographic evaluations were done by the same independent observer."

No mention of blinding. Lack of blinding less likely to affect these outcomes bias)

Death, reoperation

Incomplete outcome data (attrition bias) Low risk

Functional outcomes, pain, clinical out-

comes, complications
Participant flow diagram provided; similar and modest losses in each group 


\section{Zhu 2011 (Continued)}

\begin{tabular}{|c|c|c|}
\hline $\begin{array}{l}\text { Incomplete outcome data (attrition bias) } \\
\text { Death, reoperation }\end{array}$ & Low risk & $\begin{array}{l}\text { Participant flow diagram provided; similar } \\
\text { and modest losses in each group: data re- } \\
\text { ported }\end{array}$ \\
\hline Selective reporting (reporting bias) & Unclear risk & Insufficient information to judge this. \\
\hline Balance in baseline characteristics? & Unclear risk & $\begin{array}{l}\text { No indication of any major baseline im- } \\
\text { balance in } 51 \text { participants followed up at } 3 \\
\text { years but no data for } 6 \text { participants lost to } \\
\text { follow-up }\end{array}$ \\
\hline Free from performance bias? & Low risk & $\begin{array}{l}\text { All surgical procedures performed by senior } \\
\text { surgeon and comparable rehabilitation. Al- } \\
\text { though general anaesthesia used only for } \\
\text { the plate group, this was considered un- } \\
\text { likely to affect the findings. }\end{array}$ \\
\hline
\end{tabular}

Zyto 1997

Methods

Method of randomisation: sealed envelopes

Independent assessor at final follow-up

Loss to follow-up at 3 years: 14/43 (8 deaths, 2 could not be traced, 1 hemi-prosthesis,

3 exclusions)

Participants

Huddinge University Hospital, Stockholm, Sweden

Period of study recruitment: April 1990 to February 1993

43 "elderly" patients with proximal humeral fractures (AO classification system: A 8; B

27; C 8) - see notes.

In trial report:

40 patients with displaced 3- or 4-part fractures (Neer).

Exclusion criteria: pathological fracture, high energy trauma, $<30 \%$ contact between humeral head and shaft, other fractures, impaired ability of patient to co-operate, relevant concomitant disease

35 female, 5 male; mean age 74 years

Interventions

Interventions started: surgery within 48 hours.

1. Internal fixation (cerclage wiring (8): or surgical tension band (14)) under general anaesthesia. Antibiotic therapy. Physiotherapy.

2. non-surgical treatment: sling for 7 to 10 days. Then physiotherapy.

Assigned: 22/21; (20/20)

Completed (50 months): 15/14

Outcomes

Length of follow-up: 3 to 5 years (listed as 50 months in trial report; patient questionnaire, clinical and radiological assessment); also after treatment and at 1 year:

Subjective assessment of function including ability to carry $5 \mathrm{~kg}$, sleep on injured side, comb hair, perform personal hygiene

Constant score: overall shoulder function and components (pain, power, range of motion, activities of daily living) 
Complications: deep infection, non-union, pulmonary embolism, avascular necrosis of humeral head

Mortality

\begin{tabular}{|c|c|}
\hline \multirow[t]{2}{*}{ Notes } & Both groups had the same physiotherapy regimen. \\
\hline & $\begin{array}{l}\text { Three patients excluded from } 1995 \text { data set (Tornkvist 1995) as, on review by Zyto and } \\
\text { a radiologist, the patients did not have 3- or 4-part fractures (personal communication) } \\
\text { Zyto's response to a letter from H. A. Karladani admits that there may have been some } \\
\text { inaccuracy in their classification of the fracture patterns but stressed that the Neer clas- } \\
\text { sification system was flawed and that other factors such as osteoporotic bone need to be } \\
\text { considered too }\end{array}$ \\
\hline
\end{tabular}

Risk of bias

\begin{tabular}{|c|c|c|}
\hline Bias & Authors' judgement & Support for judgement \\
\hline $\begin{array}{l}\text { Random sequence generation (selection } \\
\text { bias) }\end{array}$ & Unclear risk & "randomised by sealed envelopes" \\
\hline Allocation concealment (selection bias) & Unclear risk & $\begin{array}{l}\text { "randomised by sealed envelopes" (at time } \\
\text { of admission) No indication of safeguards }\end{array}$ \\
\hline $\begin{array}{l}\text { Blinding (performance bias and detection } \\
\text { bias) } \\
\text { Functional outcomes, pain, clinical out- } \\
\text { comes, complications }\end{array}$ & High risk & $\begin{array}{l}\text { Some independent assessment by radiogra- } \\
\text { pher and potentially by main author but no } \\
\text { blinding }\end{array}$ \\
\hline
\end{tabular}

Blinding (performance bias and detection Unclear risk bias)

Death, reoperation
No blinding but may not have affected appraisal of mortality (which was not split by treatment group)
Incomplete outcome data (attrition bias) Unclear risk Functional outcomes, pain, clinical outcomes, complications

Incomplete outcome data (attrition bias) High risk

Death, reoperation

Selective reporting (reporting bias) High risk

Balance in baseline characteristics?

Low risk
Post-randomisation exclusions and moderately large loss to follow-up $(14 / 43=32 \%$; $(11 / 40=28 \%))$

Only whole group data presented for deaths out of 40 participants

Insufficient information to judge this but some post-randomisation exclusions and final follow-up performed by first author who does not appear in the earlier reports of the trial

No important imbalances in baseline characteristics. 


\section{Zyto 1997 (Continued)}

\begin{tabular}{|l|l|l|l|l}
\hline Free from performance bias? & Low risk & $\begin{array}{l}\text { No indications of serious performance bias: } \\
\text { surgery performed by orthopaedic special- } \\
\text { ists who were experienced in the surgical } \\
\text { technique }\end{array}$ \\
\hline
\end{tabular}

$\mathrm{AO}=$ Arbeitsgemeinschaft fur Osteosynthesefragen / Association for the Study of Internal Fixation (or ASIF)

$\mathrm{AVN}=$ avascular necrosis

$\mathrm{A} \& \mathrm{E}=$ accident and emergency

$\mathrm{MI}=$ myocardial infarction

ORIF = open reduction and internal fixation

$\mathrm{PE}=$ pulmonary embolism

\section{Characteristics of excluded studies [ordered by study ID]}

\begin{tabular}{|c|c|}
\hline Study & Reason for exclusion \\
\hline Bing 2002 & $\begin{array}{l}\text { This was a randomised clinical trial (sealed envelopes - computer-generated sequence - held in a box), } \\
\text { recruitment } 03 / 11 / 1997 \text { to } 14 / 01 / 1999 \text {, that compared Rush pins fixation with Polaris nail fixation of } \\
\text { displaced two-part fractures of the proximal humerus. Contact with a Dr Sharma in July } 2000 \text { revealed } \\
65 \text { of the } 80 \text { patients in the trial had reached 2-year follow-up. Abstract by Bing et al published in } 2002 \\
\text { indicated } 40 \text { patients of whom } 30 \text { had been followed-up for one year. Information gained via Alison } \\
\text { Armstrong from Grahame Taylor (one of the authors of the Bing abstract) indicated that there were } \\
\text { some concerns about the extent of missing data. Both groups had a high reoperation rate to remove } \\
\text { metalware causing impingement. This trial has been excluded because of insufficient data } \\
\text { It seems very likely, based on location and study dates, that the trial registration (Der Tavitian 2006) } \\
\text { formerly awaiting classification is for this trial. }\end{array}$ \\
\hline
\end{tabular}

Bolano $1995 \quad$ No proximal humeral fractures in a randomised trial of humeral shaft fracture treatment

Brownson 2001 This is listed in the National Research Register as a multicentre randomised trial of the management of displaced surgical neck and displaced shaft fractures of the humerus with the Halder humeral nail. Contact with Mr Brownson revealed this to be part of the trial run from Nottingham (see Wallace 2000) which had been abandoned. Mr Brownson indicated that the very specific inclusion criteria (2part fractures with over $50 \%$ displacement) had reduced the potential sample size; patient consent had also been a problem

Carbone 2012

This is a prospective comparison of MIROS (Minimally Invasive Reduction and Osteosynthesis System ${ }^{\circledR)}$ versus traditional percutaneous pinning, each intervention being carried out at one of two hospitals in the same town in Italy. Not randomised 
(Continued)

Cigni 2012

This study published only as a conference abstract compared two approaches for plate fixation. The abstract did not mention how the 40 patients "were divided in two groups “, but we judged it was very unlikely this was through randomisation

De Boer 2003

This is a multicentre comparative study of locked internal fixators and non-operative treatment. Not randomised

Dias 2001

Trial abandoned. This randomised trial (random number sheets that are remotely administered) compared hemiarthroplasty versus fixation (generally suture reinforced with wires) versus non-surgical treatment (manipulation, sling for 2 weeks, then mobilisation) for 3- and 4-part fractures of the proximal humerus. Trial started in 2001, with one year follow-up (outcome was assessed by independent physiotherapists). Aimed for 90 to 100 participants, aged > 45 years. Contact with Alison Armstrong revealed that recruitment stalled at 11 patients (16 refusals) in 2008; centre stopped trial when it became a trial site for the ProFHER trial

Edelson 2008

Article mentions an abandoned randomised trial comparing "operative versus conservative care" which was unsuccessful "because patients insisted on proactively choosing rather than being assigned to a treatment group by lot“.” No other details given

Elidrissi 2013

Prospective study involving 26 patients with proximal humeral fractures treated open reduction and internal fixation using an anatomical humeral plate (12 patients) or a palm tree pinning technique of Kapandji (14 patients). Inspection of the full text confirmed this was not a randomised or quasirandomised trial

Erdoğ an 2014

This study compared locking plate fixation with or without and inferomedial screw (IMS) in 36 proximal humerus fractures. Inspection of the full text showed this to be a retrospective comparison

Fan 2012

Translation of this study comparing surgical versus non-surgical treatment showed this was a retrospective comparison: "To this end, we retrospectively analyzed 20091 Month - January 201135 cases"

Flannery 2006

This is listed in the National Research Register as a randomised trial comparing non-surgical treatment and hemiarthroplasty for four-part fractures of the proximal humerus. Contact with Mr Flannery revealed his centre failed to recruit anyone into the trial. Mr Turner, the lead investigator of the multicentre trial, involving the South Thames Shoulder and Elbow Group, confirmed that the trial was abandoned due to the inability to recruit patients

Gradl 2009

Prospective study involving 152 patients with unilateral displaced and unstable proximal humeral fractures treated either with an antegrade angular and sliding stable proximal interlocking nail or an angular stable plate. Not a randomised or quasi-randomised trial

Hems 2000

This is listed in the National Research Register as a randomised trial comparing non-surgical treatment and the Halder humeral nail for displaced fractures of the surgical neck and shaft of the humerus. Contact with Mr Hems revealed this to be part of the trial run from Nottingham (see Wallace 2000) . Mr Hems indicated that they had had considerable difficulty in recruiting patients (only those with proximal humeral fractures were eligible in his centre) and had no results

IRCT2013052313435N1 The trial registration document for this study stated that it was randomised. However, a search identified a journal publication that described "prospective clinical trial, observational - Cohort studying" and 
gave no mention of random allocation. Hence this is not a randomised trial

Liao 2009

While the English abstract claims that "70 senile patients" were "randomly divided into three groups to receive different surgical methods" the distribution and characteristics (age and fracture type) of the patients in the three groups indicated serious selection bias and implied this was not a randomised trial. For example: " 21 patients in the group A receiving Kirschner tension band or screw internal fixation, 37 patients in group B receiving internal fixation of locking proximal humeral plate, and 12 patients in group $\mathrm{C}$ receiving humeral head replacement." There was no reply to request for clarification from the lead author

This was stated in the title and text of the conference abstract to be a "randomized controlled clinical trial" that compared early rehabilitation with standard rehabilitation after intramedullary nailing with a Diphos nail. However, the two groups were not concurrent and it seems that this was retrospective comparison with an historic control group. A subsequent publication of a cohort study of the nail used in this study makes no mention of this trial and adds support to our interpretation (Maniscalco 2014b).

Martetschlager 2012

The choice of intervention (deltopectoral versus anterolateral-splitting approach for locking plate fixation) was according to surgeon's preference and not "random" as suggested by the study authors. The Discussion referred to "several limitations, including its retrospective study design" of this "current study". Hence this was not a randomised or quasi-randomised controlled trial

Martin 2000

Contact with a trialist revealed that due to the discovery of problems with randomisation it was decided not to proceed with publication as the trial results could be compromised

Mechlenburg 2009

This was originally registered as a randomised controlled trial comparing a plate with a hemiarthroplasty. However, it is now registered as a prospective study of fixation with a PHILOS plate. Inger Mechlenburg confirmed that no patients had been included in the trial - the trial was abandoned because no funding was obtained

NCT00384852

The primary aim of this multicentre randomised trial was to "assess whether fracture union is accelerated in subjects with humeral fractures (proximal, diaphyseal) treated non-surgically (standard of care) and a single dose of rhBMP-2/CPM [recombinant human bone morphogenetic protein-2 (rhBMP-2)/ Calcium Phosphate Matrix] compared to subjects who receive standard of care alone". Its results were reported in a systematic review (ref 69* in Lo 2012). This reported that "While promising, the published results from the Phase II studies for humeral and femoral fractures showed little enhancement over traditional treatments $[69,70]$. A positive risk/benefit ratio for these treatments was not demonstrated leading to Pfizer no longer pursuing the clinical development of rhBMP-2/CPM for these applications. "Attempts over several months to obtain the report using the citation provided always met with the claim of 'server maintenance'. Unfortunately, Kevin Lo also could not supply a copy of this article (12/ $02 / 2015$ ) and hence the reason for exclusion

*A Phase 2, Multicenter, Double-blind, Randomized, Stratified, Controlled, Efficacy, Safety and Feasibility Study of Recombinant Human Bone Morphogenetic Protein-2 (rhBMP-2)/Calcium Phosphate Matrix (CPM) as an Adjuvant Therapy in Closed Fractures of the Humerus, Pfizer, Inc. Sep 15. 2011 ClinicalStudyResults.org,. http://www.clinicalstudyresults.org/documents/company-study_11378_0.pdf

NCT01532076

This randomised trial, which compared adipose tissue-derived mesenchymal stem cells composite graft augmentation versus acellular composite graft augmentation was terminated early after recruiting only 8 of the planned 290 participants with an isolated proximal humeral fracture 


The registration document indicates this completed randomised efficacy trial evaluated dry needling in
post-operative shoulder pain in a mixed population of people who had proximal humeral fractures fixed
with a Philos plate or people who had undergone surgical repair of a rotator cuff tear. Twenty patients
were [to be] enrolled between February to April 2013. Primary outcome measure was the Constant-
Murley score before and one week after the intervention (which was applied once with physical therapy)
. Excluded as a mixed population and very short term follow-up

NTR2186

The registration document of this study, which compared the DePuy Delta Xtend reversed shoulder prosthesis with non-surgical treatment in the management of 4-part fractures of the proximal humerus, indicates this is not a randomised trial. The non-surgical treatment arm was a historical control group

Parnes 2005

There has been no response from the lead author of this 'trial' (last contact attempted 8 June 2012), which appears to have been reported in a conference abstract only. In 2003, 50 patients with 3- and 4-part fractures and fracture dislocations of the proximal humerus were "random selected" for surgery (closed or open reduction and external fixation or hemiarthroplasty) or non-surgical treatment. The very limited results are split descriptively according to three groups (2 reflecting the 2 different surgical methods). There is currently insufficient evidence to support this being a randomised trial

Pullen 2007

This is listed in the National Research Register Archive as a randomised trial comparing the T2 proximal humeral nail with the PHILOS plate system in patients with 2- or 3-part proximal humeral fractures. the recruitment target was 100 patients (between $01 / 09 / 2005$ to $01 / 09 / 2007$ ), and follow-up was 16 weeks. We have not located any other report of this study than the details provided in the National Research Register (UK) by, at that time, a Trauma and Orthopaedic Registrar who has now moved to another hospital. There was no response to a request for further information sent $8 / 6 / 2012$. There is no indication that this study, which may not have started, will ever be reported

Rodriguez-Merchan 95 No proximal humeral fractures in a quasi-randomised trial of humeral shaft fracture treatment

Shah 2003

This is listed in the National Research Register Archive as a multicentre randomised trial of the management of four part fractures of proximal humerus that compared hemiarthroplasty versus non-surgical treatment. The recruitment target was 200 patients, with a one year follow-up using the ConstantMurley shoulder score and Oxford Shoulder score. The listed start and end dates were 01/01/2003 and $01 / 02 / 2005$. No details were received of the other centres in the very limited further information received from $\mathrm{Mr}$ Shah in April 2003. There was no response to a request for further information sent $13 / 11 / 06$. There is no indication that this study, which may not have started, will ever be reported

Sinopidis 2010

This was registered as a randomised study of reverse shoulder prosthesis and hemiarthroplasty for elderly patients with proximal humeral fractures. However, the principal investigator left the hospital (and country) before it started and a contact at Liverpool (Matthew Smith) confirmed that the study was closed after this

Wallace 2000

This is listed in the National Research Register as a multicentre randomised trial of the management of displaced surgical neck and displaced shaft fractures of the humerus with the Halder humeral nail. Contact with Prof Wallace's secretary revealed that the study had not gone ahead. The secretary mentioned three other sites (Halifax; Liverpool; and one in Scotland). No reason given. See Brownson 2001.

Wan 2005

This is a mixed population trial evaluating additional mobilisation therapy that included other fractures (e.g. clavicular and scapular fractures) as well as proximal humeral fractures. This trial was excluded 
because separate proximal humeral fracture data were not reported and the contact author is unavailable

\begin{tabular}{ll}
\hline Warnecke 1999 & A multicentre prospective study but not a randomised trial. \\
\hline Welsh 2000 & $\begin{array}{l}\text { This is listed in the National Research Register as a randomised comparison of operative and non- } \\
\text { operative management of proximal humeral fractures. This trial was abandoned due to poor recruitment, } \\
\text { mainly due to lack of patient consent }\end{array}$ \\
\hline
\end{tabular}

Yang 2006

Correspondence with the author revealed that this was not a randomised trial. The choice of surgery was dependent on the success of closed reduction

Zhang 2010

While the English abstract claims that " 58 patients with 3 parts and 4 parts fractures of proximal humerus were randomly treated with AO locked compressive plates (LCP) or humeral head replacement.

" the characteristics (fracture type) of the patients in the two groups indicated serious selection bias and implied this was not a randomised trial. Thus, 25 of 28 patients in the plate group had 3-part fractures ( 1 with a dislocation) and 3 had 4-part fractures ( 1 with dislocation) whereas 11 of 30 in the replacement group had 3-part fractures (2 with dislocation), 16 with 4-part fractures (4 with dislocation) and the other three had humeral head split fractures. There was no reply to request for clarification from the lead author

Zuckerman 2012

Commentary only on Olerud 2011b.

Characteristics of studies awaiting assessment [ordered by study ID]

Battistella 2011

\begin{tabular}{ll}
\hline Methods & "Randomized clinical study" \\
\hline Participants & $\begin{array}{l}54 \text { patients (38 female, } 26 \text { male, mean age } 61 \text { years) with 2-part surgical neck fractures or 3-part valgus impacted } \\
\text { fractures }\end{array}$ \\
\hline Interventions & $\begin{array}{l}\text { Surgery involving a titanium plate: } \\
\text { 1. Minimally invasive fixation based on anterolateral deltoid split approach and percutaneous reduction } \\
\text { 2. Open reduction and internal fixation by standard deltopectoral approach }\end{array}$ \\
\hline Outcomes & Constant score, instrumental activities of daily living, pain (VAS), range of motion, union, complications \\
\hline Notes & Requests for further information sent to Dr Battistella (8 and 14 May 2012) were unsuccessful \\
\hline
\end{tabular}

Brorson 2009

Methods Multicentre, randomised clinical trial (central randomisation unit)

Participants 25 recruited out of a planned 162 patients with displaced 4-part fractures of the proximal humerus 
Brorson 2009 (Continued)

\author{
Interventions 1. Hemiarthroplasty \\ 2. Fixed-angle plate osteosynthesis \\ 3. Non-surgical treatment
}

Outcomes

Follow-up: 3 years (primary outcome: 1 year)

Primary outcome: Constant Disability Scale

Secondary outcomes: Oxford Shoulder Score, Short Form-36

- Published protocol.
- Correspondence from Stig Brorson (June 11 2012) reveals a slower than anticipated recruitment. (Start date:
April 2009; End date: March 2013 (final date for primary outcome measure))
- Entry for trial (clinicaltrials.gov) on January 192015 , indicated that "The recruitment status of this study is
unknown because the information has not been verified recently". Status as "recruiting" had been last verified on
June 2012 by Herlev Hospital. The study completion date had been changed from March 2012 to March 2013.
- Correspondence from Stig Brorson (January 28 2015) revealed: "Unfortunately, we had to stop inclusion after
25 patients. They all followed the protocol and were evaluated accordingly. However, 9 out of 11 centres withdrew
from the study because they found allocation of patients with 4-part fractures to non-surgical treatment ethically
problematic. Ironically, we are now unable to continue the study as most surgeons find plating of 4-part fractures
problematic! All data on the 25 patients are available." Stig Brorson further clarified (February 62015$)$ : "We
enrolled 25 patients and randomly allocated them to non-surgical treatment, ORIF with locking plate or HA. Two
died before the first evaluation, no drop-outs. The remaining 23 patients were evaluated after 12 months with
blinded and non-blinded Constant Score, Oxford Shoulder Score and Short-Form 36. All data are available and
unpublished."
• Trial transferred from Ongoing to Studies awaiting classification (11/02/2015)
Discussions are taking place on the most suitable approach to take with this data set (23/05/2015)

Liu 2011

\begin{tabular}{ll}
\hline Methods & "Randomly divided" \\
\hline Participants & $\begin{array}{l}50 \text { patients aged } 60 \text { or above with proximal humeral fractures } \\
\text { Mean } 70 \text { years (range } 60 \text { to } 83 \text { years); } 36 \text { female, } 14 \text { male; } 19 \text { two-part, } 21 \text { three-part and } 10 \text { four-part fractures }\end{array}$ \\
\hline Interventions & $\begin{array}{l}\text { 1. PHILOS plate augmentation with minimally invasive injectable graft (MIIG) X3 Hivisc } \\
\text { 2. PHILOS plate alone } \\
\text { Minimally invasive percutaneous plate osteosynthesis used in both groups }\end{array}$ \\
\hline Outcomes & $\begin{array}{l}\text { Follow-up: mean } 18 \text { months (range } 12 \text { to } 25 \text { months) } \\
\text { Neer scoring system, complications, healing time, operative time, blood loss }\end{array}$ \\
\hline Notes & $\begin{array}{l}\text { No response to inquiry on method of allocation sent 07/12/14; baseline imbalance in type of fracture with more 3- } \\
\text { part and 4-part fractures in the first group }\end{array}$ \\
\hline
\end{tabular}

Interventions for treating proximal humeral fractures in adults (Review)

Copyright () 2015 The Cochrane Collaboration. Published by John Wiley \& Sons, Ltd. 
Luo 2008

\begin{tabular}{|c|c|}
\hline Methods & Patients were randomly allocated via a random numbers table. \\
\hline Participants & $\begin{array}{l}60 \text { patients ( } 32 \text { females, } 28 \text { males; age range: } 39 \text { to } 62 \text { years) treated operatively for fracture of the surgical neck of } \\
\text { the humerus }\end{array}$ \\
\hline Interventions & $\begin{array}{l}\text { 1. Acupuncture (electroacupuncture and infrared radiation) plus passive exercise of the shoulder joint } \\
\text { 2. Exercises only: passive exercise of the shoulder joint followed by active exercises } \\
\text { Treatment lasted } 1 \text { month. }\end{array}$ \\
\hline Outcomes & $\begin{array}{l}\text { Follow-up: } 1 \text { month } \\
\text { Shoulder pain score (VAS) } \\
\text { Shoulder joint activity }\end{array}$ \\
\hline Notes & $\begin{array}{l}\text { Trial in Chinese with English abstract. Translation of methods section (1.1) confirmed that this was a randomised } \\
\text { trial }\end{array}$ \\
\hline \multicolumn{2}{|c|}{ NCT02052206 } \\
\hline Methods & Randomised trial \\
\hline Participants & 80 patients with either osteoarthritis of the shoulder or complex proximal humeral fractures \\
\hline Interventions & $\begin{array}{l}\text { For shoulder replacement surgery } \\
\text { 1. computer assisted } 3 \mathrm{D} \text { planning } \\
\text { 2. conventional } 2 \mathrm{D} \text { planning. }\end{array}$ \\
\hline Outcomes & $\begin{array}{l}\text { Follow-up: } 6 \text { weeks and } 1 \text { year } \\
\text { Primary outcome: Discrepancy (difference of translation in } \mathrm{mm} \text { and rotation in degrees) in the realised position of } \\
\text { the components compared with the preoperative planning (CT scans) } \\
\text { Secondary outcomes: Clinical outcome, subjective shoulder value, Constant score }\end{array}$ \\
\hline Notes & $\begin{array}{l}\text { Study not open to recruitment (January 2014); but is now open to recruitment (May 2015) } \\
\text { Contact: Lazaros Vlachopoulos, Balgrist University Hospital, Switzerland }\end{array}$ \\
\hline
\end{tabular}

Wang 2013

\begin{tabular}{ll}
\hline Methods & "Randomly divided" \\
\hline Participants & $\begin{array}{l}80 \text { "elderly" patients with proximal humerus fractures } \\
\text { Mean } 67 \text { years (range } 60 \text { to } 83 \text { years); } 42 \text { female, } 38 \text { male; AO classification: } 12 \mathrm{~A}, 45 \mathrm{~B} \text { and } 23 \mathrm{C} \text { fractures }\end{array}$ \\
\hline Interventions & $\begin{array}{l}\text { 1. Minimally invasive percutaneous insertion of PHILOS plate with injectable bone } \\
\text { 2. Minimally invasive percutaneous insertion of PHILOS plate alone }\end{array}$ \\
\hline Outcomes & $\begin{array}{l}\text { Length of follow-up: unknown } \\
\text { Constant score, complications, healing time, bone mineral density, patient satisfaction with results of treatment }\end{array}$
\end{tabular}


Wang 2013 (Continued)

Notes No response to inquiry on method of allocation sent 15/01/14; translated title from Chinese states this is a "casecontrol" study. Data extracted from abstract. (Unusually high Constant score in the bone substitute group: 97.2 (SD 4.6)). Translation required if considered study should be included in future

Zhu 2014

\begin{tabular}{ll}
\hline Methods & patients "randomly either underwent" \\
\hline Participants & $\begin{array}{l}\text { 40 people with comminuted proximal humeral fractures (however, "patients with evidence of arthrosis or a clinical } \\
\text { follow-up shorter than } 12 \text { months were excluded") }\end{array}$ \\
\hline Interventions & $\begin{array}{l}\text { 1. Locking plate and an autologous crest bone graft } \\
\text { 2. Locking plate only }\end{array}$ \\
\hline Outcomes & $\begin{array}{l}\text { Length of follow-up: mean } 25 \text { months, range } 13 \text { to } 48 \text { months } \\
\text { Range of motion, pain, SF-36, return to previous activities or occupation, complications, time to union } \\
\text { was also recorded. }\end{array}$ \\
\hline Notes & Queries on allocation method and donor site complications/morbidity sent 22 May 2015 \\
\hline
\end{tabular}

\section{Characteristics of ongoing studies [ordered by study ID]}

\section{ACTRN12610000730000}

Trial name or title Minimally invasive versus standard open reduction of proximal humerus fractures

Official title: A prospective randomised controlled trial comparing clinical and radiographic outcomes of the deltopectoral and limited deltoid splitting approaches for fixation of displaced proximal humeral fractures in a skeletally mature population

\begin{tabular}{|c|c|}
\hline Methods & Single-centre, randomised controlled trial. Computer generated random list. Unblinded \\
\hline Participants & 90 patients with displaced proximal humeral fractures \\
\hline Interventions & $\begin{array}{l}\text { 1. Minimally invasive approach with closed reduction and temporary fixation with k-wires, then the limited } \\
\text { deltoid splitting approach (locking plate) } \\
\text { 2. Standard open reduction and internal fixation via the deltopectoral approach (locking plate) }\end{array}$ \\
\hline Outcomes & $\begin{array}{l}\text { Follow-up: } 1 \text { year } \\
\text { Primary outcome: Constant Shoulder Score and DASH (Disabilities of the Arm, Shoulder, and Hand) Score } \\
\text { Secondary outcomes: radiographic outcomes, complications, union rate }\end{array}$ \\
\hline Starting date & Anticipated start date: October 2010 (however, study is not yet recruiting) \\
\hline Contact information & $\begin{array}{l}\text { Jeremy Stanley } \\
14 \text { Grand Drive } \\
\text { Remuera }\end{array}$ \\
\hline
\end{tabular}

Interventions for treating proximal humeral fractures in adults (Review)

Copyright () 2015 The Cochrane Collaboration. Published by John Wiley \& Sons, Ltd. 


\section{ACTRN12610000730000 (Continued)}

Auckland 1050

New Zealand

jellystan@hotmail.com

Trial was not recruiting (nor ethics approval)
No change on checking February 112015

\section{DELPHI}

Trial name or title Clinical investigation for fractures of the proximal humerus in elderly patients. A randomized study of two surgical treatments: Reverse total shoulder arthroplasty versus angular stable device Philos

\begin{tabular}{ll}
\hline Methods & $\begin{array}{l}\text { Randomised trial; } 6 \text { centres } \\
\text { "semi-blinded" for the physiotherapist who will examine the patients }\end{array}$ \\
\hline Participants & $\begin{array}{l}120 \text { patients, aged } 65 \text { years to } 85 \text { years, admitted in hospital with a displaced three- or four-part proximal } \\
\text { humerus fracture of OTA/AO group 11-B2 or 11-C2 (displaced fracture of extra-articular or articular, bifocal } \\
\text { type) }\end{array}$ \\
\hline Interventions & $\begin{array}{l}\text { 1. Reversed shoulder prosthesis } \\
\text { 2. ORIF with PHILOS plate }\end{array}$ \\
\hline
\end{tabular}

Outcomes Follow-up: 3, 6, 12 and 24 months, and five years

Primary outcome: Constant score

Secondary outcomes: Oxford Shoulder score; quality of life measured with the 15D score, health economics, radiographic results

Starting date Start date: January 2013

Estimated completion date: June 2021 (June 2021 = final data collection date for primary outcome measure)

Contact information Dr Tore Fjalestad

Orthopaedic Department, Division of surgery and Clinical Neuroscience

Oslo University Hospital HF

Nydalen, Postbox 4950

Oslo, Norway

torfja@online.no

Notes

This appeared in Studies awaiting assessment in 2012 version of the review as Fjalestad (RCT proposal)

Published protocol available

Recruiting status verified in October 2014 by Oslo University Hospital 


\section{HOMERUS}

Trial name or title Hemiarthroplasty versus osteosynthesis in humeral fractures (HOMERUS): A multicentre randomised trial Official title: Three- and four-part fracture of the proximal humerus in the elderly. Angle stable locking compression plate osteosynthesis versus hemiarthroplasty

\begin{tabular}{|c|c|}
\hline Methods & Multicentre, randomised clinical trial. Single blinded (outcomes assessor) \\
\hline Participants & $\begin{array}{l}134 \text { patients aged over } 60 \text { years with displaced 3-and 4-part proximal humeral fractures with more than } 5 \\
\mathrm{~mm} \text { of dislocation in one of the fracture planes }\end{array}$ \\
\hline Interventions & $\begin{array}{l}\text { 1. Hemiarthroplasty } \\
\text { 2. Angle stable locking compression plate osteosynthesis }\end{array}$ \\
\hline Outcomes & $\begin{array}{l}\text { Follow-up: } 2 \text { years } \\
\text { Primary outcome: DASH (Disabilities of the Arm, Shoulder, and Hand) score } \\
\text { Secondary outcomes: VAS (Visual analogue score) for pain and patient satisfaction, Constant-Murley Score, } \\
\text { SF-36, Radiographic evaluation, complications }\end{array}$ \\
\hline Starting date & $\begin{array}{l}\text { Start date: September } 2010 \\
\text { Anticipated completion date: August } 2012\end{array}$ \\
\hline Contact information & $\begin{array}{l}\text { Dr PA Verbeek } \\
\text { Department of Orthopaedic Surgery } \\
\text { University Medical Centre Groningen (UMCG) } \\
\text { Groningen } \\
\text { The Netherlands } \\
\text { paulverbeek@gmail.com }\end{array}$ \\
\hline Notes & \\
\hline
\end{tabular}

\section{HURA}

Trial name or title A randomised clinical trial comparing a lateral minimally invasive approach and the traditional anterior approach for plating of proximal humerus fractures

\begin{tabular}{ll} 
Methods & Randomised, single blind (outcome assessors), clinical trial \\
\hline Participants & 90 patients, with humeral surgical neck fracture, Neer II valgus-type, and Neer III \\
\hline Interventions & $\begin{array}{l}\text { 1. Lateral minimally invasive approach (plate fixation) } \\
\text { 2. Deltopectoral approach (plate fixation) }\end{array}$ \\
\hline Outcomes & $\begin{array}{l}\text { Follow-up: 3, 6, and } 12 \text { weeks, and at 6, 12, } 18 \text { and } 24 \text { months } \\
\text { Primary outcome: Quick DASH } \\
\text { Secondary outcomes: SF-12v2 Questionnaire, Constant Shoulder Score, the Patient Scar Assessment Scale, } \\
\text { complication rate }\end{array}$ \\
\hline Starting date & $\begin{array}{l}\text { Start date: November } 2007 \\
\text { End date: January } 2018 \text { (January } 2016 \text { = final data collection date for primary outcome) }\end{array}$
\end{tabular}


HURA

(Continued)

$\begin{array}{ll}\text { Contact information } & \text { Marie-France Poirer } \\ & \text { Hopital Sacré-Coeur } \\ & \text { Montreal } \\ & \text { Quebec } \\ & \text { Canada } \\ & \text { H4J1C5 } \\ & \text { mariefrancepoirier@hotmail.com }\end{array}$

Notes

Entry for trial (clinicaltrials.gov) on April 10 2012, indicated that "The recruitment status of this study is unknown because the information has not been verified recently"

Entry for trial (clinicaltrials.gov) on January 19 2015, indicated that "This study is currently recruiting participants"; "Verified August 2014 by Université de Montreal" End date of study changed from January 2012 to January 2018

\section{NCT00438633}

Trial name or title Early vs delayed physical therapy (exercises) for non-operatively-treated proximal humerus fractures: a prospective randomized trial

\begin{tabular}{ll}
\hline Methods & Randomised trial \\
\hline Participants & 60 patients, aged 18 years or over, with non-operatively treated proximal humeral fractures \\
\hline Interventions & $\begin{array}{l}\text { 1. Physical therapy started immediately after diagnosis of injury } \\
\text { 2. Physical therapy delayed until 3 weeks after diagnosis of injury }\end{array}$ \\
\hline Outcomes & $\begin{array}{l}\text { Follow-up: } 6 \text { months } \\
\text { Primary outcome: shoulder flexion } \\
\text { Secondary outcomes: shoulder pain Likert scores; external and internal rotation; abduction; DASH and } \\
\text { Constant scores }\end{array}$ \\
\hline Starting date & $\begin{array}{l}\text { Start date: February } 2005 \\
\text { End date: December } 2013 \text { (December 2012 = final data collection date for primary outcome measure) }\end{array}$ \\
\hline Contact information & $\begin{array}{l}\text { Prof David Ring } \\
\text { Director of Research } \\
\text { Hand Service } \\
\text { Massachusetts General Hospital } \\
\text { Boston } \\
\text { Massachusetts } \\
\text { USA } \\
\text { dring@partners.org }\end{array}$ \\
\hline
\end{tabular}

Notes

Changes to NCT00438633 on 27 May 2008 seemed to indicate that, despite its official title, this is now a prospective cohort study (accessed: April 10 2012). David Ring confirmed it was still an RCT (April 16 2012)

Entry for trial (clinicaltrials.gov) on January 19 2015, indicated that "This study is enrolling participants by invitation only", last verified July 2012. The study design was still listed as a prospective cohort 


\section{NCT00818987}

\begin{tabular}{|c|c|}
\hline Trial name or title & $\begin{array}{l}\text { A multicentre prospective randomized control trial on the treatment of three-and four-part proximal humerus } \\
\text { fractures in patients } 70 \text { years and older: comparing open reduction and internal fixation with non operative } \\
\text { treatment }\end{array}$ \\
\hline Methods & Randomised controlled trial: "randomly (like flipping a coin)" \\
\hline Participants & 120 patients aged 70 years or over with a 3- or 4-part fracture \\
\hline Interventions & $\begin{array}{l}\text { 1. Open reduction and internal fixation } \\
\text { 2. Non-operative treatment (reduction and immobilisation) }\end{array}$ \\
\hline Outcomes & $\begin{array}{l}\text { Follow-up: } 1 \text { year } \\
\text { Primary outcome: patients' functional shoulder scores as measured by the Disabilities of the Arm, Shoulder, } \\
\text { and Hand (DASH) questionnaire } \\
\text { Secondary outcomes: functional and mental status instruments (i.e. SF-36/EQ-5D) used to assess the pa- } \\
\text { tient's health-related quality of life; re-operation rates; and the time required to return to pre-injury level of } \\
\text { independence }\end{array}$ \\
\hline Starting date & $\begin{array}{l}\text { November } 2010 \\
\text { Estimated completion date: October } 2011\end{array}$ \\
\hline Contact information & $\begin{array}{l}\text { Contact: Raman Johal (raman.johal@vch.ca) } \\
\text { Principal investigator: Pierre Guy, University of British Columbia, Canada }\end{array}$ \\
\hline Notes & $\begin{array}{l}\text { As of registration update }(17 / 02 / 2011) \text { the study was recruiting. Start and end dates amended. } \\
\text { Entry for trial (clinicaltrials.gov) on January } 192015 \text {, indicated that "The recruitment status of this study is } \\
\text { unknown because the information has not been verified recently". Status as "recruiting" had been last verified } \\
\text { on February } 2011\end{array}$ \\
\hline
\end{tabular}

\section{NCT00999193}

\begin{tabular}{ll} 
Trial name or title & $\begin{array}{l}\text { Effectiveness and cost-effectiveness of operative and conservative treatment of comminuted fractures of the } \\
\text { proximal humerus. A randomised, controlled study }\end{array}$ \\
\hline Methods & Randomised single blind (outcomes assessor) \\
\hline Participants & $\begin{array}{l}90 \text { older patients with comminuted, displaced fractures of the proximal humerus } \\
\text { Inclusion criteria: Age over } 65 \text { years; acute trauma with randomisation within } 7 \text { days of injury; 3- or 4-part } \\
\text { fracture with }>5 \text { mm dislocation of the anatomic neck (AO classification C1-2 for non-luxation fractures; } \\
\text { C3 for luxation fractures) }\end{array}$
\end{tabular}

Interventions

1. PHILOS locking plate: open reduction of the fracture (and GH joint), internal fixation with the PHILOS locking plate. Tuberculum fragments are sutured to the plate with thick non-absorbable suture.

2. Global FX hemiarthroplasty: replacement of the humeral articular head with hemiprosthesis. Tubercles are sutured to the prosthesis with thick nonabsorbable sutures.

3. Non-surgical treatment: immobilisation in a supporting brace for 3 weeks, then increasingly active rehabilitation programme supported by a physiotherapist until 12 weeks of the injury 


\section{NCT00999193 (Continued)}

\begin{tabular}{ll}
\hline Outcomes & $\begin{array}{l}\text { Follow-up: } 24 \text { months } \\
\text { Primary outcomes: Pain at rest and activity (Numeric Rating Scale), Constant score } \\
\text { Secondary outcomes: Simple Shoulder test (SST), Disabilities of the Arm, Shoulder and Hand (DASH), } \\
\text { quality-of-life assessment (15D), subjective patient satisfaction, complications and costs }\end{array}$ \\
\hline Starting date & $\begin{array}{l}\text { November } 2010 \\
\text { End date: December } 2018 \text { (December } 2016 \text { = final data collection date for primary outcome) }\end{array}$ \\
\hline Contact information & $\begin{array}{l}\text { Tuomas Lähdeoja, MD: tuomas.lahdeoja@hus.fi } \\
\text { Mika Paavola, MD: mika.paavola@hus.fi } \\
\text { Helsinki University, Helsinki, Finland }\end{array}$ \\
\hline Notes & $\begin{array}{l}\text { As of registration update (22/01/2012) the study was recruiting. Start and end dates amended. } \\
\text { Entry for trial (clinicaltrials.gov) on January 19 2015, indicated that "This study is currently recruiting } \\
\text { participants"; "Verified October 2014 by Helsinki University" Study completion date changed from November } \\
\text { 2014 to December 2018. The estimated enrolment dropped from } 150 \text { to } 90\end{array}$ \\
\hline
\end{tabular}

\section{NCT01086202}

Trial name or title Clinical outcome comparison between medial and lateral offset reverse shoulder arthroplasty

\begin{tabular}{|c|c|}
\hline Methods & Randomised single blinded trial \\
\hline Participants & $\begin{array}{l}40 \text { patients aged between the ages of } 50 \text { and } 95 \text { years of age who are a candidate for a reverse shoulder } \\
\text { arthroplasty. This includes patients with rotator cuff tear arthroplasty, irreparable rotator cuff tears, significant } \\
\text { proximal humerus fractures and malunions, and chronic proximal humerus dislocators }\end{array}$ \\
\hline Interventions & $\begin{array}{l}\text { Tornier reversed shoulder arthroplasty: } \\
\text { 1. Medial offset design } \\
\text { 2. Lateral offset design }\end{array}$ \\
\hline Outcomes & $\begin{array}{l}\text { Follow-up: } 2 \text { years } \\
\text { Shoulder functional score } \\
\text { Pain scores } \\
\text { Radiological outcomes }\end{array}$ \\
\hline Starting date & $\begin{array}{l}\text { May } 2010 \\
\text { End date: April } 2012\end{array}$ \\
\hline Contact information & $\begin{array}{l}\text { Wesley Phipatanakul, MD } \\
\text { wphip@hotmail.com } \\
\text { Principal investigator: Montri D Wongworawat, MD, } \\
\text { Loma Linda University Health Department of Orthopaedic Surgery, Loma Linda } \\
\text { Californa } 92354 \\
\text { USA }\end{array}$ \\
\hline
\end{tabular}




\section{NCT01086202 (Continued)}

Notes
humeral fractures.
As of registration update $(21 / 06 / 2011)$ the study was still recruiting

\section{NCT01113411}

Trial name or title

Effectiveness of intensive rehabilitation on shoulder function after a fracture of the proximal humerus treated by locked plate. A prospective randomized study

\begin{tabular}{ll}
\hline Methods & Randomised clinical trial \\
\hline Participants & $\begin{array}{l}80 \text { patients aged over } 18 \text { years treated by PHILOS locked plate system for unstable closed fracture of the } \\
\text { proximal humerus (two-part and three-part fractures according to the Neer classification) within } 7 \text { days on } \\
\text { injury }\end{array}$
\end{tabular}

Interventions $\quad$ 1. Early and intensive exercise programme

A thoraco brachial brace will be worn for 48 hours following the surgery and then removed for the remainder of treatment. Patients will then start the intensive rehabilitation programme without physical therapy. The exercise programme will be provided to the patient. The exercises consist of active and active-assisted movements of the shoulder for a period of six weeks, limiting external rotation to $0^{\circ}$. Patients are encouraged to use their affected limb for daily activities. Strengthening exercises are started the 6th week following surgery and the full programme will be completed three months after surgery. Patients who wish can then continue their rehabilitation with a physiotherapist. The patient will complete a daily diary to validate the frequency and intensity of the exercises

versus

2. Standard rehabilitation programme

The patient will wear the thoraco brachial brace for a period of four weeks following the surgery. It may be taken off for hygiene purposes and dressing. After the four weeks, the patient will take the brace off permanently and begin an exercise programme, writing down the frequency and intensity of the exercises. Physiotherapy is allowed for the remaining part of the three months rehabilitation programme

Outcomes Length of follow-up: 12 months

Primary outcome: Constant score (adjusted for age) at 6 months. A difference of 10 points is considered significant (standard deviation of 15 points).

Secondary outcomes: reoperation, redisplacement, Constant score at 12 months, Dash, return to professional activities, pain, range of motion

Starting date December 2009

Final follow-up date: December 2013 (final data collection date for primary outcome)

Contact information Hélène Côté, Reg. Nurse: helco3@hotmail.com

Stéphane Pelet, MD, PhD: spelet01@hotmail.com

Hopital de l'Enfant-Jésus, Canada

Notes

Entry for trial (clinicaltrials.gov) on January 19 2015, indicated that "The recruitment status of this study is unknown because the information has not been verified recently". Status as "recruiting" had been last verified on December 2012. The study completion date had been changed from December 2012 to December 2013 


\begin{tabular}{|c|c|}
\hline Trial name or title & $\begin{array}{l}\text { The effect of the timing of postoperative mobilisation after locking plate osteosynthesis of fractures of the } \\
\text { surgical neck of the humerus }\end{array}$ \\
\hline Methods & $\begin{array}{l}\text { Randomised controlled trial } \\
\text { Single blind (outcomes assessor) }\end{array}$ \\
\hline Participants & $\begin{array}{l}100 \text { patients, } 18 \text { years or older, with surgery performed within } 10 \text { days of injury for a dislocated }(>1 \mathrm{~cm} \text { or } \\
35 \text { degrees) AO 11-A2, - A } 3,-\mathrm{B} 1 \text { or }-\mathrm{B} 2 \text { fracture of the surgical neck of the proximal humerus with a possible } \\
\text { fracture of the greater tuberosity }\end{array}$ \\
\hline Interventions & $\begin{array}{l}\text { 1. Immediate mobilisation after open reduction and Philos plate fixation: immediate passive range of motion } \\
\text { exercises are begun postoperatively, after } 3 \text { weeks, active unloaded mobilisation begins after three weeks and } \\
\text { active, loaded use is allowed } 6 \text { weeks postoperatively } \\
\text { 2. Standard mobilisation after open reduction and Philos plate fixation: immediately postoperatively the arm } \\
\text { is held in a sling, active mobilisation of healthy joints and pendulum exercises are begun. Passive range of } \\
\text { motion exercises of the shoulder are begun } 3 \text { weeks postoperatively. Active mobilisation begins after six weeks }\end{array}$ \\
\hline Outcomes & $\begin{array}{l}\text { Follow-up: } 3 \text { and } 6 \text { weeks, 3, 6, } 12 \text { and } 24 \text { months } \\
\text { Primary outcome: DASH (Disabilities of the Arm, Shoulder, and Hand) score } \\
\text { Secondary outcomes: Constant score, simple shoulder test (SST), pain at rest and during motion, subjective } \\
\text { satisfaction, quality of life using the 15D instrument, complications }\end{array}$ \\
\hline Starting date & $\begin{array}{l}\text { May } 2011 \\
\text { Estimated completed date: December } 2016 \text { (December } 2016 \text { = final data collection date for primary outcome) }\end{array}$ \\
\hline Contact information & $\begin{array}{l}\text { Tuomas Lahdeoja, MD } \\
\text { Töölö Hospital, Helsinki University Central Hospital } \\
\text { Helsinki, Finland, 00029 } \\
\text { tuomas.lahdeoja@hus.fi }\end{array}$ \\
\hline Notes & Verified as recruiting participants in October 2014 by Helsinki University \\
\hline
\end{tabular}

\section{NCT01557413}

Trial name or title Randomised study between intramedullary locking nails and locking plates for treatment of proximal humerus fractures (HUMERUS)

Official title: Randomised study between intramedullary locking nails and locking plates for treatment of proximal humerus fractures in patients after 40-year-old

Methods Single centre, randomised controlled trial. Unblinded.

\begin{tabular}{ll}
\hline Participants & $\begin{array}{l}84 \text { patients, aged between } 40 \text { and } 85 \text { years, with a type III or IV “cephalotuberosity” proximal humeral fracture } \\
\text { (classification of Neer and DUPARC) }\end{array}$
\end{tabular}
(classification of Neer and DUPARC)

Interventions $\quad$ 1. Intramedullary nail (Multilock, Synthes)

2. Locking plate (SURFIX, Integra) 


\section{NCT01557413 (Continued)}

\begin{tabular}{ll}
\hline Outcomes & $\begin{array}{l}\text { Follow-up: 1 year } \\
\text { Primary outcome: Constant Score } \\
\text { Secondary outcomes: Quick DASH, complication (malunion, necrosis, infection) }\end{array}$ \\
\hline Starting date & $\begin{array}{l}\text { Start date: February } 2012 \\
\text { Estimated completion date: February } 2017 \text { (final data collection for primary outcome) }\end{array}$ \\
\hline Contact information & $\begin{array}{l}\text { Dr Patrick Boyer } \\
\text { Group Hospitalier Bichat - Claude Bernard 46, rue Henri-Huchard } \\
\text { Paris } \\
\text { Ile de France } \\
\text { France 75018 } \\
\text { patrick.boyer@bch.aphp.fr }\end{array}$ \\
\hline Entry for trial (clinicaltrials.gov) on January 19 2015, indicated that "This study is currently recruiting \\
participants"; "Verified August 2014 by Assistance Publique - Hôpitaux de Paris". Study completion date \\
changed from November 2015 to February 2017. The estimated enrolment dropped from 144 to 84
\end{tabular}

\section{NCT01847508}

Trial name or title A multicenter randomized controlled trial to investigate the treatment outcome of PHILOS screw augmentation compared to PHILOS without augmentation in older adult patients with proximal humerus fractures

\begin{tabular}{|c|c|}
\hline Methods & Randomised controlled trial \\
\hline Participants & $\begin{array}{l}128 \text { (planned enrolment) patients aged } 65 \text { years or older with an acute ( } \leq 10 \text { days ) closed fracture of the } \\
\text { proximal humerus after low energy trauma. Any displaced or unstable } 3 \text { - or } 4 \text {-part fracture of the proximal } \\
\left.\text { humerus (i.e. segment displacement }>0.5 \mathrm{~cm} \text { or angulated }>45^{\circ}\right) \text { except isolated displaced fractures of the } \\
\text { greater or lesser tuberosity } \\
\text { Exclusion criteria: bilateral or previous proximal humerus fractures on either side, splitting fracture of the } \\
\text { humeral head or humeral head impression fracture, cuff-arthropathy of the contra- or ipsilateral proximal } \\
\text { humerus, associated nerve or vessel injury, any known clotting disorders, severe cardiac and/or pulmonary } \\
\text { insufficiency, known hypersensitivity or allergy to any of the components of Traumacem V+ Cement Kit, any } \\
\text { severe systemic disease (class } 4 \text { - } 6 \text { of the American Society of Anesthesiologists (ASA) physical status classifi- } \\
\text { cation), any not-medically-managed severe systemic disease (class } 3 \text { of the ASA physical status classification), } \\
\text { recent history of substance abuse, prisoner }\end{array}$ \\
\hline
\end{tabular}

Interventions

1. PHILOS screw augmentation: Proximal Humeral Internal Locking System with screw tip augmentation (PHILOS+) with high viscous polymethylmethacrylate (PMMA) cement (Traumacem $\mathrm{V}_{+}$)

2. Proximal Humeral Internal Locking System (PHILOS)

Outcomes

Follow-up: 1 year (primary outcome)

Primary outcome: Any occurrence of radiographically confirmed mechanical failure during the first year after treatment

Secondary outcomes include: quality of life, intra- and postoperative adverse events related to the procedure and/or device, reoperation rate, surgical details including of augmentation, shoulder function (Shoulder Pain and Disability Index (SPADI), shoulder function (Constant score, shoulder function (Disabilities of the Arm, Shoulder and Hand Score (QuickDASH)) 


\section{NCT01847508 (Continued)}

\begin{tabular}{ll}
\hline Starting date & $\begin{array}{l}\text { October } 2013 \\
\text { Final follow-up date: September } 2016 \text { (final data collection date for primary outcome measure) }\end{array}$ \\
\hline Contact information & $\begin{array}{l}\text { Jan Ljungqvist, } \\
+41442002461 \\
\text { jan.ljungqvist@aofoundation.org }\end{array}$ \\
\hline Notes & Recruiting confirmed January 2015 \\
\hline
\end{tabular}

\section{NCT01984112}

Trial name or title Proximal humerus fractures: randomized study between intramedullary locking nails and locking plates for Neer 2- and 3-part displaced fractures

\begin{tabular}{|c|c|}
\hline Methods & Randomised controlled trial \\
\hline Participants & $\begin{array}{l}72 \text { patients, aged } 50 \text { years to } 85 \text { years, with acute closed fractures (less than } 21 \text { days since injury) of the } \\
\text { proximal humerus classified as } 2 \text { - or } 3 \text {-parts of Neer, with involvement of the humeral head and one of the } \\
\text { tuberosities }\end{array}$ \\
\hline Interventions & $\begin{array}{l}\text { 1. Intramedullary locked nail performed by antero-lateral transdeltoid minimally invasive approach and } \\
\text { rotator cuff augmentation with non-absorbable polyester suture } \\
\text { 2. Osteosynthesis with Philos plate, through deltopectoral approach and rotator cuff augmentation with non- } \\
\text { absorbable polyester suture }\end{array}$ \\
\hline Outcomes & $\begin{array}{l}\text { Follow-up: } 12 \text { months } \\
\text { Primary outcome: absolute Constant-Murley score } \\
\text { Secondary outcomes: DASH (Disability of Arm-Shoulder-Hand) score, UCLA (University of California Los } \\
\text { Angeles) score, individual relative Constant-Murley score compared with non-injured shoulder, overall com- } \\
\text { plications and need for additional surgery, post-operative integrity of the rotator cuff evaluated by ultrasonog- } \\
\text { raphy }\end{array}$ \\
\hline Starting date & $\begin{array}{l}\text { May } 2011 \\
\text { Final follow-up date: December } 2015 \text { (final data collection date for primary outcome) }\end{array}$ \\
\hline Contact information & $\begin{array}{l}\text { Mauro Gracitelli, MD } \\
\text { Instituto de Ortopedia e Traumatologia } \\
\text { São Paulo, SP, Brazil, 05403-010 } \\
\text { mgracitelli@gmail.com }\end{array}$ \\
\hline Notes & Verified in November 2013 as recruiting participants by University of Sao Paulo \\
\hline
\end{tabular}


Trial name or title Prospective, randomized and double blind study of parallel groups for evaluating the effectiveness between two surgical techniques for reconstruction of humeral proximal extremity fractures or fractures luxation in three or four fragments of Neer's classification

\begin{tabular}{ll}
\hline Methods & Randomised controlled trial \\
\hline Participants & $\begin{array}{l}40 \text { patients, aged } 70 \text { years or over, with "humeral proximal extremity fracture or fracture luxation in three or } \\
\text { four fragments of Neer's classification" }\end{array}$ \\
\hline Interventions & $\begin{array}{l}\text { 1. Reverse arthroplasty Delta Xtent (DePuy) } \\
\text { 2. Hemiarthroplasty Global Fx (DePuy) }\end{array}$ \\
\hline Outcomes & $\begin{array}{l}\text { Follow-up: 1,3,6, 12, 18 and 24 months } \\
\text { Primary outcome: ASES (American Shoulder and Elbow) score } \\
\text { Secondary outcomes: intra-operative complications; post-operative complications, surgical time, recovery } \\
\text { time }\end{array}$ \\
\hline Starting date & $\begin{array}{l}\text { May 2013 } \\
\text { Estimated completed date: May 2016 (May 2015 = final data collection date for primary outcome) }\end{array}$ \\
\hline Contact information & $\begin{array}{l}\text { Carlos Alvarez, MD } \\
\text { Hospital General Universitario Gregorio Marañon } \\
\text { Madrid, Spain, 28007 } \\
\text { calvargon@gmail.com }\end{array}$ \\
\hline Notes & \begin{tabular}{l} 
Verified as recruiting participants in March 2014 by Hospital General Universitario Gregorio Marañon \\
\hline
\end{tabular} \\
\hline
\end{tabular}

\section{NTR3208}

Trial name or title Arthroplasty in three- and four-part proximal humerus fracture: hemi or reverse? Prospective multicentre randomised clinical trial

\begin{tabular}{ll} 
Methods & Multicentre, randomised controlled trial. Unblinded. \\
\hline Participants & $\begin{array}{l}52 \text { patients aged over } 65 \text { years with displaced 3- or 4-part proximal humerus fractures who are candidates for } \\
\text { primary shoulder arthroplasty }\end{array}$ \\
\hline Interventions & $\begin{array}{l}\text { 1. Aequalis reverse fracture prosthesis } \\
\text { 2. Aequalis fracture prosthesis }\end{array}$ \\
\hline Outcomes & $\begin{array}{l}\text { Follow-up: 1 year } \\
\text { Primary outcome: Constant Shoulder Score } \\
\text { Secondary outcomes: DASH (Disabilities of the Arm, Shoulder, and Hand) Score, SF-12 questionnaire, } \\
\text { Visual Analogue Score of pain }\end{array}$ \\
\hline
\end{tabular}

Starting date Start date: July 2010

Estimated completion date: December 2014 
NTR3208 (Continued)

\begin{tabular}{ll}
\hline Contact information & Yde Engelsma \\
& Medisch Centrum Alkmaar \\
& Wilhelminalaan 12 \\
& 1815 JD Alkmaar \\
& The Netherlands \\
& y.engelsma@mca.nl \\
\hline Notes & \\
\hline
\end{tabular}

\section{NTR4019}

Trial name or title PROMOTION-trial: A PROspective randomized Multicenter trial for the treatment of dislocated 3-part proximal humerus fractures: Open reduction and internal fixaTION versus intramedullary nailing

\begin{tabular}{ll}
\hline Methods & Randomised controlled trial \\
\hline Participants & $\begin{array}{l}92 \text { patients, aged } 18 \text { years or over, with unilateral displaced 3-part proximal humeral fracture }(>45 \text { degrees } \\
\text { or }>0.5 \mathrm{~cm} \text { displacement between major fracture fragments) }\end{array}$
\end{tabular}

$\begin{array}{ll}\text { Interventions } & \text { 1. Philos plate (Proximal humeral internal locking system, Synthes) } \\ \text { 2. PHN (Proximal Humeral Nailing system, Stryker) }\end{array}$

2. PHN (Proximal Humeral Nailing system, Stryker)

\begin{tabular}{ll} 
Outcomes & $\begin{array}{l}\text { Follow-up: 1 year } \\
\text { Primary outcome: Constant-Murley score } \\
\text { Secondary outcomes: DASH-score, pain, SF-12, EQ-5D, complication and mortality rate }\end{array}$ \\
\hline Starting date & $\begin{array}{l}1 \text { August } 2013 \\
\text { Planned closing date: } 31 \text { July } 2017\end{array}$ \\
\hline Contact information & $\begin{array}{l}\text { Jeroen Bransen } \\
\text { Maastrict University Medical Centre } \\
\text { Department of Surgery PO Box } 5800 \\
\text { 6202 AZ } \\
\text { Maastricht } \\
\text { The Netherlands } \\
\text { jeroenbransen@gmail.com }\end{array}$ \\
\hline Notes & Ethics approval not received for this multicentre trial at time of registration (3 June 2013)
\end{tabular}

\section{ProCon}

Trial name or title Primary hemiarthroplasty versus conservative treatment for comminuted fractures of the proximal humerus in the elderly (ProCon) - a multicenter randomized trial

Methods Randomised trial: "variable block randomisation will be accomplished via a trial website" 
ProCon (Continued)

\begin{tabular}{|c|c|}
\hline Participants & $\begin{array}{l}\text { Patients ( } 65 \text { years or older) with a comminuted proximal humeral fracture } \\
80 \text { patients ( } 65 \text { years or older) with a comminuted proximal humeral fracture: three-part (Hertel classification } \\
\text { type } 9,10,11) \text {, four-part (Hertel type } 12) \text {, anatomical neck (Hertel type } 2) \text {, or split-head fractures of the } \\
\text { humeral head }\end{array}$ \\
\hline Interventions & $\begin{array}{l}\text { 1. Hemiarthroplasty (Affinis® Fracture shoulder endoprosthesis) } \\
\text { 2. Non-surgical treatment (collar and cuff for three weeks) }\end{array}$ \\
\hline Outcomes & $\begin{array}{l}\text { Follow-up: } 1,3 \text { and } 6 \text { weeks, and 3, 6, } 12 \text { and } 24 \text { months } \\
\text { Primary outcome (Constant Score) and secondary outcomes (DASH, pain, radiographic healing, secondary } \\
\text { intervention rates, complication rates, mortality rates, SF-36, and EQ-5D) } \\
\text { Costs for (in)formal healthcare consumption }\end{array}$ \\
\hline Starting date & $\begin{array}{l}\text { Start date: } 15 \text { June } 2009 \\
\text { Planned end date: } 31 \text { December } 2013\end{array}$ \\
\hline Contact information & $\begin{array}{l}\text { Dennis Den Hartog } \\
\text { Department of Surgery-Traumatology } \\
\text { Erasmus MC } \\
\text { University Medical Center } \\
\text { Rotterdam } \\
\text { P.O. Box } 2040 \\
3000 \text { CA Rotterdam } \\
\text { The Netherlands } \\
\text { d.denhartog@erasmusmc.nl }\end{array}$ \\
\hline Notes & Published protocol \\
\hline
\end{tabular}

\section{ROTATE}

Trial name or title Return Of funcTion And exTErnal rotation post proximal humerus fracture fixation with neutral rotation brace (ROTATE)

\begin{tabular}{ll}
\hline Methods & Randomised controlled trial \\
\hline Participants & $\begin{array}{l}100 \text { patients (planned), aged over } 18 \text { years, with proximal humeral fractures requiring operative intervention } \\
\text { with extramedullary plate fixation (i.e. fractures displaced by } 1 \mathrm{~cm} \text { and/or angulated by } 45 \text { degrees or more }\end{array}$ \\
\hline Interventions & $\begin{array}{l}\text { Post-surgery sling } \\
\text { 1. External rotation of the shoulder in a neutral rotation brace } \\
\text { 2. Polysling holding the proximal humerus in internal rotation } \\
\text { The operation, post-operative treatment and planned physiotherapy will be the same in the two groups }\end{array}$ \\
\hline Outcomes & $\begin{array}{l}\text { Follow-up: } 6 \text { weeks, } 9 \text { weeks, } 3 \text { months and } 1 \text { year } \\
\text { Primary outcomes: Oxford shoulder score (1 year), DASH (the Disabilities of the Arm, Shoulder and Hand) } \\
\text { (1 year) } \\
\text { Secondary outcomes: range of movement (flexion, extension, abduction, external and internal rotation), SF- } \\
12 \text { score, time to union of fracture, return to work post surgery, re-operations and complications }\end{array}$
\end{tabular}


ROTATE (Continued)

\begin{tabular}{ll}
\hline Starting date & $\begin{array}{l}\text { Start date: January } 2013 \\
\text { End date: December 2015 }\end{array}$ \\
\hline Contact information & $\begin{array}{l}\text { Ms Victoria Conboy, FRCS (Orth) (Principal Investigator) } \\
\text { Consultant in Trauma and Orthopaedics } \\
\text { Torbay District General Hospital } \\
\text { Lawes Bridge } \\
\text { Torquay } \\
\text { TQ2 7AA } \\
\text { United Kingdom } \\
\text { veronica.conboy@nhs.net }\end{array}$ \\
\hline Notes & Sponsor: South Devon Healthcare NHS Foundation Trust \\
\hline
\end{tabular}

\section{SHeRPA}

\begin{tabular}{|c|c|}
\hline Trial name or title & Comparison of two shoulder replacement methods after trauma \\
\hline Methods & Multicentre ( 5 centres in the UK), randomised controlled trial. Participants are blinded \\
\hline Participants & $\begin{array}{l}50 \text { patients aged over } 65 \text { years with displaced 3- or 4-part proximal humerus fractures; fit for surgery } \\
\text { Exclusion: dementia, non consent, unfit for reverse shoulder arthroplasty, glenoid fracture, axillary nerve palsy }\end{array}$ \\
\hline Interventions & $\begin{array}{l}\text { 1. Reverse total shoulder arthroplasty } \\
\text { 2. Hemiarthroplasty }\end{array}$ \\
\hline Outcomes & $\begin{array}{l}\text { Follow-up: } 2 \text { years, also } 6 \text { weeks, } 3 \text { and } 12 \text { months } \\
\text { Primary outcome: Constant Shoulder Score (at } 12 \text { months) } \\
\text { Secondary outcomes: QuickDASH (Disabilities of the Arm, Shoulder, and Hand) Score, Oxford Shoulder } \\
\text { Score, ASES score }\end{array}$ \\
\hline Starting date & $\begin{array}{l}\text { Start date: June } 2013 \\
\text { Estimated completion date: August } 2017\end{array}$ \\
\hline Contact information & $\begin{array}{l}\text { Adam Watts } \\
\text { Wrightington Hospital } \\
\text { Hall Lane } \\
\text { Appley Bridge } \\
\text { Wigan } \\
\text { Lancashire } \\
\text { WN6 9EP } \\
\text { United Kingdom }\end{array}$ \\
\hline Notes & $\begin{array}{l}\text { Retrospectively registered (applied: 13/01/2015) after end of recruitment } \\
\text { Funder: Tornier UK Limited (probably manufacturer of implants under test) }\end{array}$ \\
\hline
\end{tabular}


Trial name or title Management of conservatively treated proximal humeral fractures: prospective randomized study (taken from Torrens 2012a)

\begin{tabular}{|c|c|}
\hline Methods & Randomised controlled trial (use of computer-generated random numbers) \\
\hline Participants & 80 participants with non-surgically treated proximal humeral fractures \\
\hline Interventions & $\begin{array}{l}\text { 1. Functional one week immobilisation regimen } \\
\text { 2. Conventional four weeks immobilisation regimen } \\
\text { Both groups will follow the same progressive rehabilitation programme }\end{array}$ \\
\hline Outcomes & $\begin{array}{l}\text { Length of follow-up: } 1 \text { year (also } 1 \text { week, and } 3 \text { and } 6 \text { months) } \\
\text { Pain (analogue pain scale: EVA), Constant shoulder functional score, satisfaction score, Euroqol-5D, secondary } \\
\text { surgery (based on Torrens 2012a) }\end{array}$ \\
\hline Starting date & Late 2012 (May 25 2015: “started 6 months ago") \\
\hline Contact information & $\begin{array}{l}\text { Carlos Torrens, MD, Castelldefels, Spain } \\
\text { Email: CTorrens@parcdesalutmar.cat }\end{array}$ \\
\hline Notes & $\begin{array}{l}\text { In response to a query on the status of Torrens } 2012 \mathrm{a} \text {, Carlos Torrens ( } 25 \text { May 2015): "Unfortunately this } \\
\text { study was stopped because of lack of money so we just could recruit } 40 \text { patients. Right now we have found } \\
\text { money again and we re-started it } 6 \text { months ago, so I hope that during this year we will be able to recruit } 80 \\
\text { patients and we will follow them } 1 \text { year meaning that in } 2 \text { years we will be able to publish more consistent } \\
\text { results." } \\
\text { Confirmation received from Carlos Torrens on June } 42015 \text { that this is a new trial }\end{array}$ \\
\hline
\end{tabular}

\section{TPHF}

Trial name or title Treatment of Proximal Humeral Fractures (TPHF)

Official title: A national, prospective, randomized, multicenter, controlled head-to-head comparison of conservative, plate fixation and prosthesis in treatment of displaced 2-, 3- and 4 part fractures of proximal humerus of 60 years and older patients

\begin{tabular}{ll}
\hline Methods & Multicentre, randomised clinical trial. Single blinded (outcomes assessor) \\
\hline Participants & 290 patients aged over 60 years with displaced 2-, 3- and 4-part proximal humeral fractures \\
\hline Interventions & $\begin{array}{l}\text { 1. PHILOS locking plate } \\
\text { 2. Epoca prosthesis } \\
\text { 3. Non-surgical management }\end{array}$ \\
\hline
\end{tabular}

Outcomes

Follow-up: 2 years

Primary outcome: DASH (Disabilities of the Arm, Shoulder, and Hand) score

Secondary outcome: EQ-5D Questionnaire 
Starting date Start date: January 2011

Estimated completion date: September 2018 (December 2016 = final data collection date for primary outcome)

Contact information

Antti Launonen

Tampere University Hospital

Tampere

Pirkanmaa

Finland, 33521

antti.launonen@pshp.fi

Notes

As of registration update (01/02/2011), trial was recruiting in Tampere University Hospital, but recruitment had not started in Oulu or Kuopio

Entry for trial (clinicaltrials.gov) on January 19 2015, indicated that "This study is currently recruiting participants"; "Verified November 2014 by Tampere University Hospital". End date of study changed from September 2016 to September 2018

$\mathrm{AO}=$ Arbeitsgemeinschaft fur Osteosynthesefragen / Association for the Study of Internal Fixation (or ASIF)

LCP = Locking compression plate

NRR = National Research Register

$\mathrm{ORIF}=$ open reduction and internal fixation 
DATA AND ANALYSES

\section{Comparison 1. Early mobilisation (within or up to 1 week) versus immobilisation for 3 or 4 weeks}

\begin{tabular}{|c|c|c|c|c|}
\hline Outcome or subgroup title & $\begin{array}{l}\text { No. of } \\
\text { studies }\end{array}$ & $\begin{array}{c}\text { No. of } \\
\text { participants }\end{array}$ & Statistical method & Effect size \\
\hline $\begin{array}{l}1 \text { Shoulder disability: Croft } \\
\text { Shoulder Disability Score }\end{array}$ & 1 & & Risk Ratio (M-H, Fixed, 95\% CI) & Totals not selected \\
\hline $\begin{array}{l}1.1 \text { Disability ( } 1 \text { or more } \\
\text { problems) at } 1 \text { year }\end{array}$ & 1 & & Risk Ratio (M-H, Fixed, 95\% CI) & $0.0[0.0,0.0]$ \\
\hline $\begin{array}{l}1.2 \text { Severe disability ( } 5 \text { or } \\
\text { more problems) at } 1 \text { year }\end{array}$ & 1 & & Risk Ratio (M-H, Fixed, 95\% CI) & $0.0[0.0,0.0]$ \\
\hline $\begin{array}{l}\text { 1.3 Disability ( } 1 \text { or more } \\
\text { problems) at } 2 \text { years }\end{array}$ & 1 & & Risk Ratio (M-H, Fixed, 95\% CI) & $0.0[0.0,0.0]$ \\
\hline $\begin{array}{l}1.4 \text { Severe disability ( } 5 \text { or } \\
\text { more problems) at } 2 \text { years }\end{array}$ & 1 & & Risk Ratio (M-H, Fixed, 95\% CI) & $0.0[0.0,0.0]$ \\
\hline $\begin{array}{l}2 \text { Croft shoulder disability score: } \\
\text { individual problems at } 2 \text { years }\end{array}$ & 1 & & Risk Ratio (M-H, Fixed, 95\% CI) & Totals not selected \\
\hline 2.1 Pain on movement & 1 & & Risk Ratio (M-H, Fixed, 95\% CI) & $0.0[0.0,0.0]$ \\
\hline 2.2 Bathing difficulties & 1 & & Risk Ratio (M-H, Fixed, 95\% CI) & $0.0[0.0,0.0]$ \\
\hline $\begin{array}{l}2.3 \text { Change position at night } \\
\text { more often }\end{array}$ & 1 & & Risk Ratio (M-H, Fixed, 95\% CI) & $0.0[0.0,0.0]$ \\
\hline 2.4 Disturbed sleep & 1 & & Risk Ratio (M-H, Fixed, 95\% CI) & $0.0[0.0,0.0]$ \\
\hline $\begin{array}{l}2.5 \text { No active pastimes or } \\
\text { usual physical recreation }\end{array}$ & 1 & & Risk Ratio (M-H, Fixed, 95\% CI) & $0.0[0.0,0.0]$ \\
\hline 2.6 Lifting problems & 1 & & Risk Ratio (M-H, Fixed, 95\% CI) & $0.0[0.0,0.0]$ \\
\hline 2.7 Help needed & 1 & & Risk Ratio (M-H, Fixed, 95\% CI) & $0.0[0.0,0.0]$ \\
\hline $\begin{array}{l}2.8 \text { More accidents (e.g. } \\
\text { dropping things) }\end{array}$ & 1 & & Risk Ratio (M-H, Fixed, 95\% CI) & $0.0[0.0,0.0]$ \\
\hline $\begin{array}{l}3 \text { Number of treatment sessions } \\
\text { (until independent function } \\
\text { achieved) }\end{array}$ & 1 & & Mean Difference (IV, Fixed, 95\% CI) & Totals not selected \\
\hline $\begin{array}{l}4 \text { SF-36 scores: pain } \& \text { physical } \\
\text { dimensions }\end{array}$ & 1 & & Mean Difference (IV, Fixed, 95\% CI) & Totals not selected \\
\hline $\begin{array}{l}\text { 4.1 Physical functioning } \\
(0-100 \text { : excellent) at } 16 \text { weeks }\end{array}$ & 1 & & Mean Difference (IV, Fixed, 95\% CI) & $0.0[0.0,0.0]$ \\
\hline $\begin{array}{l}\text { 4.2 Physical functioning } \\
(0-100 \text { : excellent) at } 1 \text { year }\end{array}$ & 1 & & Mean Difference (IV, Fixed, 95\% CI) & $0.0[0.0,0.0]$ \\
\hline $\begin{array}{l}4.3 \text { Role limitation physical } \\
(0-100: \text { none }) \text { at } 16 \text { weeks }\end{array}$ & 1 & & Mean Difference (IV, Fixed, 95\% CI) & $0.0[0.0,0.0]$ \\
\hline $\begin{array}{l}\text { 4.4 Role limitation physical } \\
(0-100: \text { none }) \text { at } 1 \text { year }\end{array}$ & 1 & & Mean Difference (IV, Fixed, 95\% CI) & $0.0[0.0,0.0]$ \\
\hline $\begin{array}{l}\text { 4.5 Pain (0-100: none) at } 16 \\
\text { weeks }\end{array}$ & 1 & & Mean Difference (IV, Fixed, 95\% CI) & $0.0[0.0,0.0]$ \\
\hline $\begin{array}{l}\text { 4.6 Pain (0-100: none) at } 1 \\
\text { year }\end{array}$ & 1 & & Mean Difference (IV, Fixed, 95\% CI) & $0.0[0.0,0.0]$ \\
\hline
\end{tabular}

Interventions for treating proximal humeral fractures in adults (Review)

Copyright $\odot 2015$ The Cochrane Collaboration. Published by John Wiley \& Sons, Ltd. 
5.1 At 3 months

5.2 At 6 months

5.3 At 12 months

6 Adverse events

6.1 Frozen shoulder

6.2 Fracture displacement

6.3 Non-union

6.4 Complex regional pain

syndrome type 1

6.5 Treated (injection)

subacromial impingement

6.6 Shoulder complications

6.7 Fracture complications

7 Mortality

8 Constant shoulder score (ratio of

affected/unaffected arm)

8.18 weeks

8.216 weeks

8.31 year

9 Constant shoulder score (0 to

100: best)

9.1 At 6 weeks

9.2 At 3 months

9.3 At 6 months

9.4 At 12 months

9.56 months: subjective

assessment ( 0 to 35 : best)

9.66 months: objective

assessment range of motion

and strength (0 to 65: best)

10 Pain VAS (0 to 100: worst pain)

10.1 At 6 weeks

10.2 At 3 months

10.3 At 6 months

10.4 At 12 months

11 Changes in pain intensity $(\mathrm{mm})$

from baseline: $100 \mathrm{~mm}$ visual

analogue scale (positive change

$=$ less pain)

11.1 At 6 weeks

11.2 At 3 months

11.3 At 6 months

12 Range of motion at 6 months (degrees): difference between two shoulders

12.1 Abduction

12.2 Anterior elevation

12.3 Lateral rotation
Mean Difference (IV, Fixed, 95\% CI)

Mean Difference (IV, Fixed, 95\% CI)

Mean Difference (IV, Fixed, 95\% CI)

Risk Ratio (M-H, Fixed, 95\% CI)

80

106

106

115

Risk Ratio (M-H, Fixed, 95\% CI)

Risk Ratio (M-H, Fixed, 95\% CI)

Risk Ratio (M-H, Fixed, 95\% CI)

Risk Ratio (M-H, Fixed, 95\% CI)

64

Risk Ratio (M-H, Fixed, 95\% CI)

259

Risk Ratio (M-H, Fixed, 95\% CI)

Risk Ratio (M-H, Fixed, 95\% CI)

Risk Ratio (M-H, Fixed, 95\% CI)

Mean Difference (IV, Fixed, 95\% CI)

Mean Difference (IV, Fixed, 95\% CI)

Mean Difference (IV, Fixed, 95\% CI)

Mean Difference (IV, Fixed, 95\% CI)

Mean Difference (IV, Fixed, 95\% CI)

64

Mean Difference (IV, Fixed, 95\% CI)

Mean Difference (IV, Fixed, 95\% CI)

Mean Difference (IV, Fixed, 95\% CI)

Mean Difference (IV, Fixed, 95\% CI)

Mean Difference (IV, Fixed, 95\% CI)

Mean Difference (IV, Fixed, 95\% CI)

64

Mean Difference (IV, Fixed, 95\% CI)

Mean Difference (IV, Fixed, 95\% CI)

106

105

39

Mean Difference (IV, Fixed, 95\% CI)

Mean Difference (IV, Fixed, 95\% CI)

Mean Difference (IV, Fixed, 95\% CI)

Mean Difference (IV, Fixed, 95\% CI)

Mean Difference (IV, Fixed, 95\% CI)

Mean Difference (IV, Fixed, 95\% CI)

Mean Difference (IV, Fixed, 95\% CI)

Mean Difference (IV, Fixed, 95\% CI)

Mean Difference (IV, Fixed, 95\% CI)

Mean Difference (IV, Fixed, 95\% CI)

Mean Difference (IV, Fixed, 95\% CI)
$0.0[0.0,0.0]$

$0.0[0.0,0.0]$

$0.0[0.0,0.0]$

Subtotals only

$0.33[0.01,7.95]$

$2.2[0.22,22.45]$

$0.0[0.0,0.0]$

$1.09[0.07,16.71]$

$1.0[0.07,15.30]$

$0.73[0.15,3.63]$

$2.2[0.22,22.45]$

Totals not selected

Totals not selected

$0.0[0.0,0.0]$

$0.0[0.0,0.0]$

$0.0[0.0,0.0]$

Subtotals only

$10.10[2.02,18.18]$

$6.53[0.77,12.30]$

$3.39[-1.46,8.24]$

$1.46[-7.05,9.97]$

$1.90[-0.54,4.34]$

$4.10[-0.62,8.82]$

Subtotals only $-3.60[-20.76,13$. $56]$

$-5.13[-14.76,4.50]$

$4.29[-5.48,14.07]$

$10.8[-4.59,26.19]$

Totals not selected

$0.0[0.0,0.0]$

$0.0[0.0,0.0]$

$0.0[0.0,0.0]$

Totals not selected

$0.0[0.0,0.0]$

$0.0[0.0,0.0]$

$0.0[0.0,0.0]$ 
13 Patient dissatisfied with treatment

14 Patient satisfaction (0 to 10: higher scores - greater satisfaction)

14.1 At 3 months

14.2 At 6 months

14.3 At 12 months

\section{Comparison 2. Gilchrist bandage versus 'Classic' Desault bandage}

\begin{tabular}{|c|c|c|c|c|}
\hline Outcome or subgroup title & $\begin{array}{l}\text { No. of } \\
\text { studies }\end{array}$ & $\begin{array}{c}\text { No. of } \\
\text { participants }\end{array}$ & Statistical method & Effect size \\
\hline 1 Problems with bandages & 1 & & Risk Ratio (M-H, Fixed, 95\% CI) & Totals not selected \\
\hline $\begin{array}{l}\text { 1.1 Application of bandage } \\
\text { was uncomfortable }\end{array}$ & 1 & & Risk Ratio (M-H, Fixed, 95\% CI) & $0.0[0.0,0.0]$ \\
\hline $\begin{array}{l}\text { 1.2 Premature bandage } \\
\text { removal }\end{array}$ & 1 & & Risk Ratio (M-H, Fixed, 95\% CI) & $0.0[0.0,0.0]$ \\
\hline $\begin{array}{l}2 \text { Fracture displacement by } 3 \\
\text { weeks }\end{array}$ & 1 & & Risk Ratio (M-H, Fixed, 95\% CI) & Totals not selected \\
\hline $\begin{array}{l}3 \text { Poor or bad rating by patient at } \\
\text { fracture consolidation }\end{array}$ & 1 & & Risk Ratio (M-H, Fixed, 95\% CI) & Totals not selected \\
\hline
\end{tabular}

\section{Comparison 3. Instructed self-exercise versus conventional physiotherapy}

\begin{tabular}{|c|c|c|c|c|}
\hline Outcome or subgroup title & $\begin{array}{l}\text { No. of } \\
\text { studies }\end{array}$ & $\begin{array}{c}\text { No. of } \\
\text { participants }\end{array}$ & Statistical method & Effect size \\
\hline $\begin{array}{l}1 \text { Pain at one year (scale } 0 \text { to } 8 \text { : } \\
\text { maximum pain) }\end{array}$ & 1 & & Mean Difference (IV, Fixed, 95\% CI) & Totals not selected \\
\hline $\begin{array}{l}2 \text { Severe or moderate pain at } 3 \\
\text { months }\end{array}$ & 1 & & Risk Ratio (M-H, Fixed, 95\% CI) & Totals not selected \\
\hline 3 Requested change of therapy & 1 & & Risk Ratio (M-H, Fixed, 95\% CI) & Totals not selected \\
\hline $\begin{array}{l}4 \text { Adverse events (frozen shoulder: } \\
1 \mathrm{v} 2 \text {; unexplained prolonged } \\
\text { pain: } 0 \mathrm{v} 1 \text { ) }\end{array}$ & 1 & & Risk Ratio (M-H, Fixed, 95\% CI) & Totals not selected \\
\hline $\begin{array}{l}5 \text { Neer's rating ( } 0 \text { to } 100 \text { : best) at } \\
\text { mean } 16 \text { months (exploratory } \\
\text { analysis) }\end{array}$ & 1 & & Mean Difference (IV, Fixed, 95\% CI) & Totals not selected \\
\hline $\begin{array}{l}6 \text { Active gleno-humeral elevation } \\
\text { (degrees) }\end{array}$ & 1 & & Mean Difference (IV, Fixed, 95\% CI) & Totals not selected \\
\hline
\end{tabular}




\begin{tabular}{|c|c|c|c|c|}
\hline Outcome or subgroup title & $\begin{array}{l}\text { No. of } \\
\text { studies }\end{array}$ & $\begin{array}{c}\text { No. of } \\
\text { participants }\end{array}$ & Statistical method & Effect size \\
\hline $\begin{array}{l}1 \text { Functional scores at } 12 \text { months } \\
\text { (higher = better outcome) }\end{array}$ & 5 & 419 & Std. Mean Difference (IV, Fixed, 95\% CI) & $0.07[-0.12,0.26]$ \\
\hline $\begin{array}{l}\text { 1.1 DASH (0 to 100: worst } \\
\text { disability) (reversed) }\end{array}$ & 2 & 105 & Std. Mean Difference (IV, Fixed, 95\% CI) & $0.19[-0.19,0.57]$ \\
\hline 1.2 ASES ( 0 to $24:$ best) & 1 & 48 & Std. Mean Difference (IV, Fixed, 95\% CI) & $-0.10[-0.67,0.46]$ \\
\hline 1.3 SST ( 0 to 12 : best) & 1 & 47 & Std. Mean Difference (IV, Fixed, 95\% CI) & $0.11[-0.46,0.69]$ \\
\hline 1.4 OSS ( 0 to $48:$ best $)$ & 1 & 219 & Std. Mean Difference (IV, Fixed, 95\% CI) & $0.04[-0.22,0.31]$ \\
\hline $\begin{array}{l}2 \text { Functional scores at } 24 \text { months } \\
\text { (higher }=\text { better outcome) }\end{array}$ & 4 & 351 & Std. Mean Difference (IV, Fixed, 95\% CI) & $0.07[-0.14,0.28]$ \\
\hline $\begin{array}{l}2.1 \text { DASH (0 to } 100 \text { : worst } \\
\text { disability) (reversed) }\end{array}$ & 2 & 99 & Std. Mean Difference (IV, Fixed, 95\% CI) & $0.33[-0.07,0.73]$ \\
\hline 2.2 ASES ( 0 to $24:$ best) & 1 & 42 & Std. Mean Difference (IV, Fixed, 95\% CI) & $-0.02[-0.62,0.59]$ \\
\hline 2.3 OSS ( 0 to $48:$ best $)$ & 1 & 210 & Std. Mean Difference (IV, Fixed, 95\% CI) & $-0.03[-0.30,0.24]$ \\
\hline $\begin{array}{l}3 \text { Oxford Shoulder Score (0 to 48: } \\
\text { best outcome) }\end{array}$ & 1 & & Mean Difference (IV, Fixed, 95\% CI) & Subtotals only \\
\hline 3.1 Over 2 years & 1 & 231 & Mean Difference (IV, Fixed, 95\% CI) & $0.75[-1.68,3.18]$ \\
\hline 3.2 At 6 months & 1 & 226 & Mean Difference (IV, Fixed, 95\% CI) & $2.25[-0.42,4.92]$ \\
\hline 3.3 At 12 months & 1 & 219 & Mean Difference (IV, Fixed, 95\% CI) & $0.43[-2.10,2.96]$ \\
\hline 3.4 At 24 months & 1 & 210 & Mean Difference (IV, Fixed, 95\% CI) & $-0.29[-2.84,2.26]$ \\
\hline $\begin{array}{l}4 \text { DASH ( } 0 \text { to } 100: \text { worst } \\
\text { disability) }\end{array}$ & 2 & & Mean Difference (IV, Fixed, 95\% CI) & Subtotals only \\
\hline 4.1 at 4 months & 2 & 106 & Mean Difference (IV, Fixed, 95\% CI) & $0.91[-7.00,8.83]$ \\
\hline 4.2 at 12 months & 2 & 105 & Mean Difference (IV, Fixed, 95\% CI) & $-4.51[-13.50,4.48]$ \\
\hline 4.3 at 24 months & 2 & 99 & Mean Difference (IV, Fixed, 95\% CI) & $-7.43[-16.26,1.41]$ \\
\hline $\begin{array}{l}5 \text { American Shoulder and Elbow } \\
\text { Surgeons score ( } 0 \text { to } 24 \text { : best) }\end{array}$ & 1 & & Mean Difference (IV, Fixed, 95\% CI) & Totals not selected \\
\hline 5.1 at 6 months & 1 & & Mean Difference (IV, Fixed, 95\% CI) & $0.0[0.0,0.0]$ \\
\hline 5.2 at 12 months & 1 & & Mean Difference (IV, Fixed, 95\% CI) & $0.0[0.0,0.0]$ \\
\hline 5.3 at 24 months & 1 & & Mean Difference (IV, Fixed, 95\% CI) & $0.0[0.0,0.0]$ \\
\hline $\begin{array}{l}6 \text { Simple Shoulder Test (0 to } 12 \text { : } \\
\text { best function) }\end{array}$ & 1 & & Mean Difference (IV, Fixed, 95\% CI) & Totals not selected \\
\hline 6.1 at 3 months & 1 & & Mean Difference (IV, Fixed, 95\% CI) & $0.0[0.0,0.0]$ \\
\hline 6.2 at 12 months & 1 & & Mean Difference (IV, Fixed, 95\% CI) & $0.0[0.0,0.0]$ \\
\hline 7 Activities of daily living & 1 & & Risk Ratio (M-H, Fixed, 95\% CI) & Totals not selected \\
\hline $\begin{array}{l}7.1 \text { Unable to manage } \\
\text { personal hygiene at } 1 \text { year }\end{array}$ & 1 & & Risk Ratio (M-H, Fixed, 95\% CI) & $0.0[0.0,0.0]$ \\
\hline $\begin{array}{l}7.2 \text { Unable to comb hair at } 1 \\
\text { year }\end{array}$ & 1 & & Risk Ratio (M-H, Fixed, 95\% CI) & $0.0[0.0,0.0]$ \\
\hline $\begin{array}{l}7.3 \text { Unable to sleep on } \\
\text { fractured side at } 1 \text { year }\end{array}$ & 1 & & Risk Ratio (M-H, Fixed, 95\% CI) & $0.0[0.0,0.0]$ \\
\hline $\begin{array}{l}7.4 \text { Unable to carry } 5 \mathrm{~kg} \text { at } 1 \\
\text { year }\end{array}$ & 1 & & Risk Ratio (M-H, Fixed, 95\% CI) & $0.0[0.0,0.0]$ \\
\hline $\begin{array}{l}7.5 \text { Unable to manage } \\
\text { personal hygiene at } 50 \text { months }\end{array}$ & 1 & & Risk Ratio (M-H, Fixed, 95\% CI) & $0.0[0.0,0.0]$ \\
\hline $\begin{array}{l}\text { 7.6 Unable to comb hair at } 50 \\
\text { months }\end{array}$ & 1 & & Risk Ratio (M-H, Fixed, 95\% CI) & $0.0[0.0,0.0]$ \\
\hline
\end{tabular}

Interventions for treating proximal humeral fractures in adults (Review)

Copyright $\odot 2015$ The Cochrane Collaboration. Published by John Wiley \& Sons, Ltd. 
7.7 Unable to sleep on fractured side at 50 months

7.8 Unable to carry $5 \mathrm{~kg}$ at 50 months

8 Quality of life assessment:

EuroQol (0: dead to 1: best

health)

8.1 at 3 to 4 months

8.2 at 6 months

8.3 at 12 months

8.4 at 24 months

9 Quality of life assessment

(Fjalestad 2010 and 2014 data)

$9.115 \mathrm{D}$ at 3 months (0:

death; 1 : perfect health)

$9.215 \mathrm{D}$ at 6 months

$9.315 \mathrm{D}$ at 12 months

9.4 number of QALYs at 1

year

9.5 numbers of QALYs at 1

year (- deaths)

$9.615 \mathrm{D}$ at 24 months

10 Quality of life: SF-12 Physical

Component Score (0 to 100 :

best)

10.1 at 6 months

10.2 at 12 months

10.3 at 24 months

11 Quality of life: SF-12 Mental

Component Score (0 to 100 :

best)

11.1 at 6 months

11.2 at 12 months

11.3 at 24 months

12 Mortality

13 Additional surgery

(re-operation or secondary

surgery)

13.1 at 6 to 12 months

13.2 at 2 years

14 Adverse events / complications

14.1 Number of patients with complications

14.2 Additional

shoulder-related therapy

14.3 Infection

14.4 Nerve injury / palsy

14.5 Non-union

14.6 Avascular necrosis

14.7 Symptomatic malunion

14.8 Screw penetration into joint
Mean Difference (IV, Fixed, 95\% CI)

Mean Difference (IV, Fixed, 95\% CI)

Mean Difference (IV, Fixed, 95\% CI)

Mean Difference (IV, Fixed, 95\% CI)

Mean Difference (IV, Fixed, 95\% CI)

Mean Difference (IV, Fixed, 95\% CI)

Mean Difference (IV, Fixed, 95\% CI)

Mean Difference (IV, Fixed, 95\% CI)

Mean Difference (IV, Fixed, 95\% CI)

Mean Difference (IV, Fixed, 95\% CI)

Mean Difference (IV, Fixed, 95\% CI)

Mean Difference (IV, Fixed, 95\% CI)

Mean Difference (IV, Fixed, 95\% CI)

216

218

210

Mean Difference (IV, Fixed, 95\% CI)

Mean Difference (IV, Fixed, 95\% CI)

Mean Difference (IV, Fixed, 95\% CI)

Mean Difference (IV, Fixed, 95\% CI)
Mean Difference (IV, Fixed, 95\% CI)

Mean Difference (IV, Fixed, 95\% CI)

Mean Difference (IV, Fixed, 95\% CI)

Risk Ratio (M-H, Fixed, 95\% CI)

Risk Ratio (M-H, Fixed, 95\% CI)

Risk Ratio (M-H, Fixed, 95\% CI)

Risk Ratio (M-H, Fixed, 95\% CI)

Risk Ratio (M-H, Fixed, 95\% CI)

Risk Ratio (M-H, Fixed, 95\% CI)

Risk Ratio (M-H, Fixed, 95\% CI)

Risk Ratio (M-H, Fixed, 95\% CI)

Risk Ratio (M-H, Fixed, 95\% CI)

Risk Ratio (M-H, Fixed, 95\% CI)

Risk Ratio (M-H, Fixed, 95\% CI)

Risk Ratio (M-H, Fixed, 95\% CI)

Risk Ratio (M-H, Fixed, 95\% CI)
$0.0[0.0,0.0]$

$0.0[0.0,0.0]$

Subtotals only

$0.01[-0.02,0.04]$

0.04 [0.01, 0.08]

$0.02[-0.02,0.06]$

$0.03[-0.01,0.08]$

Totals not selected

$0.0[0.0,0.0]$

$0.0[0.0,0.0]$

$0.0[0.0,0.0]$

$0.0[0.0,0.0]$

$0.0[0.0,0.0]$

$0.0[0.0,0.0]$

Subtotals only

$2.60[-0.24,5.44]$

$1.5[-1.42,4.42]$

$1.10[-1.99,4.19]$

Subtotals only

$-0.60[-3.57,2.37]$

$-2.0[-4.81,0.81]$

$-1.40[-4.33,1.53]$

$1.40[0.69,2.83]$

$2.06[1.18,3.60]$

1.37 [0.32, 5.93]

2.20 [1.20, 4.04]

Subtotals only

$1.30[0.80,2.11]$

$1.75[0.53,5.83]$

$4.31[1.11,16.74]$

$1.16[0.37,3.59]$

$0.43[0.19,0.98]$

$0.83[0.53,1.32]$

$0.8[0.22,2.91]$

$11.49[2.25,58.76]$ 
14.9 Metalwork (internal fixation) problems

14.10 Wire penetration at 1 year

14.11 Redisplacement

resulting in an operation

14.12 Implant-related

(hemiarthroplasty) failure

14.13 Secondary dislocation

or resorption of the greater

tuberosity

14.14 Tuberosity displacement

at 50 months

14.15 Fixation failure

resulting in an operation

14.16 Refracture

14.17 Post-traumatic stiffness

14.18 Impingement

14.19 Rotator cuff tear

14.20 Post-traumatic stiffness

14.21 CRPS or severe pain

14.22 Dislocation or

instability

14.23 Heterotopic ossification

14.24 Post-traumatic

osteoarthritis (signs of)

15 Dependent in activities of daily living (or dead) at 6 months

16 Constant scores (overall: 0 to 100: best score)

16.1 at 3-4 months

16.2 at 12 months

16.3 at 24 months

16.4 at 50 months

17 Constant scores (difference

between injured and uninjured

shoulder): Normal $=0$.

17.1 at 6 months

17.2 at 12 months

17.3 at 24 months

18 Poor or unsatisfactory function at 1 year (Neer rating)

19 VAS disability (0 to 100 : no restrictions)

19.1 at 3 months

19.2 at 12 months

20 Pain: VAS (0 to 100 : worst

pain)

20.1 At 3 months

20.2 At 2 years
Risk Ratio (M-H, Fixed, 95\% CI)
Risk Ratio (M-H, Fixed, 95\% CI)

Risk Ratio (M-H, Fixed, 95\% CI)

Risk Ratio (M-H, Fixed, 95\% CI)

Risk Ratio (M-H, Fixed, 95\% CI)

Risk Ratio (M-H, Fixed, 95\% CI)

Risk Ratio (M-H, Fixed, 95\% CI)

Risk Ratio (M-H, Fixed, 95\% CI)

Risk Ratio (M-H, Fixed, 95\% CI)

Risk Ratio (M-H, Fixed, 95\% CI)

Risk Ratio (M-H, Fixed, 95\% CI)

Risk Ratio (M-H, Fixed, 95\% CI)

Risk Ratio (M-H, Fixed, 95\% CI)

Mean Difference (IV, Fixed, 95\% CI)

Mean Difference (IV, Fixed, 95\% CI)

Mean Difference (IV, Fixed, 95\% CI)

Mean Difference (IV, Fixed, 95\% CI)

Mean Difference (IV, Fixed, 95\% CI)

Mean Difference (IV, Fixed, 95\% CI)

Mean Difference (IV, Fixed, 95\% CI) Mean Difference (IV, Fixed, 95\% CI) Mean Difference (IV, Fixed, 95\% CI)

Risk Ratio (M-H, Fixed, 95\% CI)

Mean Difference (IV, Fixed, 95\% CI)

Mean Difference (IV, Fixed, 95\% CI)

Mean Difference (IV, Fixed, 95\% CI)

Mean Difference (IV, Fixed, 95\% CI)

49 101
Mean Difference (IV, Fixed, 95\% CI)

Mean Difference (IV, Fixed, 95\% CI)
$21.0[1.24,354.53]$

$3.0[0.13,69.31]$

$0.26[0.03,2.22]$

$4.0[0.45,35.18]$

$13.15[1.78,96.90]$

$0.15[0.01,2.71]$

$3.0[0.13,70.30]$

$1.0[0.07,14.05]$

$1.2[0.38,3.83]$

$1.0[0.18,5.62]$

$3.0[0.48,18.73]$

$1.2[0.38,3.83]$

$2.0[0.18,21.78]$

0.33 [0.01, 8.10]

$0.0[0.0,0.0]$

$0.68[0.27,1.70]$

Totals not selected

Subtotals only

$-2.90[-7.35,1.56]$

$2.81[-2.20,7.82]$

$-0.25[-6.75,6.25]$

$-5.0[-17.52,7.52]$

Totals not selected

$0.0[0.0,0.0]$

$0.0[0.0,0.0]$

$0.0[0.0,0.0]$

Totals not selected

Totals not selected

$0.0[0.0,0.0]$

$0.0[0.0,0.0]$

Subtotals only

$-18.0[-29.03,-6.97]$

$-6.38[-14.18,1.41]$ 
21 Constant score at 50 months: overall and components

21.1 Overall score (0-100:

best score)

21.2 Pain (maximum score

15)

21.3 Range of motion

(maximum score 40)

21.4 Power (maximum score

25)

21.5 Activities of daily living (maximum score 20)

22 Constant (often severe) pain at 6 months

23 Failure to recover $75 \%$ muscle power relative to other arm (survivors) at 6 months

23.1 Flexion

23.2 Abduction

23.3 Lateral rotation

24 Range of movement impairments in survivors at 6 months

24.1 Flexion < 45 degrees

24.2 Unable to place thumb

on mid spine (T12)

24.3 Lateral rotation $<5$ degrees

25 Costs at 1 year (Euros in 2005)

26 Total costs including indirect

costs (Euros) at 1 year
Mean Difference (IV, Fixed, 95\% CI)

Mean Difference (IV, Fixed, 95\% CI)

Mean Difference (IV, Fixed, 95\% CI)

Mean Difference (IV, Fixed, 95\% CI)

Mean Difference (IV, Fixed, 95\% CI)

Mean Difference (IV, Fixed, 95\% CI)

Risk Ratio (M-H, Fixed, 95\% CI)

Risk Ratio (M-H, Fixed, 95\% CI)

Risk Ratio (M-H, Fixed, 95\% CI)

Risk Ratio (M-H, Fixed, 95\% CI)

Risk Ratio (M-H, Fixed, 95\% CI)

Risk Ratio (M-H, Fixed, 95\% CI)

Risk Ratio (M-H, Fixed, 95\% CI)

Risk Ratio (M-H, Fixed, 95\% CI)

Risk Ratio (M-H, Fixed, 95\% CI)

Other data

Mean Difference (IV, Fixed, 95\% CI)
Totals not selected

$0.0[0.0,0.0]$

$0.0[0.0,0.0]$

$0.0[0.0,0.0]$

$0.0[0.0,0.0]$

$0.0[0.0,0.0]$

Totals not selected

Totals not selected

$0.0[0.0,0.0]$

$0.0[0.0,0.0]$

$0.0[0.0,0.0]$

Totals not selected

$0.0[0.0,0.0]$

$0.0[0.0,0.0]$

$0.0[0.0,0.0]$

No numeric data

Totals not selected

Comparison 5. Locking plate versus locking intramedullary nail

\begin{tabular}{|c|c|c|c|c|}
\hline Outcome or subgroup title & $\begin{array}{l}\text { No. of } \\
\text { studies }\end{array}$ & $\begin{array}{c}\text { No. of } \\
\text { participants }\end{array}$ & Statistical method & Effect size \\
\hline $\begin{array}{l}1 \text { American Shoulder and Elbow } \\
\text { Surgeons (ASES) score ( } 0 \text { to } \\
\text { 100: best) }\end{array}$ & 1 & & Mean Difference (IV, Fixed, 95\% CI) & Totals not selected \\
\hline 1.1 At 1 year & 1 & & Mean Difference (IV, Fixed, 95\% CI) & $0.0[0.0,0.0]$ \\
\hline 1.2 At 3 years & 1 & & Mean Difference (IV, Fixed, 95\% CI) & $0.0[0.0,0.0]$ \\
\hline $\begin{array}{l}2 \text { Death, re-operation and adverse } \\
\text { events }\end{array}$ & 1 & & Risk Ratio (M-H, Fixed, 95\% CI) & Totals not selected \\
\hline 2.1 Death & 1 & & Risk Ratio (M-H, Fixed, 95\% CI) & $0.0[0.0,0.0]$ \\
\hline 2.2 Any complication & 1 & & Risk Ratio (M-H, Fixed, 95\% CI) & $0.0[0.0,0.0]$ \\
\hline $\begin{array}{l}2.3 \text { Screw penetration } \\
\text { into humeral head (all had } \\
\text { re-operation) }\end{array}$ & 1 & & Risk Ratio (M-H, Fixed, 95\% CI) & $0.0[0.0,0.0]$ \\
\hline
\end{tabular}

Interventions for treating proximal humeral fractures in adults (Review)

Copyright $\odot 2015$ The Cochrane Collaboration. Published by John Wiley \& Sons, Ltd. 


2.4 Heterotopic ossification
2.5 Infection
2.6 Osteonecrosis
2.7 Degenerative change of
glenohumeral joint
2.8 Secondary varus collapse
2.9 Non-union
3 Pain (VAS: 0 to 10: worst)
4 Constant score (0 to 100: best)
4.1 At 1 year
4.2 At 3 year
5 Active range of motion (at 3
years)
5.1 Forward elevation
(degrees)
5.2 External rotation
6 Range of movement: internal
rotation (level on spine)
7 Strength of suprapinatus (relative
to opposite side) \% - at 3 years
7.1 At 1 year
7.2 At 3 years
8 Operation times and blood loss
8.1 Duration of surgery
(minutes)
8.2 Blood loss (ml)
9.1 Pneumothorax
9.2 Blood transfusion

Risk Ratio (M-H, Fixed, 95\% CI)

Risk Ratio (M-H, Fixed, 95\% CI)

Risk Ratio (M-H, Fixed, 95\% CI)

Risk Ratio (M-H, Fixed, 95\% CI)

Risk Ratio (M-H, Fixed, 95\% CI)

Risk Ratio (M-H, Fixed, 95\% CI)

Other data

Mean Difference (IV, Fixed, 95\% CI)

Mean Difference (IV, Fixed, 95\% CI)

Mean Difference (IV, Fixed, 95\% CI)

Mean Difference (IV, Fixed, 95\% CI)

Mean Difference (IV, Fixed, 95\% CI)

Mean Difference (IV, Fixed, 95\% CI)

Other data

Mean Difference (IV, Fixed, 95\% CI)

Mean Difference (IV, Fixed, 95\% CI)

Mean Difference (IV, Fixed, 95\% CI)

Mean Difference (IV, Fixed, 95\% CI)

Mean Difference (IV, Fixed, 95\% CI)

Mean Difference (IV, Fixed, 95\% CI)

Risk Ratio (M-H, Fixed, 95\% CI)

Risk Ratio (M-H, Fixed, 95\% CI)

Risk Ratio (M-H, Fixed, 95\% CI)
$0.0[0.0,0.0]$

$0.0[0.0,0.0]$

$0.0[0.0,0.0]$

$0.0[0.0,0.0]$

$0.0[0.0,0.0]$

$0.0[0.0,0.0]$

No numeric data

Totals not selected

$0.0[0.0,0.0]$

$0.0[0.0,0.0]$

Totals not selected

$0.0[0.0,0.0]$

$0.0[0.0,0.0]$

No numeric data

Totals not selected

$0.0[0.0,0.0]$

$0.0[0.0,0.0]$

Totals not selected $0.0[0.0,0.0]$

$0.0[0.0,0.0]$

Totals not selected $0.0[0.0,0.0]$ $0.0[0.0,0.0]$

Comparison 6. Locking plate versus intramedullary nails (Zifko method)

\begin{tabular}{|c|c|c|c|c|}
\hline Outcome or subgroup title & $\begin{array}{l}\text { No. of } \\
\text { studies }\end{array}$ & $\begin{array}{c}\text { No. of } \\
\text { participants }\end{array}$ & Statistical method & Effect size \\
\hline $\begin{array}{l}1 \text { Complications and [slight] } \\
\text { malunion }\end{array}$ & 1 & & Risk Ratio (M-H, Fixed, 95\% CI) & Totals not selected \\
\hline 1.1 Any complication & 1 & & Risk Ratio (M-H, Fixed, 95\% CI) & $0.0[0.0,0.0]$ \\
\hline 1.2 Malunion (usually slight) & 1 & & Risk Ratio (M-H, Fixed, 95\% CI) & $0.0[0.0,0.0]$ \\
\hline $\begin{array}{l}2 \text { Constant score ( } \% \text { of healthy } \\
\text { limb) at mean } 2 \text { years }\end{array}$ & 1 & & Mean Difference (IV, Fixed, 95\% CI) & Totals not selected \\
\hline $\begin{array}{l}3 \text { Time to union and time to } \\
\text { recover upper limb function } \\
\text { (weeks) }\end{array}$ & 1 & & Mean Difference (IV, Fixed, 95\% CI) & Totals not selected \\
\hline $\begin{array}{l}3.1 \text { Time to radiographic } \\
\text { union }\end{array}$ & 1 & & Mean Difference (IV, Fixed, 95\% CI) & $0.0[0.0,0.0]$ \\
\hline $\begin{array}{l}\text { 3.2 Time to recover normal } \\
\text { upper limb function }\end{array}$ & 1 & & Mean Difference (IV, Fixed, 95\% CI) & $0.0[0.0,0.0]$ \\
\hline
\end{tabular}

Interventions for treating proximal humeral fractures in adults (Review)

Copyright $\odot 2015$ The Cochrane Collaboration. Published by John Wiley \& Sons, Ltd. 


\begin{tabular}{llll}
$\begin{array}{l}\text { 4 Operation and fluoroscopic } \\
\text { times }\end{array}$ & 1 & Mean Difference (IV, Fixed, 95\% CI) & Totals not selected \\
$\begin{array}{l}\text { 4.1 Duration of operation } \\
\text { (minutes) }\end{array}$ & 1 & Mean Difference (IV, Fixed, 95\% CI) & $0.0[0.0,0.0]$ \\
$\begin{array}{l}\text { 4.2 X-ray exposure (minutes) } \\
\text { 5 Length of hospital stay (days) }\end{array}$ & 1 & $\begin{array}{l}\text { Mean Difference (IV, Fixed, 95\% CI) } \\
\text { Mean Difference (IV, Fixed, 95\% CI) }\end{array}$ & $\begin{array}{l}0.0[0.0,0.0] \\
\text { Totals not selected }\end{array}$ \\
\hline
\end{tabular}

Comparison 7. Replacement (hemiarthroplasty) versus fixation (tension band wiring; plate fixation) (4 part fractures)

\begin{tabular}{|c|c|c|c|c|}
\hline Outcome or subgroup title & $\begin{array}{l}\text { No. of } \\
\text { studies }\end{array}$ & $\begin{array}{c}\text { No. of } \\
\text { participants }\end{array}$ & Statistical method & Effect size \\
\hline $\begin{array}{l}1 \text { DASH score (0 to } 100: \text { worst } \\
\text { disability) }\end{array}$ & 1 & & Mean Difference (IV, Fixed, 95\% CI) & Totals not selected \\
\hline 1.1 At 4 months & 1 & & Mean Difference (IV, Fixed, 95\% CI) & $0.0[0.0,0.0]$ \\
\hline 1.2 At 12 months & 1 & & Mean Difference (IV, Fixed, 95\% CI) & $0.0[0.0,0.0]$ \\
\hline 1.3 At 24 months & 1 & & Mean Difference (IV, Fixed, 95\% CI) & $0.0[0.0,0.0]$ \\
\hline $\begin{array}{l}2 \text { EQ-5D score ( } 0 \text { to } 1 \text { : best } \\
\text { quality of life) }\end{array}$ & 1 & & Mean Difference (IV, Fixed, 95\% CI) & Totals not selected \\
\hline 2.1 At 4 months & 1 & & Mean Difference (IV, Fixed, 95\% CI) & $0.0[0.0,0.0]$ \\
\hline 2.2 At 12 months & 1 & & Mean Difference (IV, Fixed, 95\% CI) & $0.0[0.0,0.0]$ \\
\hline 2.3 At 24 months & 1 & & Mean Difference (IV, Fixed, 95\% CI) & $0.0[0.0,0.0]$ \\
\hline 3 Re-operation & 2 & 62 & Risk Ratio (M-H, Fixed, 95\% CI) & $0.32[0.10,1.10]$ \\
\hline $\begin{array}{l}3.1 \text { Hemiarthroplasty versus } \\
\text { tension band wiring }\end{array}$ & 1 & 30 & Risk Ratio (M-H, Fixed, 95\% CI) & $0.09[0.01,1.51]$ \\
\hline $\begin{array}{l}3.2 \text { Hemiarthroplasty versus } \\
\text { locking plate fixation }\end{array}$ & 1 & 32 & Risk Ratio (M-H, Fixed, 95\% CI) & $0.68[0.16,2.88]$ \\
\hline 4 Dead at 2 years & 1 & & Risk Ratio (M-H, Fixed, 95\% CI) & Totals not selected \\
\hline 5 Implant removal at 1 year & 1 & & Risk Ratio (M-H, Fixed, 95\% CI) & Totals not selected \\
\hline $\begin{array}{l}6 \text { Constant score ( } 0 \text { to } 100 \text { : best } \\
\text { score) }\end{array}$ & 1 & & Mean Difference (IV, Fixed, 95\% CI) & Totals not selected \\
\hline 6.1 At 4 months & 1 & & Mean Difference (IV, Fixed, 95\% CI) & $0.0[0.0,0.0]$ \\
\hline 6.2 At 12 months & 1 & & Mean Difference (IV, Fixed, 95\% CI) & $0.0[0.0,0.0]$ \\
\hline 6.3 At 24 months & 1 & & Mean Difference (IV, Fixed, 95\% CI) & $0.0[0.0,0.0]$ \\
\hline $\begin{array}{l}7 \text { Pain VAS ( } 0 \text { to } 100 \text { : worst pain) } \\
\text { at } 24 \text { months }\end{array}$ & 1 & & Mean Difference (IV, Fixed, 95\% CI) & Totals not selected \\
\hline 8 Pain at 1 year & 1 & & Risk Ratio (M-H, Fixed, 95\% CI) & Totals not selected \\
\hline 9 Range of motion at 24 months & 1 & & Mean Difference (IV, Fixed, 95\% CI) & Totals not selected \\
\hline 9.1 Flexion (degrees) & 1 & & Mean Difference (IV, Fixed, 95\% CI) & $0.0[0.0,0.0]$ \\
\hline 9.2 Extension (degrees) & 1 & & Mean Difference (IV, Fixed, 95\% CI) & $0.0[0.0,0.0]$ \\
\hline
\end{tabular}




\begin{tabular}{|c|c|c|c|c|}
\hline Outcome or subgroup title & $\begin{array}{l}\text { No. of } \\
\text { studies }\end{array}$ & $\begin{array}{c}\text { No. of } \\
\text { participants }\end{array}$ & Statistical method & Effect size \\
\hline $\begin{array}{l}1 \text { Shoulder function scores at } 24 \\
\text { to } 49 \text { months }\end{array}$ & 1 & & Mean Difference (IV, Fixed, 95\% CI) & Totals not selected \\
\hline $\begin{array}{l}\text { 1.1 Quick DASH score (0 to } \\
\text { 55: worst outcome) }\end{array}$ & 1 & & Mean Difference (IV, Fixed, 95\% CI) & $0.0[0.0,0.0]$ \\
\hline 2 Re-operation & 1 & & Risk Ratio (M-H, Fixed, 95\% CI) & Totals not selected \\
\hline 3 Death & 1 & 62 & Risk Ratio (M-H, Fixed, 95\% CI) & $0.0[0.0,0.0]$ \\
\hline $\begin{array}{l}4 \text { Composite (objective and } \\
\text { subjective) shoulder function } \\
\text { scores at } 24 \text { to } 49 \text { months }\end{array}$ & 1 & & Mean Difference (IV, Fixed, 95\% CI) & Totals not selected \\
\hline $\begin{array}{l}\text { 4.1 UCLA score ( } 0 \text { to } 35 \text { : best } \\
\text { outcome) }\end{array}$ & 1 & & Mean Difference (IV, Fixed, 95\% CI) & $0.0[0.0,0.0]$ \\
\hline $\begin{array}{l}4.2 \text { Constant score ( } 0 \text { to } 100 \text { : } \\
\text { best outcome) }\end{array}$ & 1 & & Mean Difference (IV, Fixed, 95\% CI) & $0.0[0.0,0.0]$ \\
\hline $\begin{array}{l}4.3 \text { Constant } \% \text { relative to } \\
\text { opposite side }\end{array}$ & 1 & & Mean Difference (IV, Fixed, 95\% CI) & $0.0[0.0,0.0]$ \\
\hline $\begin{array}{l}5 \text { Constant score at } 24 \text { to } \\
49 \text { months: overall and } \\
\text { components }\end{array}$ & 1 & & Mean Difference (IV, Fixed, 95\% CI) & Totals not selected \\
\hline $\begin{array}{l}5.1 \text { Overall score }(0-100 \text { : best } \\
\text { score) }\end{array}$ & 1 & & Mean Difference (IV, Fixed, 95\% CI) & $0.0[0.0,0.0]$ \\
\hline 5.2 Pain (maximum score 15) & 1 & & Mean Difference (IV, Fixed, 95\% CI) & $0.0[0.0,0.0]$ \\
\hline $\begin{array}{l}5.3 \text { Range of motion } \\
\text { (maximum score } 40 \text { ) }\end{array}$ & 1 & & Mean Difference (IV, Fixed, 95\% CI) & $0.0[0.0,0.0]$ \\
\hline $\begin{array}{l}\text { 5.4 Power (maximum score } \\
\text { 25) }\end{array}$ & 1 & & Mean Difference (IV, Fixed, 95\% CI) & $0.0[0.0,0.0]$ \\
\hline $\begin{array}{l}5.5 \text { Activities of daily living } \\
\text { (maximum score } 20 \text { ) }\end{array}$ & 1 & & Mean Difference (IV, Fixed, 95\% CI) & $0.0[0.0,0.0]$ \\
\hline 6 Complications & 1 & & Risk Ratio (M-H, Fixed, 95\% CI) & Totals not selected \\
\hline 6.1 Any complication & 1 & & Risk Ratio (M-H, Fixed, 95\% CI) & $0.0[0.0,0.0]$ \\
\hline 6.2 Intra-operative fracture & 1 & & Risk Ratio (M-H, Fixed, 95\% CI) & $0.0[0.0,0.0]$ \\
\hline 6.3 Deep infection & 1 & & Risk Ratio (M-H, Fixed, 95\% CI) & $0.0[0.0,0.0]$ \\
\hline 6.4 Superficial infection & 1 & & Risk Ratio (M-H, Fixed, 95\% CI) & $0.0[0.0,0.0]$ \\
\hline 6.5 Haematoma & 1 & & Risk Ratio (M-H, Fixed, 95\% CI) & $0.0[0.0,0.0]$ \\
\hline $\begin{array}{l}\text { 6.6 Neurological } \\
\text { complications }\end{array}$ & 1 & & Risk Ratio (M-H, Fixed, 95\% CI) & $0.0[0.0,0.0]$ \\
\hline 6.7 Severe stiffness & 1 & & Risk Ratio (M-H, Fixed, 95\% CI) & $0.0[0.0,0.0]$ \\
\hline $\begin{array}{l}\text { 6.8 Proximal migration of } \\
\text { implant }\end{array}$ & 1 & & Risk Ratio (M-H, Fixed, 95\% CI) & $0.0[0.0,0.0]$ \\
\hline 7 Radiological assessment findings & 1 & & Risk Ratio (M-H, Fixed, 95\% CI) & Totals not selected \\
\hline 7.1 Malunion of tuberosities & 1 & & Risk Ratio (M-H, Fixed, 95\% CI) & $0.0[0.0,0.0]$ \\
\hline 7.2 Resorption of tuberosities & 1 & & Risk Ratio (M-H, Fixed, 95\% CI) & $0.0[0.0,0.0]$ \\
\hline 7.3 Scapular notching & 1 & & Risk Ratio (M-H, Fixed, 95\% CI) & $0.0[0.0,0.0]$ \\
\hline 7.4 Heterotopic ossification & 1 & & Risk Ratio (M-H, Fixed, 95\% CI) & $0.0[0.0,0.0]$ \\
\hline $\begin{array}{l}8 \text { Range of motion (degrees) at } 24 \\
\text { to } 49 \text { months }\end{array}$ & 1 & & Mean Difference (IV, Fixed, 95\% CI) & Totals not selected \\
\hline 8.1 Anterior forward & 1 & & Mean Difference (IV, Fixed, 95\% CI) & $0.0[0.0,0.0]$ \\
\hline
\end{tabular}

Interventions for treating proximal humeral fractures in adults (Review)

Copyright $\odot 2015$ The Cochrane Collaboration. Published by John Wiley \& Sons, Ltd. 


\begin{tabular}{|c|c|c|c|c|}
\hline Outcome or subgroup title & $\begin{array}{l}\text { No. of } \\
\text { studies }\end{array}$ & $\begin{array}{c}\text { No. of } \\
\text { participants }\end{array}$ & Statistical method & Effect size \\
\hline 1 Re-operation & 1 & & Risk Ratio (M-H, Fixed, 95\% CI) & Totals not selected \\
\hline 1.1 For complication or a fall & 1 & & Risk Ratio (M-H, Fixed, 95\% CI) & $0.0[0.0,0.0]$ \\
\hline $\begin{array}{l}1.2 \text { Plate removal by patient } \\
\text { request }\end{array}$ & 1 & & Risk Ratio (M-H, Fixed, 95\% CI) & $0.0[0.0,0.0]$ \\
\hline 2 Dead at 1 year & 1 & & Risk Ratio (M-H, Fixed, 95\% CI) & Totals not selected \\
\hline 3 Complications & 1 & & Risk Ratio (M-H, Fixed, 95\% CI) & Totals not selected \\
\hline 3.1 Injurious fall on shoulder & 1 & & Risk Ratio (M-H, Fixed, 95\% CI) & $0.0[0.0,0.0]$ \\
\hline 3.2 Axillary nerve damage & 1 & & Risk Ratio (M-H, Fixed, 95\% CI) & $0.0[0.0,0.0]$ \\
\hline 3.3 Screw perforation & 1 & & Risk Ratio (M-H, Fixed, 95\% CI) & $0.0[0.0,0.0]$ \\
\hline 3.4 Implant (head or shaft) & 1 & & Risk Ratio (M-H, Fixed, 95\% CI) & $0.0[0.0,0.0]$ \\
\hline \multicolumn{5}{|l|}{ loosening } \\
\hline 3.5 Deep infection & 1 & & Risk Ratio (M-H, Fixed, 95\% CI) & $0.0[0.0,0.0]$ \\
\hline 3.6 Humeral head necrosis & 1 & & Risk Ratio (M-H, Fixed, 95\% CI) & $0.0[0.0,0.0]$ \\
\hline $\begin{array}{l}4 \text { Constant score (0 to } 100 \text { : best } \\
\text { score) }\end{array}$ & 1 & & Mean Difference (IV, Fixed, 95\% CI) & Totals not selected \\
\hline 4.1 At 6 months & 1 & & Mean Difference (IV, Fixed, 95\% CI) & $0.0[0.0,0.0]$ \\
\hline 4.2 At 12 months & 1 & & Mean Difference (IV, Fixed, 95\% CI) & $0.0[0.0,0.0]$ \\
\hline $\begin{array}{l}5 \text { Pain (VAS } 0 \text { to } 10 \text { : intolerable } \\
\text { pain) }\end{array}$ & 1 & & Mean Difference (IV, Fixed, 95\% CI) & Totals not selected \\
\hline 5.1 At 6 months & 1 & & Mean Difference (IV, Fixed, 95\% CI) & $0.0[0.0,0.0]$ \\
\hline 5.2 At 12 months & 1 & & Mean Difference (IV, Fixed, 95\% CI) & $0.0[0.0,0.0]$ \\
\hline $\begin{array}{l}6 \text { Operation and fluoroscopic } \\
\text { times }\end{array}$ & 1 & & Mean Difference (IV, Fixed, 95\% CI) & Totals not selected \\
\hline $\begin{array}{l}\text { 6.1 Duration of operation } \\
\text { (minutes) }\end{array}$ & 1 & & Mean Difference (IV, Fixed, 95\% CI) & $0.0[0.0,0.0]$ \\
\hline $6.2 \mathrm{X}$-ray exposure (minutes) & 1 & & Mean Difference (IV, Fixed, 95\% CI) & $0.0[0.0,0.0]$ \\
\hline 7 Length of hospital stay (days) & 1 & & Mean Difference (IV, Fixed, 95\% CI) & Totals not selected \\
\hline
\end{tabular}

Comparison 10. Polyaxial versus monoaxial screw insertion in plate fixation

\begin{tabular}{|c|c|c|c|c|}
\hline Outcome or subgroup title & $\begin{array}{l}\text { No. of } \\
\text { studies }\end{array}$ & $\begin{array}{c}\text { No. of } \\
\text { participants }\end{array}$ & Statistical method & Effect size \\
\hline $\begin{array}{l}1 \text { DASH score at } 12 \text { months ( } 0 \text { to } \\
\text { 100: greatest disability) }\end{array}$ & 1 & & Mean Difference (IV, Fixed, 95\% CI) & Totals not selected \\
\hline $\begin{array}{l}2 \text { Simple shoulder test ( } 0 \text { to } 12 \text { : } \\
\text { best outcome) }\end{array}$ & 1 & & Mean Difference (IV, Fixed, 95\% CI) & Totals not selected \\
\hline 2.1 At 3 months & 1 & & Mean Difference (IV, Fixed, 95\% CI) & $0.0[0.0,0.0]$ \\
\hline 2.2 At 6 months & 1 & & Mean Difference (IV, Fixed, 95\% CI) & $0.0[0.0,0.0]$ \\
\hline 2.3 At 12 months & 1 & & Mean Difference (IV, Fixed, 95\% CI) & $0.0[0.0,0.0]$ \\
\hline
\end{tabular}




\begin{tabular}{|c|}
\hline 3 Re-operation \\
\hline 3.1 By 6 months \\
\hline 3.2 By 1 year \\
\hline 4 Dead at 1 year \\
\hline $\begin{array}{l}5 \text { Constant score at } 12 \text { months (\% } \\
\text { of contralateral limb) }\end{array}$ \\
\hline $\begin{array}{l}6 \text { Complications (radiological } \\
\text { assessment) }\end{array}$ \\
\hline 6.1 Any complication \\
\hline $\begin{array}{l}\text { 6.2 Primary implant } \\
\text { malposition }\end{array}$ \\
\hline $\begin{array}{l}6.3 \text { Secondary loss of } \\
\text { reduction and screw perforation }\end{array}$ \\
\hline $\begin{array}{l}\text { 6.4 Non-union / delayed } \\
\text { union due to osteonecrosis ( } 6 \\
\text { months) }\end{array}$ \\
\hline $\begin{array}{l}6.5 \text { Avascular necrosis at } 1 \\
\text { year }\end{array}$ \\
\hline $\begin{array}{l}6.6 \text { Varus deformity }(>10 / \\
\geq 20 \text { degrees) }\end{array}$ \\
\hline $\begin{array}{l}\text { 6.7 Greater tuberosity } \\
\text { displacement }\end{array}$ \\
\hline $\begin{array}{l}\text { 6.8 Screw cut-out } \\
\text { (intra-articular) }\end{array}$ \\
\hline $\begin{array}{l}7 \text { Range of motion (degrees) at } 12 \\
\text { months }\end{array}$ \\
\hline 7.1 Flexion \\
\hline 7.2 Abduction \\
\hline 7.3 External rotation \\
\hline 7.4 Internal rotation \\
\hline $\begin{array}{l}8 \text { Operation and fluoroscopic } \\
\text { times }\end{array}$ \\
\hline $\begin{array}{l}\text { 8.1 Duration of operation } \\
\text { (minutes) }\end{array}$ \\
\hline $\begin{array}{l}8.2 \text { Fluoroscopic time } \\
\text { (minutes) }\end{array}$ \\
\hline
\end{tabular}

Risk Ratio (M-H, Fixed, 95\% CI)

66

Risk Ratio (M-H, Fixed, 95\% CI)

180 Risk Ratio (M-H, Fixed, 95\% CI)

Risk Ratio (M-H, Fixed, 95\% CI)

Mean Difference (IV, Fixed, 95\% CI)

Risk Ratio (M-H, Fixed, 95\% CI)

Risk Ratio (M-H, Fixed, 95\% CI)

Risk Ratio (M-H, Fixed, 95\% CI)

Risk Ratio (M-H, Fixed, 95\% CI)

Risk Ratio (M-H, Fixed, 95\% CI)

Risk Ratio (M-H, Fixed, 95\% CI)

Risk Ratio (M-H, Fixed, 95\% CI)

Risk Ratio (M-H, Fixed, 95\% CI)

Risk Ratio (M-H, Fixed, 95\% CI)

Mean Difference (IV, Fixed, 95\% CI)

Mean Difference (IV, Fixed, 95\% CI)

Mean Difference (IV, Fixed, 95\% CI)

Mean Difference (IV, Fixed, 95\% CI)

Mean Difference (IV, Fixed, 95\% CI)

Mean Difference (IV, Fixed, 95\% CI)

Mean Difference (IV, Fixed, 95\% CI)

Mean Difference (IV, Fixed, 95\% CI)
Subtotals only

$0.85[0.15,4.76]$

1.10 [0.58, 2.08]

Totals not selected

Totals not selected

Totals not selected

$0.0[0.0,0.0]$

$0.0[0.0,0.0]$

$0.0[0.0,0.0]$

$0.0[0.0,0.0]$

$0.0[0.0,0.0]$

$0.0[0.0,0.0]$

$0.0[0.0,0.0]$

$0.0[0.0,0.0]$

Totals not selected

$0.0[0.0,0.0]$

$0.0[0.0,0.0]$

$0.0[0.0,0.0]$

$0.0[0.0,0.0]$

Totals not selected

$0.0[0.0,0.0]$

$0.0[0.0,0.0]$

\section{Comparison 11. Medial support screws versus control for locking plate fixation}

\begin{tabular}{|c|c|c|c|c|}
\hline Outcome or subgroup title & $\begin{array}{l}\text { No. of } \\
\text { studies }\end{array}$ & $\begin{array}{c}\text { No. of } \\
\text { participants }\end{array}$ & Statistical method & Effect size \\
\hline 1 Adverse events & 1 & & Risk Ratio (M-H, Fixed, 95\% CI) & Totals not selected \\
\hline 1.1 Early loss of fixation & 1 & & Risk Ratio (M-H, Fixed, 95\% CI) & $0.0[0.0,0.0]$ \\
\hline $\begin{array}{l}1.2 \text { Re-operation for early } \\
\text { failure }\end{array}$ & 1 & & Risk Ratio (M-H, Fixed, 95\% CI) & $0.0[0.0,0.0]$ \\
\hline $\begin{array}{l}1.3 \text { Osteonecrosis } \\
\text { (asymptomatic) }\end{array}$ & 1 & & Risk Ratio (M-H, Fixed, 95\% CI) & $0.0[0.0,0.0]$ \\
\hline
\end{tabular}

Interventions for treating proximal humeral fractures in adults (Review)

Copyright $\odot 2015$ The Cochrane Collaboration. Published by John Wiley \& Sons, Ltd. 


\section{Comparison 12. MultiLoc Proximal Humeral Nail (MPHN) versus Polarus nail}

\begin{tabular}{|c|c|c|c|c|}
\hline Outcome or subgroup title & $\begin{array}{l}\text { No. of } \\
\text { studies }\end{array}$ & $\begin{array}{c}\text { No. of } \\
\text { participants }\end{array}$ & Statistical method & Effect size \\
\hline 1 Adverse events & 1 & & Risk Ratio (M-H, Fixed, 95\% CI) & Totals not selected \\
\hline 1.1 Re-operation & 1 & & Risk Ratio (M-H, Fixed, 95\% CI) & $0.0[0.0,0.0]$ \\
\hline 1.2 Post-op impingement & 1 & & Risk Ratio (M-H, Fixed, 95\% CI) & $0.0[0.0,0.0]$ \\
\hline 1.3 Screw loosening & 1 & & Risk Ratio (M-H, Fixed, 95\% CI) & $0.0[0.0,0.0]$ \\
\hline 1.4 Non-union & 1 & & Risk Ratio (M-H, Fixed, 95\% CI) & $0.0[0.0,0.0]$ \\
\hline 1.5 Rotator cuff symptoms & 1 & & Risk Ratio (M-H, Fixed, 95\% CI) & $0.0[0.0,0.0]$ \\
\hline $\begin{array}{l}\text { 1.6 Intra-operative } \\
\text { complications }\end{array}$ & 1 & & Risk Ratio (M-H, Fixed, 95\% CI) & $0.0[0.0,0.0]$ \\
\hline 1.7 Mortality & 1 & & Risk Ratio (M-H, Fixed, 95\% CI) & $0.0[0.0,0.0]$ \\
\hline 1.8 Radiographic malunion & 1 & & Risk Ratio (M-H, Fixed, 95\% CI) & $0.0[0.0,0.0]$ \\
\hline $\begin{array}{l}2 \text { Constant score ( } 0 \text { to } 100 \text { : best } \\
\text { outcome) at } 14 \text { months ( } 6 \text { to } \\
22 \text { months) }\end{array}$ & 1 & & Mean Difference (IV, Fixed, 95\% CI) & Totals not selected \\
\hline $\begin{array}{l}2.1 \text { Unadjusted Constant } \\
\text { score }\end{array}$ & 1 & & Mean Difference (IV, Fixed, 95\% CI) & $0.0[0.0,0.0]$ \\
\hline 2.2 Adjusted Constant score & 1 & & Mean Difference (IV, Fixed, 95\% CI) & $0.0[0.0,0.0]$ \\
\hline $\begin{array}{l}3 \text { Range of shoulder motion } \\
\text { (degrees) }\end{array}$ & 1 & & Mean Difference (IV, Fixed, 95\% CI) & Totals not selected \\
\hline 3.1 Lateral elevation & 1 & & Mean Difference (IV, Fixed, 95\% CI) & $0.0[0.0,0.0]$ \\
\hline 3.2 Forward flexion & 1 & & Mean Difference (IV, Fixed, 95\% CI) & $0.0[0.0,0.0]$ \\
\hline 3.3 External rotation & 1 & & Mean Difference (IV, Fixed, 95\% CI) & $0.0[0.0,0.0]$ \\
\hline $\begin{array}{l}4 \text { Lengths of surgery and hospital } \\
\text { stay }\end{array}$ & 1 & & Mean Difference (IV, Fixed, 95\% CI) & Totals not selected \\
\hline $\begin{array}{l}4.1 \text { Length of surgery } \\
\text { (minutes) }\end{array}$ & 1 & & Mean Difference (IV, Fixed, 95\% CI) & $0.0[0.0,0.0]$ \\
\hline $\begin{array}{l}4.2 \text { Length of hospital stay } \\
\text { (days) }\end{array}$ & 1 & & Mean Difference (IV, Fixed, 95\% CI) & $0.0[0.0,0.0]$ \\
\hline
\end{tabular}

Comparison 13. Hemiarthoplasty: EPOCA prosthesis versus HAS prosthesis

\begin{tabular}{|c|c|c|c|c|}
\hline Outcome or subgroup title & $\begin{array}{l}\text { No. of } \\
\text { studies }\end{array}$ & $\begin{array}{c}\text { No. of } \\
\text { participants }\end{array}$ & Statistical method & Effect size \\
\hline 1 Adverse events & 1 & & Risk Ratio (M-H, Fixed, 95\% CI) & Totals not selected \\
\hline 1.1 Deep infection & 1 & & Risk Ratio (M-H, Fixed, 95\% CI) & $0.0[0.0,0.0]$ \\
\hline $\begin{array}{l}1.2 \text { Persistent pain - scheduled } \\
\text { for reoperation }\end{array}$ & 1 & & Risk Ratio (M-H, Fixed, 95\% CI) & $0.0[0.0,0.0]$ \\
\hline 2 Radiological assessment findings & 1 & & Risk Ratio (M-H, Fixed, 95\% CI) & Totals not selected \\
\hline 2.1 Resorption of tuberosities & 1 & & Risk Ratio (M-H, Fixed, 95\% CI) & $0.0[0.0,0.0]$ \\
\hline
\end{tabular}

Interventions for treating proximal humeral fractures in adults (Review)

Copyright $\odot 2015$ The Cochrane Collaboration. Published by John Wiley \& Sons, Ltd. 


$\begin{array}{llll}\begin{array}{l}\text { 2.2 Secondary dislocation of } \\ \text { tuberosities }\end{array} & 1 & \text { Risk Ratio (M-H, Fixed, 95\% CI) } & 0.0[0.0,0.0] \\ \begin{array}{l}2.3 \text { Superior migration of } \\ \text { prosthesis }\end{array} & 1 & \text { Risk Ratio (M-H, Fixed, 95\% CI) } & 0.0[0.0,0.0] \\ 2.4 \text { Anterior subluxations } & 1 & \text { Risk Ratio (M-H, Fixed, 95\% CI) } & 0.0[0.0,0.0] \\ 2.5 \text { Glenoid erosion } & 1 & \text { Risk Ratio (M-H, Fixed, 95\% CI) } & 0.0[0.0,0.0] \\ \begin{array}{l}2.6 \text { Aseptic loosening of stem } \\ \text { Range of motion results at one }\end{array} & 1 & \text { Risk Ratio (M-H, Fixed, 95\% CI) } & 0.0[0.0,0.0] \\ \text { year (degrees) } & \text { Other data } & \text { No numeric data }\end{array}$

Comparison 14. Hemiarthroplasty: tenodesis of long head of biceps (LHB) versus LHB tendon left intact

\begin{tabular}{|c|c|c|c|c|}
\hline Outcome or subgroup title & $\begin{array}{l}\text { No. of } \\
\text { studies }\end{array}$ & $\begin{array}{c}\text { No. of } \\
\text { participants }\end{array}$ & Statistical method & Effect size \\
\hline $\begin{array}{l}1 \text { Complications and further } \\
\text { surgery }\end{array}$ & 1 & & Risk Ratio (M-H, Fixed, 95\% CI) & Totals not selected \\
\hline 1.1 Any complication & 1 & & Risk Ratio (M-H, Fixed, 95\% CI) & $0.0[0.0,0.0]$ \\
\hline $\begin{array}{l}1.2 \text { Further surgery for listed } \\
\text { complications }\end{array}$ & 1 & & Risk Ratio (M-H, Fixed, 95\% CI) & $0.0[0.0,0.0]$ \\
\hline 1.3 Deep infection & 1 & & Risk Ratio (M-H, Fixed, 95\% CI) & $0.0[0.0,0.0]$ \\
\hline 1.4 Tuberosity malunion & 1 & & Risk Ratio (M-H, Fixed, 95\% CI) & $0.0[0.0,0.0]$ \\
\hline $\begin{array}{l}1.5 \text { Inferior subluxation of } \\
\text { prosthesis }\end{array}$ & 1 & & Risk Ratio (M-H, Fixed, 95\% CI) & $0.0[0.0,0.0]$ \\
\hline $\begin{array}{l}\text { 1.6 Loss of reduction of } \\
\text { greater tuberosity }\end{array}$ & 1 & & Risk Ratio (M-H, Fixed, 95\% CI) & $0.0[0.0,0.0]$ \\
\hline $\begin{array}{l}2 \text { Constant score (0 to } 100 \text { : best } \\
\text { function) at } 2 \text { years }\end{array}$ & 1 & & Mean Difference (IV, Fixed, 95\% CI) & Totals not selected \\
\hline 3 Shoulder pain at 2 year follow-up & 1 & & Risk Ratio (M-H, Fixed, 95\% CI) & Totals not selected \\
\hline $\begin{array}{l}4 \text { Active shoulder elevation } \\
\text { (degrees) at } 2 \text { years }\end{array}$ & 1 & & Mean Difference (IV, Fixed, 95\% CI) & Totals not selected \\
\hline
\end{tabular}

\section{Comparison 15. Post-operative (percutaneous fixation) immobilisation for 1 week versus 3 weeks}

\begin{tabular}{|c|c|c|c|c|}
\hline Outcome or subgroup title & $\begin{array}{l}\text { No. of } \\
\text { studies }\end{array}$ & $\begin{array}{c}\text { No. of } \\
\text { participants }\end{array}$ & Statistical method & Effect size \\
\hline $\begin{array}{l}1 \text { Neer score } \leq 80 \text { points } \\
\text { (unsatisfactory or failure) at } 6 \\
\text { months }\end{array}$ & 1 & & Risk Ratio (M-H, Fixed, 95\% CI) & Totals not selected \\
\hline $\begin{array}{l}2 \text { Premature removal of Kirschner } \\
\text { wires }\end{array}$ & 1 & & Risk Ratio (M-H, Fixed, 95\% CI) & Totals not selected \\
\hline
\end{tabular}


Comparison 16. Post-operative (hemiarthroplasty) mobilisation: early ( 2 weeks immobilisation) versus late (6 weeks)

\begin{tabular}{|c|c|c|c|c|}
\hline Outcome or subgroup title & $\begin{array}{l}\text { No. of } \\
\text { studies }\end{array}$ & $\begin{array}{c}\text { No. of } \\
\text { participants }\end{array}$ & Statistical method & Effect size \\
\hline $\begin{array}{l}1 \text { Oxford Shoulder Score at } 1 \text { year } \\
\text { (adjusted: } 0 \text { to } 100 \text { best) }\end{array}$ & 1 & & Mean Difference (IV, Fixed, 95\% CI) & Totals not selected \\
\hline $\begin{array}{l}2 \text { Constant shoulder score (at } 1 \\
\text { year) }\end{array}$ & 1 & & Mean Difference (IV, Fixed, 95\% CI) & Totals not selected \\
\hline $\begin{array}{l}2.1 \text { Overall score ( } 0 \text { to } 100 \text { : } \\
\text { best) }\end{array}$ & 1 & & Mean Difference (IV, Fixed, 95\% CI) & $0.0[0.0,0.0]$ \\
\hline $\begin{array}{l}2.2 \text { Pain component ( } 0 \text { to } 15 \text { : } \\
\text { best)) }\end{array}$ & 1 & & Mean Difference (IV, Fixed, 95\% CI) & $0.0[0.0,0.0]$ \\
\hline $\begin{array}{l}2.3 \text { Activities of daily living } \\
\text { component ( } 0 \text { to } 25 \text { : best) }\end{array}$ & 1 & & Mean Difference (IV, Fixed, 95\% CI) & $0.0[0.0,0.0]$ \\
\hline $\begin{array}{l}2.4 \text { Mobility component ( } 0 \text { to } \\
\text { 40: best) }\end{array}$ & 1 & & Mean Difference (IV, Fixed, 95\% CI) & $0.0[0.0,0.0]$ \\
\hline $\begin{array}{l}2.5 \text { Strength component ( } 0 \text { to } \\
25 \text { : best) }\end{array}$ & 1 & & Mean Difference (IV, Fixed, 95\% CI) & $0.0[0.0,0.0]$ \\
\hline 3 Radiological assessment findings & 1 & & Risk Ratio (M-H, Fixed, 95\% CI) & Totals not selected \\
\hline $\begin{array}{l}3.1 \text { Non-union (with bone } \\
\text { resorption) }\end{array}$ & 1 & & Risk Ratio (M-H, Fixed, 95\% CI) & $0.0[0.0,0.0]$ \\
\hline 3.2 Malunion & 1 & & Risk Ratio (M-H, Fixed, 95\% CI) & $0.0[0.0,0.0]$ \\
\hline $\begin{array}{l}3.3 \text { Greater tuberosity } \\
\text { migration (all had severe pain } \\
\text { at } 6 \& 12 \text { months) }\end{array}$ & 1 & & Risk Ratio (M-H, Fixed, 95\% CI) & $0.0[0.0,0.0]$ \\
\hline $\begin{array}{l}3.4 \text { Superior luxation of } \\
\text { prosthesis }\end{array}$ & 1 & & Risk Ratio (M-H, Fixed, 95\% CI) & $0.0[0.0,0.0]$ \\
\hline 4 Range of motion at 1 year & 1 & & Mean Difference (IV, Fixed, 95\% CI) & Totals not selected \\
\hline 4.1 Elevation (degrees) & 1 & & Mean Difference (IV, Fixed, 95\% CI) & $0.0[0.0,0.0]$ \\
\hline 4.2 External rotation (degrees) & 1 & & Mean Difference (IV, Fixed, 95\% CI) & $0.0[0.0,0.0]$ \\
\hline
\end{tabular}


Analysis I.I. Comparison I Early mobilisation (within or up to I week) versus immobilisation for 3 or 4 weeks, Outcome I Shoulder disability: Croft Shoulder Disability Score.

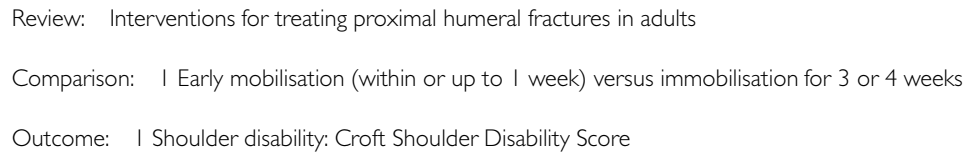

I Disability (I or more problems) at I year

2 Severe disability ( 5 or more problems) at I year

3 Disability (I or more problems) at 2 years 
Analysis I.2. Comparison I Early mobilisation (within or up to I week) versus immobilisation for 3 or 4 weeks, Outcome 2 Croft shoulder disability score: individual problems at 2 years.

Review: Interventions for treating proximal humeral fractures in adults

Comparison: | Early mobilisation (within or up to I week) versus immobilisation for 3 or 4 weeks

Outcome: 2 Croft shoulder disability score: individual problems at 2 years

\begin{tabular}{|c|c|c|c|c|}
\hline \multirow[t]{2}{*}{ Study or subgroup } & \multirow{2}{*}{$\begin{array}{r}\text { Early }(\leq \text { I week }) \\
n / N\end{array}$} & \multirow{2}{*}{$\begin{array}{r}3 \text { or } 4 \text { weeks } \\
\mathrm{n} / \mathrm{N}\end{array}$} & \multirow{2}{*}{$\begin{array}{c}\text { Risk Ratio } \\
\text { M-H,Fixed,95\% Cl }\end{array}$} & \multirow{2}{*}{$\begin{array}{r}\text { Risk Ratio } \\
\mathrm{M}-\mathrm{H}, \text { Fixed,95\% Cl }\end{array}$} \\
\hline & & & & \\
\hline \multicolumn{5}{|l|}{ I Pain on movement } \\
\hline Hodgson 2003 & $5 / 37$ & $13 / 37$ & & $0.38[0.15,0.97]$ \\
\hline \multicolumn{5}{|l|}{2 Bathing difficulties } \\
\hline Hodgson 2003 & $4 / 37$ & $7 / 37$ & & $0.57[0.18,1.79]$ \\
\hline \multicolumn{5}{|c|}{3 Change position at night more often } \\
\hline Hodgson 2003 & $6 / 37$ & $12 / 37$ & - & $0.50[0.21,1.19]$ \\
\hline \multicolumn{5}{|l|}{4 Disturbed sleep } \\
\hline Hodgson 2003 & $3 / 37$ & $6 / 37$ & & $0.50[0.14,1.85]$ \\
\hline \multicolumn{5}{|c|}{5 No active pastimes or usual physical recreation } \\
\hline Hodgson 2003 & $6 / 37$ & $3 / 37$ & & $2.00[0.54,7.40]$ \\
\hline \multicolumn{5}{|l|}{6 Lifting problems } \\
\hline Hodgson 2003 & $16 / 37$ & $15 / 37$ & $\longrightarrow$ & $1.07[0.62,1.83]$ \\
\hline \multicolumn{5}{|l|}{7 Help needed } \\
\hline Hodgson 2003 & $9 / 37$ & $6 / 37$ & & $1.50[0.59,3.79]$ \\
\hline \multicolumn{5}{|c|}{8 More accidents (e.g. dropping things) } \\
\hline Hodgson 2003 & $9 / 37$ & $5 / 37$ & & $1.80[0.67,4.86]$ \\
\hline
\end{tabular}

$\begin{array}{lllllll}0.1 & 0.2 & 0.5 & 1 & 2 & 5 & 10\end{array}$

Favours early Favours 3 weeks 
Analysis I.3. Comparison I Early mobilisation (within or up to I week) versus immobilisation for 3 or 4 weeks, Outcome 3 Number of treatment sessions (until independent function achieved).

Review: Interventions for treating proximal humeral fractures in adults

Comparison: | Early mobilisation (within or up to I week) versus immobilisation for 3 or 4 weeks

Outcome: 3 Number of treatment sessions (until independent function achieved)

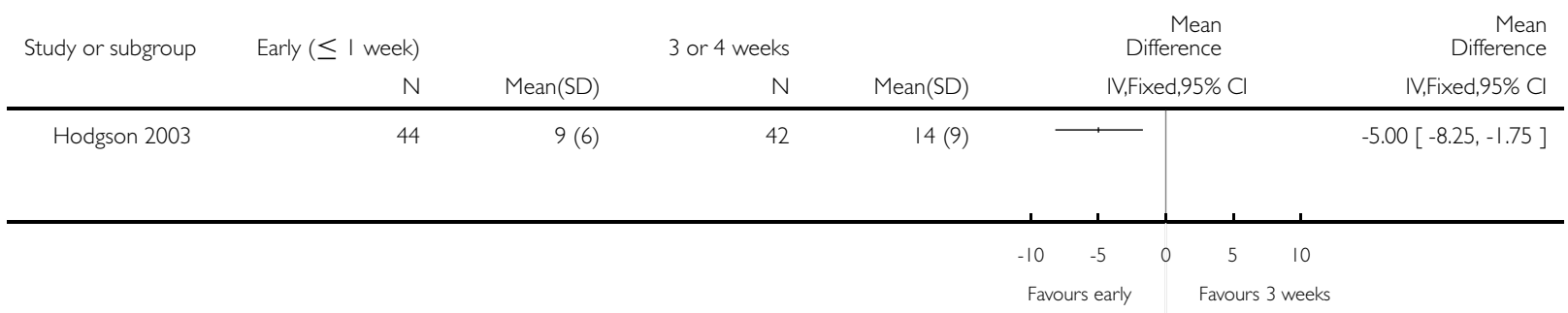

Analysis I.4. Comparison I Early mobilisation (within or up to I week) versus immobilisation for 3 or 4 weeks, Outcome 4 SF-36 scores: pain \& physical dimensions.

Review: Interventions for treating proximal humeral fractures in adults

Comparison: | Early mobilisation (within or up to I week) versus immobilisation for 3 or 4 weeks

Outcome: 4 SF-36 scores: pain \% physical dimensions

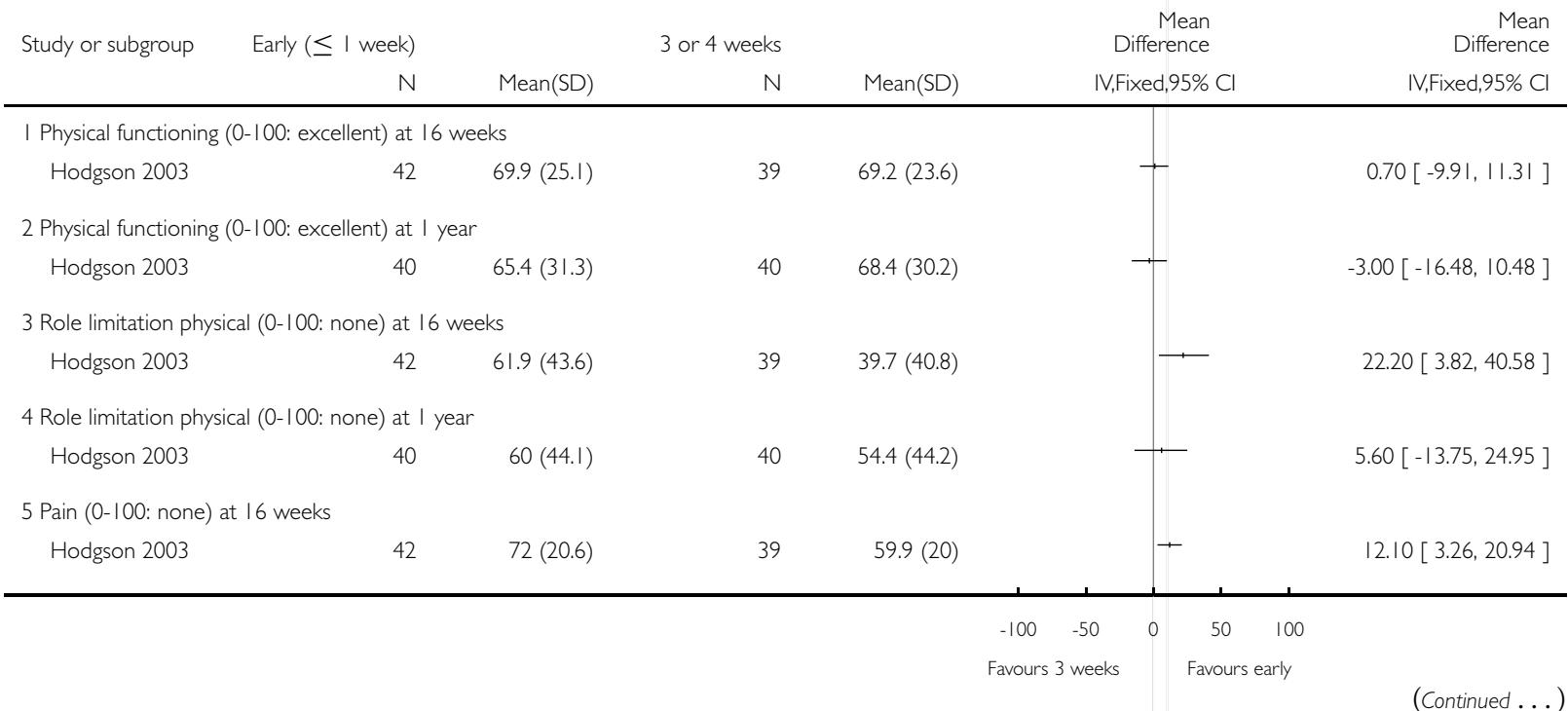




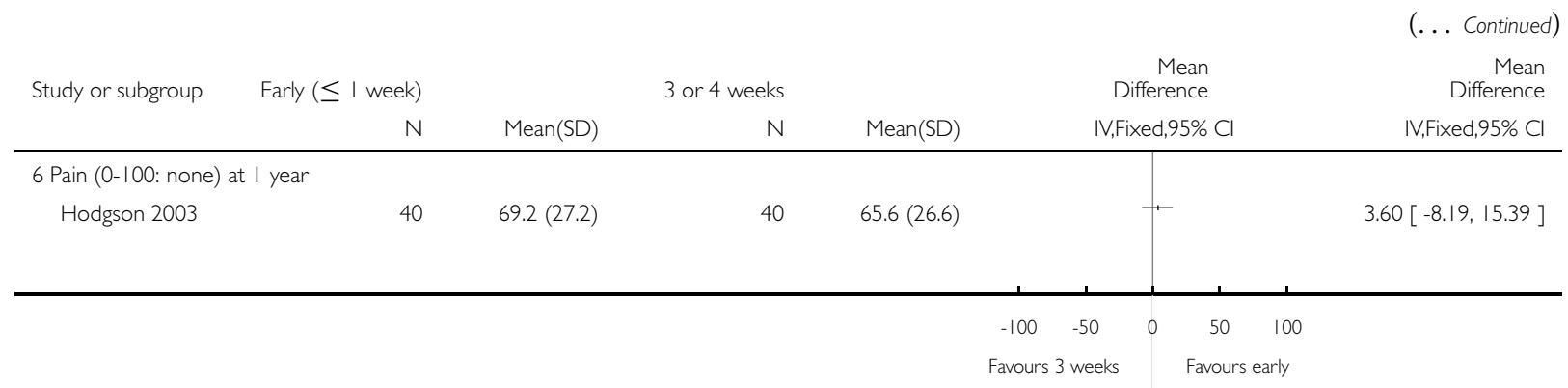

Analysis I.5. Comparison I Early mobilisation (within or up to I week) versus immobilisation for 3 or 4 weeks, Outcome 5 Quality of life assessment: EuroQol 5D (0: dead to I: best health).

Review: Interventions for treating proximal humeral fractures in adults

Comparison: | Early mobilisation (within or up to I week) versus immobilisation for 3 or 4 weeks

Outcome: 5 Quality of life assessment: EuroQol 5D (0: dead to I: best health)

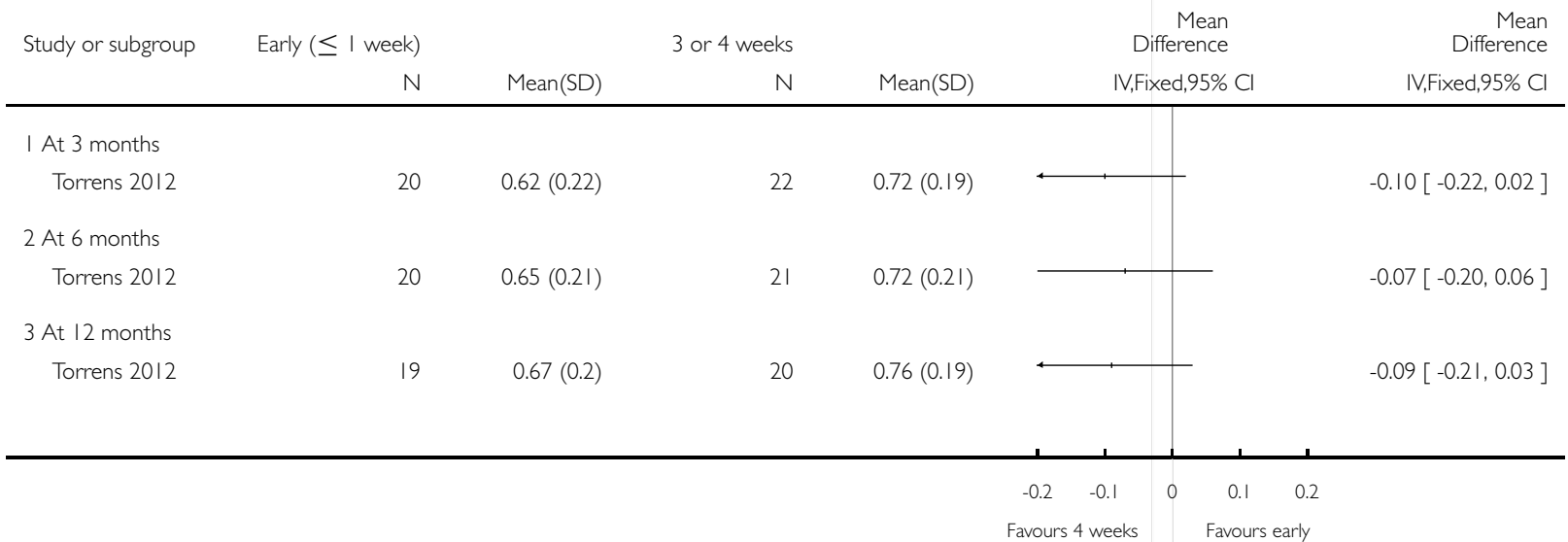


Analysis I.6. Comparison I Early mobilisation (within or up to I week) versus immobilisation for 3 or 4 weeks, Outcome 6 Adverse events.

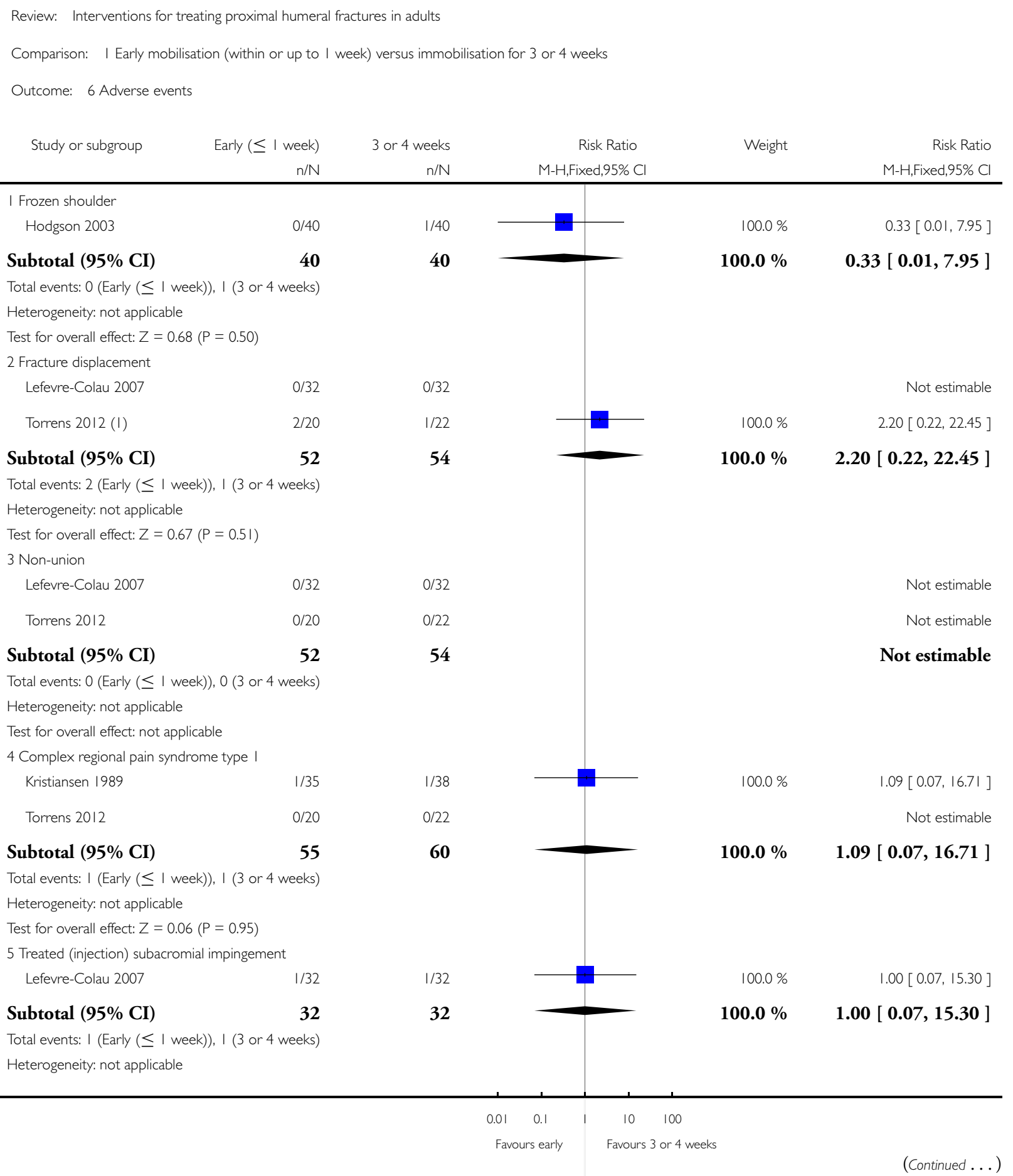




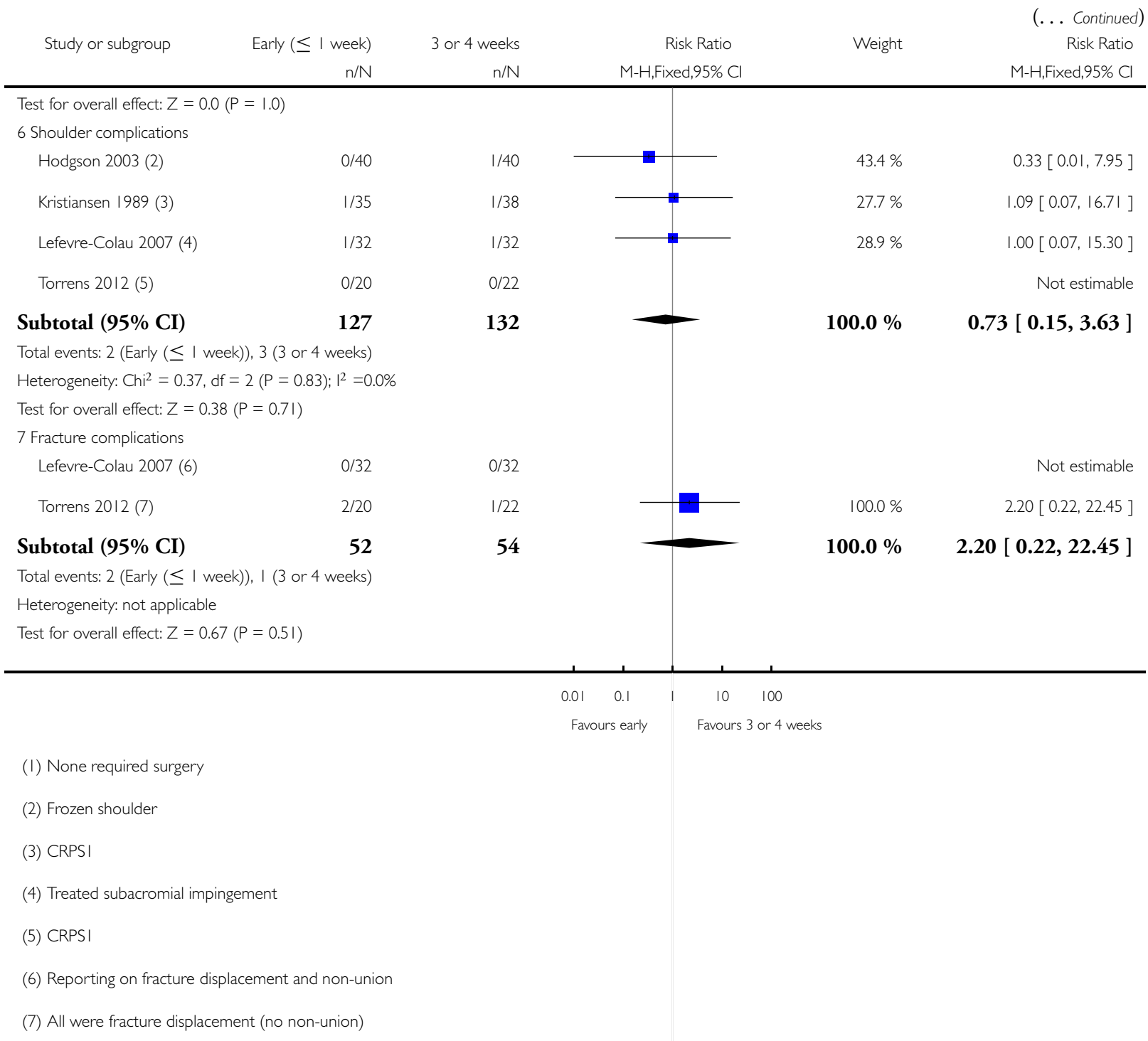


Analysis I.7. Comparison I Early mobilisation (within or up to I week) versus immobilisation for 3 or 4 weeks, Outcome 7 Mortality.

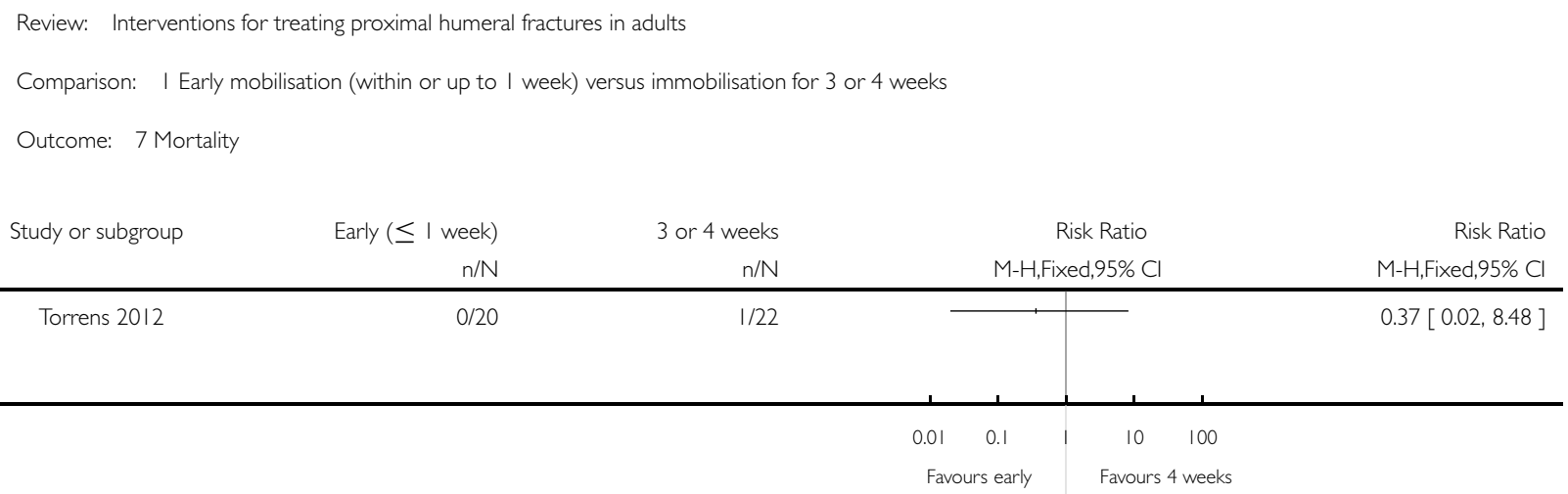

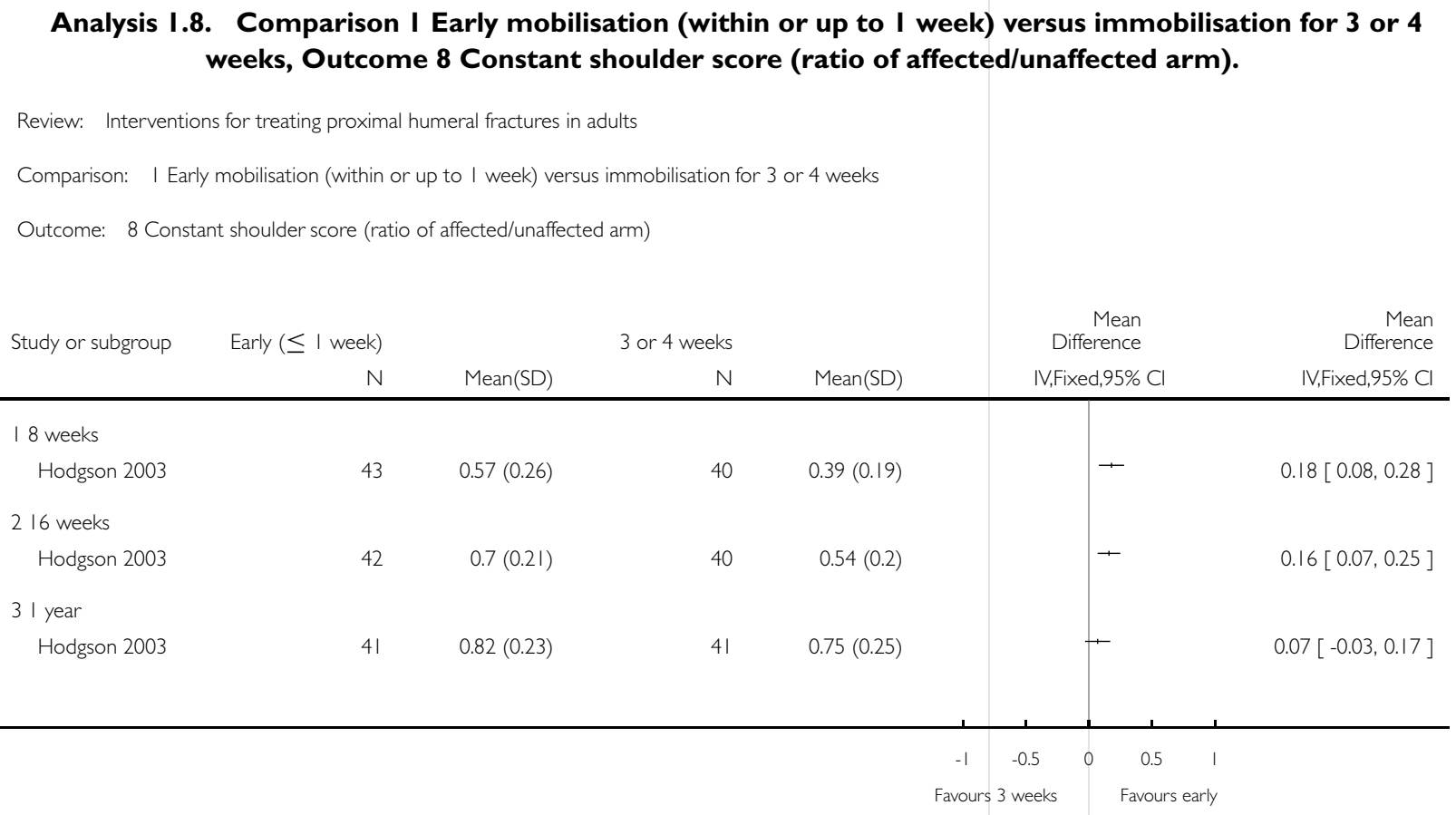


Analysis I.9. Comparison I Early mobilisation (within or up to I week) versus immobilisation for 3 or 4 weeks, Outcome 9 Constant shoulder score (0 to 100: best).

Review: Interventions for treating proximal humeral fractures in adults

Comparison: | Early mobilisation (within or up to I week) versus immobilisation for 3 or 4 weeks

Outcome: 9 Constant shoulder score (0 to 100: best)

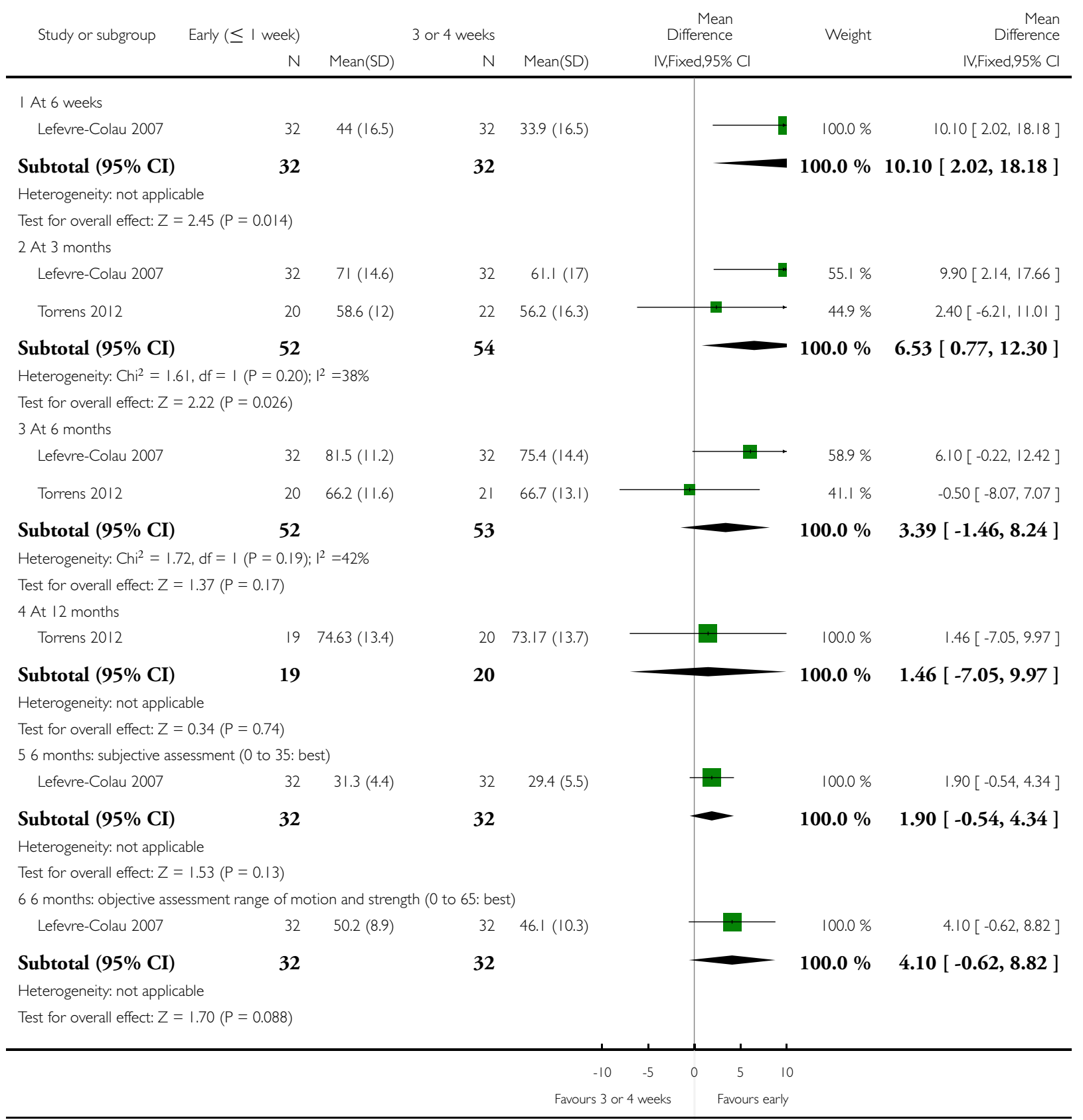

Interventions for treating proximal humeral fractures in adults (Review)

Copyright $\odot 2015$ The Cochrane Collaboration. Published by John Wiley \& Sons, Ltd. 
Analysis I.I0. Comparison I Early mobilisation (within or up to I week) versus immobilisation for 3 or 4 weeks, Outcome 10 Pain VAS (0 to 100: worst pain).

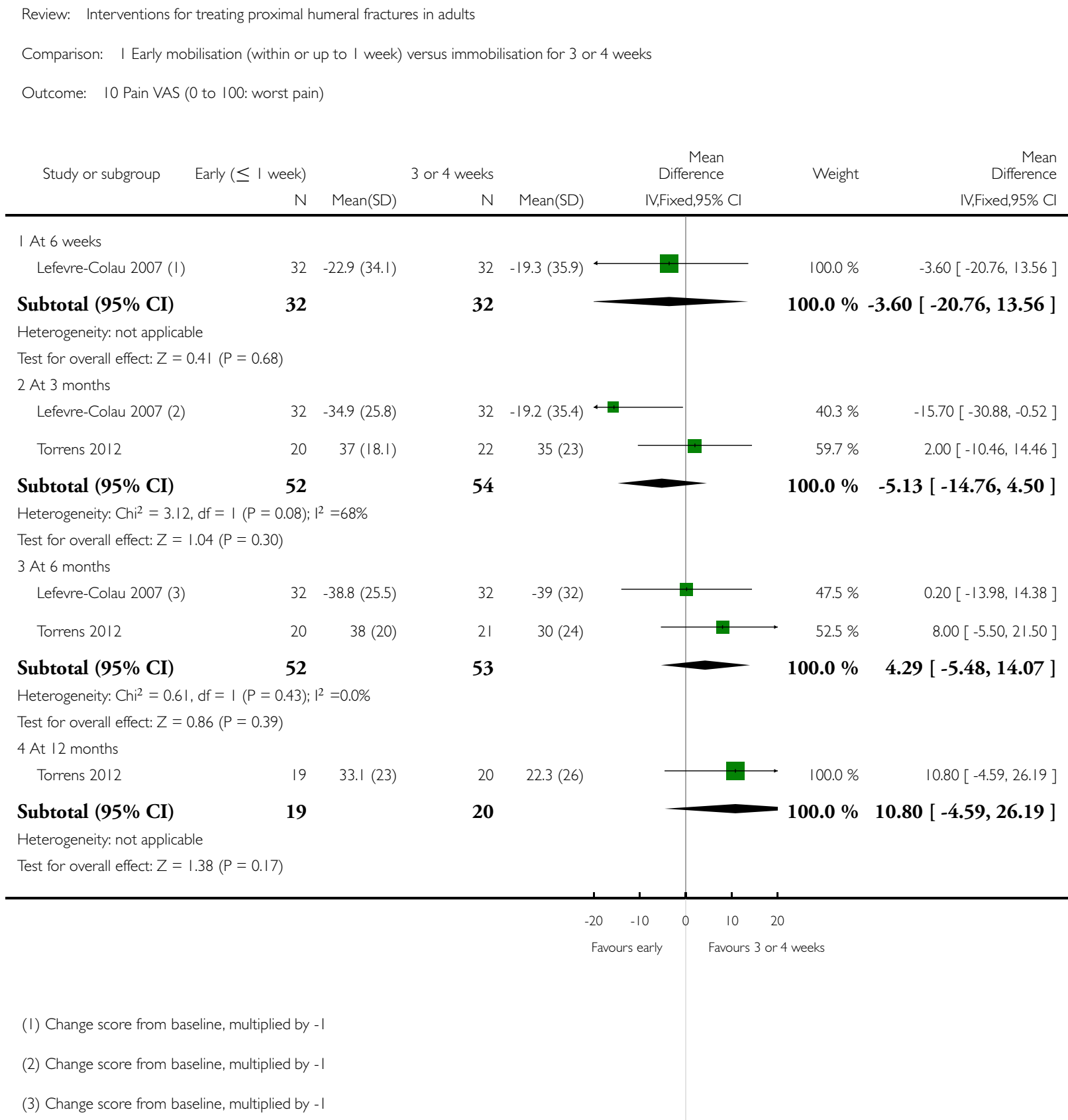

920
(I) Change score from baseline, multiplied by -I
(2) Change score from baseline, multiplied by - I
(3) Change score from baseline, multiplied by - I 
Analysis I.I I. Comparison I Early mobilisation (within or up to I week) versus immobilisation for 3 or 4 weeks, Outcome II Changes in pain intensity $(\mathrm{mm})$ from baseline: $100 \mathrm{~mm}$ visual analogue scale (positive change = less pain).

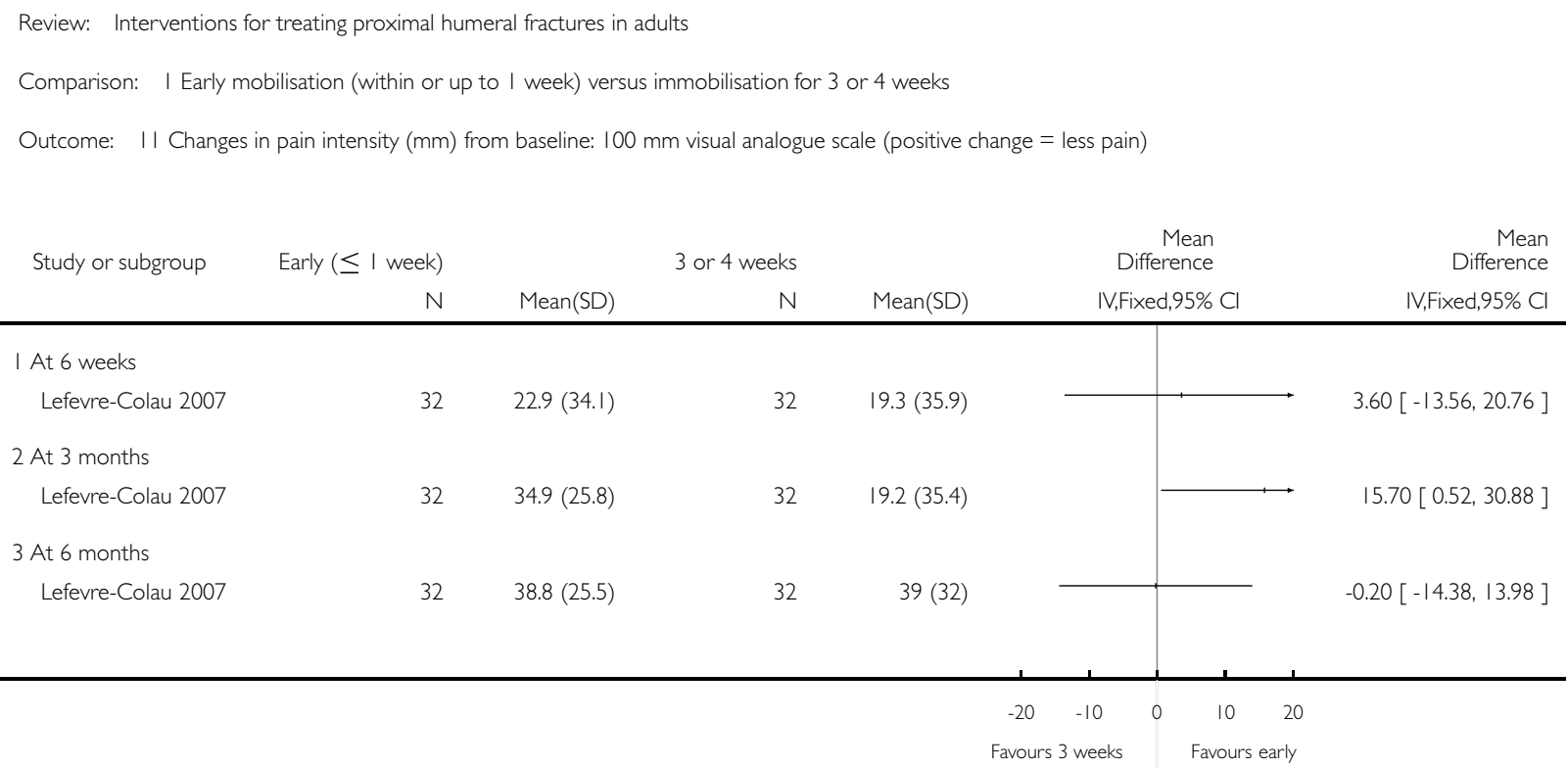


Analysis I.12. Comparison I Early mobilisation (within or up to I week) versus immobilisation for 3 or 4 weeks, Outcome 12 Range of motion at 6 months (degrees): difference between two shoulders.

Review: Interventions for treating proximal humeral fractures in adults

Comparison: | Early mobilisation (within or up to I week) versus immobilisation for 3 or 4 weeks

Outcome: 12 Range of motion at 6 months (degrees): difference between two shoulders

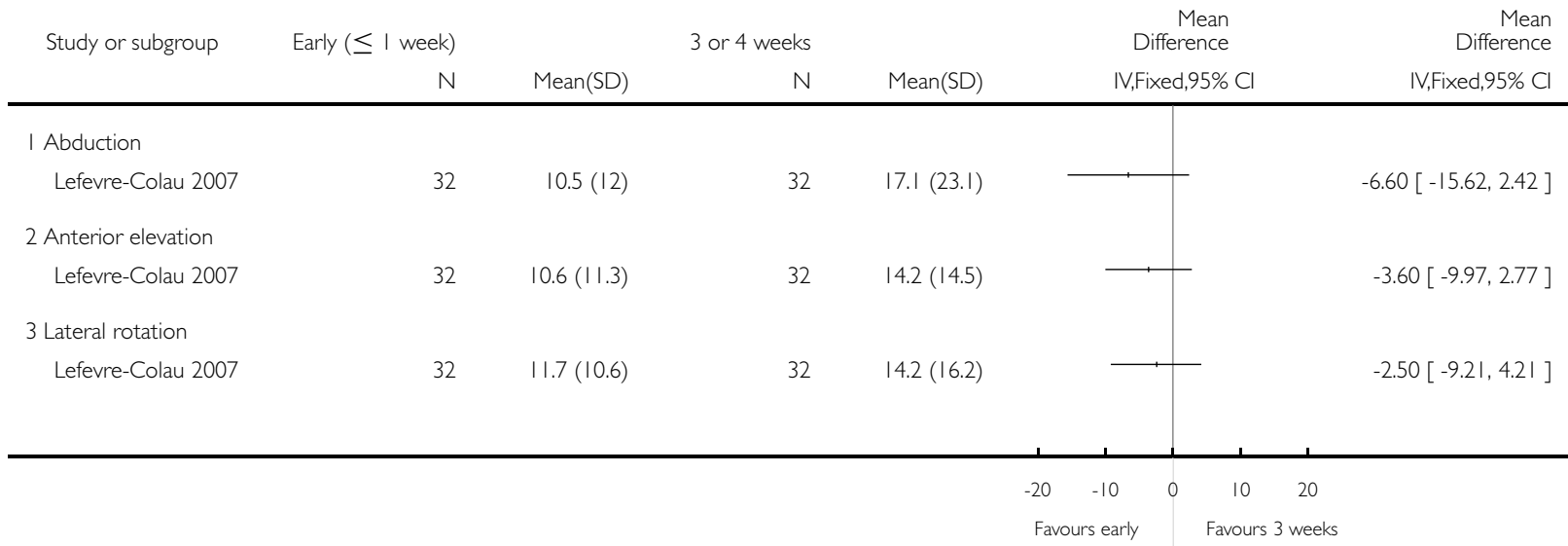

Analysis I.13. Comparison I Early mobilisation (within or up to I week) versus immobilisation for 3 or 4 weeks, Outcome 13 Patient dissatisfied with treatment.

Review: Interventions for treating proximal humeral fractures in adults

Comparison: | Early mobilisation (within or up to I week) versus immobilisation for 3 or 4 weeks

Outcome: 13 Patient dissatisfied with treatment

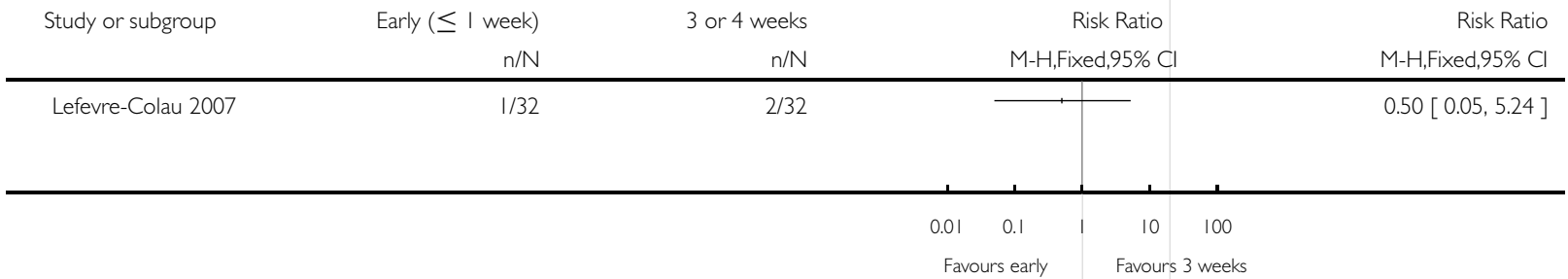


Analysis I.14. Comparison I Early mobilisation (within or up to I week) versus immobilisation for 3 or 4 weeks, Outcome I 4 Patient satisfaction (0 to I0: higher scores - greater satisfaction).

Review: Interventions for treating proximal humeral fractures in adults

Comparison: I Early mobilisation (within or up to I week) versus immobilisation for 3 or 4 weeks

Outcome: 14 Patient satisfaction (0 to 10: higher scores - greater satisfaction)

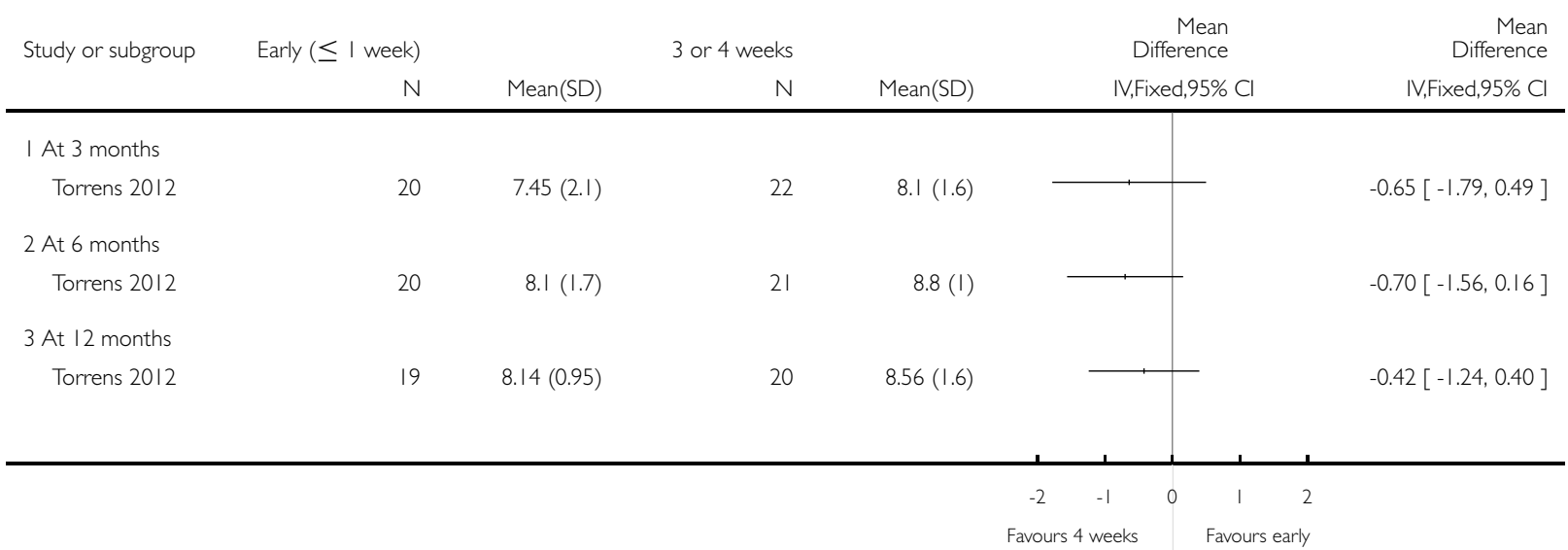

Analysis 2.I. Comparison 2 Gilchrist bandage versus 'Classic' Desault bandage, Outcome I Problems with bandages.

Review: Interventions for treating proximal humeral fractures in adults

Comparison: 2 Gilchrist bandage versus 'Classic' Desault bandage

Outcome: I Problems with bandages

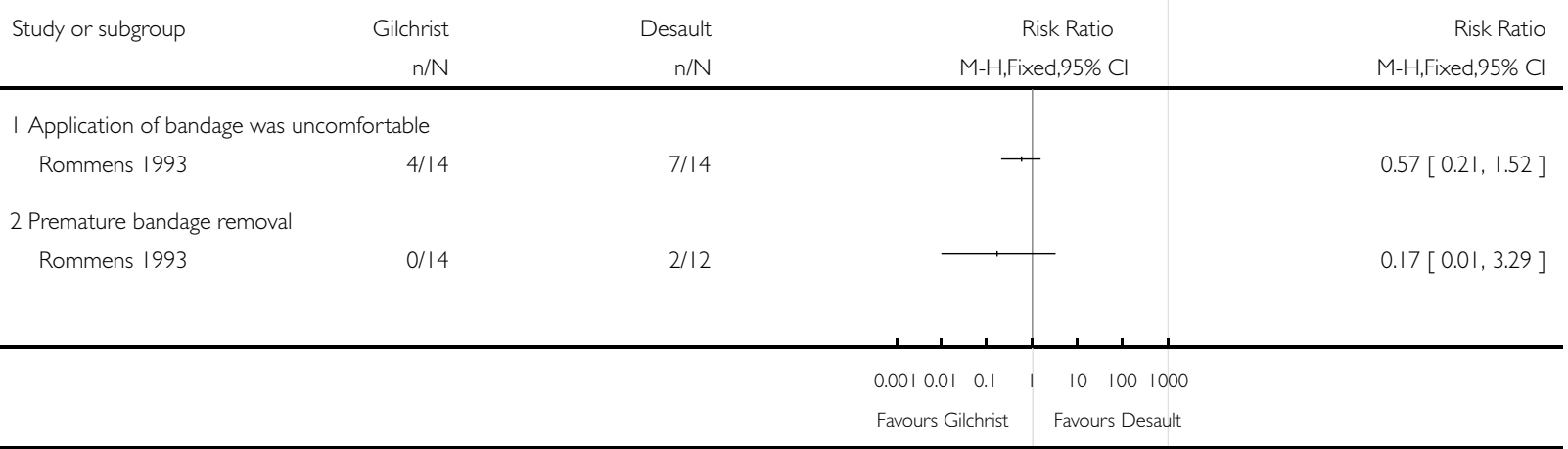

Interventions for treating proximal humeral fractures in adults (Review)

Copyright @ 2015 The Cochrane Collaboration. Published by John Wiley \& Sons, Ltd. 


\section{Analysis 2.2. Comparison 2 Gilchrist bandage versus 'Classic' Desault bandage, Outcome 2 Fracture displacement by 3 weeks.}

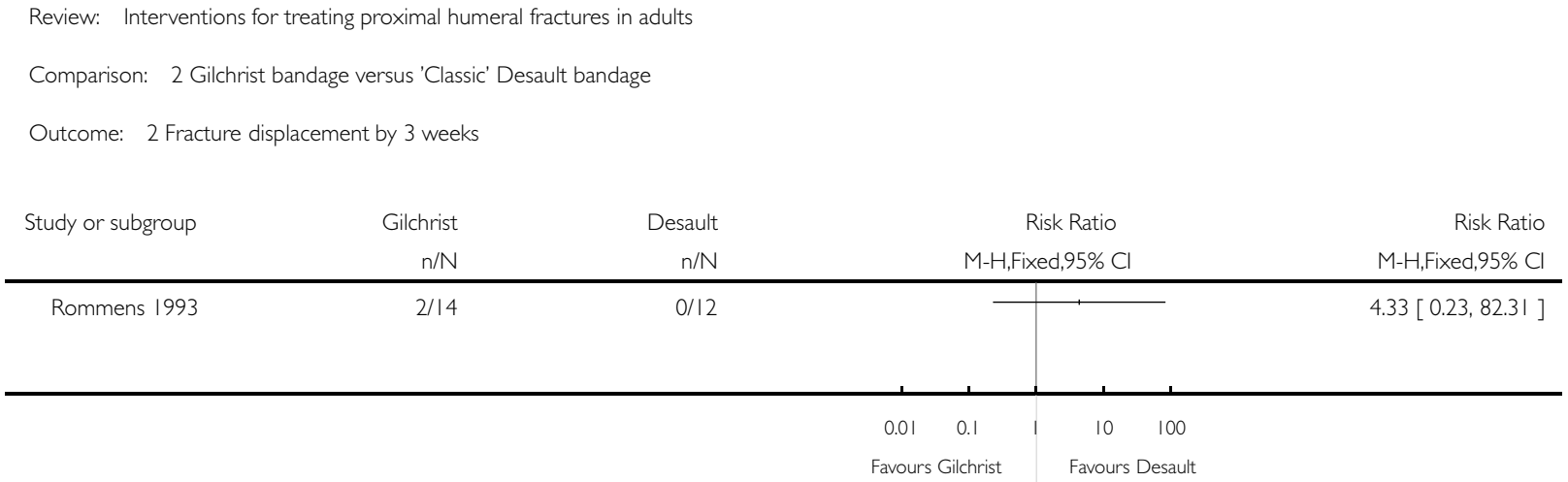

Analysis 2.3. Comparison 2 Gilchrist bandage versus 'Classic' Desault bandage, Outcome 3 Poor or bad rating by patient at fracture consolidation.

Review: Interventions for treating proximal humeral fractures in adults

Comparison: 2 Gilchrist bandage versus 'Classic' Desault bandage

Outcome: 3 Poor or bad rating by patient at fracture consolidation

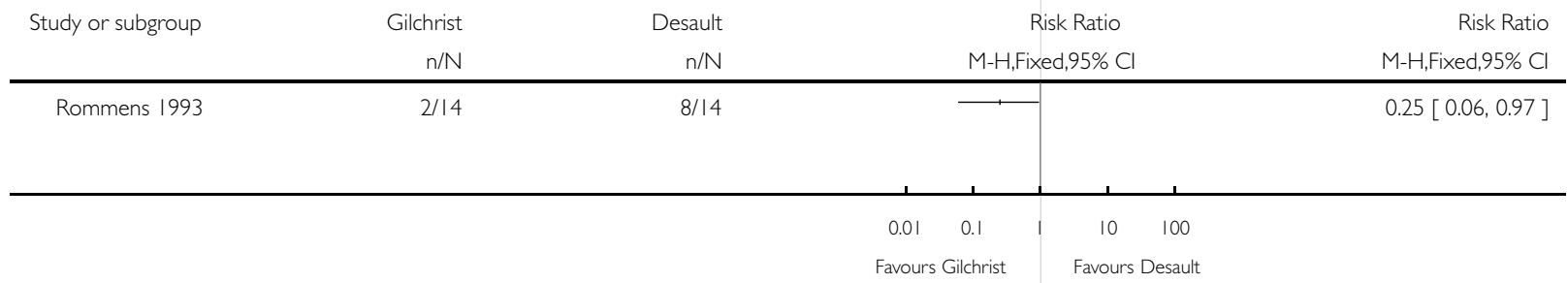


Analysis 3.I. Comparison 3 Instructed self-exercise versus conventional physiotherapy, Outcome I Pain at one year (scale 0 to 8: maximum pain).

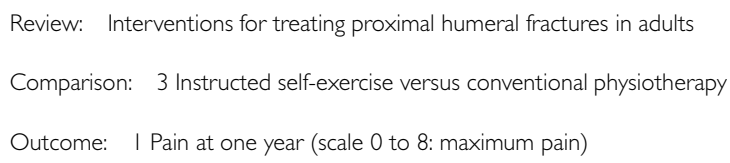

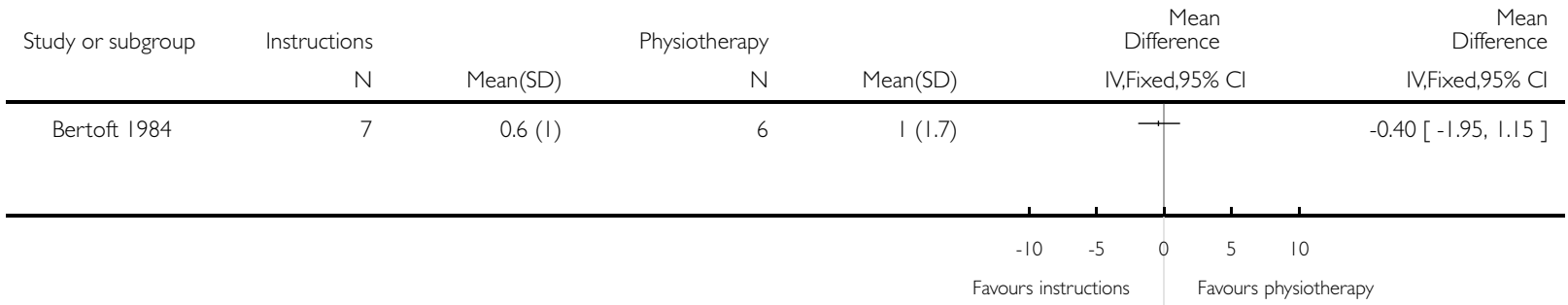

Analysis 3.2. Comparison 3 Instructed self-exercise versus conventional physiotherapy, Outcome 2 Severe or moderate pain at 3 months.

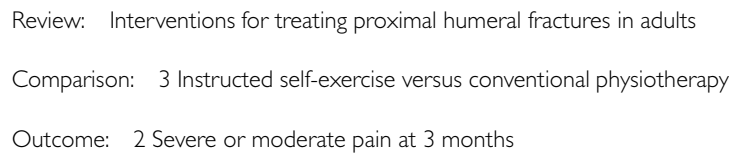

\begin{tabular}{llll} 
& $\mathrm{n} / \mathrm{N}$ & $\mathrm{n} / \mathrm{N}$ & $\mathrm{M}-\mathrm{H}, \mathrm{Fixed}, 95 \% \mathrm{Cl}$ \\
\hline Lundberg 1979 & $4 / 20$ & $2 / 22$ & $\mathrm{M}-\mathrm{H}, \mathrm{Fixed}, 95 \% \mathrm{Cl}$ \\
\hline
\end{tabular}

$\begin{array}{ccccc}0.01 & 0.1 & 1 & 10 & 100 \\ \text { Favours instructions } & & \text { Favours physiotherapy }\end{array}$


Analysis 3.3. Comparison 3 Instructed self-exercise versus conventional physiotherapy, Outcome 3 Requested change of therapy.

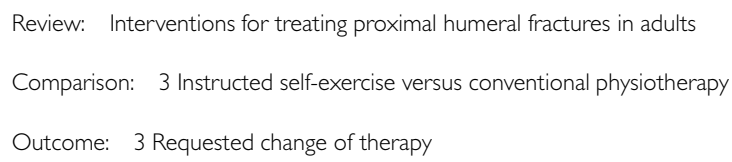

\begin{tabular}{lll} 
& $\mathrm{n} / \mathrm{N}$ & $\mathrm{n} / \mathrm{N}$ \\
\hline Bertoft 1984 & $1 / 10$ & $2 / 8$
\end{tabular}

$n / N$

Analysis 3.4. Comparison 3 Instructed self-exercise versus conventional physiotherapy, Outcome 4 Adverse events (frozen shoulder: I $v 2$; unexplained prolonged pain: 0 v I).

Review: Interventions for treating proximal humeral fractures in adults

Comparison: 3 Instructed self-exercise versus conventional physiotherapy

Outcome: 4 Adverse events (frozen shoulder: I v 2; unexplained prolonged pain: 0 v I)

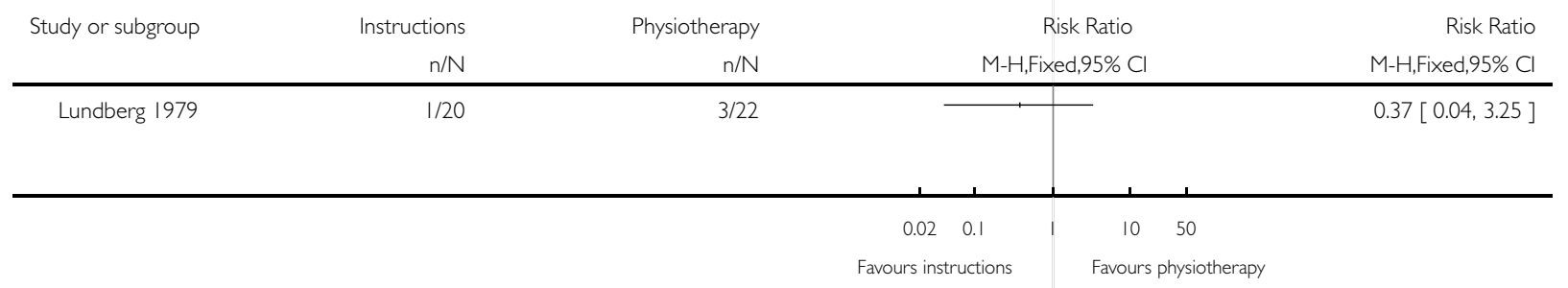


Analysis 3.5. Comparison 3 Instructed self-exercise versus conventional physiotherapy, Outcome 5 Neer's rating (0 to 100: best) at mean 16 months (exploratory analysis).

Review: Interventions for treating proximal humeral fractures in adults

Comparison: 3 Instructed self-exercise versus conventional physiotherapy

Outcome: 5 Neer's rating ( 0 to 100: best) at mean 16 months (exploratory analysis)

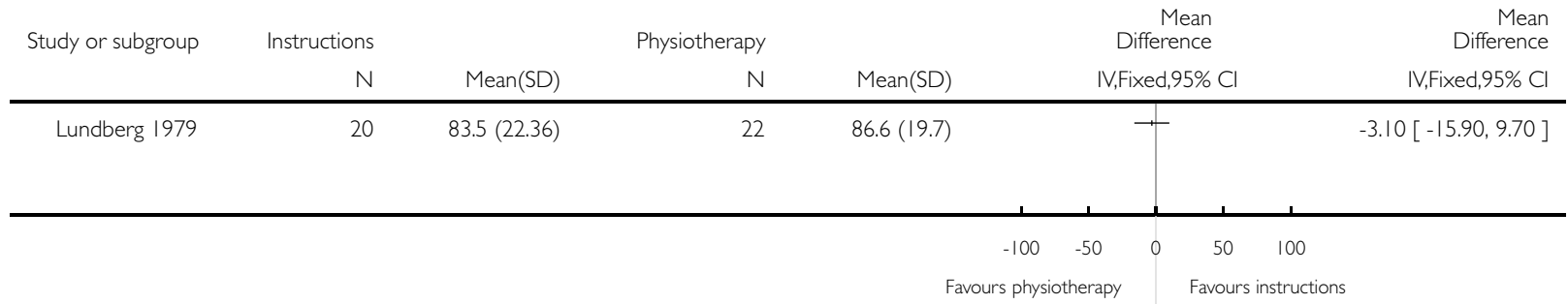

Analysis 3.6. Comparison 3 Instructed self-exercise versus conventional physiotherapy, Outcome 6 Active gleno-humeral elevation (degrees).

Review: Interventions for treating proximal humeral fractures in adults

Comparison: 3 Instructed self-exercise versus conventional physiotherapy

Outcome: 6 Active gleno-humeral elevation (degrees)

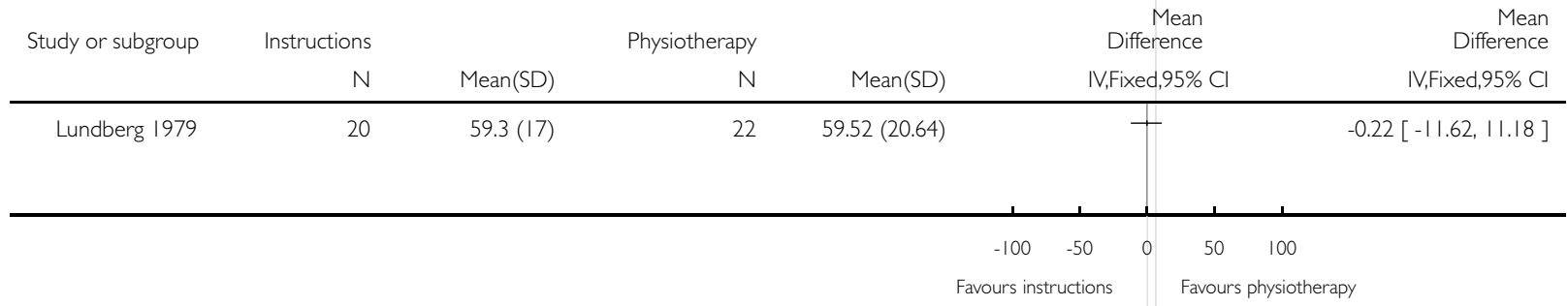




\section{Analysis 4.I. Comparison 4 Surgical versus non-surgical treatment, Outcome I Functional scores at 12 months (higher = better outcome).}

Review: Interventions for treating proximal humeral fractures in adults

Comparison: 4 Surgical versus non-surgical treatment

Outcome: I Functional scores at 12 months (higher = better outcome)

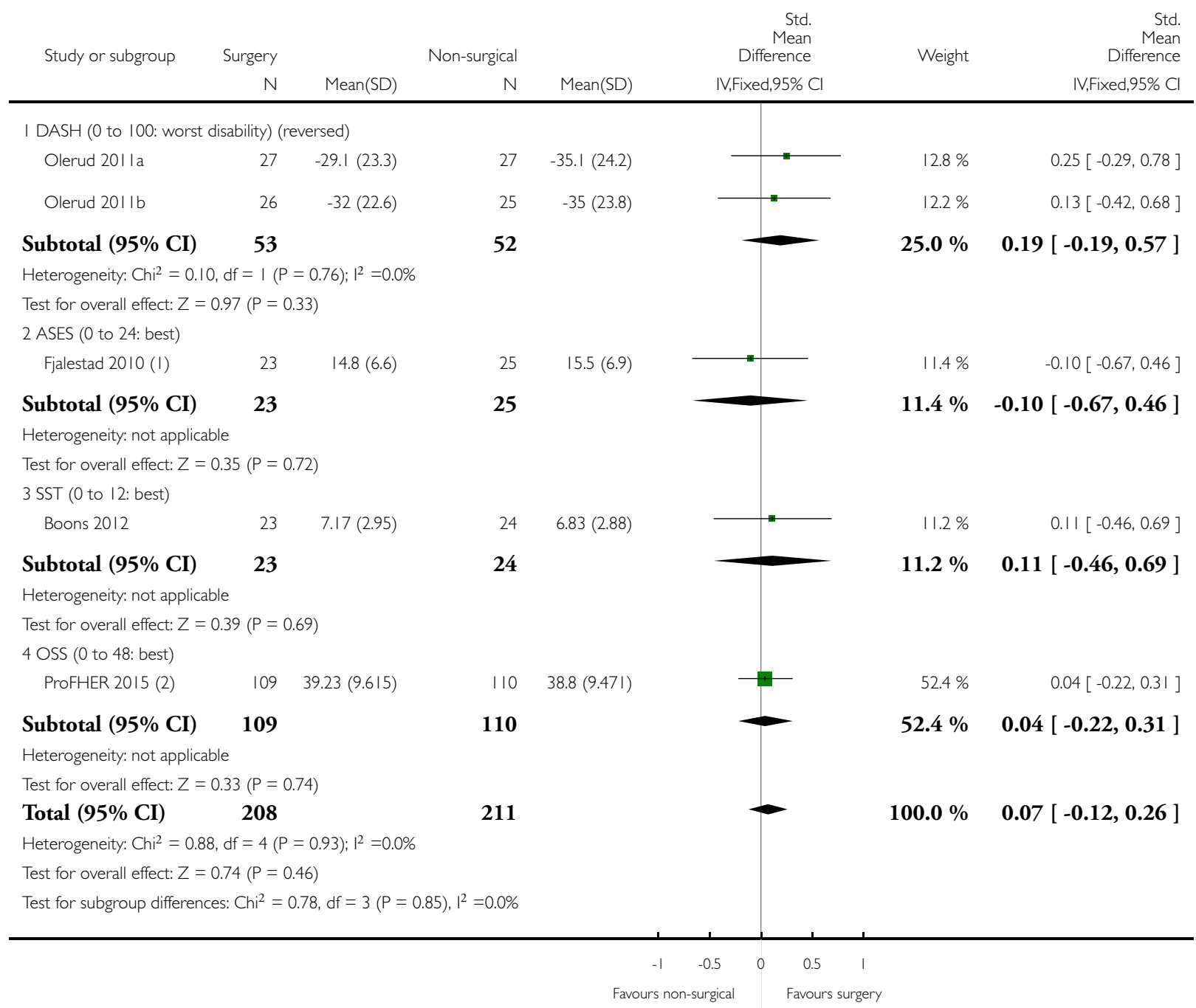

(I) Values made negative to reverse order of effect

(2) Adjusted scores 


\section{Analysis 4.2. Comparison 4 Surgical versus non-surgical treatment, Outcome 2 Functional scores at 24 months (higher = better outcome).}

Review: Interventions for treating proximal humeral fractures in adults

Comparison: 4 Surgical versus non-surgical treatment

Outcome: 2 Functional scores at 24 months (higher = better outcome)

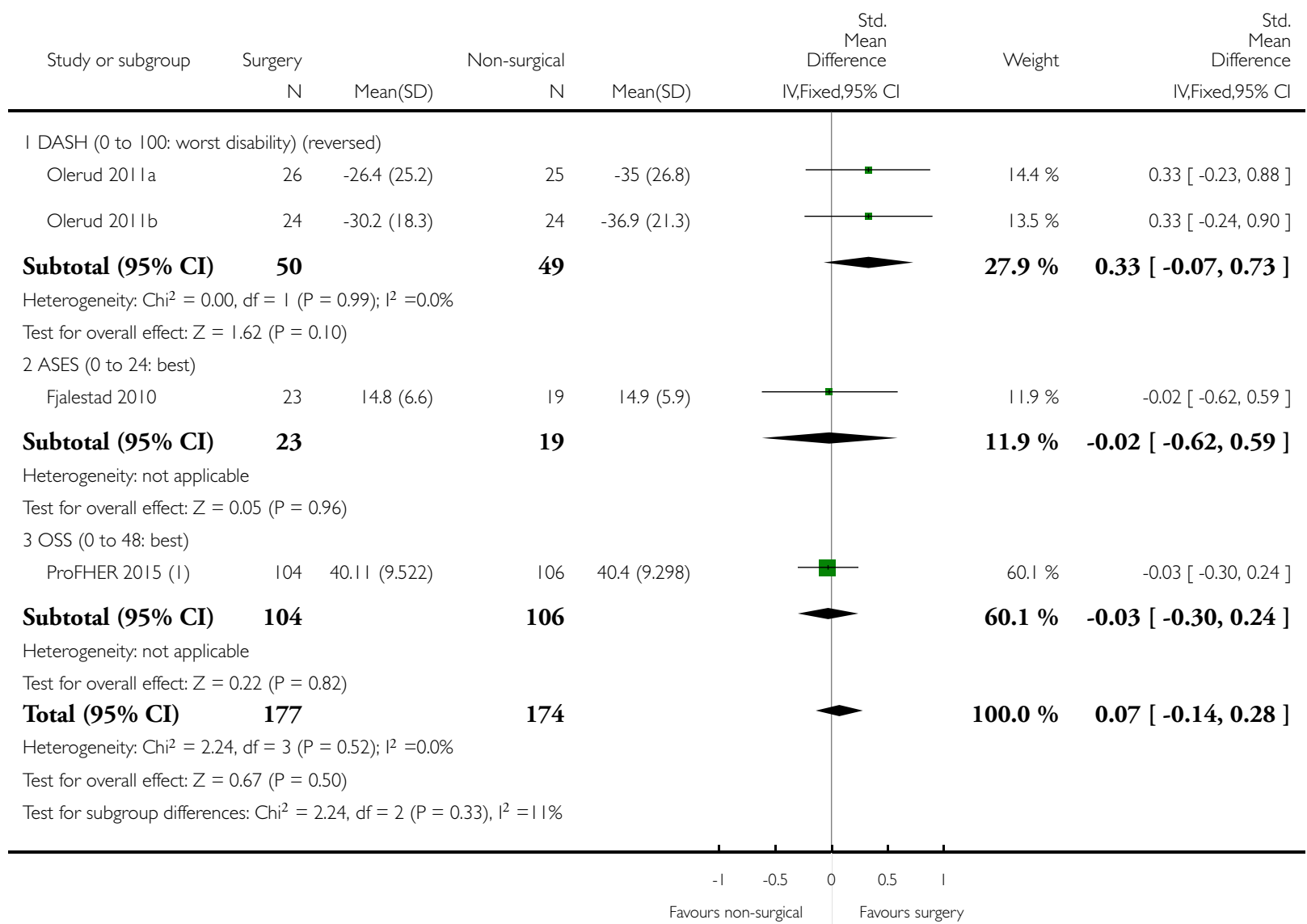

( ) Adjusted scores 
Analysis 4.3. Comparison 4 Surgical versus non-surgical treatment, Outcome 3 Oxford Shoulder Score (0 to 48: best outcome).

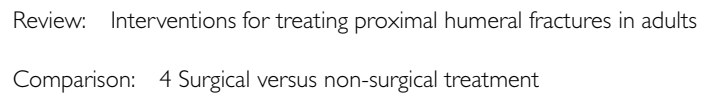

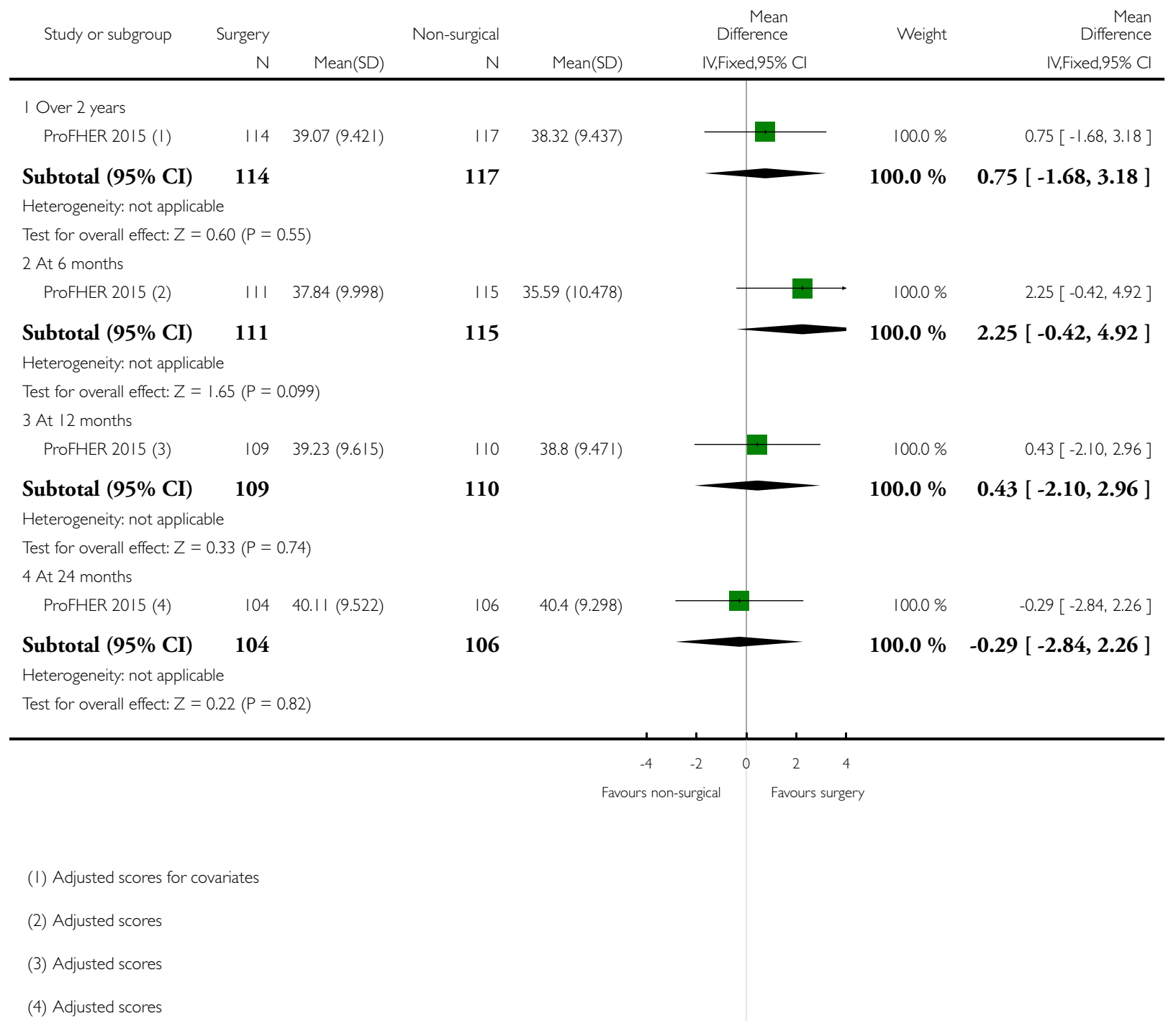


Analysis 4.4. Comparison 4 Surgical versus non-surgical treatment, Outcome 4 DASH (0 to I00: worst disability).

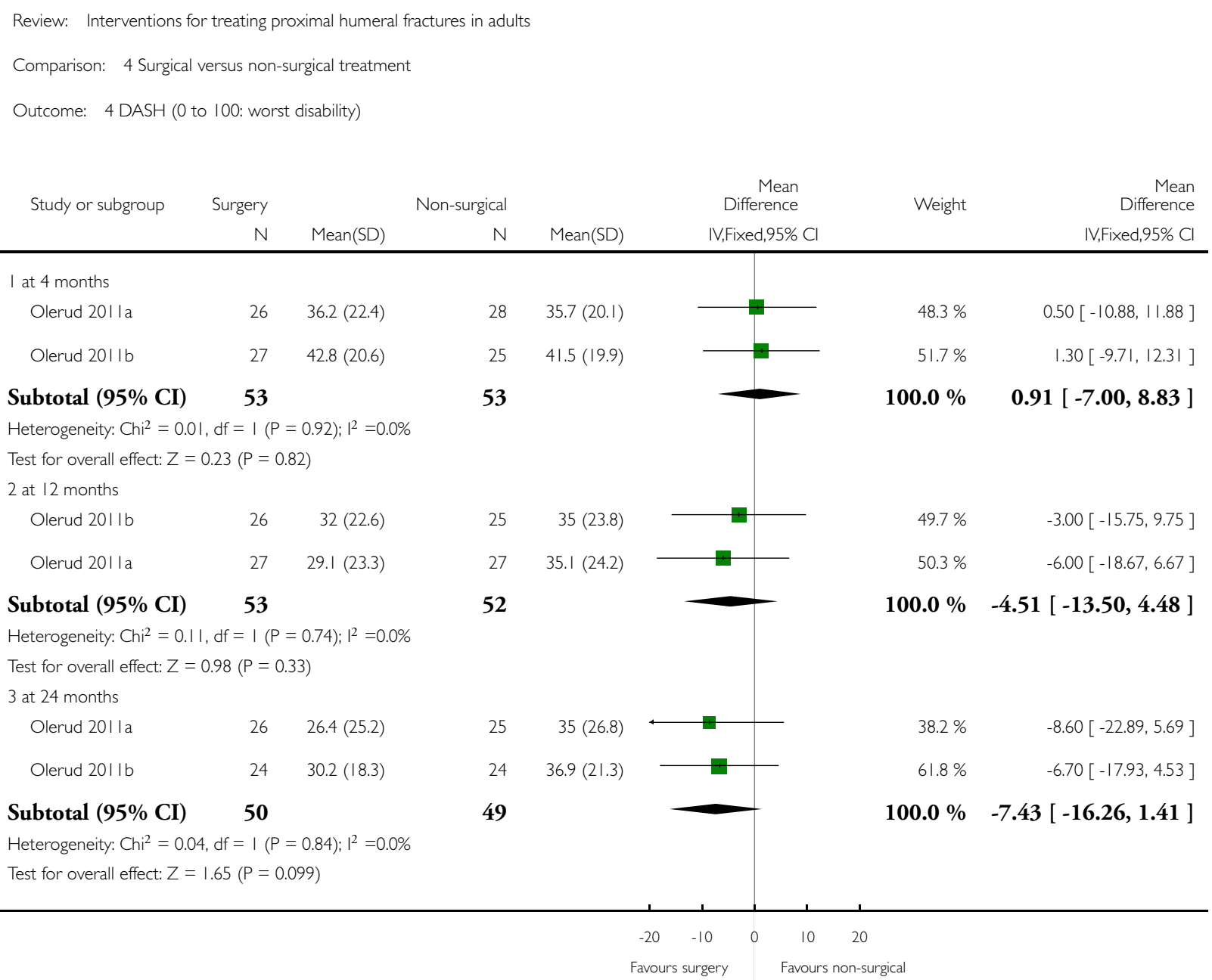


Analysis 4.5. Comparison 4 Surgical versus non-surgical treatment, Outcome 5 American Shoulder and Elbow Surgeons score (0 to 24: best).

Review: Interventions for treating proximal humeral fractures in adults

Comparison: 4 Surgical versus non-surgical treatment

Outcome: 5 American Shoulder and Elbow Surgeons score (0 to 24: best)

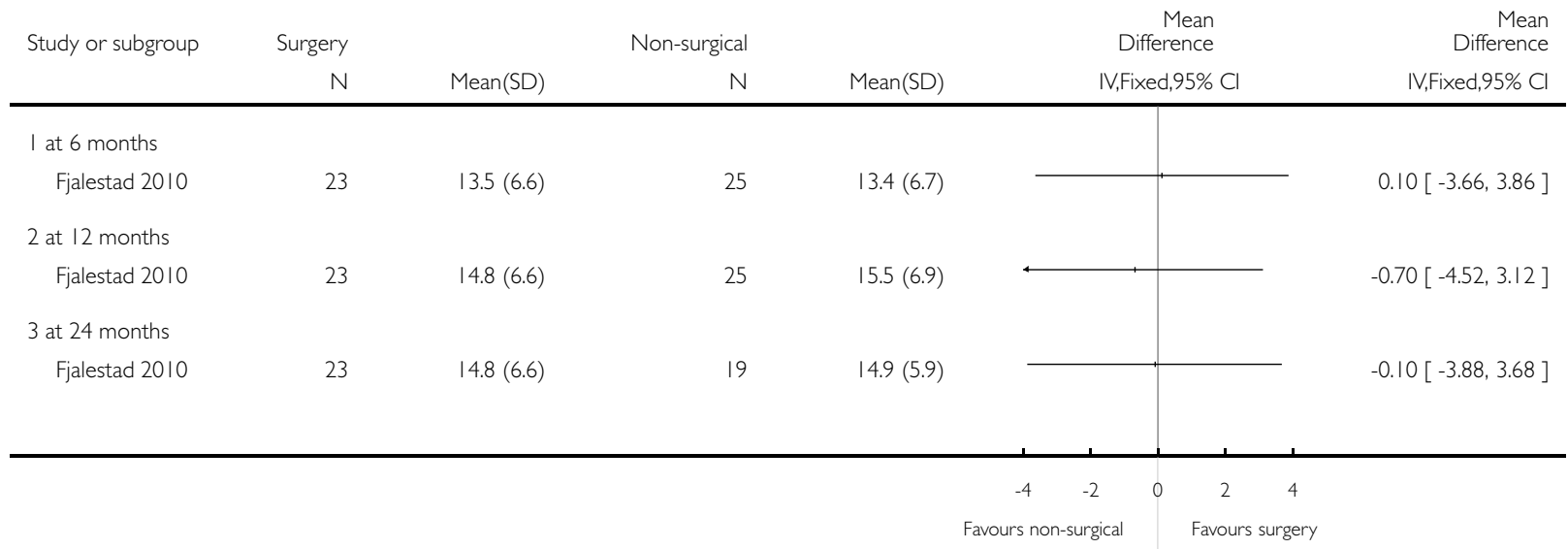

Analysis 4.6. Comparison 4 Surgical versus non-surgical treatment, Outcome 6 Simple Shoulder Test (0 to I2: best function).

Review: Interventions for treating proximal humeral fractures in adults

Comparison: 4 Surgical versus non-surgical treatment

Outcome: 6 Simple Shoulder Test (0 to 12: best function)

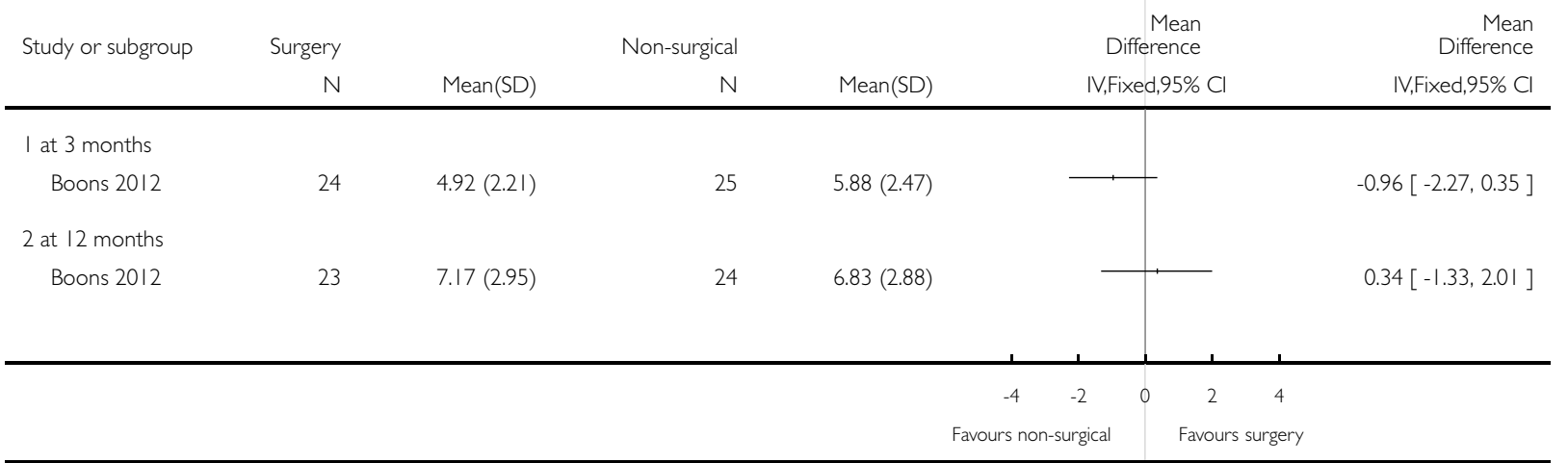

Interventions for treating proximal humeral fractures in adults (Review)

Copyright @ 2015 The Cochrane Collaboration. Published by John Wiley \& Sons, Ltd. 


\section{Analysis 4.7. Comparison 4 Surgical versus non-surgical treatment, Outcome 7 Activities of daily living.}

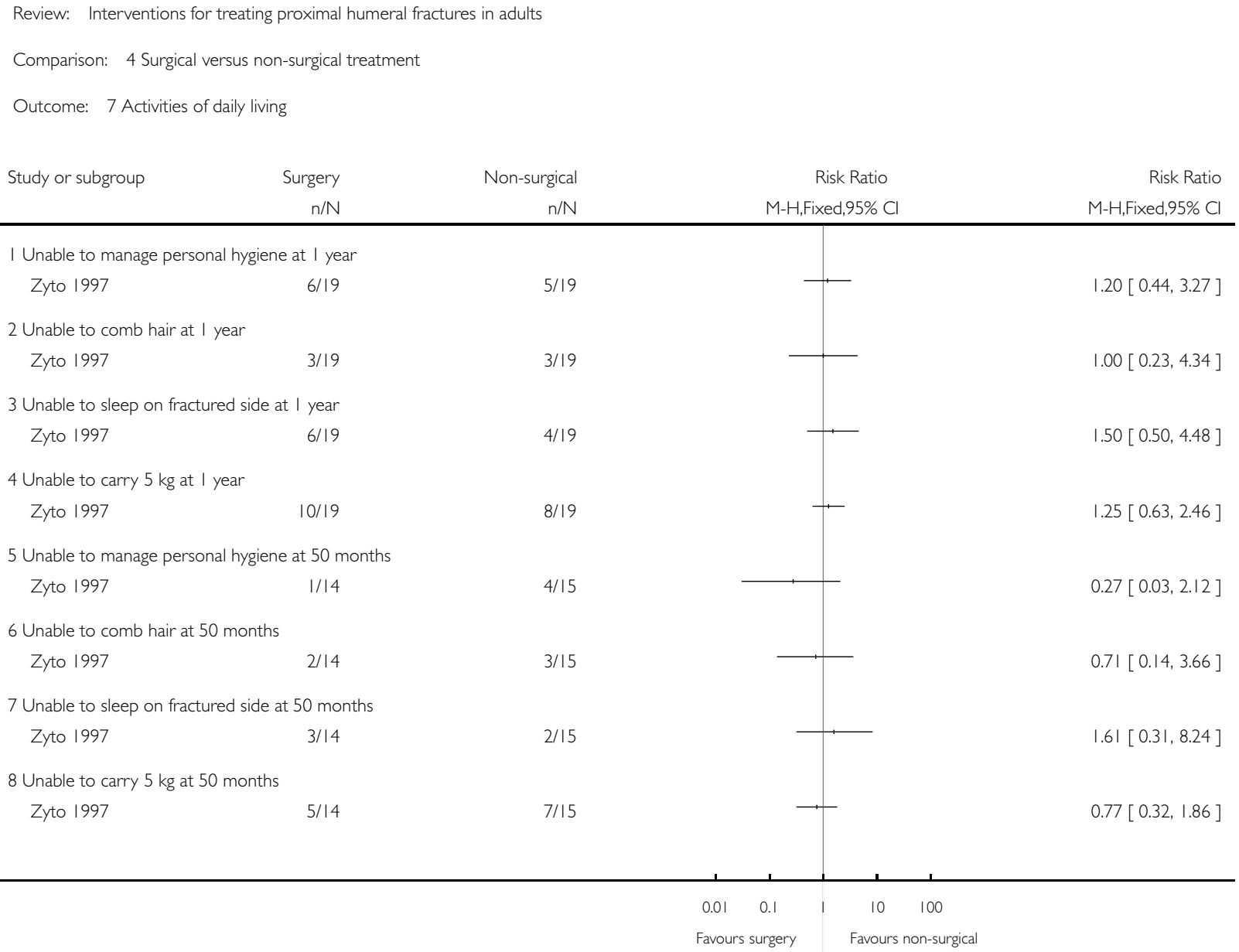




\section{Analysis 4.8. Comparison 4 Surgical versus non-surgical treatment, Outcome 8 Quality of life assessment: EuroQol (0: dead to I: best health).}

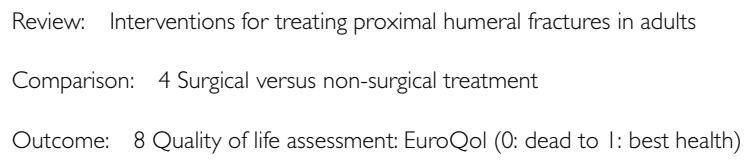

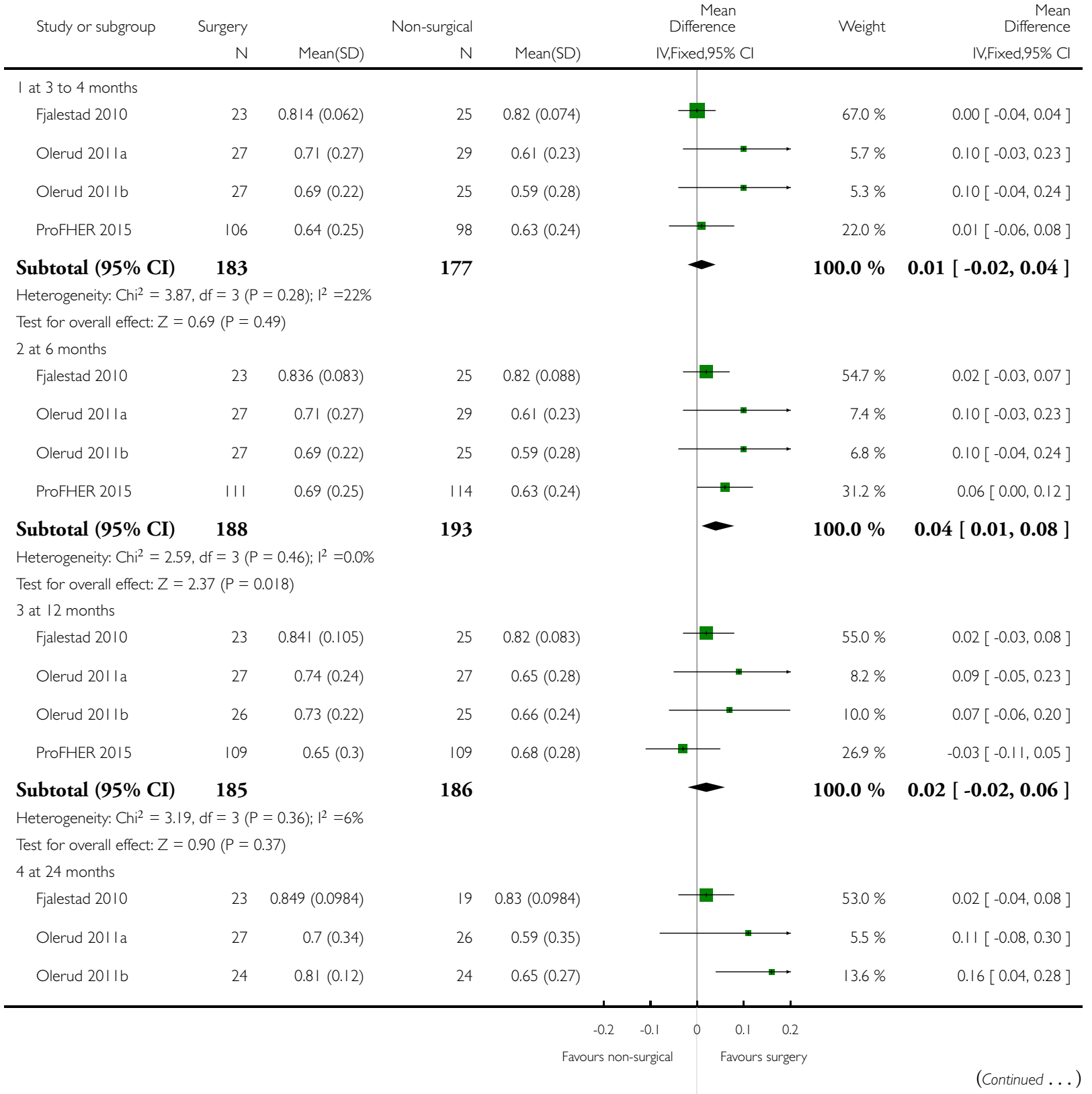




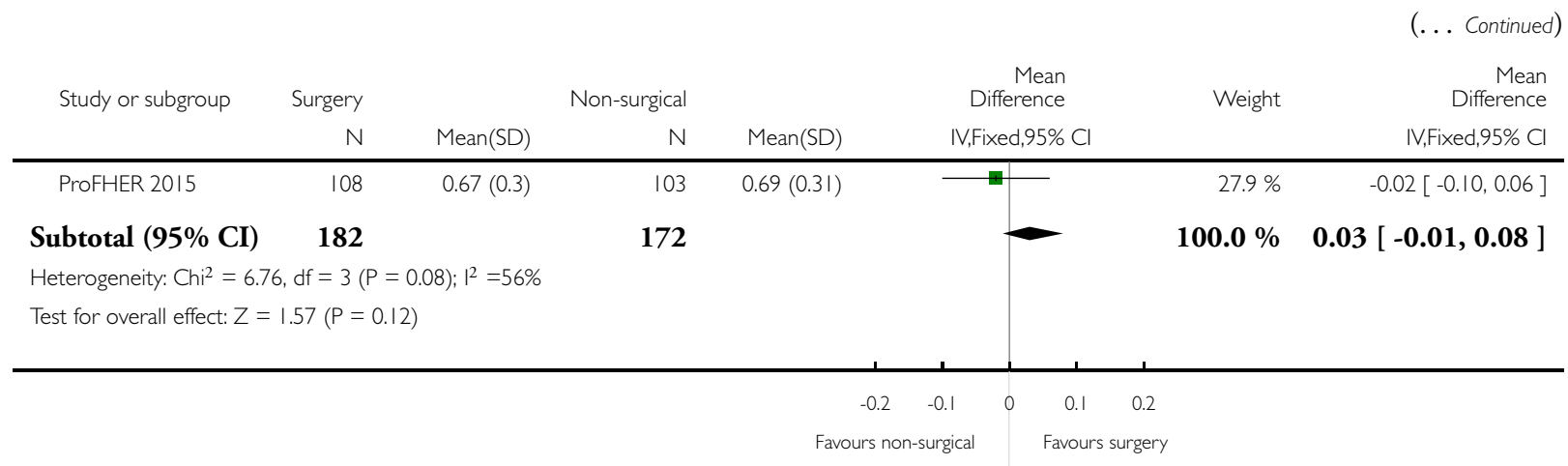

Analysis 4.9. Comparison 4 Surgical versus non-surgical treatment, Outcome 9 Quality of life assessment (Fjalestad 2010 and 2014 data).

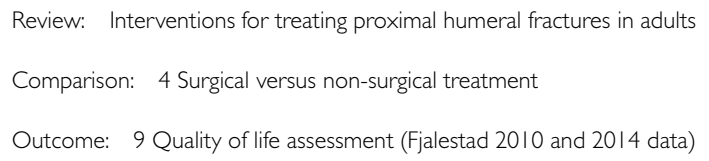

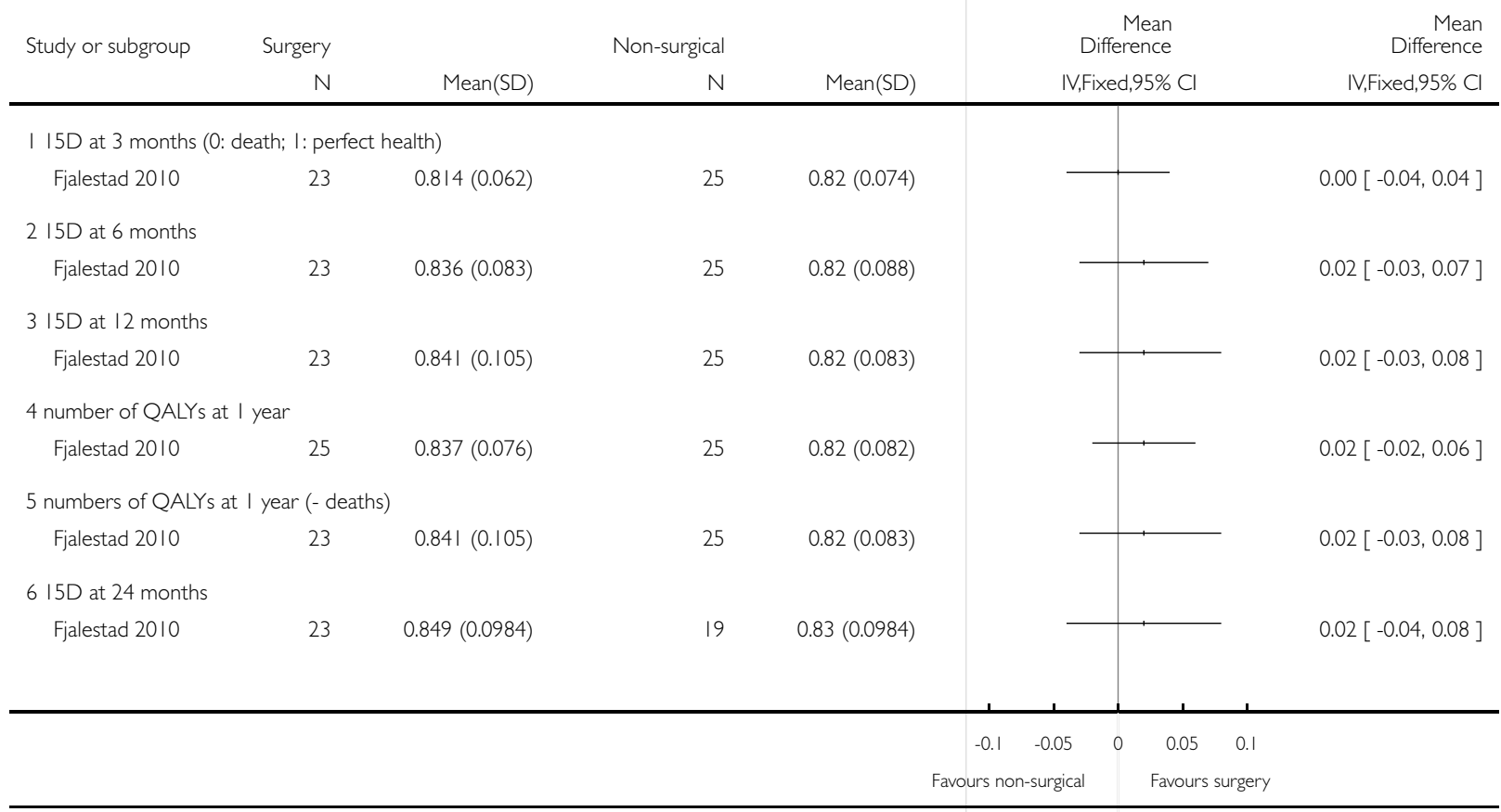


Analysis 4.10. Comparison 4 Surgical versus non-surgical treatment, Outcome 10 Quality of life: SF-I 2 Physical Component Score (0 to 100: best).

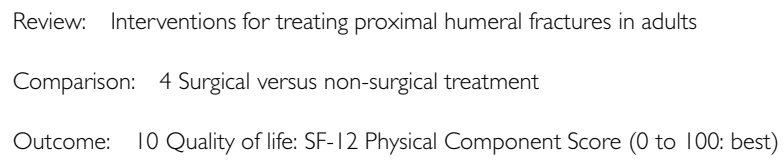

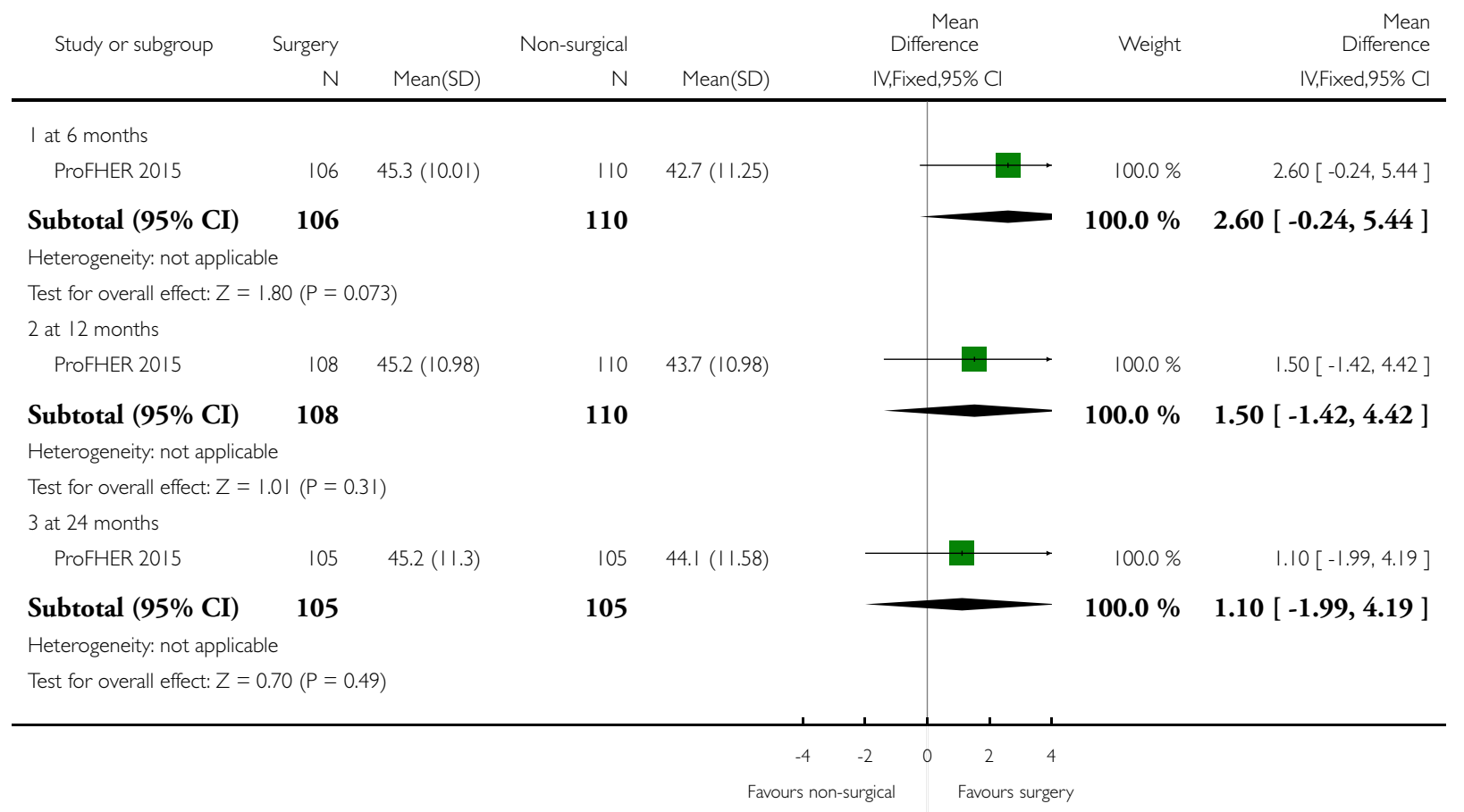


Analysis 4.I I. Comparison 4 Surgical versus non-surgical treatment, Outcome I I Quality of life: SF-I 2 Mental Component Score (0 to 100: best).

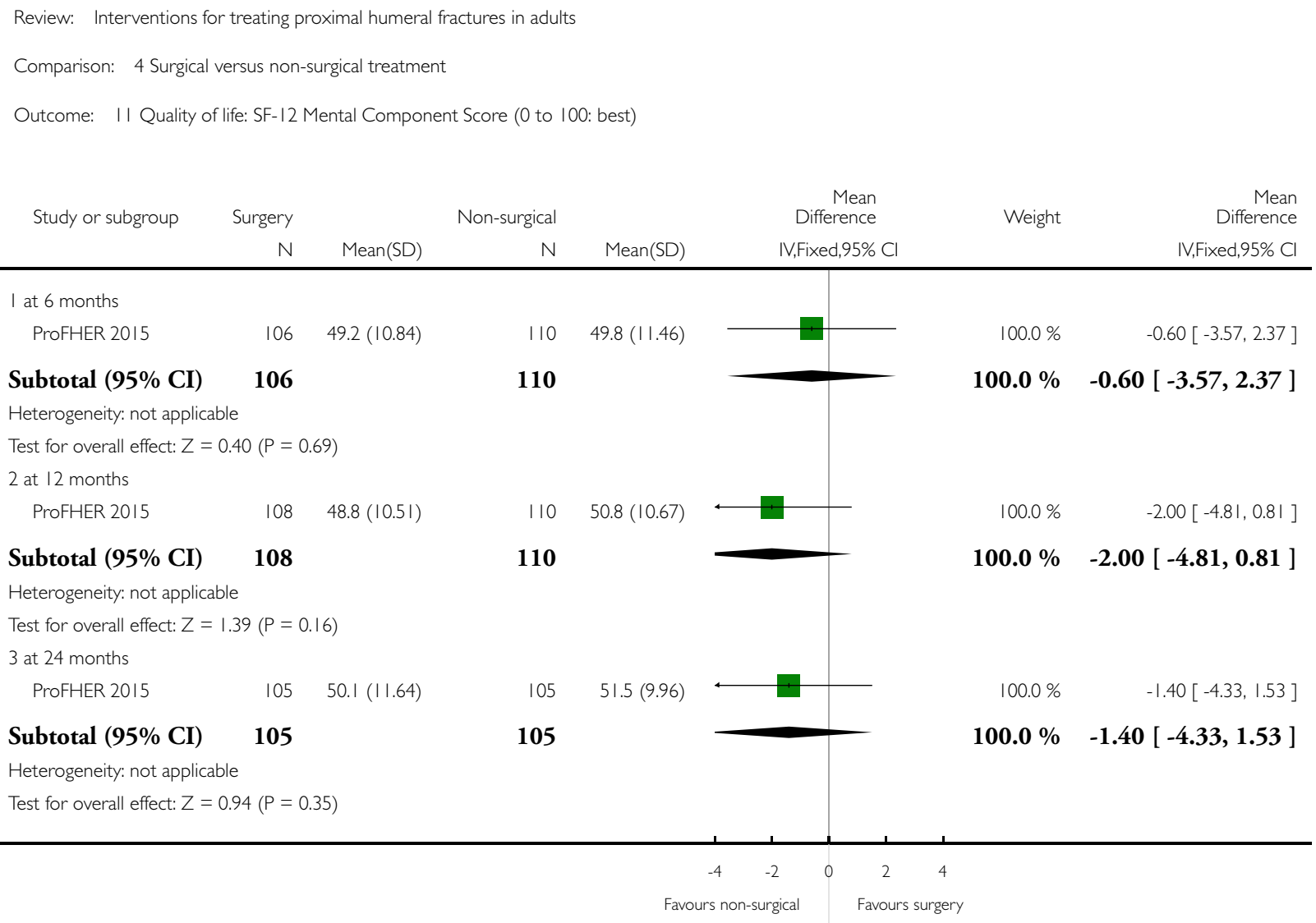




\section{Analysis 4.I2. Comparison 4 Surgical versus non-surgical treatment, Outcome I 2 Mortality.}

Review: Interventions for treating proximal humeral fractures in adults

Comparison: 4 Surgical versus non-surgical treatment

Outcome: 12 Mortality

\begin{tabular}{|c|c|c|c|c|c|}
\hline \multirow[t]{2}{*}{ Study or subgroup } & Surgery & Non-surgical & Risk Ratio & Weight & Risk Ratio \\
\hline & $\mathrm{n} / \mathrm{N}$ & \multicolumn{3}{|c|}{ M-H,Fixed,95\% Cl } & M-H,Fixed,95\% Cl \\
\hline Boons 2012 & $0 / 25$ & $1 / 25$ & $\div$ & $12.0 \%$ & $0.33[0.01,7.81]$ \\
\hline Fjalestad $2010(\mathrm{I})$ & $2 / 25$ & $1 / 25$ & $\longrightarrow$ & $8.0 \%$ & $2.00[0.19,20.67]$ \\
\hline Olerud 20I la & $2 / 30$ & $2 / 29$ & $\Longrightarrow$ & $16.3 \%$ & $0.97[0.15,6.41]$ \\
\hline Olerud $201 \mathrm{lb}$ & $3 / 27$ & $2 / 28$ & $\longrightarrow$ & $15.7 \%$ & $1.56[0.28,8.59]$ \\
\hline ProFHER 2015 & $9 / 125$ & $5 / 125$ & & $40.0 \%$ & $1.80[0.62,5.22]$ \\
\hline Stableforth 1984 & $1 / 16$ & $1 / 16$ & & $8.0 \%$ & $1.00[0.07,14.64]$ \\
\hline Total (95\% CI) & 248 & 248 & 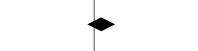 & $100.0 \%$ & $1.40[0.69,2.83]$ \\
\hline \multicolumn{6}{|c|}{ Total events: 17 (Surgery), 12 (Non-surgical) } \\
\hline \multicolumn{6}{|c|}{ Heterogeneity: $\mathrm{Chi}^{2}=1.32, \mathrm{df}=5(P=0.93) ; \mathrm{I}^{2}=0.0 \%$} \\
\hline \multicolumn{6}{|c|}{ Test for overall effect: $Z=0.94(P=0.35)$} \\
\hline \multicolumn{6}{|c|}{ Test for subgroup differences: Not applicable } \\
\hline
\end{tabular}

(I) 2 surgery at 8 and 9 weeks; I conservative after I year 
Analysis 4.13. Comparison 4 Surgical versus non-surgical treatment, Outcome I 3 Additional surgery (reoperation or secondary surgery).

Review: Interventions for treating proximal humeral fractures in adults

Comparison: 4 Surgical versus non-surgical treatment

Outcome: 13 Additional surgery (re-operation or secondary surgery)

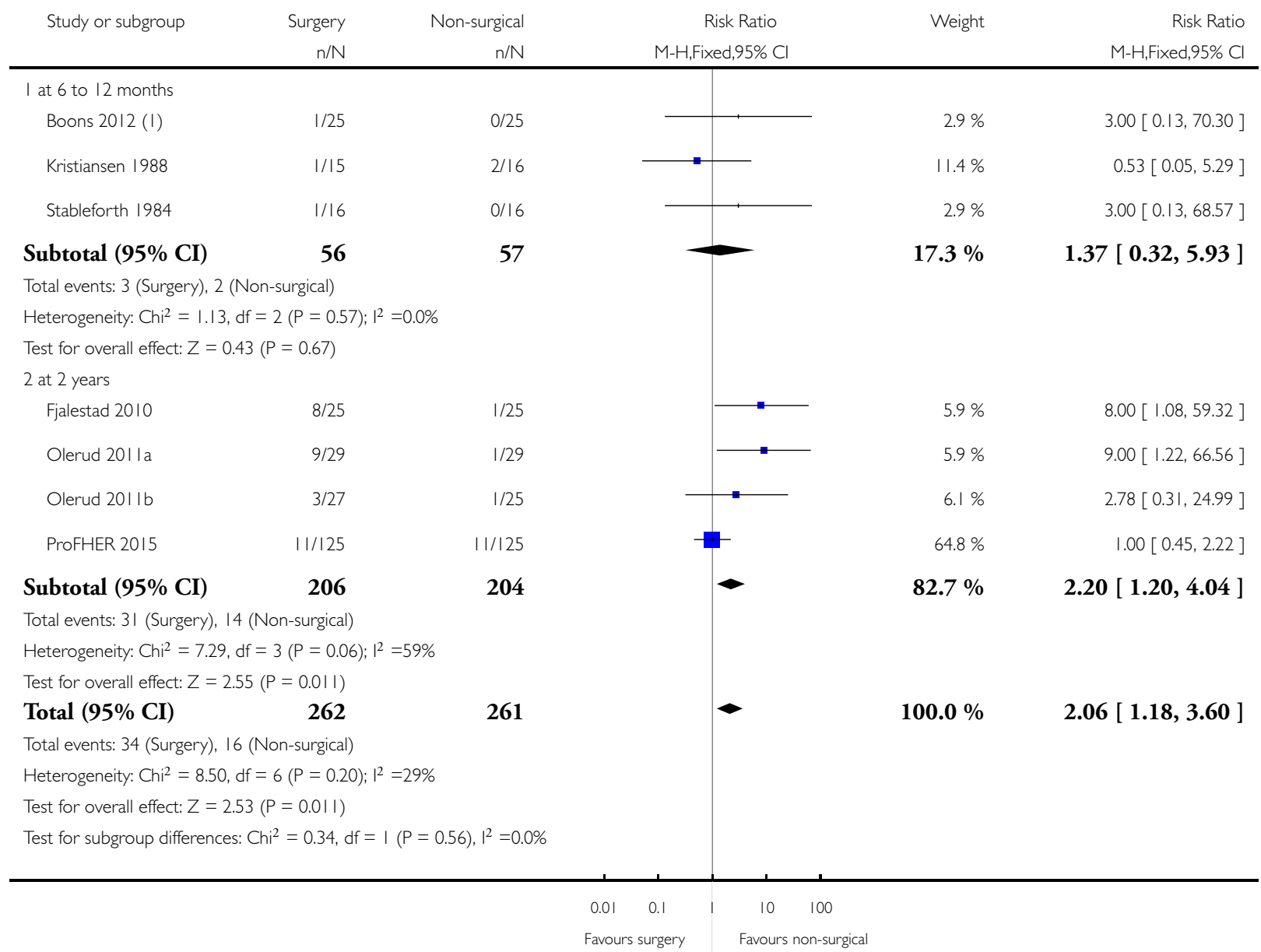

( I) I patient in the conservative treatment group had an operation at 13 months from non-union 


\section{Analysis 4.14. Comparison 4 Surgical versus non-surgical treatment, Outcome 14 Adverse events I complications.}

\begin{tabular}{|c|c|c|c|c|c|}
\hline \multicolumn{6}{|c|}{ Comparison: 4 Surgical versus non-surgical treatment } \\
\hline \multicolumn{6}{|c|}{ Outcome: 14 Adverse events / complications } \\
\hline \multirow[t]{2}{*}{ Study or subgroup } & Surgery & Non-surgical & Risk Ratio & Weight & Risk Ratio \\
\hline & $\mathrm{n} / \mathrm{N}$ & $n / N$ & M-H,Fixed, $95 \% \mathrm{Cl}$ & & M-H,Fixed, $95 \% \mathrm{Cl}$ \\
\hline \multicolumn{6}{|c|}{ I Number of patients with complications } \\
\hline ProFHER 2015 (I) & $30 / 125$ & $23 / 125$ & & $100.0 \%$ & $1.30[0.80,2.11]$ \\
\hline Subtotal $(95 \% \mathrm{CI})$ & 125 & 125 & $\bullet$ & $100.0 \%$ & $1.30[0.80,2.11]$ \\
\hline \multicolumn{6}{|c|}{ Total events: 30 (Surgery), 23 (Non-surgical) } \\
\hline \multicolumn{6}{|c|}{ Heterogeneity: not applicable } \\
\hline \multicolumn{6}{|c|}{ Test for overall effect: $Z=1.08(P=0.28)$} \\
\hline \multicolumn{6}{|c|}{2 Additional shoulder-related therapy } \\
\hline ProFHER 2015 & $7 / 125$ & $4 / 125$ & 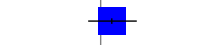 & $100.0 \%$ & $1.75[0.53,5.83]$ \\
\hline Subtotal $(95 \% \mathrm{CI})$ & 125 & 125 & - & $100.0 \%$ & $1.75[0.53,5.83]$ \\
\hline \multicolumn{6}{|c|}{ Total events: 7 (Surgery), 4 (Non-surgical) } \\
\hline \multicolumn{6}{|c|}{ Heterogeneity: not applicable } \\
\hline \multicolumn{6}{|c|}{ Test for overall effect: $Z=0.91 \quad(P=0.36)$} \\
\hline \multicolumn{6}{|l|}{3 Infection } \\
\hline Boons 2012 & $0 / 25$ & $0 / 25$ & & & Not estimable \\
\hline Fjalestad 2010 & $0 / 25$ & $0 / 25$ & & & Not estimable \\
\hline Kristiansen 1988 & $1 / 15$ & $0 / 16$ & & $19.6 \%$ & $3.19[0.14,72.69]$ \\
\hline Olerud 20I la & $2 / 27$ & 0/29 & - & $19.6 \%$ & $5.36[0.27,106.78]$ \\
\hline Olerud $201 \mathrm{lb}$ & $0 / 27$ & $0 / 25$ & & & Not estimable \\
\hline ProFHER 2015 & $2 / 125$ & $0 / 125$ & 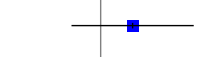 & $20.3 \%$ & $5.00[0.24,103.10]$ \\
\hline Stableforth 1984 & $1 / 16$ & $0 / 16$ & 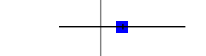 & $20.3 \%$ & $3.00[0.13,68.57]$ \\
\hline Zyto 1997 & $2 / 19$ & $0 / 19$ & 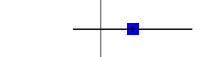 & $20.3 \%$ & $5.00[0.26,97.70]$ \\
\hline Subtotal $(95 \% \mathrm{CI})$ & 279 & 280 & - & $100.0 \%$ & $4.31[1.11,16.74]$ \\
\hline \multicolumn{6}{|c|}{ Total events: 8 (Surgery), 0 (Non-surgical) } \\
\hline \multicolumn{6}{|c|}{ Heterogeneity: $\mathrm{Ch}^{2}=0.13, \mathrm{df}=4(\mathrm{P}=1.00) ; 1^{2}=0.0 \%$} \\
\hline \multicolumn{6}{|c|}{ Test for overall effect: $Z=2.11$ ( $P=0.035)$} \\
\hline \multicolumn{6}{|l|}{4 Nerve injury / palsy } \\
\hline Fjalestad 2010 (2) & $3 / 21$ & $3 / 20$ & & $60.3 \%$ & $0.95[0.22,4.18]$ \\
\hline Olerud 201 la & $0 / 27$ & $1 / 26$ & $\longrightarrow$ & $29.9 \%$ & $0.32[0.01,7.55]$ \\
\hline Olerud $201 \mathrm{lb}$ & $0 / 27$ & $0 / 25$ & & & Not estimable \\
\hline \multirow[t]{3}{*}{ ProFHER 2015} & $2 / 125$ & $0 / 125$ & - & $9.8 \%$ & $5.00[0.24,103.10]$ \\
\hline & & & $0.1 \quad 1 \quad 10 \quad 100$ & & \\
\hline & & & Favours no & & \\
\hline
\end{tabular}




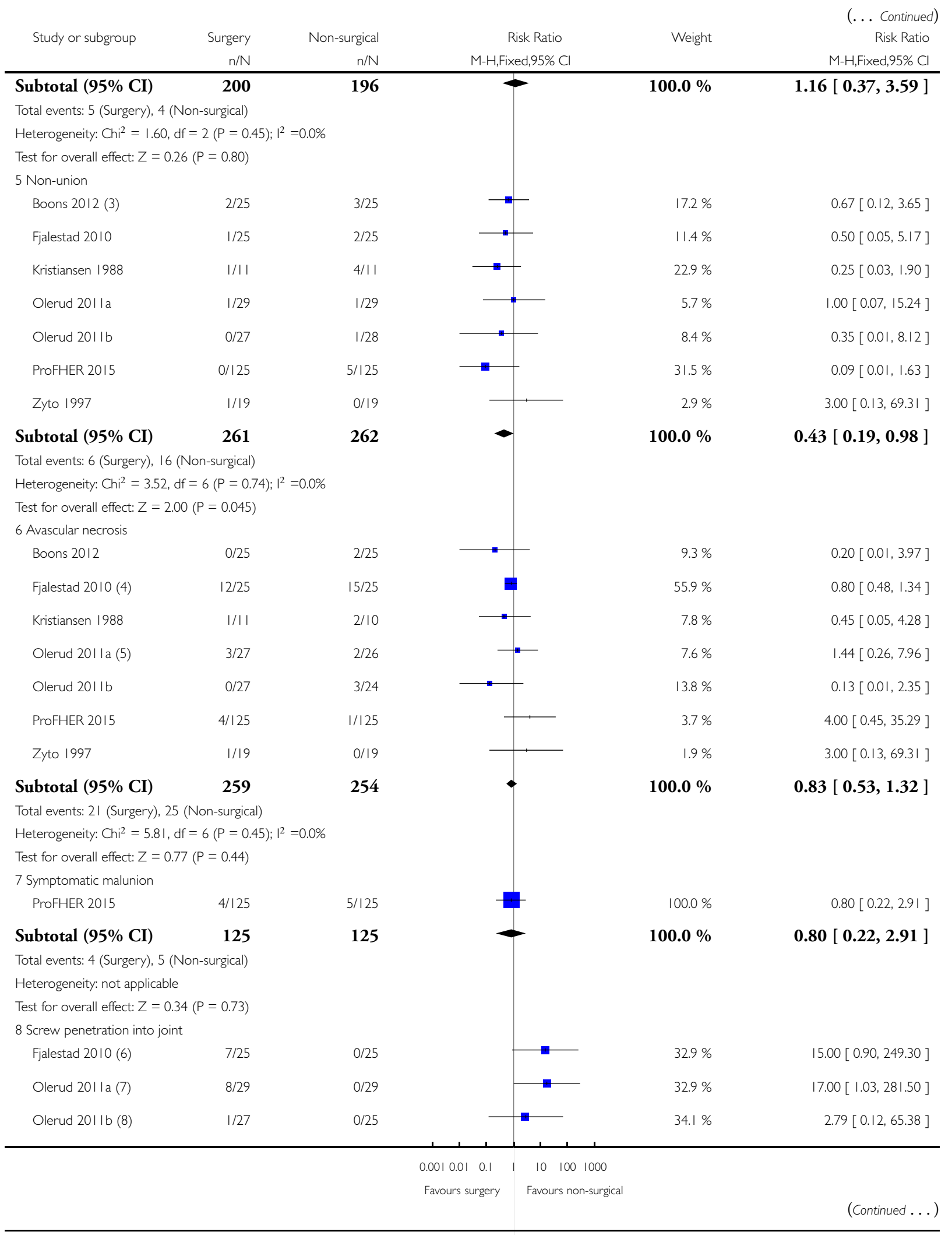

Interventions for treating proximal humeral fractures in adults (Review)

Copyright $\odot 2015$ The Cochrane Collaboration. Published by John Wiley \& Sons, Ltd. 


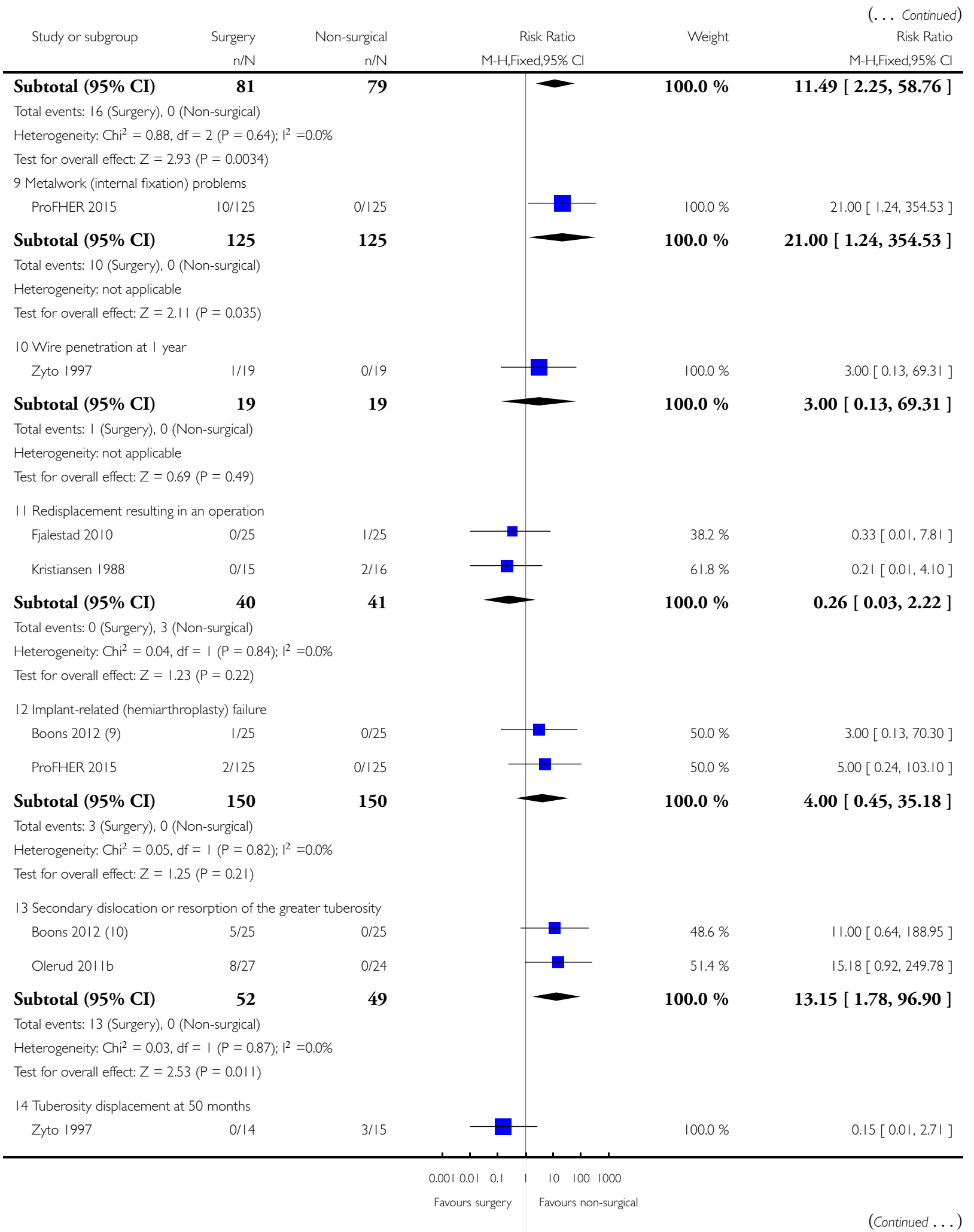

Interventions for treating proximal humeral fractures in adults (Review)

Copyright () 2015 The Cochrane Collaboration. Published by John Wiley \& Sons, Ltd. 


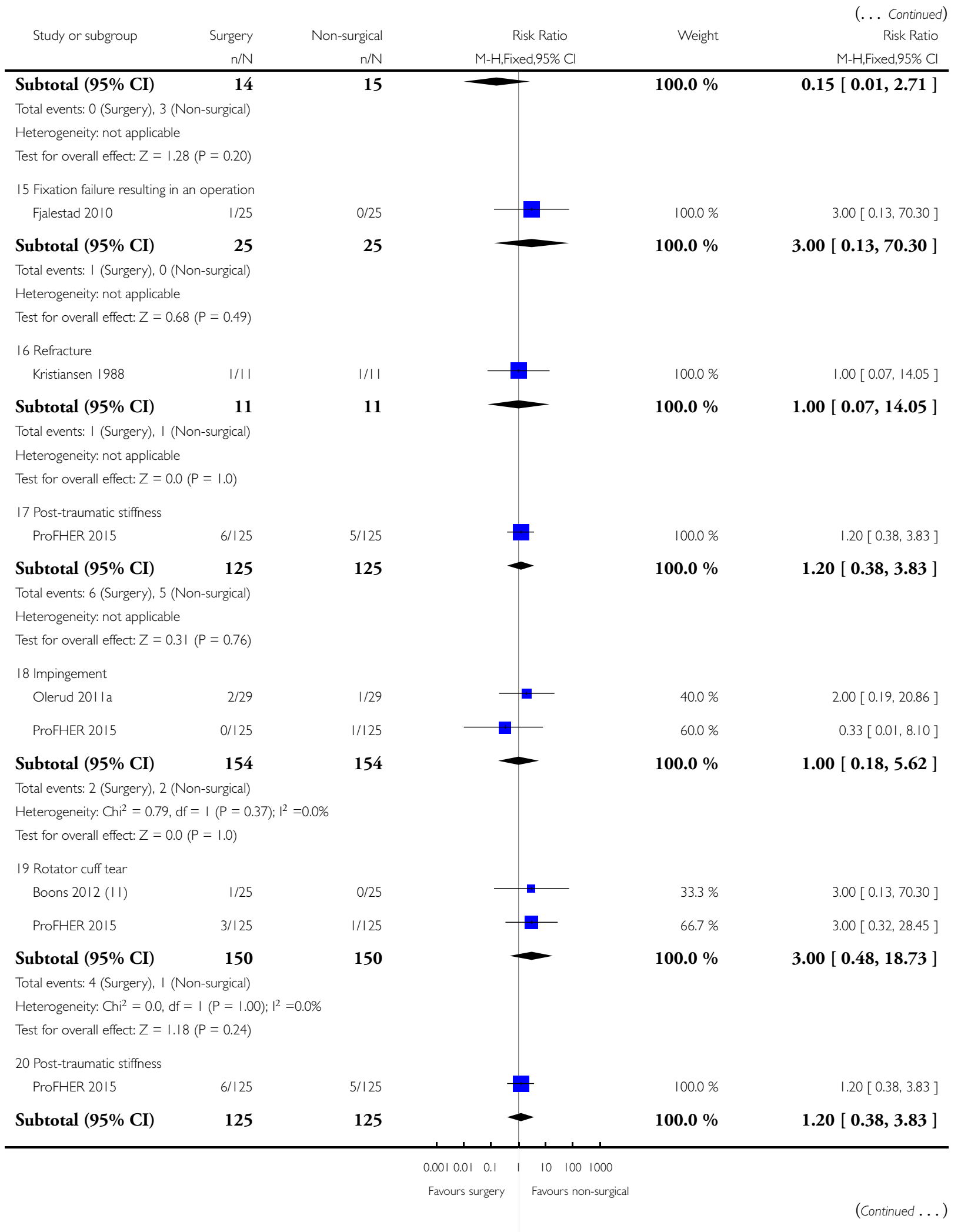

Interventions for treating proximal humeral fractures in adults (Review)

Copyright () 2015 The Cochrane Collaboration. Published by John Wiley \& Sons, Ltd. 
Total events: 6 (Surgery), 5 (Non-surgical)

Heterogeneity: not applicable

Test for overall effect: $Z=0.31(P=0.76)$

21 CRPS or severe pain

ProFHER 2015 (12)

Subtotal (95\% CI)

Total events: 2 (Surgery), I (Non-surgical)

Heterogeneity: not applicable

Test for overall effect: $Z=0.57(P=0.57)$

22 Dislocation or instability

PrOFHER 2015

\section{Subtotal (95\% CI)}

125

Total events: 0 (Surgery), I (Non-surgical)

Heterogeneity: not applicable

Test for overall effect: $Z=0.67(P=0.50)$

23 Heterotopic ossification

$$
\text { Boons } 2012
$$

\section{Subtotal (95\% CI)}

Total events: 0 (Surgery), 0 (Non-surgical)

Heterogeneity: not applicable

Test for overall effect: not applicable

24 Post-traumatic osteoarthritis (signs of)

Fjalestad 2010

Olerud 201 la

Olerud 201 Ib (13)

Zyto 1997

Subtotal $(95 \% \mathrm{CI})$

Total events: 6 (Surgery), 9 (Non-surgical)

Heterogeneity: $\mathrm{Chi}^{2}=3.99, \mathrm{df}=3(\mathrm{P}=0.26) ; \mathrm{I}^{2}=25 \%$

Test for overall effect: $Z=0.83(P=0.4 I)$

$100.0 \%$

$2.00[0.18,21.78]$

I/125

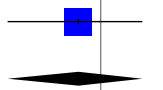

$100.0 \%$

$0.33[0.01,8.10]$

125

$100.0 \%$

$0.33[0.01,8.10]$

Not estimable

25

Not estimable

$4.9 \%$

$3.00[0.13,70.30]$
$19.8 \%$

$56.5 \%$

$18.8 \%$

$100.0 \%$

90

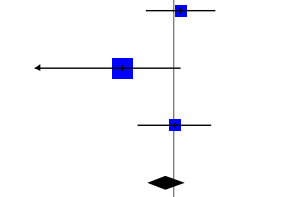

I.44 $[0.26,7.96]$

$0.08[0.00,1.40]$

$1.07[0.17,6.61]$

$0.68[0.27,1.70]$ 

(I) 6 patients in the surgical group had 2 listed complications
(2) I person in each group had persistent deltoid atrophy
(3) The 2 non-unions in the surgery group were of the greater tuberosity
(4) At 2 years (8 versus 13 at I year): mostly asymptomatic (3 versus 2 had some pain)
(5) 2 cases (I severe, I minor) in the surgical group had reoperations
(6) Three implants were removed
(7) One of the 3 'secondary screw penetrations' were operated on for this reason
(8) I patient in this trial had plate fixation instead of a prosthesis
(9) Head-stem separation; revised after I week
(10) Secondary superior migration of GT; partial bone resorption in 2
( I I) "potential" rotator cuff tear; patient had proximal migration of their hemiarthroplasty
(12) I patient in surgery group had complex regional pain syndrome (CRPS)
(13) Surgery was arthroplasty

\section{Analysis 4.I5. Comparison 4 Surgical versus non-surgical treatment, Outcome I 5 Dependent in activities of daily living (or dead) at 6 months.}

Review: Interventions for treating proximal humeral fractures in adults

Comparison: 4 Surgical versus non-surgical treatment

Outcome: 15 Dependent in activities of daily living (or dead) at 6 months

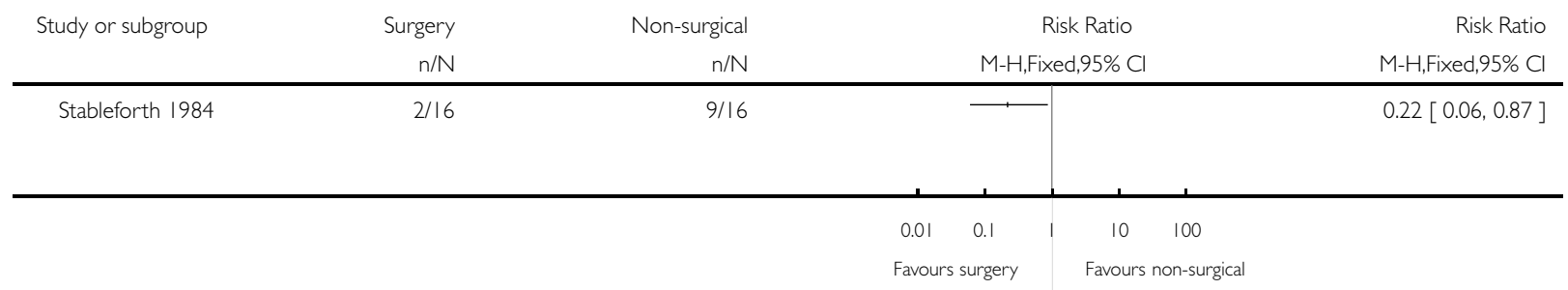


Analysis 4.16. Comparison 4 Surgical versus non-surgical treatment, Outcome 16 Constant scores (overall: 0 to 100: best score).

Review: Interventions for treating proximal humeral fractures in adults

Comparison: 4 Surgical versus non-surgical treatment

Outcome: 16 Constant scores (overall: 0 to 100: best score)

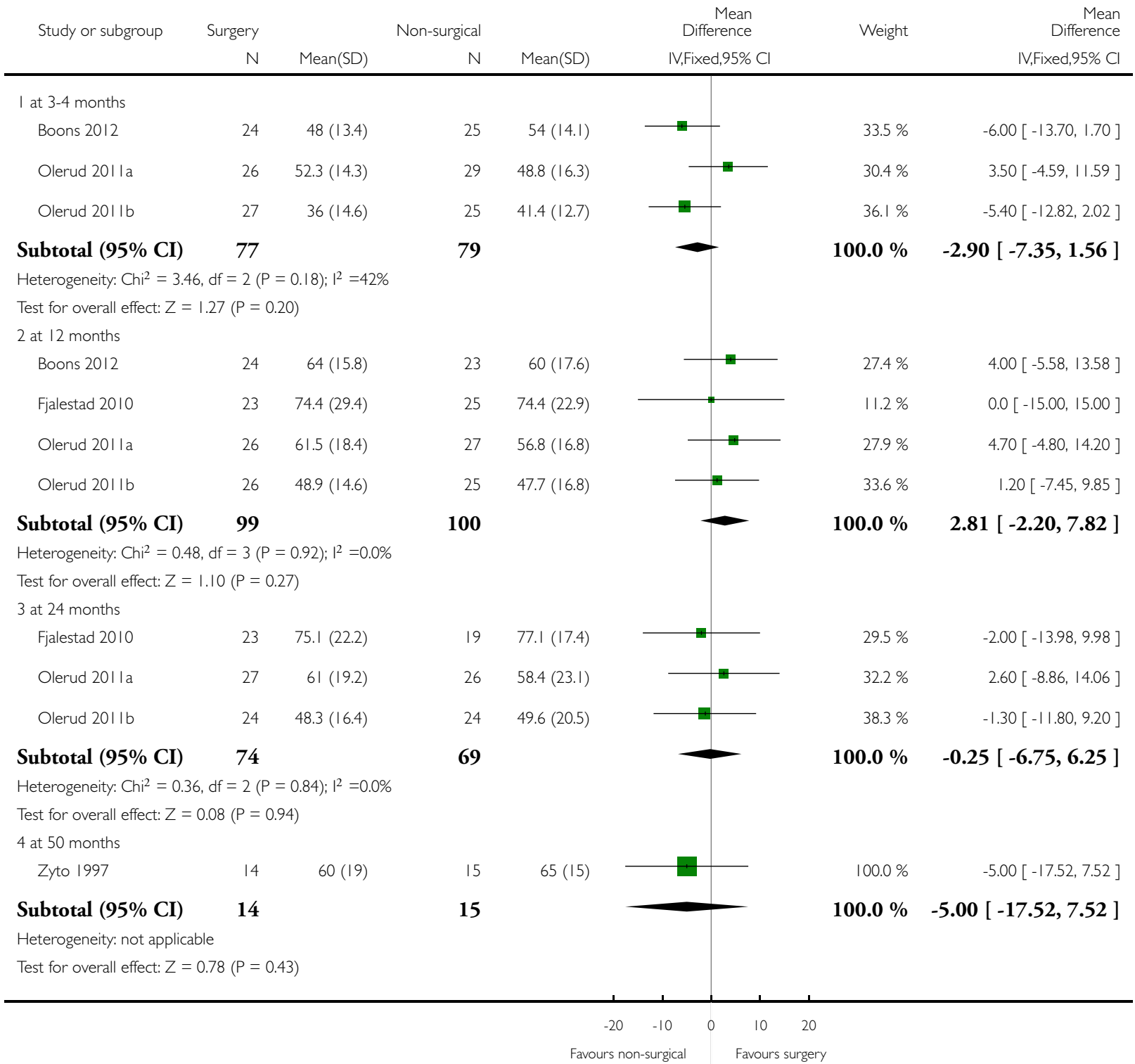




\section{Analysis 4.17. Comparison 4 Surgical versus non-surgical treatment, Outcome 17 Constant scores}

(difference between injured and uninjured shoulder): Normal $=0$..

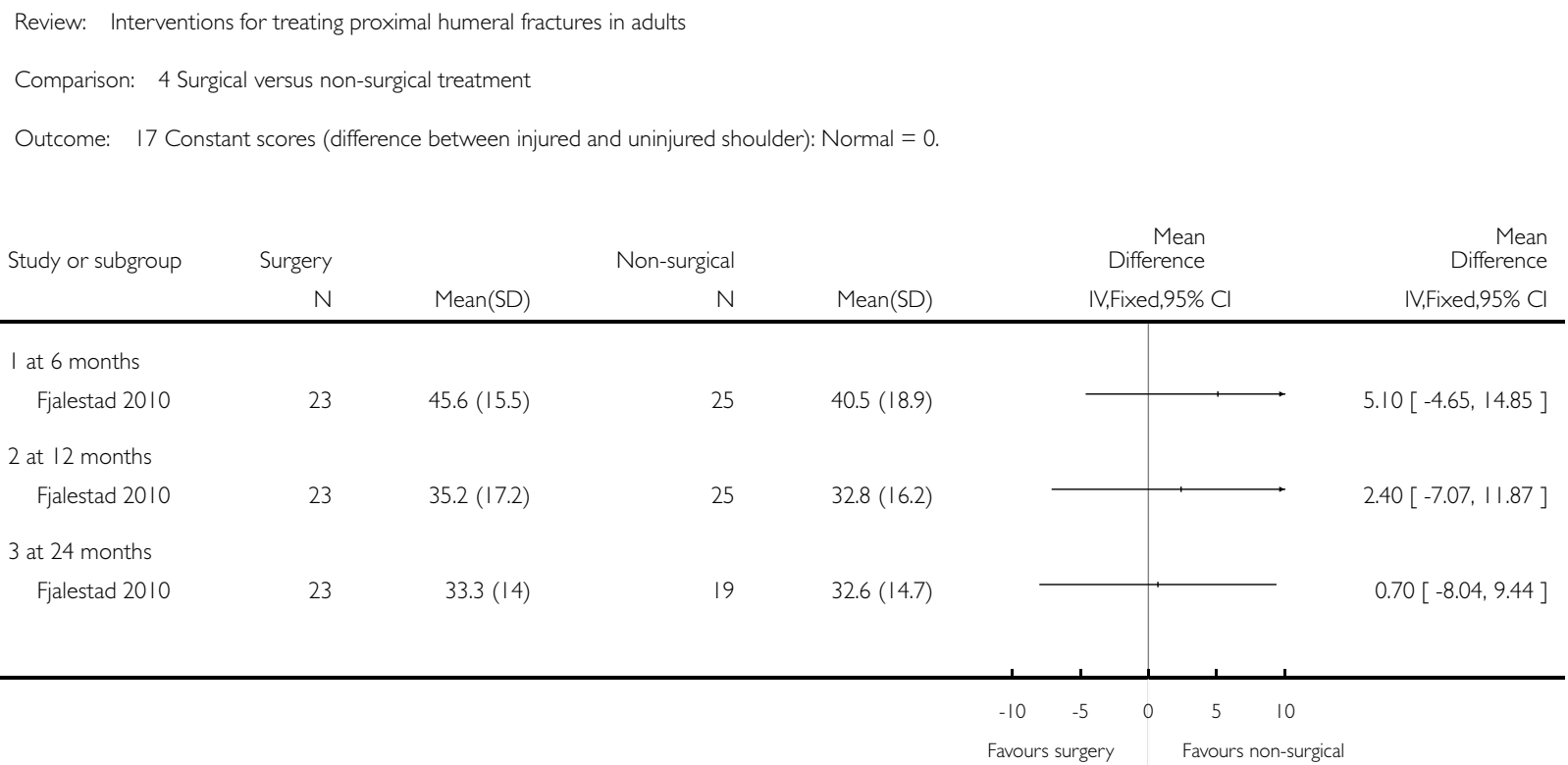

Analysis 4.18. Comparison 4 Surgical versus non-surgical treatment, Outcome 18 Poor or unsatisfactory function at I year (Neer rating).

Review: Interventions for treating proximal humeral fractures in adults

Comparison: 4 Surgical versus non-surgical treatment

Outcome: 18 Poor or unsatisfactory function at I year (Neer rating)

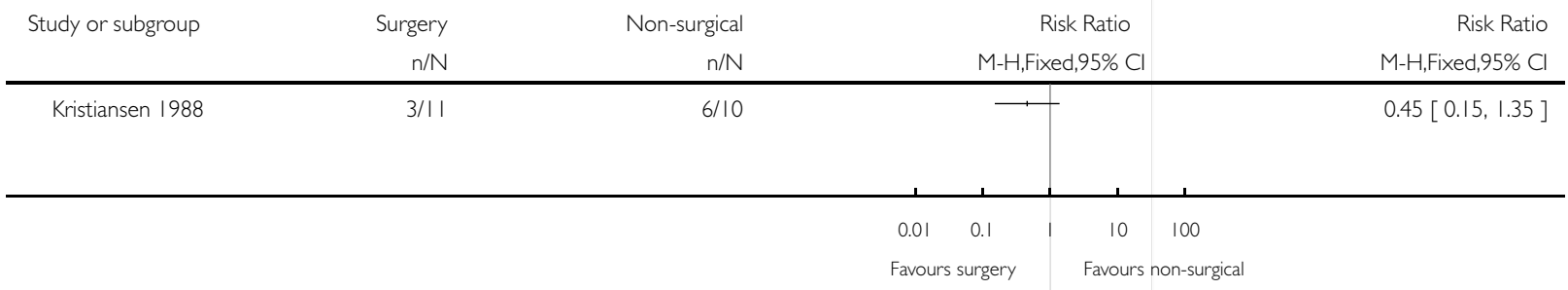


Analysis 4.19. Comparison 4 Surgical versus non-surgical treatment, Outcome I9 VAS disability (0 to 100 : no restrictions).

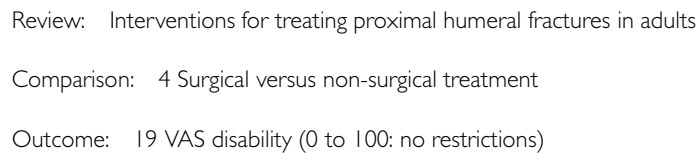

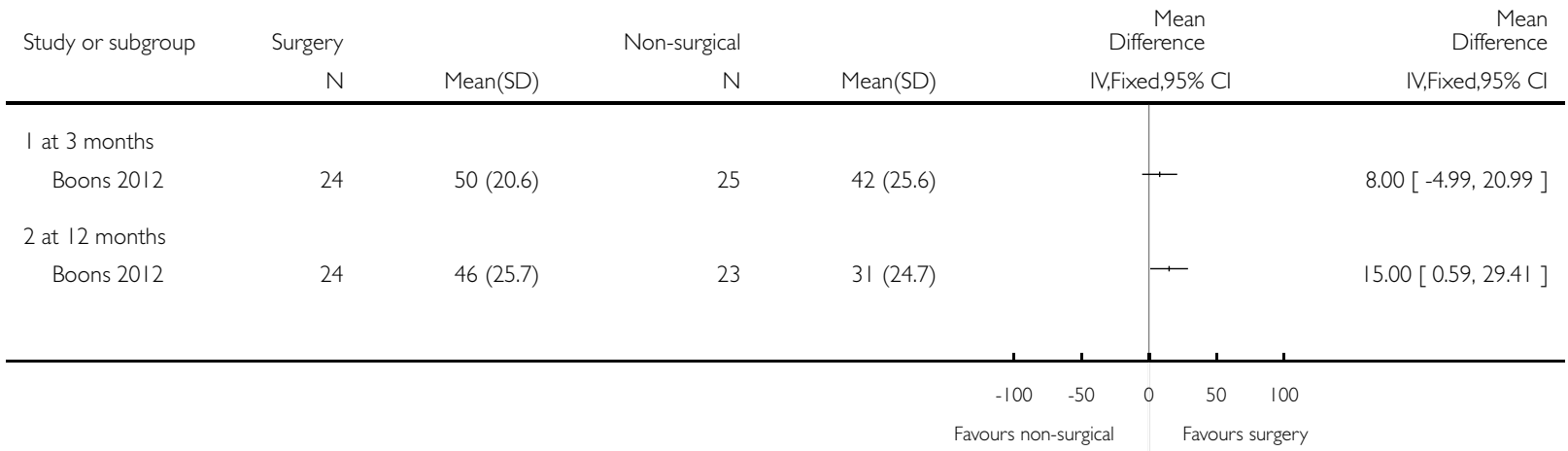


Analysis 4.20. Comparison 4 Surgical versus non-surgical treatment, Outcome 20 Pain: VAS (0 to 100 : worst pain).

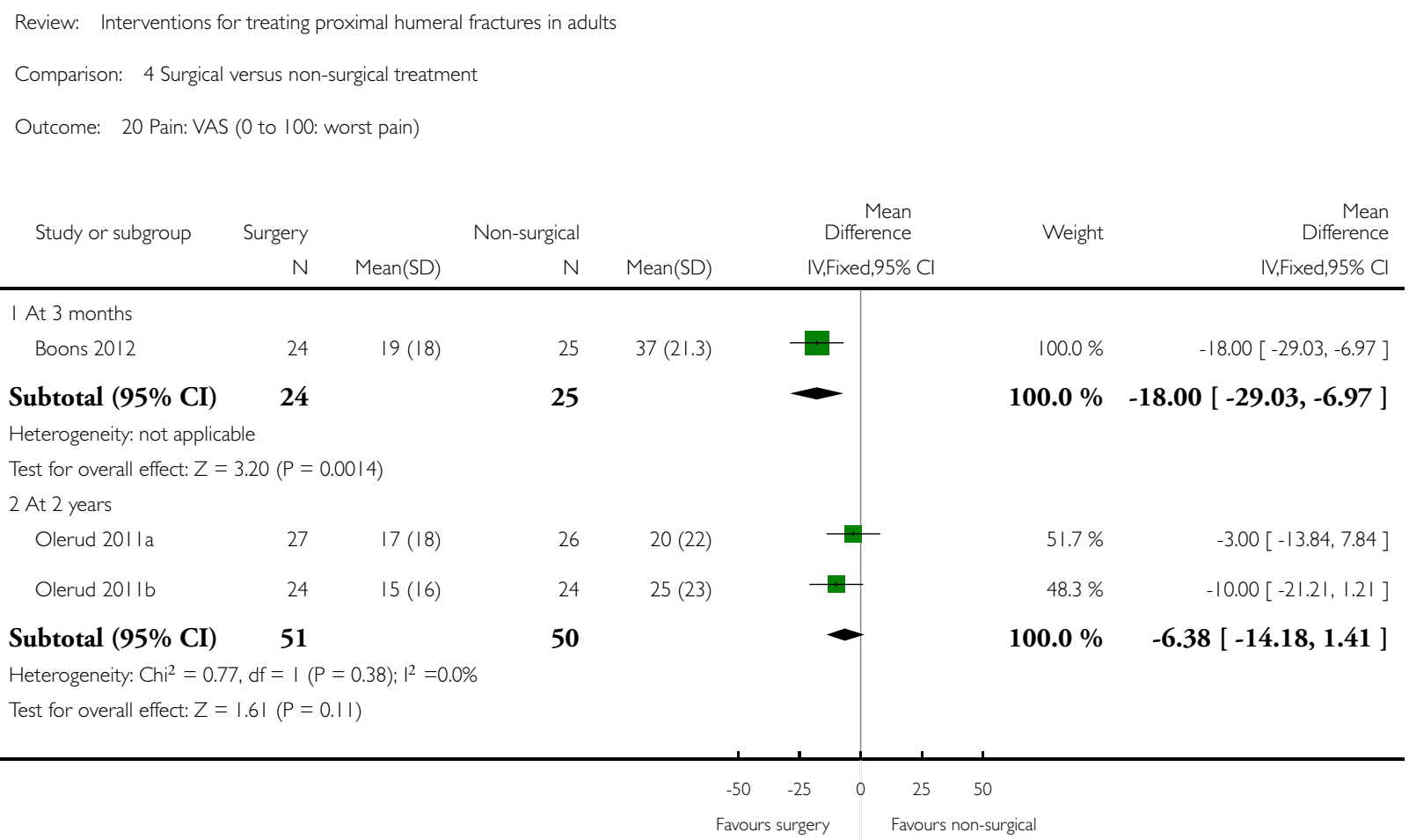


Analysis 4.2I. Comparison 4 Surgical versus non-surgical treatment, Outcome 21 Constant score at 50 months: overall and components.

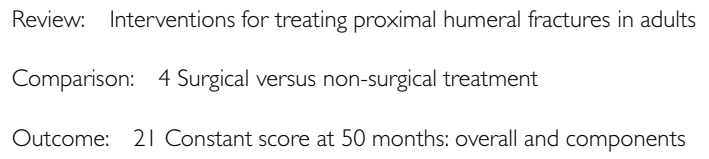

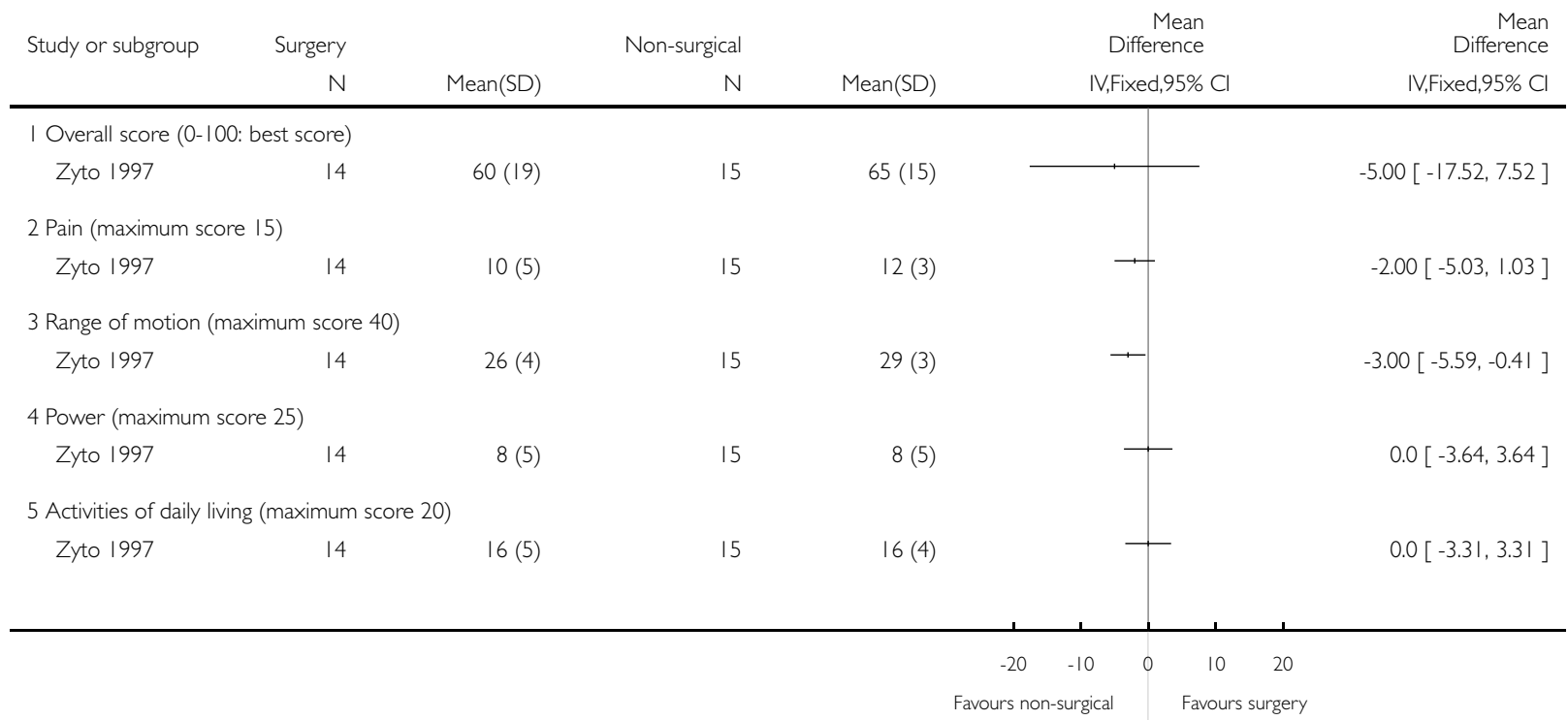

Analysis 4.22. Comparison 4 Surgical versus non-surgical treatment, Outcome 22 Constant (often severe) pain at 6 months.

Review: Interventions for treating proximal humeral fractures in adults

Comparison: 4 Surgical versus non-surgical treatment

Outcome: 22 Constant (often severe) pain at 6 months

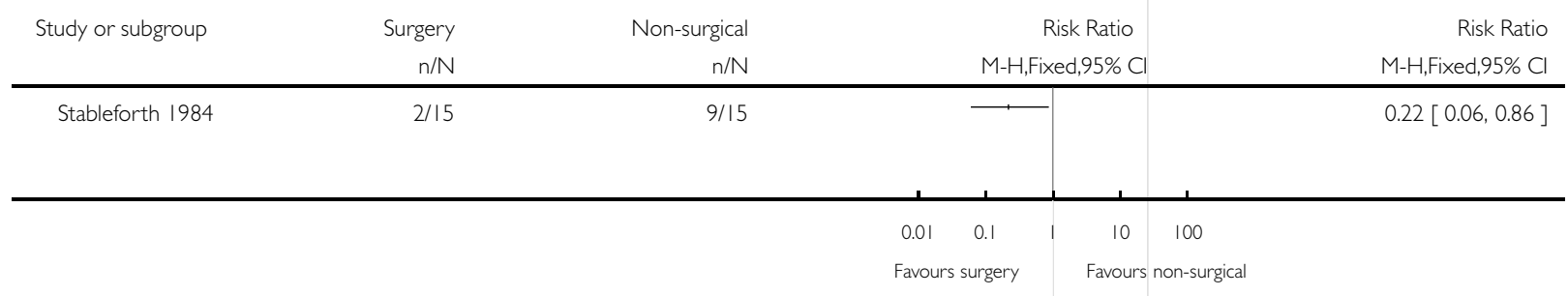


Analysis 4.23. Comparison 4 Surgical versus non-surgical treatment, Outcome 23 Failure to recover $75 \%$ muscle power relative to other arm (survivors) at 6 months.

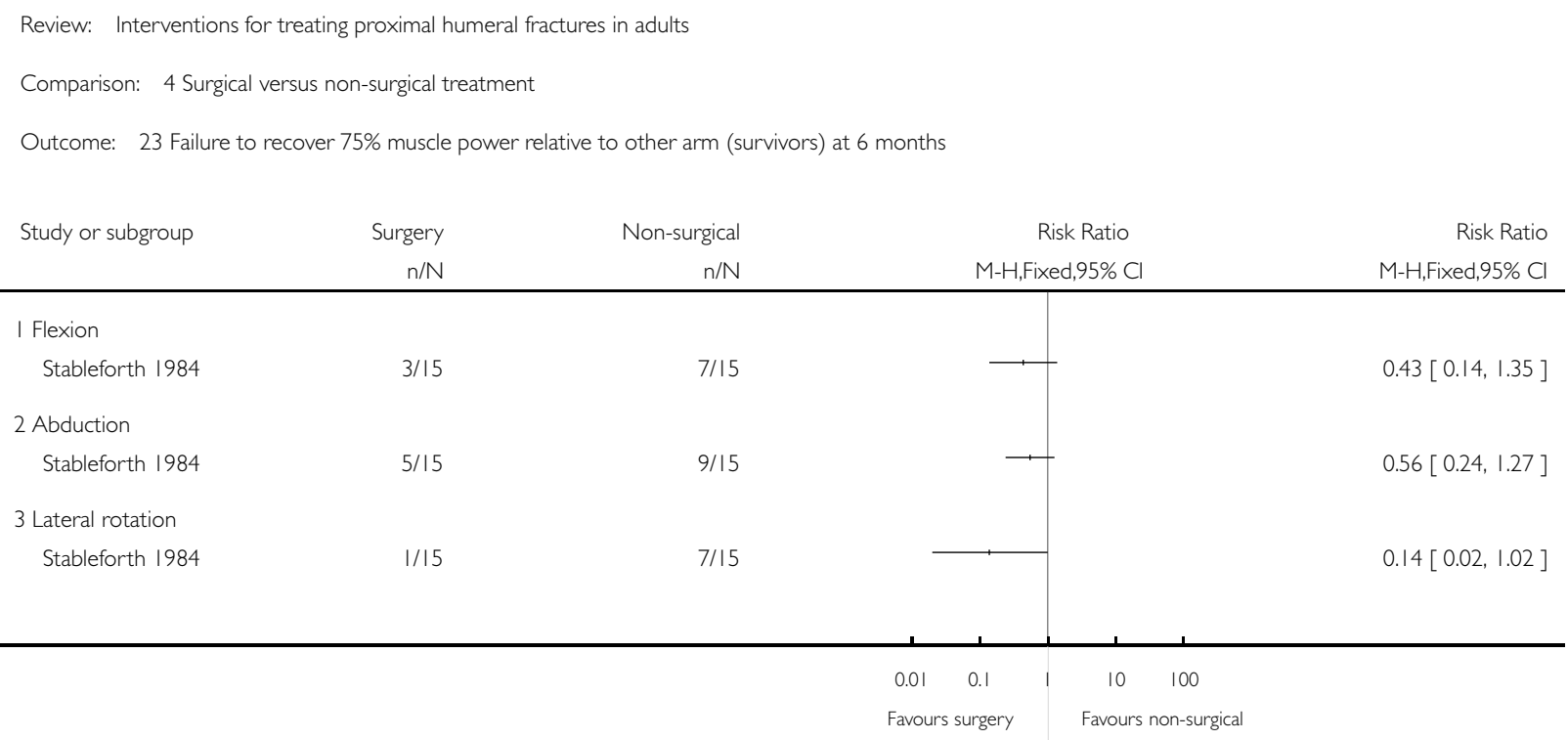


Analysis 4.24. Comparison 4 Surgical versus non-surgical treatment, Outcome 24 Range of movement impairments in survivors at 6 months.

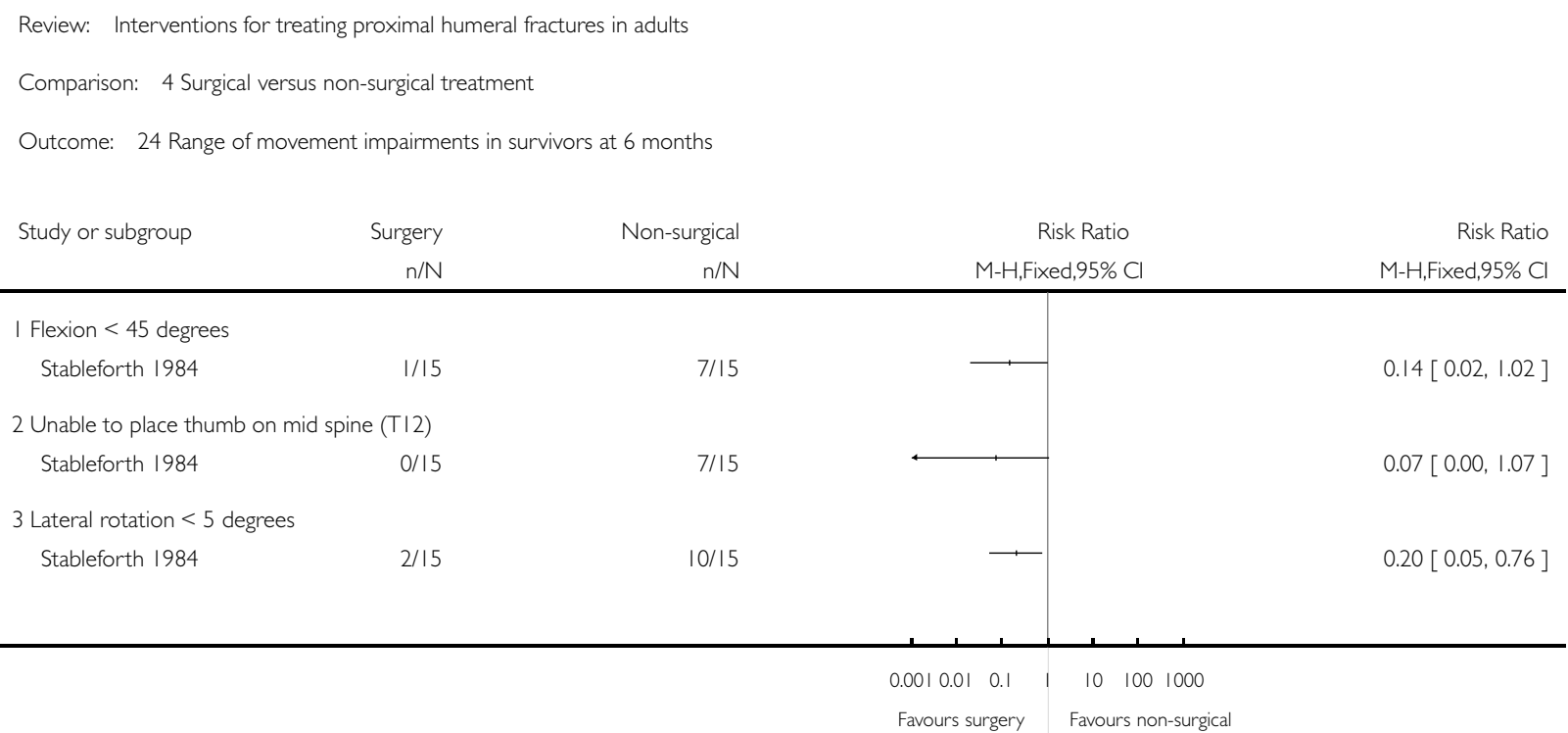

Analysis 4.25. Comparison 4 Surgical versus non-surgical treatment, Outcome 25 Costs at I year (Euros in 2005).

Costs at 1 year (Euros in 2005)

\begin{tabular}{|c|c|c|c|c|}
\hline Study & Measure & Surgery & Non-surgical treatment & Difference (conclusion) \\
\hline Fjalestad 2010 & Total health-care costs & mean $=10,367$ & mean $=10,946$ & $\begin{array}{l}\text { Abstract: "the mean difference in to- } \\
\text { tal health-care costs was } 597 \text { Euros in } \\
\text { favour of surgery ( } 95 \% \text { CI }=-5291 \text {, } \\
\text { 3777)". No significant difference }\end{array}$ \\
\hline Fjalestad 2010 & Health-care + indirect costs & mean $=23,953$ & mean $=21,878$ & $\begin{array}{l}\text { Reformatted text: "Including indirect } \\
\text { costs... the difference [was] 2,075 ( } 95 \% \\
\mathrm{CI}=-15,949 \text { to } 20,100) \text { ". No signifi- } \\
\text { cant difference, but favours the non-sur- } \\
\text { gical group }\end{array}$ \\
\hline
\end{tabular}


Analysis 4.26. Comparison 4 Surgical versus non-surgical treatment, Outcome 26 Total costs including indirect costs (Euros) at I year.

Review: Interventions for treating proximal humeral fractures in adults

Comparison: 4 Surgical versus non-surgical treatment

Outcome: 26 Total costs including indirect costs (Euros) at I year

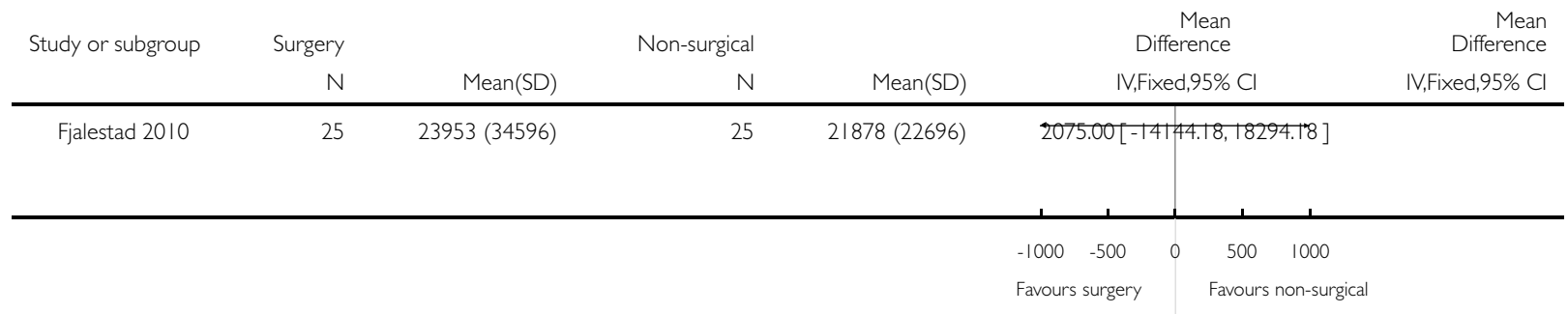

Analysis 5.I. Comparison 5 Locking plate versus locking intramedullary nail, Outcome I American Shoulder and Elbow Surgeons (ASES) score (0 to 100: best).

Review: Interventions for treating proximal humeral fractures in adults

Comparison: 5 Locking plate versus locking intramedullary nail

Outcome: I American Shoulder and Elbow Surgeons (ASES) score (0 to 100: best)

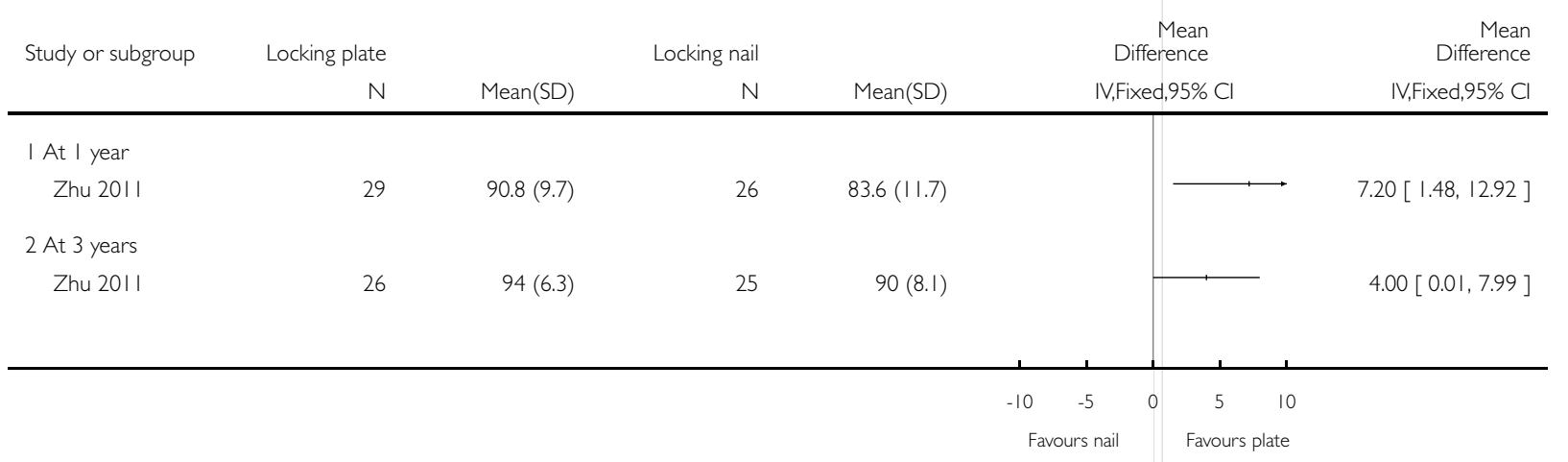


Analysis 5.2. Comparison 5 Locking plate versus locking intramedullary nail, Outcome 2 Death, reoperation and adverse events.

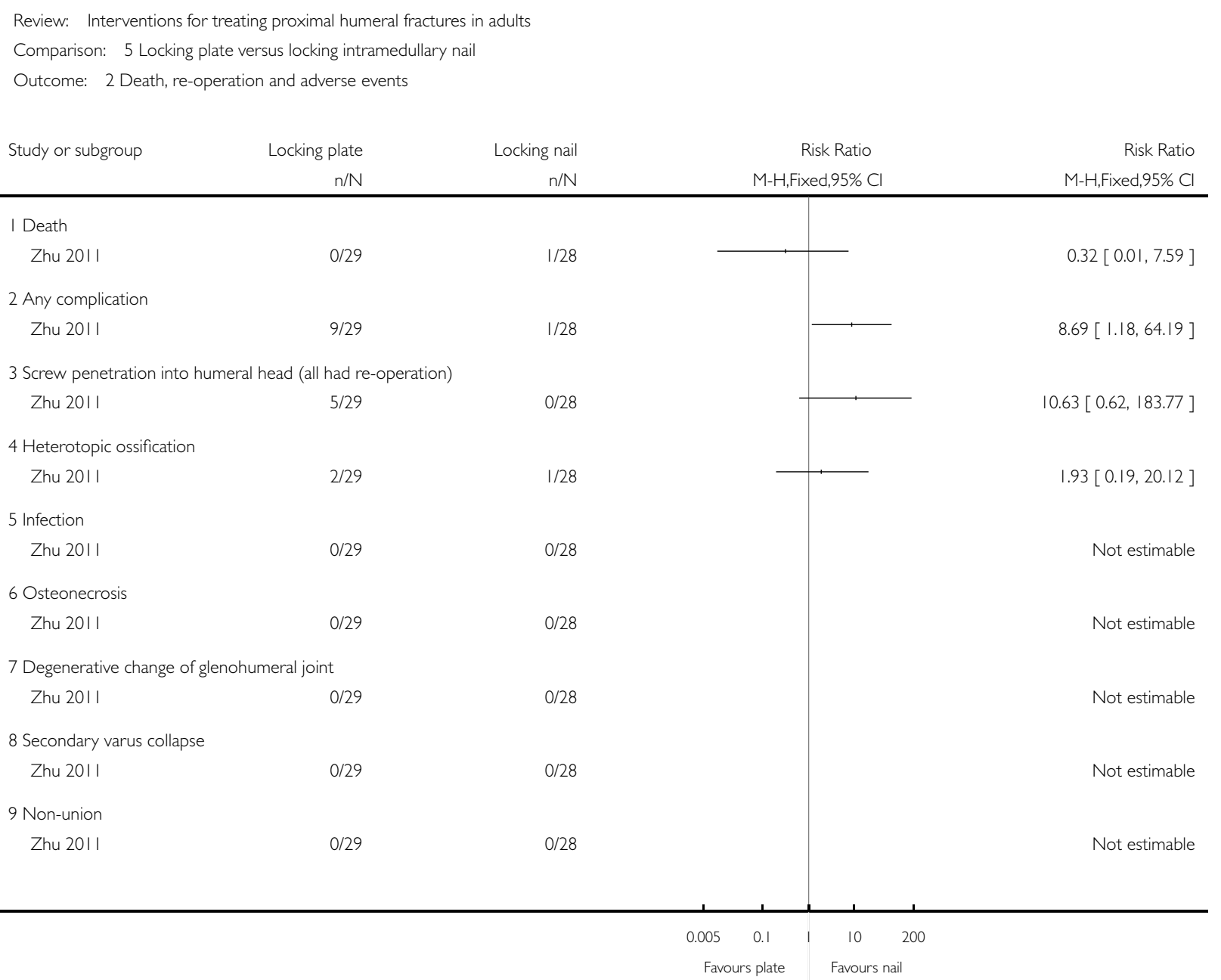

Analysis 5.3. Comparison 5 Locking plate versus locking intramedullary nail, Outcome 3 Pain (VAS: 0 to I0: worst).

Pain (VAS: 0 to 10: worst)

\begin{tabular}{|c|c|c|c|c|}
\hline Study & Measure & Locking plate & Locking nail & Reported significance \\
\hline Zhu 2011 & Pain at 1 year & $\begin{array}{l}\text { median }=0.5 \\
\text { interquartile range: } 1.8 \\
\mathrm{n}=29\end{array}$ & $\begin{array}{l}\text { median }=1.0 \\
\text { interquartile range }=1.0 \\
\mathrm{n}=26\end{array}$ & $\mathrm{P}=0.042$ \\
\hline
\end{tabular}


Pain (VAS: 0 to 10: worst) (Continued)

\begin{tabular}{|c|c|c|c|c|}
\hline Zhu 2011 & Pain at 3 years & $\begin{array}{l}\text { median }=0 \\
\text { interquartile range }=0.8 \\
\mathrm{n}=26\end{array}$ & $\begin{array}{l}\text { median }=0 \\
\text { interquartile range }=1.0 \\
\mathrm{n}=25\end{array}$ & $\mathrm{P}=0.642$ \\
\hline
\end{tabular}

Analysis 5.4. Comparison 5 Locking plate versus locking intramedullary nail, Outcome 4 Constant score (0 to 100: best).

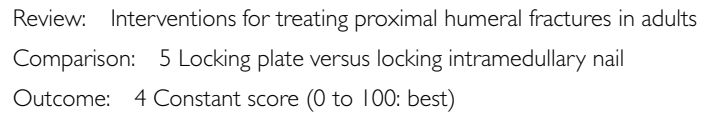

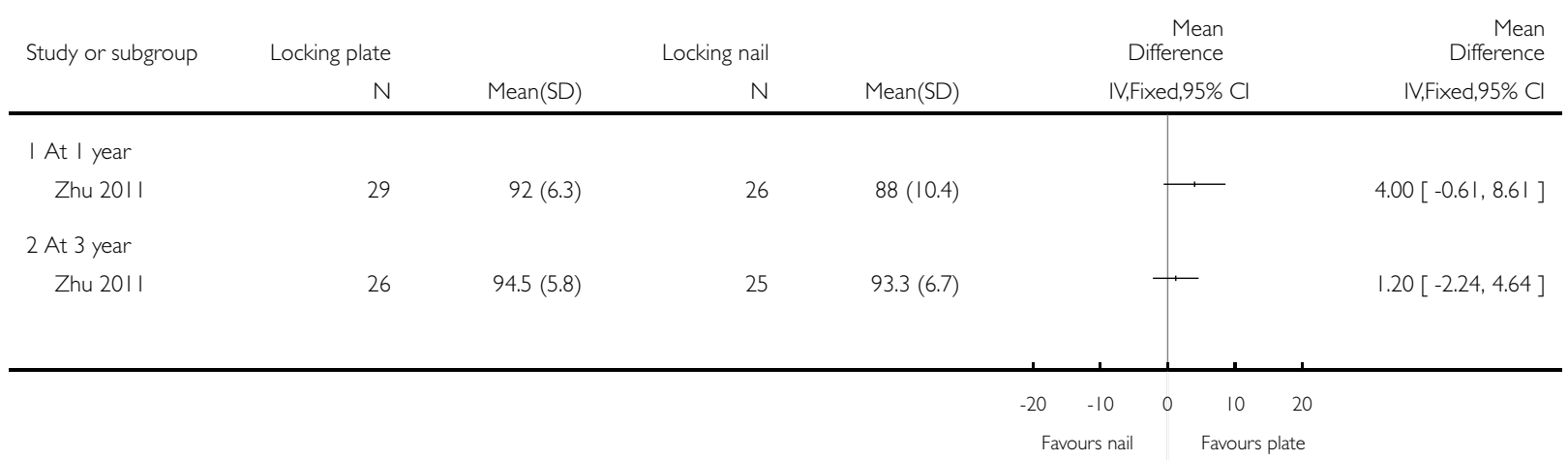


Analysis 5.5. Comparison 5 Locking plate versus locking intramedullary nail, Outcome 5 Active range of motion (at 3 years).

Review: Interventions for treating proximal humeral fractures in adults

Comparison: 5 Locking plate versus locking intramedullary nail

Outcome: 5 Active range of motion (at 3 years)

\begin{tabular}{|c|c|c|c|c|c|c|c|c|c|}
\hline \multirow[t]{2}{*}{ Study or subgroup } & Locking plate & \multicolumn{3}{|c|}{ Locking nail } & \multicolumn{4}{|c|}{$\begin{array}{r}\text { Mean } \\
\text { Difference }\end{array}$} & \multirow{2}{*}{$\begin{array}{r}\text { Mean } \\
\text { Difference } \\
\text { IV,Fixed,95\% Cl}\end{array}$} \\
\hline & $\mathrm{N}$ & Mean(SD) & N & Mean(SD) & & & xed,95\% Cl & & \\
\hline \multicolumn{10}{|c|}{ I Forward elevation (degrees) } \\
\hline Zhu 20II & 26 & $157.3(15.1)$ & 25 & $160.8(11.9)$ & & & & & $-3.50[-10.95,3.95]$ \\
\hline \multicolumn{10}{|l|}{2 External rotation } \\
\hline \multirow[t]{2}{*}{ Zhu 201I } & 26 & $40.4(17.4)$ & 25 & $47.8(17.3)$ & & & - & & $-7.40[-16.92,2.12]$ \\
\hline & & & & & -20 & -10 & 10 & 20 & \\
\hline
\end{tabular}

Analysis 5.6. Comparison 5 Locking plate versus locking intramedullary nail, Outcome 6 Range of movement: internal rotation (level on spine).

Range of movement: internal rotation (level on spine)

\begin{tabular}{lllll}
\hline Study & Measure & Locking plate & Locking nail & Reported significance \\
\hline Zhu 2011 & At 1 year & $\begin{array}{l}\text { mean location = T8 } \\
\text { range }=\mathrm{T} 4 \text { to L2 } \\
\mathrm{n}=29\end{array}$ & $\begin{array}{l}\text { mean location }=\mathrm{T} 9 \\
\text { range }=\mathrm{T} 2 \text { to buttock } \\
\mathrm{n}=26\end{array}$ & $\mathrm{P}=0.443$ \\
\hline Zhu 2011 & At 3 years & $\begin{array}{l}\text { mean location }=\mathrm{T} 8 \\
\text { range }=\mathrm{T} 2 \text { to buttock } \\
\mathrm{n}=26\end{array}$ & $\begin{array}{l}\text { mean location }=\mathrm{T} 8 \\
\text { range }=\mathrm{T} 2 \text { to buttock } \\
\mathrm{n}=25\end{array}$ & $\mathrm{P}=0.636$ \\
\hline
\end{tabular}


Analysis 5.7. Comparison 5 Locking plate versus locking intramedullary nail, Outcome 7 Strength of suprapinatus (relative to opposite side) \% - at 3 years.

Review: Interventions for treating proximal humeral fractures in adults

Comparison: 5 Locking plate versus locking intramedullary nail

Outcome: 7 Strength of suprapinatus (relative to opposite side) \% - at 3 years

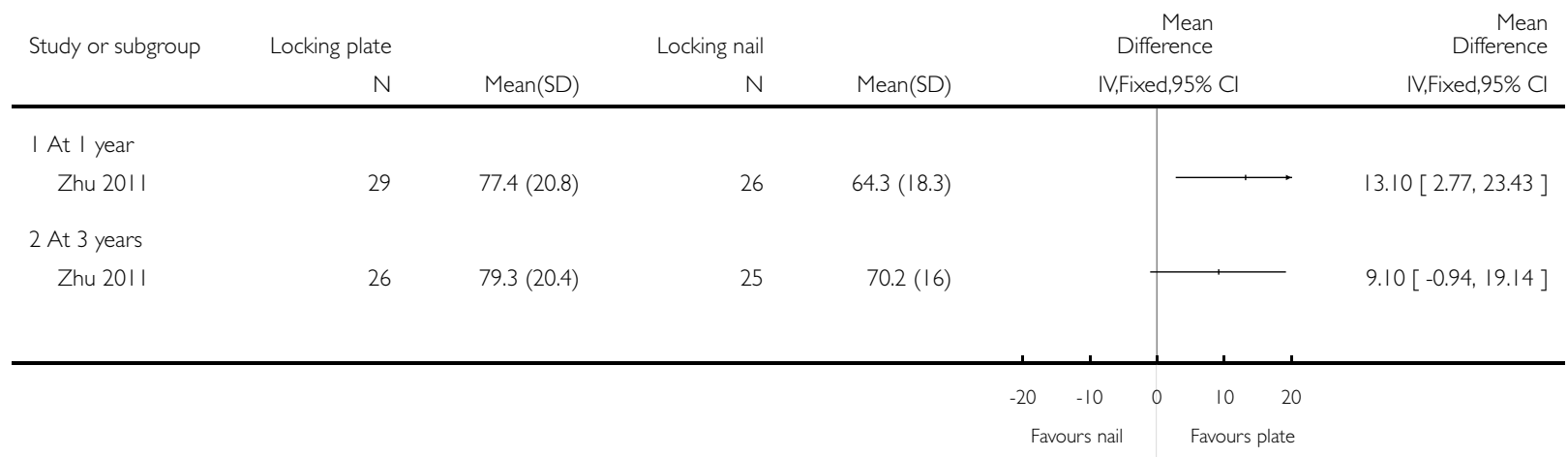

Analysis 5.8. Comparison 5 Locking plate versus locking intramedullary nail, Outcome 8 Operation times and blood loss.

Review: Interventions for treating proximal humeral fractures in adults

Comparison: 5 Locking plate versus locking intramedullary nail

Outcome: 8 Operation times and blood loss

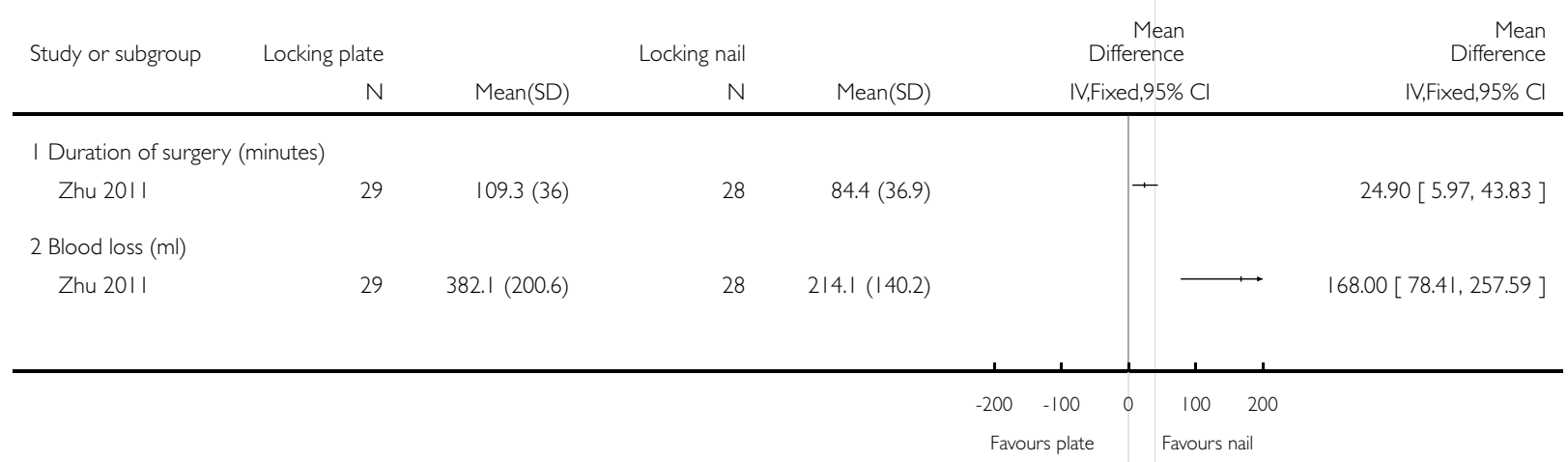


Analysis 5.9. Comparison 5 Locking plate versus locking intramedullary nail, Outcome 9 Intra-operative complication.

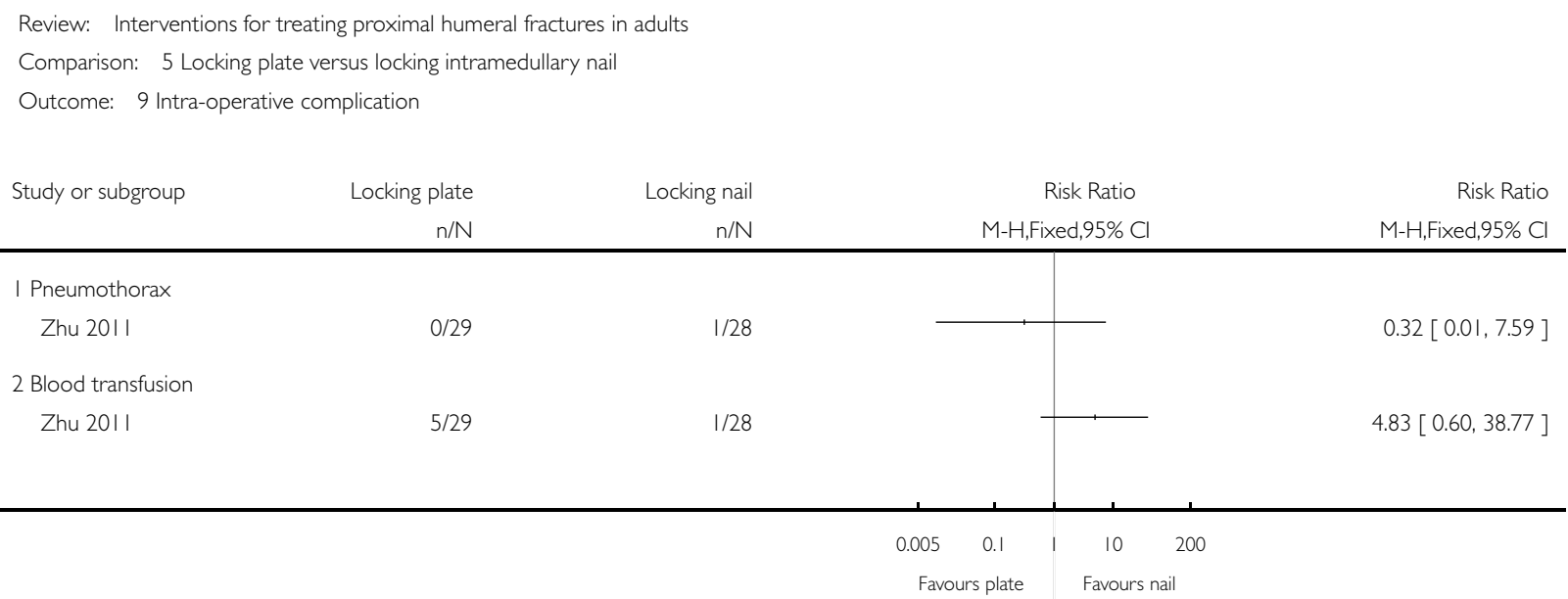

Analysis 6.I. Comparison 6 Locking plate versus intramedullary nails (Zifko method), Outcome I Complications and [slight] malunion.

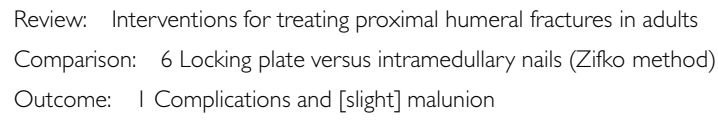

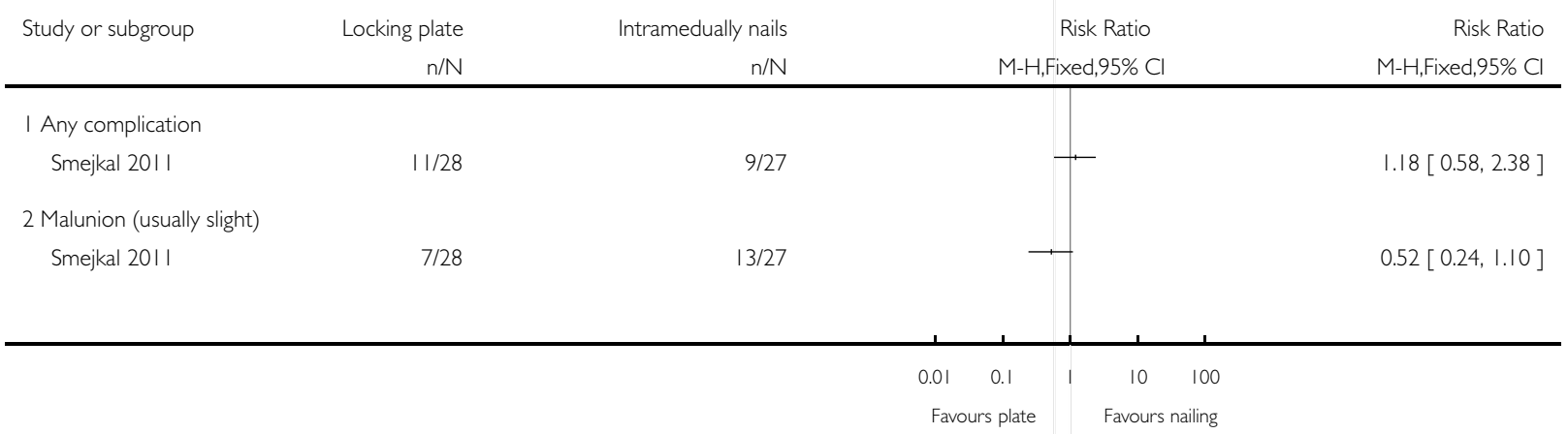


Analysis 6.2. Comparison 6 Locking plate versus intramedullary nails (Zifko method), Outcome 2 Constant score (\% of healthy limb) at mean 2 years.

Review: Interventions for treating proximal humeral fractures in adults

Comparison: 6 Locking plate versus intramedullary nails (Zifko method)

Outcome: 2 Constant score (\% of healthy limb) at mean 2 years

\begin{tabular}{|c|c|c|c|c|c|c|c|c|c|}
\hline \multirow[t]{2}{*}{ Study or subgroup } & Locking plate & \multicolumn{3}{|c|}{ Intramedually nails } & \multicolumn{4}{|c|}{$\begin{array}{r}\text { Mean } \\
\text { Difference }\end{array}$} & \multirow{2}{*}{$\begin{array}{r}\text { Mean } \\
\text { Difference } \\
\text { IV,Fixed,95\% Cl}\end{array}$} \\
\hline & $\mathrm{N}$ & Mean(SD) & $\mathrm{N}$ & Mean(SD) & & & xed,95\% Cl & & \\
\hline \multirow[t]{3}{*}{ Smejkal 201 I } & 28 & $86.64(10.83)$ & 27 & $87.45(14.03)$ & & & & & $-0.81[-7.45,5.83]$ \\
\hline & & & & & -10 & -5 & 0 & 10 & \\
\hline & & & & & Favo & & Favou & & \\
\hline
\end{tabular}

Analysis 6.3. Comparison 6 Locking plate versus intramedullary nails (Zifko method), Outcome 3 Time to union and time to recover upper limb function (weeks).

Review: Interventions for treating proximal humeral fractures in adults

Comparison: 6 Locking plate versus intramedullary nails (Zifko method)

Outcome: 3 Time to union and time to recover upper limb function (weeks)

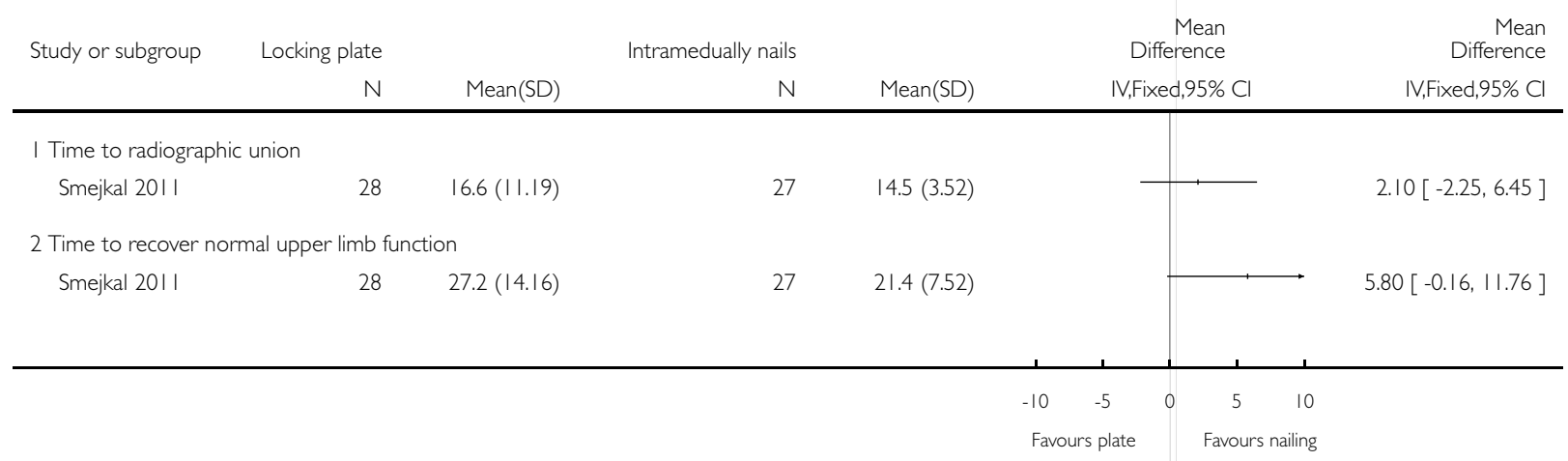




\section{Analysis 6.4. Comparison 6 Locking plate versus intramedullary nails (Zifko method), Outcome 4}

Operation and fluoroscopic times.

Review: Interventions for treating proximal humeral fractures in adults

Comparison: 6 Locking plate versus intramedullary nails (Zifko method)

Outcome: 4 Operation and fluoroscopic times

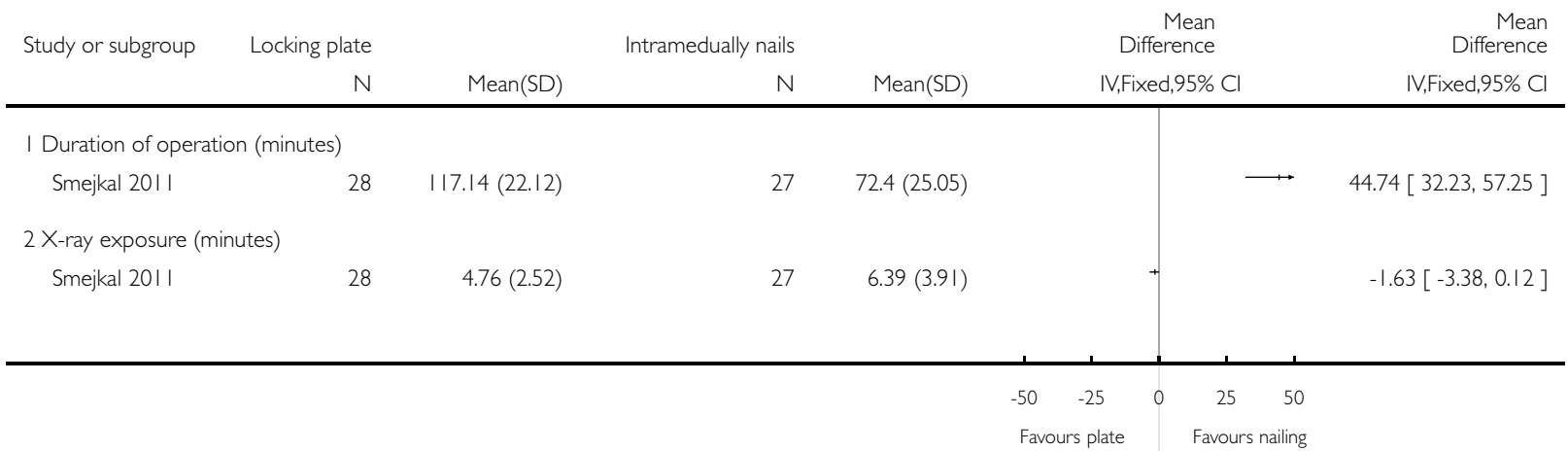

Analysis 6.5. Comparison 6 Locking plate versus intramedullary nails (Zifko method), Outcome 5 Length of hospital stay (days).

Review: Interventions for treating proximal humeral fractures in adults

Comparison: 6 Locking plate versus intramedullary nails (Zifko method)

Outcome: 5 Length of hospital stay (days)

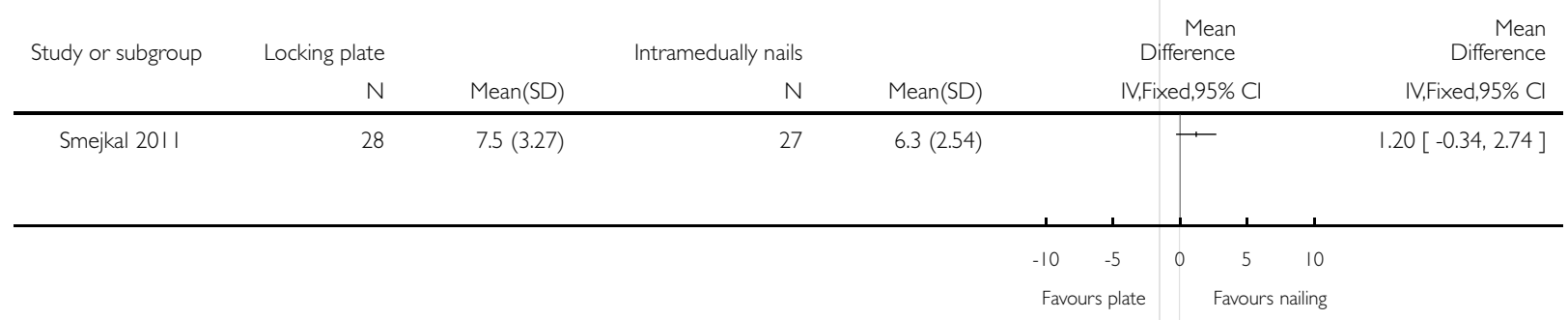


Analysis 7.I. Comparison 7 Replacement (hemiarthroplasty) versus fixation (tension band wiring; plate fixation) (4 part fractures), Outcome I DASH score (0 to I00: worst disability).

Review: Interventions for treating proximal humeral fractures in adults

Comparison: 7 Replacement (hemiarthroplasty) versus fixation (tension band wiring; plate fixation) (4 part fractures)

Outcome: I DASH score (0 to I00: worst disability)

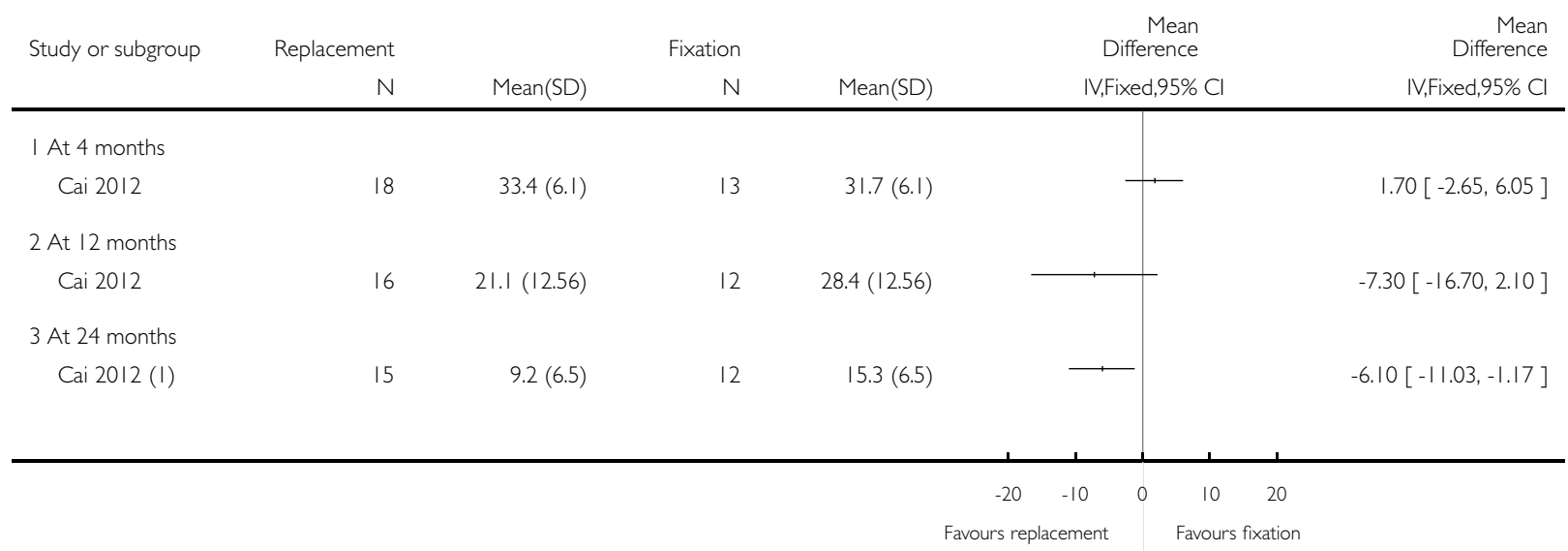

( I) SDs derived from reported $\mathrm{P}=0.023$ but report states not statistically significant

Analysis 7.2. Comparison 7 Replacement (hemiarthroplasty) versus fixation (tension band wiring; plate fixation) (4 part fractures), Outcome 2 EQ-5D score (0 to I: best quality of life).

Review: Interventions for treating proximal humeral fractures in adults

Comparison: 7 Replacement (hemiarthroplasty) versus fixation (tension band wiring; plate fixation) (4 part fractures)

Outcome: 2 EQ-5D score ( 0 to I: best quality of life)

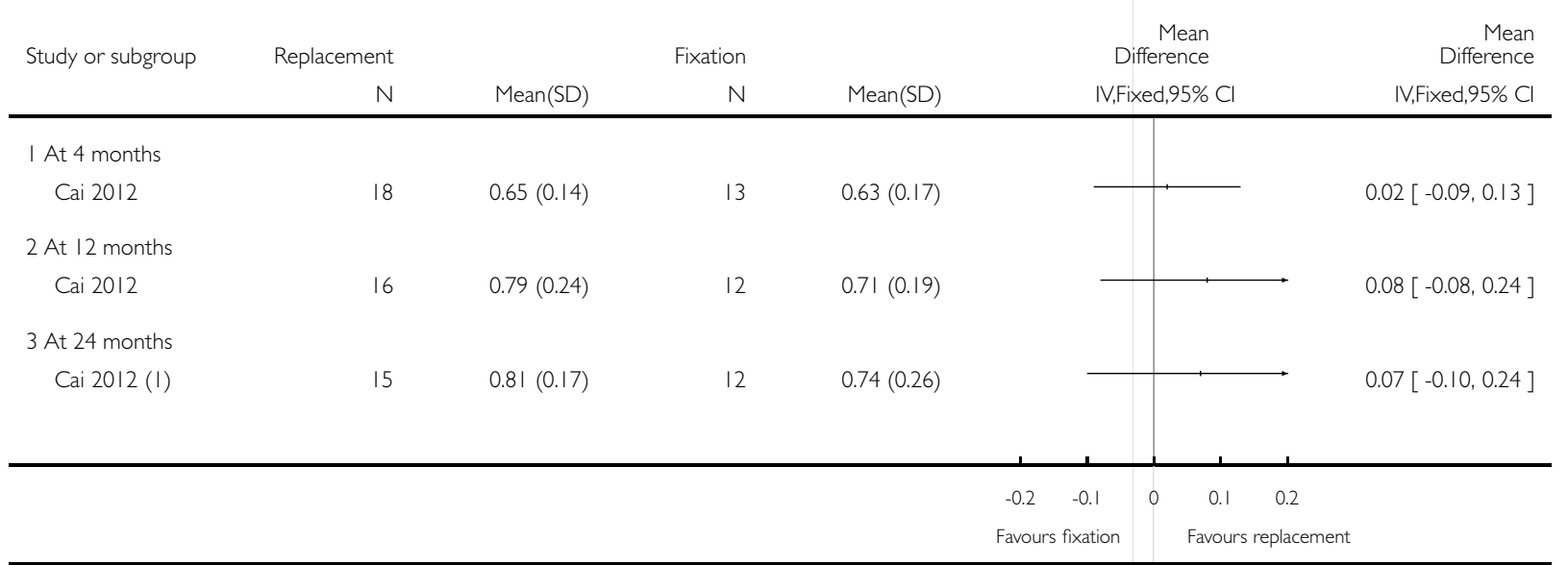

Interventions for treating proximal humeral fractures in adults (Review)

Copyright $\odot 2015$ The Cochrane Collaboration. Published by John Wiley \& Sons, Ltd. 
(I) SDs derived from reported $\mathrm{P}=0.023$ but report states not statistically significant

\section{Analysis 7.3. Comparison 7 Replacement (hemiarthroplasty) versus fixation (tension band wiring; plate} fixation) (4 part fractures), Outcome 3 Re-operation.

Review: Interventions for treating proximal humeral fractures in adults

Comparison: 7 Replacement (hemiarthroplasty) versus fixation (tension band wiring; plate fixation) (4 part fractures)

Outcome: 3 Re-operation

Study or subgroup Replacement

$n / N$

I Hemiarthroplasty versus tension band wiring

Hoellen 1997 (I)

Subtotal (95\% CI)

Total events: 0 (Replacement), 5 (Fixation)

Heterogeneity: not applicable

Test for overall effect: $Z=1.67$ ( $P=0.095)$

2 Hemiarthroplasty versus locking plate fixation

$$
\text { Cai } 2012 \text { (2) }
$$

19

Subtotal (95\% CI)

Total events: 3 (Replacement), 3 (Fixation)

Heterogeneity: not applicable

Test for overall effect: $Z=0.52(P=0.60)$

Total (95\% CI)

34

Total events: 3 (Replacement), 8 (Fixation)

Heterogeneity: $\mathrm{Ch}^{2}=1.82, \mathrm{df}=\mathrm{I}(\mathrm{P}=0.18) ; \mathrm{I}^{2}=45 \%$

Test for overall effect: $Z=1.81(P=0.070)$

Test for subgroup differences: $\mathrm{Chi}^{2}=1.57, \mathrm{df}=\mathrm{I}(\mathrm{P}=0.21), \mathrm{I}^{2}=36 \%$

15

15

13

28 $\begin{array}{lll}\text { Fixation } & \text { Risk Ratio } & \text { Weight }\end{array}$

$\mathrm{n} / \mathrm{N} \quad \mathrm{M}-\mathrm{H}$, Fixed,95\% Cl

$60.7 \%$

$0.09[0.01,1.51]$

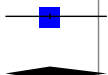

$60.7 \%$

$0.09[0.01,1.51]$

$39.3 \%$

$0.68[0.16,2.88]$

$39.3 \%$

$0.68[0.16,2.88]$

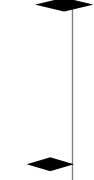

$100.0 \%$

$0.32[0.10,1.10]$

(1) At I year

(2) At 2 years 
Analysis 7.4. Comparison 7 Replacement (hemiarthroplasty) versus fixation (tension band wiring; plate fixation) (4 part fractures), Outcome 4 Dead at 2 years.

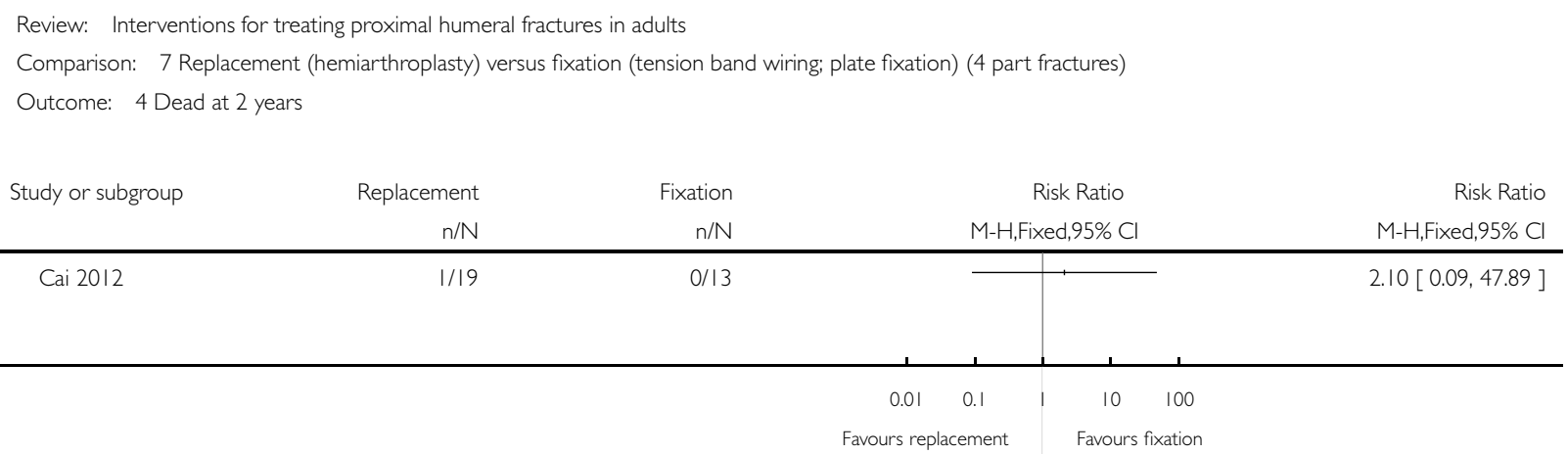

Analysis 7.5. Comparison 7 Replacement (hemiarthroplasty) versus fixation (tension band wiring; plate fixation) (4 part fractures), Outcome 5 Implant removal at I year.

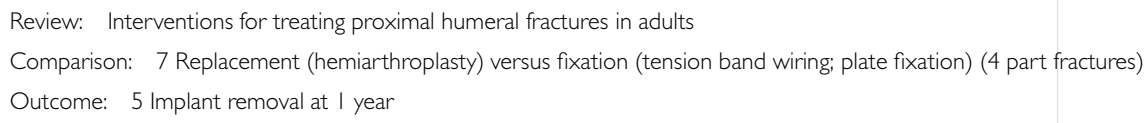

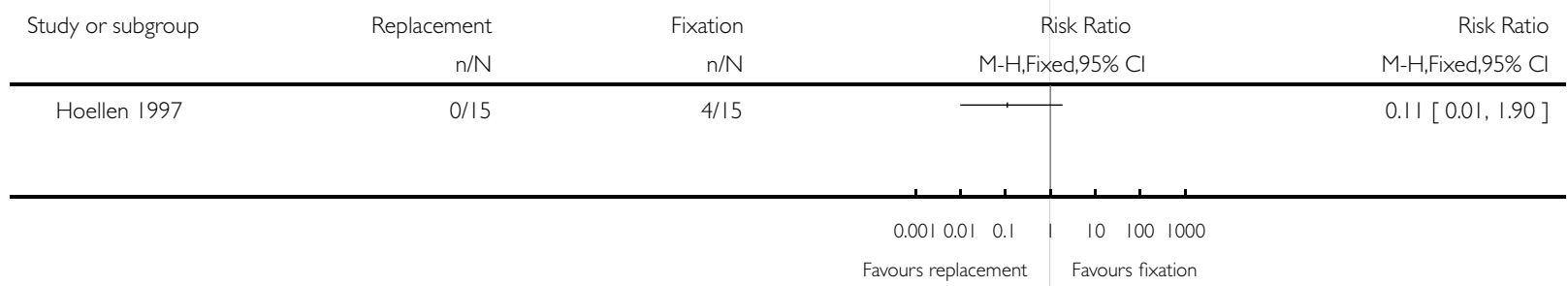


Analysis 7.6. Comparison 7 Replacement (hemiarthroplasty) versus fixation (tension band wiring; plate fixation) (4 part fractures), Outcome 6 Constant score (0 to 100: best score).

Review: Interventions for treating proximal humeral fractures in adults

Comparison: 7 Replacement (hemiarthroplasty) versus fixation (tension band wiring; plate fixation) (4 part fractures)

Outcome: 6 Constant score (0 to 100: best score)

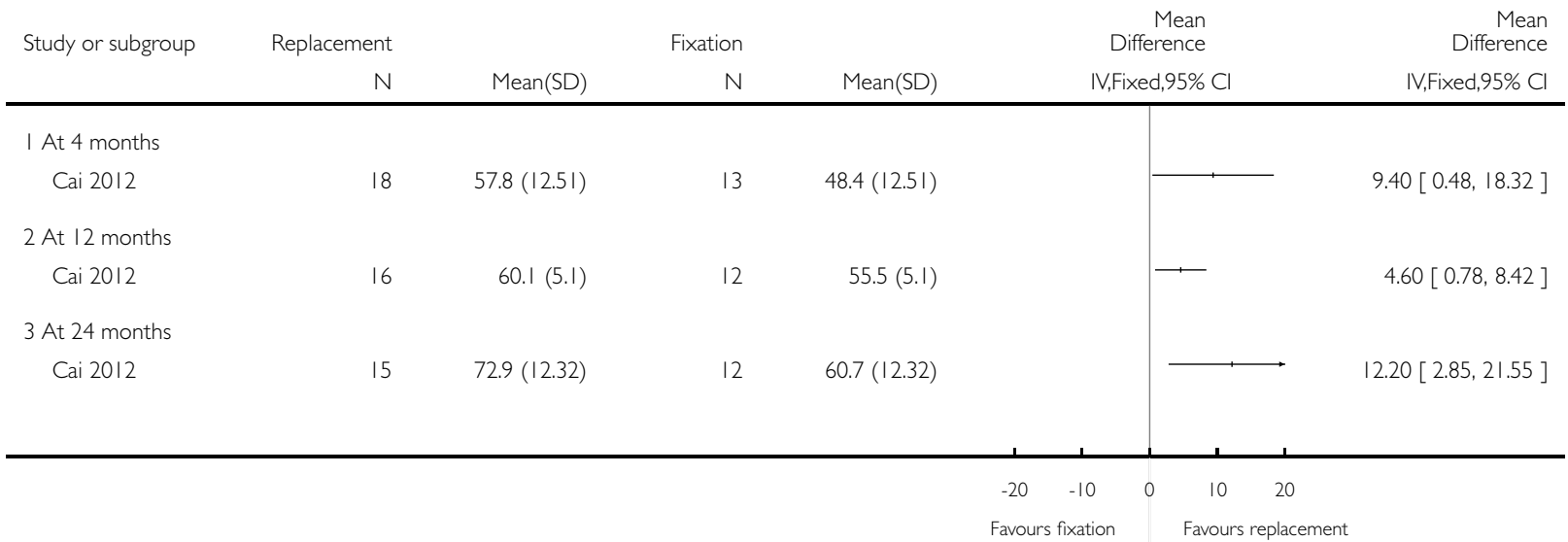

Analysis 7.7. Comparison 7 Replacement (hemiarthroplasty) versus fixation (tension band wiring; plate fixation) (4 part fractures), Outcome 7 Pain VAS (0 to 100: worst pain) at 24 months.

Review: Interventions for treating proximal humeral fractures in adults

Comparison: 7 Replacement (hemiarthroplasty) versus fixation (tension band wiring; plate fixation) (4 part fractures)

Outcome: 7 Pain VAS (0 to 100: worst pain) at 24 months

\begin{tabular}{|c|c|c|c|c|c|c|c|c|c|}
\hline \multirow[t]{2}{*}{ Study or subgroup } & \multirow{2}{*}{$\begin{array}{r}\text { Replacement } \\
N\end{array}$} & \multicolumn{3}{|c|}{ Fixation } & \multicolumn{4}{|c|}{$\begin{array}{r}\text { Mean } \\
\text { Difference }\end{array}$} & \multirow{2}{*}{$\begin{array}{r}\text { Mean } \\
\text { Difference } \\
\text { IV,Fixed,95\% Cl }\end{array}$} \\
\hline & & Mean(SD) & $\mathrm{N}$ & Mean(SD) & & & $\mathrm{ked}, 95 \% \mathrm{Cl}$ & & \\
\hline Cai 2012 (I) & 15 & $13(47.9)$ & 21 & $21(47.9)$ & & & - & & $-8.00[-39.74,23.74]$ \\
\hline & & & & & -50 & -25 & 25 & 50 & \\
\hline
\end{tabular}

( 1 ) SDs derived from reported $P=0.023$ but report states not statistically significant 
Analysis 7.8. Comparison 7 Replacement (hemiarthroplasty) versus fixation (tension band wiring; plate fixation) (4 part fractures), Outcome 8 Pain at I year.

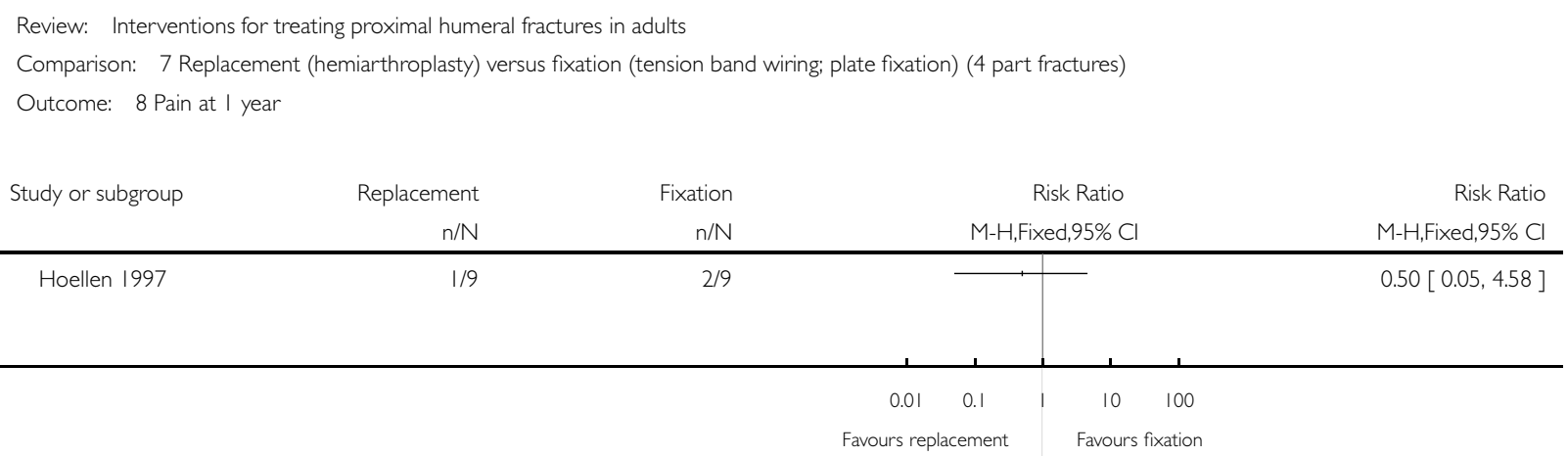

Analysis 7.9. Comparison 7 Replacement (hemiarthroplasty) versus fixation (tension band wiring; plate fixation) (4 part fractures), Outcome 9 Range of motion at 24 months.

Review: Interventions for treating proximal humeral fractures in adults

Comparison: 7 Replacement (hemiarthroplasty) versus fixation (tension band wiring; plate fixation) (4 part fractures)

Outcome: 9 Range of motion at 24 months

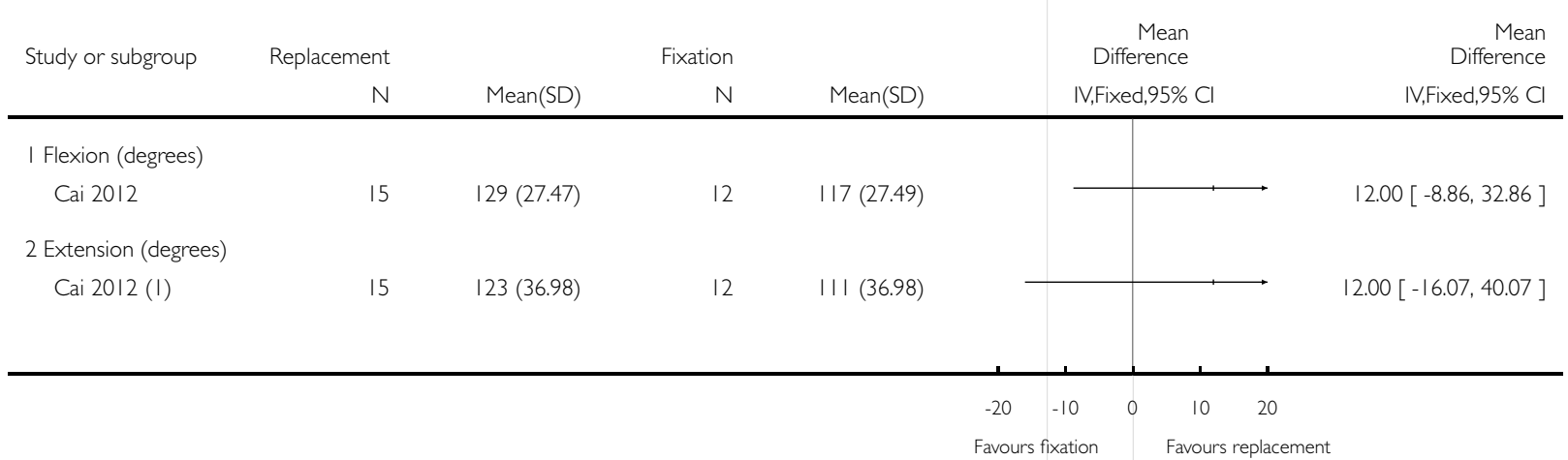

( 1 ) SDs derived from reported $P=0.023$ but report states not statistically significant 
Analysis 8.I. Comparison 8 Reverse shoulder arthroplasty (RSA) versus hemiarthroplasty (HA), Outcome I Shoulder function scores at 24 to 49 months.

Review: Interventions for treating proximal humeral fractures in adults

Comparison: 8 Reverse shoulder arthroplasty (RSA) versus hemiarthroplasty $(\mathrm{HA})$

Outcome: I Shoulder function scores at 24 to 49 months

\begin{tabular}{|c|c|c|c|c|c|c|c|c|c|}
\hline \multirow[t]{2}{*}{ Study or subgroup } & \multirow{2}{*}{$\begin{array}{r}\text { RSA } \\
\mathrm{N}\end{array}$} & \multicolumn{3}{|c|}{$\mathrm{HA}$} & \multicolumn{4}{|c|}{$\begin{array}{r}\text { Mean } \\
\text { Difference }\end{array}$} & $\begin{array}{l}\text { Mean } \\
\text { Difference }\end{array}$ \\
\hline & & Mean(SD) & $\mathrm{N}$ & Mean(SD) & \multicolumn{4}{|c|}{ IV,Fixed,95\% Cl } & IV,Fixed,95\% Cl \\
\hline \multicolumn{10}{|c|}{ I Quick DASH score (0 to 55: worst outcome) } \\
\hline \multirow[t]{3}{*}{ Sebasti -Forcada 2014} & 31 & | $7.5(7.78)$ & 30 & $24.4(7.78)$ & & $\longrightarrow$ & & & $-6.90[-10.81,-2.99]$ \\
\hline & & & & & -20 & -10 & 10 & 20 & \\
\hline & & & & & Fav & rs RSA & Favours & & \\
\hline
\end{tabular}

Analysis 8.2. Comparison 8 Reverse shoulder arthroplasty (RSA) versus hemiarthroplasty (HA), Outcome 2 Re-operation.

Review: Interventions for treating proximal humeral fractures in adults

Comparison: 8 Reverse shoulder arthroplasty (RSA) versus hemiarthroplasty $(H A)$

Outcome: 2 Re-operation

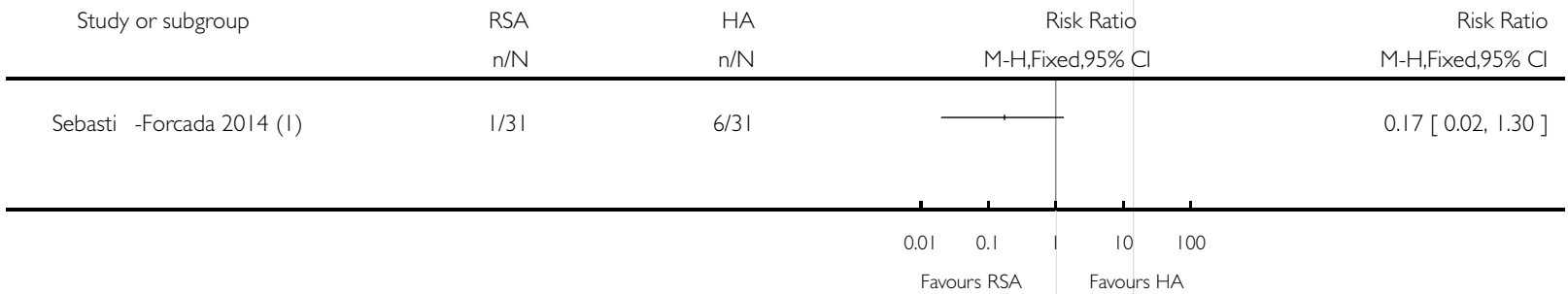

( ) All re-operations to RSA 
Analysis 8.3. Comparison 8 Reverse shoulder arthroplasty (RSA) versus hemiarthroplasty (HA), Outcome 3 Death.

Review: Interventions for treating proximal humeral fractures in adults

Comparison: 8 Reverse shoulder arthroplasty (RSA) versus hemiarthroplasty $(\mathrm{HA})$

Outcome: 3 Death

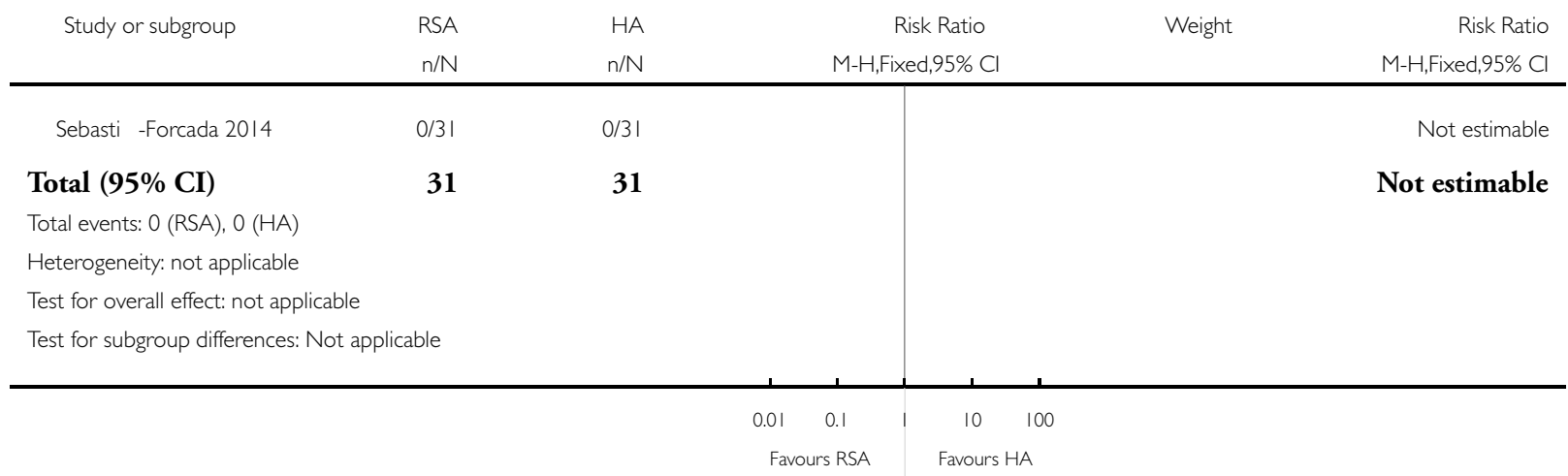

Analysis 8.4. Comparison 8 Reverse shoulder arthroplasty (RSA) versus hemiarthroplasty (HA), Outcome 4 Composite (objective and subjective) shoulder function scores at 24 to 49 months.

Review: Interventions for treating proximal humeral fractures in adults

Comparison: 8 Reverse shoulder arthroplasty (RSA) versus hemiarthroplasty (HA)

Outcome: 4 Composite (objective and subjective) shoulder function scores at 24 to 49 months

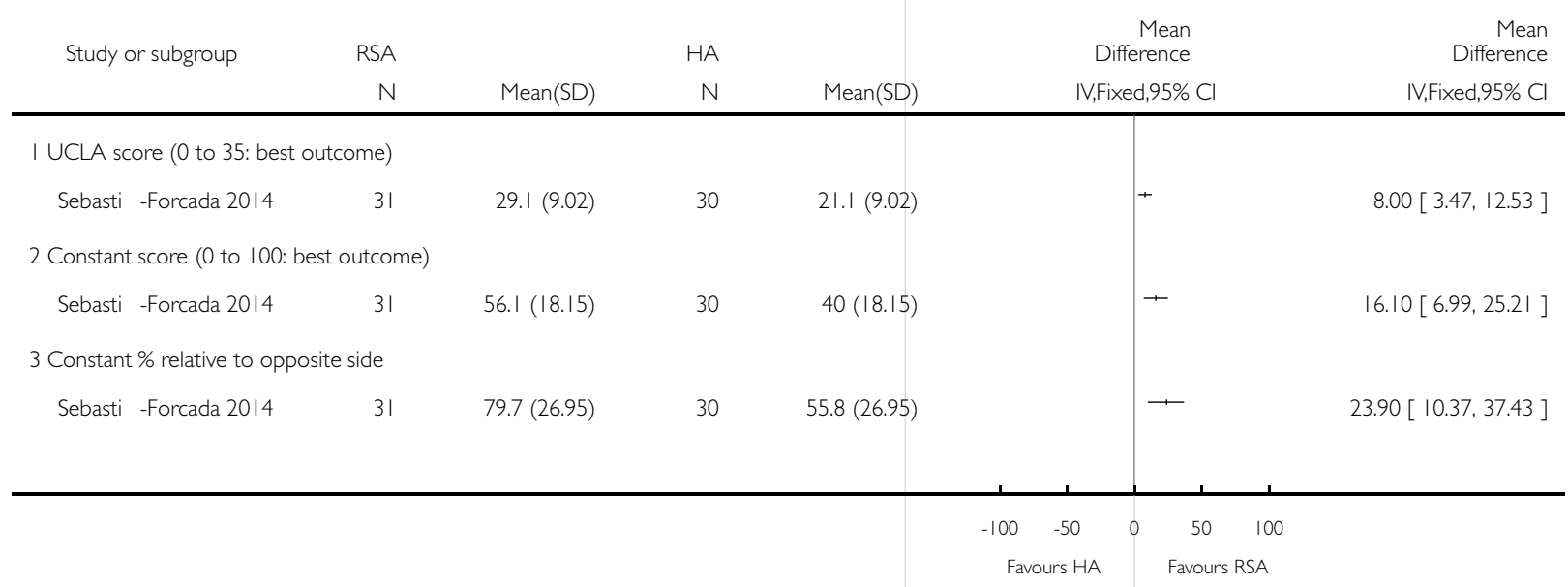


Analysis 8.5. Comparison 8 Reverse shoulder arthroplasty (RSA) versus hemiarthroplasty (HA), Outcome 5 Constant score at 24 to 49 months: overall and components.

Review: Interventions for treating proximal humeral fractures in adults

Comparison: 8 Reverse shoulder arthroplasty (RSA) versus hemiarthroplasty (HA)

Outcome: 5 Constant score at 24 to 49 months: overall and components

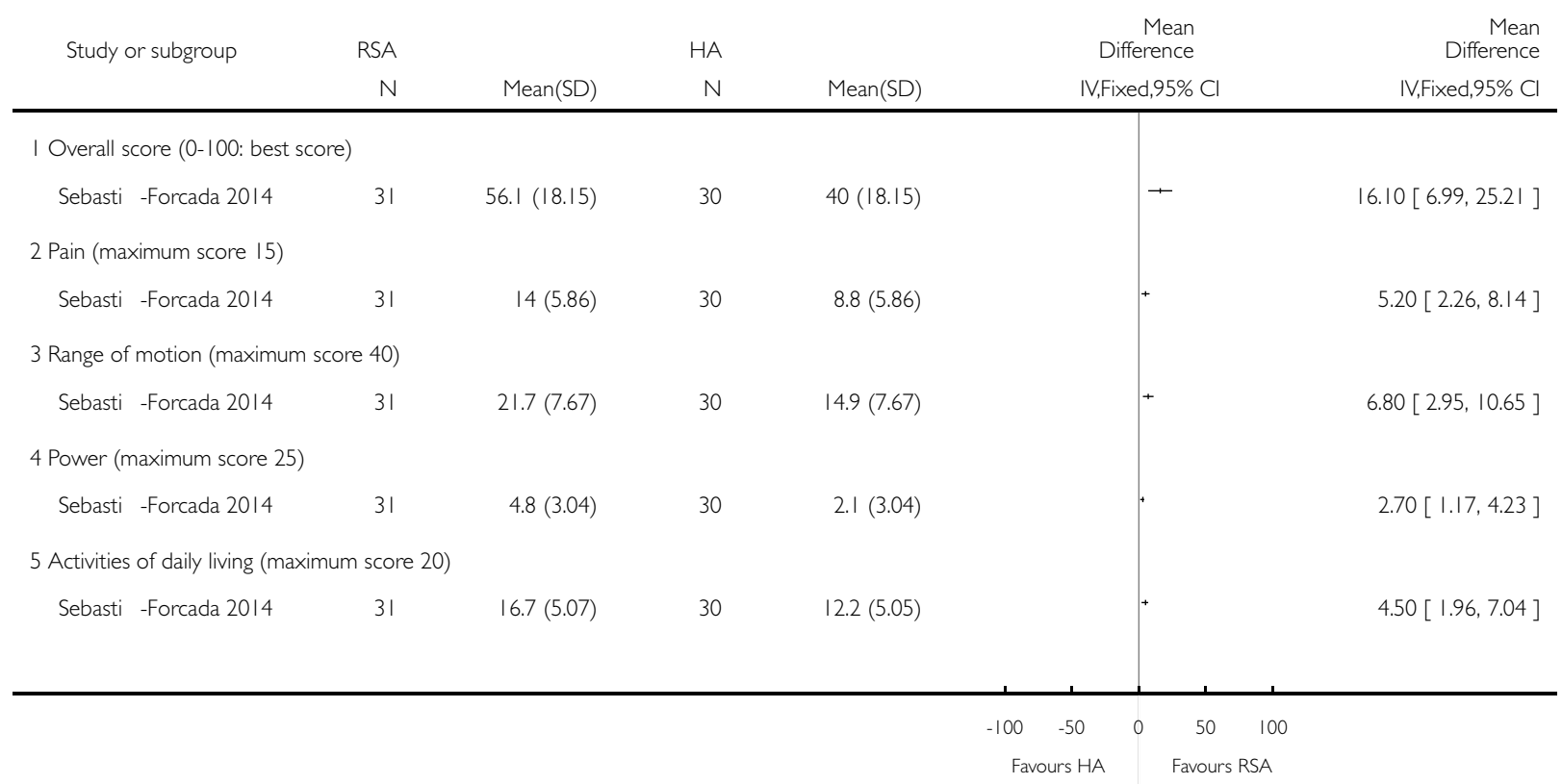




\section{Analysis 8.6. Comparison 8 Reverse shoulder arthroplasty (RSA) versus hemiarthroplasty (HA), Outcome}

6 Complications.

Review: Interventions for treating proximal humeral fractures in adults

Comparison: 8 Reverse shoulder arthroplasty (RSA) versus hemiarthroplasty (HA)

Outcome: 6 Complications

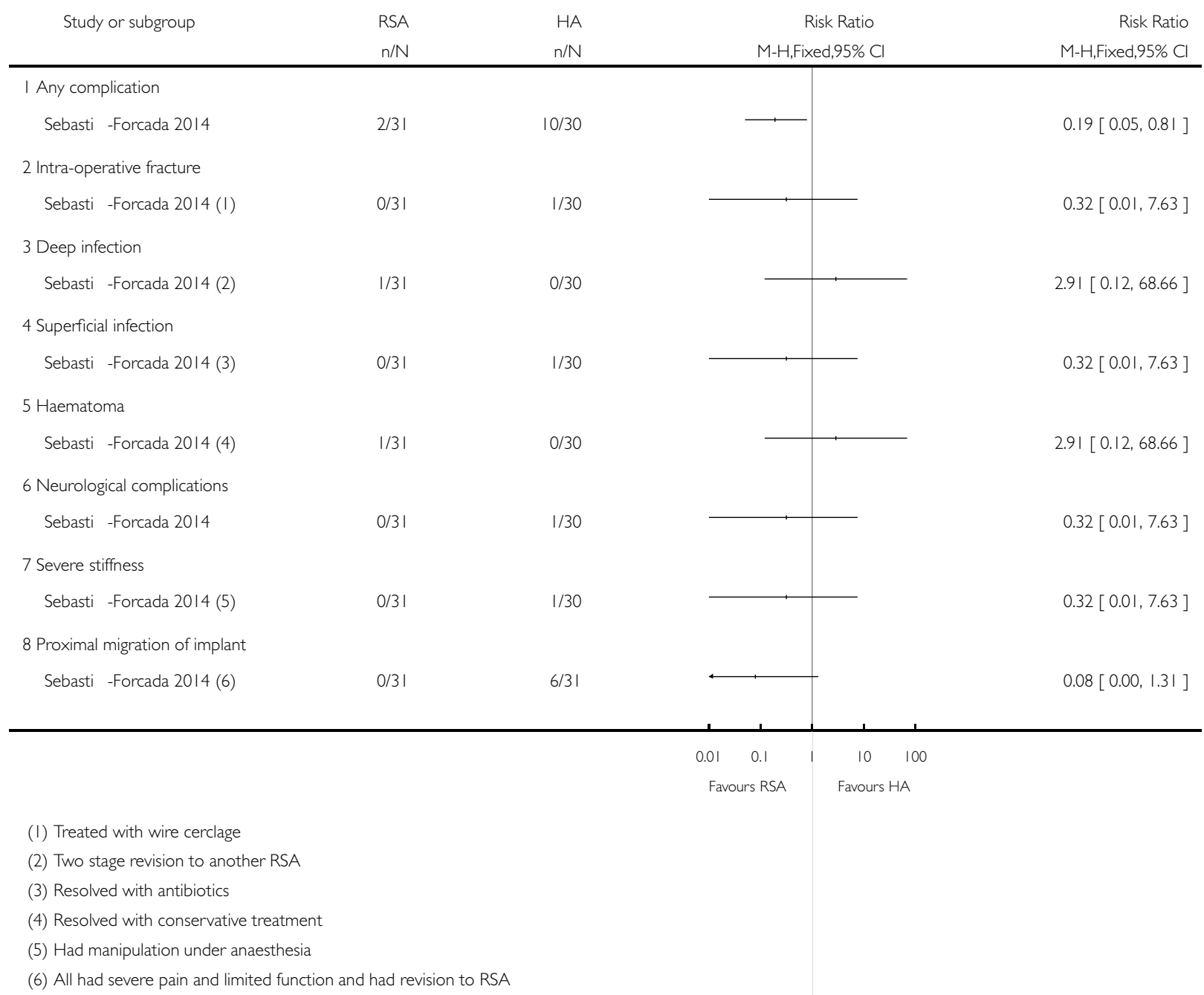


Analysis 8.7. Comparison 8 Reverse shoulder arthroplasty (RSA) versus hemiarthroplasty (HA), Outcome 7 Radiological assessment findings.

Review: Interventions for treating proximal humeral fractures in adults

Comparison: 8 Reverse shoulder arthroplasty (RSA) versus hemiarthroplasty (HA)

Outcome: 7 Radiological assessment findings

Study or subgroup RSA

Sebasti -Forcada 2014

2 Resorption of tuberosities

Sebasti -Forcada 2014

3 Scapular notching

Sebasti -Forcada 2014 (I)

4 Heterotopic ossification

Sebasti -Forcada 2014 (2) $\mathrm{n} / \mathrm{N}$

$6 / 31$

HA

$\mathrm{n} / \mathrm{N}$

$9 / 30$

$5 / 31$

$|/ 3|$

$5 / 31$
0/30

$6 / 31$
Risk Ratio

M-H,Fixed,95\% Cl

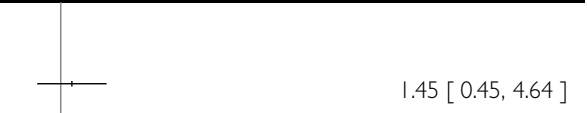

$0.54[0.20,1.42]$

$2.91[0.12,68.66]$

$0.83[0.28,2.45]$

( I) No clinical effect

(2) No clinical significance 
Analysis 8.8. Comparison 8 Reverse shoulder arthroplasty (RSA) versus hemiarthroplasty (HA), Outcome 8 Range of motion (degrees) at 24 to 49 months.

Review: Interventions for treating proximal humeral fractures in adults

Comparison: 8 Reverse shoulder arthroplasty (RSA) versus hemiarthroplasty (HA)

Outcome: 8 Range of motion (degrees) at 24 to 49 months

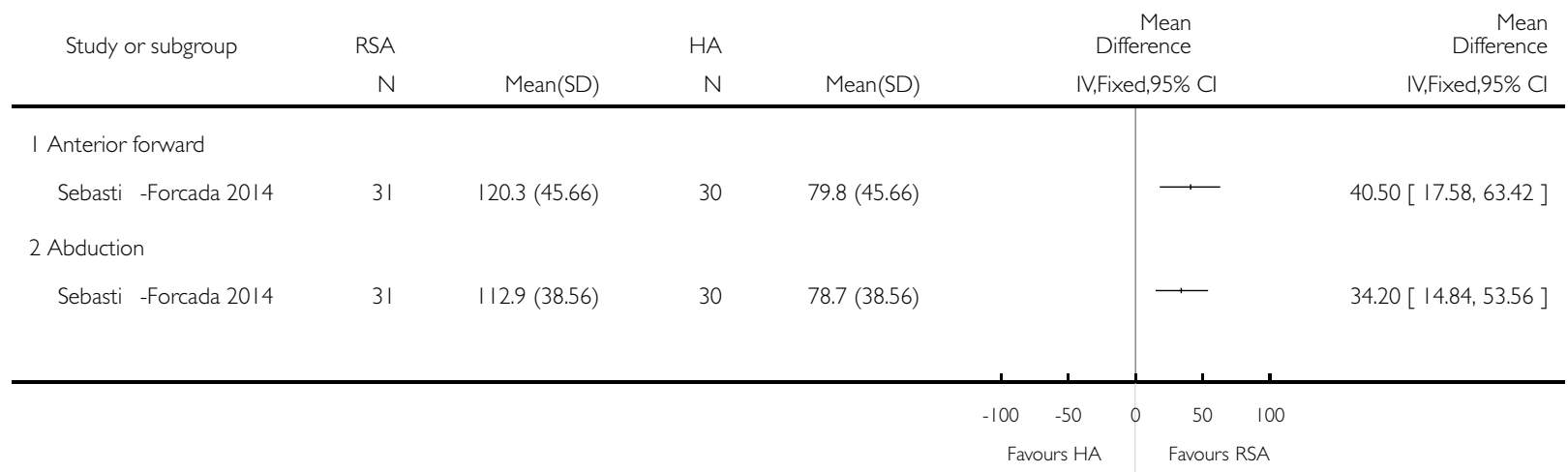

Analysis 9.I. Comparison 9 Deltoid-split versus deltopectoral approaches for plate fixation, Outcome I Reoperation.

Review: Interventions for treating proximal humeral fractures in adults

Comparison: 9 Deltoid-split versus deltopectoral approaches for plate fixation

Outcome: I Re-operation

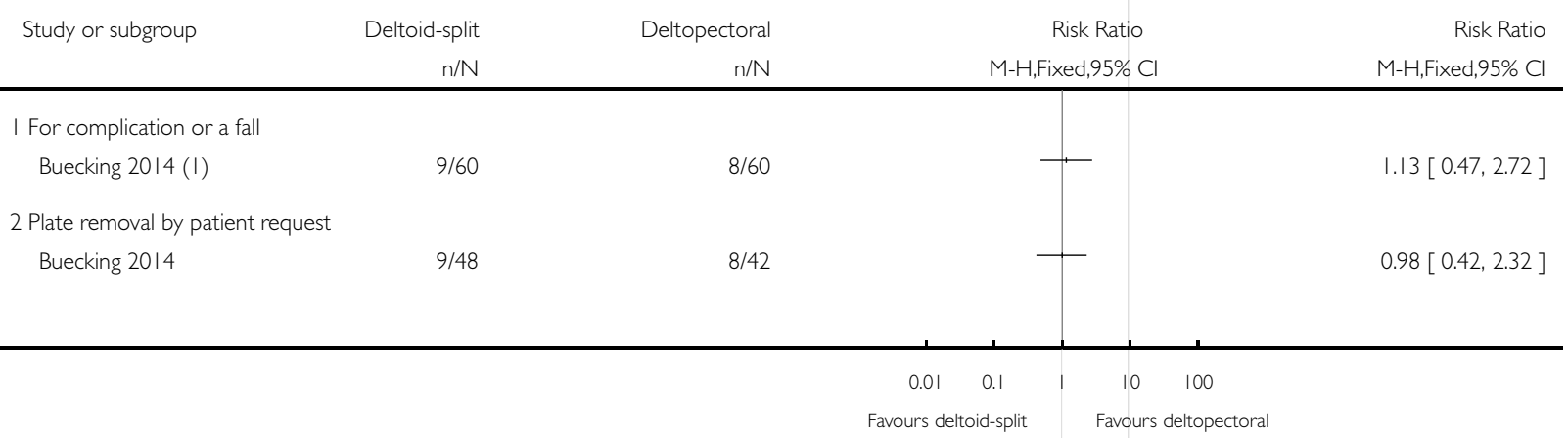

(I) in one case in each group a re-operation resulted from a fall on the shoulder 
Analysis 9.2. Comparison 9 Deltoid-split versus deltopectoral approaches for plate fixation, Outcome 2 Dead at I year.

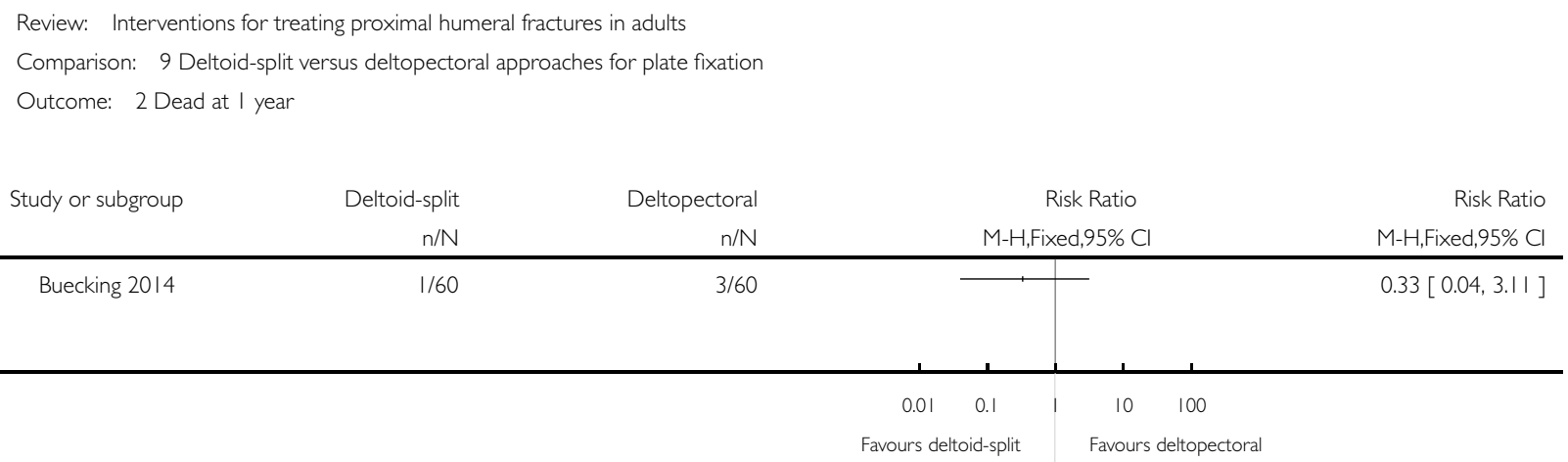

Analysis 9.3. Comparison 9 Deltoid-split versus deltopectoral approaches for plate fixation, Outcome 3 Complications.

Review: Interventions for treating proximal humeral fractures in adults

Comparison: 9 Deltoid-split versus deltopectoral approaches for plate fixation

Outcome: 3 Complications

Study or subgroup

\section{Injurious fall on shoulder}

$$
\text { Buecking } 2014 \text { (I) }
$$

$1 / 60$

2 Axillary nerve damage

Buecking 2014 (2)

3 Screw perforation

$$
\text { Buecking } 2014 \text { (3) }
$$

4 Implant (head or shaft) loosening

Buecking 2014 (4)

5 Deep infection

$$
\text { Buecking } 2014 \text { (5) }
$$

6 Humeral head necrosis

Buecking 2014 (6)

$1.00[0.06,15.62]$

Not estimable

$7.00[0.37,132.66]$

$0.83[0.27,2.58]$

0.33 [0.01, 8.02 ]

Not estimable

\subsection{1}

0.1

10

100

Favours deltoid-split

Favours deltopectoral 
(I) Both were associated with postsurgical delirium and resulted in a re-operation

(2) The I case resulted in implant removal

(3) All 3 were treated with joint replacement

(4) Group I: all treated with joint replacement; Group 2: 2 joint replacement, 4 with osteosythesis

(5) The I case resulted in implant removal

(6) The I case resulted in implant removal

\section{Analysis 9.4. Comparison 9 Deltoid-split versus deltopectoral approaches for plate fixation, Outcome 4} Constant score (0 to 100: best score).

Review: Interventions for treating proximal humeral fractures in adults

Comparison: 9 Deltoid-split versus deltopectoral approaches for plate fixation

Outcome: 4 Constant score (0 to 100: best score)

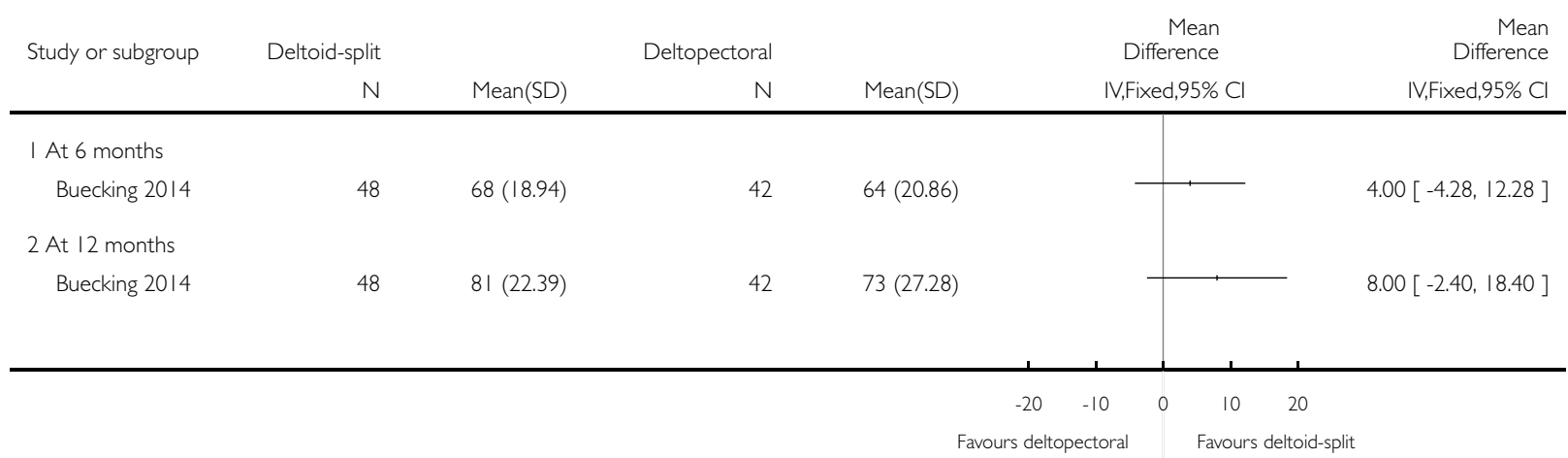


Analysis 9.5. Comparison 9 Deltoid-split versus deltopectoral approaches for plate fixation, Outcome 5 Pain (VAS 0 to 10: intolerable pain).

Review: Interventions for treating proximal humeral fractures in adults

Comparison: 9 Deltoid-split versus deltopectoral approaches for plate fixation

Outcome: 5 Pain (VAS 0 to 10: intolerable pain)

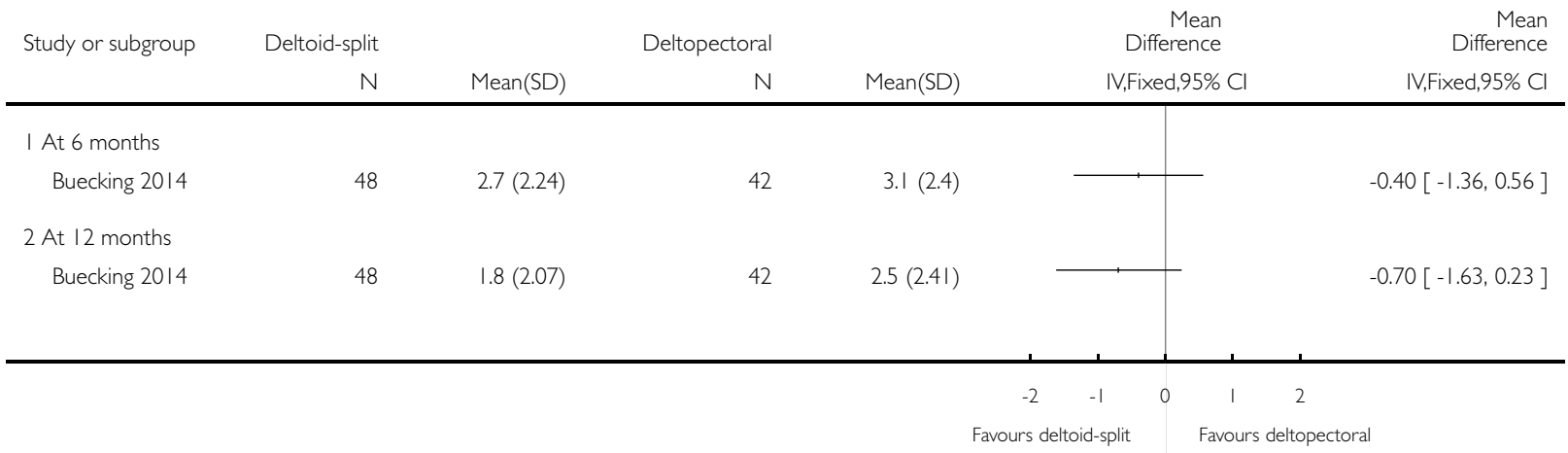

Analysis 9.6. Comparison 9 Deltoid-split versus deltopectoral approaches for plate fixation, Outcome 6 Operation and fluoroscopic times.

Review: Interventions for treating proximal humeral fractures in adults

Comparison: 9 Deltoid-split versus deltopectoral approaches for plate fixation

Outcome: 6 Operation and fluoroscopic times

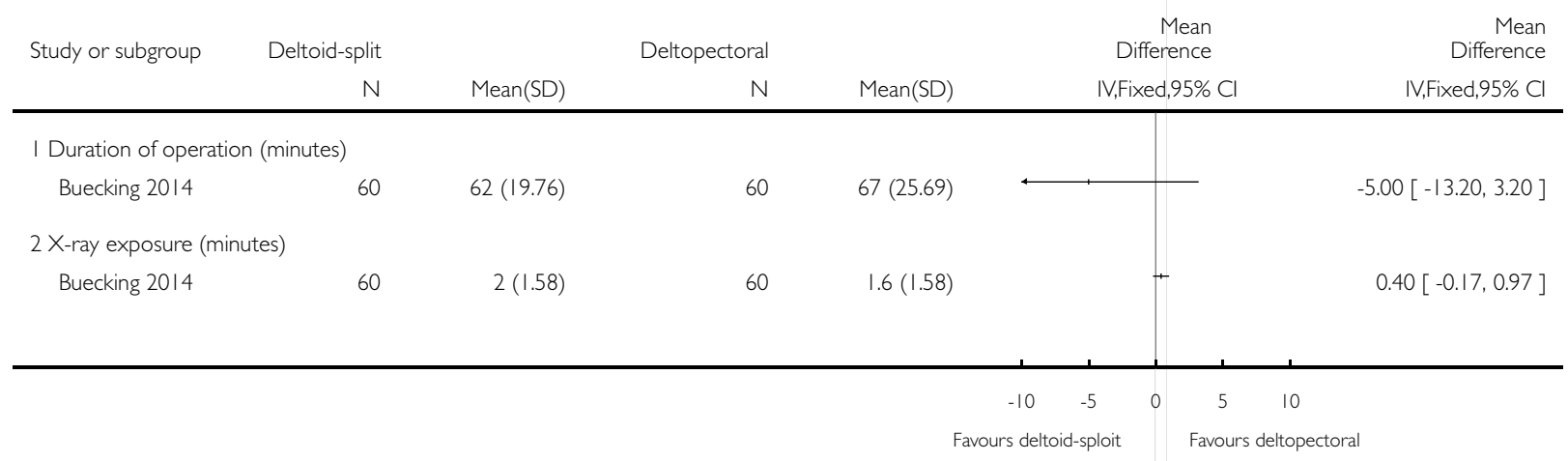


Analysis 9.7. Comparison 9 Deltoid-split versus deltopectoral approaches for plate fixation, Outcome 7 Length of hospital stay (days).

Review: Interventions for treating proximal humeral fractures in adults

Comparison: 9 Deltoid-split versus deltopectoral approaches for plate fixation

Outcome: 7 Length of hospital stay (days)

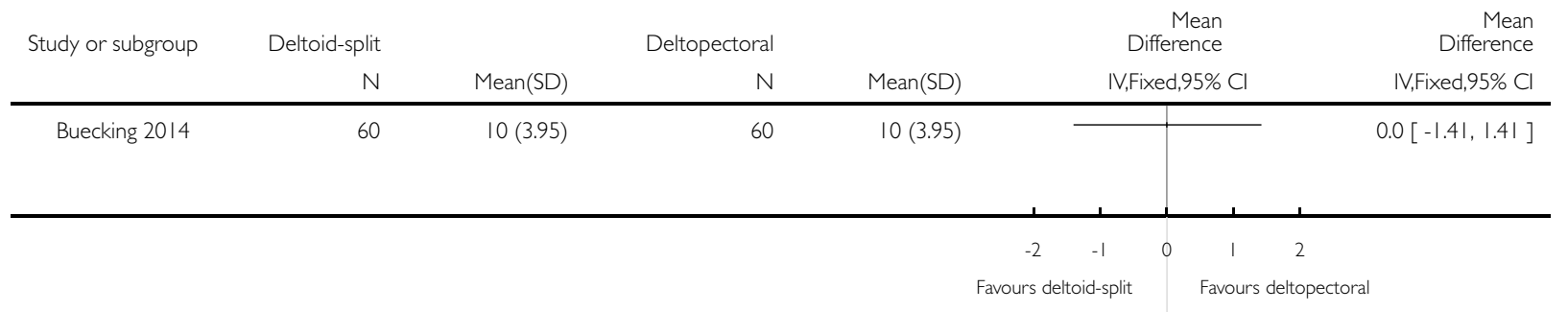

Analysis 10.1. Comparison 10 Polyaxial versus monoaxial screw insertion in plate fixation, Outcome I DASH score at 12 months (0 to I00: greatest disability).

Review: Interventions for treating proximal humeral fractures in adults

Comparison: 10 Polyaxial versus monoaxial screw insertion in plate fixation

Outcome: I DASH score at 12 months (0 to 100: greatest disability)

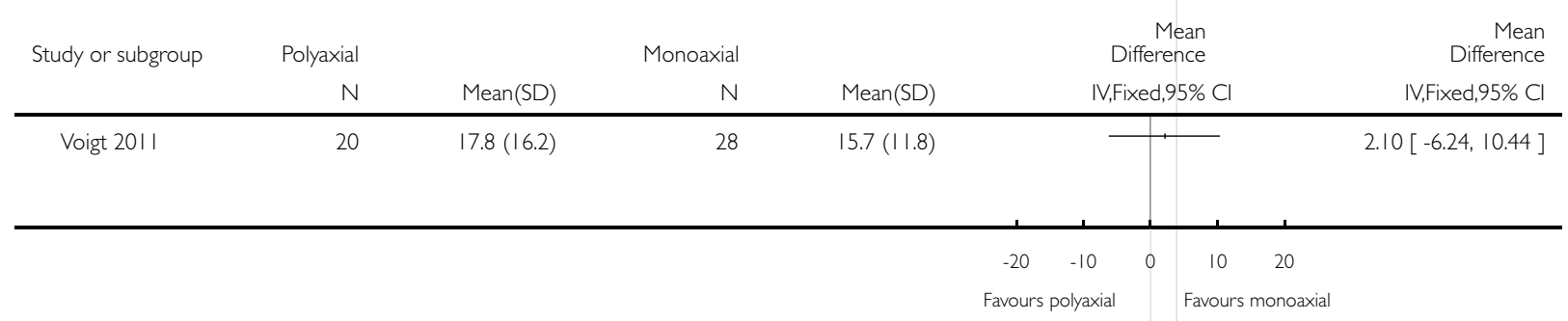


Analysis 10.2. Comparison 10 Polyaxial versus monoaxial screw insertion in plate fixation, Outcome 2 Simple shoulder test ( 0 to I2: best outcome).

Review: Interventions for treating proximal humeral fractures in adults

Comparison: 10 Polyaxial versus monoaxial screw insertion in plate fixation

Outcome: 2 Simple shoulder test (0 to 12: best outcome)

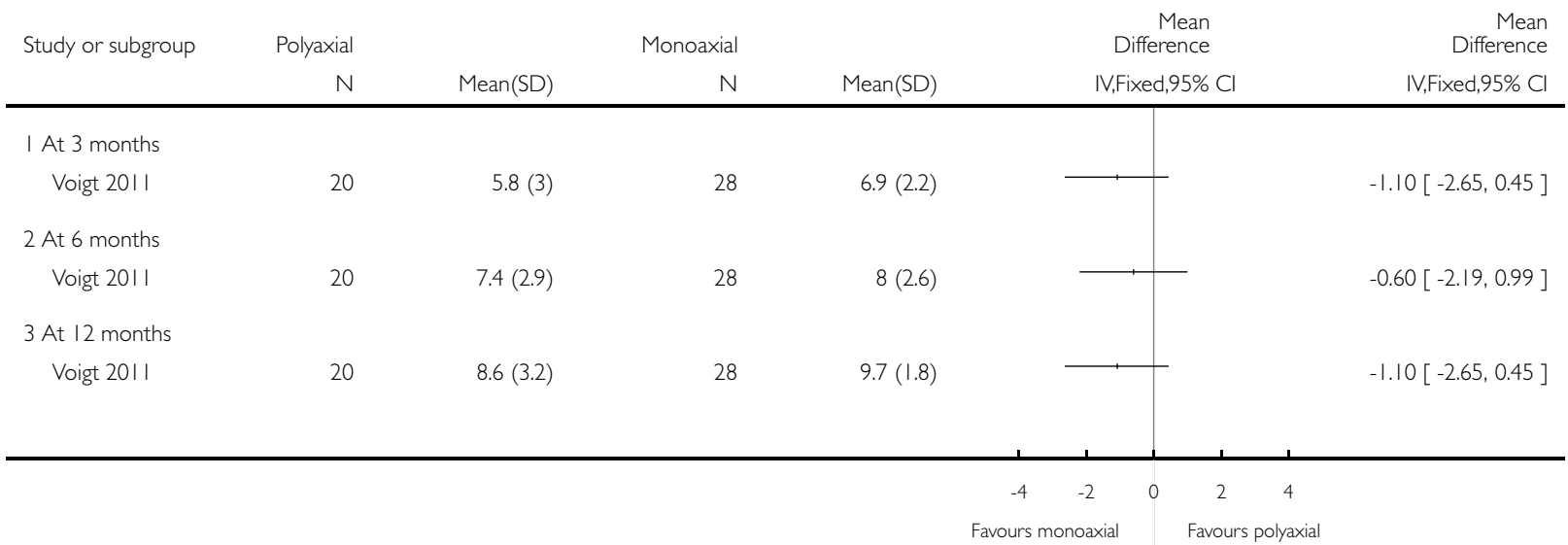


Analysis 10.3. Comparison IO Polyaxial versus monoaxial screw insertion in plate fixation, Outcome 3 Reoperation.

Review: Interventions for treating proximal humeral fractures in adults

Comparison: 10 Polyaxial versus monoaxial screw insertion in plate fixation

Outcome: 3 Re-operation

\begin{tabular}{|c|c|c|c|c|c|}
\hline \multirow[t]{2}{*}{ Study or subgroup } & Polyaxial & Monoaxial & Risk Ratio & \multirow[t]{2}{*}{ Weight } & \multirow{2}{*}{$\begin{array}{r}\text { Risk Ratic } \\
\text { M-H,Fixed,95\% C }\end{array}$} \\
\hline & $n / N$ & $n / N$ & M-H,Fixed,95\% Cl & & \\
\hline \multicolumn{6}{|l|}{ I By 6 months } \\
\hline Ockert 2010 & $2 / 29$ & $3 / 37$ & & $100.0 \%$ & $0.85[0.15,4.76]$ \\
\hline Subtotal $(95 \% \mathrm{CI})$ & 29 & 37 & & $100.0 \%$ & $0.85[0.15,4.76]$ \\
\hline \multicolumn{6}{|c|}{ Total events: 2 (Polyaxial), 3 (Monoaxial) } \\
\hline \multicolumn{6}{|c|}{ Heterogeneity: not applicable } \\
\hline \multicolumn{6}{|c|}{ Test for overall effect: $Z=0.18(P=0.85)$} \\
\hline \multicolumn{6}{|l|}{2 By I year } \\
\hline Ockert $2010(1)$ & $9 / 58$ & $12 / 66$ & & $75.9 \%$ & $0.85[0.39,1.88]$ \\
\hline Voigt 2011 & $6 / 25$ & $4 / 31$ & - & $24.1 \%$ & $1.86[0.59,5.88]$ \\
\hline Subtotal $(95 \% \mathrm{CI})$ & 83 & 97 & - & $100.0 \%$ & $1.10[0.58,2.08]$ \\
\hline \multicolumn{6}{|c|}{ Total events: 15 (Polyaxial), 16 (Monoaxial) } \\
\hline \multicolumn{6}{|c|}{ Heterogeneity: Chi $^{2}=1.20, d f=1(P=0.27) ; 1^{2}=17 \%$} \\
\hline Test for overall effect: $Z=$ & $=0.78)$ & & & & \\
\hline
\end{tabular}

(I) Data from extended trial published 2014

\section{Analysis 10.4. Comparison 10 Polyaxial versus monoaxial screw insertion in plate fixation, Outcome 4 Dead} at I year.

Review: Interventions for treating proximal humeral fractures in adults

Comparison: 10 Polyaxial versus monoaxial screw insertion in plate fixation

Outcome: 4 Dead at I year

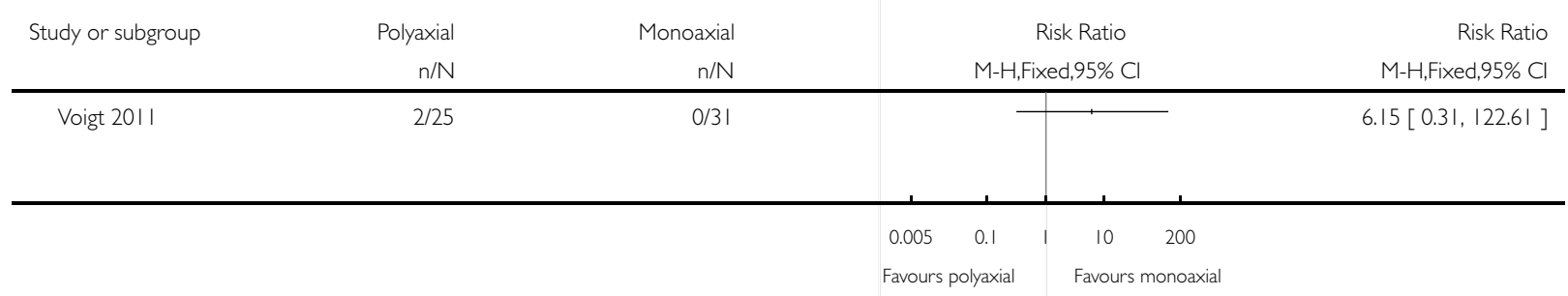


Analysis 10.5. Comparison 10 Polyaxial versus monoaxial screw insertion in plate fixation, Outcome 5 Constant score at 12 months (\% of contralateral limb).

Review: Interventions for treating proximal humeral fractures in adults

Comparison: 10 Polyaxial versus monoaxial screw insertion in plate fixation

Outcome: 5 Constant score at 12 months (\% of contralateral limb)

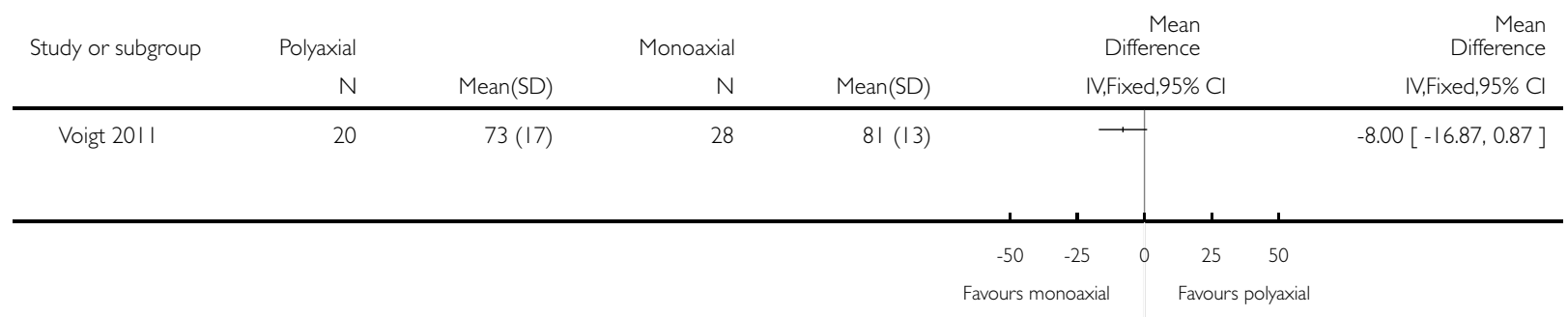

Analysis 10.6. Comparison 10 Polyaxial versus monoaxial screw insertion in plate fixation, Outcome 6 Complications (radiological assessment).

Review: Interventions for treating proximal humeral fractures in adults

Comparison: 10 Polyaxial versus monoaxial screw insertion in plate fixation

Outcome: 6 Complications (radiological assessment)

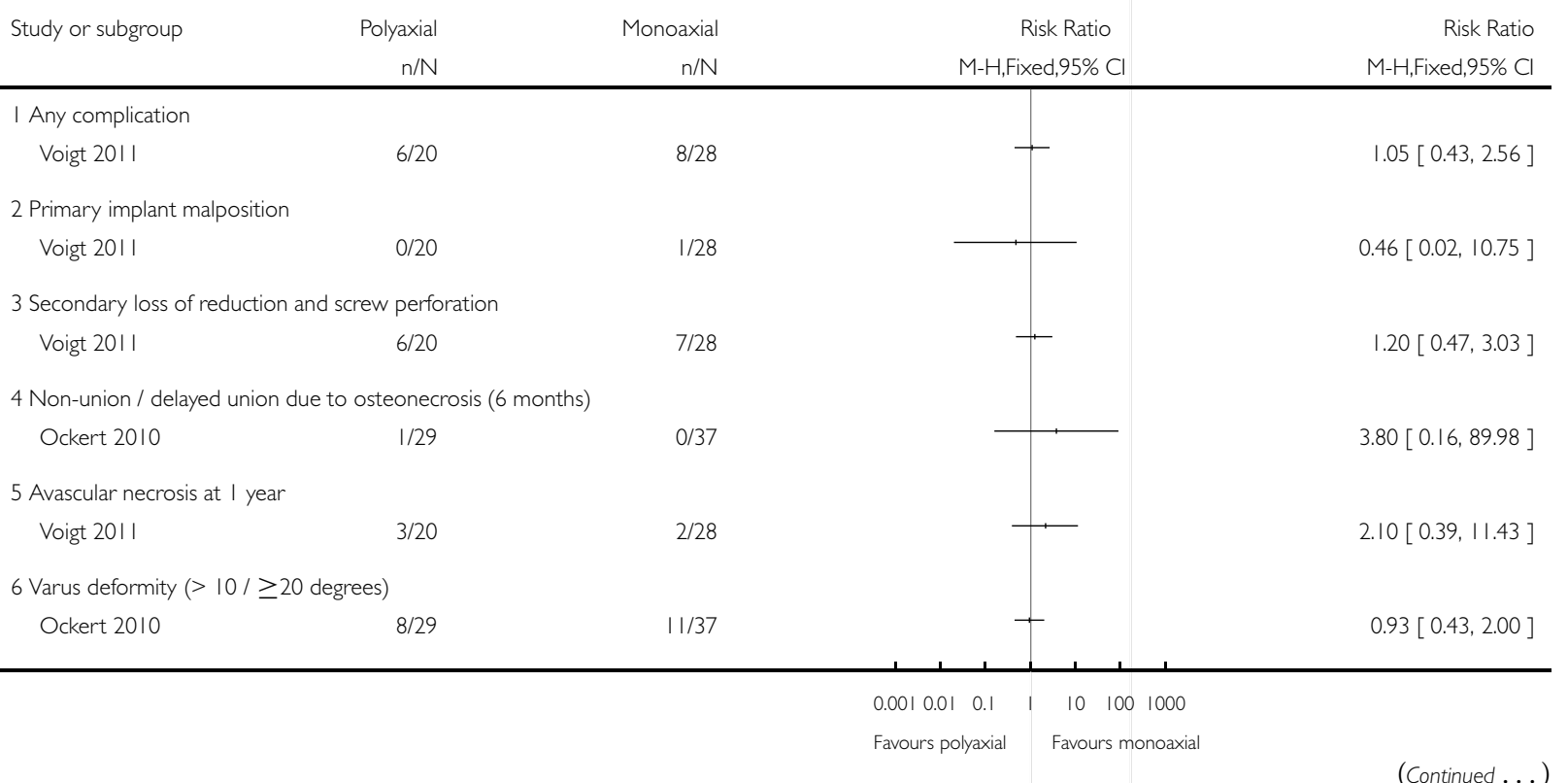




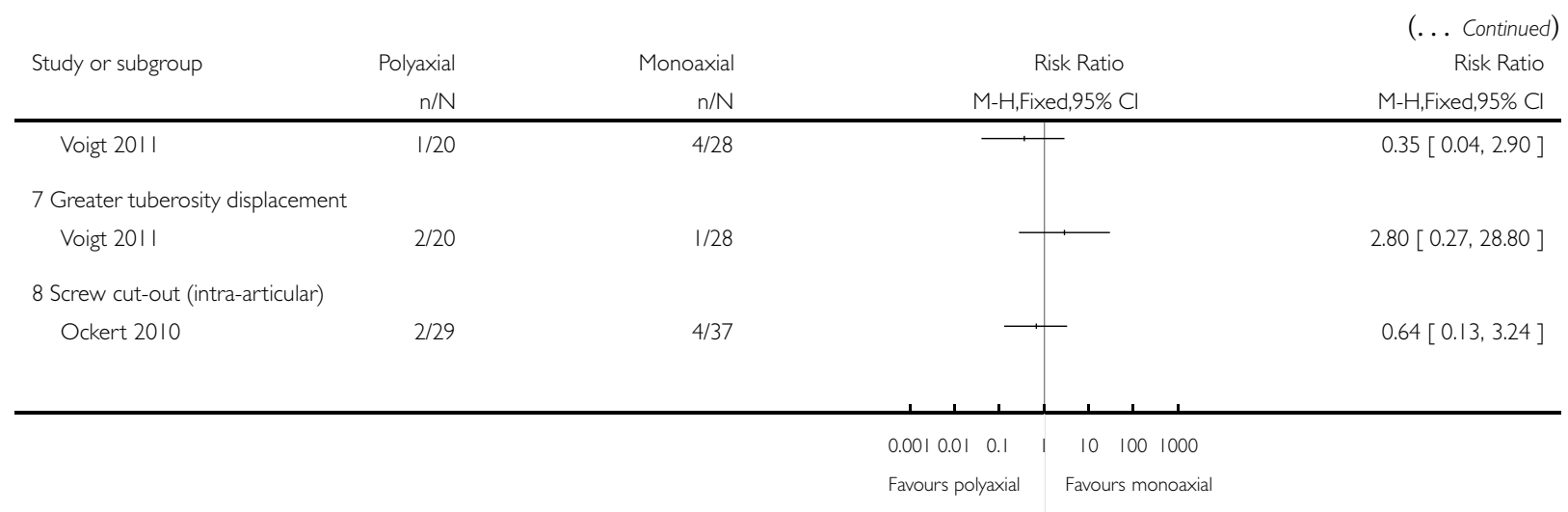

Analysis 10.7. Comparison 10 Polyaxial versus monoaxial screw insertion in plate fixation, Outcome 7 Range of motion (degrees) at 12 months.

Review: Interventions for treating proximal humeral fractures in adults

Comparison: 10 Polyaxial versus monoaxial screw insertion in plate fixation

Outcome: 7 Range of motion (degrees) at 12 months

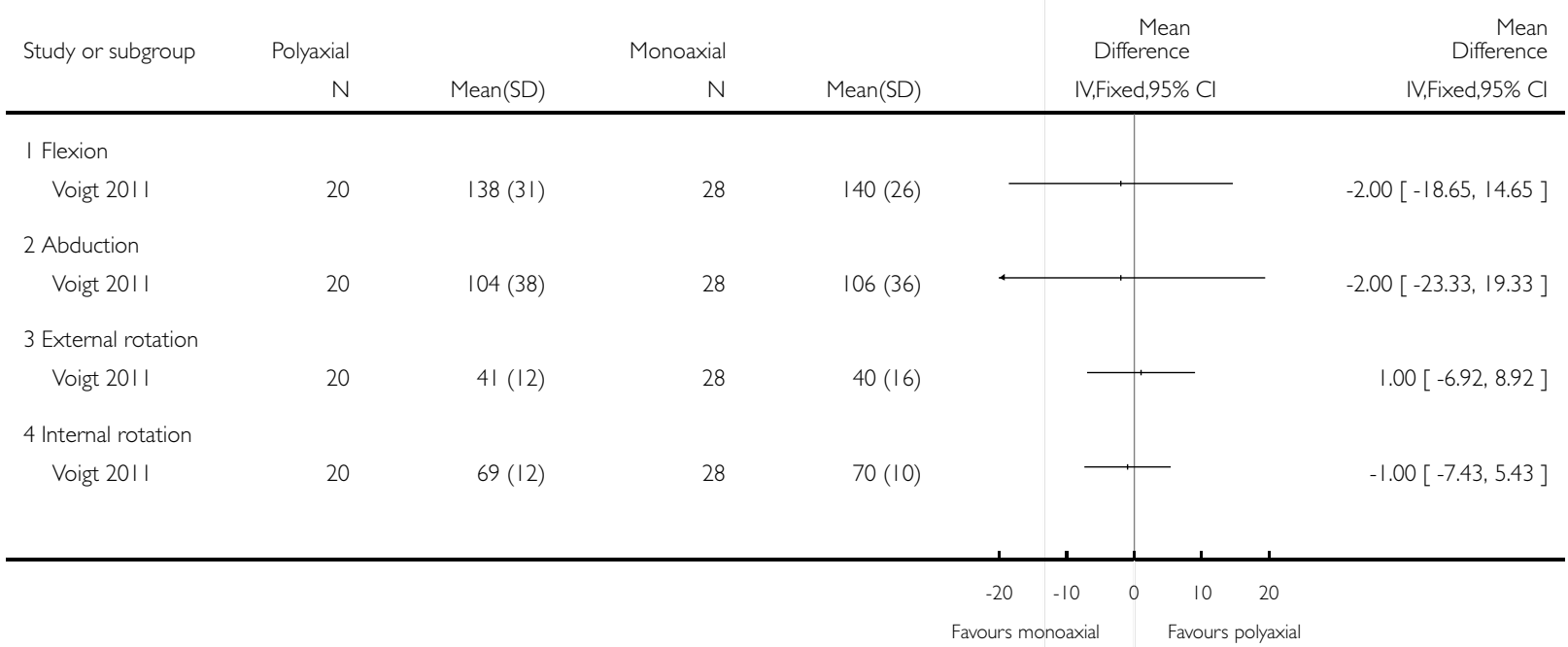


Analysis 10.8. Comparison 10 Polyaxial versus monoaxial screw insertion in plate fixation, Outcome 8 Operation and fluoroscopic times.

Review: Interventions for treating proximal humeral fractures in adults

Comparison: 10 Polyaxial versus monoaxial screw insertion in plate fixation

Outcome: 8 Operation and fluoroscopic times

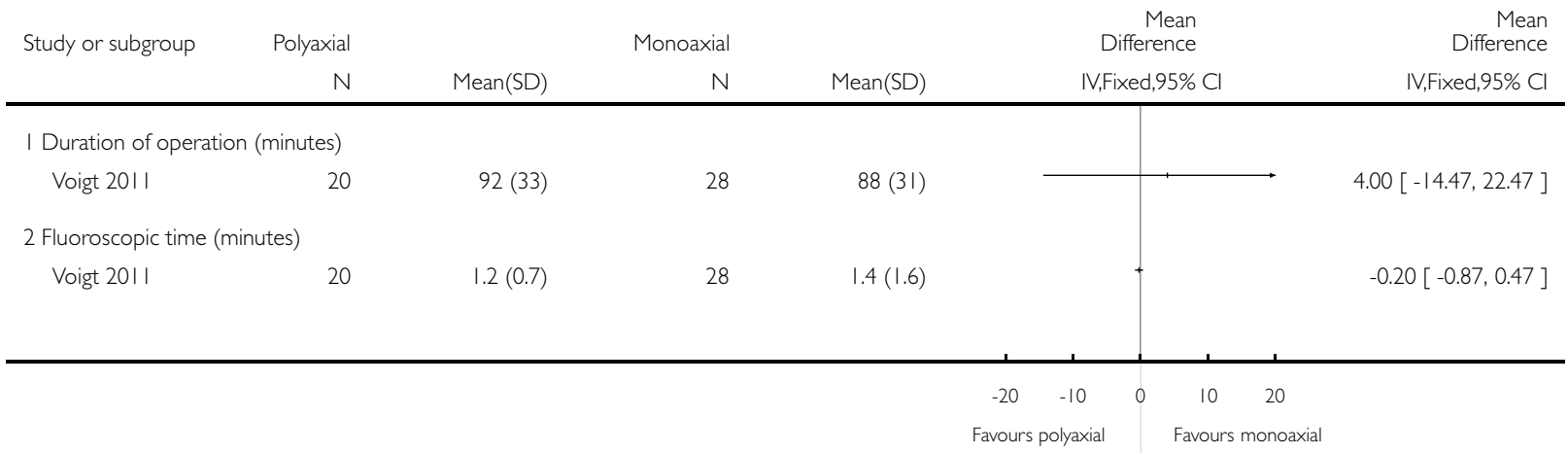

Analysis I I.I. Comparison I I Medial support screws versus control for locking plate fixation, Outcome I Adverse events.

Review: Interventions for treating proximal humeral fractures in adults

Comparison: II Medial support screws versus control for locking plate fixation

Outcome: I Adverse events

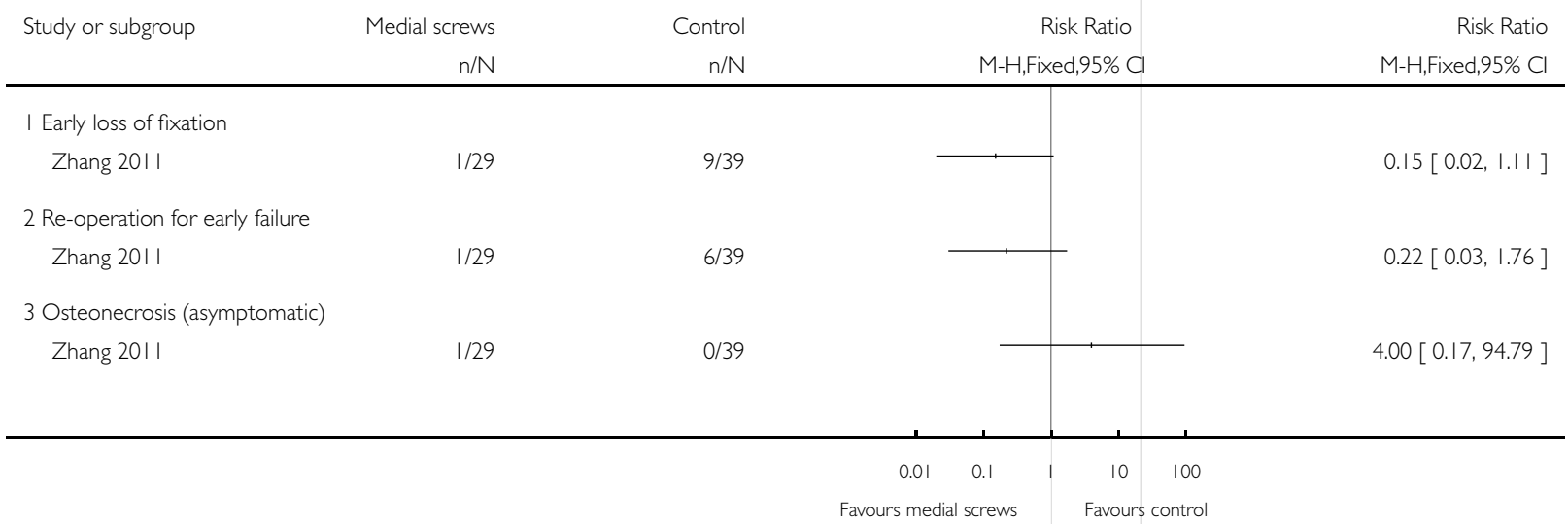


Analysis II.2. Comparison I I Medial support screws versus control for locking plate fixation, Outcome 2 Constant score ( 0 to 100: best) at 2.5 years.

Review: Interventions for treating proximal humeral fractures in adults

Comparison: II Medial support screws versus control for locking plate fixation

Outcome: 2 Constant score (0 to 100: best) at 2.5 years

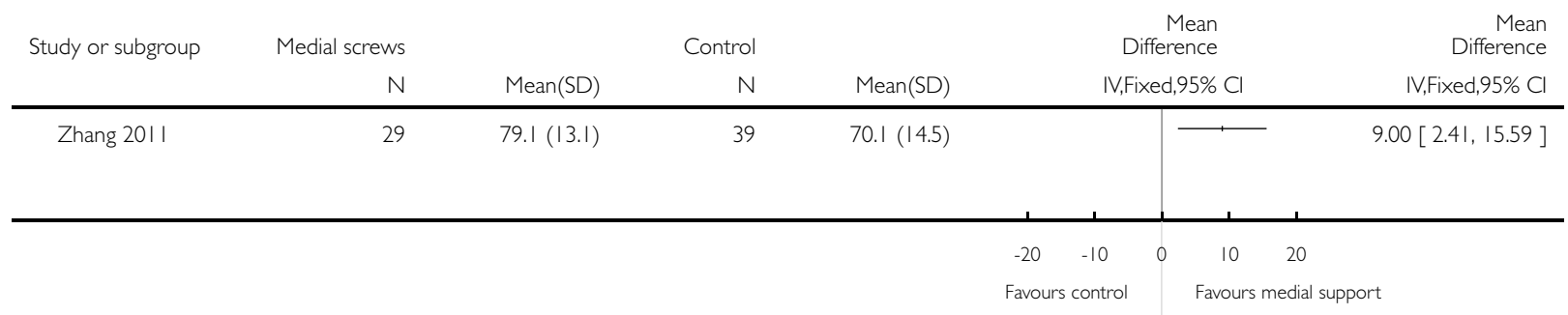

\section{Analysis I2.I. Comparison I2 MultiLoc Proximal Humeral Nail (MPHN) versus Polarus nail, Outcome I} Adverse events.

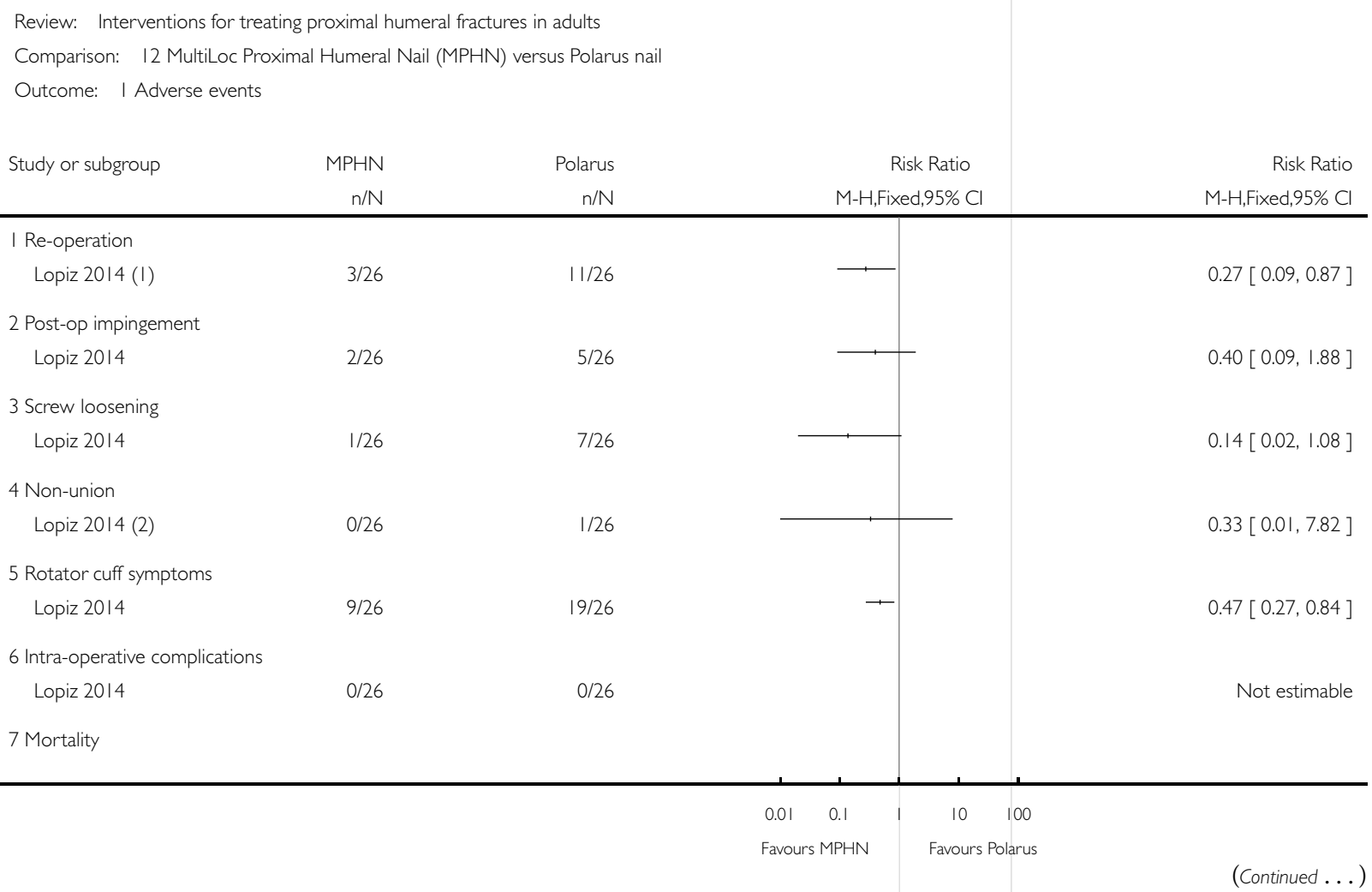




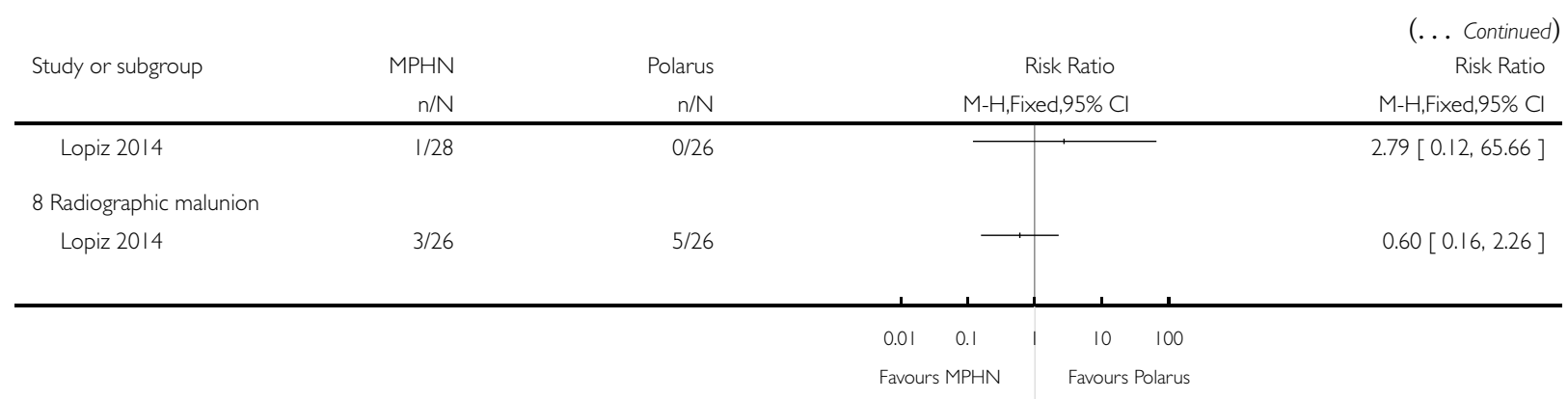

(I) Straight: I screw \% 2 nail removals; Curved: 7 screw and 4 nail removals (I to arthroplasty)

(2) Non-union eventually resulted in a reverse shoulder replacement

\section{Analysis I2.2. Comparison I 2 MultiLoc Proximal Humeral Nail (MPHN) versus Polarus nail, Outcome 2} Constant score (0 to 100: best outcome) at 14 months (6 to 22 months).

Review: Interventions for treating proximal humeral fractures in adults

Comparison: 12 MultiLoc Proximal Humeral Nail (MPHN) versus Polarus nail

Outcome: 2 Constant score ( 0 to 100: best outcome) at 14 months (6 to 22 months)

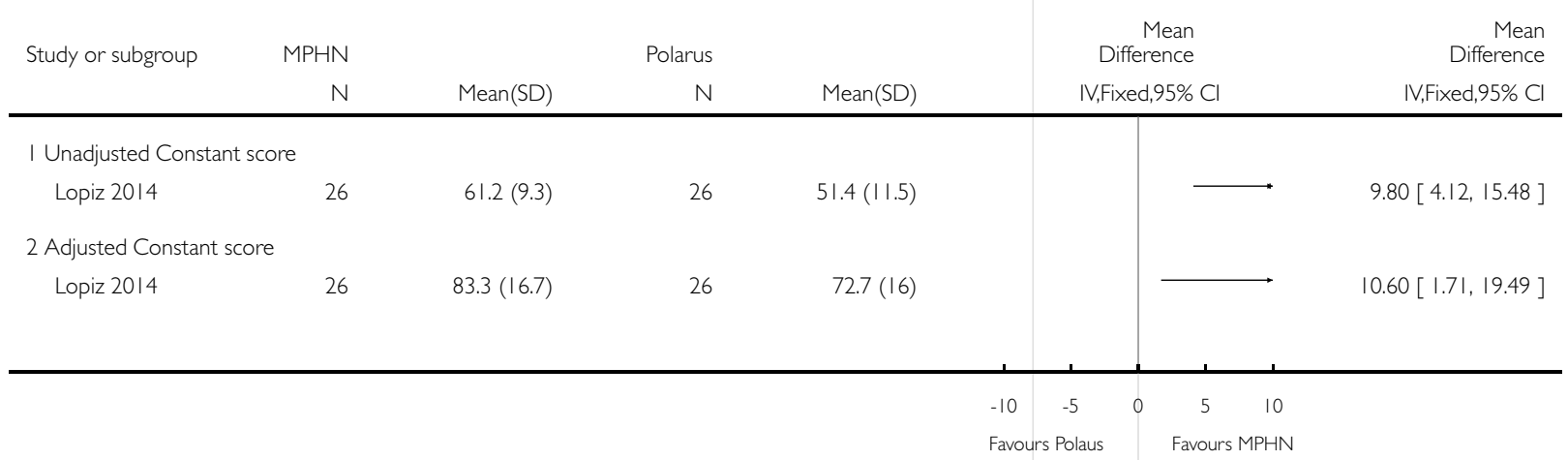


Analysis 12.3. Comparison I 2 MultiLoc Proximal Humeral Nail (MPHN) versus Polarus nail, Outcome 3 Range of shoulder motion (degrees).

Review: Interventions for treating proximal humeral fractures in adults

Comparison: 12 MultiLoc Proximal Humeral Nail (MPHN) versus Polarus nail

Outcome: 3 Range of shoulder motion (degrees)

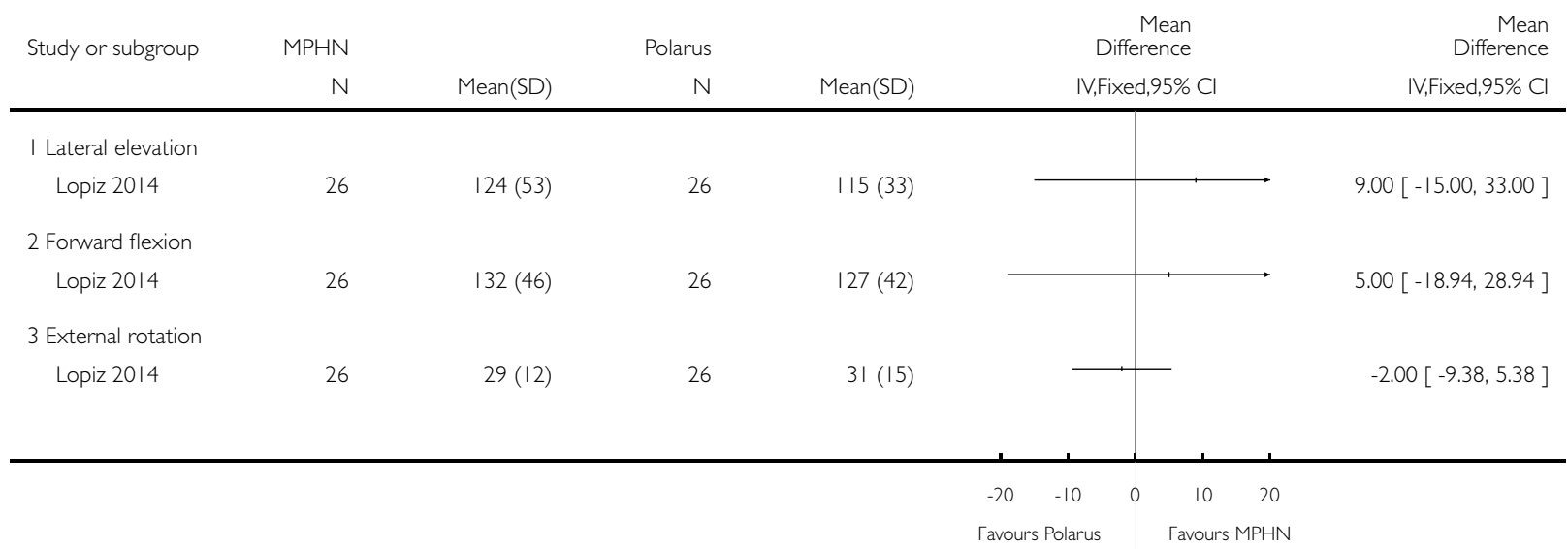

Analysis 12.4. Comparison I MultiLoc Proximal Humeral Nail (MPHN) versus Polarus nail, Outcome 4 Lengths of surgery and hospital stay.

Review: Interventions for treating proximal humeral fractures in adults

Comparison: 12 MultiLoc Proximal Humeral Nail (MPHN) versus Polarus nail

Outcome: 4 Lengths of surgery and hospital stay

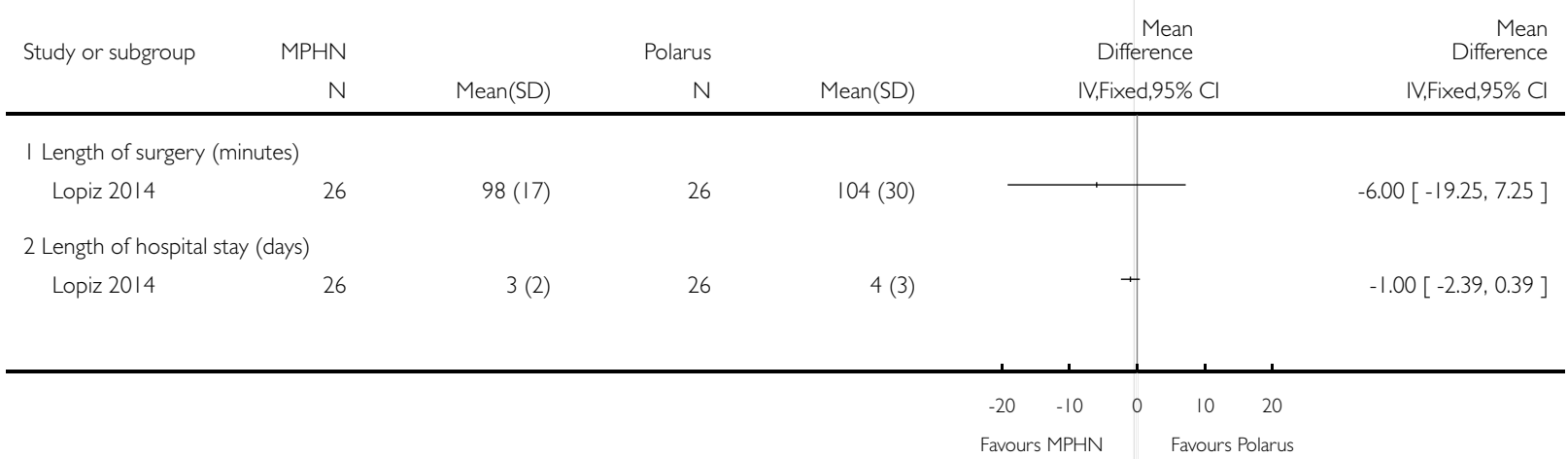




\section{Analysis 13.I. Comparison I3 Hemiarthoplasty: EPOCA prosthesis versus HAS prosthesis, Outcome I}

Adverse events.

Review: Interventions for treating proximal humeral fractures in adults

Comparison: 13 Hemiarthoplasty: EPOCA prosthesis versus HAS prosthesis

Outcome: I Adverse events

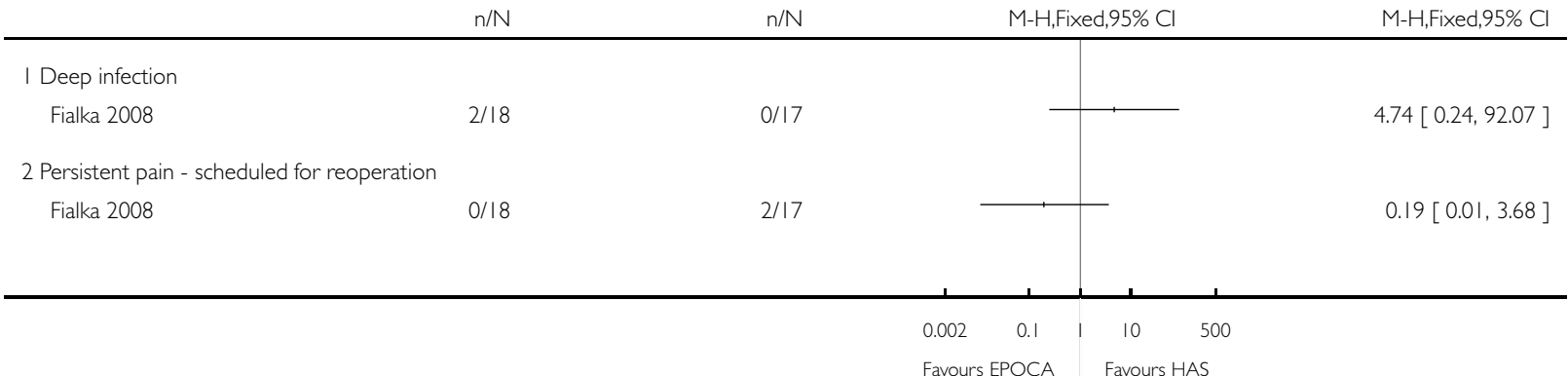

Analysis 13.2. Comparison 13 Hemiarthoplasty: EPOCA prosthesis versus HAS prosthesis, Outcome 2 Radiological assessment findings.

Review: Interventions for treating proximal humeral fractures in adults

Comparison: 13 Hemiarthoplasty: EPOCA prosthesis versus HAS prosthesis

Outcome: 2 Radiological assessment findings

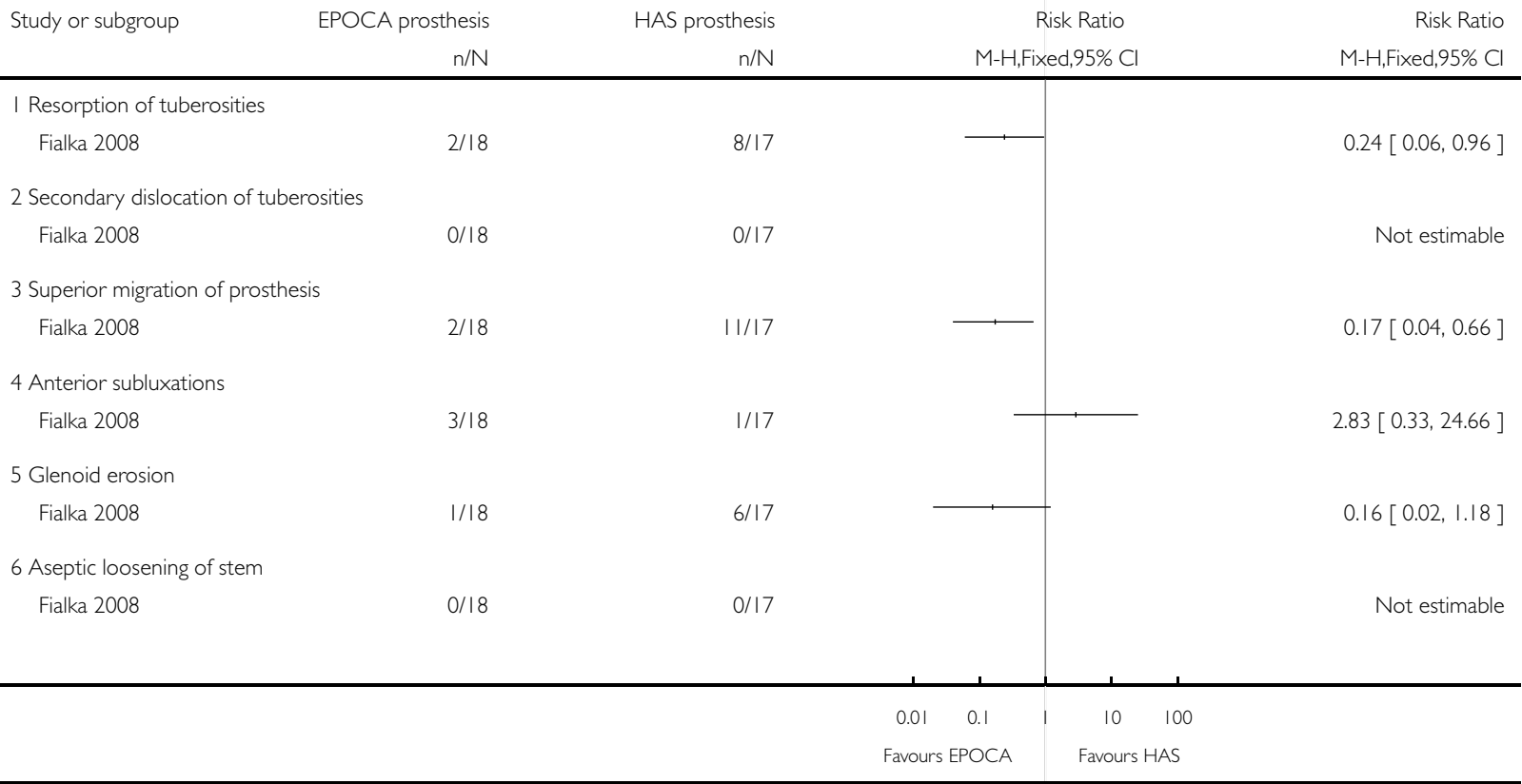


Analysis 13.3. Comparison 13 Hemiarthoplasty: EPOCA prosthesis versus HAS prosthesis, Outcome 3 Range of motion results at one year (degrees).

Range of motion results at one year (degrees)

\begin{tabular}{|c|c|c|c|c|}
\hline Study & Measure & $\begin{array}{l}\text { EPOCA prosthesis } \\
\mathrm{n}=18\end{array}$ & $\begin{array}{l}\text { HAS prosthesis } \\
\mathrm{n}=17\end{array}$ & Reported significance \\
\hline Fialka 2008 & Active forward flexion & $\begin{array}{l}\text { mean }=109^{\circ} \\
\text { range }=30^{\circ} \text { to } 150^{\circ}\end{array}$ & $\begin{array}{l}\text { mean }=62^{\circ} \\
\text { range }=20^{\circ} \text { to } 110^{\circ}\end{array}$ & $\mathrm{P}<0.001$ \\
\hline Fialka 2008 & Active abduction & $\begin{array}{l}\text { mean }=101^{\circ} \\
\text { range }=30^{\circ} \text { to } 150^{\circ}\end{array}$ & $\begin{array}{l}\text { mean }=62^{\circ} \\
\text { range }=30^{\circ} \text { to } 100^{\circ}\end{array}$ & $P=0.001$ \\
\hline Fialka 2008 & $\begin{array}{l}\text { Active external rotation in } \\
90^{\circ} \text { abduction }\end{array}$ & $\begin{array}{l}\text { mean }=30^{\circ} \\
\text { range }=0^{\circ} \text { to } 60^{\circ}\end{array}$ & $\begin{array}{l}\text { mean }=17^{\circ} \\
\text { range }=0^{\circ} \text { to } 40^{\circ}\end{array}$ & $\mathrm{P}=0.01$ \\
\hline Fialka 2008 & $\begin{array}{l}\text { Active external rotation in } \\
90^{\circ} \text { abduction }\end{array}$ & $\begin{array}{l}\text { mean }=45^{\circ} \\
\text { range }=0^{\circ} \text { to } 70^{\circ}\end{array}$ & $\begin{array}{l}\text { mean }=13^{\circ} \\
\text { range }=0^{\circ} \text { to } 40^{\circ}\end{array}$ & $P=0.001$ \\
\hline
\end{tabular}




\section{Analysis 14.I. Comparison I 4 Hemiarthroplasty: tenodesis of long head of biceps (LHB) versus LHB tendon left intact, Outcome I Complications and further surgery.}

Review: Interventions for treating proximal humeral fractures in adults

Comparison: 14 Hemiarthroplasty: tenodesis of long head of biceps (LHB) versus LHB tendon left intact

Outcome: I Complications and further surgery

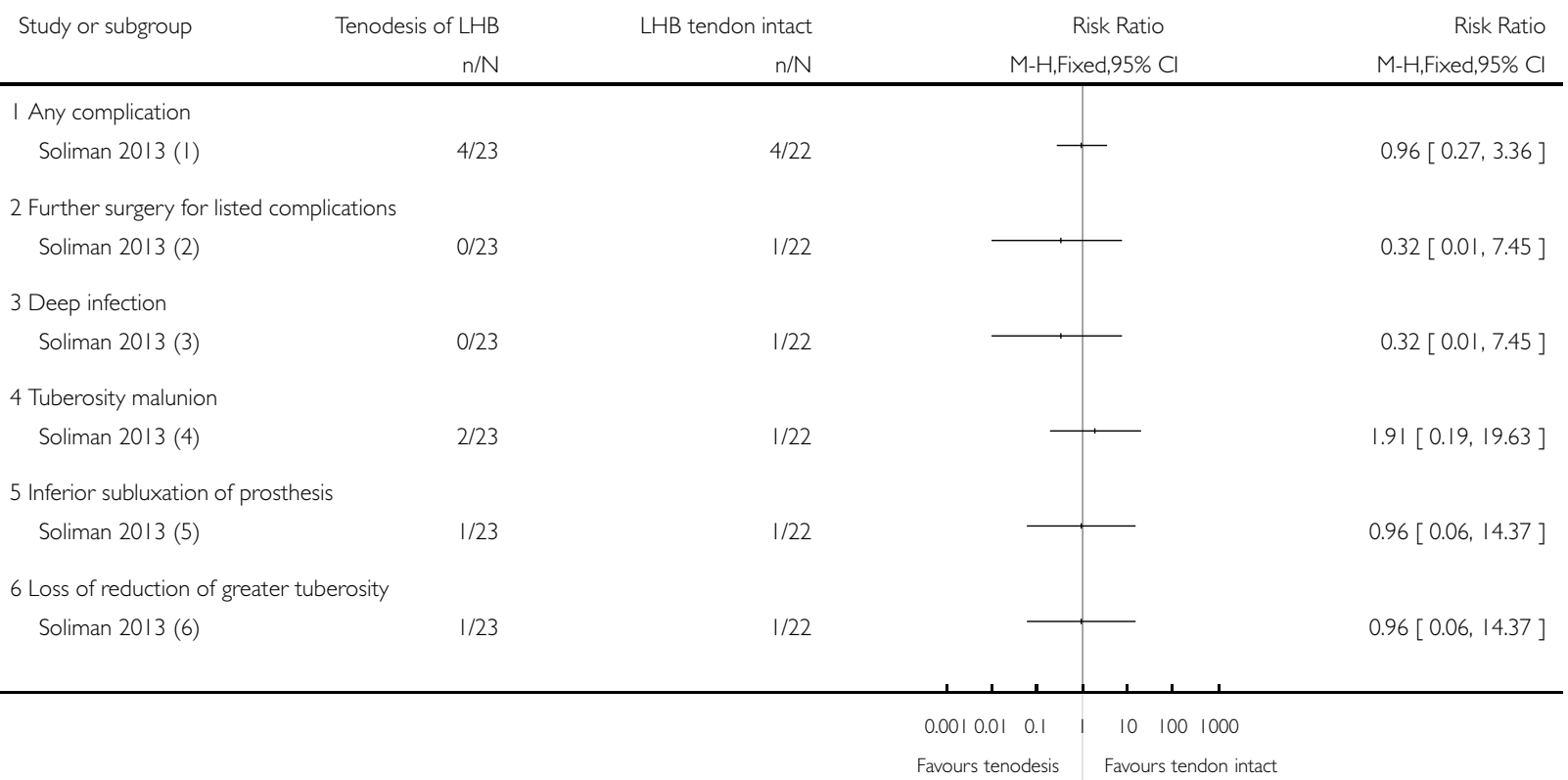

(I) There is a disparity between the number of participants with complications and total individual complications

(2) This was debridement for deep infection

(3) All cases were mild pain (discomfort) except 2 moderate pain in LBT left intact group

(4) All cases were mild pain (discomfort) except 2 moderate pain in LBT left intact group

(5) All cases were mild pain (discomfort) except 2 moderate pain in LBT left intact group

(6) All cases were mild pain (discomfort) except 2 moderate pain in LBT left intact group 
Analysis 14.2. Comparison I 4 Hemiarthroplasty: tenodesis of long head of biceps (LHB) versus LHB tendon left intact, Outcome 2 Constant score ( 0 to 100: best function) at 2 years.

Review: Interventions for treating proximal humeral fractures in adults

Comparison: 14 Hemiarthroplasty: tenodesis of long head of biceps (LHB) versus LHB tendon left intact

Outcome: 2 Constant score (0 to 100: best function) at 2 years

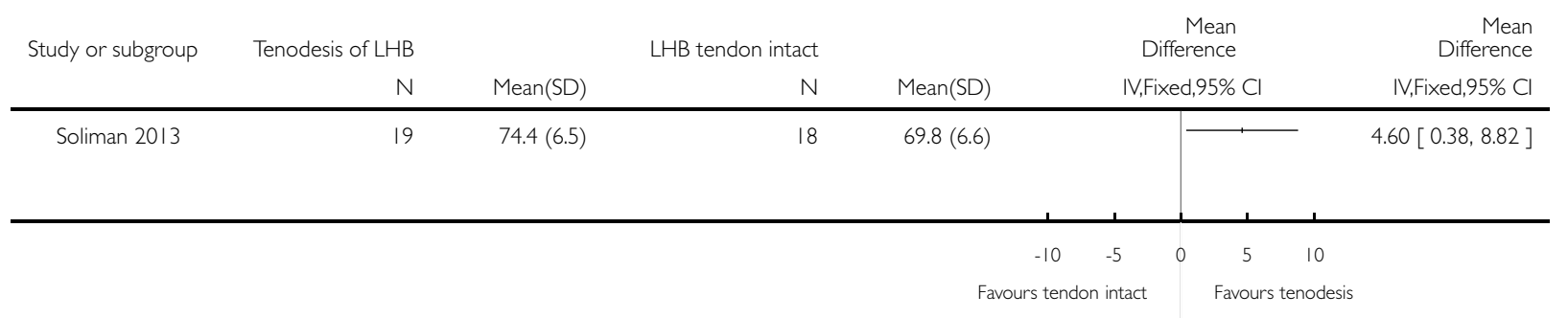

Analysis 14.3. Comparison I 4 Hemiarthroplasty: tenodesis of long head of biceps (LHB) versus LHB tendon left intact, Outcome 3 Shoulder pain at 2 year follow-up.

Review: Interventions for treating proximal humeral fractures in adults

Comparison: 14 Hemiarthroplasty: tenodesis of long head of biceps (LHB) versus LHB tendon left intact

Outcome: 3 Shoulder pain at 2 year follow-up

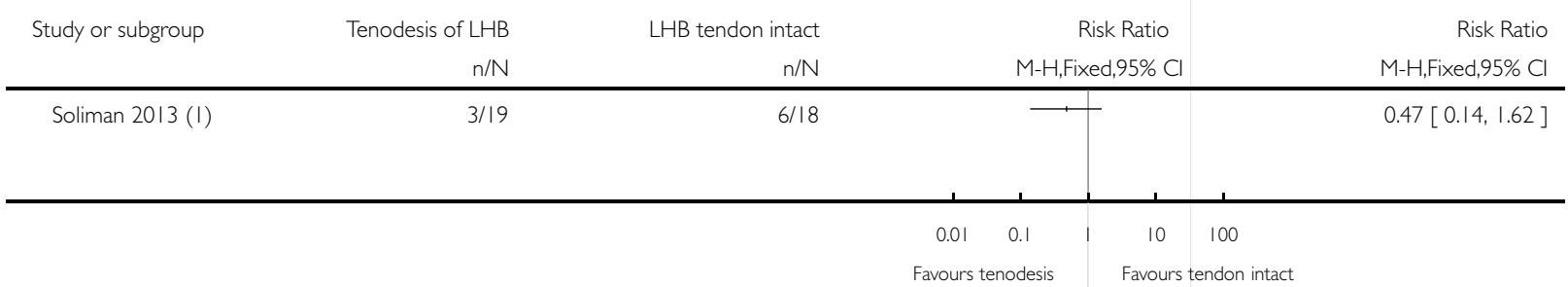

(I) All cases were mild pain (discomfort) except 2 moderate pain in LBT left intact group 


\section{Analysis 14.4. Comparison I4 Hemiarthroplasty: tenodesis of long head of biceps (LHB) versus LHB tendon}

left intact, Outcome 4 Active shoulder elevation (degrees) at 2 years.

Review: Interventions for treating proximal humeral fractures in adults

Comparison: 14 Hemiarthroplasty: tenodesis of long head of biceps (LHB) versus LHB tendon left intact

Outcome: 4 Active shoulder elevation (degrees) at 2 years

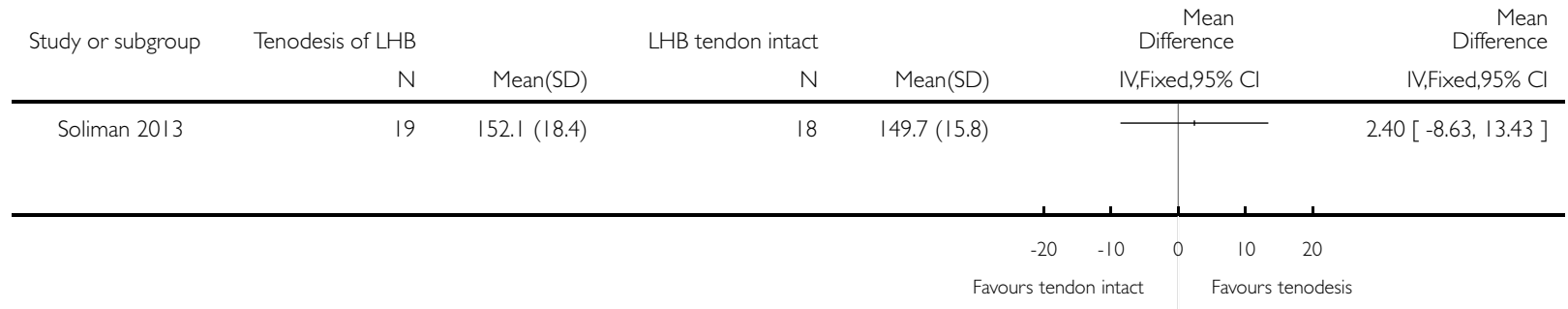

Analysis 15.I. Comparison I5 Post-operative (percutaneous fixation) immobilisation for I week versus 3 weeks, Outcome I Neer score $\leq \mathbf{8 0}$ points (unsatisfactory or failure) at 6 months.

Review: Interventions for treating proximal humeral fractures in adults

Comparison: 15 Post-operative (percutaneous fixation) immobilisation for I week versus 3 weeks

Outcome: I Neer score $\leq 80$ points (unsatisfactory or failure) at 6 months

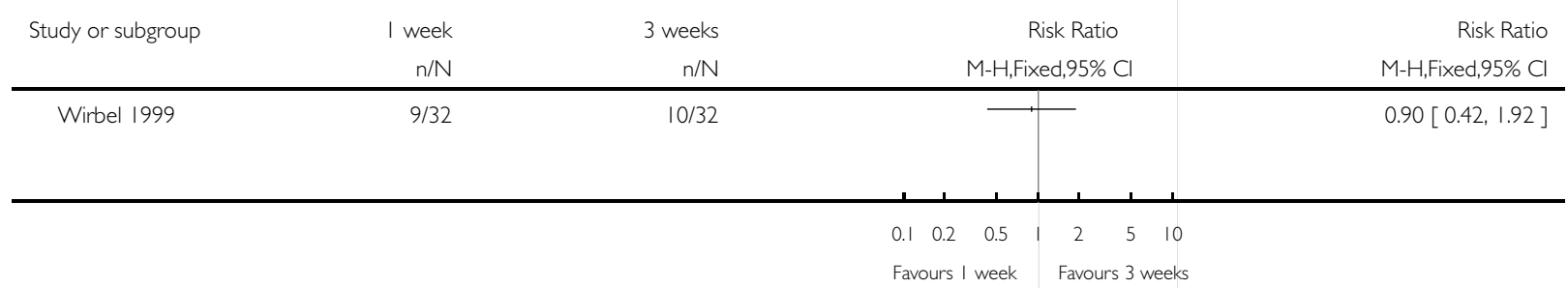


Analysis I5.2. Comparison I5 Post-operative (percutaneous fixation) immobilisation for I week versus 3 weeks, Outcome 2 Premature removal of Kirschner wires.

Review: Interventions for treating proximal humeral fractures in adults

Comparison: 15 Post-operative (percutaneous fixation) immobilisation for I week versus 3 weeks

Outcome: 2 Premature removal of Kirschner wires

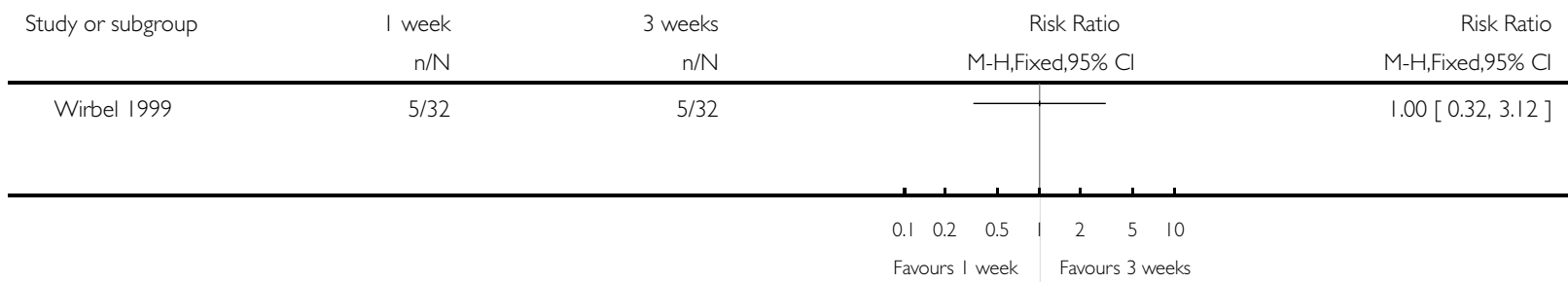

Analysis 16.1. Comparison 16 Post-operative (hemiarthroplasty) mobilisation: early (2 weeks immobilisation) versus late (6 weeks), Outcome I Oxford Shoulder Score at I year (adjusted: 0 to 100 best).

Review: Interventions for treating proximal humeral fractures in adults

Comparison: 16 Post-operative (hemiarthroplasty) mobilisation: early ( 2 weeks immobilisation) versus late (6 weeks)

Outcome: I Oxford Shoulder Score at I year (adjusted: 0 to 100 best)

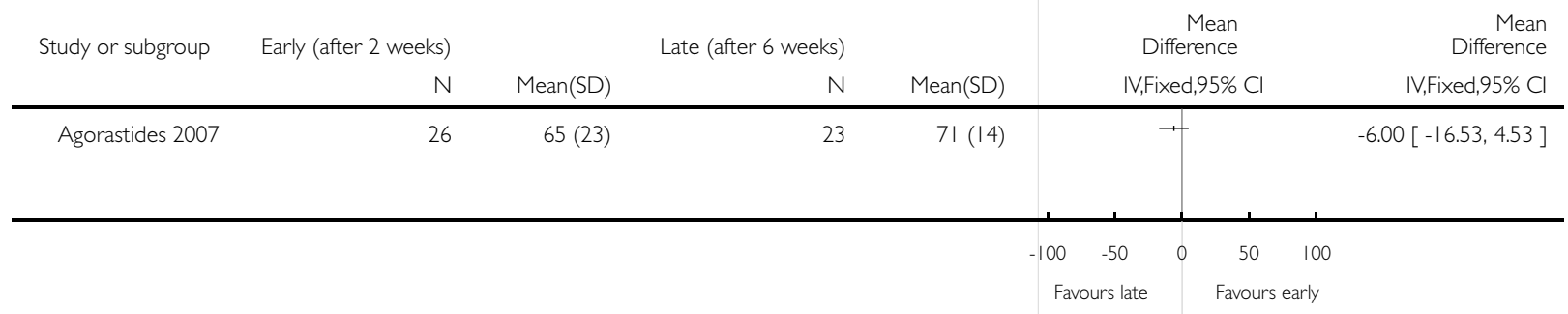


Analysis 16.2. Comparison 16 Post-operative (hemiarthroplasty) mobilisation: early ( 2 weeks immobilisation) versus late (6 weeks), Outcome 2 Constant shoulder score (at I year).

Review: Interventions for treating proximal humeral fractures in adults

Comparison: 16 Post-operative (hemiarthroplasty) mobilisation: early ( 2 weeks immobilisation) versus late (6 weeks)

Outcome: 2 Constant shoulder score (at I year)

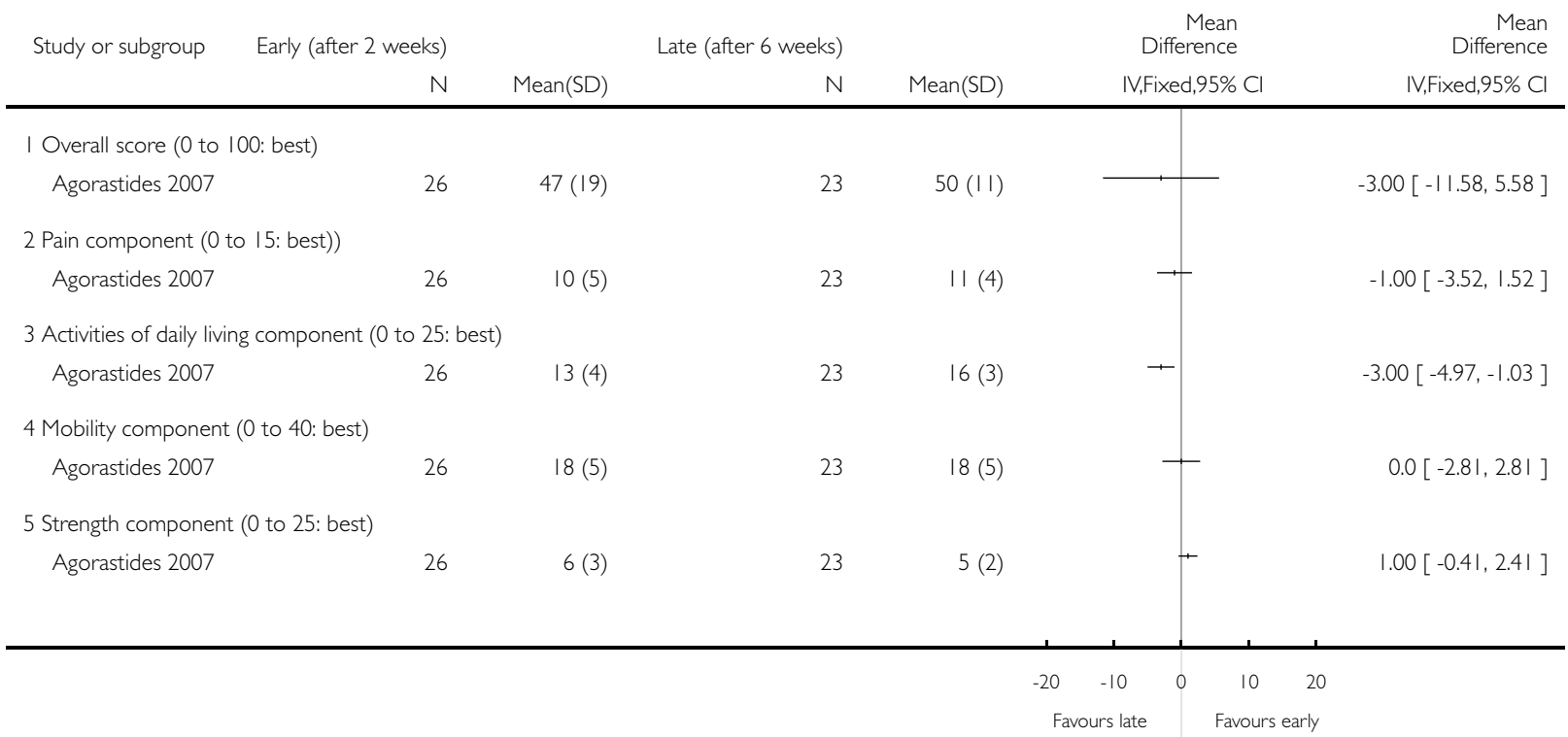


Analysis 16.3. Comparison 16 Post-operative (hemiarthroplasty) mobilisation: early ( 2 weeks immobilisation) versus late (6 weeks), Outcome 3 Radiological assessment findings.

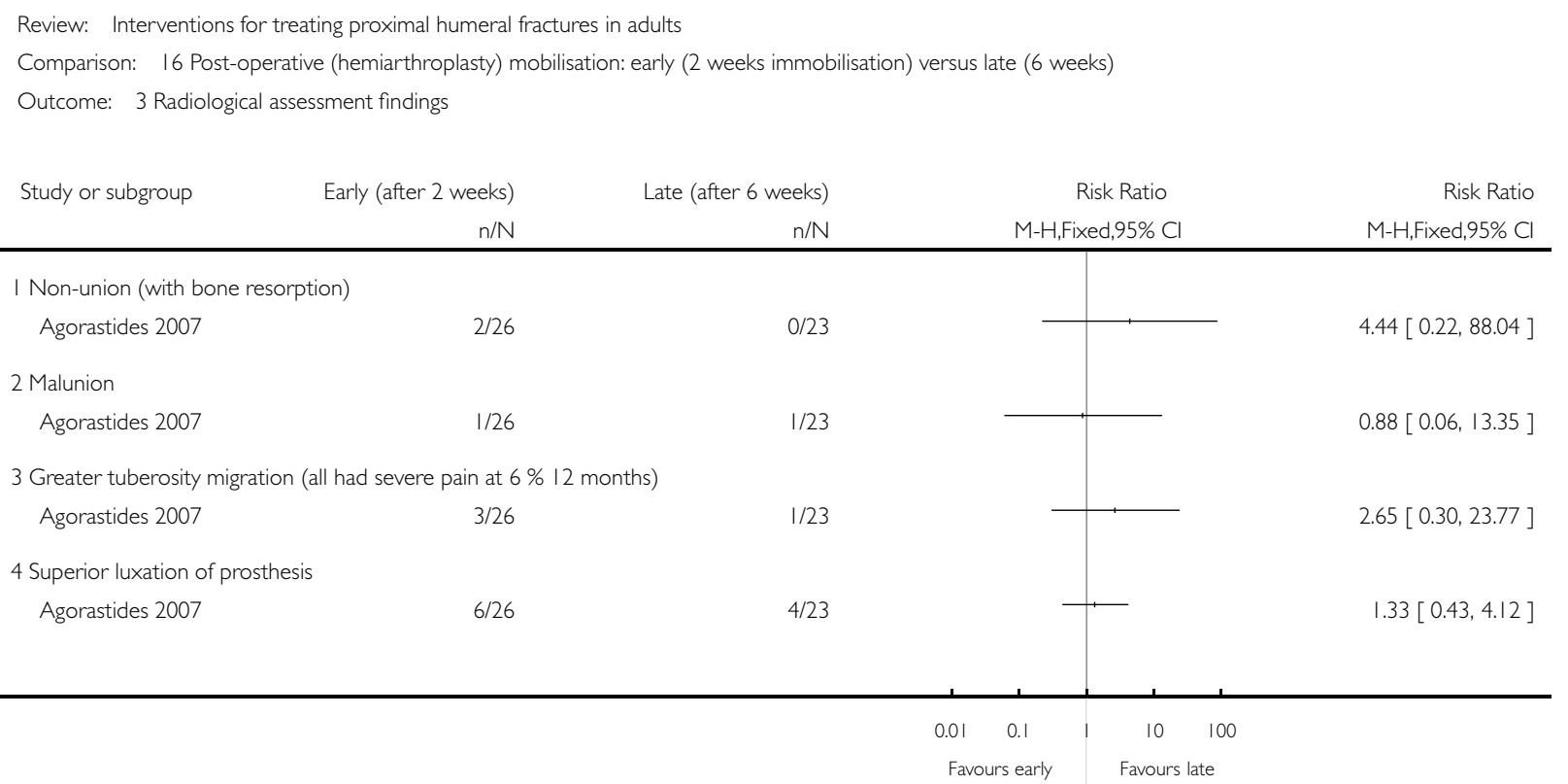

Analysis 16.4. Comparison 16 Post-operative (hemiarthroplasty) mobilisation: early (2 weeks immobilisation) versus late (6 weeks), Outcome 4 Range of motion at I year.

Review: Interventions for treating proximal humeral fractures in adults

Comparison: 16 Post-operative (hemiarthroplasty) mobilisation: early (2 weeks immobilisation) versus late (6 weeks)

Outcome: 4 Range of motion at I year

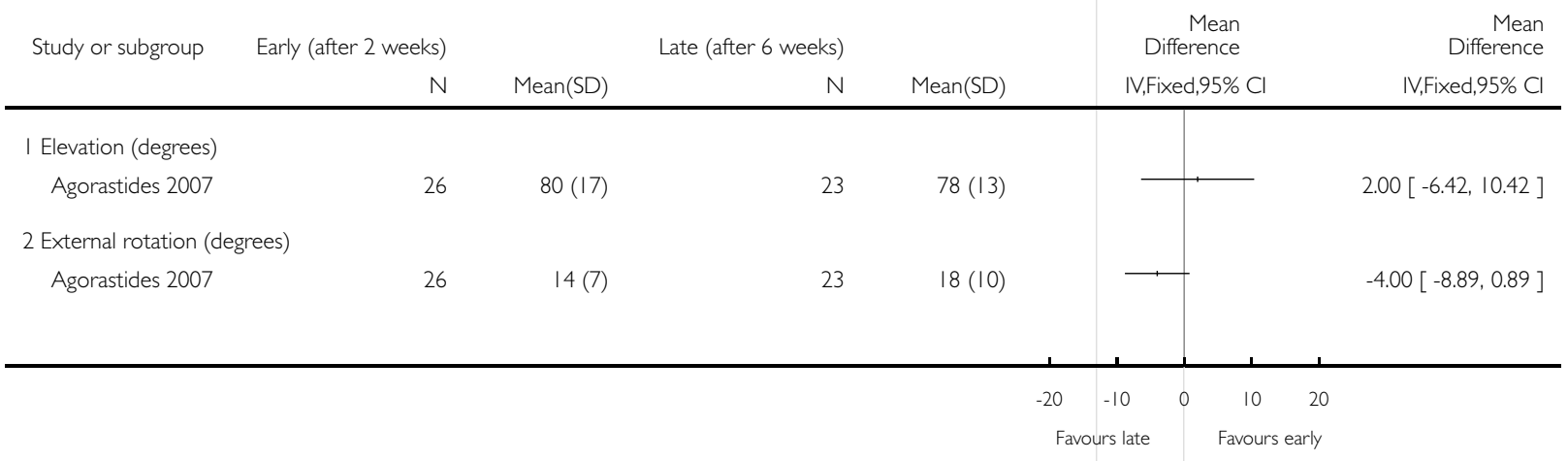


ADDITIONAL TABLES

Table 1. Surgical versus non-surgical treatment trials: brief characteristics

\begin{tabular}{|c|c|c|c|c|}
\hline Study & $\begin{array}{l}\text { Participants } \\
\text { (Neer classification) }\end{array}$ & Surgery & $\begin{array}{l}\text { Non-surgical } \\
\text { (starting with) }\end{array}$ & Follow-up \\
\hline Boons 2012 & $\begin{array}{l}50 \text { participants with 4- } \\
\text { part fractures } \\
\text { (The Netherlands) }\end{array}$ & $\begin{array}{l}\text { Humeral head replace- } \\
\text { ment with the Global } \\
\text { prostheses; cemented }\end{array}$ & Sling immobilisation & 1 year \\
\hline Fjalestad 2010 & $\begin{array}{l}50 \text { participants with } 3 \text { - or } \\
\text { 4-part fractures } \\
\text { (Norway) }\end{array}$ & $\begin{array}{l}\text { Open reduction and fixa- } \\
\text { tion with an interlocking } \\
\text { plate device and metal cer- } \\
\text { clages }\end{array}$ & $\begin{array}{l}\text { Immobilisation of the in- } \\
\text { jured arm in a modified } \\
\text { Velpeau bandage. Closed } \\
\text { reduction in } 8 \text { patients }\end{array}$ & 2 years \\
\hline Kristiansen 1988 & $\begin{array}{l}30 \text { participants with } 312 \text { - } \\
\text {, 3- or 4-part fractures. In- } \\
\text { cluded } 7 \text { 2-part, } 19 \text { 3-part } \\
\text { and } 5 \text { 4-part fractures } \\
\text { (Denmark) }\end{array}$ & $\begin{array}{l}\text { Percutaneous reduction } \\
\text { and external fixation }\end{array}$ & $\begin{array}{l}\text { Closed manipulation and } \\
\text { sling immobilisation }\end{array}$ & 2 years \\
\hline Olerud 2011a & $\begin{array}{l}60 \text { participants with 3- } \\
\text { part fractures (all had dis- } \\
\text { placed surgical neck frac- } \\
\text { ture) } \\
\text { (Sweden) }\end{array}$ & $\begin{array}{l}\text { Open reduction and fixa- } \\
\text { tion with a PHILOS plate } \\
\text { and non-absorbable su- } \\
\text { tures }\end{array}$ & Sling immobilisation & 2 years \\
\hline Olerud 2011b & $\begin{array}{l}55 \text { participants with 4- } \\
\text { part fractures } \\
\text { (Sweden) }\end{array}$ & $\begin{array}{l}\text { Humeral head replace- } \\
\text { ment with the Global Fx } \\
\text { prosthesis }\end{array}$ & Sling immobilisation & 2 years \\
\hline ProFHER 2015 & $\begin{array}{l}250 \\
\text { ticipants with "displaced } \\
\text { fracture of the proximal } \\
\text { humerus that involved the } \\
\text { surgical neck". Included } \\
18 \text { 1-part (but confirmed } \\
\text { as still "displaced"), } 1282 \text { - } \\
\text { part, 93 3-part and } 114 \text { - } \\
\text { part fractures } \\
\text { (UK) }\end{array}$ & $\begin{array}{l}\text { Either internal fixation } \\
\text { (majority were PHILOS } \\
\text { plates) or joint replace- } \\
\text { ment (hemiarthroplasty) } \\
\text { Pragmatic trial - choice } \\
\text { based on surgeon's experi- } \\
\text { ence with method }\end{array}$ & Sling immobilisation & 2 years \\
\hline Stableforth 1984 & $\begin{array}{l}32 \text { participants with } 4 \text { - } \\
\text { part fracture } \\
(\mathrm{UK})\end{array}$ & Hemiarthroplasty & $\begin{array}{l}\text { Closed manipulation and } \\
\text { sling }\end{array}$ & 6 months \\
\hline Zyto 1997 & $\begin{array}{l}40 \text { participants with } 3 \text { - or } \\
\text { 4-part fractures ( } 3 \text { others } \\
\text { excluded) } \\
\text { (Sweden) }\end{array}$ & $\begin{array}{l}\text { Internal fixa- } \\
\text { tion using surgical tension } \\
\text { band or cerclage wiring }\end{array}$ & Sling immobilisation & 50 months \\
\hline
\end{tabular}

Interventions for treating proximal humeral fractures in adults (Review)

Copyright @ 2015 The Cochrane Collaboration. Published by John Wiley \& Sons, Ltd. 
Table 2. Assessment of items relating to applicability of trial findings

\begin{tabular}{|c|c|c|c|c|}
\hline & $\begin{array}{l}\text { Clearly defined study } \\
\text { population? }\end{array}$ & $\begin{array}{l}\text { Interventions } \\
\text { sufficiently described? }\end{array}$ & $\begin{array}{l}\text { Main outcomes suffi- } \\
\text { ciently } \\
\text { described? }\end{array}$ & $\begin{array}{l}\text { Appropriate timing of } \\
\text { outcome measurement? } \\
\text { (Yes }=\geq 1 \text { year) }\end{array}$ \\
\hline Agorastides 2007 & $\begin{array}{l}\text { Partial: exclusions not } \\
\text { specified upfront }\end{array}$ & Yes & Yes & Yes: 1 year \\
\hline Bertoft 1984 & $\begin{array}{l}\text { Partial: no exclusion cri- } \\
\text { teria given (e.g. ability to } \\
\text { understand instructions } \\
\text { for exercises) }\end{array}$ & Yes & Yes & Yes: 1 year \\
\hline Boons 2012 & Yes & Yes & Yes & Yes: 1 year \\
\hline Buecking 2014 & $\begin{array}{l}\text { Partial: indication for } \\
\text { hemiarthroplasty poorly } \\
\text { defined ( } 27 \text { excluded be- } \\
\text { fore randomisation be- } \\
\text { cause "implantation of a } \\
\text { prosthesis was planned") }\end{array}$ & Yes & Yes & Yes: 1 year \\
\hline Cai 2012 & $\begin{array}{l}\text { Partial: unclear defini- } \\
\text { tion of 4-part fractures. }\end{array}$ & $\begin{array}{l}\text { Yes: however, time to } \\
\text { surgery not reported }\end{array}$ & Yes & Yes: 2 years \\
\hline Fialka 2008 & Yes & Yes & Yes & Yes: 1 year \\
\hline Fjalestad 2010 & Yes & Yes & Yes & Yes: 2 years \\
\hline Hoellen 1997 & $\begin{array}{l}\text { Yes: but some question } \\
\text { over fracture type in that } \\
\text { the Holbein } 1999 \text { report } \\
\text { included 3-part fractures } \\
\text { too }\end{array}$ & Yes & Yes & Yes: 1 year \\
\hline Hodgson 2003 & Yes & Yes & Yes & Yes: 2 years \\
\hline Kristiansen 1988 & $\begin{array}{l}\text { Partial: no exclusion cri- } \\
\text { teria given }\end{array}$ & $\begin{array}{l}\text { Partial: incomplete de- } \\
\text { scription of timing of } \\
\text { sling use and care of ex- } \\
\text { ternal fixator pin sites }\end{array}$ & $\begin{array}{l}\text { Partial: no description of } \\
\text { measurement procedures }\end{array}$ & Yes: 1 year \\
\hline Kristiansen 1989 & $\begin{array}{l}\text { Partial: no exclusion cri- } \\
\text { teria given }\end{array}$ & $\begin{array}{l}\text { Partial: } \\
\text { although sling and body } \\
\text { bandage are common ex- } \\
\text { pressions, some variation } \\
\text { possible }\end{array}$ & $\begin{array}{l}\text { Partial: no description of } \\
\text { measurement procedures }\end{array}$ & Yes: 2 years \\
\hline Lefevre-Colau 2007 & Yes & Yes & Yes & Partial: 6 months \\
\hline
\end{tabular}


Table 2. Assessment of items relating to applicability of trial findings

\begin{tabular}{|c|c|c|c|c|}
\hline Livesley 1992 & $\begin{array}{l}\text { Yes: although this in- } \\
\text { cluded } 4 \text { patients under } \\
20 \text { years with epiphyseal } \\
\text { fractures }\end{array}$ & Yes & Yes & Partial: 6 months \\
\hline Lopiz 2014 & $\begin{array}{l}\text { Partial: insufficient crite- } \\
\text { ria given in terms of suit- } \\
\text { ability for surgery }\end{array}$ & Yes & Yes & $\begin{array}{l}\text { Partial: minimum } 6 \\
\text { months }\end{array}$ \\
\hline Lundberg 1979 & $\begin{array}{l}\text { Partial: no exclusion cri- } \\
\text { teria given (e.g. ability to } \\
\text { understand instructions } \\
\text { for exercises) }\end{array}$ & Yes & Yes & $\begin{array}{l}\text { Yes: } 1 \text { year or above } \\
\text { (mean: } 16 \text { months) }\end{array}$ \\
\hline Ockert 2010 & $\begin{array}{l}\text { Partial: exclusion crite- } \\
\text { ria described in con- } \\
\text { text of post-randomisa- } \\
\text { tion exclusions }\end{array}$ & Yes & Yes & Partial: 6 months \\
\hline Olerud 2011a & Yes & Yes & Yes & Yes: 2 years \\
\hline Olerud 2011b & Yes & Yes & Yes & Yes: 2 years \\
\hline ProFHER 2015 & Yes & $\begin{array}{l}\text { Yes } \\
\text { In the context of this be- } \\
\text { ing a pragmatic trial }\end{array}$ & Yes & Yes: 2 years \\
\hline Revay 1992 & Yes & $\begin{array}{l}\text { Partial: fre- } \\
\text { quency of swimming ses- } \\
\text { sions not stated }\end{array}$ & Yes & Yes: 1 year \\
\hline Rommens 1993 & $\begin{array}{l}\text { Yes: but to note that } \\
\text { other fractures including } \\
\text { rib ( } 3 \text { participants) were } \\
\text { included }\end{array}$ & Yes & $\begin{array}{l}\text { Partial: functional out- } \\
\text { come assessment not de- } \\
\text { scribed (sufficiently) }\end{array}$ & $\begin{array}{l}\text { No: only until fracture } \\
\text { consolidation }\end{array}$ \\
\hline Sebastiá-Forcada 2014 & Yes & Yes & Yes & Yes: minimum 2 years \\
\hline Smejkal 2011 & Yes & $\begin{array}{l}\text { Partial: Only minimal in- } \\
\text { tra-operative } \\
\text { details given and noth- } \\
\text { ing regarding post-opera- } \\
\text { tive management includ- } \\
\text { ing rehabilitation }\end{array}$ & $\begin{array}{l}\text { Partial: this may have } \\
\text { been 'lost in translation' } \\
\text { (Czech article) }\end{array}$ & $\begin{array}{l}\text { Yes: mean } 2 \text { years but } \\
\text { range not stated (proba- } \\
\text { bly most/all }>1 \text { year as } \\
\text { recruitment had finished } \\
\text { January 2010) }\end{array}$ \\
\hline Soliman 2013 & $\begin{array}{l}\text { No: no explanation given } \\
\text { for a younger population; } \\
\text { insufficient criteria given } \\
\text { in terms of suitability for }\end{array}$ & Yes & $\begin{array}{l}\text { Partial: incomplete de- } \\
\text { scription of pain cat- } \\
\text { egories; no clarification }\end{array}$ & $\begin{array}{l}\text { Yes: minimum } 21 \\
\text { months follow-up }\end{array}$ \\
\hline
\end{tabular}


Table 2. Assessment of items relating to applicability of trial findings

hemiarthroplasty

\begin{tabular}{|c|c|c|}
\hline & hemiarthroplasty & \\
\hline Stableforth 1984 & Yes & Yes \\
\hline Torrens 2012 & $\begin{array}{l}\text { Partial: the }<1.5 \mathrm{~cm} \text { cri- } \\
\text { terion for posterior dis- } \\
\text { placement of the greater } \\
\text { tuberosity is unusual and } \\
\text { no justification was given } \\
\text { by the authors }\end{array}$ & $\begin{array}{l}\text { Partial: incomplete de- } \\
\text { scription of accompany- } \\
\text { ing "progressive rehabili- } \\
\text { tation program" }\end{array}$ \\
\hline
\end{tabular}

\begin{tabular}{|c|c|c|c|c|}
\hline Voigt 2011 & Yes & Yes & Yes & Yes: 1 year \\
\hline Wirbel 1999 & Yes & Yes & $\begin{array}{l}\text { Partial: no description of } \\
\text { measurement procedures }\end{array}$ & $\begin{array}{l}\text { Partial: between } 9 \text { and } \\
36 \text { months; < } 1 \text { year in } \\
10 \text { participants. Main re- } \\
\text { sults applied to } 6 \text { months }\end{array}$ \\
\hline Zhang 2011 & Yes & Yes & $\begin{array}{l}\text { Partial: Insufficient in- } \\
\text { formation on measure- } \\
\text { ment of complications } \\
\text { and timing of their mea- } \\
\text { surement }\end{array}$ & $\begin{array}{l}\text { Yes: All over } 25 \text { months } \\
\text { (mean } 30.8 \text { months) }\end{array}$ \\
\hline Zhu 2011 & Yes & Yes & Yes & Yes: 1 and 3 years \\
\hline Zyto 1997 & Yes & Yes & Yes & $\begin{array}{l}\text { Yes: } 1 \text { year, and } 3 \text { to } 5 \\
\text { years }\end{array}$ \\
\hline
\end{tabular}

of modification to Con-

stant score

Partial: no description Partial: up to 6 months, of measurement proce- then between 18 months dures, incomplete de- to 12 years. This is too scription of pain cate- spread out. Most results gories applied to the 6-month follow-up
Partial:
incom- Yes: 1 year

plete description of mea-

surement procedures

$$
\text { surement procedures }
$$




\section{A P P E N D I C E S}

\section{Appendix I. Search strategies January 2012 to November 2014}

\section{The Cochrane Library (Wiley Online Library)}

\#1 MeSH descriptor: [Shoulder Fractures] explode all trees (63) \#2 MeSH descriptor: [Humeral Fractures] explode all trees (85)

\#3 MeSH descriptor: [Humerus] explode all trees (63)

\#4 (shoulder* or humor*) (5000)

\#5 fract* $^{*}$ (27727)

\#6 (\#3 or \#4) (5000)

\#7 (\#1 or \#2) (148)

\#8 (\#5 and \#6) (759)

\#9 (\#7 or \#8) Publication Year from 2011 to 2012 (91) [Trials]

\section{MEDLINE (OVID Online)}

1 Shoulder Fractures/ (2387)

2 Humeral Fractures/ (6074)

3 ((humor\$ or shoulder\$) adj10 (fracture $\$$ or fixat $\$)$ ).tw. (8248)

4 or/2-3 (10515)

5 (proximal or neck\$1 or sub?capital).tw. (316523)

6 and $/ 4-5$ (2718)

7 or/1,6 (3865)

8 Randomized controlled trial.pt. (399438)

9 Controlled clinical trial.pt. (90638)

10 randomized.ab. (318613)

11 placebo.ab. (163586)

12 Drug therapy.fs. (1782347)

13 randomly.ab. (228361)

14 trial.ab. (332305)

15 groups.ab. (1435899)

16 or/8-15 (3524227)

17 exp Animals/ not Humans/ (4090273)

1816 not 17 (3026698)

197 and 18 (402)

$20(2012 \$$ or $2013 \$$ or $2014 \$)$.ed. (2951048)

2119 and 20 (114)

\section{EMBASE (OVID Online)}

1 Humerus Fracture/ (7941)

2 ((humer\$ or shoulder\$) adj10 (fract\$ or fixat\$)).tw. (9383)

3 or/1-2 (12152)

4 (proximal or neck $\$ 1$ or sub?capital).tw. (366908)

5 and/3-4 (3262)

6 exp Randomized Controlled Trial/ (352803)

7 exp Double Blind Procedure/ (116053)

8 exp Single Blind Procedure/ (18999)

9 exp Crossover Procedure/ (40548)

10 Controlled Study/ (4443980) 
11 or/6-10 (4522468)

12 ((clinical or controlled or comparative or placebo or prospective\$ or randomi\#ed) adj3 (trial or study)).tw. (773369)

13 (random\$ adj7 (allocat\$ or allot\$ or assign $\$$ or basis\$ or divid\$ or order\$)).tw. (191210)

14 ((singl\$ or doubl\$ or trebl\$ or tripl\$) adj7 (blind\$ or mask\$)).tw. (164016)

15 (cross?over\$ or (cross adj1 over\$)).tw. (70584)

16 ( (allocat\$ or allot\$ or assign\$ or divid\$) adj3 (condition\$ or experiment $\$$ or intervention $\$$ or treatment $\$$ or therap $\$$ or control\$ or group\$)).tw. (251188)

17 or/12-16 (1156434)

18 or/ 11,17 (5116362)

19 limit 18 to human (3115123)

20 and/5,19 (688)

21 ( $2012 \$$ or $2013 \$$ or $2014 \$)$.em. (4100346)

2220 and 21 (199)

\section{CINAHL (EBSCO)}

S1 (MH "Shoulder Fractures") (642)

S2 (MH "Humeral Fractures") $(1,135)$

S3 (MH "Humerus/IN/SU") (410)

S4 TX ( ( humer* or shoulder* ) ) AND TX ( ( fracture* or fixat* $)$ ) $(3,568)$

S5 S2 or S3 or S4 $(3,751)$

S6 TX proximal or neck or subcapital or sub-capital $(58,806)$

S7 S5 and S6 (935)

S8 S1 or S7 $(1,256)$

S9 (MH “Clinical Trials+”) $(180,311)$

S10 (MH "Evaluation Research+") (20,829)

S11 (MH "Comparative Studies") $(78,838)$

S12 (MH “Crossover Design”) (12,280)

S13 PT Clinical Trial $(76,753)$

S14 (MH "Random Assignment") (38,116)

S15 S9 or $S 10$ or $S 11$ or $S 12$ or S13 or $S 14(285,418)$

S16 TX ((clinical or controlled or comparative or placebo or prospective or randomi?ed) and (trial or study)) $(512,070)$

S17 TX (random* and (allocat* or allot* or assign* or basis* or divid* or order*)) $(68,589)$

S18 TX ((singl* or doubl* or trebl* or tripl*) and (blind* or mask $\left.\left.{ }^{*}\right)\right)(757,946)$

S19 TX ( crossover* or 'cross over' ) or TX cross n1 over $(15,336)$

S20 TX ((allocat* or allot* or assign* or divid*) and (condition* or experiment* or intervention* or treatment* or therap* or control* or group*)) $(86,431)$

S21 S16 or S17 or S18 or S19 or S20 $(1,172,263)$

S22 S15 or S21 $(1,241,245)$

S23 S8 and S22 (474)

S24 EM 2012 OR EM 2013 OR EM $2014(993,625)$

S25 S23 AND S24 (129)

\section{AMED (OVID Online)}

1 exp Shoulder/ (1171)

2 exp Humerus/ (115)

3 Fractures Bone/ (790)

4 (fract\$ or break\$ or broken or ruptur\$).tw. (9763)

51 or $2(1265)$

63 or $4(9763)$

75 and $6(63)$

8 ((humer\$ or shoulder\$) adj10 (fracture\$ or fixat\$)).tw. (130)

Interventions for treating proximal humeral fractures in adults (Review)

Copyright $\odot 2015$ The Cochrane Collaboration. Published by John Wiley \& Sons, Ltd. 
97 or $8(143)$

10 (proximal or neck $\$ 1$ or sub?capital).tw. (5084)

119 and $10(42)$

12 (2012\$ or $2013 \$$ or $2014 \$)$.up. (30025)

1311 and $12(5)$

\section{Other searches}

PEDro

Simple search

1. proximal AND humer* (17 records)

2. neck AND humer* ${ }^{*}(8$ records $)$

Advanced search

3. Abstract and title: fracture*

Body part: upper arm, shoulder or shoulder girdle

Method: Clinical trial (30 records)

The Bone and Joint Journal Orthopaedic Proceedings

Title "proximal humer*" and full text or abstract or title "random*" limited to Orthopaedic Proceedings (13 records)

WHO International Clinical Trials Registry Platform Search Portal

1. proximal and humer* $(58$ records)

2. neck and humer* (3 records)

ISRCTN registry

1. proximal and humer* (38 records)

2. neck AND humer* (28 records)

ClinicalTrials.gov

(proximal OR neck) AND humerus (50 records)

\section{Appendix 2. Numbers and status of studies in the published versions of the review}

\begin{tabular}{l|l|l}
\hline Version & Trial status & Changes \\
\hline $\begin{array}{l}\text { Ist version } \\
\text { Issue 1, } 2001\end{array}$ & $\begin{array}{l}\text { The original review had } 9 \text { included studies, } \\
\text { 4 excluded studies and } 6 \text { studies listed as } \\
\text { ongoing }\end{array}$ \\
\hline $\begin{array}{l}\text { 2nd version (substantive update) } \\
\text { Issue 2, 2002 }\end{array}$ & $\begin{array}{l}\text { This update had } 10 \text { included studies, } 9 \text { ex- } \\
\text { cluded studies, } 3 \text { studies listed as ongoing } \\
\text { and 1 study awaiting assessment }\end{array}$ & $\begin{array}{l}\text { (Stableforth 1984) was included, one (War- } \\
\text { necke 1999) was excluded, one (Dias 2001) } \\
\text { listed as ongoing, and the other (Martin } \\
\text { 2000) placed in Studies Awaiting Assess- } \\
\text { ment. Further information obtained from } \\
\text { trialists resulted in the exclusion of four tri- } \\
\text { als that had been previously listed as ongo- } \\
\text { ing studies. Three (Brownson 2001; Hems } \\
\text { 2000; Wallace 2000) of these had been set } \\
\text { up as a multicentre study to test the Halder } \\
\text { nail (Halder 2001), and one (Welsh 2000) } \\
\text { had been set up to compare surgical with } \\
\text { conservative treatment }\end{array}$ \\
\hline
\end{tabular}

Interventions for treating proximal humeral fractures in adults (Review)

Copyright () 2015 The Cochrane Collaboration. Published by John Wiley \& Sons, Ltd. 


\begin{tabular}{l|l}
$\begin{array}{l}\text { 3rd version (minor update) } \\
\text { Issue 3, } 2002\end{array}$ & As above
\end{tabular} \begin{tabular}{l}
$\begin{array}{l}\text { Note: this update included some changes } \\
\text { to the Discussion in response to comments } \\
\text { received from an external reviewer }\end{array}$ \\
\hline
\end{tabular}

4th version (substantive update) Issue 4, 2003
This update had 12 included studies, 11 excluded studies, and 4 studies listed as ongoing
Of four newly identified studies, one (Wirbel 1999) was included, one (de Boer 2003) excluded, and two (Frostick 2003; Shah 2003) are listed as ongoing. The other newly included trial (Hodgson 2003) was formerly listed as an ongoing trial. A trial (Martin 2000), previously in 'Studies awaiting assessment', was excluded. Limited additional findings from newly identified trial reports were included for Hoellen 1997
5 th version (minor update)

Issue 2, 2007
This update had 12 included studies, 12 excluded studies, 5 studies listed as ongoing and 4 pending assessment
Six new studies were identified, one (Fjalestad 2007) was listed as ongoing, one (Flannery 2006) was excluded and the other four were placed in 'Studies awaiting assessment', pending further information 6th version

(new citation update)

Issue 12, 2010
This update had 16 included studies, 18 excluded studies, 11 studies listed as ongoing and 4 pending assessment
Sixteen new studies were identified. Of these, one (Fialka 2008) was included, four (Gradl 2009; Mechlenburg 2009; Wan 2005; Yang 2006) were excluded, 10 (Brorson 2009; HURA; Liverpool (renamed as Sinopidis 2010 in the next update); NCT00438633; NCT00818987; NCT00999193; $\quad$ NCT01086202; NCT01113411; ProCon; ProFHER 2015) were placed in ongoing trials and one (Luo 2008) awaits assessment. New reports or information resulted in the inclusion of three more trials (Agorastides 2007: former ongoing study Frostick 2003; Fjalestad 2010: former ongoing study Fjalestad 2007; and Lefevre-Colau 2007: formerly Lefevre-colau 2006, a study awaiting assessment); and the exclusion of two studies (Bing 2002: former ongoing trial Sharma 2000; Dias 2001: former ongoing trial Dias 2001 and study awaiting assessment Der Tavitian 2006).

7th version (new citation update) Issue 12, This update had 23 included studies, 26 2012 excluded studies, 14 studies listed as ongoing and 3 pending assessment
Overall, 18 new studies were identified. Of these, seven (Ockert 2010; Olerud 2011a; Olerud 2011b; Smejkal 2011; Voigt 2011; Zhang 2011; Zhu 2011) were included, 
four (Carbone 2012; Edelson 2008; Liao 2009; Zhang 2010) were excluded, five ( ACTRN12610000730000; HOMERUS; NCT01557413; NTR3208; TPHF) were placed in ongoing trials and two (Battistella 2011; Fjalestad (RCT proposal)a) await assessment. Further information was obtained for several studies in the previous version (Handoll 2010); this included the one year follow-up report of functional outcome for Fjalestad 2010, and information resulting in the exclusion of Sinopidis 2010, a former ongoing study. Further consideration of Shah 2003, which was listed as an ongoing study, and Pullen 2007, which was awaiting classification, led to their exclusion: it is very unlikely that any further information will be obtained for these trials, including whether they started. Also excluded was Parnes 2005, another study awaiting classification in Handoll 2010; there is currently insufficient evidence to support this being a randomised trial

8th version (new citation update) Issue $\mathrm{x}$, This update had 31 included studies, 38 2015 excluded studies, 21 studies listed as ongoing and 7 awaiting classification
Overall, 32 new studies were identified. Of these, eight were included (Boons 2012; Buecking 2014; Cai 2012; Lopiz 2014; ProFHER 2015 (5 references, including 1 trial registration and trial protocol); Sebastiá-Forcada 2014; Soliman 2013 (2 references, including 1 trial registration); Torrens 2012 (1 reference and unpublished data)), 12 were excluded (Cigni 2012; Elidrissi 2013; Erdoğ an 2014; Fan 2012; IRCT2013052313435N1; Maniscalco 2014a (2 references); Martetschlager 2012; NCT00384852; NCT01532076; NCT02122315; NTR2186; Zuckerman 2012), eight were placed in ongoing trials (NCT01524965; NCT01847508; NCT01984112; $\quad$ NCT02075476; NTR4019; ROTATE (2 references, including 1 trial registration); SHeRPA; Torrens) and four await classification (Liu 2011 (2 papers); NCT02052206; Wang 2013; Zhu 2014).

Further information was obtained for several studies in the previous version ( Handoll 2012); this included the two- 

of functional outcome for Fjalestad 2010, and an additional article (Ockert 2014) , which reported on 48 additional participants for Ockert 2010. A trial registration document and published protocol (Fjalestad 2014b) were found for a newly designated ongoing trial (DELPHI) , previously Fjalestad (RCT proposal), in studies awaiting classification. Published protocols were also found for two ongoing trials (HOMERUS (Verbeek 2012); TPHF (Launonen 2012)). Additional information from updated trial registration documentation was added in for seven ongoing trials (HURA; NCT00438633; NCT00818987; $\quad$ NCT00999193; NCT01113411; NCT01557413; TPHF) . Additional information was also available for Brorson 2009 which was moved from ongoing to studies awaiting classification

\section{Appendix 3. Previous acknowledgements and contribution of authors}

\section{Acknowledgements}

We thank Panos Thomas for his contribution to the protocol, and Linda Digance, Christopher Muller and Sonia Stewart for foreign translations. We thank Bill Gillespie, Peter Herbison, Leeann Morton, David Sonnabend, John Stothard, Marc Swiontkowski and Janet Wale for their help at editorial review of the first version. We thank David Sonnabend for sharing his observations on the second version, and Bill Gillespie for his suggestions for incorporating these into the third version. We thank Lesley Gillespie, Peter Herbison, Nicola Maffulli and Janet Wale for their help at editorial review of the fourth version. We thank Joanne Elliott, Bill Gillespie, Lindsey Shaw and Janet Wale for their contributions at editorial review of the fifth version. We thank also Lesley Gillespie for her help in developing the revised search strategy and trial retrieval for the updates and advice on presentation. We thank Laurent Audige of the AO-ASIF Foundation and Anette Blümle of the German Cochrane Centre for the supply of several trial reports.

For the sixth version: We thank Nigel Hanchard, Annie Herbert and David Limb for their helpful feedback at editorial review. We thank Joanne Elliott for patiently supplying several search downloads for this version and Lindsey Elstub for her editorial support. We thank Ling-Hsiang Chuang for her help with translations from Chinese. We thank Graham Tytherleigh-Strong for his early input on this update.

For the seventh version: We thank Xavier Griffin, David Limb and Yemisi Takwoingi for their helpful feedback at editorial review. We thank Catherine Deering for patiently supplying several search downloads for this version, Joanne Elliott for advice on searching and Lindsey Elstub for her editorial support.

We thank Alastair Gibson for his major contributions to the first four versions of this review and Rajan Madhok for his major contributions to the first five versions of the review.

We thank the following for further information on their research in this area before the current update: Alison Armstrong, Cathy Booth, Stig Brorson, Peter Brownson, Piet de Boer, Joe Dias, Tore Fjalestad, Mark Flannery, Tim Hems, Stephen Hodgson, Roo Kulkarni, Inger Mechlenburg, Per Olerud, Shea Palmer, Rajiv Sharma, Matt Smith, Robin Turner, Christine Voigt, Lei Yang and Lei Zhang. Helen Handoll's work on the first version of the review was supported by the Chief Scientist Office, Department of Health, The Scottish Office, UK. Her work on the first and second updates was supported by the East Riding and Hull Health Authority, UK. 


\section{Contribution of authors}

Rajan Madhok (RM) and Panos Thomas initiated the review and wrote the protocol. Helen Handoll (HH) searched for trials and provided a set of potential studies for inclusion. Alastair Gibson (JNAG) and HH assessed trial quality, tabulated the data and were the main authors of first published version of the review. JNAG, HH and RM contributed to the final manuscript.

For the first and third (both substantive) updates, Helen Handoll initiated the update by extending the search for trials and relevant materials, contacting trialists and preparing the first drafts. JNAG, HH and RM assessed the newly identified trials and contributed to the final manuscripts. All authors contributed to rewording of the discussion in the second minor update (amendment).

For the fourth (minor) update, Helen Handoll initiated the update by extending the search for trials and relevant materials, contacting trialists and preparing the first drafts. RM performed study selection and contributed to the final manuscript.

For the fifth update (sixth version), Helen Handoll initiated the update by extending the search for trials and relevant materials, contacted trialists, revised text and tables to conform to new methodology and formatting requirements, performed risk of bias assessment for already included trials and prepared the first full draft. Both authors piloted forms, performed study selection, and assessed risk of bias and extracted data for the new included trials. Benjamin Ollivere provided feedback on interim drafts and contributed to the final manuscript.

For the sixth update (seventh version), Helen Handoll initiated the update by extending the search for trials and relevant materials, contacted trialists, performed most of data entry and prepared the first full draft. All three authors screened and selected studies, and assessed risk of bias and extracted data for the newly included trials. Katie Rollins also entered data into RevMan. Both Benjamin Ollivere and Katie Rollins provided feedback on interim drafts and contributed to the final manuscript.

\section{WHAT'S NEW}

Last assessed as up-to-date: 10 November 2014.

\begin{tabular}{|c|c|c|}
\hline Date & Event & Description \\
\hline 30 October 2015 & New citation required and conclusions have changed & $\begin{array}{l}\text { 1. Changed conclusions for the surgical versus non-sur- } \\
\text { gical intervention comparison. } \\
\text { 2. Conclusions changed to accommodate findings of the } \\
\text { new comparisons. } \\
\text { 3. Change in authorship. }\end{array}$ \\
\hline 30 October 2015 & New search has been performed & $\begin{array}{l}\text { In this update, published in Issue } 11,2015 \text {, the following } \\
\text { changes were made: } \\
\text { 1. The full search was updated to November } 2014 \text {. } \\
\text { 2. Overall, } 32 \text { new studies were identified. Of these, eight } \\
\text { were included, } 12 \text { were excluded, eight were placed in } \\
\text { ongoing trials and four await classification. Two further } \\
\text { reports were identified for two already included trials. } \\
\text { Upon identification of a trial registration document and } \\
\text { published protocol, one study previously awaiting clas- } \\
\text { sification was moved to ongoing. Published protocols } \\
\text { were found for two ongoing trials; and additional infor- } \\
\text { mation from updated trial registration documentation } \\
\text { added in for seven ongoing trials. Additional informa- } \\
\text { tion for one ongoing trial resulted in its transfer from } \\
\text { ongoing to studies awaiting classification. } \\
\text { 3. Quality of the evidence was assessed using GRADE; } \\
\text { two 'Summary of findings' tables added and the Discus- } \\
\text { sion revised and updated. }\end{array}$ \\
\hline
\end{tabular}

Interventions for treating proximal humeral fractures in adults (Review)

Copyright () 2015 The Cochrane Collaboration. Published by John Wiley \& Sons, Ltd. 


\section{H I S T O R Y}

Protocol first published: Issue 1, 1996

Review first published: Issue 1, 2001

\begin{tabular}{lll}
\hline Date & Event & Description \\
\hline 22 October 2012 & New citation required and conclusions have changed & $\begin{array}{l}\text { 1. Conclusions changed to accommodate findings of } \\
\text { the new comparisons. } \\
\text { 2. Change in authorship. }\end{array}$ \\
\hline 22 October 2012 & New search has been performed & $\begin{array}{l}\text { In this update, published in Issue 12, 2012, the fol- } \\
\text { lowing changes were made: } \\
1 . \text { The full search was updated to January 2012, with } \\
\text { other searches extended to June 2012. }\end{array}$ \\
$\begin{array}{l}\text { 2. Eighteen new studies were identified. Of these, } \\
\text { seven were included, four were excluded, five were } \\
\text { placed in ongoing studies and two await assessment. A } \\
\text { new report was available for one already included trial, } \\
\text { and contact with a trialist resulted in the exclusion of } \\
\text { one study that had been previously listed as ongoing. } \\
\text { In the absence of further information after attempts } \\
\text { at contact, consideration of one former ongoing study } \\
\text { and two studies formerly in 'Studies awaiting assess- } \\
\text { ment' led to their exclusion. } \\
\text { 3. Discussion revised and updated. } \\
\text { 4. Changes made to the conclusions. }\end{array}$
\end{tabular}

1 November 2010 New citation required and conclusions have changed

1. Conclusions changed to accommodate findings of the new comparisons.

2. Change in authorship.

1 November $2010 \quad$ New search has been performed

In this update, published in Issue 12, 2010, the following changes occurred:

1. The full search was updated to March 2010; with other searches extended to August 2010.

2. Sixteen new studies were identified, of which one was included, four were excluded, 10 were placed in ongoing trials and one awaits assessment. New reports or information resulted in the inclusion of three more trials and the exclusion of two studies that had been identified previously. 
3. Review methods and formatting were updated.

4. Background and Discussion revised and updated.

5. Changes made to the conclusions.

5 August $2008 \quad$ Amended Converted to new review format.

28 September 2007 New search has been performed

The fourth update (Issue 2, 2007) included the following:

1. Trial search extended from May 2003 to September 2006.

2. Identification of six new studies: one of which was placed in 'Ongoing studies', one was excluded and the other four are in 'Studies awaiting assessment', pending further information or publication.

3. Various adjustments were made to text, tables and presentation of the analyses to conform to revised methodology and the Cochrane Style Guide: the 'Synopsis' was amended to a 'Plain language summary'; the 'Abstract' was shortened; the 'Objectives' were reworded; methodological quality scores of individual criteria are no longer summed; all totals were removed from the Analyses (Forest plots) and the number of Analyses were reduced by presenting similar outcome measures (e.g. complications) together from the same trial.

There was no change to the conclusions of the review.

12 August $2003 \quad$ New search has been performed

The third update (Issue 4, 2003) included the following:

1. Trial search extended from November 2001 to May 2003.

2. Inclusion of two new trials, one of which had been listed as ongoing.

3. Inclusion of two new ongoing trials.

4. Exclusion of four trials previously listed as ongoing.

5. One trial, previously in pending, was excluded.

6. Addition of limited findings from newly identified trial reports of an already included trial.

7. The conclusions of the review were slightly modified to include the possibility of immediate physiotherapy, without immobilisation, for some types of undisplaced fractures

8 May $2002 \quad$ Amended

15 February 2002 New search has been performed
The second update (Issue 3, 2002) included some changes to the Discussion in response to comments received from an external reviewer

The first update (Issue 2, 2002) included the following: 
1. Trial search extended from July 2000 to November 2001.

2. Inclusion of one new trial.

3. Inclusion of one new ongoing trial.

4. Exclusion of four trials previously listed as ongoing.

5. One trial excluded and another placed in pending.

6. Addition of material from a newly available epidemiological study and commentary on a newly available systematic review.

There was no change to the conclusions of the review.

\section{CONTRIBUTIONSOFAUTHORS}

For the seventh update (eighth version), Helen Handoll initiated the update by extending the search for trials and relevant materials, contacted trialists, performed most of data entry and prepared the first full draft. Both authors screened and selected studies, assessed risk of bias and extracted data for the newly included trials, and assessed the quality of the evidence using GRADE. Stig Brorson provided feedback on interim drafts and contributed to the final manuscript.

Helen Handoll is the guarantor of the review.

The summaries of the contributions of authors for previous versions of the review are presented in Appendix 3.

\section{DECLARATIONSOF INTEREST}

Helen Handoll is a member of the trial management group of ProFHER 2015; an independent review of this trial was performed by Stig Brorson. No other interests to declare.

Stig Brorson was the lead investigator on Brorson 2009. No other interests to declare.

Both authors performed independent study selection on the trial for which the other author was an investigator.

\section{SOURCES OF SUPPORT}

\section{Internal sources}

- University of Teesside, Middlesbrough, UK. 


\section{External sources}

- National Institute for Health Research, UK.

This project was supported by the National Institute for Health Research, via funding to the ProFHER trial (http:// www.nets.nihr.ac.uk/projects/hta/0640453). The views and opinions expressed therein are those of the authors and do not necessarily reflect those of the NIHR, NHS or the Department of Health.

\section{DIFFERENCES BETWEEN PROTOCOLANDREVIEW}

\section{Update in 2015}

The first primary outcome was split into patient-reported shoulder-related scores and patient-reported quality of life scores (see Types of outcome measures).

A key change in terminology was replacing 'conservative' treatment with 'non-surgical' treatment.

We assessed the quality of the evidence using GRADE and, where sufficient evidence was available, prepared 'Summary of findings' tables.

\section{Update in 2012}

Statement added to Types of participants clarifying the inclusion of trials with a small proportion of children.

A new secondary outcome was added to Types of outcome measures (composite scores, whether validated or not, of subjectivelyand objectively-rated function and overall outcome). This was to distinguish explicitly between validated measures of patient-reported function and activities of daily living and other commonly used composite scores such as the Constant score.

Examples of the secondary outcome 'Other complications' added.

\section{Update in 2010}

Most of the changes to methods in Issue 12, 2010 reflected the uptake of new methodology and reporting as described in the Handbook (Higgins 2008b). These include risk of bias assessment and more explicit reporting of data analysis and collection. Types of outcome measures have been revised to define primary and secondary outcomes. Patient-reported measures of upper-limb function and a separate category for serious adverse events have been added.

\section{Update in 2007}

The order of the main categories of outcome measures was altered in Issue 2, 2007 to reflect the greater priority given to functional and clinical outcomes.

\section{INDEX TERMS}

\section{Medical Subject Headings (MeSH)}

Bandages; Fracture Fixation [methods]; Immobilization [methods]; Physical Therapy Modalities; Randomized Controlled Trials as Topic; Self Care; Shoulder Fractures [surgery; *therapy]; Treatment Outcome 


\section{MeSH check words}

Adult; Humans 